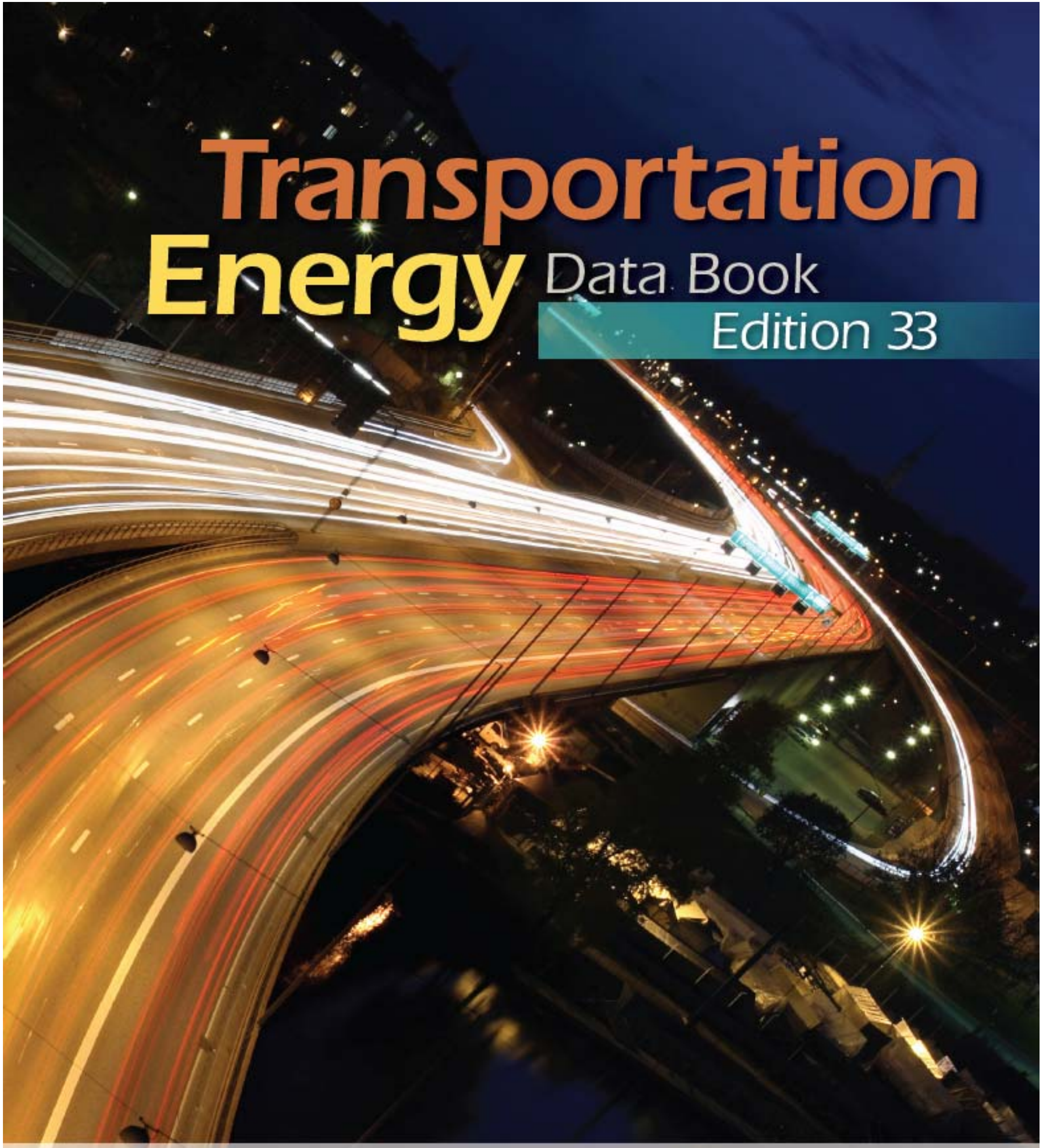

Stacy C. Davis

Susan W. Diegel

Robert G. Boundy 


\section{Transportation Energy Data Book Quick Facts}

\section{Petroleum}

- The U.S. produces 10 million barrels of petroleum per day ( $\mathrm{M} \mathrm{bpd),} \mathrm{or} 12 \%$ of the world's 85.35M bpd.

- The U.S. consumes $18.6 \mathrm{M}$ bpd, or $21 \%$ of the world's $88.3 \mathrm{M}$ bpd.

- U.S. transportation petroleum use is $67 \%$ of total U.S. petroleum use.

- U.S. transportation petroleum use is $126 \%$ of total U.S. petroleum production.

- Petroleum comprises $92 \%$ of U.S. transportation energy use.

- Cars and light trucks account for $63 \%$ of U.S. transportation petroleum use.

- Medium trucks (Class 3-6) account for $4 \%$ of U.S. transportation petroleum use.

- Heavy trucks (Class 7-8) account for $17 \%$ of U.S. transportation petroleum use.

\section{Energy}

- U.S. transportation energy use accounts for $28 \%$ of total U.S. energy use.

- In 2011, 98\% of ethanol consumed in the U.S. is consumed as ethanol in gasohol (E10).

- Cars and light trucks account for $59 \%$ of U.S. transportation energy use.

- Medium trucks account for $5 \%$ of U.S. transportation energy use.

- Heavy trucks account for $17 \%$ of U.S. transportation energy use.

\section{Light Vehicle Characteristics}

- There are 111,290 cars and 120,847 light trucks in the U.S. (232,137 total light vehicles).

- U.S. cars:

○ 7,586,000 cars were sold in 2013.

o The average age of a U.S. car is 11.4 years; the average car lifetime is 16.9 years.

- The average fuel economy for the U.S. car fleet (all cars on the road today) is $24.9 \mathrm{mpg}$.

○ Cars comprise $50 \%$ of new light vehicle sales.

- U.S. light trucks:

o 7,693,000 light trucks were sold in 2013.

○ The average age of a U.S. light truck is 11.3 years; the average light truck lifetime is 15.5 years.

O The average fuel economy for the U.S. light truck fleet (all light trucks on the road today) is $18.5 \mathrm{mpg}$.

○ Light trucks comprise $50 \%$ of new light vehicle sales.

- There were 8,716,720 fleet vehicles in 2013: 4,438,330 cars and 4,278,390 trucks.

- U.S. car registrations account for $16 \%$ of total world car registrations.

- U.S. truck and bus registrations account for $38 \%$ of total world truck and bus registrations.

- The average U.S. household vehicle travels 11,300 miles per year (2009 NHTS).

\section{Heavy Truck Characteristics}

- 10,659,000 heavy trucks were registered in the U.S. in 2012.

- In 2002 (the last time a survey was conducted), heavy trucks accounted for $80 \%$ of medium and heavy truck fuel use.

Note: Data are for calendar year 2012 or 2013 unless otherwise noted. 
ORNL-6990

(Edition 33 of ORNL-5198)

Center for Transportation Analysis

Energy and Transportation Science Division

\title{
TRANSPORTATION ENERGY DATA BOOK: EDITION 33
}

\author{
Stacy C. Davis \\ Susan W. Diegel
}

Oak Ridge National Laboratory

Robert G. Boundy

Roltek, Inc.

July 2014

Transportation Energy Data Book: Edition 33

can be found online at:

cta.ornl.gov/data

\author{
Prepared for the \\ Vehicle Technologies Office \\ Office of Energy Efficiency and Renewable Energy \\ U.S. Department of Energy
}

Prepared by the

Oak Ridge National Laboratory

Oak Ridge, Tennessee 37831-6073

Managed by

UT-BATTELLE, LLC

for the

U.S. DEPARTMENT OF ENERGY

under Contract No. DE-AC05-00OR22725 


\section{DOCUMENT AVAILABILITY}

Reports produced after January 1, 1996, are generally available free via the U.S. Department of Energy (DOE) Information Bridge:

Website: http://www.osti.gov/bridge

Reports produced before January 1, 1996, may be purchased by members of the public from the following source:

National Technical Information Service

5285 Port Royal Road

Springfield, VA 22161

Telephone: 703-605-6000 (1-800-553-6847)

TDD: 703-487-4639

Fax: 703-605-6900

E-mail: info@ntis.fedworld.gov

Website: http://www.ntis.gov/support/ordernowabout.htm

Reports are available to DOE employees, DOE contractors, Energy Technology Data Exchange (ETDE) representatives, and International Nuclear Information System (INIS) representatives from the following source:

Office of Scientific and Technical Information

P.O. Box 62

Oak Ridge, TN 37831

Telephone: $865-576-8401$

Fax: 865-576-5728

E-mail: reports@adonis.osti.gov

Website: http://www.osti.gov/contact.html

This report was prepared as an account of work sponsored by an agency of the United States Government. Neither the United States government nor any agency thereof, nor any of their employees, makes any warranty, express or implied, or assumes any legal liability or responsibility for the accuracy, completeness, or usefulness of any information, apparatus, product, or process disclosed, or represents that its use would not infringe privately owned rights. Reference herein to any specific commercial product, process, or service by trade name, trademark, manufacturer, or otherwise, does not necessarily constitute or imply its endorsement, recommendation, or favoring by the United States Government or any agency thereof. The views and opinions of authors expressed herein do not necessarily state or reflect those of the United States Government or any agency thereof. 
Users of the Transportation Energy Data Book are encouraged to comment on errors, omissions, emphases, and organization of this report to one of the persons listed below. Requests for additional complementary copies of this report, additional data, or information on an existing table should be referred to Ms. Stacy Davis, Oak Ridge National Laboratory.

\author{
Stacy C. Davis \\ Oak Ridge National Laboratory \\ National Transportation Research Center \\ 2360 Cherahala Boulevard \\ Knoxville, Tennessee 37932 \\ Telephone: (865) 946-1256 \\ FAX: (865) 946-1314 \\ E-mail: DAVISSC@ornl.gov \\ Website Location: cta.ornl.gov/data \\ Jacob W. Ward \\ Vehicle Technologies Office \\ Energy Efficiency and Renewable Energy \\ Department of Energy, EE-2G \\ Forrestal Building \\ 1000 Independence Avenue, S.W. \\ Washington, D.C. 20585 \\ Telephone: (202) 586-7606 \\ FAX: (202) 586-1600 \\ E-mail: JACOB.WARD@ee.doe.gov \\ Website Location: vehicles.energy.gov
}

Spreadsheets of the tables in the

Transportation Energy Data Book

can be found on the web at:

cta.ornl.gov/data 
Find useful data and information in other U.S. Department of Energy, Office of Energy Efficiency and Renewable Energy Data Books.

Vehicle Technologies Market Report:

cta.ornl.gov/vtmarketreport

Biomass Energy Data Book:

cta.ornl.gov/bedb

Buildings Energy Data Book:

www.btscoredatabook.net

Hydrogen Data Book:

hydrogen.pnl.gov/cocoon/morf/hydrogen/article/103 


\section{TABLE OF CONTENTS}

FOREWORD $\ldots$

ACKNOWLEDGMENTS .........................................................................................

ABSTRACT

INTRODUCTION.........................................................................................................................Xv

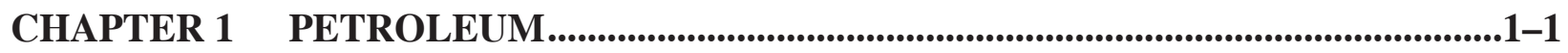

Table 1.1 Proved Reserves of Crude Oil and Natural Gas, 1980-2012............................1-2

Table 1.2 World Crude Oil Production, 1960-2013 ….......................................................1-3

Table 1.3 World Petroleum Production, 1973-2013 ……………………....................1-4

Table $1.4 \quad$ World Petroleum Consumption, 1960-2012 ………………………............1-5

Figure 1.1 World Oil Reserves, Production and Consumption, 2012 ...............................1-6

Table 1.5 World Oil Reserves, Production and Consumption, 2012 ……….......................6

Figure 1.2 World Natural Gas Reserves, Production and Consumption, 2012 .................1-7

Table 1.6 World Natural Gas Reserves, Production and Consumption, 2012 ...................1-7

Table 1.7 U.S. Petroleum Imports, 1960-2013 ….........................................................1-8

Table $1.8 \quad$ Imported Crude Oil by Country of Origin, 1973-2013 .....................................1-9

Table 1.9 Crude Oil Supplies, 1973-2013 .................................................................1-10

Figure 1.3 Oil Price and Economic Growth, 1970-2013..............................................1-11

Figure 1.4 Costs of Oil Dependence to the U.S. Economy, 1970-2012 ……...................1-12

Figure 1.5 Refinery Gross Output by World Region, 2003 and 2013 ............................1-13

Table 1.10 U.S. Refinery Input of Crude Oil and Petroleum Products, 1987-2012.........1-14

Table 1.11 U.S. Refinery Yield of Petroleum Products from a Barrel of Crude Oil,

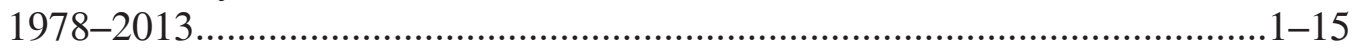

Table 1.12 United States Petroleum Production, Imports and Exports, 1950-2013 ........1-16 
Table 1.13 Petroleum Production and Transportation Petroleum Consumption in Context, 1950-2013. $1-17$

Figure 1.6 United States Petroleum Production and Consumption - All Sectors, 1973-2040. $1-18$

Figure 1.7 United States Petroleum Production, and Transportation Consumption, 1970-2040. $1-19$

Table 1.14 Consumption of Petroleum by End-Use Sector, 1973-2013 $1-20$

Table 1.15 Highway Transportation Petroleum Consumption by Mode, 1970-2012 ......1-21

Table 1.16 Nonhighway Transportation Petroleum Consumption by Mode, 1970-2012. $1-22$

Table 1.17 Transportation Petroleum Use by Mode, 2011-2012. $1-23$

CHAPTER 2 ENERGY $.2-1$

Figure 2.1 World Consumption of Primary Energy, 2011 $.2-2$

Table 2.1

U.S. Consumption of Total Energy by End-Use Sector, 1973-2013 $.2-3$

Table 2.2 Distribution of Energy Consumption by Source, 1973 and 2013. $.2-4$

Table 2.3 Alternative Fuel and Oxygenate Consumption, 2005-2011 $.2-5$

Table 2.4 Ethanol Consumption, 1995-2011

Table 2.5

Domestic Consumption of Transportation Energy by Mode and Fuel Type, 2012 . $.2-7$

Table 2.6 Transportation Energy Use by Mode, 2011-2012 _....................................2-8

Table 2.7 Highway Transportation Energy Consumption by Mode, 1970-2012 ............2-9

Table 2.8 Nonhighway Transportation Energy Consumption by Mode, 1970-2012 .....2-10

Table 2.9 Off-Highway Transportation-Related Fuel Consumption from the Nonroad Model, 2012 . $.2-11$

Table 2.10 Fuel Consumption from Lawn and Garden Equipment, 2012 $.2-12$

Table 2.11 Highway Usage of Gasoline and Diesel, 1973-2012 ..... $.2-13$

Table 2.12 Passenger Travel and Energy Use, 2012 $.2-14$ 
Table 2.13 Energy Intensities of Highway Passenger Modes, 1970-2012.....................2-15

Table 2.14 Energy Intensities of Nonhighway Passenger Modes, 1970-2012...............2-16

Figure 2.2 Energy Intensity of Light Rail Transit Systems, 2012...............................2-17

Figure 2.3 Energy Intensity of Heavy Rail Systems, 2012.........................................2-18

Figure 2.4 Energy Intensity of Commuter Rail Systems, 2012 _.................................2-18

Table 2.15 Energy Intensities of Freight Modes, 1970-2012 .....................................2-19

CHAPTER 3 ALL HIGHWAY VEHICLES AND CHARACTERISTICS ......................3-1

Table 3.1 World Production of Cars and Trucks, 2000-2012 _...................................3-2

Table 3.2 Car Registrations for Selected Countries, 1960-2012 ..................................3-3

Table 3.3 Truck and Bus Registrations for Selected Countries, 1960-2012 ..................3-4

Table $3.4 \quad$ U.S. Cars and Trucks in Use, 1970-2012 ....................................................6

Figure $3.1 \quad$ Vehicles per Thousand People: U.S. (Over Time) Compared to Other Countries (in 2002 and 2012) ....................................................................... $3-7$

Table 3.5 Vehicles per Thousand People in Other Countries, 2002 and 2012 .................3-9

Table $3.6 \quad$ Vehicles per Thousand People in the United States, 1990-2012 ..................3-10

Table 3.7 Shares of Highway Vehicle-Miles Traveled by Vehicle Type,

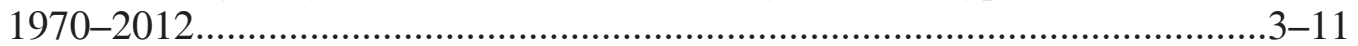

Table $3.8 \quad$ Cars in Operation by Age, 1970, 2001, and 2013......................................12

Table $3.9 \quad$ Trucks in Operation by Age, 1970, 2001, and 2013 ...................................13

Table $3.10 \quad$ U.S. Average Vehicle Age, 1995-2013 ..................................................14

Table $3.11 \quad$ New Retail Vehicle Sales, 1970-2013........................................................15

Table 3.12 Car Scrappage and Survival Rates, 1970, 1980 and 1990 Model Years ........3-16

Table 3.13 Light Truck Scrappage and Survival Rates .............................................

Table $3.14 \quad$ Heavy Truck Scrappage and Survival Rates............................................3-18 
Table 4.1 Summary Statistics for Cars, 1970-2012 ................................................. $4-2$

Table 4.2 Summary Statistics for Two-Axle, Four-Tire Trucks, 1970-2012 _................4-3

Table 4.3 Summary Statistics on Class 1, Class 2a, and Class 2b Light Trucks .............4-4

Table 4.4 Sales Estimates of Class 1, Class 2a, and Class 2b Light Trucks,

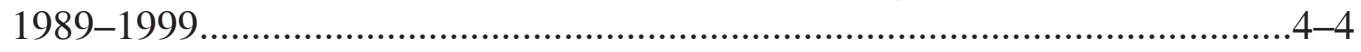

Table 4.5 New Retail Car Sales in the United States, 1970-2013 ...............................4-5

Table 4.6 New Retail Sales of Trucks 10,000 Pounds GVW and Less in the United States, 1970-2013 ....................................................................4-6

Table 4.7 Production Sales, Production Shares, and Production-Weighted Fuel Economies of New Domestic and Import Cars, Selected Model Years 1975-2013

Table 4.8 Definition of Car Sport Utility Vehicles in Model Year 2013........................4-8

Table 4.9 Production, Production Shares, and Production-Weighted Fuel Economies of New Domestic and Import Light Trucks, Model Years 1975-2013 ...........4-9

Table 4.10 Production and Production-Weighted Fuel Economies of New Domestic and Import Cars, Light Trucks and Light Vehicles, Model Years

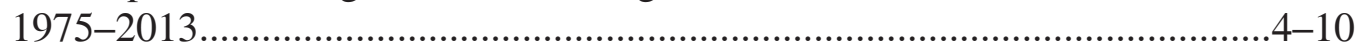

Table 4.11 Light Vehicle Production Shares, Model Years 1975-2013 .......................4-11

Figure 4.1 Light Vehicle Production Shares, Model Years 1975-2013........................4-12

Table 4.12 Production-Weighted Engine Size of New Domestic and Import Cars, Model Years 1975-2013 ............................................................................4-13

Table 4.13 Production-Weighted Engine Size of New Domestic and Import Light Trucks, Model Years 1975-2013 $.4-14$

Table 4.14 Production-Weighted Curb Weight of New Domestic and Import Cars, Model Years 1975-2013 ........................................................................4-15

Table 4.15 Average Material Consumption for a Domestic Light Vehicle, Model

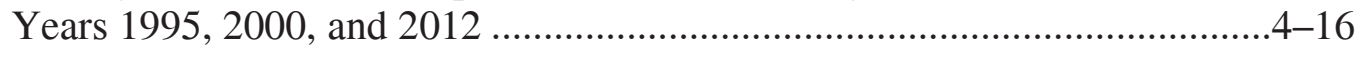

Table 4.16 New Light Vehicle Dealerships and Sales, 1970-2012 _.............................4-17

Table 4.17 Conventional Refueling Stations, 1993-2012 ….....................................4-18 
Table 4.18 Fuel Economy and Carbon Dioxide Emissions Standards, MY 2012-2025 .4-19

Table 4.19 Fuel Economy and Carbon Dioxide Targets for Model Year 2025

Table 4.20 Car Corporate Average Fuel Economy (CAFE) Standards versus

Sales-Weighted Fuel Economy Estimates, 1978-2013

Table 4.21 Light Truck Corporate Average Fuel Economy (CAFE) Standards versus Sales-Weighted Fuel Economy Estimates, 1978-2013.

Table 4.22 Corporate Average Fuel Economy (CAFE) Fines Collected, 1983-2012.....4-23

Table 4.23 The Gas Guzzler Tax on New Cars .4-24

Table 4.24 List of Model Year 2013 Cars with Gas Guzzler Taxes $.4-25$

Table 4.25 Tax Receipts from the Sale of Gas Guzzlers, 1980-2012 ...... $4-27$

Table 4.26 Fuel Economy by Speed, PSAT Model Results $4-28$

Table 4.27 Fuel Economy by Speed, 1973, 1984, 1997, and 2012 Studies $.4-29$

Figure 4.2 Fuel Economy by Speed, 1973, 1984, 1997, and 2012 Studies ....... $4-30$

Table 4.28 Driving Cycle Attributes 4-31

Figure 4.3

City Driving Cycle $4-32$

Figure 4.4

Highway Driving Cycle. $4-32$

Figure 4.5 Air Conditioning (SC03) Driving Cycle $4-33$

Figure 4.6 Cold Temperature (Cold FTP) Driving Cycle... 4-33

Figure 4.7 High Speed (US06) Driving Cycle $.4-34$

Figure 4.8 New York City Driving Cycle. 4-35

Figure 4.9 Representative Number Five Driving Cycle .4-35

Table 4.29 Comparison of U.S., European, and Japanese Driving Cycles Attributes ......4-36

Table 4.30 Example of Differing Results Using the U.S., European, and Japanese Driving Cycles. $.4-36$

Table 4.31 Summary Statistics on Demand Response Vehicles, 1994-2012. $.4-37$ 


\section{CHAPTER 5 HEAVY VEHICLES AND CHARACTERISTICS.................................5-1}

Table 5.1 Summary Statistics for Class 3-8 Single-Unit Trucks, 1970-2012 ................5-2

Table 5.2 Summary Statistics for Class 7-8 Combination Trucks, 1970-2012 _..............5-3

Table 5.3 New Retail Truck Sales by Gross Vehicle Weight, 1970-2013 .....................5-4

Table 5.4 Truck Statistics by Gross Vehicle Weight Class, 2002 ................................5-6

Table 5.5 Truck Harmonic Mean Fuel Economy by Size Class, 1992, 1997,

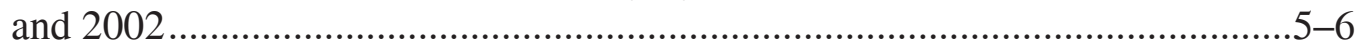

Table $5.6 \quad$ Truck Statistics by Size, 2002 ............................................................. $5-7$

Table 5.7 Percentage of Trucks by Size Ranked by Major Use, 2002 .........................5-8

Table 5.8 Percentage of Trucks by Fleet Size and Primary Fueling Facility, 2002.........5-9

Table 5.9 Share of Trucks by Major Use and Primary Fueling Facility, 2002 ..............5-10

Figure 5.1 Distribution of Trucks over 26,000 lbs. by Vehicle-Miles Traveled..............5-11

Figure 5.2 Share of Heavy Trucks with Selected Electronic Features, 2002 …..............5-12

Table 5.10 Effect of Terrain on Class 8 Truck Fuel Economy .....................................5-14

Table 5.11 Fuel Economy for Class 8 Trucks as Function of Speed and

Tractor-Trailer Tire Combination .........................................................5-15

Figure 5.3 Class 8 Truck Fuel Economy as a Function of Speed and Tractor-Trailer Tire Combination and Percentage of Total Distance Traveled as a Function of Speed ......................................................................................5-16

Figure 5.4 Class 8 Truck Percent of Total Fuel Consumed as a Function of Speed and Tractor-Trailer Tire Combination ......................................................5-17

Table 5.12 Class 8 Truck Weight by Component........................................................5-18

Table 5.13 Gross Vehicle Weight vs. Empty Vehicle Weight.......................................5-19

Figure 5.5 Distribution of Class 8 Trucks by On-Road Vehicle Weight, 2008 ...............5-20

Table 5.14 Value of Goods in the United States: Comparison of the 1997, 2002, 2007, and 2012 Commodity Flow Surveys.

Table 5.15 Tons of Freight in the United States: Comparison of the 1997, 2002, 2007, and 2012 Commodity Flow Surveys 
Table 5.16 Growth of Ton Miles in the United States: Comparison of the 1997, 2002, 2007, and 2012 Commodity Flow Surveys

Table 5.17 Average Miles per Shipment in the United States: Comparison of the 1997, 2002, 2007, and 2012 Commodity Flow Surveys

Table 5.18 Summary Statistics on Transit Buses and Trolleybuses, 1994-2012 $.5-26$

\section{CHAPTER 6 ALTERNATIVE FUEL AND ADVANCED TECHNOLOGY VEHICLES AND CHARACTERISTICS $.06-1$}

Table 6.1 Estimates of Alternative Fuel Highway Vehicles in Use, 1995-2011............6-3

Table $6.2 \quad$ Alternative Fuel Transit Vehicles, 2013 ..................................................6-4

Table 6.3 E85 Flex-Fuel Vehicles Available by Manufacturer, Model Year 2014 .........6-5

Table 6.4 Non-E85 Alternative Fuel Vehicles Available by Manufacturer, Model Year 2014 .....................................................................................6-7

Table 6.5 Hybrid and Plug-In Vehicle Sales, 1999-2013 ….......................................6-9

Table 6.6 Electric Drive Vehicles Available by Manufacturer, Model Year 2014 ........6-10

Table 6.7 Electric Drive Medium/Heavy Trucks and Buses Available by

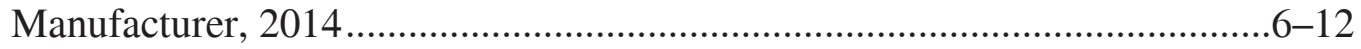

Table 6.8 Number of Alternative Refuel Sites by State and Fuel Type, 2014..............6-13

Figure $6.1 \quad$ Clean Cities Coalitions...................................................................... $6-14$

Figure 6.2 Top Ten Natural Gas Producing Countries, 2012.....................................6-15

Table 6.9 Properties of Conventional and Alternative Liquid Fuels ..........................6-16

Table 6.10 Properties of Conventional and Alternative Gaseous Fuels ........................6-17

CHAPTER 7 FLEET VEHICLES AND CHARACTERISTICS...............................................7-1

Figure 7.1 Fleet Vehicles in Service as of January 1, 2013 …......................................7-2

Table 7.1 New Light Fleet Vehicle Registrations by Vehicle Type, Model

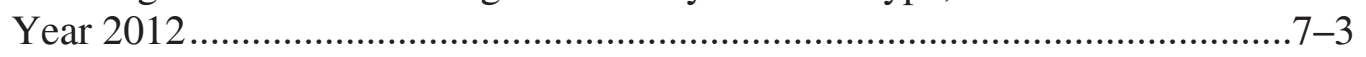

Table 7.2 Average Length of Time Commercial Fleet Vehicles are in Service, 2012 .....7-3

Table 7.3 Average Annual Vehicle-Miles of Travel for Commercial Fleet Vehicles,

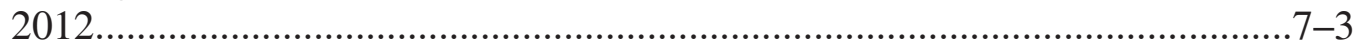


Figure 7.2 Average Miles per Domestic Federal Vehicle by Vehicle Type, 2012.............7-4

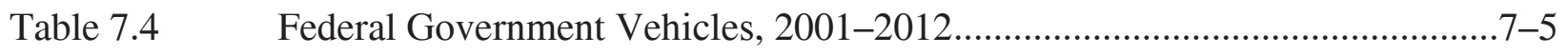

Table 7.5 Federal Fleet Vehicle Acquisitions by Fuel Type, FY 2002- 2012 _...............7-6

Table 7.6 Fuel Consumed by Federal Government Fleets, FY 2000-2012....................7-6

Table $7.7 \quad$ Federal Government Vehicles by Agency, FY 2012 ................................7-7

CHAPTER 8 HOUSEHOLD VEHICLES AND CHARACTERISTICS........................8-1

Table $8.1 \quad$ Population and Vehicle Profile, 1950-2012 …............................................ $8-2$

Table 8.2 Vehicles and Vehicle-Miles per Capita, 1950-2012 ..................................8-3

Table 8.3 Average Annual Expenditures of Households by Income, 2012 ..................8-4

Table 8.4 Annual Household Expenditures for Transportation, 1985-2012 _..................8-5

Table 8.5 Household Vehicle Ownership, 1960-2012 Census.....................................8-6

Table 8.6 Demographic Statistics from the 1969, 1977, 1983, 1990, 1995 NPTS

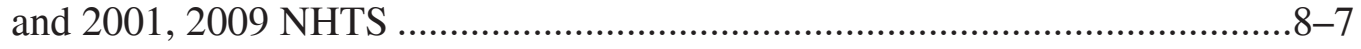

Table 8.7 Average Annual Vehicle-Miles, Vehicle Trips and Trip Length per Household 1969, 1977, 1983, 1990, 1995 NPTS and 2001, 2009 NHTS .......8-8

Table 8.8 Average Number of Vehicles and Vehicle Travel per Household,

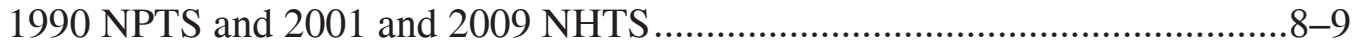

Table $8.9 \quad$ Trip Statistics by Trip Purpose, 2001 and 2009 NHTS ............................8-10

Figure 8.1 Average Vehicle Occupancy by Vehicle Type, 1995 NPTS and

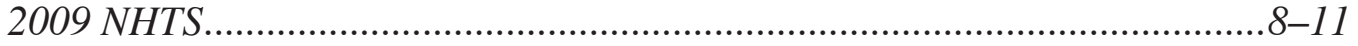

Figure 8.2 Average Vehicle Occupancy by Trip Purpose, 1977 NPTS and

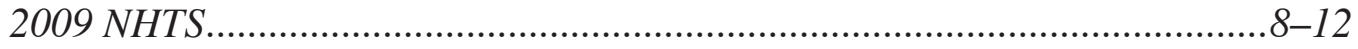

Table 8.10 Average Annual Miles per Household Vehicle by Vehicle Age ..................8-13

Table 8.11 Self-Reported vs. Odometer Average Annual Miles, 1995 NPTS and

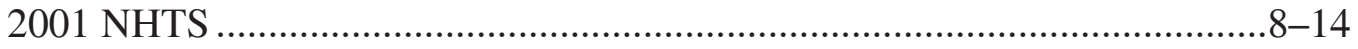

Figure $8.3 \quad$ Share of Vehicle Trips by Trip Distance, 2009 NHTS................................8-15

Figure $8.4 \quad$ Share of Vehicle Trips to Work by Trip Distance, 2009 NHTS ....................8-15 
Table 8.12 Share of Vehicles by Annual Miles of Travel and Vehicle Age, 2009 NHTS $.8-16$

Table $8.13 \quad$ Household Vehicle Trips, 2009 NHTS ...........................................................

Figure 8.5 Average Daily Miles Driven (per Driver), 2009 NHTS ..................................8-17

Table 8.14 Daily Vehicle Miles of Travel (per Vehicle) by Number of Vehicles in the Household, 2009 NHTS .............................................................................8-18

Table 8.15 Daily and Annual Vehicle Miles of Travel and Average Age for Each Vehicle in a Household, 2009 NHTS .............................................................8-18

Figure 8.6 Daily Vehicle Miles of Travel for Each Vehicle in a Household,

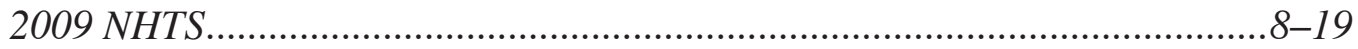

Figure 8.7 Annual Vehicle Miles of Travel for Each Vehicle in a Household, 2009 NHTS. $.8-19$

Table 8.16 Means of Transportation to Work, 1980, 1990, 2000 and 2012 $.8-20$

Table 8.17 Characteristics of U.S. Daily per Vehicle Driving vs. Dwelling Unit Type and Density $.8-21$

Table 8.18 Housing Unit Characteristics, 2010 $.8-21$

Table 8.19 Workers by Commute Time, 1990, 2000, and 2011 ………….....................8-22

Table 8.20 Bicycle Sales, 1981-2011..........................................................................

Figure 8.8 Walk and Bike Trips by Trip Purpose, 2009 NHTS......................................8-24

Table 8.21 Long-Distance Trip Characteristics, 2001 NHTS ………………………..........8-26

CHAPTER 9 NONHIGHWAY MODES .........................................................................................9-1

Table 9.1 Nonhighway Energy Use Shares, 1970-2012 ……………………................9-2

Table 9.2 Summary Statistics for U.S. Domestic and International Certificated Route Air Carriers (Combined Totals), 1970-2013 ..........................................9-3

Table 9.3 Summary Statistics for General Aviation, 1970-2012 ……………................9-4

Table 9.4 Tonnage Statistics for Domestic and International Waterborne Commerce, 1970-2012 …………………………………………………....9-5

Table 9.5 Summary Statistics for Domestic Waterborne Commerce, 1970-2012 _..........9-6 
Table 9.6 Recreational Boat Energy Use, 1970-2012 ………………………................9-7

Table 9.7 Class I Railroad Freight Systems in the United States Ranked by Revenue Ton-Miles, 2012 ...................................................................... $9-8$

Table 9.8 Summary Statistics for Class I Freight Railroads, 1970-2012 .........................9-9

Table 9.9 Intermodal Rail Traffic, 1965-2012 …….............................................

Table 9.10 Summary Statistics for the National Railroad Passenger Corporation (Amtrak), 1971-2012 ..................................................................................9-11

Table 9.11 Summary Statistics for Commuter Rail Operations, 1984-2012.....................9-12

Table 9.12 Summary Statistics for Rail Transit Operations, 1970-2012 ..........................9-13

CHAPTER 10 TRANSPORTATION AND THE ECONOMY ...........................................10-1

Figure 10.1 Transportation Services Index, January 1990-January 2014 .........................10-2

Table 10.1 Gasoline Prices for Selected Countries, 1990-2013 ..........................................10-3

Table 10.2 Diesel Fuel Prices for Selected Countries, 1998-2013 _....................................10-4

Figure 10.2 Gasoline Prices for Selected Countries, 1990 and 2013 ..............................10-5

Figure 10.3 Diesel Prices for Selected Countries, 1990 and 2013 .................................10-6

Table 10.3 Prices for a Barrel of Crude Oil and a Gallon of Gasoline, 1978-2013 ..........10-7

Table 10.4 Retail Prices for Motor Fuel, 1978-2013 ………………………................10-8

Table 10.5 Refiner Sales Prices for Propane and No. 2 Diesel, 1978-2013 ......................10-9

Table 10.6 Refiner Sales Prices for Aviation Gasoline and Jet Fuel, 1978-2013 ...........10-10

Table 10.7 Federal Excise Taxes on Motor Fuels, 2011 _..............................................10-11

Table 10.8 Federal and State Alternative Fuel Incentives, 2014 …...............................10-12

Table 10.9 Federal and State Advanced Technology Incentives, 2014 ……..................10-13

Table 10.10 Average Price of a New Car, 1913-2012 …...............................................10-14

Table 10.11 Average Price of a New Car (Domestic and Import), 1970-2012................10-15

Table 10.12 Car Operating Cost per Mile, 1985-2013 ……….......................................10-16 
Table 10.13 Fixed Car Operating Costs per Year, 1975-2013 ………………...............10-17

Table 10.14 Personal Consumption Expenditures, 1970-2013 ………………...............10-18

Table 10.15 Consumer Price Indices, 1970-2013 ………………...........................10-18

Table 10.16 Transportation-Related Employment, 2000 and 2013 ….............................10-19

Table $10.17 \quad$ U.S. Employment for Motor Vehicles and Motor Vehicle Parts

Manufacturing, 1990-2013 ........................................................................10-20

CHAPTER 11 GREENHOUSE GAS EMISSIONS...................................................................11-1

Table 11.1 World Carbon Dioxide Emissions, 1990 and 2013 ......................................11-2

Table 11.2 Numerical Estimates of Global Warming Potentials Compared with Carbon Dioxide.........................................................................................11-3

Table $11.3 \quad$ U.S. Emissions of Greenhouse Gases, based on Global Warming

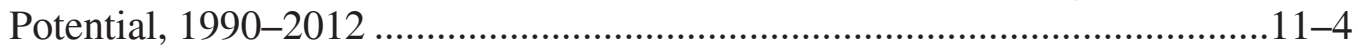

Table 11.4 Total U.S. Greenhouse Gas Emissions by End-Use Sector, 2012 …...............11-5

Table 11.5 U.S. Carbon Emissions from Fossil Fuel Consumption by End-Use Sector, 1990-2012 ………………………………………….....11-6

Table 11.6 U.S. Carbon Emissions from Fossil Fuel Combustion in the Transportation End-Use Sector .....................................................................11-7

Table 11.7 Transportation Greenhouse Gas Emissions by Mode, 1990 and 2012 ............11-8

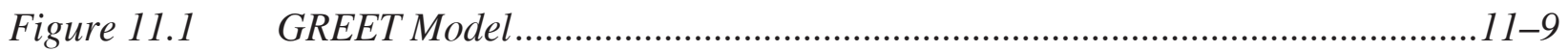

Figure 11.2 GREET Model Feedstocks and Fuels .................................................11-10

Figure 11.3 Well-to-Wheel Emissions for Various Fuels and Vehicle Technologies.......11-11

Table 11.8 Production-Weighted Annual Carbon Footprint of New Domestic and Import Cars, Model Years 1975-2013 ............................................................11-13

Table $11.9 \quad$ Production-Weighted Annual Carbon Footprint of New Domestic and Import Light Trucks, Model Years 1975-2013 ...............................................11-14

Table 11.10 Average Annual Carbon Footprint by Vehicle Classification, 1975 and 2013 $11-15$

Table 11.11 Direct Carbon Dioxide Emissions from a Gallon of Fuel...............................11-16 
Table 12.1 Total National Emissions of the Criteria Air Pollutants by Sector, 2013 .......12-2

Table 12.2 Total National Emissions of Carbon Monoxide, 1970-2013 _......................12-3

Table 12.3 Emissions of Carbon Monoxide from Highway Vehicles, 1970-2005 ..........12-4

Table 12.4 Total National Emissions of Nitrogen Oxides, 1970-2013 ........................12-5

Table 12.5 Emissions of Nitrogen Oxides from Highway Vehicles, 1970-2005............12-6

Table 12.6 Total National Emissions of Volatile Organic Compounds, 1970-2013 .......12-7

Table 12.7 Emissions of Volatile Organic Compounds from Highway Vehicles,

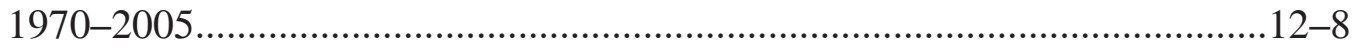

Table 12.8 Total National Emissions of Particulate Matter (PM-10), 1970-2013 _.........12-9

Table 12.9 Emissions of Particulate Matter (PM-10) from Highway Vehicles,

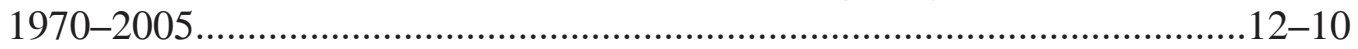

Table 12.10 Total National Emissions of Particulate Matter (PM-2.5), 1990-2013 _.......12-11

Table 12.11 Emissions of Particulate Matter (PM-2.5) from Highway Vehicles,

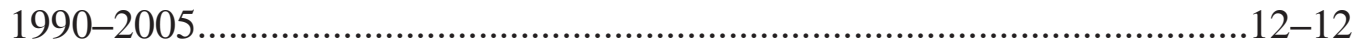

Table 12.12 Tier 3 Non-Methane Organic Gases and Nitrogen Oxide Standards............12-14

Table 12.13 Tier 3 Particulate Matter Emission Standards for Light Gasoline Vehicles, MY 2017 and Beyond .................................................................12-15

Table 12.14 Tier 3 Evaporative Emission Standards ..............................................12-15

Table 12.15 Light-Duty Vehicle, Light-Duty Truck, and Medium-Duty Passenger Vehicle - Tier 2 Exhaust Emission Standards .........................................12-16

Table 12.16 Light-Duty Vehicle, Light-Duty Truck, and Medium-Duty Passenger Vehicle - Tier 2 Evaporative Emission Standards ..................................12-17

Table 12.17 Heavy-Duty Highway Compression-Ignition Engines and Urban Buses Exhaust Emission Standards ............................................................12-18

Table 12.18 Heavy-Duty Highway Spark-Ignition Engines - Exhaust Emission Standards $12-20$

Table 12.19 Heavy-Duty Highway Compression-Ignition and Spark-Ignition Engines Evaporative Emission Standards. 
Table 12.20 California Car, Light Truck and Medium Truck Emission Certification Standards

Table 12.21 Aircraft - Exhaust Emission Standards

Table 12.22 Nonroad Compression-Ignition Engines - Exhaust Emission Standards .....12-25

Table 12.23 Nonroad Large Spark-Ignition Engines - Exhaust and Evaporative Emission Standards.

Table 12.24 Locomotives - Exhaust Emission Standards $12-28$

Table 12.25 Marine Compression-Ignition (CI) Engines - Exhaust Emission Standards

Table 12.26 Marine Spark-Ignition Engines and Vessels - Exhaust Emission

Standards $12-34$

Table 12.27 Nonroad Recreational Engines and Vehicles - Exhaust Emission Standards $12-36$

Table 12.28 Gasoline Sulfur Standards $12-38$

Table 12.29 Highway, Nonroad, Locomotive, and Marine (NRLM) Diesel Fuel Sulfur Standards....

APPENDIX A. SOURCES \& METHODOLOGIES .....................................................A-1

APPENDIX B. CONVERSIONS .......................................................................................

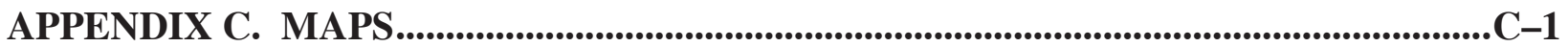

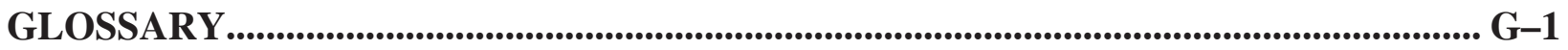

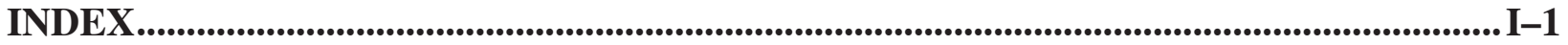


xviii

TRANSPORTATION ENERGY DATA BOOK: EDITION 33-2014 


\section{FOREWORD}

Welcome to this 33rd edition of the Transportation Energy Data Book. Twenty-four editions of this Data Book have been produced by Stacy Davis; DOE is grateful for the dedication, consistency, and skill she has brought to this effort.

I would like to bring to your attention some of the data that are new in this edition:

- Tables 3.8 and 3.9 have 2013 data on the cars and trucks in operation by age.

- Tables 4.7 - 4.14 have been changed significantly due to changes in the source data. The Environmental Protection Agency (EPA) changed the vehicle classifications, combining wagons with the rest of the cars and dropping the small, medium, large categories of cars, pickups, sportutilities, and vans. The fuel economy displayed in those tables has been adjusted by the EPA to provide the best estimate of real-world performance.

- Figure 5.1, which is the distribution of trucks over 26,000 lbs. by vehicle-miles traveled, now includes a graph on all trucks in additional to the $<2$-year old trucks.

- Tables 5.14-5.17 show data from the newly released 2012 Commodity Flow Survey (CFS) in comparison with previous CFS data.

- Tables 6.3 and 6.4 were split from one table in last year's edition because there are so many alternative fuel vehicles in model year 2014 .

- Table 6.7 names the model year 2014 electric-drive medium/heavy trucks and buses.

- Figure 6.2 shows the top ten natural gas-producing countries, along with the natural gas reserves of those countries.

- $\quad$ Figure 11.2 displays data on greenhouse gas emissions from the latest GREET model.

- Tables 12.13 and 12.14 present information on the Tier 3 emission standards which were finalized in April 2014.

I hope you find value in this data book. Stacy and I welcome suggestions on how to improve it.

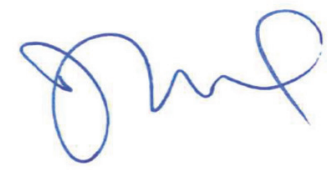

Jacob W. Ward

Analysis Manager, Vehicle Technologies Office

U.S. Department of Energy 


\section{ACKNOWLEDGMENTS}

The authors would like to express their gratitude to the many individuals who assisted in the preparation of this document. First, we would like to thank Jacob Ward and the Vehicle Technologies Office staff for their continued support of the Transportation Energy Data Book project. We would also like to thank Mark Robbins for the cover. This book would not have been possible without the dedication of Debbie Bain, who has masterfully prepared the manuscript since 1998.

This book would not be possible without the leadership, guidance, and vision of Phil Patterson, who began this book in the 1970's. We hope to continue this report into the future with the same level of excellence. The authors and the transportation research community will be forever grateful for his efforts. 


\begin{abstract}
The Transportation Energy Data Book: Edition 33 is a statistical compendium prepared and published by Oak Ridge National Laboratory (ORNL) under contract with the U.S. Department of Energy, Office of Energy Efficiency and Renewable Energy, Vehicle Technologies Office. Designed for use as a desk-top reference, the Data Book represents an assembly and display of statistics and information that characterize transportation activity, and presents data on other factors that influence transportation energy use. The purpose of this document is to present relevant statistical data in the form of tables and graphs. The latest edition of the Data Book is available to a larger audience via the Internet (cta.ornl.gov/data).

This edition of the Data Book has 12 chapters which focus on various aspects of the transportation industry. Chapter 1 focuses on petroleum; Chapter 2 - energy; Chapter 3 highway vehicles; Chapter 4 - light vehicles; Chapter 5 - heavy vehicles; Chapter 6 - alternative fuel vehicles; Chapter 7 - fleet vehicles; Chapter 8 - household vehicles; Chapter 9 nonhighway modes; Chapter 10 - transportation and the economy; Chapter 11 - greenhouse gas emissions; and Chapter 12 - criteria pollutant emissions. The sources used represent the latest available data. There are also three appendices which include detailed source information for some tables, measures of conversion, and the definition of Census divisions and regions. A glossary of terms and a title index are also included for the reader's convenience.
\end{abstract}




\section{INTRODUCTION}

In January 1976, the Transportation Energy Conservation (TEC) Division of the Energy Research and Development Administration contracted with Oak Ridge National Laboratory (ORNL) to prepare a Transportation Energy Conservation Data Book to be used by TEC staff in their evaluation of current and proposed conservation strategies. The major purposes of the Data Book were to draw together, under one cover, transportation data from diverse sources, to resolve data conflicts and inconsistencies, and to produce a comprehensive document. The first edition of the TEC Data Book was published in October 1976. With the passage of the Department of Energy (DOE) Organization Act, the work being conducted by the former Transportation Energy Conservation Division fell under the purview of the DOE's Office of Transportation Programs. This work continues today in the Vehicle Technologies Office.

Policymakers and analysts need to be well-informed about activity in the transportation sector. The organization and scope of the data book reflect the need for different kinds of information. For this reason, Edition 33 updates much of the same type of data that is found in previous editions.

In any attempt to compile a comprehensive set of statistics on transportation activity, numerous instances of inadequacies and inaccuracies in the basic data are encountered. Where such problems occur, estimates are developed by ORNL. To minimize the misuse of these statistics, an appendix (Appendix A) is included to document the estimation procedures. The attempt is to provide sufficient information for the conscientious user to evaluate the estimates and to form their own opinions as to their utility. Clearly, the accuracy of the estimates cannot exceed the accuracy of the primary data, an accuracy which in most instances is unknown. In cases where data accuracy is known or substantial errors are strongly suspected in the data, the reader is alerted. In all cases it should be recognized that the estimates are not precise.

The majority of the statistics contained in the data book are taken directly from published sources, although these data may be reformatted for presentation by ORNL. Consequently, neither ORNL nor DOE endorses the validity of these data. 
xxvi

TRANSPORTATION ENERGY DATA BOOK: EDITION 33-2014 


\section{Chapter 1 \\ Petroleum}

Summary Statistics from Tables/Figures in this Chapter

\begin{tabular}{|c|c|c|c|}
\hline \multicolumn{4}{|l|}{ Source } \\
\hline Table 1.3 & World Petroleum Production, 2013 (million barrels per day) ${ }^{\mathrm{a}}$ & & 88.35 \\
\hline \multirow{5}{*}{ Table 1.4} & U.S. Production (million barrels per day) & & 10.01 \\
\hline & U.S. Share & & $11.7 \%$ \\
\hline & World Petroleum Consumption, 2012 (million barrels per day) & & 88.31 \\
\hline & U.S. Consumption (million barrels per day) & & 18.55 \\
\hline & U.S. Share & & $21.0 \%$ \\
\hline \multirow[t]{6}{*}{ Figure 1.5} & Average Refinery Yield, 2013 & $\begin{array}{l}\text { OECD } \\
\text { Europe }\end{array}$ & $\begin{array}{c}\text { North } \\
\text { America }\end{array}$ \\
\hline & Gasoline & $19.0 \%$ & $42.2 \%$ \\
\hline & Diesel oil & $41.3 \%$ & $27.4 \%$ \\
\hline & Residual fuel & $11.2 \%$ & $4.7 \%$ \\
\hline & Kerosene & $7.4 \%$ & $7.3 \%$ \\
\hline & Other & $12.7 \%$ & $14.8 \%$ \\
\hline Table 1.13 & $\begin{array}{l}\text { U.S. transportation petroleum use as a percent of U.S. petroleum } \\
\text { production, } 2013\end{array}$ & & $125.9 \%$ \\
\hline Table 1.13 & Net imports as a percentage of U.S. petroleum consumption, 2013 & & $32.8 \%$ \\
\hline Table 1.14 & Transportation share of U.S. petroleum consumption, 2013 & & $68.9 \%$ \\
\hline Table 1.17 & Highway share of transportation petroleum consumption, 2012 & & $85.9 \%$ \\
\hline Table 1.17 & Light vehicle share of transportation petroleum consumption, 2012 & & $63.6 \%$ \\
\hline
\end{tabular}

In this document, petroleum is defined as crude oil (including lease condensate) and natural gas plant liquids.

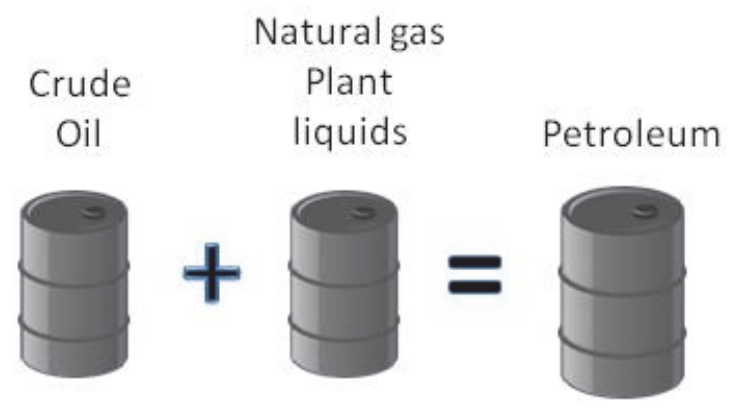

${ }^{a}$ Because other liquids and processing gain are not included, the world production is smaller than world petroleum consumption. 
As new technologies appear and new areas are explored, the amount of proved reserves of crude oil and natural gas has grown. Although the reserves of natural gas in the United States were 66\% higher in 2012 than it was in 1980, the U.S. share of World natural gas reserves is lower. The U.S. share of crude oil reserves has been under $2 \%$ since 2003 .

Table 1.1

Proved Reserves of Crude Oil and Natural Gas, 1980-2012

\begin{tabular}{|c|c|c|c|c|c|c|}
\hline \multirow[b]{2}{*}{ Year } & \multicolumn{2}{|c|}{$\begin{array}{l}\text { Crude Oil Reserves } \\
\text { (billion barrels) }\end{array}$} & \multirow{2}{*}{$\begin{array}{c}\text { U.S. Share of } \\
\text { Crude Oil } \\
\text { Reserves }\end{array}$} & \multicolumn{2}{|c|}{$\begin{array}{l}\text { Natural Gas Reserves } \\
\text { (trillion cubic feet) }\end{array}$} & \multirow{2}{*}{$\begin{array}{l}\text { U.S. Share of } \\
\text { Natural Gas } \\
\text { Reserves }\end{array}$} \\
\hline & World & United States & & World & United States & \\
\hline 1980 & 641.9 & 29.8 & $4.6 \%$ & $2,592.0$ & 201.0 & $7.8 \%$ \\
\hline 1981 & 649.1 & 29.8 & $4.6 \%$ & $2,653.9$ & 199.0 & $7.5 \%$ \\
\hline 1982 & 667.4 & 29.4 & $4.4 \%$ & $2,927.0$ & 201.7 & $6.9 \%$ \\
\hline 1983 & 665.6 & 27.9 & $4.2 \%$ & $3,038.4$ & 201.5 & $6.6 \%$ \\
\hline 1984 & 666.5 & 27.7 & $4.2 \%$ & $3,208.5$ & 200.2 & $6.2 \%$ \\
\hline 1985 & 697.8 & 28.4 & $4.1 \%$ & $3,407.2$ & 197.5 & $5.8 \%$ \\
\hline 1986 & 698.6 & 28.4 & $4.1 \%$ & $3,490.1$ & 193.4 & $5.5 \%$ \\
\hline 1987 & 697.9 & 26.9 & $3.9 \%$ & $3,648.7$ & 191.6 & $5.3 \%$ \\
\hline 1988 & 887.5 & 27.3 & $3.1 \%$ & $3,796.6$ & 187.2 & $4.9 \%$ \\
\hline 1989 & 906.0 & 26.8 & $3.0 \%$ & $3,933.2$ & 168.0 & $4.3 \%$ \\
\hline 1990 & $1,000.5$ & 26.5 & $2.6 \%$ & $3,987.5$ & 167.1 & $4.2 \%$ \\
\hline 1991 & 998.7 & 26.3 & $2.6 \%$ & $4,215.6$ & 169.3 & $4.0 \%$ \\
\hline 1992 & 989.4 & 24.7 & $2.5 \%$ & $4,376.7$ & 167.1 & $3.8 \%$ \\
\hline 1993 & 996.1 & 23.7 & $2.4 \%$ & $4,884.4$ & 165.0 & $3.4 \%$ \\
\hline 1994 & 998.3 & 23.0 & $2.3 \%$ & $5,013.8$ & 162.4 & $3.2 \%$ \\
\hline 1995 & 999.3 & 22.5 & $2.2 \%$ & $4,981.9$ & 163.8 & $3.3 \%$ \\
\hline 1996 & $1,007.4$ & 22.4 & $2.2 \%$ & $4,935.3$ & 165.1 & $3.3 \%$ \\
\hline 1997 & $1,018.5$ & 22.0 & $2.2 \%$ & $4,947.0$ & 166.5 & $3.4 \%$ \\
\hline 1998 & $1,020.1$ & 22.5 & $2.2 \%$ & $5,087.6$ & 167.2 & $3.3 \%$ \\
\hline 1999 & $1,032.8$ & 21.0 & $2.0 \%$ & $5,141.9$ & 164.0 & $3.2 \%$ \\
\hline 2000 & $1,016.8$ & 21.8 & $2.1 \%$ & $5,150.0$ & 167.4 & $3.3 \%$ \\
\hline 2001 & $1,028.1$ & 22.0 & $2.1 \%$ & $5,288.9$ & 177.4 & $3.4 \%$ \\
\hline 2002 & $1,032.0$ & 22.4 & $2.2 \%$ & $5,457.6$ & 183.5 & $3.4 \%$ \\
\hline 2003 & $1,213.1$ & 22.7 & $1.9 \%$ & $5,505.4$ & 186.9 & $3.4 \%$ \\
\hline 2004 & $1,265.0$ & 21.9 & $1.7 \%$ & 6,079.1 & 189.0 & $3.1 \%$ \\
\hline 2005 & $1,277.2$ & 21.4 & $1.7 \%$ & $6,046.6$ & 192.5 & $3.2 \%$ \\
\hline 2006 & $1,292.9$ & 21.8 & $1.7 \%$ & $6,124.6$ & 204.4 & $3.3 \%$ \\
\hline 2007 & $1,316.7$ & 21.0 & $1.6 \%$ & $6,190.9$ & 211.1 & $3.4 \%$ \\
\hline 2008 & $1,332.0$ & 21.3 & $1.6 \%$ & $6,213.7$ & 237.7 & $3.8 \%$ \\
\hline 2009 & $1,340.0$ & 19.1 & $1.4 \%$ & $6,262.4$ & 244.7 & $3.9 \%$ \\
\hline 2010 & $1,355.7$ & 20.7 & $1.5 \%$ & $6,638.2$ & 272.5 & $4.1 \%$ \\
\hline 2011 & $1,473.8$ & 23.3 & $1.6 \%$ & $6,708.2$ & 304.6 & $4.5 \%$ \\
\hline 2012 & $1,526.0$ & 26.5 & $1.7 \%$ & $6,844.6$ & 334.1 & $4.9 \%$ \\
\hline \multicolumn{7}{|c|}{ Average Annual Percentage Change } \\
\hline $1970-2012$ & $2.7 \%$ & $-0.4 \%$ & & $3.1 \%$ & $1.6 \%$ & \\
\hline 2002-2012 & $4.0 \%$ & $1.7 \%$ & & $2.3 \%$ & $6.2 \%$ & \\
\hline
\end{tabular}

Source:

U.S. Department of Energy, Energy Information Administration, International Energy Statistics, April 2014. 
In 2013, the Organization of Petroleum Exporting Countries (OPEC) accounted for $42.5 \%$ of world oil production. The U.S. production of crude oil increased to 7.46 million barrels per day, which is the highest production since 1989. The U.S. production is $9.8 \%$ of World production.

Table 1.2

World Crude Oil Production, 1960-2013 ${ }^{\mathrm{a}}$ (million barrels per day)

\begin{tabular}{|c|c|c|c|c|c|c|}
\hline Year & $\begin{array}{l}\text { United } \\
\text { States }\end{array}$ & U.S. share & Total OPEC & OPEC share & $\begin{array}{c}\text { Total non- } \\
\text { OPEC }\end{array}$ & World \\
\hline 1960 & 7.04 & $33.5 \%$ & 8.70 & $41.4 \%$ & 12.29 & 20.99 \\
\hline 1965 & 7.80 & $25.7 \%$ & 14.35 & $47.3 \%$ & 15.98 & 30.33 \\
\hline 1970 & 9.64 & $21.0 \%$ & 23.30 & $50.8 \%$ & 22.59 & 45.89 \\
\hline 1975 & 8.38 & $15.9 \%$ & 26.79 & $50.3 \%$ & 27.04 & 52.83 \\
\hline 1980 & 8.60 & $14.4 \%$ & 26.38 & $44.3 \%$ & 34.18 & 59.56 \\
\hline 1985 & 8.97 & $16.6 \%$ & 15.37 & $28.5 \%$ & 38.60 & 53.97 \\
\hline 1986 & 8.68 & $15.4 \%$ & 18.28 & $32.5 \%$ & 37.95 & 56.23 \\
\hline 1987 & 8.35 & $14.7 \%$ & 18.52 & $32.7 \%$ & 38.15 & 56.67 \\
\hline 1988 & 8.14 & $13.9 \%$ & 20.32 & $34.6 \%$ & 38.42 & 58.74 \\
\hline 1989 & 7.61 & $12.7 \%$ & 22.07 & $36.9 \%$ & 37.79 & 59.86 \\
\hline 1990 & 7.36 & $12.2 \%$ & 22.49 & $37.2 \%$ & 38.00 & 60.50 \\
\hline 1991 & 7.42 & $12.3 \%$ & 23.27 & $38.7 \%$ & 36.86 & 60.13 \\
\hline 1992 & 7.17 & $11.9 \%$ & 24.40 & $40.6 \%$ & 35.70 & 60.10 \\
\hline 1993 & 6.85 & $11.4 \%$ & 25.12 & $41.7 \%$ & 35.05 & 60.17 \\
\hline 1994 & 6.66 & $10.9 \%$ & 25.51 & $41.7 \%$ & 35.66 & 61.17 \\
\hline 1995 & 6.56 & $10.5 \%$ & 25.54 & $40.9 \%$ & 36.89 & 62.43 \\
\hline 1996 & 6.47 & $10.1 \%$ & 26.02 & $40.8 \%$ & 37.80 & 63.82 \\
\hline 1997 & 6.45 & $9.8 \%$ & 27.29 & $41.5 \%$ & 38.51 & 65.81 \\
\hline 1998 & 6.25 & $9.3 \%$ & 28.37 & $42.3 \%$ & 38.67 & 67.03 \\
\hline 1999 & 5.88 & $8.9 \%$ & 27.22 & $41.3 \%$ & 38.74 & 65.97 \\
\hline 2000 & 5.82 & $8.5 \%$ & 28.94 & $42.2 \%$ & 39.58 & 68.53 \\
\hline 2001 & 5.80 & $8.5 \%$ & 28.13 & $41.3 \%$ & 40.00 & 68.13 \\
\hline 2002 & 5.74 & $8.5 \%$ & 26.47 & $39.3 \%$ & 40.83 & 67.29 \\
\hline 2003 & 5.65 & $8.1 \%$ & 27.98 & $40.3 \%$ & 41.48 & 69.46 \\
\hline 2004 & 5.44 & $7.5 \%$ & 30.43 & $41.9 \%$ & 42.16 & 72.59 \\
\hline 2005 & 5.18 & $7.0 \%$ & 31.90 & $43.2 \%$ & 41.87 & 73.77 \\
\hline 2006 & 5.09 & $6.9 \%$ & 31.61 & $43.1 \%$ & 41.79 & 73.40 \\
\hline 2007 & 5.08 & $6.9 \%$ & 31.35 & $42.9 \%$ & 41.73 & 73.08 \\
\hline 2008 & 5.00 & $6.8 \%$ & 32.67 & $44.2 \%$ & 41.26 & 73.94 \\
\hline 2009 & 5.35 & $7.4 \%$ & 30.83 & $42.5 \%$ & 41.78 & 72.61 \\
\hline 2010 & 5.47 & $7.4 \%$ & 31.80 & $42.7 \%$ & 42.59 & 74.39 \\
\hline 2011 & 5.65 & $7.6 \%$ & 32.02 & $43.0 \%$ & 42.49 & 74.50 \\
\hline 2012 & 6.49 & $8.5 \%$ & 33.17 & $43.7 \%$ & 42.71 & 75.89 \\
\hline 2013 & 7.46 & $9.8 \%$ & 32.34 & $42.5 \%$ & 43.71 & 76.05 \\
\hline \multicolumn{7}{|c|}{ Average annual percentage change } \\
\hline 1960-2013 & $0.1 \%$ & & $2.5 \%$ & & $2.4 \%$ & $2.5 \%$ \\
\hline 1970-2013 & $-0.6 \%$ & & $0.8 \%$ & & $1.5 \%$ & $1.2 \%$ \\
\hline 2003-2013 & $2.8 \%$ & & $1.5 \%$ & & $0.5 \%$ & $0.9 \%$ \\
\hline
\end{tabular}

\section{Source:}

U.S. Department of Energy, Energy Information Administration, International Energy Statistics Website, April 2014. (Additional resources: www.eia.doe.gov)

\footnotetext{
${ }^{a}$ Includes lease condensate. Excludes natural gas plant liquids.

${ }^{\mathrm{b}}$ See Glossary for membership.
} 
This table shows petroleum production, which includes both crude oil and natural gas plant liquids. Because other liquids and processing gain are not included, the world total is smaller than world petroleum consumption (Table 1.4). The United States was responsible for 11.7\% of the world's petroleum production in 2013 and $9.8 \%$ of the world's crude oil production (Table 1.2).

Table 1.3

World Petroleum Production, 1973-2013 (million barrels per day)

\begin{tabular}{|c|c|c|c|c|c|c|c|}
\hline Year & $\begin{array}{l}\text { United } \\
\text { States }\end{array}$ & $\begin{array}{c}\text { U.S. } \\
\text { share }\end{array}$ & $\begin{array}{c}\text { Total } \\
\text { OPEC }^{b}\end{array}$ & $\begin{array}{c}\text { OPEC } \\
\text { share }\end{array}$ & $\begin{array}{c}\text { Total } \\
\text { non- } \\
\text { OPEC }\end{array}$ & $\begin{array}{l}\text { Non- } \\
\text { OPEC } \\
\text { share }\end{array}$ & World \\
\hline 1973 & 10.95 & $18.7 \%$ & 29.99 & $51.3 \%$ & 28.48 & $48.7 \%$ & 58.47 \\
\hline 1975 & 10.01 & $18.0 \%$ & 26.16 & $47.0 \%$ & 28.48 & $51.2 \%$ & 55.62 \\
\hline 1980 & 10.17 & $16.1 \%$ & 26.05 & $41.3 \%$ & 35.77 & $56.8 \%$ & 63.00 \\
\hline 1981 & 10.18 & $17.1 \%$ & 21.95 & $36.8 \%$ & 37.73 & $63.2 \%$ & 59.68 \\
\hline 1982 & 10.20 & $17.9 \%$ & 18.54 & $32.5 \%$ & 38.55 & $67.5 \%$ & 57.09 \\
\hline 1983 & 10.25 & $18.0 \%$ & 17.25 & $30.3 \%$ & 39.64 & $69.7 \%$ & 56.89 \\
\hline 1984 & 10.51 & $18.0 \%$ & 17.29 & $29.6 \%$ & 41.09 & $70.4 \%$ & 58.37 \\
\hline 1985 & 10.58 & $18.3 \%$ & 16.20 & $28.0 \%$ & 40.90 & $70.6 \%$ & 57.90 \\
\hline 1986 & 10.23 & $16.9 \%$ & 18.53 & $30.6 \%$ & 41.17 & $68.1 \%$ & 60.49 \\
\hline 1987 & 9.94 & $16.3 \%$ & 18.70 & $30.7 \%$ & 41.47 & $68.1 \%$ & 60.93 \\
\hline 1988 & 9.77 & $15.5 \%$ & 20.80 & $32.9 \%$ & 41.86 & $66.2 \%$ & 63.20 \\
\hline 1989 & 9.16 & $14.2 \%$ & 22.52 & $35.0 \%$ & 41.18 & $64.0 \%$ & 64.31 \\
\hline 1990 & 8.91 & $13.7 \%$ & 23.71 & $36.4 \%$ & 40.80 & $62.6 \%$ & 65.14 \\
\hline 1991 & 9.08 & $14.0 \%$ & 23.65 & $36.4 \%$ & 40.53 & $62.4 \%$ & 64.95 \\
\hline 1992 & 8.87 & $13.7 \%$ & 25.02 & $38.5 \%$ & 39.37 & $60.6 \%$ & 64.95 \\
\hline 1993 & 8.58 & $13.2 \%$ & 25.83 & $39.6 \%$ & 38.82 & $59.5 \%$ & 65.23 \\
\hline 1994 & 8.39 & $12.6 \%$ & 26.52 & $39.8 \%$ & 39.21 & $58.9 \%$ & 66.55 \\
\hline 1995 & 8.32 & $12.2 \%$ & 27.19 & $40.0 \%$ & 40.21 & $59.1 \%$ & 68.01 \\
\hline 1996 & 8.30 & $11.9 \%$ & 27.70 & $39.8 \%$ & 41.26 & $59.3 \%$ & 69.52 \\
\hline 1997 & 8.27 & $11.5 \%$ & 29.05 & $40.5 \%$ & 42.05 & $58.7 \%$ & 71.65 \\
\hline 1998 & 8.01 & $11.0 \%$ & 30.21 & $41.4 \%$ & 42.35 & $58.0 \%$ & 73.04 \\
\hline 1999 & 7.73 & $10.7 \%$ & 29.13 & $40.4 \%$ & 43.02 & $59.6 \%$ & 72.15 \\
\hline 2000 & 7.73 & $10.3 \%$ & 30.95 & $41.3 \%$ & 43.95 & $58.7 \%$ & 74.90 \\
\hline 2001 & 7.67 & $10.3 \%$ & 30.35 & $40.6 \%$ & 44.47 & $59.4 \%$ & 74.83 \\
\hline 2002 & 7.63 & $10.3 \%$ & 28.79 & $38.9 \%$ & 45.31 & $61.1 \%$ & 74.10 \\
\hline 2003 & 7.40 & $9.7 \%$ & 30.44 & $39.8 \%$ & 46.08 & $60.2 \%$ & 76.52 \\
\hline 2004 & 7.23 & $9.1 \%$ & 33.04 & $41.4 \%$ & 46.83 & $58.6 \%$ & 79.87 \\
\hline 2005 & 6.90 & $8.5 \%$ & 34.74 & $42.7 \%$ & 46.58 & $57.3 \%$ & 81.32 \\
\hline 2006 & 6.83 & $8.4 \%$ & 34.47 & $42.5 \%$ & 46.65 & $57.5 \%$ & 81.12 \\
\hline 2007 & 6.86 & $8.5 \%$ & 34.26 & $42.3 \%$ & 46.67 & $57.7 \%$ & 80.93 \\
\hline 2008 & 6.78 & $8.3 \%$ & 35.60 & $43.5 \%$ & 46.17 & $56.5 \%$ & 81.77 \\
\hline 2009 & 7.26 & $9.0 \%$ & 33.93 & $42.0 \%$ & 46.78 & $58.0 \%$ & 80.72 \\
\hline 2010 & 7.54 & $9.1 \%$ & 35.16 & $42.4 \%$ & 47.74 & $57.6 \%$ & 82.90 \\
\hline 2011 & 7.87 & $9.5 \%$ & 35.42 & $42.6 \%$ & 47.76 & $57.4 \%$ & 83.18 \\
\hline 2012 & 8.90 & $10.5 \%$ & 36.80 & $43.3 \%$ & 48.20 & $56.7 \%$ & 84.99 \\
\hline 2013 & 10.01 & $11.7 \%$ & 35.98 & $42.2 \%$ & 49.37 & $57.8 \%$ & 85.35 \\
\hline \multicolumn{8}{|c|}{ Average annual percentage change } \\
\hline $1973-2013$ & $-0.2 \%$ & & $0.5 \%$ & & $1.4 \%$ & & $1.0 \%$ \\
\hline $2003-2013$ & $3.1 \%$ & & $1.7 \%$ & & $0.7 \%$ & & $1.1 \%$ \\
\hline
\end{tabular}

Source:

U.S. Department of Energy, Energy Information Administration, International Energy Statistics Website, April 2014. (Additional resources: www.eia.doe.gov)

${ }^{\mathrm{b}}$ Organization of Petroleum Exporting Countries. See Glossary for membership. 
During the 1980s and 1990s, the United States accounted for about one-quarter of the world's petroleum consumption, but since 2000 that share has been decreasing. In 2011 the United States accounted for only 21.6\%. World petroleum consumption decreased in 2009 but rose in 2010. Non-OECD consumption has continued to increase.

Table 1.4

World Petroleum Consumption, 1960-2012 (million barrels per day)

\begin{tabular}{|c|c|c|c|c|c|}
\hline Year & United States & U.S. share & Total OECD ${ }^{\mathrm{a}}$ & Total non-OECD & World \\
\hline 1960 & 9.80 & $45.9 \%$ & 15.78 & 5.56 & 21.34 \\
\hline 1965 & 11.51 & $37.0 \%$ & 22.81 & 8.33 & 31.14 \\
\hline 1970 & 14.70 & $31.4 \%$ & 34.69 & 12.12 & 46.81 \\
\hline 1975 & 16.32 & $29.0 \%$ & 39.14 & 17.06 & 56.20 \\
\hline 1980 & 17.06 & $27.0 \%$ & 41.87 & 21.25 & 63.12 \\
\hline 1981 & 16.06 & $26.3 \%$ & 39.60 & 21.36 & 60.95 \\
\hline 1982 & 15.30 & $25.7 \%$ & 37.87 & 21.68 & 59.55 \\
\hline 1983 & 15.23 & $25.9 \%$ & 37.00 & 21.78 & 58.78 \\
\hline 1984 & 15.73 & $26.3 \%$ & 37.90 & 21.89 & 59.80 \\
\hline 1985 & 15.73 & $26.2 \%$ & 37.70 & 22.39 & 60.08 \\
\hline 1986 & 16.28 & $26.3 \%$ & 38.83 & 22.99 & 61.82 \\
\hline 1987 & 16.67 & $26.4 \%$ & 39.59 & 23.51 & 63.11 \\
\hline 1988 & 17.28 & $26.6 \%$ & 40.92 & 24.05 & 64.98 \\
\hline 1989 & 17.33 & $26.2 \%$ & 41.62 & 24.47 & 66.09 \\
\hline 1990 & 16.99 & $25.5 \%$ & 41.78 & 24.77 & 66.55 \\
\hline 1991 & 16.71 & $24.9 \%$ & 42.25 & 24.96 & 67.21 \\
\hline 1992 & 17.03 & $25.3 \%$ & 43.28 & 24.10 & 67.38 \\
\hline 1993 & 17.24 & $25.5 \%$ & 43.72 & 23.87 & 67.59 \\
\hline 1994 & 17.72 & $25.7 \%$ & 44.95 & 23.98 & 68.93 \\
\hline 1995 & 17.72 & $25.3 \%$ & 45.44 & 24.69 & 70.13 \\
\hline 1996 & 18.31 & $25.5 \%$ & 46.57 & 25.15 & 71.71 \\
\hline 1997 & 18.62 & $25.3 \%$ & 47.34 & 26.12 & 73.46 \\
\hline 1998 & 18.92 & $25.5 \%$ & 47.53 & 26.59 & 74.12 \\
\hline 1999 & 19.52 & $25.7 \%$ & 48.56 & 27.32 & 75.88 \\
\hline 2000 & 19.70 & $25.7 \%$ & 48.54 & 28.24 & 76.78 \\
\hline 2001 & 19.65 & $25.4 \%$ & 48.57 & 28.91 & 77.48 \\
\hline 2002 & 19.76 & $25.3 \%$ & 48.55 & 29.62 & 78.17 \\
\hline 2003 & 20.03 & $25.1 \%$ & 49.23 & 30.48 & 79.71 \\
\hline 2004 & 20.73 & $25.1 \%$ & 50.09 & 32.48 & 82.57 \\
\hline 2005 & 20.80 & $24.7 \%$ & 50.43 & 33.64 & 84.08 \\
\hline 2006 & 20.69 & $24.3 \%$ & 50.13 & 35.01 & 85.14 \\
\hline 2007 & 20.68 & $24.1 \%$ & 50.00 & 35.91 & 85.91 \\
\hline 2008 & 19.50 & $23.1 \%$ & 48.34 & 36.16 & 84.50 \\
\hline 2009 & 18.77 & $22.1 \%$ & 46.31 & 38.45 & 84.76 \\
\hline 2010 & 19.18 & $22.0 \%$ & 46.94 & 40.43 & 87.36 \\
\hline 2011 & 18.95 & $21.5 \%$ & 46.49 & 41.76 & 88.25 \\
\hline 2012 & 18.55 & $21.0 \%$ & 45.90 & 42.41 & 88.31 \\
\hline \multicolumn{6}{|c|}{ Average annual percentage change } \\
\hline $1960-2012$ & $1.2 \%$ & & $2.1 \%$ & $4.0 \%$ & $2.8 \%$ \\
\hline $1970-2012$ & $0.6 \%$ & & $0.7 \%$ & $3.0 \%$ & $1.5 \%$ \\
\hline 2002-2012 & $-0.6 \%$ & & $-0.6 \%$ & $3.7 \%$ & $1.2 \%$ \\
\hline
\end{tabular}

\section{Source:}

U.S. Department of Energy, Energy Information Administration, International Energy Statistics Website, May 2013. (Additional resources: www.eia.doe.gov)

\footnotetext{
${ }^{a}$ Organization for Economic Cooperation and Development. See Glossary for membership.
} 
Figure 1.1. World Oil Reserves, Production and Consumption, 2012

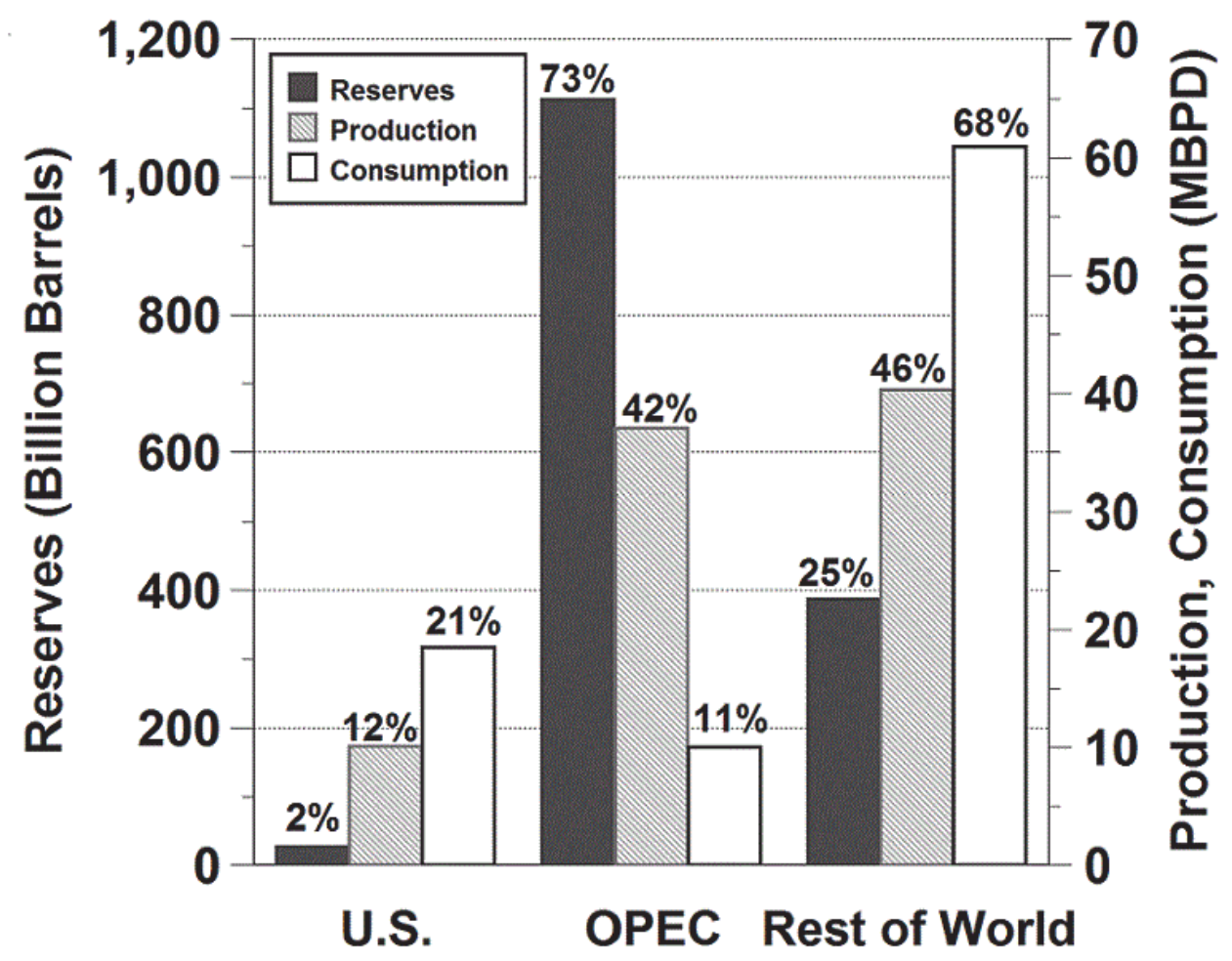

Table 1.5

World Oil Reserves, Production and Consumption, 2012

\begin{tabular}{lcccccc}
\hline & $\begin{array}{c}\text { Crude oil } \\
\text { reserves } \\
\text { (billion } \\
\text { barrels) }\end{array}$ & $\begin{array}{c}\text { Reserve } \\
\text { share }\end{array}$ & $\begin{array}{c}\text { Petroleum } \\
\text { production } \\
\text { (million } \\
\text { barrels per } \\
\text { day) }\end{array}$ & $\begin{array}{c}\text { Production } \\
\text { share }\end{array}$ & $\begin{array}{c}\text { Petroleum } \\
\text { consumption } \\
\text { (million } \\
\text { barrels per } \\
\text { day) }\end{array}$ & $\begin{array}{c}\text { Consumption } \\
\text { share }\end{array}$ \\
\hline United States & 26.5 & $2 \%$ & 10.1 & $12 \%$ & 18.5 & $21 \%$ \\
OPEC & $1,112.9$ & $73 \%$ & 37.0 & $42 \%$ & 10.0 & $11 \%$ \\
Rest of world & 386.6 & $25 \%$ & 40.3 & $46 \%$ & 60.9 & $68 \%$ \\
\hline
\end{tabular}

Note: Total consumption is higher than total production due to refinery gains including alcohol and liquid products produced from coal and other sources. OPEC countries include Venezuela, Iran, Iraq, Kuwait, Qatar, Saudi Arabia, Angola, United Arab Emirates, Algeria, Libya, Nigeria, Indonesia, Gabon, and Ecuador.

\section{Sources:}

Energy Information Administration, International Energy Statistics, April 2014. (Additional resources: www.eia.doe.gov) 
Figure 1.2. World Natural Gas Reserves, Production and Consumption, 2012

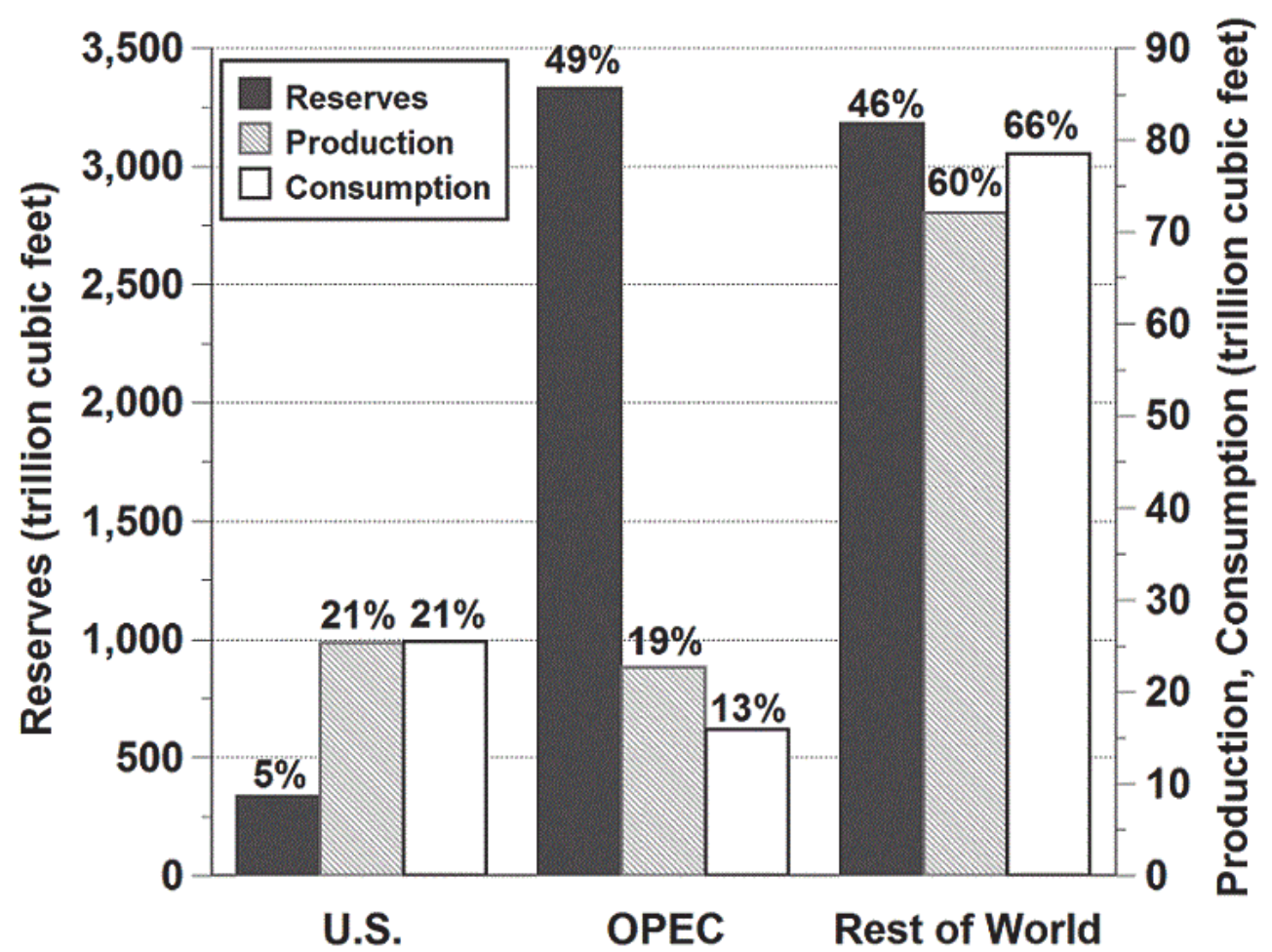

Table 1.6

World Natural Gas Reserves, Production and Consumption, 2012 (trillion cubic feet)

\begin{tabular}{lrrcccc}
\hline & $\begin{array}{c}\text { Natural } \\
\text { gas } \\
\text { reserves }\end{array}$ & $\begin{array}{c}\text { Reserve } \\
\text { share }\end{array}$ & $\begin{array}{c}\text { Natural gas } \\
\text { production }\end{array}$ & $\begin{array}{c}\text { Production } \\
\text { share }\end{array}$ & $\begin{array}{c}\text { Natural gas } \\
\text { consumption }\end{array}$ & $\begin{array}{c}\text { Consumption } \\
\text { share }\end{array}$ \\
\hline U.S. & 334.1 & $5 \%$ & 25.3 & $21 \%$ & 25.5 & $21 \%$ \\
OPEC & $3,330.1$ & $49 \%$ & 22.7 & $19 \%$ & 15.9 & $13 \%$ \\
Rest of world & $3,180.4$ & $46 \%$ & 72.1 & $60 \%$ & 78.5 & $66 \%$ \\
\hline
\end{tabular}

Note: Production data are dry gas production.

Source:

Energy Information Administration, International Energy Statistics, 2014. (Additional resources: www.eia.doe.gov) 
The share of petroleum imported to the United States can be calculated using total imports or net imports. Net imports, which are the preferred data, rose to over $50 \%$ of U.S. petroleum consumption for the first time in 1998, while total imports reached 50\% for the first time in 1993. OPEC share of net imports has been below $50 \%$ since 1993. Net imports as a share of consumption decreased to 32.8\% in 2013-the lowest since 1985.

Table 1.7

U.S. Petroleum Imports, 1960-2013 (million barrels per day)

\begin{tabular}{|c|c|c|c|c|c|}
\hline Year & $\begin{array}{l}\text { Net OPEC } \\
\text { imports }\end{array}$ & $\begin{array}{l}\text { Net OPEC } \\
\text { share }\end{array}$ & Net imports & $\begin{array}{l}\text { Net imports as a share } \\
\text { of U.S. consumption }\end{array}$ & Total imports \\
\hline 1960 & 1.31 & $81.3 \%$ & 1.61 & $\mathrm{~b}$ & 1.82 \\
\hline 1965 & 1.48 & $64.7 \%$ & 2.28 & $\mathrm{~b}$ & 2.47 \\
\hline 1970 & 1.34 & $42.5 \%$ & 3.16 & 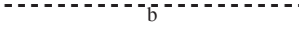 & 3.42 \\
\hline 1975 & 3.60 & $59.5 \%$ & 5.89 & $35.8 \%$ & 6.06 \\
\hline 1980 & 4.30 & $62.2 \%$ & 6.36 & $37.3 \%$ & 6.91 \\
\hline 1985 & 1.83 & $36.1 \%$ & 4.29 & $27.3 \%$ & 5.07 \\
\hline 1986 & 2.84 & $45.6 \%$ & 5.44 & $33.4 \%$ & 6.22 \\
\hline 1987 & 3.06 & $45.8 \%$ & 5.91 & $35.4 \%$ & 6.68 \\
\hline 1988 & 3.52 & $47.6 \%$ & 6.59 & $38.0 \%$ & 7.40 \\
\hline 1989 & 4.14 & $51.4 \%$ & 7.20 & $41.3 \%$ & 8.06 \\
\hline 1990 & 4.30 & $53.6 \%$ & 7.16 & $42.2 \%$ & 8.02 \\
\hline 1991 & 4.09 & $53.7 \%$ & 6.63 & $38.9 \%$ & 7.63 \\
\hline 1992 & 4.09 & $51.9 \%$ & 6.94 & $40.9 \%$ & 7.89 \\
\hline 1993 & 4.27 & $49.6 \%$ & 7.62 & $44.9 \%$ & 8.62 \\
\hline 1994 & 4.25 & $47.2 \%$ & 8.05 & $45.7 \%$ & 9.00 \\
\hline 1995 & 4.00 & $45.3 \%$ & 7.89 & $44.5 \%$ & 8.84 \\
\hline 1996 & 4.21 & $44.4 \%$ & 8.50 & $46.4 \%$ & 9.48 \\
\hline 1997 & 4.57 & $45.0 \%$ & 9.16 & $49.2 \%$ & 10.16 \\
\hline 1998 & 4.91 & $45.8 \%$ & 9.76 & $51.6 \%$ & 10.71 \\
\hline 1999 & 4.95 & $45.6 \%$ & 9.91 & $50.8 \%$ & 10.85 \\
\hline 2000 & 5.20 & $45.4 \%$ & 10.42 & $52.9 \%$ & 11.46 \\
\hline 2001 & 5.53 & $46.6 \%$ & 10.90 & $55.5 \%$ & 11.87 \\
\hline 2002 & 4.61 & $39.9 \%$ & 10.55 & $53.4 \%$ & 11.53 \\
\hline 2003 & 5.16 & $42.1 \%$ & 11.24 & $56.1 \%$ & 12.26 \\
\hline 2004 & 5.70 & $43.4 \%$ & 12.10 & $58.4 \%$ & 13.15 \\
\hline 2005 & 5.59 & $40.7 \%$ & 12.55 & $60.3 \%$ & 13.71 \\
\hline 2006 & 5.52 & $40.2 \%$ & 12.39 & $59.9 \%$ & 13.71 \\
\hline 2007 & 5.98 & $44.4 \%$ & 12.04 & $58.2 \%$ & 13.47 \\
\hline 2008 & 5.95 & $46.1 \%$ & 11.11 & $57.0 \%$ & 12.92 \\
\hline 2009 & 4.78 & $40.9 \%$ & 9.67 & $51.5 \%$ & 11.69 \\
\hline 2010 & 4.91 & $41.6 \%$ & 9.44 & $49.2 \%$ & 11.79 \\
\hline 2011 & 4.56 & $39.8 \%$ & 8.45 & $44.8 \%$ & 11.44 \\
\hline 2012 & 4.27 & $40.3 \%$ & 7.39 & $40.0 \%$ & 10.60 \\
\hline 2013 & 3.71 & $37.8 \%$ & 6.20 & $32.8 \%$ & 9.79 \\
\hline \multicolumn{6}{|c|}{ Average annual percentage change } \\
\hline 1960-2013 & $2.0 \%$ & & $2.6 \%$ & & $3.2 \%$ \\
\hline $1970-2013$ & $2.4 \%$ & & $1.6 \%$ & & $2.5 \%$ \\
\hline $2003-2013$ & $-3.2 \%$ & & $-5.8 \%$ & & $-2.2 \%$ \\
\hline
\end{tabular}

\section{Source:}

U.S. Department of Energy, Energy Information Administration, Monthly Energy Review, Washington, DC, March 2014, Table 3.3a. (Additional resources: www.eia.gov)

${ }^{a}$ Organization of Petroleum Exporting Countries. See Glossary for membership.

${ }^{\mathrm{b}}$ Data are not available. 
Just over half of the oil imported to the United States in 2013 was from the western hemisphere. Canada, Mexico, and Venezuela provided most of the oil from the western hemisphere, along with small amounts from Brazil,

Columbia, Ecuador, and the U.S. Virgin Islands (these countries are not listed separately).

Table 1.8

Imported Crude Oil by Country of Origin, 1973-2013 (million barrels per day)

\begin{tabular}{|c|c|c|c|c|c|c|c|c|c|}
\hline Year & $\begin{array}{c}\text { Saudi } \\
\text { Arabia }\end{array}$ & Venezuela & Nigeria & 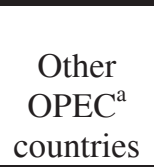 & Canada & Mexico & Russia & $\begin{array}{c}\text { Other } \\
\text { non- } \\
\text { OPEC } \\
\text { countries }\end{array}$ & $\begin{array}{c}\text { Total } \\
\text { imports }\end{array}$ \\
\hline 1973 & 0.49 & 1.13 & 0.46 & 0.91 & 1.32 & 0.02 & 0.03 & 1.90 & 6.26 \\
\hline 1975 & 0.71 & 0.70 & 0.76 & 1.42 & 0.85 & 0.07 & 0.01 & 1.52 & 6.06 \\
\hline 1980 & 1.26 & 0.48 & 0.86 & 1.70 & 0.45 & 0.53 & 0.00 & 1.62 & 6.91 \\
\hline 1981 & 1.13 & 0.41 & 0.62 & 1.17 & 0.45 & 0.52 & 0.00 & 1.70 & 6.00 \\
\hline 1982 & 0.55 & 0.41 & 0.51 & 0.67 & 0.48 & 0.68 & 0.00 & 1.80 & 5.11 \\
\hline 1983 & 0.34 & 0.42 & 0.30 & 0.80 & 0.55 & 0.83 & 0.00 & 1.81 & 5.05 \\
\hline 1984 & 0.32 & 0.55 & 0.22 & 0.96 & 0.63 & 0.75 & 0.01 & 2.00 & 5.44 \\
\hline 1985 & 0.17 & 0.60 & 0.29 & 0.76 & 0.77 & 0.82 & 0.01 & 1.64 & 5.07 \\
\hline 1986 & 0.68 & 0.79 & 0.44 & 0.92 & 0.81 & 0.70 & 0.02 & 1.86 & 6.22 \\
\hline 1987 & 0.75 & 0.80 & 0.53 & 0.97 & 0.85 & 0.65 & 0.01 & 2.10 & 6.68 \\
\hline 1988 & 1.07 & 0.79 & 0.62 & 1.03 & 1.00 & 0.75 & 0.03 & 2.11 & 7.40 \\
\hline 1989 & 1.22 & 0.87 & 0.82 & 1.23 & 0.93 & 0.77 & 0.05 & 2.17 & 8.06 \\
\hline 1990 & 1.34 & 1.02 & 0.80 & 1.13 & 0.93 & 0.76 & 0.04 & 1.99 & 8.02 \\
\hline 1991 & 1.80 & 1.03 & 0.70 & 0.55 & 1.03 & 0.81 & 0.03 & 1.67 & 7.63 \\
\hline 1992 & 1.72 & 1.17 & 0.68 & 0.52 & 1.07 & 0.83 & 0.02 & 1.88 & 7.89 \\
\hline 1993 & 1.41 & 1.30 & 0.74 & 0.82 & 1.18 & 0.92 & 0.05 & 2.19 & 8.62 \\
\hline 1994 & 1.40 & 1.33 & 0.64 & 0.87 & 1.27 & 0.98 & 0.03 & 2.46 & 9.00 \\
\hline 1995 & 1.34 & 1.48 & 0.63 & 0.55 & 1.33 & 1.07 & 0.02 & 2.41 & 8.83 \\
\hline 1996 & 1.36 & 1.68 & 0.62 & 0.56 & 1.42 & 1.24 & 0.03 & 2.57 & 9.48 \\
\hline 1997 & 1.41 & 1.77 & 0.70 & 0.69 & 1.56 & 1.39 & 0.01 & 2.63 & 10.16 \\
\hline 1998 & 1.49 & 1.72 & 0.70 & 1.00 & 1.60 & 1.35 & 0.02 & 2.83 & 10.71 \\
\hline 1999 & 1.48 & 1.49 & 0.66 & 1.33 & 1.54 & 1.32 & 0.09 & 2.95 & 10.85 \\
\hline 2000 & 1.57 & 1.55 & 0.90 & 1.19 & 1.81 & 1.37 & 0.07 & 3.00 & 11.46 \\
\hline 2001 & 1.66 & 1.55 & 0.89 & 1.43 & 1.83 & 1.44 & 0.09 & 2.98 & 11.87 \\
\hline 2002 & 1.55 & 1.40 & 0.62 & 1.03 & 1.97 & 1.55 & 0.21 & 3.20 & 11.53 \\
\hline 2003 & 1.77 & 1.38 & 0.87 & 1.14 & 2.07 & 1.62 & 0.25 & 3.15 & 12.26 \\
\hline 2004 & 1.56 & 1.55 & 1.14 & 1.45 & 2.14 & 1.66 & 0.30 & 3.34 & 13.15 \\
\hline 2005 & 1.54 & 1.53 & 1.17 & 1.36 & 2.18 & 1.66 & 0.41 & 3.87 & 13.71 \\
\hline 2006 & 1.46 & 1.42 & 1.11 & 1.52 & 2.35 & 1.71 & 0.37 & 3.76 & 13.71 \\
\hline 2007 & 1.48 & 1.36 & 1.13 & 2.00 & 2.45 & 1.53 & 0.41 & 3.09 & 13.47 \\
\hline 2008 & 1.53 & 1.19 & 0.99 & 2.25 & 2.49 & 1.30 & 0.47 & 2.70 & 12.92 \\
\hline 2009 & 1.00 & 1.06 & 0.81 & 1.90 & 2.48 & 1.21 & 0.56 & 2.66 & 11.69 \\
\hline 2010 & 1.10 & 0.99 & 1.02 & 1.80 & 2.54 & 1.28 & 0.61 & 2.46 & 11.79 \\
\hline 2011 & 1.19 & 0.95 & 0.82 & 1.59 & 2.73 & 1.21 & 0.62 & 2.32 & 11.44 \\
\hline 2012 & 1.37 & 0.96 & 0.44 & 1.51 & 2.95 & 1.03 & 0.48 & 1.87 & 10.60 \\
\hline 2013 & 1.33 & 0.80 & 0.28 & 1.30 & 3.13 & 0.92 & 0.46 & 1.58 & 9.79 \\
\hline
\end{tabular}

Sources:

U.S. Department of Energy, Energy Information Administration, Monthly Energy Review, Washington, DC, March 2014, Tables 3.3c and 3.3d. (Additional resources: www.eia.gov)

${ }^{a}$ Organization of Petroleum Exporting Countries. See Glossary for membership. 
The Strategic Petroleum Reserve (SPR) began in October 1977 as a result of the 1975 Energy Policy and Conservation Act. Its purpose is to provide protection against oil supply disruptions. The U.S. consumed nearly 19 million barrels per day in 2013. At that rate of consumption, the SPR supply would last 37 days if used exclusively and continuously.

Table 1.9

Crude Oil Supplies, 1973-2013

\begin{tabular}{|c|c|c|c|c|c|}
\hline \multirow[b]{2}{*}{ Year } & $\begin{array}{l}\text { Strategic } \\
\text { Petroleum } \\
\text { Reserve } \\
\end{array}$ & $\begin{array}{c}\text { Other } \\
\text { crude oil } \\
\text { stocks }^{\mathrm{a}} \\
\end{array}$ & $\begin{array}{c}\text { Total } \\
\text { crude oil stocks }\end{array}$ & \multirow{2}{*}{$\begin{array}{c}\text { U.S. petroleum } \\
\text { consumption } \\
\text { (million barrels per day) }\end{array}$} & \multirow{2}{*}{$\begin{array}{l}\text { Number of days } \\
\text { the SPR would } \\
\text { supply the U.S. }\end{array}$} \\
\hline & \multicolumn{3}{|c|}{ (Million Barrels) } & & \\
\hline 1973 & 0.0 & 242.5 & 242.5 & 17.3 & 0 \\
\hline $1977^{-}$ & 7.5 & $340.2^{-}$ & 347.7 & 18.4 & 0 \\
\hline 1978 & 66.9 & 309.4 & 376.3 & 18.8 & 4 \\
\hline 1979 & 91.2 & 339.1 & 430.3 & 18.5 & 5 \\
\hline 1980 & 107.8 & 358.2 & 466.0 & 17.1 & 6 \\
\hline 1981 & 230.3 & 363.5 & 593.8 & 16.1 & 14 \\
\hline 1982 & 293.8 & 349.7 & 643.6 & 15.3 & 19 \\
\hline 1983 & 379.1 & 343.9 & 722.9 & 15.2 & 25 \\
\hline 1984 & 450.5 & 345.4 & 795.9 & 15.7 & 29 \\
\hline 1985 & 493.3 & 320.9 & 814.2 & 15.7 & 31 \\
\hline 1986 & 511.6 & 331.2 & 842.8 & 16.3 & 31 \\
\hline 1987 & 540.6 & 349.0 & 889.6 & 16.7 & 32 \\
\hline 1988 & 559.5 & 330.4 & 889.9 & 17.3 & 32 \\
\hline 1989 & 579.9 & 341.3 & 921.1 & 17.3 & 33 \\
\hline 1990 & 585.7 & 322.7 & 908.4 & 17.0 & 34 \\
\hline 1991 & 568.5 & 324.6 & 893.1 & 16.7 & 34 \\
\hline 1992 & 574.7 & 318.1 & 892.9 & 17.0 & 34 \\
\hline 1993 & 587.1 & 335.4 & 922.5 & 17.2 & 34 \\
\hline 1994 & 591.7 & 337.2 & 928.9 & 17.7 & 33 \\
\hline 1995 & 591.6 & 303.3 & 895.0 & 17.7 & 33 \\
\hline 1996 & 565.8 & 283.9 & 849.7 & 18.3 & 31 \\
\hline 1997 & 563.4 & 304.7 & 868.1 & 18.6 & 30 \\
\hline 1998 & 571.4 & 323.5 & 894.9 & 18.9 & 30 \\
\hline 1999 & 567.2 & 284.5 & 851.7 & 19.5 & 29 \\
\hline 2000 & 540.7 & 285.5 & 826.2 & 19.7 & 27 \\
\hline 2001 & 550.2 & 312.0 & 862.2 & 19.6 & 28 \\
\hline 2002 & 599.1 & 277.6 & 876.7 & 19.8 & 30 \\
\hline 2003 & 638.4 & 268.9 & 907.3 & 20.0 & 32 \\
\hline 2004 & 675.6 & 285.7 & 961.3 & 20.7 & 33 \\
\hline 2005 & 684.5 & 323.7 & $1,008.2$ & 20.8 & 33 \\
\hline 2006 & 688.6 & 312.3 & $1,000.9$ & 20.7 & 33 \\
\hline 2007 & 696.9 & 286.1 & 983.0 & 20.7 & 34 \\
\hline 2008 & 701.8 & 325.8 & $1,027.7$ & 19.5 & 36 \\
\hline 2009 & 726.6 & 325.2 & $1,051.8$ & 18.8 & 39 \\
\hline 2010 & 726.5 & 333.4 & $1,060.0$ & 19.2 & 38 \\
\hline 2011 & 696.0 & 330.7 & $1,026.6$ & 18.9 & 37 \\
\hline 2012 & 695.3 & 365.0 & $1,060.3$ & 18.5 & 38 \\
\hline 2013 & 696.0 & 357.6 & $1,053.6$ & 18.9 & 37 \\
\hline
\end{tabular}

Sources:

U.S. Department of Energy, Energy Information Administration, Monthly Energy Review, Washington, DC, April 2014, Tables 3.1 and 3.4. (Additional resources: www.eia.gov) in transit.

${ }^{a}$ Other crude oil stocks include stocks held by petroleum companies, as well as stocks of Alaskan crude oil

${ }^{\mathrm{b}}$ Strategic Petroleum Reserves divided by U.S. consumption per day. This would only hold true if the SPR were the only oil used for that many days. 
Major oil price shocks have disrupted world energy markets five times in the past 30 years (1973-74, 1979-80, 1990-91, 1999-2000, 2008). Most of the oil price shocks were followed by an economic recession in the United States.

Figure 1.3. Oil Price and Economic Growth, 1970-2013

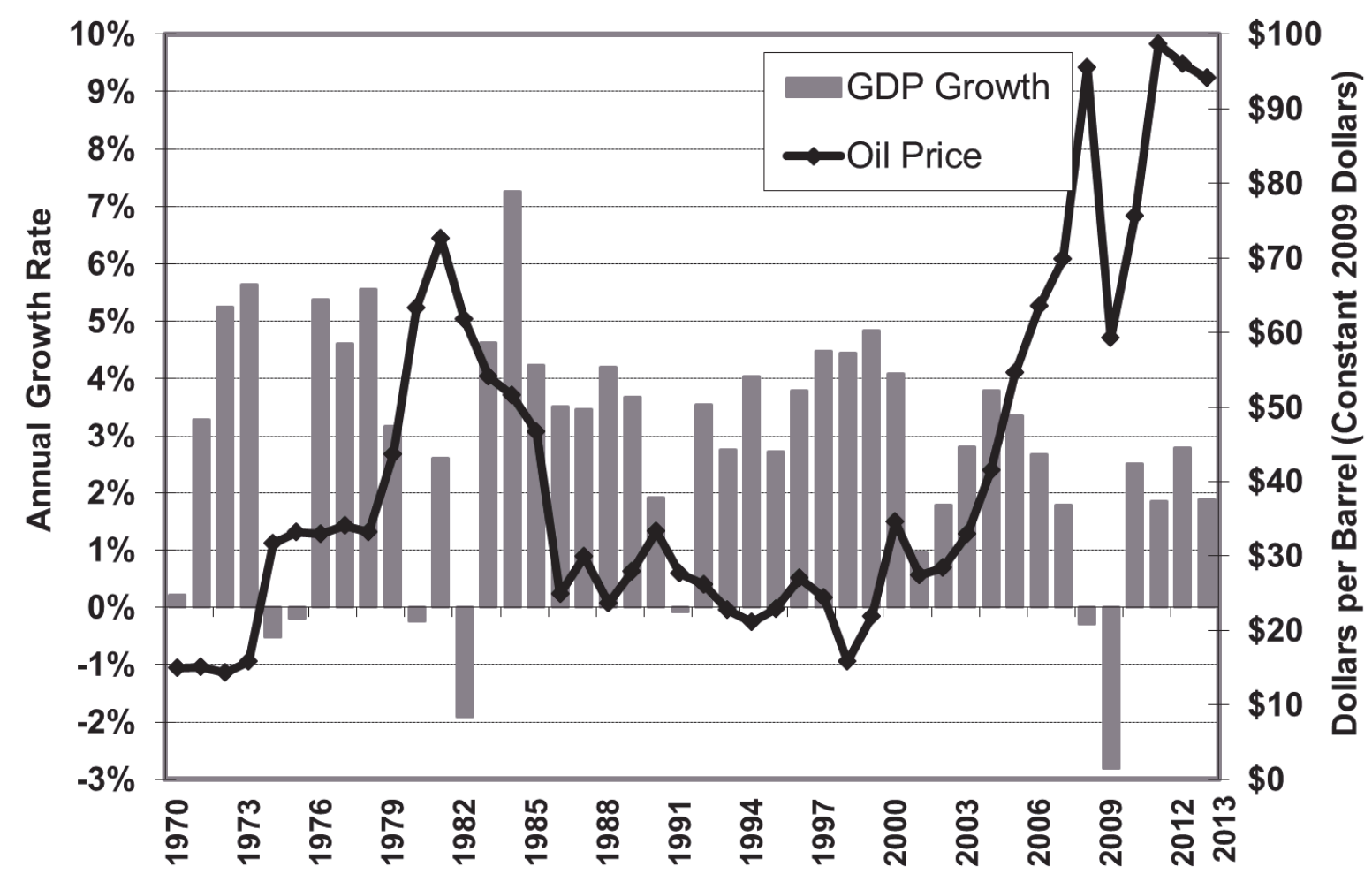

Source:

Greene, D.L. and N. I. Tishchishyna, Costs of Oil Dependence: A 2000 Update, Oak Ridge National Laboratory, ORNL/TM-2000/152, Oak Ridge, TN, 2000, and data updates, 2013. (Additional resources: cta.ornl.gov/cta/publications.shtml) 
The United States has long recognized the problem of oil dependence and the economic problems that arise from it. According to Oak Ridge National Laboratory (ORNL) researchers Greene and Hopson, oil dependence is a combination of four factors: (1) a noncompetitive world oil market strongly influenced by the OPEC cartel, (2) high levels of U.S. imports, (3) the importance of oil to the U.S. economy, and (4) the lack of economical and readily available substitutes for oil. ORNL developed a model to estimate the historical cost of oil dependence and analyze the potential effectiveness of policies on likely future costs. The most recent study using this model shows that the U.S. economy suffered the greatest losses in 2008 when wealth transfer and GDP losses (combined) amounted to approximately half a trillion dollars. However, when comparing oil dependence to the size of the economy, the year 1980 is the highest. Oil dependence costs were almost 5\% of GDP in 1980, but were $6 \%$ in 2008. In 2009, the average oil price fell to about $\$ 60$ per barrel and oil dependence costs fell to about $\$ 300$ billion for 2009 and 2010. However, the cost rose again in 2011 and 2012.

Figure 1.4. Costs of Oil Dependence to the U.S. Economy, 1970-2012

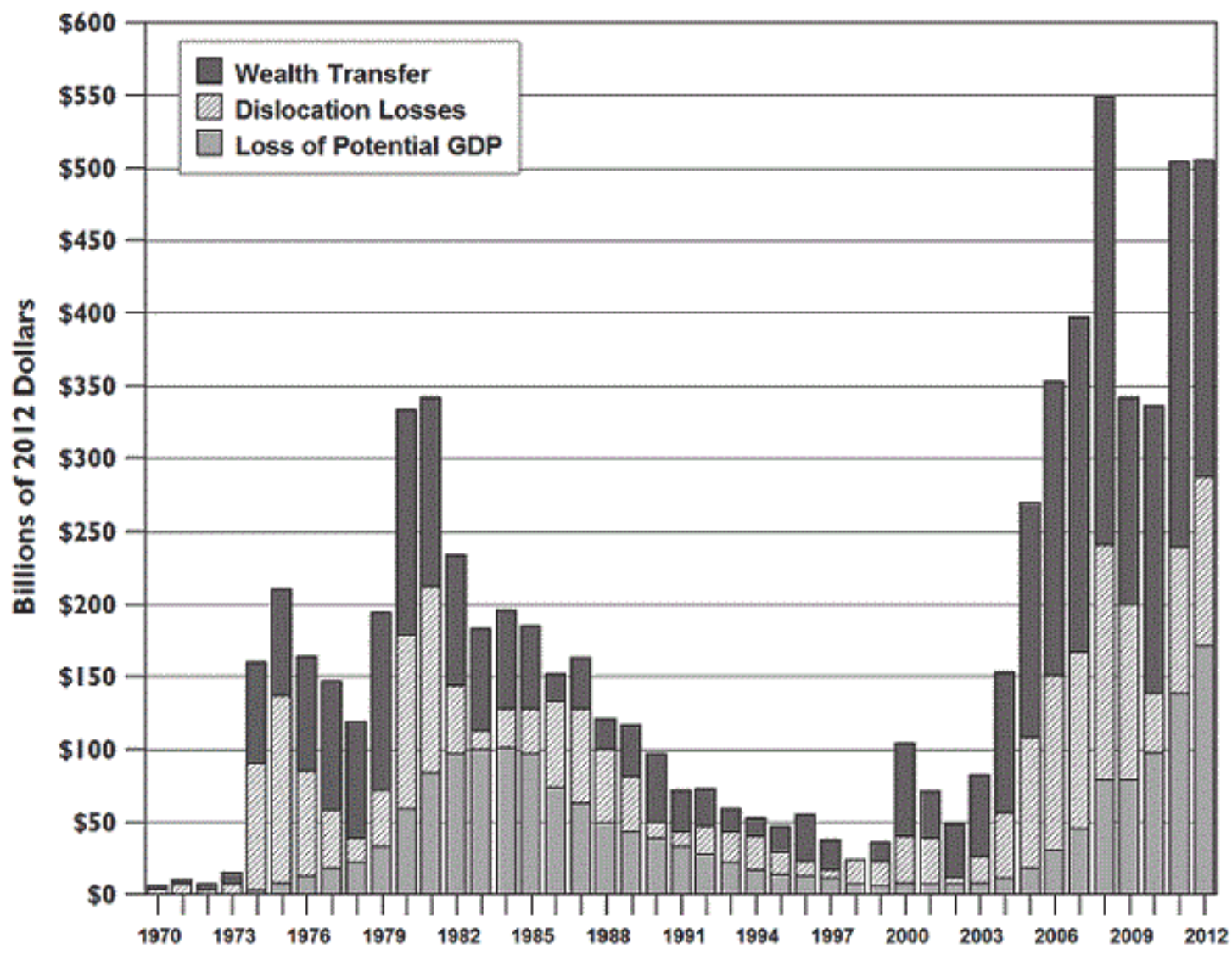

Notes:

Wealth Transfer is the product of total U.S. oil imports and the difference between the actual market price of oil (influenced by market power) and what the price would have been in a competitive market.

Dislocation Losses are temporary reductions in GDP as a result of oil price shocks.

Loss of Potential Gross Domestic Product (GDP) results because a basic resource used by the economy to produce output has become more expensive. As a consequence, with the same endowment of labor, capital, and other resources, our economy cannot produce quite as much as it could have at a lower oil price.

\section{Source:}

Greene, David L., Roderick Lee, and Janet L. Hopson, "OPEC and the Costs to the U.S. Economy of Oil Dependence: 1970-2010,” Oak Ridge National Laboratory Memorandum, 2011, and updates. 
Other parts of the world refine crude oil to produce more diesel fuel and less gasoline than does North America. The OECD Europe countries produce the lowest share of gasoline in 2013.

Figure 1.5. Refinery Gross Output by World Region, 2003 and 2013

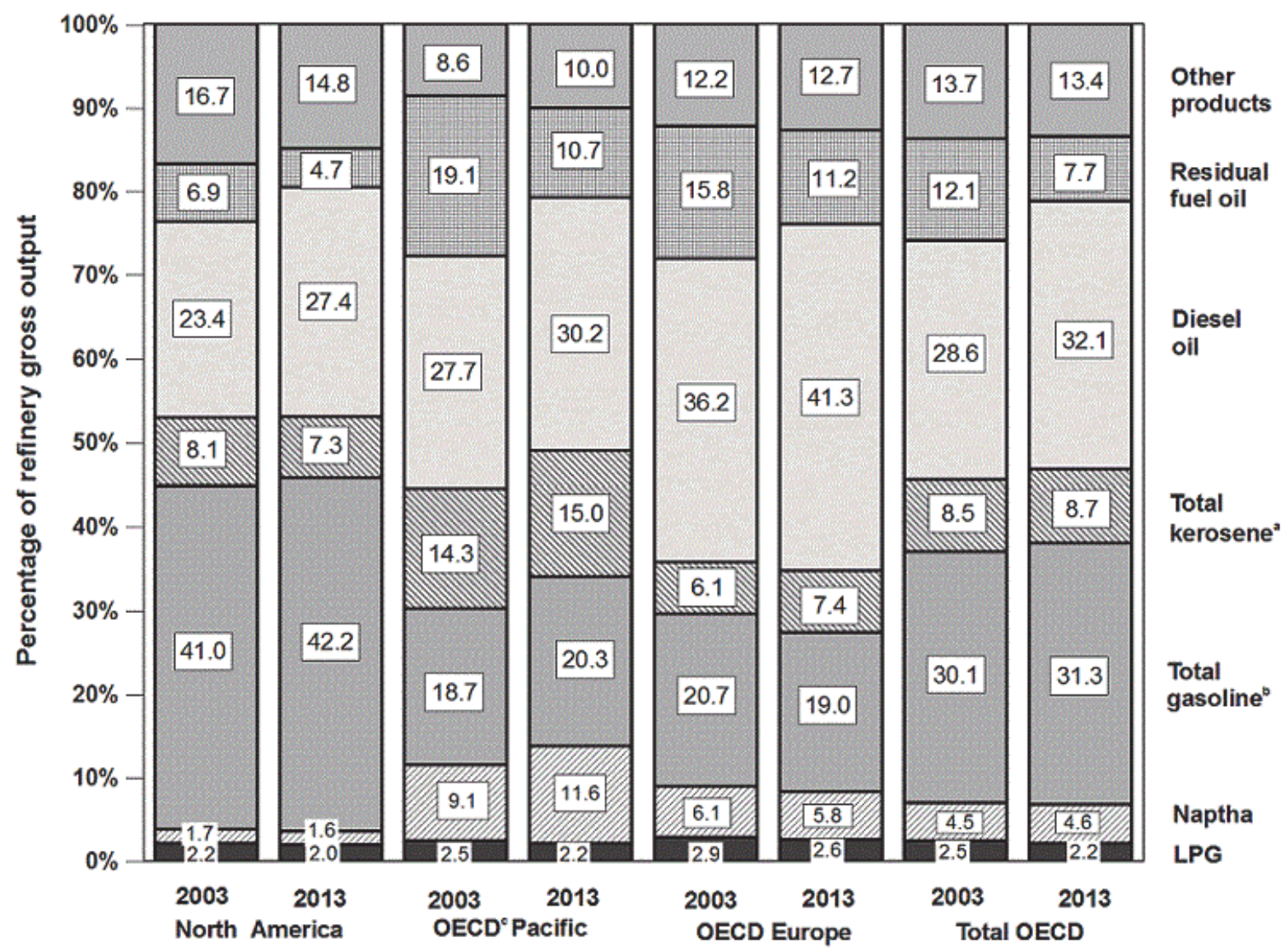

Source:

International Energy Agency, Monthly Oil Survey, January 2014. (Additional resources: www.iea.org)

\footnotetext{
${ }^{\mathrm{a}}$ Includes jet kerosene and other kerosene.

${ }^{\mathrm{b}}$ Includes motor gasoline, jet gasoline, and aviation gasoline.

${ }^{\mathrm{c}}$ Organization for Economic Cooperation and Development. See Glossary for membership.
} 
Oxygenate refinery input increased significantly in 1995, most certainly due to the Clean Air Act Amendments of 1990 which mandated the sale of reformulated gasoline in certain areas beginning in January 1995. The use of MTBE has declined in recent years due to many states banning the additive. The other hydrocarbons and liquids category includes unfinished oils, motor gasoline blending components and aviation gasoline blending components.

Table 1.10

U.S. Refinery Input of Crude Oil and Petroleum Products, 1987-2012 (thousand barrels)

\begin{tabular}{|c|c|c|c|c|c|c|c|}
\hline \multirow[b]{2}{*}{ Year } & \multirow[b]{2}{*}{ Crude oil } & \multirow[b]{2}{*}{$\begin{array}{c}\text { Natural gas } \\
\text { liquids }\end{array}$} & \multicolumn{3}{|c|}{ Oxygenates } & \multirow{2}{*}{$\begin{array}{c}\text { Other } \\
\text { hydrocarbons } \\
\text { and liquids }\end{array}$} & \multirow[b]{2}{*}{$\begin{array}{l}\text { Total input to } \\
\text { refineries }\end{array}$} \\
\hline & & & $\begin{array}{c}\text { Fuel } \\
\text { ethanol }\end{array}$ & $\mathrm{MTBE}^{\mathrm{a}}$ & $\begin{array}{c}\text { Other } \\
\text { oxygenates }^{\mathrm{b}}\end{array}$ & & \\
\hline 1987 & $4,691,783$ & 280,889 & $\mathrm{c}$ & $\mathrm{c}$ & d & 132,720 & $5,105,392$ \\
\hline 1988 & $4,848,175$ & 304,566 & $\mathrm{c}$ & $\mathrm{c}$ & d & 105,645 & $5,258,386$ \\
\hline 1989 & $4,891,381$ & 182,109 & $\mathrm{c}$ & $\mathrm{c}$ & d & 223,797 & $5,297,287$ \\
\hline 1990 & $4,894,379$ & 170,589 & $\mathrm{c}$ & $\mathrm{c}$ & d & 260,108 & $5,325,076$ \\
\hline 1991 & $4,855,016$ & 172,306 & $\mathrm{c}$ & $\mathrm{c}$ & d & 280,265 & $5,307,587$ \\
\hline 1992 & $4,908,603$ & 171,701 & c & $\mathrm{c}$ & d & 272,676 & $5,352,980$ \\
\hline 1993 & $4,968,641$ & 179,213 & 3,351 & 49,393 & 1,866 & 280,074 & $5,482,538$ \\
\hline 1994 & $5,061,111$ & 169,868 & 3,620 & 52,937 & 1,918 & 193,808 & $5,483,262$ \\
\hline 1995 & $5,100,317$ & 172,026 & 9,055 & 79,396 & 4,122 & 190,411 & $5,555,327$ \\
\hline 1996 & $5,195,265$ & 164,552 & 11,156 & 79,407 & 3,570 & 214,282 & $5,668,232$ \\
\hline 1997 & $5,351,466$ & 151,769 & 11,803 & 86,240 & 4,246 & 201,268 & $5,806,792$ \\
\hline 1998 & $5,434,383$ & 146,921 & 11,722 & 89,362 & 4,038 & 206,135 & $5,892,561$ \\
\hline 1999 & $5,403,450$ & 135,756 & 13,735 & 94,784 & 4,147 & 225,779 & $5,877,651$ \\
\hline 2000 & $5,514,395$ & 138,921 & 15,268 & 90,288 & 4,005 & 201,135 & $5,964,012$ \\
\hline 2001 & $5,521,637$ & 156,479 & 16,929 & 87,116 & 4,544 & 192,632 & $5,979,337$ \\
\hline 2002 & $5,455,530$ & 155,429 & 26,320 & 90,291 & 2,338 & 224,567 & $5,955,475$ \\
\hline 2003 & $5,585,875$ & 152,763 & 55,626 & 67,592 & 1,937 & 163,459 & $6,027,252$ \\
\hline 2004 & $5,663,861$ & 154,356 & 74,095 & 47,600 & 940 & 194,203 & $6,135,055$ \\
\hline 2005 & $5,555,332$ & 161,037 & 84,088 & 39,751 & 612 & 295,064 & $6,135,884$ \\
\hline 2006 & $5,563,354$ & 182,924 & 117,198 & 11,580 & 57 & 322,989 & $6,198,102$ \\
\hline 2007 & $5,532,097$ & 184,383 & 136,603 & 1,610 & 0 & 349,807 & $6,204,500$ \\
\hline 2008 & $5,361,287$ & 177,559 & 190,084 & 480 & 0 & 548,843 & $6,277,893$ \\
\hline 2009 & $5,232,656$ & 177,194 & 240,955 & 90 & 0 & 518,998 & $6,169,893$ \\
\hline 2010 & $5,374,094$ & 161,479 & 285,883 & 901 & 0 & 523,015 & $6,345,372$ \\
\hline 2011 & $5,404,347$ & 178,884 & 297,266 & 1,154 & 0 & 541,059 & $6,422,710$ \\
\hline 2012 & $5,489,516$ & 186,270 & 304,155 & 806 & 0 & 425,946 & $6,406,693$ \\
\hline \multicolumn{8}{|c|}{ Average annual percentage change } \\
\hline 1987-2012 & $0.6 \%$ & $-1.6 \%$ & & & & $4.8 \%$ & $0.9 \%$ \\
\hline 2002-2012 & $0.1 \%$ & $1.8 \%$ & $27.7 \%$ & $-37.6 \%$ & $-100.0 \%$ & $6.6 \%$ & $0.9 \%$ \\
\hline
\end{tabular}

\section{Source:}

U.S. Department of Energy, Energy Information Administration, Petroleum Supply Annual 2012, Vol. 1, September 2013, Table 16, and annual. (Additional resources: www.eia.doe.gov)

\footnotetext{
${ }^{a}$ Methyl tertiary butyl ether (MTBE).

${ }^{\mathrm{b}}$ Includes methanol and other oxygenates.

c Reported in "Other" category in this year.

${ }^{\mathrm{d}}$ Data are not available.
} 
When crude oil and other hydrocarbons are processed into products that are, on average, less dense than the input, a processing volume gain occurs. Due to this gain, the product yield from a barrel of crude oil is more than 100\%. The processing volume gain has been growing over the years.

Table 1.11

U.S. Refinery Yield of Petroleum Products from a Barrel of Crude Oil, 1978-2013 (percentage)

\begin{tabular}{|c|c|c|c|c|c|c|}
\hline Year & $\begin{array}{c}\text { Motor } \\
\text { gasoline }\end{array}$ & $\begin{array}{c}\text { Distillate } \\
\text { fuel oil }\end{array}$ & Jet fuel & $\begin{array}{c}\text { Liquefied } \\
\text { petroleum gas }\end{array}$ & Other $^{\mathrm{a}}$ & Total $^{\mathrm{b}}$ \\
\hline 1978 & 44.1 & 21.4 & 6.6 & 2.3 & 29.6 & 104.0 \\
\hline 1979 & 43.0 & 21.5 & 6.9 & 2.3 & 30.3 & 104.0 \\
\hline 1980 & 44.5 & 19.7 & 7.4 & 2.4 & 30.0 & 104.0 \\
\hline 1981 & 44.8 & 20.5 & 7.6 & 2.4 & 28.7 & 104.0 \\
\hline 1982 & 46.4 & 21.5 & 8.1 & 2.2 & 26.2 & 104.4 \\
\hline 1983 & 47.6 & 20.5 & 8.5 & 2.7 & 24.8 & 104.1 \\
\hline 1984 & 46.7 & 21.5 & 9.1 & 2.9 & 24.2 & 104.4 \\
\hline 1985 & 45.6 & 21.6 & 9.6 & 3.1 & 24.6 & 104.5 \\
\hline 1986 & 45.7 & 21.2 & 9.8 & 3.2 & 24.8 & 104.7 \\
\hline 1987 & 46.4 & 20.5 & 10.0 & 3.4 & 24.5 & 104.8 \\
\hline 1988 & 46.0 & 20.8 & 10.0 & 3.6 & 24.4 & 104.8 \\
\hline 1989 & 45.7 & 20.8 & 10.1 & 4.0 & 24.2 & 104.8 \\
\hline 1990 & 45.6 & 20.9 & 10.7 & 3.6 & 24.1 & 104.9 \\
\hline 1991 & 45.7 & 21.3 & 10.3 & 3.8 & 24.1 & 105.2 \\
\hline 1992 & 46.0 & 21.2 & 9.9 & 4.3 & 24.0 & 105.4 \\
\hline 1993 & 46.1 & 21.9 & 9.2 & 4.1 & 23.3 & 104.6 \\
\hline 1994 & 45.5 & 22.3 & 9.8 & 4.2 & 23.2 & 105.0 \\
\hline 1995 & 46.4 & 21.8 & 9.7 & 4.5 & 22.8 & 105.2 \\
\hline 1996 & 45.7 & 22.7 & 10.4 & 4.5 & 22.4 & 105.7 \\
\hline 1997 & 45.7 & 22.5 & 10.3 & 4.6 & 22.4 & 105.5 \\
\hline 1998 & 46.2 & 22.3 & 9.9 & 4.4 & 22.9 & 105.7 \\
\hline 1999 & 46.5 & 22.3 & 10.2 & 4.5 & 22.4 & 105.9 \\
\hline 2000 & 46.2 & 23.1 & 10.3 & 4.5 & 22.0 & 106.1 \\
\hline 2001 & 46.2 & 23.8 & 9.8 & 4.3 & 21.6 & 105.7 \\
\hline 2002 & 47.3 & 23.2 & 9.8 & 4.3 & 21.5 & 106.1 \\
\hline 2003 & 46.9 & 23.7 & 9.5 & 4.2 & 22.1 & 106.4 \\
\hline 2004 & 46.8 & 23.9 & 9.7 & 4.0 & 22.2 & 106.6 \\
\hline 2005 & 46.2 & 25.0 & 9.8 & 3.6 & 21.6 & 106.2 \\
\hline 2006 & 45.8 & 25.4 & 9.3 & 3.9 & 21.7 & 106.1 \\
\hline 2007 & 45.5 & 26.1 & 9.1 & 4.1 & 21.5 & 106.3 \\
\hline 2008 & 44.2 & 27.8 & 9.7 & 4.1 & 20.7 & 106.5 \\
\hline 2009 & 46.1 & 26.9 & 9.3 & 4.1 & 20.2 & 106.6 \\
\hline 2010 & 45.7 & 27.5 & 9.3 & 4.3 & 20.3 & 107.1 \\
\hline 2011 & 44.9 & 28.9 & 9.4 & 4.0 & 19.8 & 107.0 \\
\hline 2012 & 45.0 & 29.1 & 9.5 & 4.1 & 19.2 & 106.9 \\
\hline 2013 & 45.0 & 29.5 & 9.5 & 4.0 & 19.0 & 107.0 \\
\hline
\end{tabular}

Source:

Department of Energy, Energy Information Administration, Petroleum Supply Navigator, April 2014. (Additional resources: www.eia.doe.gov)

${ }^{a}$ Includes aviation gasoline $(0.1 \%)$, kerosene $(0.1 \%)$, residual fuel oil $(2.9 \%)$, naphtha and other oils for petrochemical feedstock use $(1.5 \%)$, other oils for petrochemical feedstock use $(0.6 \%)$, special naphthas $(0.3 \%)$, lubricants (1.0\%), waxes $(0.1 \%)$, petroleum coke (5.5\%) asphalt and road oil (2.0\%), still gas (4.4\%), and miscellaneous products $(0.5 \%)$.

${ }^{\mathrm{b}}$ Products sum greater than $100 \%$ due to processing gain. The processing gain for years 1978 to 1980 is assumed to be 4 percent. 
Domestic petroleum production increased in 2009 for the first time in 20 years and has continued to increase. Most of the petroleum imported by the United States is in the form of crude oil. The United States does export small amounts of petroleum, mainly refined petroleum products which go to Canada and Mexico.

Table 1.12

United States Petroleum Production, Imports and Exports, 1950-2013 (million barrels per day)

\begin{tabular}{|c|c|c|c|c|c|c|c|c|c|}
\hline & \multicolumn{3}{|c|}{ Domestic production } & \multicolumn{3}{|c|}{ Net imports } & \multicolumn{3}{|c|}{ Exports } \\
\hline & $\begin{array}{c}\text { Crude } \\
\text { oil }\end{array}$ & $\begin{array}{c}\text { Natural } \\
\text { gas } \\
\text { plant } \\
\text { liquids }\end{array}$ & Total $^{\mathrm{a}}$ & $\begin{array}{c}\text { Crude } \\
\text { oil }\end{array}$ & $\begin{array}{c}\text { Petroleum } \\
\text { products }\end{array}$ & Total & $\begin{array}{c}\text { Crude } \\
\text { oil }\end{array}$ & $\begin{array}{l}\text { Petroleum } \\
\text { products }\end{array}$ & Total \\
\hline 1950 & 5.41 & 0.50 & 5.91 & 0.49 & 0.36 & 0.85 & 0.10 & 0.21 & 0.31 \\
\hline 1955 & 6.81 & 0.77 & 7.58 & 0.78 & 0.47 & 1.25 & 0.03 & 0.34 & 0.37 \\
\hline 1960 & 7.05 & 0.93 & 7.98 & 1.02 & 0.80 & 1.82 & 0.01 & 0.19 & 0.20 \\
\hline 1965 & 7.80 & 1.21 & 9.01 & 1.24 & 1.23 & 2.47 & 0.00 & 0.18 & 0.19 \\
\hline 1970 & 9.64 & 1.66 & 11.30 & 1.32 & 2.10 & 3.42 & 0.01 & 0.25 & 0.26 \\
\hline 1975 & 8.38 & 1.63 & 10.01 & 4.11 & 1.95 & 6.06 & 0.01 & 0.20 & 0.21 \\
\hline 1980 & 8.60 & 1.57 & 10.17 & 5.26 & 1.65 & 6.91 & 0.29 & 0.26 & 0.54 \\
\hline 1985 & 8.97 & 1.61 & 10.58 & 3.20 & 1.87 & 5.07 & 0.20 & 0.58 & 0.78 \\
\hline 1986 & 8.68 & 1.55 & 10.23 & 4.18 & 2.04 & 6.22 & 0.15 & 0.63 & 0.79 \\
\hline 1987 & 8.35 & 1.60 & 9.95 & 4.67 & 2.01 & 6.68 & 0.15 & 0.61 & 0.76 \\
\hline 1988 & 8.16 & 1.63 & 9.97 & 5.11 & 2.29 & 7.40 & 0.16 & 0.66 & 0.82 \\
\hline 1989 & 7.61 & 1.55 & 9.16 & 5.84 & 2.22 & 8.06 & 0.14 & 0.72 & 0.86 \\
\hline 1990 & 7.36 & 1.56 & 8.91 & 5.89 & 2.13 & 8.02 & 0.11 & 0.75 & 0.86 \\
\hline 1991 & 7.42 & 1.66 & 9.08 & 5.78 & 1.85 & 7.63 & 0.12 & 0.89 & 1.00 \\
\hline 1992 & 7.18 & 1.70 & 8.88 & 6.08 & 1.81 & 7.89 & 0.09 & 0.86 & 0.95 \\
\hline 1993 & 6.85 & 1.74 & 8.59 & 6.79 & 1.83 & 8.62 & 0.10 & 0.90 & 1.00 \\
\hline 1994 & 6.66 & 1.73 & 8.39 & 7.06 & 1.94 & 9.00 & 0.10 & 0.84 & 0.94 \\
\hline 1995 & 6.56 & 1.76 & 8.32 & 7.23 & 1.61 & 8.84 & 0.10 & 0.86 & 0.95 \\
\hline 1996 & 6.47 & 1.83 & 8.30 & 7.51 & 1.97 & 9.48 & 0.11 & 0.87 & 0.98 \\
\hline 1997 & 6.45 & 1.82 & 8.27 & 8.23 & 1.93 & 10.16 & 0.11 & 0.90 & 1.00 \\
\hline 1998 & 6.25 & 1.76 & 8.01 & 8.71 & 2.00 & 10.71 & 0.11 & 0.84 & 0.95 \\
\hline 1999 & 5.88 & 1.85 & 7.73 & 8.73 & 2.12 & 10.85 & 0.12 & 0.82 & 0.94 \\
\hline 2000 & 5.82 & 1.91 & 7.73 & 9.07 & 2.39 & 11.46 & 0.05 & 0.99 & 1.04 \\
\hline 2001 & 5.80 & 1.87 & 7.67 & 9.33 & 2.54 & 11.87 & 0.02 & 0.95 & 0.97 \\
\hline 2002 & 5.74 & 1.88 & 7.62 & 9.14 & 2.39 & 11.53 & 0.01 & 0.98 & 0.98 \\
\hline 2003 & 5.65 & 1.72 & 7.37 & 9.67 & 2.60 & 12.26 & 0.01 & 1.01 & 1.03 \\
\hline 2004 & 5.44 & 1.81 & 7.25 & 10.09 & 3.06 & 13.15 & 0.03 & 1.02 & 1.05 \\
\hline 2005 & 5.18 & 1.72 & 6.90 & 10.13 & 3.58 & 13.71 & 0.03 & 1.13 & 1.17 \\
\hline 2006 & 5.09 & 1.74 & 6.83 & 10.12 & 3.59 & 13.71 & 0.03 & 1.29 & 1.32 \\
\hline 2007 & 5.08 & 1.78 & 6.86 & 10.03 & 3.44 & 13.47 & 0.03 & 1.41 & 1.43 \\
\hline 2008 & 5.00 & 1.78 & 6.78 & 9.78 & 3.13 & 12.92 & 0.03 & 1.77 & 1.80 \\
\hline 2009 & 5.35 & 1.91 & 7.26 & 9.01 & 2.68 & 11.69 & 0.04 & 1.98 & 2.02 \\
\hline 2010 & 5.47 & 2.07 & 7.55 & 9.21 & 2.58 & 11.79 & 0.04 & 2.31 & 2.35 \\
\hline 2011 & 5.65 & 2.22 & 7.87 & 8.94 & 2.50 & 11.44 & 0.05 & 2.94 & 2.99 \\
\hline 2012 & 6.49 & 2.41 & 8.90 & 8.53 & 2.07 & 10.60 & 0.07 & 3.14 & 3.21 \\
\hline 2013 & 7.46 & 2.56 & 10.01 & 7.72 & 2.08 & 9.79 & 0.12 & 3.47 & 3.59 \\
\hline \multicolumn{10}{|c|}{ Average annual percentage change } \\
\hline $1950-2013$ & $0.5 \%$ & $2.6 \%$ & $0.8 \%$ & $4.5 \%$ & $2.8 \%$ & $4.0 \%$ & $0.3 \%$ & $4.6 \%$ & $4.0 \%$ \\
\hline 1970-2013 & $-0.6 \%$ & $1.0 \%$ & $-0.3 \%$ & $4.2 \%$ & $0.0 \%$ & $2.5 \%$ & $0.1 \%$ & $6.3 \%$ & $6.3 \%$ \\
\hline 2003-2013 & $2.8 \%$ & $4.1 \%$ & $3.1 \%$ & $-2.2 \%$ & $-2.2 \%$ & $-2.2 \%$ & $28.2 \%$ & $13.1 \%$ & $13.3 \%$ \\
\hline
\end{tabular}

Source:

U.S. Department of Energy, Energy Information Administration, Monthly Energy Review, March 2014, Tables 3.1 and 3.3b. (Additional resources: www.eia.gov)

${ }^{a}$ Total domestic production includes crude oil, natural gas plant liquids and small amounts of other liquids. 
U.S. petroleum production has been increasing and petroleum imports decreasing over the last six years. Net imports of petroleum in 2013 were at a level not seen since the late 1980's.

Table 1.13

Petroleum Production and Transportation Petroleum Consumption in Context, 1950-2013

\begin{tabular}{|c|c|c|c|c|c|c|c|c|}
\hline & $\begin{array}{c}\text { Domestic } \\
\text { petroleum } \\
\text { production }^{\mathrm{a}}\end{array}$ & $\begin{array}{c}\text { Net } \\
\text { petroleum } \\
\text { imports }\end{array}$ & $\begin{array}{l}\text { Transportation } \\
\text { petroleum } \\
\text { consumption }\end{array}$ & $\begin{array}{c}\text { U.S. } \\
\text { petroleum } \\
\text { consumption }\end{array}$ & $\begin{array}{c}\text { World } \\
\text { petroleum } \\
\text { consumption }\end{array}$ & $\begin{array}{l}\text { Net imports } \\
\text { as a share of } \\
\text { U.S. }\end{array}$ & $\begin{array}{l}\text { U.S. petroleum } \\
\text { consumption as } \\
\text { a share of world }\end{array}$ & $\begin{array}{c}\text { Transportation } \\
\text { petroleum use as } \\
\text { a share of } \\
\text { domestic }\end{array}$ \\
\hline & \multicolumn{5}{|c|}{ (million barrels per day) } & consumption & consumption & production \\
\hline 1950 & 5.91 & 0.55 & 3.36 & 6.46 & $\mathrm{~b}$ & $8.4 \%$ & & $56.8 \%$ \\
\hline 1955 & 7.58 & 0.88 & 4.46 & 8.46 & $\mathrm{~b}$ & $10.4 \%$ & $\mathrm{~b}$ & $58.8 \%$ \\
\hline 1960 & 7.99 & 1.62 & $5.15^{-}$ & $9.82^{-}$ & 21.34 & $16.5 \%$ & $46.0 \%$ & $64.5 \%$ \\
\hline 1965 & 9.01 & 2.28 & 6.04 & 11.51 & 31.14 & $19.8 \%$ & $37.0 \%$ & $67.0 \%$ \\
\hline 1970 & 11.30 & 3.16 & 7.78 & 14.70 & 46.81 & $21.5 \%$ & $31.4 \%$ & $68.9 \%$ \\
\hline 1975 & 10.01 & 5.85 & 8.95 & 16.32 & 56.20 & $35.8 \%$ & $29.0 \%$ & $89.4 \%$ \\
\hline 1980 & 10.17 & 6.36 & 9.57 & 17.06 & 63.11 & $37.3 \%$ & $27.0 \%$ & $94.1 \%$ \\
\hline 1985 & 10.58 & 4.29 & 9.84 & 15.73 & 60.08 & $27.3 \%$ & $26.2 \%$ & $93.0 \%$ \\
\hline 1986 & 10.23 & 5.44 & 10.19 & 16.28 & 61.82 & $33.4 \%$ & $26.3 \%$ & $99.6 \%$ \\
\hline 1987 & 9.94 & 5.91 & 10.50 & 16.67 & 63.11 & $35.5 \%$ & $26.4 \%$ & $105.7 \%$ \\
\hline 1988 & 9.76 & 6.59 & 10.88 & 17.28 & 64.98 & $38.1 \%$ & $26.6 \%$ & $111.4 \%$ \\
\hline 1989 & 9.16 & 7.20 & 10.94 & 17.33 & 66.09 & $41.6 \%$ & $26.2 \%$ & $119.4 \%$ \\
\hline 1990 & 8.91 & 7.16 & 10.89 & 16.99 & 66.55 & $42.2 \%$ & $25.5 \%$ & $122.2 \%$ \\
\hline 1991 & 9.08 & 6.63 & 10.76 & 16.71 & 67.21 & $39.6 \%$ & $24.9 \%$ & $118.5 \%$ \\
\hline 1992 & 8.87 & 6.94 & 10.91 & 17.03 & 67.38 & $40.8 \%$ & $25.3 \%$ & $123.0 \%$ \\
\hline 1993 & 8.58 & 7.62 & 11.12 & 17.24 & 67.59 & $44.2 \%$ & $25.5 \%$ & $129.7 \%$ \\
\hline 1994 & 8.39 & 8.05 & 11.13 & 17.72 & 68.93 & $45.5 \%$ & $25.7 \%$ & $132.6 \%$ \\
\hline 1995 & 8.32 & 7.89 & 11.61 & 17.73 & 70.13 & $44.5 \%$ & $25.3 \%$ & $139.5 \%$ \\
\hline 1996 & 8.30 & 8.50 & 11.91 & 18.31 & 71.71 & $46.4 \%$ & $25.5 \%$ & $143.5 \%$ \\
\hline 1997 & 8.27 & 9.16 & 12.05 & 18.62 & 73.46 & $49.2 \%$ & $25.3 \%$ & $145.7 \%$ \\
\hline 1998 & 8.01 & 9.76 & 12.36 & 18.92 & 74.12 & $51.6 \%$ & $25.5 \%$ & $154.3 \%$ \\
\hline 1999 & 7.73 & 9.91 & 12.70 & 19.52 & 75.88 & $50.8 \%$ & $25.7 \%$ & $164.3 \%$ \\
\hline 2000 & 7.73 & 10.42 & 12.98 & 19.70 & 76.78 & $52.9 \%$ & $25.7 \%$ & $167.9 \%$ \\
\hline 2001 & 7.67 & 10.90 & 12.86 & 19.65 & 77.48 & $55.5 \%$ & $25.4 \%$ & $167.7 \%$ \\
\hline 2002 & 7.62 & 10.55 & 13.12 & 19.76 & 78.17 & $53.4 \%$ & $25.3 \%$ & $172.1 \%$ \\
\hline 2003 & 7.36 & 11.24 & 13.20 & 20.03 & 79.71 & $56.1 \%$ & $25.1 \%$ & $178.7 \%$ \\
\hline 2004 & 7.24 & 12.10 & 13.61 & 20.73 & 82.57 & $58.4 \%$ & $25.1 \%$ & $187.7 \%$ \\
\hline 2005 & 6.90 & 12.55 & 13.79 & 20.80 & 84.08 & $60.3 \%$ & $24.7 \%$ & $199.9 \%$ \\
\hline 2006 & 6.83 & 12.39 & 13.95 & 20.69 & 85.20 & $59.9 \%$ & $24.3 \%$ & $204.3 \%$ \\
\hline 2007 & 6.86 & 12.04 & 14.00 & 20.68 & 86.02 & $58.2 \%$ & $24.0 \%$ & $204.0 \%$ \\
\hline 2008 & 6.78 & 11.11 & 13.25 & 19.50 & 84.70 & $57.0 \%$ & $23.0 \%$ & $195.3 \%$ \\
\hline 2009 & 7.26 & 9.67 & 12.84 & 18.77 & 84.92 & $51.5 \%$ & $22.1 \%$ & $176.8 \%$ \\
\hline 2010 & 7.55 & 9.44 & 12.98 & 19.18 & 87.53 & $49.2 \%$ & $21.9 \%$ & $172.0 \%$ \\
\hline 2011 & 7.87 & 8.45 & 12.76 & 18.88 & 88.74 & $44.8 \%$ & $21.3 \%$ & $162.1 \%$ \\
\hline 2012 & 8.89 & 7.39 & 12.52 & 18.49 & 89.41 & $40.0 \%$ & $20.7 \%$ & $140.7 \%$ \\
\hline 2013 & 10.00 & 6.20 & 12.59 & 18.89 & $\mathrm{~b}$ & $32.8 \%$ & $\mathrm{~b}$ & $125.9 \%$ \\
\hline & & & Average ar & nual percenta & ge change & & & \\
\hline 1950-2013 & $0.8 \%$ & $3.9 \%$ & $2.1 \%$ & $1.7 \%$ & b & & & \\
\hline 1970-2013 & $-0.3 \%$ & $1.6 \%$ & $1.1 \%$ & $0.6 \%$ & $\mathrm{~b}$ & & & \\
\hline 2003-2013 & $3.1 \%$ & $-5.8 \%$ & $-0.5 \%$ & $-0.6 \%$ & b & & & \\
\hline
\end{tabular}

Sources:

U.S. Department of Energy, Energy Information Administration, Monthly Energy Review, April 2014, Tables 2.5, 3.1, and A3. (Pre-1973 data from the Annual Energy Review). World petroleum consumption - U.S. Department of Energy, Energy Information Administration, International Energy Statistics Website, May 2013. (Additional resources: www.eia.doe.gov)

a Total domestic production includes crude oil, natural gas plant liquids and small amounts of other liquids.

${ }^{\mathrm{b}}$ Data are not available. 
Before 1989 the U.S. produced enough petroleum to meet the needs of the transportation sector, but was still short of meeting the petroleum needs of all the sectors, including industrial, residential and commercial, and electric utilities. In 1973 the gap between what the U.S. produced and what was consumed was 5.6 million barrels per day. By 2040, the gap is expected to be at least 6.0 million barrels per day if all sources of petroleum are included or 7.3 million barrels per day if only conventional petroleum sources are used.

Figure 1.6. United States Petroleum Production and Consumption - All Sectors, 1973-2040

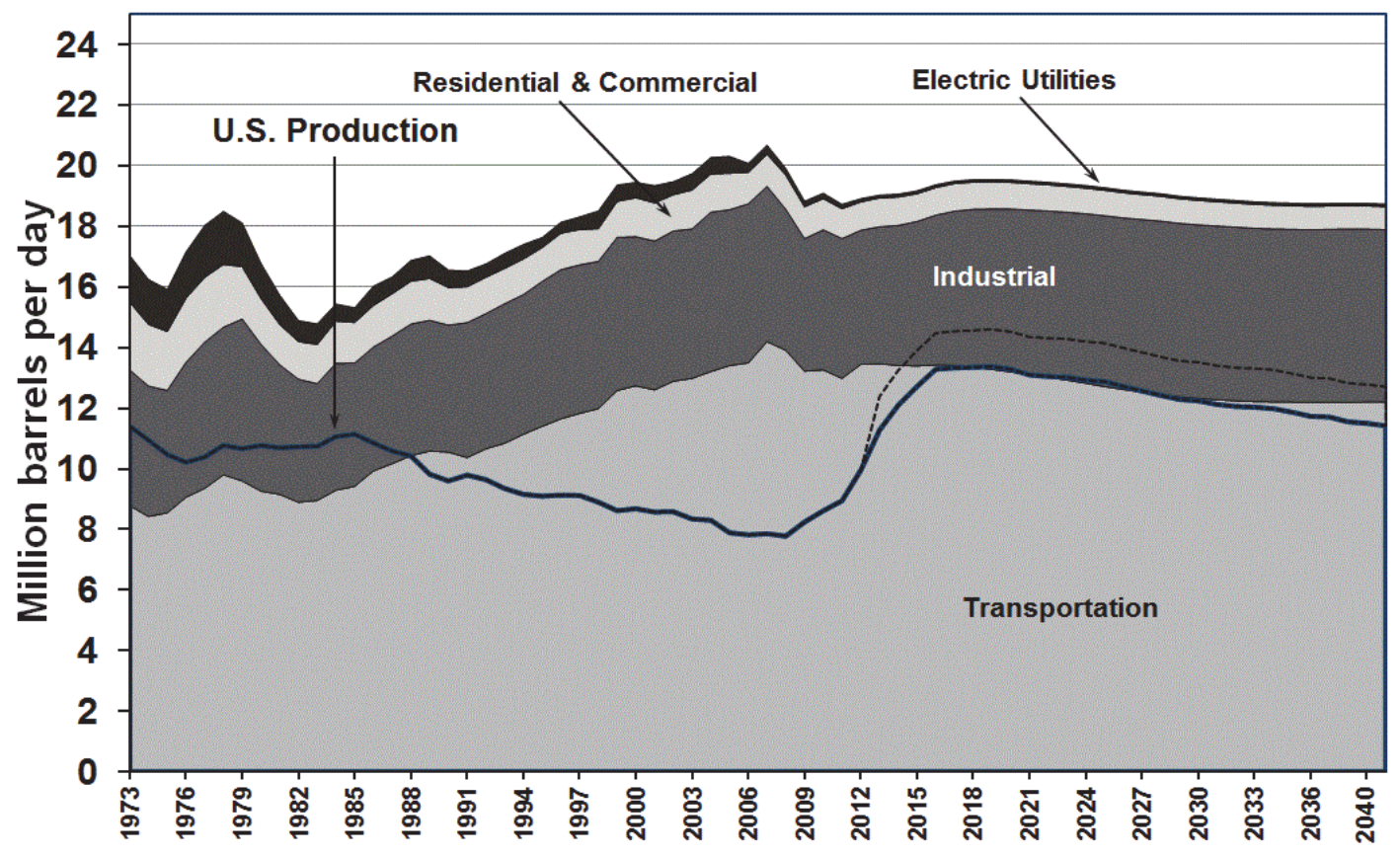

Notes: The U.S. Production has two lines after 2011. The solid line is conventional sources of petroleum, including crude oil, natural gas plant liquids, and refinery gains. The dashed line adds in other non-petroleum sources, including ethanol, biomass, liquids from coal, other blending components, other hydrocarbons, and ethers.

The sharp increase in values between 2006 and 2007 is the result of the FHWA's methodology change. The data change from historical to projected values occurs between 2012 and 2013.

\section{Source:}

See Table 1.12, 2.7 and 2.8. Projections are from the Energy Information Administration, Annual Energy Outlook 2014, May 2014. 
In 1989 the transportation sector petroleum consumption surpassed U.S. petroleum production for the first time, creating a gap that must be met with imports of petroleum. In 2009, however, the U.S. production of petroleum began to increase. The Energy Information Administration expects petroleum production to be nearly equal to transportation consumption by about 2020. When including non-petroleum sources, the production will exceed transportation demand.

Figure 1.7. United States Petroleum Production and Transportation Consumption, 1970-2040

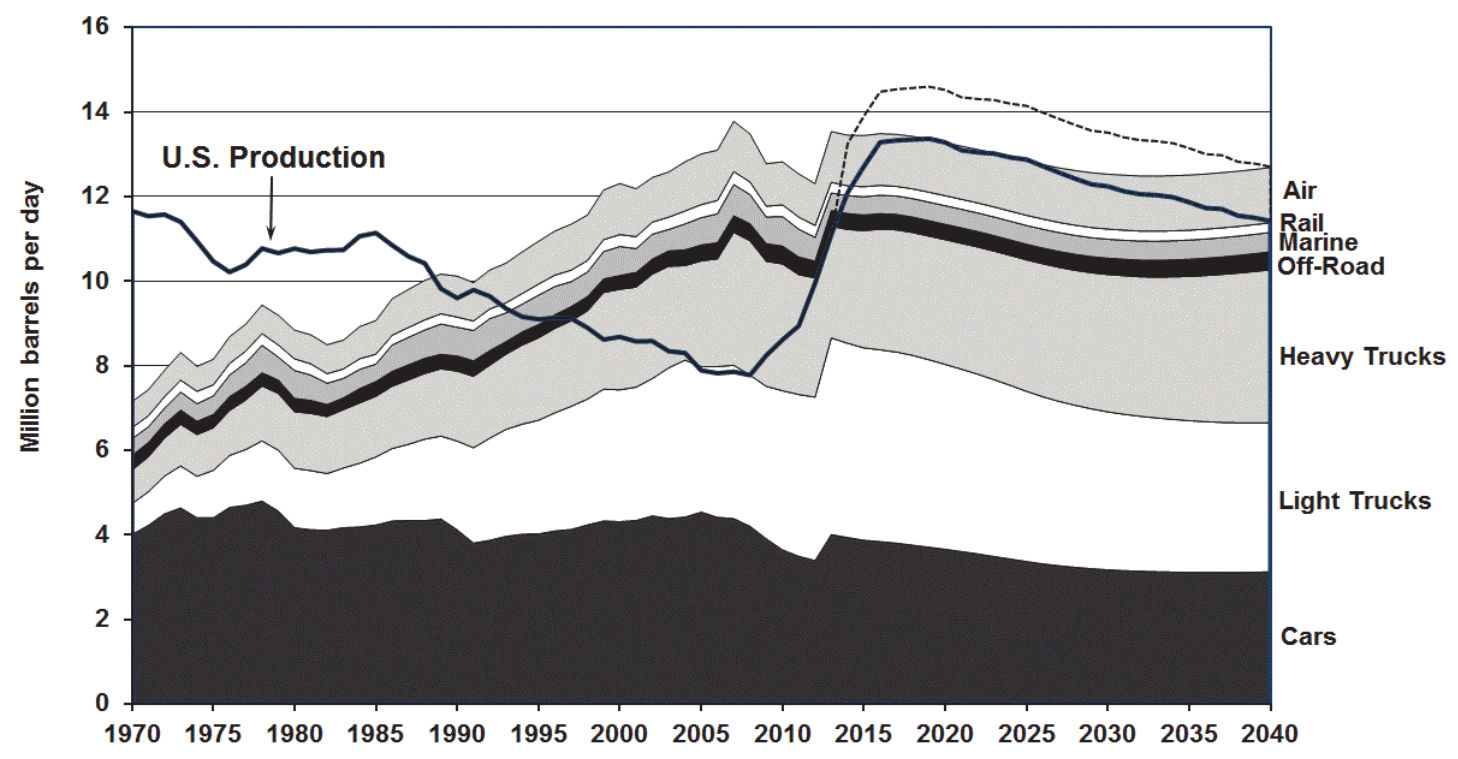

Notes: The U.S. Production has two lines after 2011. The solid line is conventional sources of petroleum, including crude oil, natural gas plant liquids, and refinery gains. The dashed line adds in other non-petroleum sources, including ethanol, biomass, liquids from coal, other blending components, other hydrocarbons, and ethers.

The sharp increase in values between 2012 and 2013 are caused by the data change from historical to projected values. The sharp increase in the value for heavy trucks between 2006 and 2007 is the result of the FHWA's methodology change.

\section{Source:}

See Table 1.12, 2.7 and 2.8. Projections are from the Energy Information Administration, Annual Energy Outlook 2014, May 2014. 
Transportation accounted for almost 70\% of the U.S. petroleum use from 2008 to 2013. Total petroleum consumption reached more than 20 million barrels per day from 2003 to 2007, but has been below that level from 2008 through present.

Table 1.14

Consumption of Petroleum by End-Use Sector, 1973-2013 (million barrels per day)

\begin{tabular}{|c|c|c|c|c|c|c|c|}
\hline Year & Transportation & Percentage & Residential & Commercial & Industrial & $\begin{array}{l}\text { Electric } \\
\text { utilities }\end{array}$ & Total \\
\hline 1973 & 9.05 & $52.3 \%$ & 1.46 & 0.77 & 4.48 & 1.54 & 17.31 \\
\hline 1974 & 8.84 & $53.1 \%$ & 1.33 & 0.70 & 4.30 & 1.48 & 16.65 \\
\hline 1975 & 8.95 & $54.8 \%$ & 1.29 & 0.65 & 4.04 & 1.39 & 16.32 \\
\hline 1976 & 9.40 & $53.7 \%$ & 1.40 & 0.72 & 4.46 & 1.52 & 17.46 \\
\hline 1977 & 9.76 & $53.0 \%$ & 1.39 & 0.75 & 4.82 & 1.71 & 18.43 \\
\hline 1978 & 10.16 & $53.9 \%$ & 1.35 & 0.72 & 4.87 & 1.75 & 18.85 \\
\hline 1979 & 10.00 & $54.0 \%$ & 1.07 & 0.65 & 5.34 & 1.44 & 18.51 \\
\hline 1980 & 9.55 & $56.0 \%$ & 0.89 & 0.63 & 4.84 & 1.15 & 17.06 \\
\hline 1981 & 9.49 & $59.1 \%$ & 0.79 & 0.54 & 4.27 & 0.96 & 16.06 \\
\hline 1982 & 9.31 & $60.8 \%$ & 0.75 & 0.50 & 4.06 & 0.69 & 15.30 \\
\hline 1983 & 9.41 & $61.8 \%$ & 0.72 & 0.57 & 3.85 & 0.68 & 15.23 \\
\hline 1984 & 9.59 & $61.0 \%$ & 0.79 & 0.60 & 4.19 & 0.56 & 15.73 \\
\hline 1985 & 9.84 & $62.6 \%$ & 0.82 & 0.53 & 4.07 & 0.48 & 15.73 \\
\hline 1986 & 10.19 & $62.6 \%$ & 0.80 & 0.57 & 4.09 & 0.64 & 16.28 \\
\hline 1987 & 10.51 & $63.0 \%$ & 0.85 & 0.55 & 4.21 & 0.55 & 16.67 \\
\hline 1988 & 10.84 & $62.7 \%$ & 0.87 & 0.54 & 4.34 & 0.68 & 17.28 \\
\hline 1989 & 10.89 & $62.8 \%$ & 0.87 & 0.51 & 4.31 & 0.74 & 17.33 \\
\hline 1990 & 10.99 & $64.7 \%$ & 0.75 & 0.50 & 4.19 & 0.57 & 16.99 \\
\hline 1991 & 10.56 & $63.2 \%$ & 0.73 & 0.46 & 4.45 & 0.52 & 16.71 \\
\hline 1992 & 10.93 & $64.2 \%$ & 0.76 & 0.45 & 4.46 & 0.44 & 17.02 \\
\hline 1993 & 10.98 & $63.7 \%$ & 0.77 & 0.40 & 4.60 & 0.49 & 17.24 \\
\hline 1994 & 11.46 & $64.7 \%$ & 0.76 & 0.41 & 4.61 & 0.47 & 17.72 \\
\hline 1995 & 11.50 & $64.9 \%$ & 0.74 & 0.38 & 4.78 & 0.33 & 17.73 \\
\hline 1996 & 11.83 & $64.6 \%$ & 0.81 & 0.40 & 4.92 & 0.36 & 18.31 \\
\hline 1997 & 12.14 & $65.2 \%$ & 0.79 & 0.38 & 4.90 & 0.41 & 18.62 \\
\hline 1998 & 12.40 & $65.6 \%$ & 0.72 & 0.36 & 4.86 & 0.58 & 18.92 \\
\hline 1999 & 12.74 & $65.3 \%$ & 0.82 & 0.37 & 5.05 & 0.54 & 19.52 \\
\hline 2000 & 12.99 & $65.9 \%$ & 0.87 & 0.42 & 4.92 & 0.51 & 19.70 \\
\hline 2001 & 12.91 & $65.7 \%$ & 0.85 & 0.41 & 4.91 & 0.57 & 19.65 \\
\hline 2002 & 13.18 & $66.7 \%$ & 0.82 & 0.38 & 4.95 & 0.43 & 19.76 \\
\hline 2003 & 13.28 & $66.3 \%$ & 0.85 & 0.43 & 4.93 & 0.54 & 20.03 \\
\hline 2004 & 13.67 & $65.9 \%$ & 0.84 & 0.42 & 5.26 & 0.54 & 20.73 \\
\hline 2005 & 13.90 & $66.8 \%$ & 0.82 & 0.39 & 5.14 & 0.55 & 20.80 \\
\hline 2006 & 14.11 & $68.2 \%$ & 0.69 & 0.35 & 5.25 & 0.29 & 20.69 \\
\hline 2007 & 14.20 & $68.7 \%$ & 0.72 & 0.34 & 5.12 & 0.30 & 20.68 \\
\hline 2008 & 13.50 & $69.2 \%$ & 0.78 & 0.36 & 4.65 & 0.21 & 19.50 \\
\hline 2009 & 13.17 & $70.1 \%$ & 0.70 & 0.35 & 4.37 & 0.18 & 18.77 \\
\hline 2010 & 13.35 & $69.6 \%$ & 0.68 & 0.35 & 4.62 & 0.18 & 19.18 \\
\hline 2011 & 13.15 & $69.6 \%$ & 0.64 & 0.35 & 4.61 & 0.14 & 18.88 \\
\hline 2012 & 12.87 & $69.6 \%$ & 0.53 & 0.31 & 4.67 & 0.10 & 18.49 \\
\hline 2013 & 13.01 & $68.9 \%$ & 0.49 & 0.27 & 5.00 & 0.12 & 18.89 \\
\hline \multicolumn{8}{|c|}{ Average annual percentage change } \\
\hline 1973-2013 & $0.9 \%$ & & $-2.7 \%$ & $-2.6 \%$ & $0.3 \%$ & $-6.2 \%$ & $0.2 \%$ \\
\hline 2003-2013 & $-0.2 \%$ & & $-5.4 \%$ & $-4.5 \%$ & $0.1 \%$ & $-14.0 \%$ & $-0.9 \%$ \\
\hline
\end{tabular}

\section{Source:}

U.S. Department of Energy, Energy Information Administration, Monthly Energy Review, April 2014, Tables 2.22.6. Converted to million barrels per day using Table A3. (Additional resources: www.eia.doe.gov) 
Light trucks include pick-ups, minivans, sport-utility vehicles, and vans. See Table 2.7 for highway energy use in trillion Btu.

Table 1.15.

Highway Transportation Petroleum Consumption by Mode, 1970-2012 (thousand barrels per day)

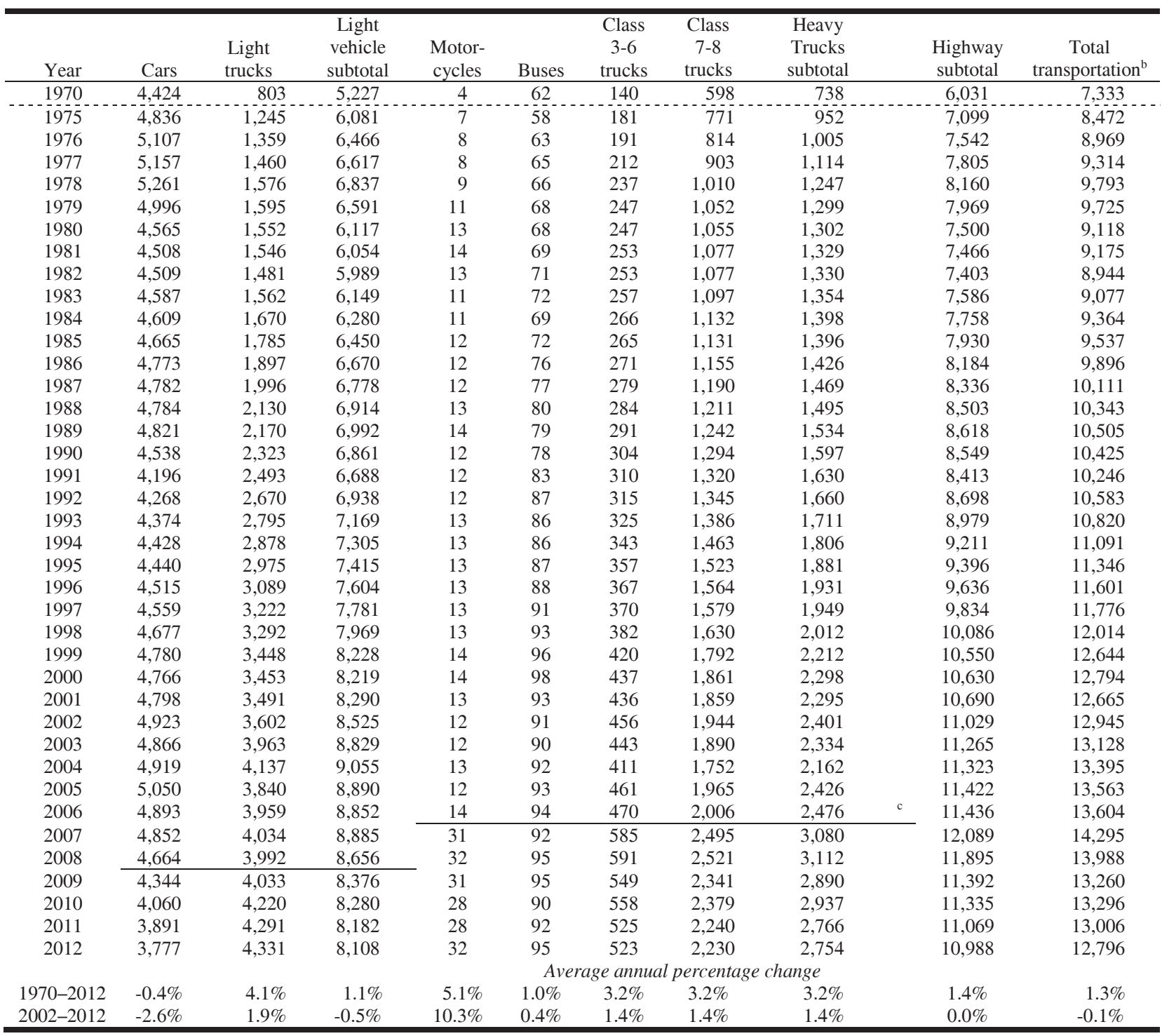

Source:

See Appendix A for Highway Energy Use.

${ }^{\text {a }}$ Each gallon of petroleum product was assumed to equal one gallon of crude oil. The oil used to produce electricity is also estimated. See Appendix A, p. 23 for details.

${ }^{b}$ Total transportation figures do not include military and off-highway energy use and may not include all possible uses of fuel for transportation (e.g., snowmobiles).

${ }^{c}$ Due to changes in the FHWA fuel use methodology, motorcycle, bus, and heavy truck data are not comparable with data before the year 2007. Car and light truck data changed after 2008; see Appendix A for car/light truck shares. 
Although about $19 \%$ of transportation energy use is for nonhighway modes, only $14 \%$ of transportation petroleum use is for nonhighway. This is because some nonhighway modes, such as pipelines and transit rail, use electricity. An estimate for the petroleum used to make electricity is included in the data. See Table 2.8 for nonhighway transportation energy use in trillion Btu.

Table 1.16

Nonhighway Transportation Petroleum Consumption by Mode, 1970-2012 ${ }^{\mathrm{a}}$ (thousand barrels per day)

\begin{tabular}{|c|c|c|c|c|c|c|}
\hline Year & Air & Water & Pipeline & Rail & $\begin{array}{c}\text { Nonhighway } \\
\text { subtotal }\end{array}$ & $\begin{array}{c}\text { Total } \\
\text { transportation }\end{array}$ \\
\hline 1970 & 625 & 381 & 43 & 253 & 1,302 & 7,333 \\
\hline 1975 & 651 & 423 & 50 & 249 & 1,373 & 8,472 \\
\hline 1980 & 697 & $625^{-}$ & 35 & 262 & 1,618 & 9,118 \\
\hline 1981 & 706 & 722 & 29 & 253 & 1,709 & 9,175 \\
\hline 1982 & 701 & 604 & 21 & 214 & 1,541 & 8,944 \\
\hline 1983 & 699 & 561 & 20 & 212 & 1,491 & 9,077 \\
\hline 1984 & 781 & 577 & 16 & 232 & 1,606 & 9,364 \\
\hline 1985 & 814 & 564 & 13 & 216 & 1,606 & 9,537 \\
\hline 1986 & 884 & 601 & 17 & 210 & 1,712 & 9,896 \\
\hline 1987 & 920 & 626 & 15 & 213 & 1,775 & 10,111 \\
\hline 1988 & 958 & 644 & 18 & 220 & 1,840 & 10,343 \\
\hline 1989 & 960 & 688 & 18 & 221 & 1,887 & 10,505 \\
\hline 1990 & 991 & 655 & 14 & 216 & 1,876 & 10,425 \\
\hline 1991 & 928 & 690 & 12 & 202 & 1,833 & 10,246 \\
\hline 1992 & 942 & 724 & 10 & 208 & 1,885 & 10,583 \\
\hline 1993 & 961 & 653 & 11 & 215 & 1,841 & 10,820 \\
\hline 1994 & 1,004 & 635 & 11 & 230 & 1,880 & 11,091 \\
\hline 1995 & 1,036 & 668 & 7 & 239 & 1,950 & 11,346 \\
\hline 1996 & 1,068 & 644 & 8 & 245 & 1,965 & 11,601 \\
\hline 1997 & 1,113 & 574 & 9 & 246 & 1,942 & 11,776 \\
\hline 1998 & 1,102 & 566 & 12 & 248 & 1,927 & 12,014 \\
\hline 1999 & 1,202 & 625 & 11 & 257 & 2,095 & 12,644 \\
\hline 2000 & 1,236 & 662 & 10 & 256 & 2,164 & 12,794 \\
\hline 2001 & 1,161 & 546 & 11 & 257 & 1,975 & 12,665 \\
\hline 2002 & 1,079 & 572 & 8 & 257 & 1,917 & 12,945 \\
\hline 2003 & 1,094 & 496 & 10 & 263 & 1,863 & 13,128 \\
\hline 2004 & 1,188 & 596 & 10 & 278 & 2,073 & 13,395 \\
\hline 2005 & 1,226 & 625 & 10 & 281 & 2,142 & 13,563 \\
\hline 2006 & 1,216 & 661 & 5 & 286 & 2,168 & 13,604 \\
\hline 2007 & 1,215 & 709 & 5 & 277 & 2,206 & 14,295 \\
\hline 2008 & 1,160 & 664 & 4 & 266 & 2,094 & 13,988 \\
\hline 2009 & 1,029 & 613 & 3 & 221 & 1,867 & 13,260 \\
\hline 2010 & 1,040 & 677 & 3 & 241 & 1,961 & 13,296 \\
\hline 2011 & 1,044 & 638 & 3 & 253 & 1,938 & 13,006 \\
\hline 2012 & 1,013 & 545 & 2 & 248 & 1,807 & 12,796 \\
\hline \multicolumn{7}{|c|}{ Average annual percentage change } \\
\hline 1970-2012 & $1.2 \%$ & $0.9 \%$ & $-7.0 \%$ & $0.0 \%$ & $0.8 \%$ & $1.3 \%$ \\
\hline 2002-2012 & $-0.6 \%$ & $-0.5 \%$ & $-12.9 \%$ & $-0.4 \%$ & $-0.6 \%$ & $-0.1 \%$ \\
\hline
\end{tabular}

Source:

See Appendix A for Nonhighway Energy Use.

a Each gallon of petroleum product was assumed to equal one gallon of crude oil. The oil used to produce electricity is also estimated. See Appendix A, p. 23 for details.

${ }^{b}$ Total transportation figures do not include military and off-highway energy use and may not include all possible uses of fuel for transportation (e.g., snowmobiles). 
Highway vehicles were responsible for $85.9 \%$ of all transportation petroleum use in 2012. See Table 2.6 for transportation energy use in trillion Btu.

Table 1.17

Transportation Petroleum Use by Mode, 2011-2012

\begin{tabular}{|c|c|c|c|c|c|c|}
\hline & \multicolumn{2}{|c|}{$\begin{array}{c}\text { Thousand barrels } \\
\text { per day }\end{array}$} & \multicolumn{2}{|c|}{ Percentage of total ${ }^{\mathrm{b}}$} & \multicolumn{2}{|c|}{$\begin{array}{c}\text { Percentage of total U.S. } \\
\text { petroleum } \\
\text { consumption }\end{array}$} \\
\hline & 2011 & 2012 & 2011 & 2012 & 2011 & 2012 \\
\hline HIGHWAY & $11,068.8$ & $10,988.3$ & $85.1 \%$ & $85.9 \%$ & $58.6 \%$ & $59.4 \%$ \\
\hline Light vehicles & $8,209.9$ & $8,139.8$ & $63.1 \%$ & $63.6 \%$ & $43.5 \%$ & $44.0 \%$ \\
\hline Cars & $3,890.8$ & $3,776.8$ & $29.9 \%$ & $29.5 \%$ & $20.6 \%$ & $20.4 \%$ \\
\hline Light trucks ${ }^{\mathrm{c}}$ & $4,291.3$ & $4,331.1$ & $33.0 \%$ & $33.8 \%$ & $22.7 \%$ & $23.4 \%$ \\
\hline Motorcycles & 27.8 & 31.9 & $0.2 \%$ & $0.2 \%$ & $0.1 \%$ & $0.2 \%$ \\
\hline Buses & 93.4 & 94.9 & $0.7 \%$ & $0.7 \%$ & $0.5 \%$ & $0.5 \%$ \\
\hline Transit & 43.6 & 42.7 & $0.3 \%$ & $0.3 \%$ & $0.2 \%$ & $0.2 \%$ \\
\hline Intercity & 15.0 & 15.0 & $0.1 \%$ & $0.1 \%$ & $0.1 \%$ & $0.1 \%$ \\
\hline School & 34.8 & 37.2 & $0.3 \%$ & $0.3 \%$ & $0.2 \%$ & $0.2 \%$ \\
\hline Medium/heavy trucks & $2,765.6$ & $2,753.6$ & $21.3 \%$ & $21.5 \%$ & $14.6 \%$ & $14.9 \%$ \\
\hline Class 3-6 & 525.5 & 523.2 & $4.0 \%$ & $4.1 \%$ & $2.8 \%$ & $2.8 \%$ \\
\hline Class 7-8 & $2,240.1$ & $2,230.4$ & $17.2 \%$ & $17.4 \%$ & $11.9 \%$ & $12.1 \%$ \\
\hline NONHIGHWAY & $1,937.6$ & $1,807.3$ & $14.9 \%$ & $14.1 \%$ & $10.3 \%$ & $9.8 \%$ \\
\hline Air & $1,043.9$ & $1,012.7$ & $8.0 \%$ & $7.9 \%$ & $5.5 \%$ & $5.5 \%$ \\
\hline General aviation & 111.3 & 112.5 & $0.9 \%$ & $0.9 \%$ & $0.6 \%$ & $0.6 \%$ \\
\hline Domestic air carriers & 719.9 & 685.6 & $5.5 \%$ & $5.4 \%$ & $3.8 \%$ & $3.7 \%$ \\
\hline International air carriers & 212.7 & 214.6 & $1.6 \%$ & $1.7 \%$ & $1.1 \%$ & $1.2 \%$ \\
\hline Water & 638.0 & 544.7 & $4.9 \%$ & $4.3 \%$ & $3.4 \%$ & $2.9 \%$ \\
\hline Freight & 512.7 & 419.6 & $3.9 \%$ & $3.3 \%$ & $2.7 \%$ & $2.3 \%$ \\
\hline Recreational & 125.3 & 125.2 & $1.0 \%$ & $1.0 \%$ & $0.7 \%$ & $0.7 \%$ \\
\hline Pipeline & 2.6 & 1.9 & $0.0 \%$ & $0.0 \%$ & $0.0 \%$ & $0.0 \%$ \\
\hline Rail & 253.1 & 247.9 & $1.9 \%$ & $1.9 \%$ & $1.3 \%$ & $1.3 \%$ \\
\hline Freight (Class I) & 242.0 & 237.1 & $1.9 \%$ & $1.9 \%$ & $1.3 \%$ & $1.3 \%$ \\
\hline Passenger & 11.1 & 10.8 & $0.1 \%$ & $0.1 \%$ & $0.1 \%$ & $0.1 \%$ \\
\hline Transit & 0.5 & 0.4 & $0.0 \%$ & $0.0 \%$ & $0.0 \%$ & $0.0 \%$ \\
\hline Commuter & 6.4 & 6.3 & $0.0 \%$ & $0.0 \%$ & $0.0 \%$ & $0.0 \%$ \\
\hline Intercity & 4.2 & 4.2 & $0.0 \%$ & $0.0 \%$ & $0.0 \%$ & $0.0 \%$ \\
\hline $\begin{array}{l}\text { HWY \& NONHWY } \\
\text { TOTAL }^{\text {d }}\end{array}$ & $13,006.4$ & $12,795.6$ & $100.0 \%$ & $100.0 \%$ & $68.9 \%$ & $69.2 \%$ \\
\hline Off-Highway & $1,026.5$ & $1,045.2$ & & & & \\
\hline
\end{tabular}

Source:

See Appendix A for Energy Use Sources.

${ }^{a}$ Each gallon of petroleum product was assumed to equal one gallon of crude oil. The oil used to produce electricity is also estimated. See Appendix A, p. 23 for details.

${ }^{\mathrm{b}}$ Percentages may not sum to totals due to rounding.

${ }^{c}$ Two-axle, four-tire trucks.

d Civilian consumption only. Totals may not include all possible uses of fuels for transportation (e.g., snowmobiles). 


\section{Chapter 2 \\ Energy}

Summary Statistics from Tables in this Chapter

\begin{tabular}{|c|c|c|c|}
\hline \multicolumn{4}{|l|}{ Source } \\
\hline Table 2.1 & $\begin{array}{l}\text { Transportation share of U.S. energy } \\
\text { consumption, } 2012\end{array}$ & & $28.1 \%$ \\
\hline Table 2.2 & $\begin{array}{l}\text { Petroleum share of transportation energy } \\
\text { consumption, } 2013\end{array}$ & & $92.2 \%$ \\
\hline \multirow[t]{8}{*}{ Table 2.3} & Alternative fuel and oxygenate consumptic & $\begin{array}{l}2011 \\
\text { (thousand } \\
\text { gasoline } \\
\text { equivalent } \\
\text { gallons) }\end{array}$ & $\begin{array}{r}\text { (share of Total } \\
\text { alt fuel/oxygenates) }\end{array}$ \\
\hline & Ethanol in gasohol & $8,563,841$ & $85.7 \%$ \\
\hline & Compressed natural gas & 220,247 & $2.2 \%$ \\
\hline & E85 & 137,165 & $1.4 \%$ \\
\hline & Liquefied petroleum gas & 124,457 & $1.2 \%$ \\
\hline & Liquefied natural gas & 26,242 & $0.3 \%$ \\
\hline & Electricity & 7,635 & $0.1 \%$ \\
\hline & $M T B E$ & 0 & $0.0 \%$ \\
\hline \multirow[t]{10}{*}{ Table 2.6} & Transportation energy use by mode, 2012 & (trillion Btu) & $\begin{array}{r}\text { (transportation } \\
\text { energy share) }\end{array}$ \\
\hline & Cars & 7,121 & $27.2 \%$ \\
\hline & Light trucks & 8,179 & $31.2 \%$ \\
\hline & Medium/heavy trucks & 5,776 & $22.0 \%$ \\
\hline & Buses & 200 & $0.8 \%$ \\
\hline & Total Highway & 21,337 & $81.4 \%$ \\
\hline & Air & 2,092 & $8.0 \%$ \\
\hline & Water & 1,187 & $4.5 \%$ \\
\hline & Pipeline & 997 & $3.8 \%$ \\
\hline & Rail & 598 & $2.3 \%$ \\
\hline
\end{tabular}


Petroleum accounted for $34 \%$ of the world's energy use in 2011. Though petroleum is the dominant energy source for OECD countries, the non-OECD countries rely on coal and petroleum.

Figure 2.1. World Consumption of Primary Energy, 2011

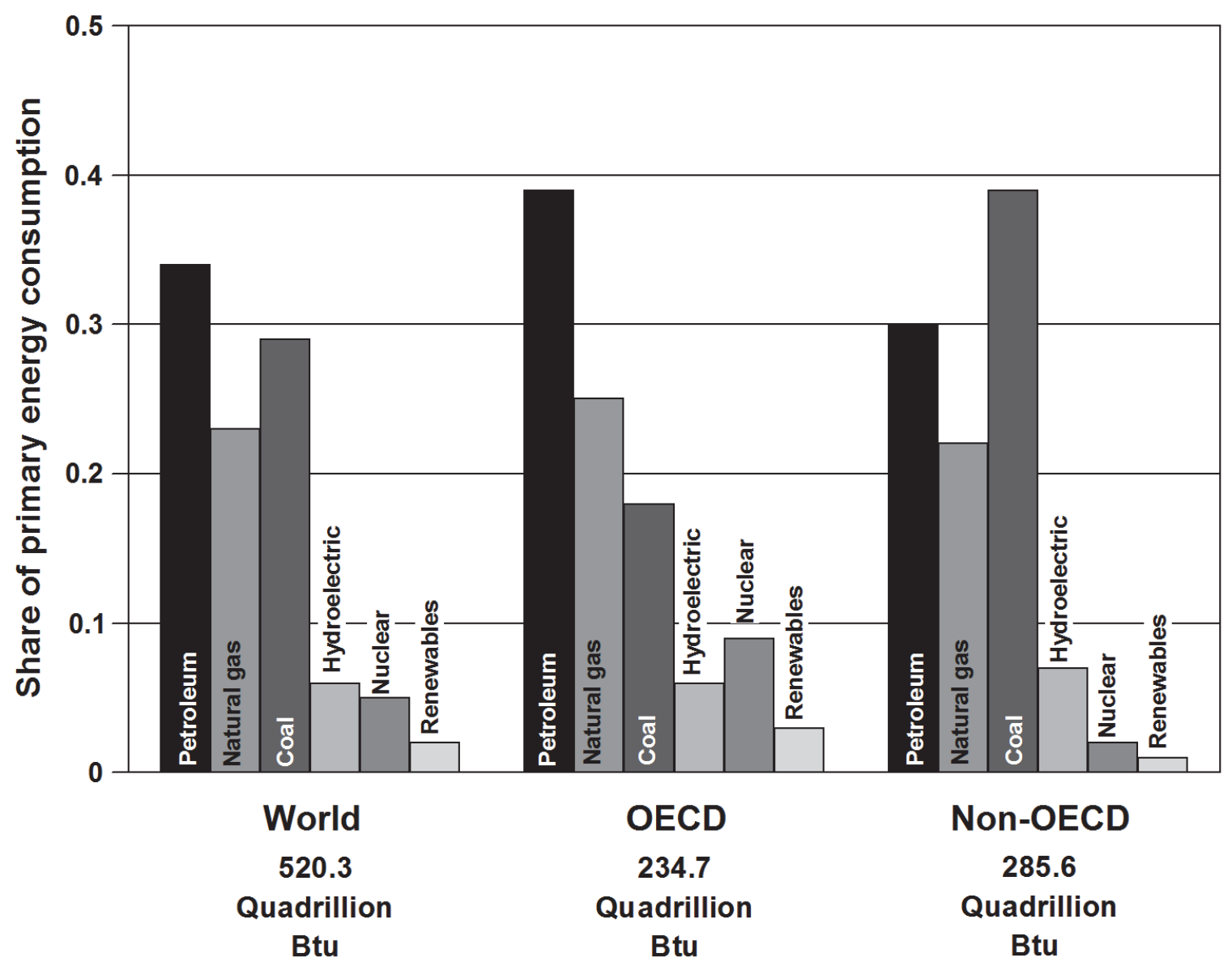

Source:

U.S. Department of Energy, Energy Information Administration, International Energy Statistics Database, April 2014. (Additional resources: www.eia.doe.gov) 
Total energy use was 97.3 quads in 2013 with transportation using 27.0\%. The Energy Information Administration includes renewable energy in each sector.

Table 2.1

\section{U. S. Consumption of Total Energy by End-Use Sector, 1973-2013 (quadrillion Btu)}

\begin{tabular}{|c|c|c|c|c|c|c|}
\hline Year & Transportation & $\begin{array}{c}\text { Percentage } \\
\text { transportation of } \\
\text { total }\end{array}$ & Industrial & Commercial & Residential & Total $^{\mathrm{a}}$ \\
\hline 1973 & 18.6 & $24.6 \%$ & 32.6 & 9.5 & 14.9 & 75.7 \\
\hline 1974 & 18.1 & $24.5 \%$ & 31.8 & 9.4 & 14.7 & 74.0 \\
\hline 1975 & 18.2 & $25.4 \%$ & 29.4 & 9.5 & 14.8 & 72.0 \\
\hline 1976 & 19.1 & $25.1 \%$ & 31.4 & 10.1 & 15.4 & 76.0 \\
\hline 1977 & 19.8 & $25.4 \%$ & 32.3 & 10.2 & 15.7 & 78.0 \\
\hline 1978 & 20.6 & $25.8 \%$ & 32.7 & 10.5 & 16.1 & 80.0 \\
\hline 1979 & 20.5 & $25.3 \%$ & 33.9 & 10.6 & 15.8 & 80.9 \\
\hline 1980 & 19.7 & $25.2 \%$ & 32.0 & 10.6 & 15.8 & 78.1 \\
\hline 1981 & 19.5 & $25.6 \%$ & 30.7 & 10.6 & 15.3 & 76.1 \\
\hline 1982 & 19.1 & $26.1 \%$ & 27.6 & 10.9 & 15.5 & 73.1 \\
\hline 1983 & 19.2 & $26.3 \%$ & 27.4 & 10.9 & 15.4 & 73.0 \\
\hline 1984 & 19.7 & $25.7 \%$ & 29.6 & 11.4 & 16.0 & 76.7 \\
\hline 1985 & 20.1 & $26.3 \%$ & 28.8 & 11.5 & 16.0 & 76.4 \\
\hline 1986 & 20.8 & $27.1 \%$ & 28.3 & 11.6 & 16.0 & 76.7 \\
\hline 1987 & 21.5 & $27.2 \%$ & 28.4 & 11.9 & 16.3 & 79.1 \\
\hline 1988 & 22.3 & $27.0 \%$ & 30.7 & 12.6 & 17.1 & 82.7 \\
\hline 1989 & 22.5 & $26.5 \%$ & 31.3 & 13.2 & 17.8 & 84.8 \\
\hline 1990 & 22.4 & $26.5 \%$ & 31.8 & 13.3 & 16.9 & 84.5 \\
\hline 1991 & 22.1 & $26.2 \%$ & 31.4 & 13.4 & 17.4 & 84.4 \\
\hline 1992 & 22.4 & $26.1 \%$ & 32.6 & 13.4 & 17.4 & 85.8 \\
\hline 1993 & 22.8 & $26.1 \%$ & 32.6 & 13.8 & 18.2 & 87.4 \\
\hline 1994 & 23.4 & $26.3 \%$ & 33.5 & 14.1 & 18.1 & 89.1 \\
\hline 1995 & 23.8 & $26.2 \%$ & 34.0 & 14.7 & 18.5 & 91.0 \\
\hline 1996 & 24.4 & $26.0 \%$ & 34.9 & 15.2 & 19.5 & 94.0 \\
\hline 1997 & 24.8 & $26.2 \%$ & 35.2 & 15.7 & 19.0 & 94.6 \\
\hline 1998 & 25.3 & $26.8 \%$ & 34.8 & 16.0 & 19.0 & 95.0 \\
\hline 1999 & 25.9 & $26.8 \%$ & 34.8 & 16.4 & 19.6 & 96.7 \\
\hline 2000 & 26.5 & $26.9 \%$ & 34.7 & 17.2 & 20.4 & 98.8 \\
\hline 2001 & 26.3 & $27.3 \%$ & 32.7 & 17.1 & 20.0 & 96.2 \\
\hline 2002 & 26.8 & $27.5 \%$ & 32.7 & 17.3 & 20.8 & 97.6 \\
\hline 2003 & 26.9 & $27.5 \%$ & 32.6 & 17.3 & 21.3 & 97.9 \\
\hline 2004 & 27.9 & $27.9 \%$ & 33.5 & 17.7 & 21.1 & 100.2 \\
\hline 2005 & 28.4 & $28.3 \%$ & 32.4 & 17.9 & 21.6 & 100.3 \\
\hline 2006 & 28.8 & $28.9 \%$ & 32.4 & 17.7 & 20.7 & 99.6 \\
\hline 2007 & 29.1 & $28.7 \%$ & 32.4 & 18.3 & 21.5 & 101.3 \\
\hline 2008 & 27.8 & $28.0 \%$ & 31.4 & 18.4 & 21.7 & 99.3 \\
\hline 2009 & 27.1 & $28.7 \%$ & 28.5 & 17.9 & 21.1 & 94.6 \\
\hline 2010 & 27.6 & $28.1 \%$ & 30.5 & 18.1 & 21.9 & 98.0 \\
\hline 2011 & 27.2 & $27.9 \%$ & 30.9 & 18.0 & 21.4 & 97.5 \\
\hline 2012 & 26.8 & $28.2 \%$ & 30.8 & 17.4 & 20.1 & 95.0 \\
\hline 2013 & 27.0 & $27.7 \%$ & 31.3 & 17.9 & 21.1 & 97.3 \\
\hline \multicolumn{7}{|c|}{ Average annual percentage change } \\
\hline 1973-2013 & $0.9 \%$ & & $-0.1 \%$ & $1.6 \%$ & $0.9 \%$ & $0.6 \%$ \\
\hline $2003-2013$ & $0.0 \%$ & & $-0.4 \%$ & $0.3 \%$ & $-0.1 \%$ & $-0.1 \%$ \\
\hline
\end{tabular}

\section{Source:}

U.S. Department of Energy, Energy Information Administration, Monthly Energy Review, March 2014, Washington, DC, Table 2.1. (Additional resources: www.eia.doe.gov)

\footnotetext{
${ }^{a}$ Electrical energy losses have been distributed among the sectors.
} 
In transportation, the alcohol fuels blended into gasoline to make gasohol (10\% ethanol or less) are counted under "renewables" and are not in with petroleum. The petroleum category, however, still contains other blending agents, such as MTBE, that are not actually petroleum, but are not broken out into a separate category.

Table 2.2

Distribution of Energy Consumption by Source, 1973 and 2013 (percentage)

\begin{tabular}{|c|c|c|c|c|c|c|}
\hline \multirow{2}{*}{$\begin{array}{l}\text { Energy } \\
\text { source }\end{array}$} & \multicolumn{2}{|c|}{ Transportation } & \multicolumn{2}{|c|}{ Residential } & \multicolumn{2}{|c|}{ Commercial } \\
\hline & 1973 & 2013 & 1973 & 2013 & 1973 & 2013 \\
\hline Petroleum $^{\mathrm{a}}$ & 95.8 & 92.2 & 18.8 & 4.2 & 16.8 & 2.7 \\
\hline Natural gas ${ }^{\mathrm{b}}$ & 4.0 & 2.9 & 33.4 & 24.0 & 27.8 & 18.7 \\
\hline Coal & 0.0 & 0.0 & 0.6 & 0.0 & 1.7 & 0.3 \\
\hline Renewable & 0.0 & 4.6 & 2.4 & 3.3 & 0.1 & 0.8 \\
\hline Nuclear & 0.0 & 0.0 & 0.0 & 0.0 & 0.0 & 0.0 \\
\hline Electricity $^{\mathrm{c}}$ & 0.2 & 0.3 & 44.8 & 68.5 & 53.6 & 77.5 \\
\hline Total & 100.0 & 100.0 & 100.0 & 100.0 & 100.0 & 100.0 \\
\hline
\end{tabular}

\begin{tabular}{lrrrrr} 
Energy & \multicolumn{2}{c}{ Industrial } & & \multicolumn{2}{c}{ Electric utilities } \\
\cline { 2 - 3 } \cline { 5 - 6 } source & 1973 & 2013 & & 1973 & 2013 \\
\hline Petroleum $^{\mathrm{a}}$ & 27.9 & 27.4 & & 17.9 & 0.7 \\
Natural gas $^{\mathrm{b}}$ & 31.8 & 28.9 & & 19.0 & 21.7 \\
Coal & 12.4 & 4.8 & & 43.9 & 43.0 \\
Renewable & 3.7 & 7.3 & & 14.4 & 12.6 \\
Nuclear & 0.0 & 0.0 & & 4.6 & 21.5 \\
Electricity $^{\mathrm{c}}$ & 24.2 & 31.6 & & 0.2 & 0.5 \\
Total & 100.0 & 100.0 & & 100.0 & 100.0 \\
\hline
\end{tabular}

Source:

U.S. Department of Energy, Energy Information Administration, Monthly Energy Review, March 2014, Washington, DC, Tables 2.2, 2.3, 2.4, 2.5, and 2.6. (Additional resources: www.eia.doe.gov)

\footnotetext{
${ }^{\text {a }}$ In transportation, the petroleum category contains some blending agents which are not petroleum.

${ }^{\mathrm{b}}$ Includes supplemental gaseous fuels. Transportation sector includes pipeline fuel and natural gas vehicle use.

${ }^{\mathrm{c}}$ Includes electrical system energy losses.
} 
Oxygenates are blended with gasoline to be used in conventional vehicles. The amount of oxygenate use dwarfs the alternative fuel use. Gasoline-equivalent gallons are used in this table to allow comparisons of different fuel types.

Table 2.3

Alternative Fuel and Oxygenate Consumption, 2005-2011 (thousand gasoline-equivalent gallons)

\begin{tabular}{|c|c|c|c|c|c|c|c|}
\hline & 2005 & 2006 & 2007 & 2008 & 2009 & 2010 & 2011 \\
\hline \multicolumn{8}{|l|}{ Alternative fuel } \\
\hline Liquefied petroleum gas & 188,171 & 173,130 & 152,360 & 147,784 & 129,631 & 126,354 & 124,457 \\
\hline Compressed natural gas & 166,878 & 172,011 & 178,585 & 189,358 & 199,513 & 210,007 & 220,247 \\
\hline Liquefied natural gas & 22,409 & 23,474 & 24,594 & 25,554 & 25,652 & 26,072 & 26,242 \\
\hline $\mathrm{E} 85^{\mathrm{a}}$ & 38,074 & 44,041 & 54,091 & 62,464 & 71,213 & 90,323 & 137,165 \\
\hline Electricity ${ }^{\mathrm{b}}$ & 5,219 & 5,104 & 5,037 & 5,050 & 4,956 & 4,847 & 7,635 \\
\hline Hydrogen & 25 & 41 & 66 & 117 & 140 & 152 & 174 \\
\hline Biodiesel & 91,649 & 267,623 & 367,764 & 324,329 & 334,809 & 270,170 & 910,968 \\
\hline Other & 2 & 2 & 2 & 2 & 2 & 0 & 0 \\
\hline Subtotal & 512,427 & 685,426 & 782,479 & 754,658 & 756,916 & 727,925 & $1,426,888$ \\
\hline \multicolumn{8}{|l|}{ Oxygenates } \\
\hline $\mathrm{MTBE}^{\mathrm{c}}$ & $1,654,500$ & 435,000 & 0 & 0 & 0 & 0 & 0 \\
\hline Ethanol in gasohol & $2,756,663$ & $3,729,168$ & $4,694,304$ & $6,442,781$ & $7,343,133$ & $8,527,431$ & $8,563,841$ \\
\hline Total & $4,923,590$ & $4,849,594$ & $5,476,783$ & $7,197,439$ & $8,099,342$ & $9,255,356$ & $9,990,729$ \\
\hline
\end{tabular}

Note: These are the latest data available from the Energy Information Administration.

\section{Source:}

U.S. Department of Energy, Energy Information Administration, Alternative Fuel Vehicle Data website, May 2013, www.eia.doe.gov/renewable. (Additional resources: www.eia.doe.gov)

${ }^{\mathrm{a}}$ Consumption includes gasoline portion of the mixture.

${ }^{\mathrm{b}}$ Vehicle consumption only; does not include power plant inputs.

${ }^{c}$ Methyl Tertiary Butyl Ether. This category includes a very small amount of other ethers, primarily Tertiary Amyl Methyl Ether (TAME) and Ethyl Tertiary Butyl Ether (ETBE). 
Ethanol is used as an oxygenate, blended with gasoline to be used as gasohol in conventional vehicles. The amount of ethanol used in gasohol dwarfs the amount used in E85. Production of E95 ended in 2000.

Table 2.4

Ethanol Consumption, 1995-2011 (thousand gallons)

\begin{tabular}{|c|c|c|c|c|}
\hline & \multicolumn{2}{|c|}{ Ethanol blends } & \multirow[b]{2}{*}{ Ethanol in gasohol } & \multirow[b]{2}{*}{ Total } \\
\hline & E85 & E95 & & \\
\hline 1995 & 166 & 970 & 934,615 & 935,751 \\
\hline 2000 & 10,530 & 12 & $1,114,313$ & $1,124,855$ \\
\hline 2001 & 12,756 & 0 & $1,173,323$ & $1,186,079$ \\
\hline 2002 & 15,513 & 0 & $1,450,721$ & $1,466,234$ \\
\hline 2003 & 26,376 & 0 & $1,919,572$ & $1,945,948$ \\
\hline 2004 & 31,581 & 0 & $2,414,167$ & $2,445,748$ \\
\hline 2005 & 38,074 & 0 & $2,756,663$ & $2,794,737$ \\
\hline 2006 & 44,041 & 0 & $3,729,168$ & $3,773,209$ \\
\hline 2007 & 54,091 & 0 & $4,694,304$ & $4,748,395$ \\
\hline 2008 & 62,464 & 0 & $6,442,781$ & $6,505,245$ \\
\hline 2009 & 71,213 & 0 & $7,343,133$ & $7,414,346$ \\
\hline 2010 & 90,323 & 0 & $8,527,431$ & $8,617,754$ \\
\hline 2011 & 137,165 & 0 & $8,563,841$ & $8,701,006$ \\
\hline 2011 Percentage & $1.6 \%$ & $0.0 \%$ & $98.4 \%$ & $100.0 \%$ \\
\hline
\end{tabular}

Note: Gallons of E85 and E95 include the gasoline portion of the blended fuel. Although this estimate for ethanol blend consumption (E85 and E95) is the best available, it is based solely on data from selected fleets (federal and state governments, alternative fuel providers, and transit companies). The ethanol in gasohol column does not include gasoline.

\section{Source:}

U.S. Department of Energy, Energy Information Administration, Alternative Fuel Vehicle website data, Washington, DC, May 2013, website: www.eia.doe.gov/renewable/afv/index.cfm. (Additional resources: www.eia.doe.gov) 
As data about alternative fuel use become available, an attempt is made to incorporate them into this table. Sometimes assumptions must be made in order to use the data. Please see Appendix A for a description of the methodology used to develop these data. See Table 1.17 for transportation petroleum use in thousand barrels per day.

Table 2.5

Domestic Consumption of Transportation Energy by Mode and Fuel Type, $2012^{\mathrm{a}}$ (trillion Btu)

\begin{tabular}{|c|c|c|c|c|c|c|c|c|}
\hline & Gasoline & $\begin{array}{c}\text { Diesel } \\
\text { fuel }\end{array}$ & $\begin{array}{l}\text { Liquefied } \\
\text { petroleum } \\
\text { gas }\end{array}$ & Jet fuel & $\begin{array}{l}\text { Residual } \\
\text { fuel oil }\end{array}$ & $\begin{array}{l}\text { Natural } \\
\text { gas }\end{array}$ & Electricity & Total $^{\mathrm{b}}$ \\
\hline HIGHWAY & $15,493.3$ & $5,755.4$ & 67.8 & - & - & 19.4 & 0.6 & $21,336.6$ \\
\hline$\overline{\text { Light vehicles }}$ & $14,904.2$ & 408.5 & 48.5 & & & - & c & $15,361.2$ \\
\hline Cars & $7,080.5$ & 40.2 & & & & & & $7,120.6$ \\
\hline Light trucks ${ }^{\mathrm{d}}$ & $7,762.6$ & 368.4 & 48.5 & & & & & $8,179.4$ \\
\hline Motorcycles & 61.1 & & & & & & & 61.1 \\
\hline Buses & 8.7 & 171.1 & - & & & 19.4 & 0.6 & 199.8 \\
\hline Transit & 1.6 & 68.0 & - & & & 19.4 & 0.6 & 89.7 \\
\hline Intercity & & 31.9 & & & & & & 31.9 \\
\hline School & 7.1 & 71.2 & & & & & & 78.3 \\
\hline Medium/heavy trucks & 580.4 & $5,175.8$ & 19.3 & - & - & - & - & $5,775.5$ \\
\hline Class 3-6 trucks & 534.0 & 724.6 & 19.1 & & & & & $1,277.7$ \\
\hline Class 7-8 trucks & 46.4 & $4,451.2$ & 0.2 & & & & & $4,497.8$ \\
\hline NONHIGHWAY & 222.0 & 831.7 & - & $2,064.5$ & 686.8 & 750.7 & 318.2 & $4,874.0$ \\
\hline$\overline{\text { Air }}$ & 27.4 & - & - & $2,064.5$ & - & - & - & $2,091.9$ \\
\hline General aviation & 27.4 & & & 201.4 & & & & 228.8 \\
\hline Domestic air carriers & & & & $1,418.9$ & & & & $1,418.9$ \\
\hline International air carriers ${ }^{\mathrm{e}}$ & & & & 444.2 & & & & 444.2 \\
\hline Water & 194.7 & 305.9 & & & 686.8 & & & $1,187.4$ \\
\hline Freight & & 255.8 & & & 686.8 & & & 942.6 \\
\hline Recreational & 194.7 & 50.1 & & & & & & 244.8 \\
\hline Pipeline & - & - & - & - & - & 750.7 & 246.2 & 997.0 \\
\hline Rail & - & 525.9 & - & - & - & - & 72.0 & 597.8 \\
\hline Freight (Class I) & & 504.0 & & & & & & 504.0 \\
\hline Passenger & & 21.8 & & & & & 72.0 & 93.8 \\
\hline Transit & & - & & & & & 47.6 & 47.6 \\
\hline Commuter & & 13.0 & & & & & 18.7 & 31.7 \\
\hline Intercity & & 8.7 & & & & & 5.7 & 14.4 \\
\hline $\begin{array}{l}\text { TOTAL HWY \& } \\
\text { NONHWY }^{b}\end{array}$ & $15,715.3$ & $6,587.1$ & 67.8 & $2,064.5$ & 686.8 & 770.2 & 318.8 & $26,210.6$ \\
\hline
\end{tabular}

Source:

See Appendix A for Energy Use Sources.

a Civilian consumption only. Totals may not include all possible uses of fuels for transportation (e.g., snowmobiles).

b Totals may not sum due to rounding.

${ }^{c}$ Data on electricity of light vehicles are not available.

d Two-axle, four-tire trucks.

${ }^{\mathrm{e}}$ One half of fuel used by domestic carriers in international operation. 
Highway vehicles were responsible for $81.4 \%$ of all transportation energy use in 2012. See Table 1.17 for transportation energy use in thousand barrels per day.

Table 2.6

Transportation Energy Use by Mode, 2011-2012

\begin{tabular}{|c|c|c|c|c|}
\hline & \multicolumn{2}{|c|}{ Trillion Btu } & \multicolumn{2}{|c|}{ Percentage of total based on Btus } \\
\hline & 2011 & 2012 & 2011 & 2012 \\
\hline HIGHWAY & $21,488.4$ & $21,336.6$ & $80.8 \%$ & $81.4 \%$ \\
\hline$\overline{\text { Light vehicles }}$ & $15,493.3$ & $15,361.2$ & $58.2 \%$ & $58.6 \%$ \\
\hline Cars & $7,335.5$ & $7,120.6$ & $27.6 \%$ & $27.2 \%$ \\
\hline Light trucks ${ }^{\mathrm{b}}$ & $8,104.4$ & $8,179.4$ & $30.5 \%$ & $31.2 \%$ \\
\hline Motorcycles & 53.3 & 61.1 & $0.2 \%$ & $0.2 \%$ \\
\hline Buses & 194.6 & 199.9 & $0.7 \%$ & $0.8 \%$ \\
\hline Transit & 91.5 & 89.7 & $0.3 \%$ & $0.3 \%$ \\
\hline Intercity & 29.9 & 31.9 & $0.1 \%$ & $0.1 \%$ \\
\hline School & 73.3 & 78.3 & $0.3 \%$ & $0.3 \%$ \\
\hline Medium/heavy trucks & $5,800.5$ & $\mathbf{5 , 7 7 5 . 5}$ & $21.8 \%$ & $22.0 \%$ \\
\hline Class 3-6 trucks & $1,283.2$ & $1,277.7$ & $4.8 \%$ & $4.9 \%$ \\
\hline Class 7-8 trucks & $4,517.3$ & $4,497.8$ & $17.0 \%$ & $17.2 \%$ \\
\hline NONHIGHWAY & $5,112.0$ & $4,874.0$ & $19.2 \%$ & $18.6 \%$ \\
\hline$\overline{\text { Air }}$ & $2,157.2$ & $2,091.9$ & $8.1 \%$ & $8.0 \%$ \\
\hline General aviation & 227.1 & 228.8 & $0.9 \%$ & $0.9 \%$ \\
\hline Domestic air carriers & $1,489.8$ & $1,418.9$ & $5.6 \%$ & $5.4 \%$ \\
\hline International air & 440.3 & 444.2 & $1.7 \%$ & $1.7 \%$ \\
\hline Water & $1,392.3$ & $1,187.4$ & $5.2 \%$ & $4.5 \%$ \\
\hline Freight & $1,147.4$ & 942.6 & $4.3 \%$ & $3.6 \%$ \\
\hline Recreational & 244.9 & 244.8 & $0.9 \%$ & $0.9 \%$ \\
\hline Pipeline & 953.4 & 997.0 & $3.6 \%$ & $3.8 \%$ \\
\hline Rail & 609.1 & 597.7 & $2.3 \%$ & $2.3 \%$ \\
\hline Freight (Class I) & 514.6 & 504.0 & $1.9 \%$ & $1.9 \%$ \\
\hline Passenger & 94.4 & 93.7 & $0.4 \%$ & $0.4 \%$ \\
\hline Transit & 48.0 & 47.6 & $0.2 \%$ & $0.2 \%$ \\
\hline Commuter & 31.9 & 31.7 & $0.1 \%$ & $0.1 \%$ \\
\hline Intercity & 14.5 & 14.4 & $0.1 \%$ & $0.1 \%$ \\
\hline HWY \& NONHWY TOTAL & $26,600.4$ & $26,210.6$ & $100.0 \%$ & $100.0 \%$ \\
\hline Off-highway & $2,053.9$ & $2,092.6$ & & \\
\hline
\end{tabular}

Source:

See Appendix A for Energy Use Sources.

a Civilian consumption only. Totals may not include all possible uses of fuels for transportation (e.g., snowmobiles).

${ }^{\mathrm{b}}$ Two-axle, four-tire trucks. 
Light trucks include pick-ups, minivans, sport-utility vehicles, and vans. See Table 1.15 for highway petroleum use in thousand barrels per day.

Table 2.7

Highway Transportation Energy Consumption by Mode, 1970-2012 (trillion Btu)

\begin{tabular}{|c|c|c|c|c|c|c|c|c|c|c|c|}
\hline Year & Cars & $\begin{array}{l}\text { Light } \\
\text { trucks }\end{array}$ & $\begin{array}{c}\text { Light } \\
\text { vehicles } \\
\text { subtotal }\end{array}$ & $\begin{array}{l}\text { Motor- } \\
\text { cycles }\end{array}$ & Buses & $\begin{array}{c}\text { Class } \\
3-6 \\
\text { trucks }\end{array}$ & $\begin{array}{c}\text { Class } \\
7-8 \\
\text { trucks }\end{array}$ & $\begin{array}{c}\text { Heavy } \\
\text { trucks } \\
\text { subtotal }\end{array}$ & & $\begin{array}{l}\text { Highway } \\
\text { subtotal }\end{array}$ & $\begin{array}{c}\text { Total } \\
\text { transportation }^{\mathrm{a}}\end{array}$ \\
\hline 1970 & 8,479 & 1,539 & 10,018 & 7 & 129 & 333 & 1,220 & 1,553 & & 11,707 & 15,395 \\
\hline 1975 & 9,298 & 2,384 & 11,682 & 14 & 124 & 430 & 1,574 & 2,003 & & 13,823 & 17,424 \\
\hline 1976 & 9,826 & 2,602 & 12,428 & 15 & 134 & 453 & 1,661 & 2,114 & & 14,691 & 18,492 \\
\hline 1977 & 9,928 & 2,797 & 12,725 & 16 & 137 & 503 & 1,841 & 2,344 & & 15,222 & 19,126 \\
\hline 1978 & 10,134 & 3,020 & 13,154 & 18 & 141 & 672 & 1,935 & 2,607 & & 15,920 & 20,097 \\
\hline 1979 & 9,629 & 3,056 & 12,685 & 22 & 144 & 813 & 1,884 & 2,697 & & 15,548 & 19,652 \\
\hline 1980 & 8,800 & 2,975 & 11,775 & 26 & 143 & 929 & 1,757 & 2,686 & & 14,630 & 18,940 \\
\hline 1981 & 8,693 & 2,963 & 11,656 & 27 & 145 & 1,065 & 1,659 & 2,724 & & 14,552 & 18,741 \\
\hline 1982 & 8,673 & 2,837 & 11,510 & 25 & 151 & 1,182 & 1,525 & 2,707 & & 14,393 & 18,237 \\
\hline 1983 & 8,802 & 2,990 & 11,792 & 22 & 152 & 1,121 & 1,649 & 2,770 & & 14,736 & 18,368 \\
\hline 1984 & 8,837 & 3,197 & 12,034 & 22 & 146 & 1,072 & 1,801 & 2,873 & & 15,075 & 18,962 \\
\hline 1985 & 8,932 & 3,413 & 12,345 & 23 & 153 & 986 & 1,897 & 2,883 & & 15,404 & 19,205 \\
\hline 1986 & 9,138 & 3,629 & 12,767 & 23 & 160 & 920 & 2,038 & 2,958 & & 15,908 & 20,276 \\
\hline 1987 & 9,157 & 3,819 & 12,976 & 24 & 164 & 858 & 2,203 & 3,061 & & 16,225 & 20,771 \\
\hline 1988 & 9,158 & 4,078 & 13,236 & 25 & 169 & 860 & 2,257 & 3,118 & & 16,548 & 21,327 \\
\hline 1989 & 9,232 & 4,156 & 13,388 & 26 & 169 & 869 & 2,330 & 3,199 & & 16,782 & 21,685 \\
\hline 1990 & 8,688 & 4,451 & 13,139 & 24 & 167 & 891 & 2,442 & 3,334 & & 16,664 & 21,581 \\
\hline 1991 & 8,029 & 4,774 & 12,803 & 23 & 177 & 895 & 2,507 & 3,402 & & 16,405 & 21,182 \\
\hline 1992 & 8,169 & 5,117 & 13,286 & 24 & 184 & 897 & 2,570 & 3,468 & & 16,962 & 21,841 \\
\hline 1993 & 8,368 & 5,356 & 13,724 & 25 & 183 & 906 & 2,671 & 3,577 & & 17,509 & 22,322 \\
\hline 1994 & 8,470 & 5,515 & 13,985 & 26 & 183 & 936 & 2,842 & 3,778 & & 17,972 & 22,930 \\
\hline 1995 & 8,489 & 5,695 & 14,184 & 25 & 184 & 954 & 2,983 & 3,937 & & 18,330 & 23,465 \\
\hline 1996 & 8,634 & 5,917 & 14,551 & 24 & 186 & 958 & 3,088 & 4,045 & & 18,806 & 23,974 \\
\hline 1997 & 8,710 & 6,168 & 14,878 & 25 & 192 & 945 & 3,141 & 4,086 & & 19,181 & 24,327 \\
\hline 1998 & 8,936 & 6,304 & 15,240 & 26 & 196 & 967 & 3,251 & 4,218 & & 19,680 & 24,662 \\
\hline 1999 & 9,134 & 6,602 & 15,736 & 26 & 203 & 1,054 & 3,584 & 4,638 & & 20,603 & 25,960 \\
\hline 2000 & 9,100 & 6,607 & 15,707 & 26 & 209 & 1,085 & 3,734 & 4,819 & & 20,761 & 26,273 \\
\hline 2001 & 9,161 & 6,678 & 15,839 & 24 & 196 & 1,074 & 3,738 & 4,813 & & 20,872 & 25,945 \\
\hline 2002 & 9,391 & 6,883 & 16,274 & 24 & 192 & 1,114 & 3,921 & 5,035 & & 21,525 & 26,536 \\
\hline 2003 & 9,255 & 7,551 & 16,806 & 24 & 190 & 1,083 & 3,812 & 4,895 & & 21,915 & 26,715 \\
\hline 2004 & 9,331 & 7,861 & 17,192 & 25 & 194 & 1,003 & 3,532 & 4,535 & & 21,946 & 27,173 \\
\hline 2005 & 9,579 & 7,296 & 16,875 & 24 & 196 & 1,126 & 3,963 & 5,088 & & 22,183 & 27,582 \\
\hline 2006 & 9,296 & 7,534 & 16,830 & 28 & 199 & 1,149 & 4,045 & 5,193 & $\mathrm{~b}$ & 22,286 & 27,760 \\
\hline 2007 & 9,221 & 7,679 & 16,900 & 59 & 195 & 1,429 & 5,031 & 6,460 & & 23,615 & 29,223 \\
\hline 2008 & 8,831 & 7,572 & 16,404 & 61 & 200 & 1,444 & 5,083 & 6,527 & & 23,192 & 28,592 \\
\hline 2009 & 8,209 & 7,635 & 15,843 & 60 & 199 & 1,341 & 4,720 & 6,061 & & 22,164 & 27,107 \\
\hline 2010 & 7,657 & 7,971 & 15,628 & 53 & 190 & 1,363 & 4,797 & 6,160 & & 22,032 & 27,185 \\
\hline 2011 & 7,336 & 8,104 & 15,440 & 53 & 195 & 1,283 & 4,517 & 5,801 & & 21,488 & 26,600 \\
\hline 2012 & 7,121 & 8,179 & 15,300 & 61 & 200 & 1,278 & 4,498 & 5,775 & & 21,337 & 26,211 \\
\hline \multicolumn{12}{|c|}{ Average annual percentage change } \\
\hline 1970-2012 & $-0.4 \%$ & $4.1 \%$ & $1.0 \%$ & $5.3 \%$ & $1.0 \%$ & $3.3 \%$ & $3.2 \%$ & $3.2 \%$ & & $1.4 \%$ & $1.3 \%$ \\
\hline 2002-2012 & $-2.7 \%$ & $1.7 \%$ & $-0.6 \%$ & $9.8 \%$ & $-0.3 \%$ & $1.4 \%$ & $1.4 \%$ & $1.4 \%$ & & $-0.1 \%$ & $-0.1 \%$ \\
\hline
\end{tabular}

Note: Totals may not add due to rounding.

Source:

See Appendix A for Highway Energy Use.

${ }^{a}$ Total transportation figures do not include military and off-highway energy use and may not include all possible uses of fuel for transportation (e.g., snowmobiles). These data have been revised due to a new data series for recreational boats.

${ }^{\mathrm{b}}$ Due to changes in the FHWA fuel use methodology, motorcycle, bus, and heavy truck data are not comparable with data before the year 2007. Car and light truck data changed after 2008; see Appendix A for car/light truck shares. 
About $19 \%$ of transportation energy use is for nonhighway modes. Air travel accounts for almost $43 \%$ of nonhighway energy use. See Table 1.16 for nonhighway petroleum use in thousand barrels per day.

Table 2.8

Nonhighway Transportation Energy Consumption by Mode, 1970-2012 (trillion Btu)

\begin{tabular}{|c|c|c|c|c|c|c|}
\hline Year & Air & Water & Pipeline & Rail & $\begin{array}{c}\text { Nonhighway } \\
\text { subtotal }\end{array}$ & $\begin{array}{c}\text { Total } \\
\text { transportation }^{\mathrm{a}}\end{array}$ \\
\hline 1970 & 1,307 & 836 & 990 & 555 & 3,688 & 15,395 \\
\hline 1975 & 1,274 & 927 & 840 & 559 & 3,601 & 17,424 \\
\hline 1976 & 1,333 & 1,083 & 803 & 581 & 3,800 & 18,492 \\
\hline 1977 & 1,350 & 1,177 & 786 & 591 & 3,904 & 19,126 \\
\hline 1978 & 1,423 & 1,382 & 784 & 588 & 4,177 & 20,097 \\
\hline 1979 & 1,488 & 1,149 & 860 & 607 & 4,104 & 19,652 \\
\hline 1980 & 1,434 & 1,393 & 896 & 588 & 4,310 & 18,940 \\
\hline 1981 & 1,453 & 1,270 & 904 & 561 & 4,189 & 18,741 \\
\hline 1982 & 1,445 & 1,063 & 855 & 481 & 3,844 & 18,237 \\
\hline 1983 & 1,440 & 974 & 740 & 478 & 3,632 & 18,368 \\
\hline 1984 & 1,609 & 964 & 782 & 532 & 3,887 & 18,962 \\
\hline 1985 & 1,677 & 871 & 755 & 498 & 3,801 & 19,205 \\
\hline 1986 & 1,823 & 1,323 & 735 & 487 & 4,368 & 20,276 \\
\hline 1987 & 1,899 & 1,378 & 772 & 498 & 4,546 & 20,771 \\
\hline 1988 & 1,978 & 1,417 & 874 & 511 & 4,779 & 21,327 \\
\hline 1989 & 1,981 & 1,516 & 890 & 515 & 4,903 & 21,685 \\
\hline 1990 & 2,046 & 1,442 & 923 & 506 & 4,918 & 21,581 \\
\hline 1991 & 1,916 & 1,523 & 860 & 478 & 4,777 & 21,182 \\
\hline 1992 & 1,945 & 1,599 & 846 & 490 & 4,879 & 21,841 \\
\hline 1993 & 1,986 & 1,437 & 885 & 505 & 4,813 & 22,322 \\
\hline 1994 & 2,075 & 1,394 & 951 & 539 & 4,958 & 22,930 \\
\hline 1995 & 2,141 & 1,468 & 967 & 559 & 5,135 & 23,465 \\
\hline 1996 & 2,206 & 1,411 & 979 & 572 & 5,167 & 23,974 \\
\hline 1997 & 2,300 & 1,250 & 1,022 & 574 & 5,146 & 24,327 \\
\hline 1998 & 2,275 & 1,232 & 897 & 578 & 4,982 & 24,662 \\
\hline 1999 & 2,483 & 1,367 & 908 & 599 & 5,357 & 25,960 \\
\hline 2000 & 2,554 & 1,454 & 904 & 601 & 5,512 & 26,273 \\
\hline 2001 & 2,397 & 1,186 & 886 & 603 & 5,073 & 25,945 \\
\hline 2002 & 2,229 & 1,247 & 931 & 605 & 5,012 & 26,536 \\
\hline 2003 & 2,260 & 1,074 & 850 & 617 & 4,800 & 26,715 \\
\hline 2004 & 2,456 & 1,299 & 822 & 650 & 5,227 & 27,173 \\
\hline 2005 & 2,532 & 1,368 & 842 & 657 & 5,399 & 27,582 \\
\hline 2006 & 2,511 & 1,450 & 842 & 670 & 5,473 & 27,760 \\
\hline 2007 & 2,509 & 1,559 & 882 & 657 & 5,608 & 29,223 \\
\hline 2008 & 2,396 & 1,460 & 911 & 634 & 5,400 & 28,592 \\
\hline 2009 & 2,127 & 1,341 & 934 & 540 & 4,943 & 27,107 \\
\hline 2010 & 2,149 & 1,485 & 939 & 581 & 5,153 & 27,185 \\
\hline 2011 & 2,157 & 1,392 & 953 & 609 & 5,112 & 26,600 \\
\hline 2012 & 2,092 & 1,187 & 997 & 598 & 4,874 & 26,211 \\
\hline \multicolumn{7}{|c|}{ Average annual percentage change } \\
\hline 1970-2012 & $1.1 \%$ & $0.8 \%$ & $0.0 \%$ & $0.2 \%$ & $0.7 \%$ & $1.3 \%$ \\
\hline 2002-2012 & $-0.6 \%$ & $-0.5 \%$ & $0.7 \%$ & $-0.1 \%$ & $-0.3 \%$ & $-0.1 \%$ \\
\hline
\end{tabular}

Note: Totals may not add due to rounding.

\section{Source:}

See Appendix A for Nonhighway Energy Use.

${ }^{a}$ Total transportation figures do not include military and off-highway energy use and may not include all possible uses of fuel for transportation (e.g., snowmobiles). 
The Environmental Protection Agency's NONROAD2008a model estimates fuel use for different types of equipment and off-highway vehicles. Most of these vehicles/equipment use diesel fuel. Recreational equipment, such as off-highway motorcycles, snowmobiles, and ATVs, are mainly fueled by gasoline.

Table 2.9

Off-Highway Transportation-Related Fuel Consumption from the Nonroad Model, 2012 (trillion Btus)

\begin{tabular}{|c|c|c|c|c|c|}
\hline & Gasoline & Diesel & LPG & $\mathrm{CNG}$ & Total \\
\hline $\begin{array}{l}\text { Agricultural equipment } \\
\text { Tractors, mowers, combines, balers, and other farm } \\
\text { equipment which has utility in its movement. }\end{array}$ & 8.5 & 576.7 & 0.0 & 0.0 & 585.2 \\
\hline Airport ground equipment & 0.3 & 15.1 & 0.2 & $\mathrm{a}$ & 15.6 \\
\hline $\begin{array}{l}\text { Construction and mining equipment } \\
\text { Pavers, rollers, drill rigs, graders, backhoes, } \\
\text { excavators, cranes, mining equipment }\end{array}$ & 11.3 & 924.6 & 1.9 & a & 937.8 \\
\hline $\begin{array}{l}\text { Industrial equipment } \\
\quad \text { Forklifts, terminal tractors, sweeper/scrubbers }\end{array}$ & 7.1 & 120.8 & 200.4 & 15.3 & 343.6 \\
\hline $\begin{array}{l}\text { Logging equipment } \\
\text { Feller/buncher/skidder }\end{array}$ & 1.7 & 22.8 & $a$ & a & 24.6 \\
\hline Railroad maintenance equipment & 0.2 & 3.6 & 0.0 & a & 3.8 \\
\hline $\begin{array}{l}\text { Recreational equipment } \\
\text { Off-road motorcycles, snowmobiles, all-terrain } \\
\text { vehicles, golf carts, specialty vehicles }\end{array}$ & 179.8 & 2.0 & 0.1 & a & 182.0 \\
\hline Total & 208.9 & $1,665.7$ & 202.7 & 15.3 & $2,092.6$ \\
\hline
\end{tabular}

Source:

Environmental Protection Agency, NONROAD2008a model, www.epa.go/oms/nonrdmdl.htm.

${ }^{a}$ There is no equipment listed for this fuel type. 
Mowing equipment consumes nearly half of all the fuel used by lawn and garden equipment. The gasoline used in lawn and garden equipment is $2.0 \%$ of total gasoline use.

Table 2.10

Fuel Consumption from Lawn and Garden Equipment, 2012 (million gallons ${ }^{\mathrm{a}}$ )

\begin{tabular}{|c|c|c|c|c|c|}
\hline Equipment & Classification & Gasoline & Diesel & LPG & $\begin{array}{c}\text { Total fuel } \\
\text { consumption }\end{array}$ \\
\hline \multicolumn{6}{|l|}{ Mowing equipment } \\
\hline Front mowers & Commercial & 20.06 & 118.40 & 0.00 & 138.46 \\
\hline Lawn \& garden tractors & Commercial & 236.72 & 24.44 & 0.00 & 261.16 \\
\hline Lawn \& garden tractors & Residential & 555.28 & 0.00 & 0.00 & 555.28 \\
\hline Lawn mowers & Commercial & 157.90 & 0.00 & 0.00 & 157.90 \\
\hline Lawn mowers & Residential & 211.18 & 0.00 & 0.00 & 211.18 \\
\hline Rear engine riding mowers & Commercial & 17.42 & 0.00 & 0.00 & 17.42 \\
\hline Rear engine riding mowers & Residential & 41.42 & 0.00 & 0.00 & 41.42 \\
\hline Total & & $1,239.98$ & 138.09 & 0.00 & $1,382.81$ \\
\hline \multicolumn{6}{|l|}{ Soil and turf equipment } \\
\hline Commercial turf equipment ${ }^{\mathrm{b}}$ & Commercial & 762.79 & 19.00 & 0.00 & 781.79 \\
\hline Rotary tillers $<6 \mathrm{HP}$ & Commercial & 87.70 & 0.00 & 0.00 & 87.70 \\
\hline Rotary tillers $<6 \mathrm{HP}$ & Residential & 19.08 & 0.00 & 0.00 & 19.08 \\
\hline Total & & 869.57 & 19.00 & 0.00 & 888.57 \\
\hline \multicolumn{6}{|l|}{ Wood cutting equipment } \\
\hline Chain saws $<6 \mathrm{HP}$ & Commercial & 76.33 & 0.00 & 0.00 & 76.33 \\
\hline Chain saws $<6 \mathrm{HP}$ & Residential & 18.26 & 0.00 & 0.00 & 18.26 \\
\hline Chippers/stump grinders & Commercial & 39.52 & 161.12 & 19.03 & 219.66 \\
\hline Shredders < $6 \mathrm{HP}$ & Commercial & 9.41 & 0.00 & 0.00 & 9.41 \\
\hline Total & & 143.52 & 161.12 & 19.03 & 323.67 \\
\hline \multicolumn{6}{|l|}{ Blowers and vacuums } \\
\hline Leafblowers/vacuums & Commercial & 209.72 & 0.02 & 0.00 & 209.74 \\
\hline Leafblowers/vacuums & Residential & 18.54 & 0.00 & 0.00 & 18.54 \\
\hline Snowblowers & Commercial & 36.06 & 2.10 & 0.00 & 38.16 \\
\hline Snowblowers & Residential & 19.09 & 0.00 & 0.00 & 19.09 \\
\hline Total & & 283.42 & 2.12 & 0.00 & 285.53 \\
\hline \multicolumn{6}{|l|}{ Trimming equipment } \\
\hline Trimmers/edgers/brush cutter & Commercial & 64.46 & 0.00 & 0.00 & 63.46 \\
\hline Trimmers/edgers/brush cutter & Residential & 26.50 & 0.00 & 0.00 & 26.50 \\
\hline Other lawn \& garden equipment ${ }^{\mathrm{c}}$ & Commercial & 23.90 & 0.45 & 0.00 & 24.35 \\
\hline Other lawn \& garden equipment ${ }^{\mathrm{c}}$ & Residential & 20.03 & 0.00 & 0.00 & 20.03 \\
\hline Total & & $\mathbf{1 3 4 . 8 8}$ & 0.45 & 0.00 & 135.33 \\
\hline Total all equipment & & $2,671.38$ & 325.52 & 19.03 & $3,015.92$ \\
\hline
\end{tabular}

Source:

U.S. Environmental Protection Agency, NONROAD2008a Model, www.epa.gov/oms/nonrdmdl.htm.

\footnotetext{
${ }^{\text {a }}$ Numbers may not sum due to rounding.

${ }^{\mathrm{b}}$ Includes equipment such as aerators, dethatchers, sod cutters, hydro-seeders, turf utility vehicles, golf course greens mowers, and sand trap groomers.

${ }^{c}$ Includes equipment not otherwise classified such as augers, sickle-bar mowers, and wood splitters.
} 
The Federal Highway Administration (FHWA) cautions that data from 1993 on may not be directly comparable to earlier years. Some states have improved reporting procedures in recent years, and the estimation procedures were revised in 1994. The FHWA no longer publishes separate estimates of gasohol or ethanol used in gasohol. See Table 2.3 for details on oxygenate usage.

Table 2.11

Highway Usage of Gasoline and Diesel, 1973-2012 (billion gallons)

\begin{tabular}{|c|c|c|c|c|}
\hline Year & Total gasoline and gasohol & Diesel $^{\mathrm{a}}$ & Percent diesel & Total highway fuel use \\
\hline 1973 & 100.6 & 9.8 & $8.9 \%$ & 110.5 \\
\hline 1975 & 99.4 & 9.6 & $8.8 \%$ & 109.0 \\
\hline 1980 & 101.2 & 13.8 & $12.0 \%$ & 115.0 \\
\hline 1981 & 99.6 & 14.9 & $13.0 \%$ & 114.5 \\
\hline 1982 & 98.5 & 14.9 & $13.1 \%$ & 113.4 \\
\hline 1983 & 100.1 & 16.0 & $13.8 \%$ & 116.1 \\
\hline 1984 & 101.4 & 17.3 & $14.6 \%$ & 118.7 \\
\hline 1985 & 103.6 & 17.8 & $14.6 \%$ & 121.3 \\
\hline 1986 & 106.8 & 18.4 & $14.7 \%$ & 125.2 \\
\hline 1987 & 108.7 & 19.0 & $14.9 \%$ & 127.7 \\
\hline 1988 & 109.8 & 20.1 & $15.5 \%$ & 129.9 \\
\hline 1989 & 110.6 & 21.2 & $16.1 \%$ & 131.9 \\
\hline 1990 & 110.2 & 21.4 & $16.3 \%$ & 131.6 \\
\hline 1991 & 107.9 & 20.7 & $16.1 \%$ & 128.6 \\
\hline 1992 & 111.0 & 22.0 & $16.5 \%$ & 132.9 \\
\hline 1993 & 113.7 & 23.5 & $17.1 \%$ & 137.2 \\
\hline 1994 & 115.0 & 25.1 & $17.9 \%$ & 140.1 \\
\hline 1995 & 117.1 & 26.2 & $18.3 \%$ & 143.3 \\
\hline 1996 & 119.5 & 27.2 & $18.5 \%$ & 146.7 \\
\hline 1997 & 120.9 & 29.4 & $19.6 \%$ & 150.3 \\
\hline 1998 & 124.7 & 30.2 & $19.5 \%$ & 154.9 \\
\hline 1999 & 128.7 & 31.9 & $19.9 \%$ & 160.7 \\
\hline 2000 & 128.9 & 33.4 & $20.6 \%$ & 162.3 \\
\hline 2001 & 129.7 & 33.4 & $20.5 \%$ & 163.1 \\
\hline 2002 & 133.0 & 34.8 & $20.7 \%$ & 167.8 \\
\hline 2003 & 134.1 & 35.5 & $20.9 \%$ & 169.6 \\
\hline 2004 & 136.5 & 37.4 & $21.5 \%$ & 173.9 \\
\hline 2005 & 135.2 & 39.1 & $22.4 \%$ & 174.3 \\
\hline 2006 & 134.8 & 40.1 & $22.9 \%$ & 174.9 \\
\hline 2007 & 135.4 & 40.7 & $23.1 \%$ & 176.1 \\
\hline 2008 & 132.2 & 38.6 & $22.6 \%$ & 170.8 \\
\hline 2009 & 132.9 & 35.3 & $21.0 \%$ & 168.1 \\
\hline 2010 & 133.1 & 36.6 & $21.6 \%$ & 169.7 \\
\hline 2011 & 131.5 & 37.1 & $22.0 \%$ & 168.6 \\
\hline 2012 & 130.9 & 37.4 & $22.2 \%$ & 168.3 \\
\hline & \multicolumn{4}{|c|}{ Average annual percentage change } \\
\hline 1973-2012 & $0.7 \%$ & $3.5 \%$ & & $1.1 \%$ \\
\hline 2002-2012 & $-0.2 \%$ & $0.7 \%$ & & $0.0 \%$ \\
\hline
\end{tabular}

\section{Source:}

U.S. Department of Transportation, Federal Highway Administration, Highway Statistics 2012, Washington, DC, 2014, Table MF-21 and annual. (Additional resources: www.fhwa.dot.gov) E85.

${ }^{a}$ Consists primarily of diesel fuel, with small quantities of other fuels, such as liquefied petroleum gas and 
Great care should be taken when comparing modal energy intensity data among modes. Because of the inherent differences among the transportation modes in the nature of services, routes available, and many additional factors, it is not possible to obtain truly comparable national energy intensities among modes. These values are averages, and there is a great deal of variability even within a mode.

Table 2.12

Passenger Travel and Energy Use, 2012

\begin{tabular}{|c|c|c|c|c|c|c|c|}
\hline & \multirow[b]{2}{*}{$\begin{array}{c}\text { Number of } \\
\text { vehicles } \\
\text { (thousands) }\end{array}$} & \multirow[b]{2}{*}{$\begin{array}{l}\text { Vehicle- } \\
\text { miles } \\
\text { (millions) }\end{array}$} & \multirow[b]{2}{*}{$\begin{array}{l}\text { Passenger- } \\
\text { miles } \\
\text { (millions) }\end{array}$} & \multirow[b]{2}{*}{$\begin{array}{l}\text { Load factor } \\
\text { (persons/ } \\
\text { vehicle) }\end{array}$} & \multicolumn{2}{|c|}{ Energy intensities } & \multirow[b]{2}{*}{$\begin{array}{c}\text { Energy use } \\
\text { (trillion Btu) }\end{array}$} \\
\hline & & & & & $\begin{array}{l}\text { (Btu per } \\
\text { vehicle- } \\
\text { mile) }\end{array}$ & $\begin{array}{l}\text { (Btu per } \\
\text { passenger- } \\
\text { mile) }\end{array}$ & \\
\hline Cars & $111,290.0$ & $1,438,800$ & $2,230,140$ & 1.55 & 4,949 & 3,193 & $7,120.6$ \\
\hline Personal trucks & $106,312.4$ & $1,026,660$ & $\mathbf{1 , 8 8 9 , 0 5 5}$ & 1.84 & 6,552 & 3,561 & $6,726.5$ \\
\hline Motorcycles & $8,455.0$ & 21,298 & 24,706 & 1.16 & 2,871 & 2,475 & 61.1 \\
\hline Demand response $^{a}$ & 68.6 & 1,618 & 1,756 & 1.1 & 15,348 & 14,143 & 24.8 \\
\hline Transit & 70.8 & 2,417 & 22,251 & 9.2 & 37,105 & 4,030 & 89.7 \\
\hline Intercity $^{\mathrm{c}}$ & b & b & b & b & b & b & 31.9 \\
\hline School $^{\mathrm{c}}$ & 675.1 & $\mathrm{~b}$ & b & b & b & b & 78.3 \\
\hline Air & b & b & b & $\mathrm{b}$ & b & b & $1,647.7$ \\
\hline Certificated route $^{\mathrm{d}}$ & b & 5,500 & 571,146 & 103.8 & 257,965 & 2,484 & $1,418.9$ \\
\hline General aviation & 209.0 & b & $\mathrm{b}$ & b & b & $\mathrm{b}$ & 228.8 \\
\hline Recreational boats & $13,602.0$ & b & b & b & b & b & 244.8 \\
\hline Rail & 20.0 & 1,415 & 37,820 & 26.7 & 66,324 & 2,481 & 93.8 \\
\hline Transit & 12.5 & 750 & 19,835 & 26.5 & 63,469 & 2,398 & 47.6 \\
\hline Commuter & 7.1 & 346 & 11,181 & 32.3 & 91,613 & 2,838 & 31.7 \\
\hline
\end{tabular}

Source:

See Appendix A for Passenger Travel and Energy Use.

${ }^{\text {a }}$ Includes passenger cars, vans, and small buses operating in response to calls from passengers to the transit operator who dispatches the vehicles.

${ }^{\mathrm{b}}$ Data are not available.

${ }^{\mathrm{c}}$ Energy use is estimated.

${ }^{\mathrm{d}}$ Only domestic service and domestic energy use are shown on this table. (Previous editions included half of international energy.) These energy intensities may be inflated because all energy use is attributed to passengerscargo energy use is not taken into account. 
Great care should be taken when comparing modal energy intensity data among modes. Because of the inherent differences among the transportation modes in the nature of services, routes available, and many additional factors, it is not possible to obtain truly comparable national energy intensities among modes. These values are averages, and there is a great deal of variability even within a mode.

Table 2.13

Energy Intensities of Highway Passenger Modes, 1970-2012

\begin{tabular}{|c|c|c|c|c|c|}
\hline \multirow[b]{2}{*}{ Year } & \multicolumn{2}{|c|}{ Cars } & \multirow{2}{*}{$\begin{array}{l}\text { Light truck }^{\mathrm{a}} \\
\text { (Btu per } \\
\text { vehicle-mile) }\end{array}$} & \multicolumn{2}{|c|}{ Transit Buses ${ }^{b}$} \\
\hline & $\begin{array}{c}\text { (Btu per } \\
\text { vehicle-mile) }\end{array}$ & $\begin{array}{c}\text { (Btu per } \\
\text { passenger-mile) }\end{array}$ & & $\begin{array}{c}\text { (Btu per } \\
\text { vehicle-mile) }\end{array}$ & $\begin{array}{c}\text { (Btu per } \\
\text { passenger-mile) }\end{array}$ \\
\hline 1970 & 9,250 & 4,868 & 12,480 & 31,796 & 2,472 \\
\hline 1975 & 8,993 & 4,733 & 11,879 & 33,748 & 2,814 \\
\hline 1980 & 7,916 & 4,279 & 10,224 & 36,553 & 2,813 \\
\hline 1981 & 7,670 & 4,184 & 9,997 & 37,745 & 3,027 \\
\hline 1982 & 7,465 & 4,109 & 9,268 & 38,766 & 3,237 \\
\hline 1983 & 7,365 & 4,092 & 9,124 & 37,962 & 3,177 \\
\hline 1984 & 7,202 & 4,066 & 8,931 & 38,705 & 3,307 \\
\hline 1985 & 7,164 & 4,110 & 8,730 & 38,876 & 3,423 \\
\hline 1986 & 7,194 & 4,197 & 8,560 & 37,889 & 3,545 \\
\hline 1987 & 6,959 & 4,128 & 8,359 & 36,247 & 3,594 \\
\hline 1988 & 6,683 & 4,033 & 8,119 & 36,673 & 3,706 \\
\hline 1989 & 6,589 & 4,046 & 7,746 & 36,754 & 3,732 \\
\hline 1990 & 6,169 & 3,856 & 7,746 & 37,374 & 3,794 \\
\hline 1991 & 5,912 & 3,695 & 7,351 & 37,732 & 3,877 \\
\hline 1992 & 5,956 & 3,723 & 7,239 & 40,243 & 4,310 \\
\hline 1993 & 6,087 & 3,804 & 7,182 & 39,043 & 4,262 \\
\hline 1994 & 6,024 & 3,765 & 7,212 & 37,259 & 4,262 \\
\hline 1995 & 5,902 & 3,689 & 7,208 & 37,251 & 4,307 \\
\hline 1996 & 5,874 & 3,683 & 7,247 & 37,452 & 4,340 \\
\hline 1997 & 5,797 & 3,646 & 7,251 & 38,861 & 4,434 \\
\hline 1998 & 5,767 & 3,638 & 7,260 & 41,296 & 4,399 \\
\hline 1999 & 5,821 & 3,684 & 7,327 & 40,578 & 4,344 \\
\hline 2000 & 5,687 & 3,611 & 7,158 & 41,695 & 4,531 \\
\hline 2001 & 5,626 & 3,583 & 7,080 & 38,535 & 4,146 \\
\hline 2002 & 5,662 & 3,607 & 7,125 & 37,548 & 4,133 \\
\hline 2003 & 5,535 & 3,525 & 7,673 & 37,096 & 4,213 \\
\hline 2004 & 5,489 & 3,496 & 7,653 & 37,855 & 4,364 \\
\hline 2005 & 5,607 & 3,571 & 7,009 & 37,430 & 4,250 \\
\hline 2006 & 5,511 & 3,510 & 6,974 & 39,568 & 4,316 \\
\hline 2007 & 5,513 & 3,512 & 6,904 & 39,931 & 4,372 \\
\hline 2008 & 5,466 & 3,526 & 6,830 & 39,906 & 4,348 \\
\hline 2009 & 5,239 & 3,380 & 7,158 & 39,160 & 4,242 \\
\hline 2010 & 5,117 & 3,301 & 6,919 & 35,953 & 4,118 \\
\hline 2011 & 5,032 & 3,246 & 6,795 & 37,718 & 4,240 \\
\hline 2012 & 4,949 & 3,193 & 6,674 & 37,105 & 4,030 \\
\hline \multicolumn{6}{|c|}{ Average annual percentage change } \\
\hline 1970-2012 & $-1.5 \%$ & $-1.0 \%$ & $-1.5 \%$ & $0.4 \%$ & $1.2 \%$ \\
\hline 2002-2012 & $-1.3 \%$ & $-1.2 \%$ & $-0.7 \%$ & $-0.1 \%$ & $-0.3 \%$ \\
\hline
\end{tabular}

\section{Source:}

See Appendix A for Highway Passenger Mode Energy Intensities.

${ }^{a}$ All two-axle, four-tire trucks.

${ }^{\mathrm{b}}$ Series not continuous between 1983 and 1984 because of a change in data source by the American Public Transportation Association (APTA). 
Great care should be taken when comparing modal energy intensity data among modes. Because of the inherent differences between the transportation modes in the nature of services, routes available, and many additional factors, it is not possible to obtain truly comparable national energy intensities among modes.

Table 2.14

Energy Intensities of Nonhighway Passenger Modes, 1970-2012

\begin{tabular}{|c|c|c|c|c|}
\hline & Air & & Rail & \\
\hline Year & $\begin{array}{c}\text { Certificated air carriers }{ }^{\mathrm{a}} \\
\text { (Btu per } \\
\text { passenger-mile) }\end{array}$ & $\begin{array}{l}\text { Intercity Amtrak } \\
\text { (Btu per } \\
\text { passenger-mile) }\end{array}$ & $\begin{array}{c}\text { Rail transit } \\
\text { (Btu per } \\
\text { passenger-mile) }\end{array}$ & $\begin{array}{l}\text { Commuter rail } \\
\text { (Btu per } \\
\text { passenger-mile) }\end{array}$ \\
\hline 1970 & . . . . . 10,115 & b & 2,157 & $\mathrm{~b}$ \\
\hline 1975 & 7,625 & 3,548 & 2,625 & 5 \\
\hline 1976 & 7,282 & 3,278 & 2,633 & $\mathrm{~b}$ \\
\hline 1977 & 6,990 & 3,443 & 2,364 & $\mathrm{~b}$ \\
\hline 1978 & 6,144 & 3,554 & 2,144 & b \\
\hline 1979 & 5,607 & 3,351 & 2,290 & $\mathrm{~b}$ \\
\hline 1980 & 5,561 & 3,065 & 2,312 & $\mathrm{~b}$ \\
\hline 1981 & 5,774 & 2,883 & 2,592 & $\mathrm{~b}$ \\
\hline 1982 & 5,412 & 3,052 & 2,699 & $\mathrm{~b}$ \\
\hline 1983 & 5,133 & 2,875 & 2,820 & $\mathrm{~b}$ \\
\hline 1984 & 5,298 & 2,923 & 3,037 & 2,804 \\
\hline 1985 & 5,053 & 2,703 & 2,809 & 2,826 \\
\hline 1986 & 5,011 & 2,481 & 3,042 & 2,926 \\
\hline 1987 & 4,827 & 2,450 & 3,039 & 2,801 \\
\hline 1988 & 4,861 & 2,379 & 3,072 & 2,872 \\
\hline 1989 & 4,844 & 2,614 & 2,909 & 2,864 \\
\hline 1990 & 4,797 & 2,505 & 3,024 & 2,822 \\
\hline 1991 & 4,602 & 2,417 & 3,254 & 2,770 \\
\hline 1992 & 4,455 & 2,534 & 3,155 & 2,629 \\
\hline 1993 & 4,490 & 2,565 & 3,373 & 2,976 \\
\hline 1994 & 4,407 & 2,282 & 3,338 & 2,682 \\
\hline 1995 & 4,349 & 2,501 & 3,340 & 2,632 \\
\hline 1996 & 4,199 & 2,690 & 3,017 & 2,582 \\
\hline 1997 & 4,173 & 2,811 & 2,856 & 2,724 \\
\hline 1998 & 3,987 & 2,788 & 2,823 & 2,646 \\
\hline 1999 & 4,108 & 2,943 & 2,785 & 2,714 \\
\hline 2000 & 3,960 & 3,235 & 2,797 & 2,551 \\
\hline 2001 & 3,943 & 3,257 & 2,803 & 2,515 \\
\hline 2002 & 3,718 & 3,212 & 2,872 & 2,514 \\
\hline 2003 & 3,614 & 2,800 & 2,837 & 2,545 \\
\hline 2004 & 3,505 & 2,760 & 2,750 & 2,569 \\
\hline 2005 & 3,346 & 2,709 & 2,783 & 2,743 \\
\hline 2006 & 3,250 & 2,650 & 2,707 & 2,527 \\
\hline 2007 & 3,153 & 2,516 & 2,577 & 2,638 \\
\hline 2008 & 3,055 & 2,398 & 2,521 & 2,656 \\
\hline 2009 & 2,901 & 2,435 & 2,516 & 2,811 \\
\hline 2010 & 2,825 & 2,271 & 2,520 & 2,897 \\
\hline 2011 & 2,772 & 2,214 & 2,459 & 2,794 \\
\hline 2012 & 2,654 & 2,214 & 2,398 & 2,838 \\
\hline \multicolumn{5}{|c|}{ Average annual percentage change ${ }^{c}$} \\
\hline 1970-2012 & $-3.1 \%$ & $-1.3 \%$ & $0.3 \%$ & $0.0 \%$ \\
\hline 2002-2012 & $-3.3 \%$ & $-3.7 \%$ & $-1.8 \%$ & $1.2 \%$ \\
\hline
\end{tabular}

Source:

See Appendix A for Nonhighway Passenger Mode Energy Intensities.

${ }^{a}$ These data differ from the data on Table 2.12 because they include half of international services. These energy intensities may be inflated because all energy use is attributed to passengers-cargo energy use is not taken into account.

${ }^{\mathrm{b}}$ Data are not available.

${ }^{c}$ Average annual percentage calculated to earliest year possible. 
The energy intensity of light rail systems, measured in btu per passenger-mile varies greatly. The weighted average of all light rail systems in 2012 is 3,400 btu/passenger-mile.

\section{Figure 2.2. Energy Intensity of Light Rail Transit Systems, 2012}

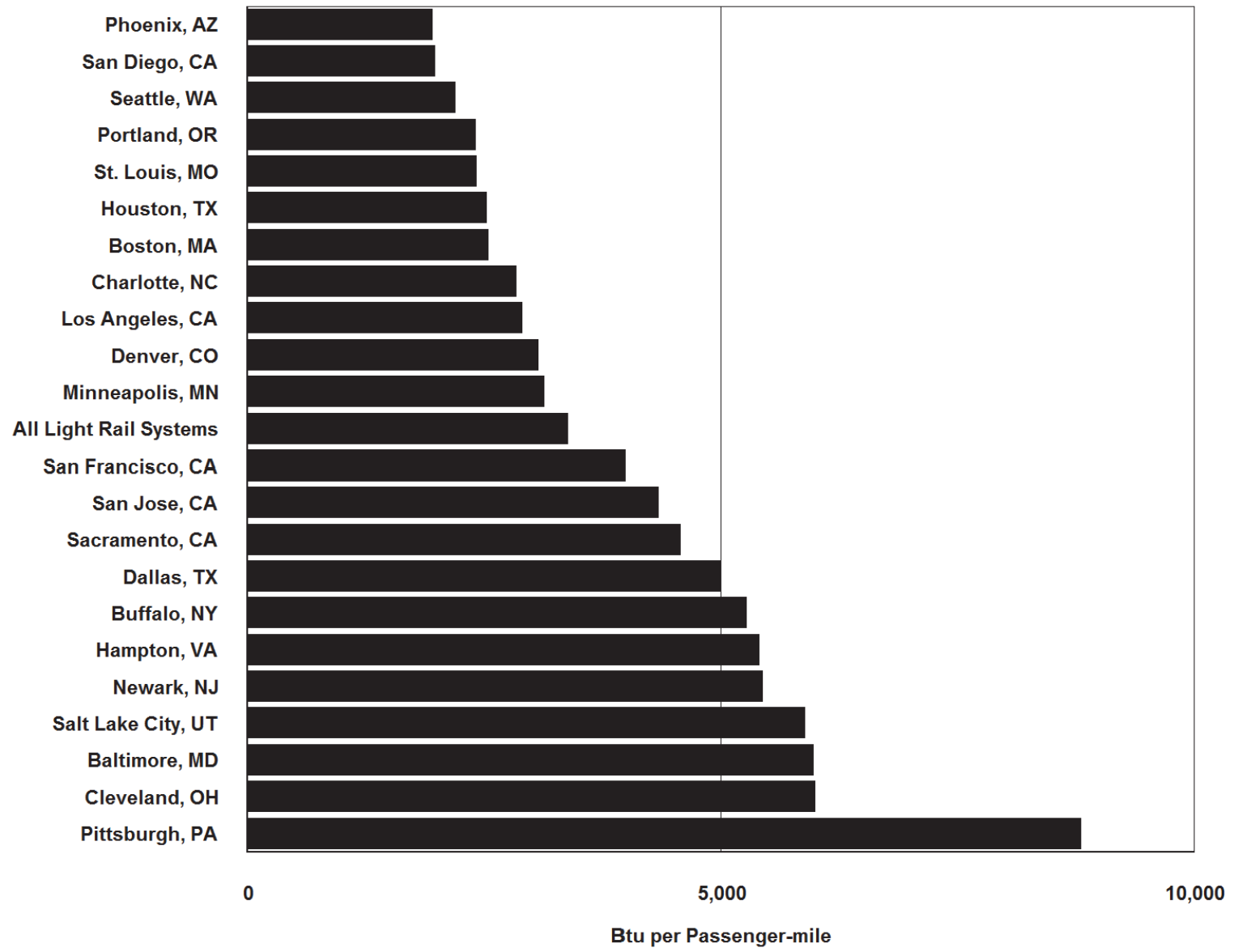

Source:

U.S. Department of Transportation, National Transit Database, May 2014. (Additional resources: www.ntdprogram.gov) 
Figure 2.3. Energy Intensity of Heavy Rail Systems, 2012

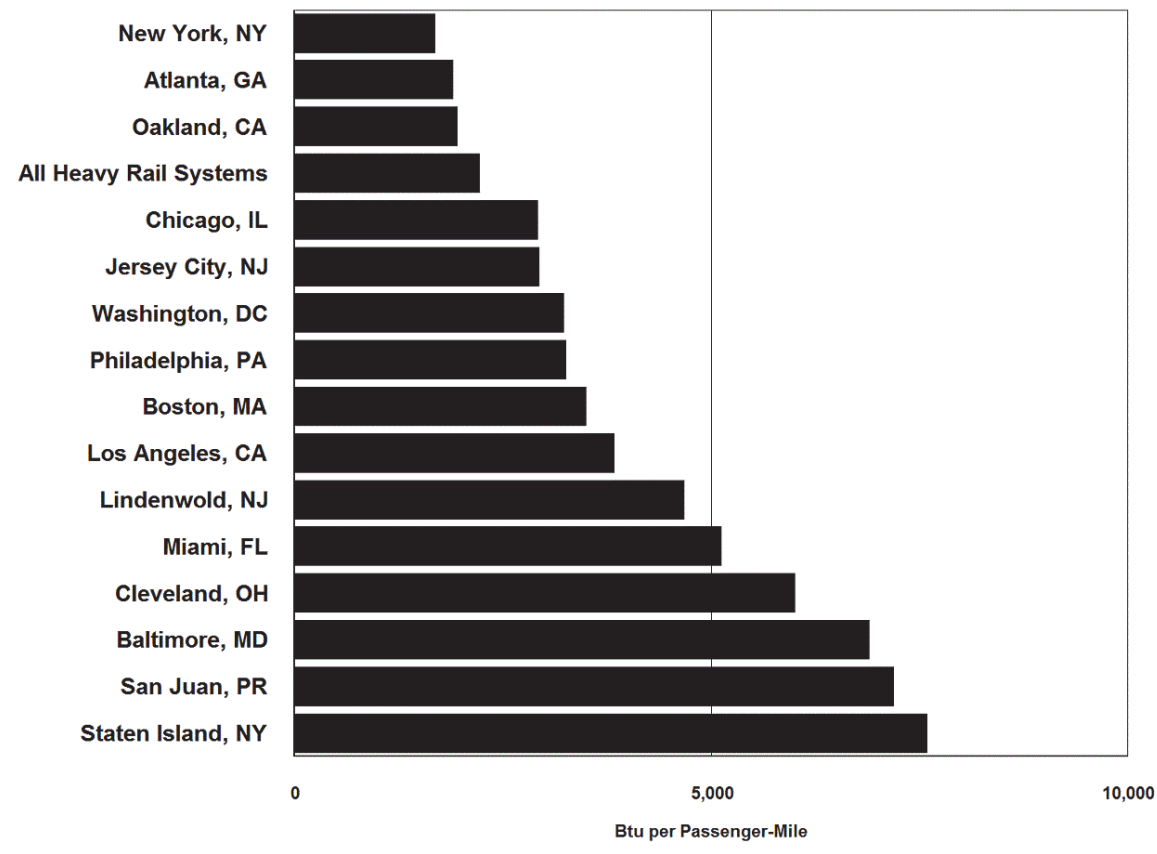

Source:

U.S. Department of Transportation, National Transit Database, May 2014. (Additional resources: www.ntdprogram.gov)

Figure 2.4. Energy Intensity of Commuter Rail Systems, 2012

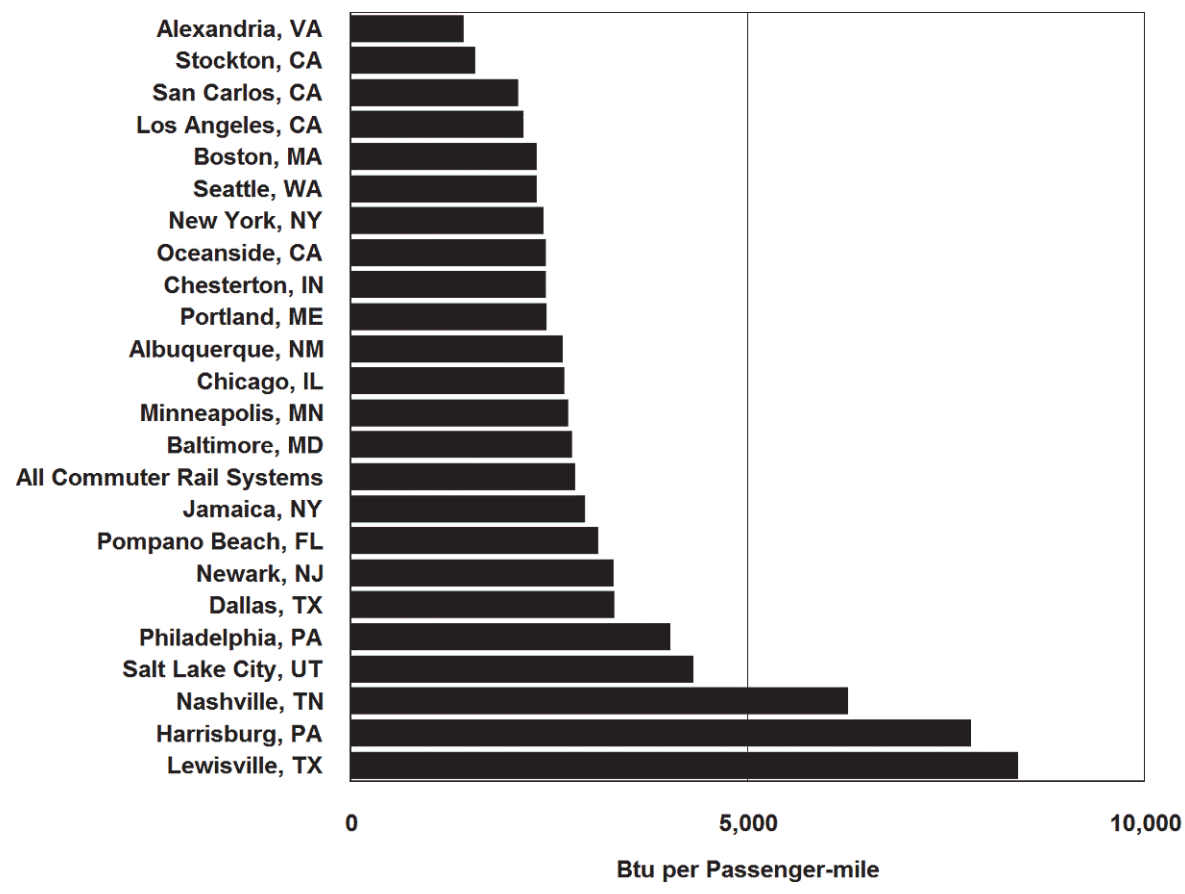

Source:

U.S. Department of Transportation, National Transit Database, May 2014. (Additional resources: www.ntdprogram.gov) 
Great care should be taken when comparing modal energy intensity data among modes. Because of the inherent differences between the transportation modes in the nature of services, routes available, and many additional factors, it is not possible to obtain truly comparable national energy intensities among modes.

Table 2.15

Energy Intensities of Freight Modes, 1970-2012

\begin{tabular}{|c|c|c|c|c|}
\hline \multirow[b]{2}{*}{ Year } & \multirow{2}{*}{$\begin{array}{l}\text { Heavy single-unit and } \\
\text { combination trucks } \\
\text { (Btu per vehicle-mile) }\end{array}$} & \multicolumn{2}{|c|}{ Class I freight railroad } & \multirow{2}{*}{$\begin{array}{c}\text { Waterborne commerce on } \\
\text { taxable waterways } \\
\text { (Btu per ton-mile) }\end{array}$} \\
\hline & & (Btu per freight car-mile) & (Btu per ton-mile) & \\
\hline 1970 & 24,960 & 17,669 & 691 & $\mathrm{a}$ \\
\hline 1975 & 24,631 & 18,739 & 687 & $\bar{a}$ \\
\hline 1976 & 24,567 & 18,938 & 680 & a \\
\hline 1977 & 24,669 & 19,226 & 669 & a \\
\hline 1978 & 24,655 & 18,928 & 641 & a \\
\hline 1979 & 24,746 & 19,188 & 618 & a \\
\hline 1980 & 24,758 & 18,742 & 597 & a \\
\hline 1981 & 25,059 & 18,629 & 572 & a \\
\hline 1982 & 24,297 & 18,404 & 553 & a \\
\hline 1983 & 23,853 & 17,864 & 525 & a \\
\hline 1984 & 23,585 & 17,795 & 510 & a \\
\hline 1985 & 23,343 & 17,500 & 497 & a \\
\hline 1986 & 23,352 & 17,265 & 486 & a \\
\hline 1987 & 22,923 & 16,790 & 456 & a \\
\hline 1988 & 22,596 & 16,758 & 443 & $\mathrm{a}$ \\
\hline 1989 & 22,411 & 16,894 & 437 & a \\
\hline 1990 & 22,795 & 16,619 & 420 & a \\
\hline 1991 & 22,749 & 15,835 & 391 & a \\
\hline 1992 & 22,609 & 16,043 & 393 & a \\
\hline 1993 & 22,373 & 16,056 & 389 & $\mathrm{a}$ \\
\hline 1994 & 22,193 & 16,340 & 388 & a \\
\hline 1995 & 22,097 & 15,992 & 372 & a \\
\hline 1996 & 22,109 & 15,747 & 368 & a \\
\hline 1997 & 21,340 & 15,784 & 370 & 266 \\
\hline 1998 & 21,516 & 15,372 & 365 & 256 \\
\hline 1999 & 22,884 & 15,363 & 363 & 266 \\
\hline 2000 & 23,449 & 14,917 & 352 & 270 \\
\hline 2001 & 23,024 & 15,108 & 346 & 253 \\
\hline 2002 & 23,462 & 15,003 & 345 & 253 \\
\hline 2003 & 22,461 & 15,016 & 344 & 251 \\
\hline 2004 & 20,540 & 15,274 & 341 & 241 \\
\hline 2005 & 22,866 & 15,152 & 337 & 241 \\
\hline 2006 & 23,340 & 14,990 & 330 & 235 \\
\hline 2007 & 21,238 & 14,846 & 320 & 225 \\
\hline 2008 & 21,008 & 14,573 & 305 & 252 \\
\hline 2009 & 21,024 & 13,907 & 291 & 225 \\
\hline 2010 & 21,499 & 13,733 & 289 & 217 \\
\hline 2011 & 21,677 & 14,043 & 298 & 211 \\
\hline \multirow[t]{2}{*}{2012} & 21,525 & 13,800 & 294 & 210 \\
\hline & \multicolumn{3}{|c|}{ Average annual percentage change } & \\
\hline 1970-2012 & $-0.4 \%$ & $-0.6 \%$ & $-2.0 \%$ & a \\
\hline 2002-2012 & $-0.9 \%$ & $-0.8 \%$ & $-1.6 \%$ & $-1.8 \%$ \\
\hline
\end{tabular}

Source:

See Appendix A for Freight Mode Energy Intensities.

${ }^{a}$ Data are not available.

${ }^{\mathrm{b}}$ Due to changes in the FHWA fuel use methodology, truck data are not comparable with data before the year 2007. 


\section{Chapter 3 \\ All Highway Vehicles and Characteristics}

Summary Statistics from Tables in this Chapter

\begin{tabular}{rlr}
\hline Source & & \\
\hline Table 3.2 & U.S. share of world car registrations, 2012 & $15.6 \%$ \\
Table 3.3 & U.S. share of world truck \& bus registrations, 2012 & $38.3 \%$ \\
Table 3.4 & Number of U.S. cars, 2012 (thousands) & 111,290 \\
Table 3.4 & Number of U.S. trucks, 2012 (thousands) & 133,130 \\
Table 3.7 & Vehicle miles traveled, 2012 (million miles) & $2,968,771$ \\
& Cars & $48.5 \%$ \\
& Two-axle, four-tire trucks & $41.3 \%$ \\
& Combination trucks & $5.5 \%$ \\
Table 3.10 & Other single-unit trucks & $3.5 \%$ \\
& Motorcycles & $0.7 \%$ \\
& Buses & $0.5 \%$ \\
& Cars (years) & 11.4 \\
& Light trucks (years) & 11.3 \\
& All light vehicles (years) & 11.4 \\
\hline
\end{tabular}


The top countries producing the world's cars and trucks have changed over the last ten years. In 2012, China was the largest producer of cars and trucks. In 2000, Japan produced the most cars and the United States produced the most trucks (includes light trucks).

Table 3.1

World Production of Cars and Trucks, 2000 and 2012 (thousands)

\begin{tabular}{lrrr}
\hline \multicolumn{1}{c}{ Cars } & & & Percent change \\
China & 2000 & 2012 & $1,670 \%$ \\
Japan & 605 & 10,705 & $2 \%$ \\
Germany & 8,363 & 8,554 & $5 \%$ \\
U.S. & 5,132 & 5,338 & $-26 \%$ \\
Brazil & 5,542 & 4,106 & $90 \%$ \\
India & 1,362 & 2,591 & $317 \%$ \\
Russia & 605 & 2,520 & $103 \%$ \\
Mexico & 969 & 1,969 & $60 \%$ \\
France & 1,130 & 1,810 & $-42 \%$ \\
Spain & 2,880 & 1,683 & $-33 \%$ \\
UK & 2,366 & 1,595 & $-11 \%$ \\
Czech Republic & 1,641 & 1,464 & $174 \%$ \\
All other countries & 428 & 1,172 & $15 \%$ \\
\hline Total world & 10,205 & 1,691 & $34 \%$ \\
\hline & 41,229 & 55,248 & Percent change \\
\multicolumn{1}{c}{ Trucks } & & & $2000-2012$ \\
\hline China & 2000 & 2012 & $485 \%$ \\
U.S. & 1,464 & 8,567 & $-14 \%$ \\
South Korea & 7,263 & 6,227 & $229 \%$ \\
India & 513 & 1,690 & $475 \%$ \\
Thailand & 283 & 1,629 & $376 \%$ \\
Canada & 315 & 1,496 & $1 \%$ \\
Japan & 1,411 & 1,423 & $-22 \%$ \\
All other countries & 1,781 & 1,388 & $26 \%$ \\
\hline Total world & 4,685 & 5,913 & $60 \%$ \\
\hline
\end{tabular}

\section{Source:}

Ward's Communications, Ward's World Motor Vehicle Data, 2013 Edition, Southfield, MI, 2013, pp. 283-288 and annual. (Additional resources: www.wardsauto.com)

${ }^{\text {a }}$ Includes all trucks and buses. In the United States, light trucks, such as pickups, vans, and sport-utility vehicles are included with trucks. 
Use caution comparing historical data because of disconnects in data series. Also, the United States is unique in how many light trucks (SUVs, minivans, pickups) are used for personal travel. Those light trucks are not included on this table. The U.S. share of world cars continues to decline. The growth in the World total comes mainly from developing countries, like China, Indonesia, India, and South Korea.

Table 3.2

Car Registrations for Selected Countries, 1960-2012 (thousands)

\begin{tabular}{|c|c|c|c|c|c|c|c|c|c|c|}
\hline Country & 1960 & 1970 & 1980 & 1990 & 2000 & 2005 & 2008 & 2010 & 2012 & $\begin{array}{c}\text { Average } \\
\text { annual } \\
\text { percentage } \\
\text { change } \\
1990-2012\end{array}$ \\
\hline Argentina & 474 & 1,482 & 3,112 & 4,284 & 5,060 & 5,340 & 6,244 & 7,605 & 9,100 & $3.5 \%$ \\
\hline Brazil & a & a & a & 12,127 & 15,393 & 18,370 & 21,884 & 25,541 & 29,566 & $4.1 \%$ \\
\hline Canada $^{\mathrm{b}}$ & 4,104 & 6,602 & 10,256 & 12,622 & 16,832 & 18,124 & 19,613 & 20,121 & 20,750 & $2.3 \%$ \\
\hline China & a & a & 351 & 1,897 & 3,750 & 8,900 & 18,270 & 34,430 & 52,165 & $16.3 \%$ \\
\hline France & 4,950 & 11,860 & 18,440 & 23,550 & 28,060 & 30,100 & 30,850 & 31,300 & 31,600 & $1.3 \%$ \\
\hline Germany $^{c}$ & 4,856 & 14,376 & 23,236 & 35,512 & 43,772 & 46,090 & 41,321 & 42,302 & 43,431 & $0.9 \%$ \\
\hline India & a & a & a & 2,300 & 5,150 & 7,654 & 9,400 & 13,300 & 18,796 & $10.0 \%$ \\
\hline Indonesia & a & a & $\mathrm{a}$ & 1,200 & a & 3,850 & 4,750 & 8,891 & 10,494 & $10.4 \%$ \\
\hline Japan & 457 & 8,779 & 23,660 & 34,924 & 52,437 & 57,091 & 57,865 & 58,347 & 58,421 & $2.4 \%$ \\
\hline Malaysia & a & a & $\mathrm{a}$ & 1,811 & 4,213 & 6,402 & 7,190 & 9,115 & 9,833 & $8.0 \%$ \\
\hline Pakistan & a & a & a & 738 & 375 & 411 & 445 & 1,726 & 1,997 & $4.6 \%$ \\
\hline Russia & a & a & a & a & 20,353 & 25,285 & 32,021 & 34,350 & 38,482 & $5.5 \%{ }^{\mathrm{d}}$ \\
\hline $\begin{array}{l}\text { South Korea } \\
\text { United }\end{array}$ & a & a & a & 2,075 & 8,084 & 11,122 & 12,484 & 13,632 & 14,577 & $9.9 \%$ \\
\hline Kingdom & 5,650 & 11,802 & 15,438 & 22,528 & 27,185 & 30,652 & 31,252 & 31,258 & 31,482 & $1.5 \%$ \\
\hline United States & 61,671 & 89,244 & 121,601 & 143,550 & 127,721 & 132,909 & 135,882 & 129,053 & 120,902 & $-0.8 \%$ \\
\hline $\begin{array}{l}\text { U.S. percentage } \\
\text { of world }\end{array}$ & $62.7 \%$ & $46.1 \%$ & $38.0 \%$ & $32.3 \%$ & $23.3 \%$ & $21.5 \%$ & $20.4 \%$ & $17.8 \%$ & $15.6 \%$ & \\
\hline World total & 98,305 & 193,479 & 320,390 & 444,900 & 548,558 & 617,914 & 667,630 & 723,567 & 773,323 & $2.5 \%$ \\
\hline
\end{tabular}

\section{Source:}

Ward's Communications, Ward's World Motor Vehicle Data, 2013 Edition, Southfield, MI, 2013, pp. 308-311 and annual. (Additional resources: www.wardsauto.com)

${ }^{\mathrm{a}}$ Data are not available.

${ }^{\mathrm{b}}$ Data from 2000 and later are not comparable to prior data. Canada reclassified autos and trucks prior to 2000.

${ }^{c}$ Data for 1990 and prior include West Germany only. Kraftwagen are included with automobiles.

${ }^{\mathrm{d}}$ Data for earliest year available. 
The United States totals include SUVs, minivans, and light trucks, many of which are used for personal travel.

Table 3.3

Truck and Bus Registrations for Selected Countries, 1960-2012 (thousands)

\begin{tabular}{|c|c|c|c|c|c|c|c|c|c|}
\hline Country & 1960 & 1970 & 1980 & 1990 & 2000 & 2005 & 2010 & 2012 & $\begin{array}{c}\text { Average } \\
\text { annual } \\
\text { percentage } \\
\text { change } \\
\text { 1990-2012 }\end{array}$ \\
\hline Argentina & 392 & 788 & 1,217 & 1,501 & 1,554 & 1,730 & 2,511 & 3,000 & $3.2 \%$ \\
\hline Brazil & a & a & a & 936 & 3,917 & 4,653 & 6,524 & 7,705 & $10.1 \%$ \\
\hline Canada $^{\mathrm{b}}$ & 1,056 & 1,481 & 2,955 & 3,931 & 739 & 786 & 933 & 995 & $-6.1 \%$ \\
\hline China & a & a & 1,480 & 4,314 & 9,650 & 21,750 & 43,590 & 57,275 & $12.5 \%$ \\
\hline France & 1,650 & 1,850 & 2,550 & 4,910 & 5,733 & 6,198 & 6,444 & 6,538 & $1.3 \%$ \\
\hline Germany $^{\mathrm{c}}$ & 786 & 1,228 & 1,617 & 2,764 & 3,534 & 3,133 & 2,960 & 3,107 & $0.5 \%$ \\
\hline India & a & a & a & 2,050 & 2,390 & 4,145 & 9,500 & 10,558 & $7.7 \%$ \\
\hline Indonesia & a & a & a & 1,391 & 2,373 & 2,950 & 6,938 & 7,510 & $8.0 \%$ \\
\hline Japan & 896 & 8,803 & 14,197 & 22,773 & 20,211 & 16,734 & 15,512 & 15,061 & $-1.9 \%$ \\
\hline Malaysia & a & a & a & 616 & 1,030 & 1,323 & 1,138 & 1,171 & $3.0 \%$ \\
\hline Pakistan & a & a & a & 172 & 385 & 414 & 538 & 588 & $5.7 \%$ \\
\hline Russia & a & a & a & 7,200 & 5,041 & 5,705 & 6,304 & 6,901 & $-0.2 \%$ \\
\hline South Korea & a & a & a & 1,320 & 3,956 & 4,275 & 4,310 & 4,293 & $5.5 \%$ \\
\hline United Kingdom & 1,534 & 1,769 & 1,920 & 3,774 & 3,361 & 3,943 & 4,220 & 4,279 & $0.6 \%$ \\
\hline United States & 12,186 & 19,175 & 34,195 & 45,106 & 85,579 & 104,788 & 119,179 & 130,595 & $5.0 \%$ \\
\hline $\begin{array}{l}\text { U.S. percentage } \\
\text { of world }\end{array}$ & $42.6 \%$ & $36.2 \%$ & $37.7 \%$ & $32.7 \%$ & $42.1 \%$ & $42.6 \%$ & $38.5 \%$ & $38.3 \%$ & \\
\hline World total & 28,583 & 52,899 & 90,592 & 138,082 & 203,272 & 245,798 & 309,395 & 341,235 & $4.2 \%$ \\
\hline
\end{tabular}

Source:

Ward's Communications, Ward's World Motor Vehicle Data, 2013 Edition, Southfield, MI, 2013, pp. 308-311 and annual. (Additional resources: www.wardsauto.com)

${ }^{a}$ Data are not available.

${ }^{b}$ Data from 2000 and later are not comparable to prior data. Canada reclassified autos and trucks prior to 2000.

${ }^{c}$ Data for 1990 and prior include West Germany only. Kraftwagen are included with automobiles. 


\section{VEHICLES IN USE}

Both the Federal Highway Administration (FHWA) and The Polk Company report figures on the car and truck population each year. The two estimates, however, differ by as much as $11.2 \%$ (1981). The differences can be attributed to several factors:

- The FHWA data include all vehicles which have been registered at any time throughout the calendar year. Therefore, the data include vehicles which were retired during the year and may double count vehicles which have been registered in different states or the same states to different owners. The Polk Company data include only those vehicles which are registered on July 1 of the given year.

- The classification of mini-vans, station wagons on truck chassis, and utility vehicles as cars or trucks causes important differences in the two estimates. The Polk Company data included passenger vans in the car count until 1980; since 1980 all vans have been counted as trucks.

- Starting in 1993, the FHWA reclassified some minivans and sport utility vehicles into the truck category which were previously included with cars. This change produced a dramatic change in the individual percentage differences of cars and trucks. The difference in total vehicles has been less than 5\% each year since 1990 and does not appear to be significantly affected by the FHWA reclassifications. Beginning with 2009, the FHWA discontinued the car/2-axle, 4-tire truck designations on Table VM-1. The data since 2009 come from Tables MV-1 and MV-9.

- The FHWA data include all non-military Federal vehicles, while The Polk Company data include only those Federal vehicles which are registered within a state. Federal vehicles are not required to have State registrations, and, according to the General Services Administration, most Federal Vehicles are not registered.

- In 2012 both Polk and FHWA changed their methodologies for the car/light truck split which created a significant decrease in the number of cars reported and a corresponding increase in the number of light trucks. 
In the early 1980's, researchers had to make a conscious choice of which data series to use, since they differed by as much as 11\%. In 2005 the two sources differed by less than 1\%. Both sources changed their methodologies for the car/light truck split causing significant decreases to the number of cars.

Table 3.4

U.S. Cars and Trucks in Use, 1970-2012 (thousands)

\begin{tabular}{|c|c|c|c|c|c|c|c|c|c|}
\hline \multirow[b]{2}{*}{ Year } & \multicolumn{3}{|c|}{ Cars } & \multicolumn{3}{|c|}{ Trucks } & \multicolumn{3}{|c|}{ Total } \\
\hline & FHWA & $\begin{array}{l}\text { The Polk } \\
\text { Company }\end{array}$ & $\begin{array}{l}\text { Percentage } \\
\text { difference }\end{array}$ & FHWA & $\begin{array}{l}\text { The Polk } \\
\text { Company }\end{array}$ & $\begin{array}{l}\text { Percentage } \\
\text { difference }\end{array}$ & FHWA & $\begin{array}{l}\text { The Polk } \\
\text { Company }\end{array}$ & $\begin{array}{l}\text { Percentage } \\
\text { difference }\end{array}$ \\
\hline 1970 & 89,243 & 80,448 & $10.9 \%$ & 18,797 & 17,688 & $6.3 \%$ & 108,040 & 98,136 & $10.1 \%$ \\
\hline 1975 & 106,706 & 95,241 & $12.0 \%$ & 25,781 & 24,813 & $3.9 \%$ & 132,487 & 120,054 & $10.4 \%$ \\
\hline 1976 & 110,189 & 97,818 & $12.6 \%$ & 27,876 & 26,560 & $5.0 \%$ & 138,065 & 124,378 & $11.0 \%$ \\
\hline 1977 & 112,288 & 99,904 & $12.4 \%$ & 29,314 & 28,222 & $3.9 \%$ & 141,602 & 128,126 & $10.5 \%$ \\
\hline 1978 & 116,573 & 102,957 & $13.2 \%$ & 31,336 & 30,565 & $2.5 \%$ & 147,909 & 133,522 & $10.8 \%$ \\
\hline 1979 & 118,429 & 104,677 & $13.1 \%$ & 32,914 & 32,583 & $1.0 \%$ & 151,343 & 137,260 & $10.3 \%$ \\
\hline 1980 & 121,601 & 104,564 & $16.3 \%$ & 33,667 & 35,268 & $-4.5 \%$ & 155,267 & 139,832 & $11.0 \%$ \\
\hline 1981 & 123,098 & 105,839 & $16.3 \%$ & 34,644 & 36,069 & $-4.0 \%$ & 157,743 & 141,908 & $11.2 \%$ \\
\hline 1982 & 123,702 & 106,867 & $15.8 \%$ & 35,382 & 36,987 & $-4.3 \%$ & 159,084 & 143,854 & $10.6 \%$ \\
\hline 1983 & 126,444 & 108,961 & $16.0 \%$ & 36,723 & 38,143 & $-3.7 \%$ & 163,166 & 147,104 & $10.9 \%$ \\
\hline 1984 & 128,158 & 112,019 & $14.4 \%$ & 37,507 & 40,143 & $-6.6 \%$ & 165,665 & 152,162 & $8.9 \%$ \\
\hline 1985 & 127,885 & 114,662 & $11.5 \%$ & 43,210 & 42,387 & $1.9 \%$ & 171,095 & 157,049 & $8.9 \%$ \\
\hline 1986 & 130,004 & 117,268 & $10.9 \%$ & 45,103 & 44,826 & $0.6 \%$ & 175,106 & 162,094 & $8.0 \%$ \\
\hline 1987 & 131,482 & 119,849 & $9.7 \%$ & 46,826 & 47,344 & $-1.1 \%$ & 178,308 & 167,193 & $6.6 \%$ \\
\hline 1988 & 133,836 & 121,519 & $10.1 \%$ & 49,941 & 50,221 & $-0.6 \%$ & 183,777 & 171,740 & $7.0 \%$ \\
\hline 1989 & 134,559 & 122,758 & $9.6 \%$ & 52,172 & 53,202 & $-1.9 \%$ & 186,731 & 175,960 & $6.1 \%$ \\
\hline 1990 & 133,700 & 123,276 & $8.5 \%$ & 54,470 & 56,023 & $-2.8 \%$ & 188,171 & 179,299 & $4.9 \%$ \\
\hline 1991 & 128,300 & 123,268 & $4.1 \%$ & 59,206 & 58,179 & $1.8 \%$ & 187,505 & 181,447 & $3.3 \%$ \\
\hline 1992 & 126,581 & 120,347 & $5.2 \%$ & 63,136 & 61,172 & $3.2 \%$ & 189,717 & 181,519 & $4.5 \%$ \\
\hline 1993 & 127,327 & 121,055 & $5.2 \%$ & 66,082 & 65,260 & $1.3 \%$ & 193,409 & 186,315 & $3.8 \%$ \\
\hline 1994 & 127,883 & 121,997 & $4.8 \%$ & 69,491 & 66,717 & $4.2 \%$ & 197,375 & 188,714 & $4.6 \%$ \\
\hline 1995 & 128,387 & 123,242 & $4.2 \%$ & 72,458 & 70,199 & $3.2 \%$ & 200,845 & 193,441 & $3.8 \%$ \\
\hline 1996 & 129,728 & 124,613 & $4.1 \%$ & 75,940 & 73,681 & $3.1 \%$ & 205,669 & 198,294 & $3.7 \%$ \\
\hline 1997 & 129,749 & 124,673 & $4.1 \%$ & 77,307 & 76,398 & $1.2 \%$ & 207,056 & 201,071 & $3.0 \%$ \\
\hline 1998 & 131,839 & 125,966 & $4.7 \%$ & 79,062 & 79,077 & $0.0 \%$ & 210,901 & 205,043 & $2.9 \%$ \\
\hline 1999 & 132,432 & 126,869 & $4.4 \%$ & 83,148 & 82,640 & $0.6 \%$ & 215,580 & 209,509 & $2.9 \%$ \\
\hline 2000 & 133,621 & 127,721 & $4.6 \%$ & 87,108 & 85,579 & $1.8 \%$ & 220,729 & 213,300 & $3.5 \%$ \\
\hline 2001 & 137,633 & 128,714 & $6.9 \%$ & 92,045 & 87,969 & $4.6 \%$ & 229,678 & 216,683 & $6.0 \%$ \\
\hline 2002 & 135,921 & 129,907 & $4.6 \%$ & 92,939 & 91,120 & $2.0 \%$ & 228,860 & 221,027 & $3.5 \%$ \\
\hline 2003 & 135,670 & 131,072 & $3.5 \%$ & 94,944 & 94,810 & $0.1 \%$ & 230,614 & 225,882 & $2.1 \%$ \\
\hline 2004 & 136,431 & 132,469 & $3.0 \%$ & 100,016 & 99,698 & $0.3 \%$ & 236,447 & 232,167 & $1.8 \%$ \\
\hline 2005 & 136,568 & 132,909 & $2.8 \%$ & 103,819 & 105,475 & $-1.6 \%$ & 240,387 & 238,384 & $0.8 \%$ \\
\hline 2006 & 135,400 & 135,047 & $0.3 \%$ & 107,944 & 109,596 & $-1.5 \%$ & 243,344 & 244,643 & $-0.5 \%$ \\
\hline 2007 & 135,933 & 135,222 & $0.5 \%$ & 110,498 & 113,479 & $-2.6 \%$ & 246,431 & 248,701 & $-0.9 \%$ \\
\hline 2008 & 137,080 & 135,882 & $0.9 \%$ & 110,242 & 113,931 & $-3.2 \%$ & 247,322 & 249,813 & $-1.0 \%$ \\
\hline 2009 & 134,880 & 132,500 & $1.8 \%$ & 110,561 & 116,472 & $-5.1 \%$ & 245,441 & 248,972 & $-1.4 \%$ \\
\hline 2010 & 130,892 & 129,053 & $1.4 \%$ & 110,322 & 119,179 & $-7.4 \%$ & 241,214 & 248,232 & $-2.8 \%$ \\
\hline 2011 & 125,657 & 127,577 & $-1.5 \%$ & 118,483 & 121,355 & $-2.4 \%$ & 244,140 & 248,932 & $-1.9 \%$ \\
\hline 2012 & 111,290 & 120,902 & $-8.0 \%$ & 133,130 & 130,595 & $1.9 \%$ & 244,420 & 251,497 & $-2.8 \%$ \\
\hline
\end{tabular}

\section{Source:}

FHWA - U.S. Department of Transportation, Federal Highway Administration, 1970-2008, Highway Statistics 2008 and earlier, Washington, DC, 2009, Table VM-1 and annual. 2009-2012 data from tables MV-1 and MV-9, Highway Statistics 2012. (Additional resources: www.fhwa.dot.gov)

Polk - The Polk Company, Detroit, Michigan. FURTHER REPRODUCTION PROHIBITED. (Additional resources: www.polk.com) 
The graphs below show the number of motor vehicles per thousand people for various countries. The data for the United States are displayed in the line which goes from 1900 to 2012. The points labeled on that line show data for the other countries/regions around the world and how their vehicles per thousand people compare to the

United States at two different points in time, 2002 and 2012. For instance, the graph shows that in 2002, Eastern Europe's vehicles per thousand people was about where the United States was in 1935, but by 2012 it is about where the United States was in 1950. The lower part of the graph (1900-1940) is shown enlarged on the facing page.

Figure 3.1. Vehicles per Thousand People: U.S. (Over Time) Compared to Other Countries (in 2002 and 2012)

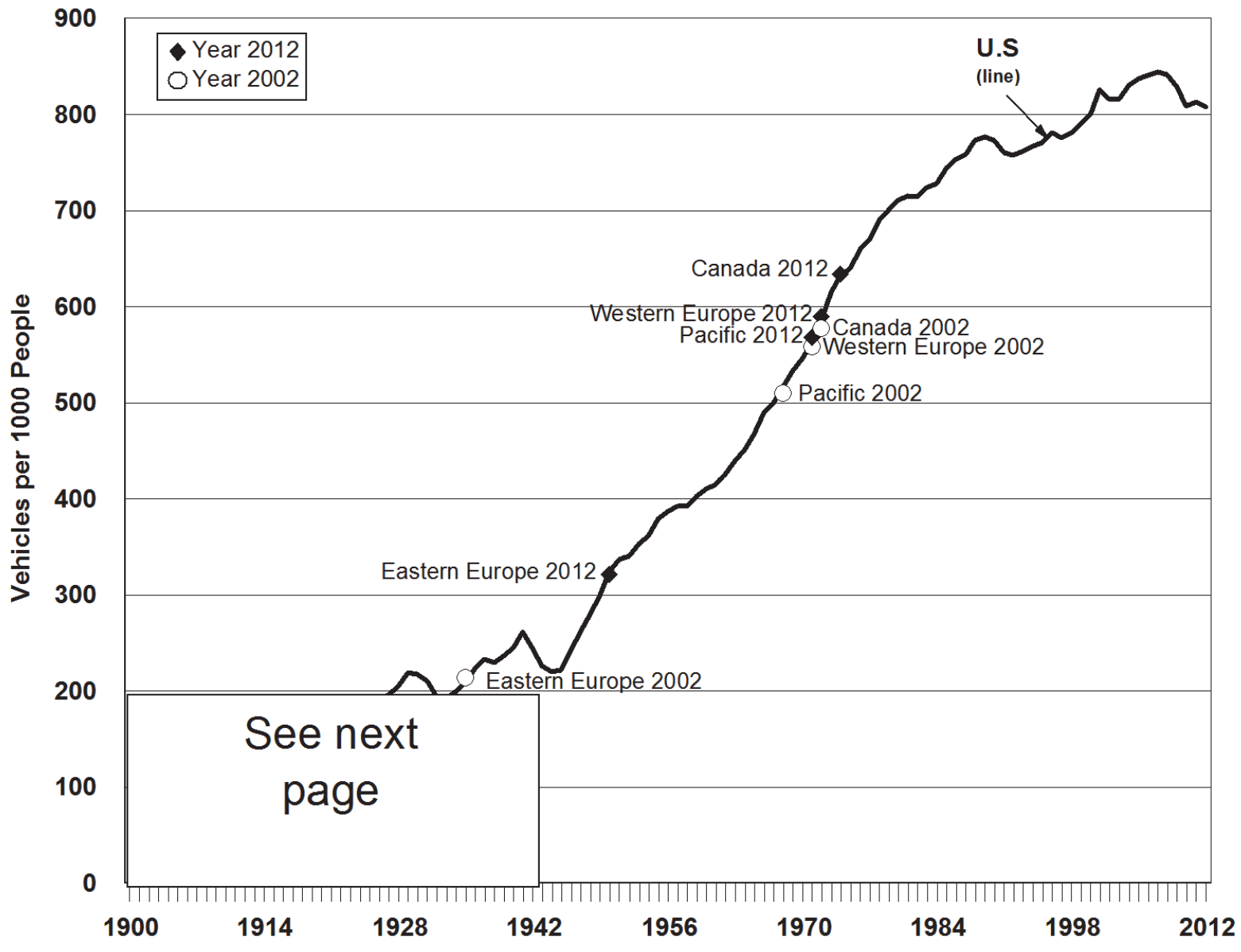




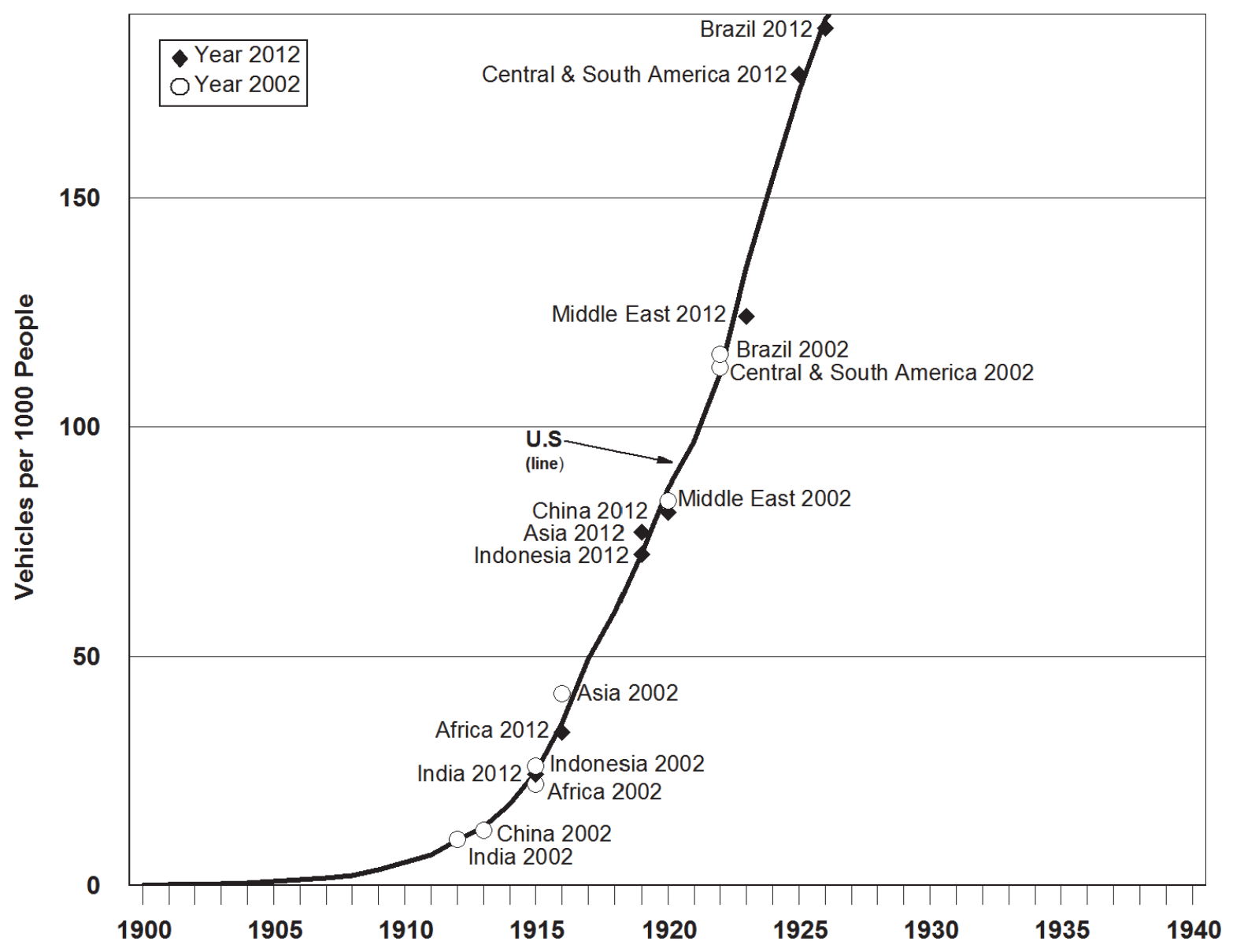

Source:

See Tables 3.4 and 3.5. 
Though some countries are listed separately in this table, those countries are also included in the regional total.

For instance, China is listed separately, but is also included in the Asia, Far East region.

Table 3.5

Vehicles per Thousand People in Other Countries, 2002 and 2012

\begin{tabular}{lcc}
\hline & \multicolumn{2}{c}{ Vehicles per 1,000 people } \\
\cline { 2 - 3 } Country/Region & 2002 & 2012 \\
\hline Africa & 22.5 & 33.6 \\
Asia, Far East & 42.0 & 77.2 \\
Asia, Middle East & 84.0 & 124.2 \\
Brazil & 115.9 & 187.0 \\
Canada & 577.3 & 634.0 \\
Central \& South America & 112.9 & 177.0 \\
China & 12.1 & 81.5 \\
Europe, East & 213.9 & 321.6 \\
Europe, West & 558.7 & 589.4 \\
India & 10.1 & 24.4 \\
Indonesia & 26.1 & 72.4 \\
Pacific & 510.2 & 568.5 \\
\hline
\end{tabular}

\section{Sources:}

Population -U.S. Census Bureau, Population Division, International Data Base (IDB) World, May 8, 2014. (Additional resources: www.census.gov/population/international)

Vehicles -Ward's Communications, Ward's World Motor Vehicle Data 2013, pp. 308-311. (Additional resources: www.fhwa.dot.gov, www.wardsauto.com) 
The number of vehicles per thousand people in the United States has grown significantly from 1900 to 2007. In 2008 to 2012, however, the number decreased from a high of 844.54 in 2007. There was a slight increase in 2011, followed by another decrease in 2012.

Table 3.6

Vehicles per Thousand People in the United States, 1990-2012

\begin{tabular}{|c|c|c|c|c|c|c|c|c|c|}
\hline Year & $\begin{array}{c}\text { U.S. } \\
\text { vehicles } \\
\text { per } 1,000 \\
\text { people }\end{array}$ & Year & $\begin{array}{c}\text { U.S. } \\
\text { vehicles } \\
\text { per } 1,000 \\
\text { people }\end{array}$ & Year & $\begin{array}{c}\text { U.S. } \\
\text { vehicles } \\
\text { per } 1,000 \\
\text { people }\end{array}$ & Year & $\begin{array}{c}\text { U.S. } \\
\text { vehicles } \\
\text { per } 1,000 \\
\text { people }\end{array}$ & Year & $\begin{array}{c}\text { U.S. } \\
\text { vehicles } \\
\text { per } 1,000 \\
\text { people }\end{array}$ \\
\hline 1900 & 0.11 & 1923 & 134.90 & 1946 & 243.11 & 1969 & 533.37 & 1992 & 757.96 \\
\hline 1901 & 0.19 & 1924 & 154.35 & 1947 & 262.56 & 1970 & 545.35 & 1993 & 761.94 \\
\hline 1902 & 0.29 & 1925 & 173.26 & 1948 & 280.20 & 1971 & 562.45 & 1994 & 766.94 \\
\hline 1903 & 0.41 & 1926 & 189.10 & 1949 & 299.56 & 1972 & 585.60 & 1995 & 770.99 \\
\hline 1904 & 0.67 & 1927 & 195.77 & 1950 & 323.71 & 1973 & 615.19 & 1996 & 781.16 \\
\hline 1905 & 0.94 & 1928 & 204.87 & 1951 & 337.14 & 1974 & 632.32 & 1997 & 776.02 \\
\hline 1906 & 1.27 & 1929 & 219.31 & 1952 & 340.57 & 1975 & 640.07 & 1998 & 781.20 \\
\hline 1907 & 1.65 & 1930 & 217.34 & 1953 & 353.67 & 1976 & 659.47 & 1999 & 790.07 \\
\hline 1908 & 2.24 & 1931 & 210.37 & 1954 & 361.40 & 1977 & 669.03 & 2000 & 800.32 \\
\hline 1909 & 3.45 & 1932 & 195.38 & 1955 & 379.77 & 1978 & 690.17 & 2001 & 825.81 \\
\hline 1910 & 5.07 & 1933 & 192.38 & 1956 & 387.58 & 1979 & 700.42 & 2002 & 815.73 \\
\hline 1911 & 6.81 & 1934 & 199.90 & 1957 & 392.11 & 1980 & 710.71 & 2003 & 816.11 \\
\hline 1912 & 9.90 & 1935 & 208.61 & 1958 & 392.17 & 1981 & 715.22 & 2004 & 829.94 \\
\hline 1913 & 12.94 & 1936 & 222.62 & 1959 & 402.83 & 1982 & 713.95 & 2005 & 837.25 \\
\hline 1914 & 17.79 & 1937 & 233.33 & 1960 & 410.37 & 1983 & 724.30 & 2006 & 840.69 \\
\hline 1915 & 24.77 & 1938 & 229.65 & 1961 & 415.11 & 1984 & 728.20 & 2007 & 844.54 \\
\hline 1916 & 35.48 & 1939 & 236.93 & 1962 & 426.06 & 1985 & 744.50 & 2008 & 841.57 \\
\hline 1917 & 49.57 & 1940 & 245.63 & 1963 & 438.75 & 1986 & 753.33 & 2009 & 828.67 \\
\hline 1918 & 59.69 & 1941 & 261.57 & 1964 & 451.57 & 1987 & 758.58 & 2010 & 808.43 \\
\hline 1919 & 72.50 & 1942 & 244.73 & 1965 & 466.90 & 1988 & 772.92 & 2011 & 812.66 \\
\hline 1920 & 86.78 & 1943 & 225.89 & 1966 & 489.34 & 1989 & 776.99 & 2012 & 807.99 \\
\hline 1921 & 96.68 & 1944 & 220.23 & 1967 & 500.66 & 1990 & 773.40 & & \\
\hline 1922 & 111.53 & 1945 & 221.80 & 1968 & 516.49 & 1991 & 760.19 & & \\
\hline
\end{tabular}

\section{Sources:}

Population - U.S. Census Bureau, Population Division, International Data Base (IDB) World, May 7, 2014. (Additional resources: www.census.gov/ipc/www/idb/)

Vehicles - (2012) U.S.: U.S. Department of Transportation, Federal Highway Administration, Highway Statistics 2012, Washington, DC, 2014. 
Total vehicle-miles traveled increased slightly from 2011 to 2012. The trend of using two-axle, four-tire trucks, such as pickups, vans, and sport-utility vehicles, for personal travel is evident in these data; two-axle, four-tire trucks account for 30.2\% more travel in 2012 than in 1970, and cars account for $34.1 \%$ less travel in that time period.

Table 3.7

Shares of Highway Vehicle-Miles Traveled by Vehicle Type, 1970-2012

\begin{tabular}{|c|c|c|c|c|c|c|c|}
\hline Year & Cars & Motorcycles & $\begin{array}{c}\text { Two-axle, } \\
\text { four-tire } \\
\text { trucks }\end{array}$ & $\begin{array}{c}\text { Other } \\
\text { single-unit } \\
\text { trucks }\end{array}$ & $\begin{array}{l}\text { Combination } \\
\text { trucks }\end{array}$ & Buses & $\begin{array}{c}\text { Total } \\
\text { vehicle-miles } \\
\text { traveled } \\
\text { (million miles) }\end{array}$ \\
\hline 1970 & $82.6 \%$ & $0.3 \%$ & $11.1 \%$ & $2.4 \%$ & $3.2 \%$ & $0.4 \%$ & $1,109,724$ \\
\hline 1975 & $77.9 \%$ & $0.4 \%$ & $15.1 \%$ & $2.6 \%$ & $3.5 \%$ & $0.5 \%$ & $1,327,664$ \\
\hline 1980 & $72.8 \%$ & $0.7 \%$ & $19.0 \%$ & $2.6 \%$ & $4.5 \%$ & $0.4 \%$ & $1,527,295$ \\
\hline 1981 & $72.9 \%$ & $0.7 \%$ & $19.1 \%$ & $2.5 \%$ & $4.4 \%$ & $0.4 \%$ & $1,555,308$ \\
\hline 1982 & $72.8 \%$ & $0.6 \%$ & $19.2 \%$ & $2.5 \%$ & $4.4 \%$ & $0.4 \%$ & $1,595,010$ \\
\hline 1983 & $72.3 \%$ & $0.5 \%$ & $19.8 \%$ & $2.6 \%$ & $4.5 \%$ & $0.3 \%$ & $1,652,788$ \\
\hline 1984 & $71.3 \%$ & $0.5 \%$ & $20.8 \%$ & $2.6 \%$ & $4.5 \%$ & $0.3 \%$ & $1,720,269$ \\
\hline 1985 & $70.2 \%$ & $0.5 \%$ & $22.0 \%$ & $2.6 \%$ & $4.4 \%$ & $0.3 \%$ & $1,774,826$ \\
\hline 1986 & $69.2 \%$ & $0.5 \%$ & $23.1 \%$ & $2.5 \%$ & $4.4 \%$ & $0.3 \%$ & $1,834,872$ \\
\hline 1987 & $68.5 \%$ & $0.5 \%$ & $23.8 \%$ & $2.5 \%$ & $4.5 \%$ & $0.3 \%$ & $1,921,204$ \\
\hline 1988 & $67.6 \%$ & $0.5 \%$ & $24.8 \%$ & $2.4 \%$ & $4.4 \%$ & $0.3 \%$ & $2,025,962$ \\
\hline 1989 & $66.8 \%$ & $0.5 \%$ & $25.6 \%$ & $2.4 \%$ & $4.4 \%$ & $0.3 \%$ & $2,096,487$ \\
\hline 1990 & $65.7 \%$ & $0.4 \%$ & $26.8 \%$ & $2.4 \%$ & $4.4 \%$ & $0.3 \%$ & $2,144,362$ \\
\hline 1991 & $62.5 \%$ & $0.4 \%$ & $29.9 \%$ & $2.4 \%$ & $4.4 \%$ & $0.3 \%$ & $2,172,050$ \\
\hline 1992 & $61.0 \%$ & $0.4 \%$ & $31.5 \%$ & $2.4 \%$ & $4.4 \%$ & $0.3 \%$ & $2,247,151$ \\
\hline 1993 & $59.9 \%$ & $0.4 \%$ & $32.5 \%$ & $2.5 \%$ & $4.5 \%$ & $0.3 \%$ & $2,296,378$ \\
\hline 1994 & $59.6 \%$ & $0.4 \%$ & $32.4 \%$ & $2.6 \%$ & $4.6 \%$ & $0.3 \%$ & $2,357,588$ \\
\hline 1995 & $59.4 \%$ & $0.4 \%$ & $32.6 \%$ & $2.6 \%$ & $4.8 \%$ & $0.3 \%$ & $2,422,696$ \\
\hline 1996 & $59.1 \%$ & $0.4 \%$ & $32.8 \%$ & $2.6 \%$ & $4.8 \%$ & $0.3 \%$ & $2,485,848$ \\
\hline 1997 & $58.7 \%$ & $0.4 \%$ & $33.2 \%$ & $2.6 \%$ & $4.9 \%$ & $0.3 \%$ & $2,561,695$ \\
\hline 1998 & $58.9 \%$ & $0.4 \%$ & $33.0 \%$ & $2.6 \%$ & $4.9 \%$ & $0.3 \%$ & $2,631,522$ \\
\hline 1999 & $58.3 \%$ & $0.4 \%$ & $33.5 \%$ & $2.6 \%$ & $4.9 \%$ & $0.3 \%$ & $2,691,056$ \\
\hline 2000 & $58.3 \%$ & $0.4 \%$ & $33.6 \%$ & $2.6 \%$ & $4.9 \%$ & $0.3 \%$ & $2,746,925$ \\
\hline 2001 & $58.2 \%$ & $0.3 \%$ & $33.7 \%$ & $2.6 \%$ & $4.9 \%$ & $0.3 \%$ & $2,797,287$ \\
\hline 2002 & $58.1 \%$ & $0.3 \%$ & $33.8 \%$ & $2.7 \%$ & $4.9 \%$ & $0.2 \%$ & $2,855,508$ \\
\hline 2003 & $57.8 \%$ & $0.3 \%$ & $34.0 \%$ & $2.7 \%$ & $4.8 \%$ & $0.2 \%$ & $2,890,412$ \\
\hline 2004 & $57.3 \%$ & $0.3 \%$ & $34.6 \%$ & $2.6 \%$ & $4.8 \%$ & $0.2 \%$ & $2,964,788$ \\
\hline 2005 & $57.1 \%$ & $0.3 \%$ & $34.8 \%$ & $2.6 \%$ & $4.8 \%$ & $0.2 \%$ & $2,989,430$ \\
\hline 2006 & $56.1 \%$ & $0.4 \%$ & $35.9 \%$ & $2.7 \%$ & $4.7 \%$ & $0.2 \%$ & $3,014,369$ a \\
\hline 2007 & $53.7 \%$ & $0.7 \%$ & $35.7 \%$ & $3.8 \%$ & $5.9 \%$ & $0.2 \%$ & $3,117,292$ \\
\hline 2008 & $52.8 \%$ & $0.7 \%$ & $36.2 \%$ & $4.1 \%$ & $6.0 \%$ & $0.2 \%$ & $3,063,059$ \\
\hline 2009 & $53.0 \%$ & $0.7 \%$ & $36.1 \%$ & $4.1 \%$ & $5.7 \%$ & $0.5 \%$ & $2,956,816$ \\
\hline 2010 & $50.4 \%$ & $0.6 \%$ & $38.8 \%$ & $3.7 \%$ & $5.9 \%$ & $0.5 \%$ & $2,967,310$ \\
\hline 2011 & $49.4 \%$ & $0.6 \%$ & $40.4 \%$ & $3.5 \%$ & $5.6 \%$ & $0.5 \%$ & $2,950,443$ \\
\hline 2012 & $48.5 \%$ & $0.7 \%$ & $41.3 \%$ & $3.5 \%$ & $5.5 \%$ & $0.5 \%$ & $2,968,771$ \\
\hline \multicolumn{8}{|c|}{ Average annual percentage change } \\
\hline 1970-2012 & & & & & & & $2.4 \%$ \\
\hline 2002-2012 & & & & & & & $0.4 \%$ \\
\hline
\end{tabular}

\section{Source:}

U.S. Department of Transportation, Federal Highway Administration, Highway Statistics 2012, Washington, DC, 2014, Table VM-1 and annual. (Additional resources: www.fhwa.dot.gov). 2009-2011 cars and 2-axle 4-tire trucks - see Appendix A for car/light truck shares.

${ }^{a}$ Due to FHWA methodology changes, data from 2007-on are not comparable with previous data. 
In 1970 only $2.9 \%$ of the car population was 15 years old or older; by 2013 that number rose to nearly $20 \%$.

Table 3.8

Cars in Operation by Age, 1970, 2001, and 2013

\begin{tabular}{|c|c|c|c|c|c|c|c|c|c|}
\hline \multirow[b]{2}{*}{ Age (years) } & \multicolumn{3}{|c|}{1970} & \multicolumn{3}{|c|}{2001} & \multicolumn{3}{|c|}{2013} \\
\hline & $\begin{array}{c}\text { Vehicles } \\
\text { (thousands) }\end{array}$ & Percentage $^{\mathrm{a}}$ & $\begin{array}{l}\text { Cumulative } \\
\text { percentage }^{\mathrm{a}}\end{array}$ & $\begin{array}{c}\text { Vehicles } \\
\text { (thousands) }\end{array}$ & Percentage $^{\mathrm{a}}$ & $\begin{array}{l}\text { Cumulative } \\
\text { percentage }^{\mathrm{a}}\end{array}$ & $\begin{array}{c}\text { Vehicles } \\
\text { (thousands) }\end{array}$ & Percentage $^{a}$ & $\begin{array}{l}\text { Cumulative } \\
\text { percentage }^{a}\end{array}$ \\
\hline Under $1^{\mathrm{b}}$ & 6,288 & $7.8 \%$ & $7.8 \%$ & 6,183 & $4.8 \%$ & $4.8 \%$ & 9,287 & $7.1 \%$ & $7.1 \%$ \\
\hline 1 & 9,299 & $11.6 \%$ & $19.4 \%$ & 8,882 & $6.9 \%$ & $11.7 \%$ & 7,700 & $5.9 \%$ & $13.1 \%$ \\
\hline 2 & 8,816 & $11.0 \%$ & $30.3 \%$ & 8,093 & $6.3 \%$ & $18.0 \%$ & 5,957 & $4.6 \%$ & $17.6 \%$ \\
\hline 3 & 7,878 & $9.8 \%$ & $40.1 \%$ & 7,555 & $5.9 \%$ & $23.9 \%$ & 6,159 & $4.7 \%$ & $22.4 \%$ \\
\hline 4 & 8,538 & $10.6 \%$ & $50.8 \%$ & 7,860 & $6.1 \%$ & $30.0 \%$ & 5,484 & $4.2 \%$ & $26.6 \%$ \\
\hline 5 & 8,506 & $10.6 \%$ & $61.3 \%$ & 7,337 & $5.7 \%$ & $35.7 \%$ & 7,226 & $5.6 \%$ & $32.1 \%$ \\
\hline 6 & 7,116 & $8.8 \%$ & $70.2 \%$ & 8,555 & $6.6 \%$ & $42.3 \%$ & 7,896 & $6.1 \%$ & $38.2 \%$ \\
\hline 7 & 6,268 & $7.8 \%$ & $78.0 \%$ & 7,471 & $5.8 \%$ & $48.1 \%$ & 7,706 & $5.9 \%$ & $44.1 \%$ \\
\hline 8 & 5,058 & $6.3 \%$ & $84.3 \%$ & 7,420 & $5.8 \%$ & $53.9 \%$ & 7,843 & $6.0 \%$ & $50.2 \%$ \\
\hline 9 & 3,267 & $4.1 \%$ & $88.3 \%$ & 6,807 & $5.3 \%$ & $59.2 \%$ & 6,924 & $5.3 \%$ & $55.5 \%$ \\
\hline 10 & 2,776 & $3.5 \%$ & $91.8 \%$ & 6,810 & $5.3 \%$ & $64.5 \%$ & 7,237 & $5.6 \%$ & $61.1 \%$ \\
\hline 11 & 1,692 & $2.1 \%$ & $93.9 \%$ & 6,692 & $5.2 \%$ & $69.7 \%$ & 7,167 & $5.5 \%$ & $66.6 \%$ \\
\hline 12 & 799 & $1.0 \%$ & $94.9 \%$ & 6,742 & $5.2 \%$ & $74.9 \%$ & 6,660 & $5.1 \%$ & $71.7 \%$ \\
\hline 13 & 996 & $1.2 \%$ & $96.1 \%$ & 6,189 & $4.8 \%$ & $79.7 \%$ & 6,889 & $5.3 \%$ & $77.0 \%$ \\
\hline 14 & 794 & $1.0 \%$ & $97.1 \%$ & 5,345 & $4.2 \%$ & $83.9 \%$ & 5,487 & $4.2 \%$ & $81.2 \%$ \\
\hline 15 and older & 2,336 & $2.9 \%$ & $100.0 \%$ & 20,773 & $16.1 \%$ & $100.0 \%$ & 24,457 & $18.8 \%$ & $100.0 \%$ \\
\hline Subtotal & 80,427 & $100.0 \%$ & & 128,714 & $100.0 \%$ & & 130,078 & & \\
\hline Age not given & 22 & & & $\mathbf{0}$ & & & 0 & & \\
\hline Total & 80,449 & & & 128,714 & & & 130,078 & & \\
\hline
\end{tabular}

Source:

The Polk Company, Detroit, MI. FURTHER REPRODUCTION PROHIBITED.

${ }^{\text {a }}$ Percentages may not sum to totals due to rounding.

${ }^{\mathrm{b}}$ Includes cars from model year 2002 and 2001 which were sold prior to July 1, 2002, and similarly, model years 1971 and 1970 sold prior to July 1, 1970. For 2013, cars sold prior to December 31, 2013 which were model year 2013, 2014 or 2015 were included. 
The number of trucks in the United States has grown significantly since 1970, some of it due to the use of light trucks (pickups, vans, sport utility vehicles) as personal passenger vehicles. Those light trucks, as well as medium and heavy trucks, are included in the data. In 1970 about $15 \%$ of trucks were age 15 or older; by 2013, that increased to $20.8 \%$.

Table 3.9

Trucks in Operation by Age, 1970, 2001, and 2013

\begin{tabular}{|c|c|c|c|c|c|c|c|c|c|}
\hline \multirow[b]{2}{*}{ Age (years) } & \multicolumn{3}{|c|}{1970} & \multicolumn{3}{|c|}{2001} & \multicolumn{3}{|c|}{2013} \\
\hline & $\begin{array}{c}\text { Vehicles } \\
\text { (thousands) }\end{array}$ & Percentage $^{\mathrm{a}}$ & $\begin{array}{l}\text { Cumulative } \\
\text { percentage }^{\mathrm{a}}\end{array}$ & $\begin{array}{c}\text { Vehicles } \\
\text { (thousands) }\end{array}$ & Percentage $^{\mathrm{a}}$ & $\begin{array}{l}\text { Cumulative } \\
\text { percentage }^{\text {a }}\end{array}$ & $\begin{array}{c}\text { Vehicles } \\
\text { (thousands) }\end{array}$ & Percentage $^{\mathrm{a}}$ & $\begin{array}{l}\text { Cumulative } \\
\text { percentage }^{\text {a }}\end{array}$ \\
\hline Under $1^{\mathrm{b}}$ & 1,262 & $7.1 \%$ & $7.1 \%$ & 6,213 & $7.1 \%$ & $7.1 \%$ & 8,097 & $6.5 \%$ & $6.5 \%$ \\
\hline 1 & 1,881 & $10.6 \%$ & $17.8 \%$ & 7,958 & $9.0 \%$ & $16.1 \%$ & 6,391 & $5.1 \%$ & $11.6 \%$ \\
\hline 2 & 1,536 & $8.7 \%$ & $26.5 \%$ & 7,522 & $8.6 \%$ & $24.7 \%$ & 6,417 & $5.2 \%$ & $16.8 \%$ \\
\hline 3 & 1,428 & $8.1 \%$ & $34.6 \%$ & 6,398 & $7.3 \%$ & $31.9 \%$ & 4,972 & $4.0 \%$ & $20.8 \%$ \\
\hline 4 & 1,483 & $8.4 \%$ & $43.0 \%$ & 6,109 & $6.9 \%$ & $38.9 \%$ & 3,991 & $3.2 \%$ & $24.0 \%$ \\
\hline 5 & 1,339 & $7.6 \%$ & $50.5 \%$ & 5,122 & $5.8 \%$ & $44.7 \%$ & 6,927 & $5.6 \%$ & $29.5 \%$ \\
\hline 6 & 1,154 & $6.5 \%$ & $57.1 \%$ & 5,574 & $6.3 \%$ & $51.0 \%$ & 7,587 & $6.1 \%$ & $35.6 \%$ \\
\hline 7 & 975 & $5.5 \%$ & $62.6 \%$ & 5,042 & $5.7 \%$ & $56.8 \%$ & 7,580 & $6.1 \%$ & $41.7 \%$ \\
\hline 8 & 826 & $4.7 \%$ & $67.3 \%$ & 4,148 & $4.7 \%$ & $61.5 \%$ & 7,585 & $6.1 \%$ & $47.8 \%$ \\
\hline 9 & 621 & $3.5 \%$ & $70.8 \%$ & 3,395 & $3.9 \%$ & $65.3 \%$ & 7,978 & $6.4 \%$ & $54.2 \%$ \\
\hline 10 & 658 & $3.7 \%$ & $74.5 \%$ & 3,221 & $3.7 \%$ & $69.0 \%$ & 7,201 & $5.8 \%$ & $60.0 \%$ \\
\hline 11 & 583 & $3.3 \%$ & $77.8 \%$ & 3,039 & $3.5 \%$ & $72.5 \%$ & 6,850 & $5.5 \%$ & $65.5 \%$ \\
\hline 12 & 383 & $2.2 \%$ & $80.0 \%$ & 3,345 & $3.8 \%$ & $76.3 \%$ & 6,163 & $4.9 \%$ & $70.4 \%$ \\
\hline 13 & 417 & $2.4 \%$ & $82.3 \%$ & 3,112 & $3.5 \%$ & $79.8 \%$ & 5,673 & $4.6 \%$ & $75.0 \%$ \\
\hline 14 & 414 & $2.3 \%$ & $84.7 \%$ & 2,544 & $2.9 \%$ & $82.7 \%$ & 5,217 & $4.2 \%$ & $79.2 \%$ \\
\hline 15 and older & 2,710 & $15.3 \%$ & $100.0 \%$ & 15,227 & $17.3 \%$ & $100.0 \%$ & 25,917 & $20.8 \%$ & $100.0 \%$ \\
\hline Subtotal & 17,670 & $100.0 \%$ & & 87,969 & $100.0 \%$ & & 124,545 & & \\
\hline Age note given & 15 & & & $\mathbf{0}$ & & & $\mathbf{0}$ & & \\
\hline Total & 17,685 & & & 87,969 & & & 124,545 & & \\
\hline
\end{tabular}

Source:

The Polk Company, Detroit, MI. FURTHER REPRODUCTION PROHIBITED.

${ }^{\text {a }}$ Percentages may not sum to totals due to rounding.

${ }^{\mathrm{b}}$ Includes trucks from model year 2002 and 2001 which were sold prior to July 1, 2002, and similarly, model years 1971 and 1970 sold prior to July 1, 1970. 
The average age of cars and light trucks has continued to grow in recent years. Light trucks, which include pickups, vans, and sport utility vehicles, typically have a lower average age than cars. In 2013, however, there is a very small difference.

Table 3.10

U.S. Average Vehicle Age, 1995-2013

\begin{tabular}{cccc}
\hline & Passenger cars & Light trucks & All light vehicles \\
\hline 1995 & 8.4 & 8.3 & 8.4 \\
1996 & 8.5 & 8.3 & 8.5 \\
1997 & 8.7 & 8.5 & 8.6 \\
1998 & 8.9 & 8.5 & 8.8 \\
1999 & 9.1 & 8.5 & 8.8 \\
2000 & 9.1 & 8.4 & 8.9 \\
2001 & 9.3 & 8.4 & 8.9 \\
\hline 2002 & 9.8 & 9.4 & 9.6 \\
2003 & 9.9 & 9.0 & 9.7 \\
2004 & 10.0 & 9.5 & 9.8 \\
2005 & 10.1 & 9.5 & 9.8 \\
2006 & 10.2 & 9.5 & 9.9 \\
2007 & 10.3 & 9.6 & 10.0 \\
2008 & 10.4 & 9.8 & 10.1 \\
2009 & 10.5 & 10.1 & 10.3 \\
2010 & 10.8 & 10.5 & 10.6 \\
2011 & 11.1 & 10.8 & 10.9 \\
2012 & 11.3 & 11.1 & 11.2 \\
2013 & 11.4 & 11.3 & 11.4 \\
\hline
\end{tabular}

\section{Source:}

R.L. Polk and Company, Detroit, MI. FURTHER REPRODUCTION PROHIBITED. (Additional resources: www.polk.com)

${ }^{a}$ In 2013, R.L. Polk and Company published a data series showing vehicle age from 2002-2013. These data did not match the previous data published by Polk. The data prior to 2002 came from earlier Polk news releases and is not comparable to the revised data. 
Table 3.11

New Retail Vehicle Sales, 1970-2013 (thousands)

\begin{tabular}{|c|c|c|c|c|c|}
\hline $\begin{array}{c}\text { Calendar } \\
\text { Year }\end{array}$ & Cars & $\begin{array}{c}\text { Light } \\
\text { Trucks }^{\text {a }}\end{array}$ & $\begin{array}{c}\text { Subtotal } \\
\text { Light Vehicles }\end{array}$ & $\begin{array}{l}\text { Heavy } \\
\text { Trucks }\end{array}$ & $\begin{array}{c}\text { Total } \\
\text { Vehicle } \\
\text { Sales } \\
\end{array}$ \\
\hline 1970 & 8,399 & 1,457 & 9,856 & 334 & 10,191 \\
\hline 1971 & 10,242 & 1,673 & 11,915 & 340 & 12,255 \\
\hline 1972 & 10,941 & 2,097 & 13,038 & 438 & 13,475 \\
\hline 1973 & 11,424 & 2,512 & 13,936 & 497 & 14,433 \\
\hline 1974 & 8,853 & 2,163 & 11,016 & 424 & 11,440 \\
\hline 1975 & 8,624 & 2,053 & 10,677 & 298 & 10,975 \\
\hline 1976 & 10,110 & 2,719 & 12,829 & 324 & 13,153 \\
\hline 1977 & 11,183 & 3,109 & 14,292 & 376 & 14,668 \\
\hline 1978 & 11,314 & 3,474 & 14,788 & 441 & 15,229 \\
\hline 1979 & 10,673 & 2,845 & 13,518 & 391 & 13,909 \\
\hline 1980 & 8,949 & 1,960 & 10,909 & 265 & 11,174 \\
\hline 1981 & 8,489 & 1,746 & 10,235 & 235 & 10,470 \\
\hline 1982 & 7,956 & 2,063 & 10,019 & 183 & 10,202 \\
\hline 1983 & 9,148 & 2,521 & 11,669 & 189 & 11,858 \\
\hline 1984 & 10,324 & 3,255 & 13,579 & 277 & 13,856 \\
\hline 1985 & 10,979 & 3,688 & 14,667 & 285 & 14,952 \\
\hline 1986 & 11,404 & 4,594 & 15,998 & 265 & 16,263 \\
\hline 1987 & 10,192 & 4,610 & 14,802 & 287 & 15,089 \\
\hline 1988 & 10,547 & 4,800 & 15,347 & 334 & 15,681 \\
\hline 1989 & 9,779 & 4,610 & 14,389 & 312 & 14,700 \\
\hline 1990 & 9,303 & 4,548 & 13,851 & 277 & 14,129 \\
\hline 1991 & 8,185 & 4,122 & 12,307 & 221 & 12,528 \\
\hline 1992 & 8,213 & 4,629 & 12,842 & 249 & 13,091 \\
\hline 1993 & 8,518 & 5,351 & 13,869 & 303 & 14,172 \\
\hline 1994 & 8,991 & 6,033 & 15,024 & 353 & 15,376 \\
\hline 1995 & 8,620 & 6,053 & 14,673 & 388 & 15,061 \\
\hline 1996 & 8,479 & 6,519 & 14,998 & 359 & 15,356 \\
\hline 1997 & 8,217 & 6,797 & 15,014 & 376 & 15,391 \\
\hline 1998 & 8,085 & 7,299 & 15,384 & 424 & 15,808 \\
\hline 1999 & 8,638 & 8,073 & 16,711 & 521 & 17,232 \\
\hline 2000 & 8,778 & 8,386 & 17,164 & 462 & 17,626 \\
\hline 2001 & 8,352 & 8,598 & 16,950 & 350 & 17,300 \\
\hline 2002 & 8,042 & 8,633 & 16,675 & 322 & 16,998 \\
\hline 2003 & 7,556 & 8,938 & 16,494 & 328 & 16,822 \\
\hline 2004 & 7,483 & 9,254 & 16,737 & 432 & 17,168 \\
\hline 2005 & 7,660 & 9,114 & 16,774 & 497 & 17,271 \\
\hline 2006 & 7,762 & 8,574 & 16,336 & 545 & 16,880 \\
\hline 2007 & 7,562 & 8,305 & 15,867 & 371 & 16,238 \\
\hline 2008 & 6,769 & 6,246 & 13,015 & 298 & 13,314 \\
\hline 2009 & 5,402 & 4,834 & 10,236 & 200 & 10,435 \\
\hline 2010 & 5,636 & 5,758 & 11,394 & 218 & 11,611 \\
\hline 2011 & 6,090 & 6,449 & 12,539 & 306 & 12,845 \\
\hline 2012 & 7,244 & 6,975 & 14,218 & 346 & 14,564 \\
\hline 2013 & 7,586 & 7,693 & 15,278 & 352 & 15,630 \\
\hline \multicolumn{6}{|c|}{ Average annual percentage change } \\
\hline 1970-2013 & $-0.2 \%$ & $3.9 \%$ & $1.0 \%$ & $0.1 \%$ & $1.0 \%$ \\
\hline 2003-2013 & $0.0 \%$ & $-1.5 \%$ & $-0.8 \%$ & $0.7 \%$ & $-0.7 \%$ \\
\hline
\end{tabular}

\section{Source:}

1970-2013: Ward's Communications, www.wardsauto.com.

${ }^{\mathrm{a}}$ Includes light trucks of 10,000 lb. gross vehicle weight and less. 
Using current registration data and a scrappage model by Greenspan and Cohen, [1996 paper: www.federalreserve.gov/pubs/feds/1996/199640/199640pap.pdf], ORNL calculated car scrappage rates for 1970, 1980, and 1990. These data are fitted model values which assume constant economic conditions. Using 19772002 data, the Federal Highway Administration completed a separate survivability study in 2006.

Table 3.12

Car Scrappage and Survival Rates 1970, 1980 and 1990 Model Years

\begin{tabular}{|c|c|c|c|c|c|c|c|}
\hline \multirow{2}{*}{$\begin{array}{l}\text { Vehicle } \\
\text { age }^{\mathrm{a}} \\
\text { (years) }\end{array}$} & \multicolumn{2}{|c|}{1970 model year } & \multicolumn{2}{|c|}{1980 model year } & \multicolumn{2}{|c|}{1990 model year } & \multirow{2}{*}{$\begin{array}{c}2002 \\
\text { Survival } \\
\text { rate }\end{array}$} \\
\hline & $\begin{array}{l}\text { Survival } \\
\text { rate }^{\mathrm{b}}\end{array}$ & $\begin{array}{l}\text { Scrappage } \\
\text { rate }^{\text {c }}\end{array}$ & $\begin{array}{c}\text { Survival } \\
\text { rate }^{b}\end{array}$ & $\begin{array}{c}\text { Scrappage } \\
\text { rate }^{\mathrm{c}}\end{array}$ & $\begin{array}{l}\text { Survival } \\
\text { rate }^{\mathrm{b}}\end{array}$ & $\begin{array}{l}\text { Scrappage } \\
\text { rate }^{\text {c }}\end{array}$ & \\
\hline 4 & 99.0 & 1.0 & 100.0 & 0.0 & 100.0 & 0.0 & 95.9 \\
\hline 5 & 94.1 & 5.0 & 96.3 & 3.7 & 100.0 & 0.0 & 94.1 \\
\hline 6 & 88.4 & 6.1 & 91.3 & 5.1 & 99.4 & 0.6 & 91.9 \\
\hline 7 & 82.0 & 7.2 & 85.7 & 6.1 & 96.3 & 3.2 & 89.2 \\
\hline 8 & 75.2 & 8.3 & 79.7 & 7.1 & 92.7 & 3.7 & 86.0 \\
\hline 9 & 68.1 & 9.5 & 73.3 & 8.1 & 88.7 & 4.3 & 82.5 \\
\hline 10 & 60.9 & 10.6 & 66.6 & 9.0 & 84.4 & 4.9 & 78.7 \\
\hline 11 & 53.8 & 11.7 & 60.0 & 10.0 & 79.8 & 5.5 & 71.7 \\
\hline 12 & 46.9 & 12.8 & 53.3 & 11.0 & 75.0 & 6.1 & 61.3 \\
\hline 13 & 40.3 & 14.0 & 46.9 & 12.0 & 70.0 & 6.7 & 50.9 \\
\hline 14 & 34.2 & 15.1 & 40.8 & 13.0 & 64.9 & 7.3 & 41.4 \\
\hline 15 & 28.7 & 16.2 & 35.1 & 14.0 & 59.7 & 7.9 & 33.1 \\
\hline 16 & 23.7 & 17.4 & 29.8 & 15.0 & 54.6 & 8.6 & 26.0 \\
\hline 17 & 19.3 & 18.5 & 25.0 & 16.1 & 49.5 & 9.3 & 20.3 \\
\hline 18 & 15.5 & 19.6 & 20.8 & 17.1 & 44.6 & 9.9 & 15.7 \\
\hline 19 & 12.3 & 20.8 & 17.0 & 18.1 & 39.9 & 10.6 & 12.0 \\
\hline 20 & 9.6 & 21.9 & 13.8 & 19.1 & 35.4 & 11.3 & 9.2 \\
\hline 21 & 7.4 & 23.0 & 11.0 & 20.1 & 31.1 & 12.0 & 7.0 \\
\hline 22 & 5.6 & 24.2 & 8.7 & 21.2 & 27.2 & 12.7 & 5.3 \\
\hline 23 & 4.2 & 25.3 & 6.7 & 22.2 & 23.5 & 13.5 & 4.0 \\
\hline 24 & 3.1 & 26.4 & 5.2 & 23.2 & 20.2 & 14.2 & 3.0 \\
\hline 25 & 2.2 & 27.5 & 3.9 & 24.2 & 17.1 & 15.0 & 2.3 \\
\hline 26 & 1.6 & 28.6 & 2.9 & 25.3 & 14.5 & 15.7 & d \\
\hline 27 & 1.1 & 29.7 & 2.2 & 26.3 & 12.1 & 16.5 & d \\
\hline 28 & 0.8 & 30.8 & 1.6 & 27.3 & 10.0 & 17.2 & d \\
\hline 29 & 0.5 & 31.9 & 1.1 & 28.4 & 8.2 & 18.0 & d \\
\hline 30 & 0.4 & 33.0 & 0.8 & 29.4 & 6.6 & 18.8 & d \\
\hline $\begin{array}{l}\text { Median } \\
\text { lifetime }\end{array}$ & \multicolumn{2}{|c|}{11.5 years } & \multicolumn{2}{|c|}{12.5 years } & \multicolumn{2}{|c|}{16.9 years } & $\begin{array}{c}152,137 \\
\text { Lifetime } \\
\text { miles }\end{array}$ \\
\hline
\end{tabular}

Sources:

Schmoyer, Richard L., unpublished study on scrappage rates, Oak Ridge National Laboratory, Oak Ridge, TN, 2001.

U.S. Department of Transportation, National Highway Traffic Safety Administration, Vehicle Survivability and Travel Mileage Schedules, January 2006.

\footnotetext{
${ }^{a}$ It was assumed that scrappage for vehicles less than 4 years old is 0 .

${ }^{\mathrm{b}}$ The percentage of automobiles which will be in use at the end of the year.

${ }^{\mathrm{c}}$ The percentage of automobiles which will be retired from use during the year.

${ }^{\mathrm{d}}$ Data are not available.
} 
Using current registration data and a scrappage model by Greenspan and Cohen 11996 paper: www.federalreserve.gov/pubs/feds/1996/199640/199640pap.pdf], ORNL calculated light truck scrappage rates for 1970, 1980, and 1990. These data are fitted model values which assume constant economic conditions. Using 1977-2002 data, the Federal Highway Administration completed a separate survivability study in 2006.

Table 3.13

Light Truck ${ }^{\mathrm{a}}$ Scrappage and Survival Rates 1970, 1980 and 1990 Model Years

\begin{tabular}{|c|c|c|c|c|c|c|c|}
\hline \multirow{2}{*}{$\begin{array}{l}\text { Vehicle } \\
\text { age } \\
\text { (years) }\end{array}$} & \multicolumn{2}{|c|}{1970 model year } & \multicolumn{2}{|c|}{1980 model year } & \multicolumn{2}{|c|}{1990 model year } & \multirow{2}{*}{$\begin{array}{c}2002 \\
\text { Survival } \\
\text { rate }^{\mathrm{c}}\end{array}$} \\
\hline & $\begin{array}{c}\text { Survival } \\
\text { rate }^{c}\end{array}$ & $\begin{array}{c}\text { Scrappage } \\
\text { rate }^{\mathrm{d}}\end{array}$ & $\begin{array}{c}\text { Survival } \\
\text { rate }^{c}\end{array}$ & $\begin{array}{l}\text { Scrappage } \\
\text { rate }^{\mathrm{d}}\end{array}$ & $\begin{array}{c}\text { Survival } \\
\text { rate }^{c}\end{array}$ & $\begin{array}{c}\text { Scrappage } \\
\text { rate }^{\mathrm{d}}\end{array}$ & \\
\hline 4 & 99.7 & 0.3 & 99.1 & 0.9 & $\begin{array}{l}99.3 \\
\end{array}$ & 0.7 & 91.9 \\
\hline 5 & 97.5 & 2.2 & 96.6 & 2.5 & 96.9 & 2.4 & 89.1 \\
\hline 6 & 94.9 & 2.7 & 93.7 & 3.1 & 94.1 & 3.0 & 85.9 \\
\hline 7 & 91.8 & 3.2 & 90.2 & 3.7 & 90.7 & 3.6 & 82.3 \\
\hline 8 & 88.3 & 3.8 & 86.3 & 4.3 & 86.9 & 4.2 & 78.3 \\
\hline 9 & 84.4 & 4.4 & 82.0 & 5.0 & 82.7 & 4.8 & 74.0 \\
\hline 10 & 80.2 & 5.0 & 77.3 & 5.7 & 78.2 & 5.5 & 69.6 \\
\hline 11 & 75.7 & 5.6 & 72.4 & 6.4 & 73.4 & 6.1 & 65.0 \\
\hline 12 & 70.9 & 6.3 & 67.3 & 7.1 & 68.4 & 6.8 & 60.4 \\
\hline 13 & 66.0 & 6.9 & 62.1 & 7.8 & 63.3 & 7.5 & 55.2 \\
\hline 14 & 61.0 & 7.6 & 56.8 & 8.5 & 58.0 & 8.2 & 50.1 \\
\hline 15 & 55.9 & 8.3 & 51.5 & 9.3 & 52.8 & 9.0 & 45.2 \\
\hline 16 & 50.8 & 9.0 & 46.3 & 10.1 & 47.7 & 9.7 & 40.6 \\
\hline 17 & 45.9 & 9.8 & 41.3 & 10.8 & 42.7 & 10.5 & 36.3 \\
\hline 18 & 41.1 & 10.5 & 36.5 & 11.6 & 37.9 & 11.3 & 32.4 \\
\hline 19 & 36.4 & 11.3 & 32.0 & 12.4 & 33.3 & 12.1 & 28.7 \\
\hline 20 & 32.1 & 12.0 & 27.7 & 13.3 & 29.0 & 12.9 & 25.4 \\
\hline 21 & 28.0 & 12.8 & 23.8 & 14.1 & 25.0 & 13.7 & 22.4 \\
\hline 22 & 24.2 & 13.6 & 20.3 & 14.9 & 21.4 & 14.5 & 19.8 \\
\hline 23 & 20.7 & 14.4 & 17.1 & 15.8 & 18.1 & 15.4 & 17.4 \\
\hline 24 & 17.5 & 15.2 & 14.2 & 16.7 & 15.2 & 16.2 & 15.2 \\
\hline 25 & 14.7 & 16.1 & 11.7 & 17.5 & 12.6 & 17.1 & 13.3 \\
\hline 26 & 12.2 & 16.9 & 9.6 & 18.4 & 10.3 & 18.0 & 11.7 \\
\hline 27 & 10.1 & 17.8 & 7.7 & 19.3 & 8.4 & 18.8 & 10.2 \\
\hline 28 & 8.2 & 18.6 & 6.2 & 20.2 & 6.7 & 19.7 & 8.9 \\
\hline 29 & 6.6 & 19.5 & 4.9 & 21.1 & 5.3 & 20.6 & 7.7 \\
\hline 30 & 5.2 & 20.4 & 3.8 & 22.1 & 4.2 & 21.5 & 6.7 \\
\hline $\begin{array}{l}\text { Median } \\
\text { lifetime }\end{array}$ & & 2 years & & years & & years & $\begin{array}{c}179,954 \\
\text { Lifetime } \\
\text { miles }\end{array}$ \\
\hline
\end{tabular}

Sources:

Schmoyer, Richard L., unpublished study on scrappage rates, Oak Ridge National Laboratory, Oak Ridge, TN, 2001.

U.S. Department of Transportation, National Highway Traffic Safety Administration, Vehicle Survivability and Travel Mileage Schedules, January 2006.

\footnotetext{
${ }^{\text {a }}$ Light trucks are trucks less than 10,000 lbs. gross vehicle weight.

${ }^{\mathrm{b}}$ It was assumed that scrappage for vehicles less than 4 years old is 0 .

${ }^{\mathrm{c}}$ The percentage of light trucks which will be in use during the year.

${ }^{\mathrm{d}}$ The percentage of light trucks which will be retired from use at the end of the year.
} 
Using current registration data and a scrappage model by Greenspan and Cohen [1996 paper:

www.federalreserve.gov/pubs/feds/1996/199640/199640pap.pdf], ORNL calculated heavy truck (trucks over 26,000 lbs. gross vehicle weight) scrappage rates. The expected median lifetime for a 1990 model year heavy truck is 29 years. These data are fitted model values which assume constant economic conditions.

Table 3.14

Heavy Truck ${ }^{\text {a }}$ Scrappage and Survival Rates 1970, 1980 and 1990 Model Years

\begin{tabular}{|c|c|c|c|c|c|c|}
\hline \multirow{2}{*}{$\begin{array}{l}\text { Vehicle } \\
\text { age }^{\mathrm{b}} \\
\text { (years) }\end{array}$} & \multicolumn{2}{|c|}{1970 model year } & \multicolumn{2}{|c|}{1980 model year } & \multicolumn{2}{|c|}{1990 model year } \\
\hline & $\begin{array}{c}\text { Survival } \\
\text { rate }^{c}\end{array}$ & $\begin{array}{l}\text { Scrappage } \\
\text { rate }^{\mathrm{d}}\end{array}$ & $\begin{array}{l}\text { Survival } \\
\text { rate }^{c}\end{array}$ & $\begin{array}{l}\text { Scrappage } \\
\text { rate }^{\mathrm{d}}\end{array}$ & $\begin{array}{l}\text { Survival } \\
\text { rate }^{c}\end{array}$ & $\begin{array}{l}\text { Scrappage } \\
\text { rate }^{\mathrm{d}}\end{array}$ \\
\hline 4 & 98.8 & 1.2 & 98.5 & 1.5 & 99.4 & 0.6 \\
\hline 5 & 97.2 & 1.6 & 96.7 & 1.9 & 98.6 & 0.8 \\
\hline 6 & 95.3 & 1.9 & 94.5 & 2.3 & 97.6 & 1.0 \\
\hline 7 & 93.2 & 2.3 & 92.0 & 2.7 & 96.5 & 1.2 \\
\hline 8 & 90.7 & 2.6 & 89.1 & 3.1 & 95.2 & 1.3 \\
\hline 9 & 88.1 & 3.0 & 86.0 & 3.5 & 93.8 & 1.5 \\
\hline 10 & 85.2 & 3.3 & 82.7 & 3.9 & 92.2 & 1.7 \\
\hline 11 & 82.1 & 3.6 & 79.1 & 4.3 & 90.5 & 1.9 \\
\hline 12 & 78.8 & 4.0 & 75.4 & 4.7 & 88.6 & 2.0 \\
\hline 13 & 75.4 & 4.3 & 71.6 & 5.1 & 86.7 & 2.2 \\
\hline 14 & 71.9 & 4.7 & 67.7 & 5.5 & 84.6 & 2.4 \\
\hline 15 & 68.3 & 5.0 & 63.7 & 5.9 & 82.4 & 2.6 \\
\hline 16 & 64.6 & 5.3 & 59.7 & 6.3 & 80.2 & 2.7 \\
\hline 17 & 61.0 & 5.7 & 55.7 & 6.7 & 77.9 & 2.9 \\
\hline 18 & 57.3 & 6.0 & 51.8 & 7.1 & 75.5 & 3.1 \\
\hline 19 & 53.7 & 6.3 & 47.9 & 7.4 & 73.0 & 3.3 \\
\hline 20 & 50.1 & 6.7 & 44.2 & 7.8 & 70.5 & 3.4 \\
\hline 21 & 46.6 & 7.0 & 40.6 & 8.2 & 68.0 & 3.6 \\
\hline 22 & 43.2 & 7.3 & 37.1 & 8.6 & 65.4 & 3.8 \\
\hline 23 & 39.9 & 7.6 & 33.7 & 9.0 & 62.8 & 3.9 \\
\hline 24 & 36.7 & 8.0 & 30.6 & 9.4 & 60.3 & 4.1 \\
\hline 25 & 33.7 & 8.3 & 27.6 & 9.7 & 57.7 & 4.3 \\
\hline 26 & 30.8 & 8.6 & 24.8 & 10.1 & 55.1 & 4.5 \\
\hline 27 & 28.0 & 8.9 & 22.2 & 10.5 & 52.6 & 4.6 \\
\hline 28 & 25.4 & 9.3 & 19.8 & 10.9 & 50.0 & 4.8 \\
\hline 29 & 23.0 & 9.6 & 17.6 & 11.2 & 47.6 & 5.0 \\
\hline 30 & 20.7 & 9.9 & 15.5 & 11.6 & 45.1 & 5.1 \\
\hline $\begin{array}{l}\text { Median } \\
\text { lifetime }\end{array}$ & \multicolumn{2}{|c|}{20.0 years } & \multicolumn{2}{|c|}{18.5 years } & \multicolumn{2}{|c|}{28.0 years } \\
\hline
\end{tabular}

Source:

Schmoyer, Richard L., unpublished study on scrappage rates, Oak Ridge National Laboratory, Oak Ridge, TN, 2001.

\footnotetext{
${ }^{a}$ Heavy trucks are trucks over 26,000 lbs. gross vehicle weight.

${ }^{\mathrm{b}}$ It was assumed that scrappage for vehicles less than 4 years old is 0 .

${ }^{c}$ The percentage of heavy trucks which will be in use at the end of the year.

${ }^{d}$ The percentage of heavy trucks which will be retired from use during the year.
} 


\section{Chapter 4 \\ Light Vehicles and Characteristics}

Summary Statistics from Tables in this Chapter

\begin{tabular}{ccr}
\hline Source & & \\
\hline Table 4.1 & Cars, 2012 & \\
& Registrations (thousands) & 111,290 \\
& Vehicle miles (billion miles) & $1,438.8$ \\
Table 4.2 & Fuel economy (miles per gallon) & 24.9 \\
& Two-axle, four-tire trucks, 2012 & \\
& Registrations (thousands) & 120,847 \\
Table 4.6 & Vehicle miles (billion miles) & $1,225.6$ \\
& Fuel economy (miles per gallon) & 18.5 \\
& Light truck share of total light vehicle sales & \\
Table 4.7 & 1970 calendar year & $14.8 \%$ \\
& Car production, 2013 model year (thousands) & $50.3 \%$ \\
Table 4.8 & Car & 9,257 \\
& Car SUV & 7,731 \\
& Light truck production, 2013 model year (thousands) & 1,526 \\
& Pickup & 5,113 \\
Tables 4.20 & Van & 1,525 \\
and 4.21 & Truck SUV & 568 \\
& Corporate average fuel economy & 3,020 \\
& Car standard, MY 2013 & $(\mathrm{mpg})$ \\
& Car fuel economy, MY 2013 & 33.5 \\
Table 4.27 & Light truck standard, MY 2013 (unreformed) & 36.0 \\
& Light truck fuel economy, MY 2013 \\
& & 25.7 \\
& Average fuel economy loss from 50 to 70 mph & 25.3 \\
& & $24.5 \%$ \\
\hline
\end{tabular}


Car registrations, along with vehicle travel and fuel use, all declined from 2008 to 2012. The data in this table from 1985-on DO NOT include minivans, pickups, or sport utility vehicles. Much of the data for 2009-on were estimated; the FHWA no longer publishes travel and fuel data for cars.

Table 4.1

Summary Statistics for Cars, 1970-2012

\begin{tabular}{|c|c|c|c|c|c|c|}
\hline Year & $\begin{array}{c}\text { Registrations }{ }^{\mathrm{a}} \\
\text { (thousands) }\end{array}$ & $\begin{array}{l}\text { Vehicle travel } \\
\text { (billion miles) }\end{array}$ & $\begin{array}{c}\text { Miles } \\
\text { (per vehicle) }\end{array}$ & $\begin{array}{c}\text { Fuel use } \\
\text { (million gallons) }\end{array}$ & $\begin{array}{l}\text { Fuel economy } \\
\text { (miles per gallon) }\end{array}$ & \\
\hline 1970 & 89,244 & 916.7 & 10,272 & 67,820 & 13.5 & \\
\hline 1975 & 106,706 & $1,034.0$ & 9,690 & 74,140 & 13.9 & \\
\hline 1980 & 121,601 & $1,111.6$ & 9,141 & 69,981 & 15.9 & \\
\hline 1981 & 123,098 & $1,133.3$ & 9,207 & 69,112 & 16.4 & \\
\hline 1982 & 123,702 & $1,161.7$ & 9,391 & 69,116 & 16.8 & \\
\hline 1983 & 126,444 & $1,195.1$ & 9,451 & 70,322 & 17.0 & \\
\hline 1984 & 128,158 & $1,227.0$ & 9,574 & 70,663 & 17.4 & \\
\hline $1985^{\mathrm{c}}$ & 127,885 & $1,246.8$ & 9,749 & 71,518 & 17.4 & \\
\hline 1986 & 130,004 & $1,270.2$ & 9,770 & 73,174 & 17.4 & \\
\hline 1987 & 131,482 & $1,316.0$ & 10,009 & 73,308 & 18.0 & \\
\hline 1988 & 133,836 & $1,370.3$ & 10,238 & 73,345 & 18.7 & \\
\hline 1989 & 134,559 & $1,401.2$ & 10,413 & 73,913 & 19.0 & \\
\hline 1990 & 133,700 & $1,408.3$ & 10,533 & 69,568 & 20.2 & \\
\hline 1991 & 128,300 & $1,358.2$ & 10,586 & 64,318 & 21.1 & \\
\hline 1992 & 126,581 & $1,371.6$ & 10,836 & 65,436 & 21.0 & \\
\hline 1993 & 127,327 & $1,374.7$ & 10,797 & 67,047 & 20.5 & \\
\hline 1994 & 127,883 & $1,406.1$ & 10,995 & 67,874 & 20.7 & \\
\hline 1995 & 128,387 & $1,438.3$ & 11,203 & 68,072 & 21.1 & \\
\hline 1996 & 129,728 & $1,469.9$ & 11,330 & 69,221 & 21.2 & \\
\hline 1997 & 129,749 & $1,502.6$ & 11,580 & 69,892 & 21.5 & \\
\hline 1998 & 131,839 & $1,549.6$ & 11,754 & 71,695 & 21.6 & \\
\hline 1999 & 132,432 & $1,569.1$ & 11,848 & 73,283 & 21.4 & \\
\hline 2000 & 133,621 & $1,600.3$ & 11,976 & 73,065 & 21.9 & \\
\hline 2001 & 137,633 & $1,628.3$ & 11,831 & 73,559 & 22.1 & \\
\hline 2002 & 135,921 & $1,658.5$ & 12,202 & 75,471 & 22.0 & \\
\hline 2003 & 135,670 & $1,672.1$ & 12,325 & 74,590 & 22.4 & \\
\hline 2004 & 136,431 & $1,699.9$ & 12,460 & 75,402 & 22.5 & \\
\hline 2005 & 136,568 & $1,708.4$ & 12,510 & 77,418 & 22.1 & \\
\hline 2006 & 135,400 & $1,690.5$ & 12,485 & 75,009 & 22.5 & \\
\hline 2007 & 135,933 & $1,672.5$ & 12,304 & 74,377 & 22.5 & \\
\hline 2008 & 137,080 & $1,615.9$ & 11,788 & 71,497 & 22.6 & d \\
\hline 2009 & 134,880 & $1,566.8$ & 11,616 & 66,587 & 23.5 & \\
\hline 2010 & 130,892 & $1,496.4$ & 11,432 & 62,245 & 24.0 & \\
\hline 2011 & 125,657 & $1,457.8$ & 11,601 & 59,646 & 24.4 & \\
\hline 2012 & 111,290 & $1,438.8$ & 12,928 & 57,899 & 24.9 & \\
\hline & \multicolumn{6}{|c|}{ Average annual percentage change } \\
\hline 1970-2012 & $0.5 \%$ & $1.1 \%$ & $0.5 \%$ & $-0.4 \%$ & $1.5 \%$ & \\
\hline 2002-2012 & $-2.0 \%$ & $-1.4 \%$ & $0.6 \%$ & $-2.6 \%$ & $1.2 \%$ & \\
\hline
\end{tabular}

\section{Source:}

1970-2008: U.S. Department of Transportation, Federal Highway Administration, Highway Statistics 2009, Washington, DC, 2011, Table VM-1 and annual. 2009-on: See Appendix A for Car/Light Truck Shares.

(Additional resources: www.fhwa.dot.gov)

a This number differs from R.L. Polk's estimates of "number of cars in use." See Table 3.3.

${ }^{\mathrm{b}}$ Fuel economy for car population.

${ }^{c}$ Beginning in this year the data were revised to exclude minivans, pickups and sport utility vehicles which may have been previously included.

${ }^{\mathrm{d}}$ Due to FHWA methodology changes, data from 2009-on are not comparable with previous data. 
Much of the data for 2009-on were estimated; the FHWA no longer publishes travel and fuel use data for twoaxle, four tire trucks.

Table 4.2

Summary Statistics for Two-Axle, Four-Tire Trucks, 1970-2012

\begin{tabular}{|c|c|c|c|c|c|c|}
\hline Year & $\begin{array}{c}\text { Registrations } \\
\text { (thousands) }\end{array}$ & $\begin{array}{l}\text { Vehicle travel } \\
\text { (billion miles) }\end{array}$ & $\begin{array}{c}\text { Miles } \\
\text { (per vehicle) }\end{array}$ & $\begin{array}{c}\text { Fuel use } \\
\text { (million gallons) }\end{array}$ & $\begin{array}{c}\text { Fuel economy } \\
\text { (miles per gallon) }\end{array}$ & \\
\hline 1970 & 14,211 & 123.3 & 8,675 & 12,313 & 10.0 & \\
\hline 1975 & 20,418 & 200.7 & 9,830 & 19,081 & 10.5 & \\
\hline 1980 & 27,876 & 290.9 & 10,437 & 23,796 & 12.2 & \\
\hline 1981 & 28,928 & 296.3 & 10,244 & 23,697 & 12.5 & \\
\hline 1982 & 29,792 & 306.1 & 10,276 & 22,702 & 13.5 & \\
\hline 1983 & 31,214 & 327.6 & 10,497 & 23,945 & 13.7 & \\
\hline 1984 & 32,106 & 358.0 & 11,151 & 25,604 & 14.0 & \\
\hline $1985^{\mathrm{a}}$ & 37,214 & 391.0 & 10,506 & 27,363 & 14.3 & \\
\hline 1986 & 39,382 & 423.9 & 10,764 & 29,074 & 14.6 & \\
\hline 1987 & 41,107 & 456.9 & 11,114 & 30,598 & 14.9 & \\
\hline 1988 & 43,805 & 502.2 & 11,465 & 32,653 & 15.4 & \\
\hline 1989 & 45,945 & 536.5 & 11,676 & 33,271 & 16.1 & \\
\hline 1990 & 48,275 & 574.6 & 11,902 & 35,611 & 16.1 & \\
\hline 1991 & 53,033 & 649.4 & 12,245 & 38,217 & 17.0 & \\
\hline 1992 & 57,091 & 706.9 & 12,381 & 40,929 & 17.3 & \\
\hline 1993 & 59,994 & 745.8 & 12,430 & 42,851 & 17.4 & \\
\hline 1994 & 62,904 & 764.6 & 12,156 & 44,112 & 17.3 & \\
\hline 1995 & 65,738 & 790.0 & 12,018 & 45,605 & 17.3 & \\
\hline 1996 & 69,134 & 816.5 & 11,811 & 47,354 & 17.2 & \\
\hline 1997 & 70,224 & 850.7 & 12,115 & 49,389 & 17.2 & \\
\hline 1998 & 71,330 & 868.3 & 12,173 & 50,462 & 17.2 & \\
\hline 1999 & 75,356 & 901.0 & 11,957 & 52,859 & 17.0 & \\
\hline 2000 & 79,085 & 923.1 & 11,672 & 52,939 & 17.4 & \\
\hline 2001 & 84,188 & 943.2 & 11,204 & 53,522 & 17.6 & \\
\hline 2002 & 85,011 & 966.0 & 11,364 & 55,220 & 17.5 & \\
\hline 2003 & 87,187 & 984.1 & 11,287 & 60,758 & 16.2 & \\
\hline 2004 & 91,845 & $1,027.2$ & 11,184 & 63,417 & 16.2 & \\
\hline 2005 & 95,337 & $1,041.1$ & 10,920 & 58,869 & 17.7 & \\
\hline 2006 & 99,125 & $1,082.5$ & 10,920 & 60,685 & 17.8 & \\
\hline 2007 & 101,470 & $1,112.3$ & 10,962 & 61,836 & 18.0 & \\
\hline 2008 & 101,235 & $1,108.6$ & 10,951 & 61,199 & 18.1 & $\mathrm{~b}$ \\
\hline 2009 & 100,154 & $1,066.5$ & 10,649 & 61,824 & 17.3 & \\
\hline 2010 & 102,702 & $1,152.1$ & 11,218 & 64,687 & 17.8 & \\
\hline 2011 & 105,571 & $1,192.7$ & 11,298 & 65,786 & 18.1 & \\
\hline 2012 & 120,847 & $1,225.6$ & 10,142 & 66,395 & 18.5 & \\
\hline & \multicolumn{6}{|c|}{ Average annual percentage change } \\
\hline 1970-2012 & $5.2 \%$ & $5.6 \%$ & $0.4 \%$ & $4.1 \%$ & $1.5 \%$ & \\
\hline 2002-2012 & $3.6 \%$ & $2.4 \%$ & $-1.1 \%$ & $1.9 \%$ & $0.6 \%$ & \\
\hline
\end{tabular}

Source:

1970-2008: U.S. Department of Transportation, Federal Highway Administration, Highway Statistics 2009, Washington, DC, 2011, Table MV-9. Previous years Table VM-1. 2009-on: See Appendix A for Car/Light Truck Shares. (Additional resources: www.fhwa.dot.gov)

${ }^{a}$ Beginning in this year the data were revised to include all vans (including mini-vans), pickups and sport utility vehicles.

${ }^{\mathrm{b}}$ Due to FHWA methodology changes, data from 2009-on are not comparable with previous data. 
Because data on Class $2 b$ trucks are scarce, the U.S. DOE funded a study to investigate available sources of data. In the final report, four methodologies are described to estimate the sales of Class $2 b$ trucks. Until another study is funded, the 1999 data are the latest available.

Table 4.3

Summary Statistics on Class 1, Class 2a, and Class 2b Light Trucks

\begin{tabular}{lccccccc}
\hline & $\begin{array}{c}\text { CY 1999 } \\
\text { truck sales } \\
\text { (millions) }\end{array}$ & $\begin{array}{c}\text { MY 2000 } \\
\text { truck } \\
\text { population } \\
\text { (millions) }\end{array}$ & $\begin{array}{c}\text { Percent } \\
\text { diesel trucks } \\
\text { in } \\
\text { population }\end{array}$ & $\begin{array}{c}\text { Average } \\
\text { age } \\
\text { (years) }\end{array}$ & $\begin{array}{c}\text { Estimated } \\
\text { annual } \\
\text { miles }^{\mathrm{a}} \\
\text { (billions) }\end{array}$ & $\begin{array}{c}\text { Estimated } \\
\text { fuel use } \\
\text { (billion }^{\mathrm{a}} \\
\text { gallons) }\end{array}$ & $\begin{array}{c}\text { Estimated fuel } \\
\text { economy } \\
\text { (miles per } \\
\text { gallon) }\end{array}$ \\
\hline Class 1 & 5.7 & 49.7 & $0.3 \%$ & 7.3 & 672.7 & 37.4 & 18.0 \\
Class 2a & 1.8 & 19.2 & $2.5 \%$ & 7.4 & 251.9 & 18.0 & 14.0 \\
Class 2b & 0.5 & 5.8 & $24.0 \%$ & 8.6 & 76.7 & 5.5 & 13.9 \\
\hline
\end{tabular}

Note: CY - calendar year. MY - model year.

\section{Source:}

Davis, S.C. and L.F. Truett, Investigation of Class $2 b$ Trucks (Vehicles of 8,500 to 10,000 lbs GVWR), ORNL/TM2002/49, March 2002, Table 16.

Table 4.4

Sales Estimates of Class 1, Class 2a, and Class 2b Light Trucks, 1989-1999

\begin{tabular}{ccccc}
\hline & \multicolumn{4}{c}{ Sales estimates (thousands) } \\
\cline { 2 - 5 } Calendar year & Class 1 & $\begin{array}{c}\text { Class 2a } \\
(6,001-8,500 \mathrm{lbs})\end{array}$ & $\begin{array}{c}\text { Class 2b } \\
(8,501-10,000 \mathrm{lbs})\end{array}$ & Total \\
\hline 1989 & 3,313 & 918 & 379 & 4,610 \\
1990 & 3,451 & 829 & 268 & 4,548 \\
1991 & 3,246 & 670 & 206 & 4,122 \\
1992 & 3,608 & 827 & 194 & 4,629 \\
1993 & 4,119 & 975 & 257 & 5,351 \\
1994 & 4,527 & 1,241 & 265 & 6,033 \\
1995 & 4,422 & 1,304 & 327 & 6,053 \\
1996 & 4,829 & 1,356 & 334 & 6,519 \\
1997 & 5,085 & 1,315 & 397 & 6,797 \\
1998 & 5,263 & 1,694 & 342 & 7,299 \\
1999 & 5,707 & 1,845 & 521 & 8,073 \\
$1989-1999$ & & & & \\
& $72.3 \%$ & Percent change & $37.5 \%$ & $75.1 \%$ \\
\hline
\end{tabular}

Note: These data were calculated using Methodology 4 from the report.

\section{Source:}

Davis, S.C. and L.F. Truett, Investigation of Class $2 b$ Trucks (Vehicles of 8,500 to 10,000 lbs GVWR), ORNL/TM2002/49, March 2002, Table 1.

${ }^{\text {a }}$ Estimates derived using 2000 population data and 1997 usage data. See source for details. 
Car sales in 2009 and 2010 were below 6 million but increased to more than 7 million by 2012. In 1980, the Big 3 (Chrysler, Ford and General Motors) held 73.8\% of the market; by 2013, that had dropped to $32.4 \%$.

Table 4.5

New Retail Car Sales in the United States, 1970-2013

\begin{tabular}{|c|c|c|c|c|c|c|}
\hline \multirow{2}{*}{$\begin{array}{c}\text { Calendar } \\
\text { year }\end{array}$} & Domestic $^{\mathrm{a}}$ & Import $^{\mathrm{b}}$ & Total & \multirow{2}{*}{$\begin{array}{c}\text { Percentage } \\
\text { imports }\end{array}$} & \multirow{2}{*}{$\begin{array}{c}\text { Percentage } \\
\text { Big } 3 \\
\text { sales }^{c}\end{array}$} & \multirow{2}{*}{$\begin{array}{c}\text { Percentage } \\
\text { diesel }\end{array}$} \\
\hline & \multicolumn{3}{|c|}{ (thousands) $^{\mathrm{e}}$} & & & \\
\hline 1970 & 7,119 & 1,280 & 8,399 & $15.2 \%$ & d & $0.07 \%$ \\
\hline 1975 & 7,053 & 1,571 & 8,624 & $18.2 \%$ & d & $0.31 \%$ \\
\hline 1980 & 6,580 & 2,369 & 8,949 & $26.5 \%$ & $73.8 \%$ & $4.32 \%$ \\
\hline 1985 & 8,205 & 2,775 & 10,979 & $25.3 \%$ & $72.9 \%$ & $0.83 \%$ \\
\hline 1986 & 8,215 & 3,189 & 11,404 & $28.0 \%$ & $70.9 \%$ & $0.37 \%$ \\
\hline 1987 & 7,085 & 3,107 & 10,192 & $30.5 \%$ & $67.6 \%$ & $0.17 \%$ \\
\hline 1988 & 7,543 & 3,004 & 10,547 & $28.5 \%$ & $69.3 \%$ & $0.02 \%$ \\
\hline 1989 & 7,098 & 2,680 & 9,779 & $27.4 \%$ & $67.9 \%$ & $0.13 \%$ \\
\hline 1990 & 6,919 & 2,384 & 9,303 & $25.6 \%$ & $65.7 \%$ & $0.08 \%$ \\
\hline 1991 & 6,162 & 2,023 & 8,185 & $24.7 \%$ & $64.2 \%$ & $0.10 \%$ \\
\hline 1992 & 6,286 & 1,927 & 8,213 & $23.5 \%$ & $65.8 \%$ & $0.06 \%$ \\
\hline 1993 & 6,742 & 1,776 & 8,518 & $20.9 \%$ & $67.3 \%$ & $0.04 \%$ \\
\hline 1994 & 7,255 & 1,735 & 8,991 & $19.3 \%$ & $65.9 \%$ & $0.04 \%$ \\
\hline 1995 & 7,114 & 1,506 & 8,620 & $17.5 \%$ & $65.3 \%$ & $0.03 \%$ \\
\hline 1996 & 7,206 & 1,272 & 8,479 & $15.0 \%$ & $64.1 \%$ & $0.09 \%$ \\
\hline 1997 & 6,862 & 1,355 & 8,217 & $16.5 \%$ & $62.2 \%$ & $0.09 \%$ \\
\hline 1998 & 6,705 & 1,380 & 8,085 & $17.1 \%$ & $59.7 \%$ & $0.14 \%$ \\
\hline 1999 & 6,919 & 1,719 & 8,638 & $19.9 \%$ & $58.3 \%$ & $0.16 \%$ \\
\hline 2000 & 6,762 & 2,016 & 8,778 & $23.0 \%$ & $55.0 \%$ & $0.26 \%$ \\
\hline 2001 & 6,254 & 2,098 & 8,352 & $25.1 \%$ & $51.4 \%$ & $0.18 \%$ \\
\hline 2002 & 5,817 & 2,226 & 8,042 & $27.7 \%$ & $48.4 \%$ & $0.39 \%$ \\
\hline 2003 & 5,473 & 2,083 & 7,556 & $27.6 \%$ & $47.1 \%$ & $0.52 \%$ \\
\hline 2004 & 5,333 & 2,149 & 7,483 & $28.7 \%$ & $44.9 \%$ & $0.40 \%$ \\
\hline 2005 & 5,473 & 2,187 & 7,660 & $28.5 \%$ & $43.1 \%$ & $0.63 \%$ \\
\hline 2006 & 5,417 & 2,345 & 7,762 & $30.2 \%$ & $40.5 \%$ & $0.82 \%$ \\
\hline 2007 & 5,197 & 2,365 & 7,562 & $31.3 \%$ & $36.9 \%$ & $0.11 \%$ \\
\hline 2008 & 4,491 & 2,278 & 6,769 & $33.7 \%$ & $34.2 \%$ & $0.11 \%$ \\
\hline 2009 & 3,558 & 1,843 & 5,402 & $34.1 \%$ & $31.3 \%$ & $2.94 \%$ \\
\hline 2010 & 3,791 & 1,844 & 5,636 & $32.7 \%$ & $31.7 \%$ & $2.69 \%$ \\
\hline 2011 & 4,143 & 1,947 & 6,090 & $32.0 \%$ & $33.3 \%$ & $1.47 \%$ \\
\hline 2012 & 5,118 & 2,125 & 7,244 & $29.3 \%$ & $31.6 \%$ & $2.69 \%$ \\
\hline 2013 & 5,432 & 2,154 & 7,586 & $28.4 \%$ & $32.4 \%$ & $2.45 \%$ \\
\hline \multicolumn{7}{|c|}{ Average annual percentage change } \\
\hline 1970-2013 & $-0.6 \%$ & $1.2 \%$ & $-0.4 \%$ & & & \\
\hline 2003-2013 & $-0.1 \%$ & $0.3 \%$ & $0.0 \%$ & & & \\
\hline
\end{tabular}

\section{Source:}

Domestic and import data - 1970-97: American Automobile Manufacturers Association, Motor Vehicle Facts and Figures 1998, Detroit, MI, 1998, p. 15, and annual. 1997 data from Economic Indicators, 4th Quarter 1997. 1998-2010: Ward's Communication, Ward's Automotive Yearbook, Detroit, MI, 2009, p. 249. 2011-2013: Ward's Communications, www.wardsauto.com.

Diesel data - Ward's Communications, Ward's Automotive Yearbook, Detroit, MI, 2009, p. 31, and Ward's Communications, www.wardsauto.com.

${ }^{a}$ Any vehicle built in North America regardless of manufacturer.

b Any vehicle built outside of North America regardless of manufacturer. Does not include import tourist deliveries.

${ }^{\mathrm{c}}$ Big 3 includes Chrysler, Ford and General Motors.

${ }^{\mathrm{d}}$ Data are not available.

e Sums may not add to totals due to rounding. 
Light trucks, which include pick-ups, minivans, sport-utility vehicles, and other trucks less than 10,000 pounds gross vehicle weight $(G V W)$, accounted for more than half of light vehicle sales from 2001 to 2007 and again in 2011 and 2013.

Table 4.6

New Retail Sales of Trucks 10,000 Pounds GVW and Less in the United States, 1970-2013

\begin{tabular}{|c|c|c|c|c|c|c|}
\hline \multirow[b]{2}{*}{$\begin{array}{c}\text { Calendar } \\
\text { year }\end{array}$} & \multirow[b]{2}{*}{$\begin{array}{l}\text { Light truck } \\
\text { sales }{ }^{\mathrm{a}} \\
\text { (thousands) }\end{array}$} & \multicolumn{5}{|c|}{ Percentages } \\
\hline & & Import $^{\mathrm{b}}$ & $\begin{array}{l}\text { Big } 3 \\
\text { sales }^{c}\end{array}$ & Diesel $^{\mathrm{d}}$ & $\begin{array}{l}\text { Light trucks of } \\
\text { light-duty } \\
\text { vehicle sales }\end{array}$ & $\begin{array}{l}\text { Light trucks } \\
\text { of total } \\
\text { truck sales }\end{array}$ \\
\hline 1970 & 1,457 & $4.5 \%$ & Not available & $f^{f}$ & $14.8 \%$ & $80.5 \%$ \\
\hline 1975 & 2,053 & $10.0 \%$ & Not available & & $20.9 \%$ & $82.8 \%$ \\
\hline 1980 & 1,960 & $24.4 \%$ & Not available & $4.0 \%$ & $17.5 \%$ & $78.6 \%$ \\
\hline $1985^{\circ}$ & 3,688 & $22.6 \%$ & $78.2 \%$ & $4.0 \%$ & $25.1 \%$ & $77.7 \%$ \\
\hline 1986 & 4,594 & $21.3 \%$ & $76.9 \%$ & $3.7 \%$ & $28.7 \%$ & $93.4 \%$ \\
\hline 1987 & 4,610 & $20.0 \%$ & $78.3 \%$ & $2.3 \%$ & $31.1 \%$ & $92.2 \%$ \\
\hline 1988 & 4,800 & $14.8 \%$ & $81.6 \%$ & $2.3 \%$ & $31.3 \%$ & $91.5 \%$ \\
\hline 1989 & 4,610 & $13.9 \%$ & $81.9 \%$ & $2.9 \%$ & $32.0 \%$ & $91.0 \%$ \\
\hline 1990 & 4,548 & $13.5 \%$ & $80.9 \%$ & $2.3 \%$ & $32.8 \%$ & $93.8 \%$ \\
\hline 1991 & 4,122 & $13.1 \%$ & $79.4 \%$ & $3.2 \%$ & $33.5 \%$ & $94.4 \%$ \\
\hline 1992 & 4,629 & $8.8 \%$ & $83.1 \%$ & $2.5 \%$ & $36.0 \%$ & $94.4 \%$ \\
\hline 1993 & 5,351 & $7.1 \%$ & $83.4 \%$ & $2.3 \%$ & $38.6 \%$ & $94.2 \%$ \\
\hline 1994 & 6,033 & $6.8 \%$ & $82.9 \%$ & $2.5 \%$ & $40.2 \%$ & $94.0 \%$ \\
\hline 1995 & 6,053 & $6.6 \%$ & $83.4 \%$ & $3.8 \%$ & $41.3 \%$ & $93.2 \%$ \\
\hline 1996 & 6,519 & $6.7 \%$ & $83.8 \%$ & $3.1 \%$ & $43.5 \%$ & $93.4 \%$ \\
\hline 1997 & 6,797 & $8.5 \%$ & $81.9 \%$ & $2.7 \%$ & $45.3 \%$ & $93.4 \%$ \\
\hline 1998 & 7,299 & $9.0 \%$ & $80.5 \%$ & $2.6 \%$ & $47.4 \%$ & $92.6 \%$ \\
\hline 1999 & 8,073 & $9.6 \%$ & $78.0 \%$ & $2.9 \%$ & $48.3 \%$ & $92.0 \%$ \\
\hline 2000 & 8,386 & $10.2 \%$ & $76.1 \%$ & $3.4 \%$ & $48.9 \%$ & $92.8 \%$ \\
\hline 2001 & 8,598 & $11.4 \%$ & $75.3 \%$ & $2.9 \%$ & $50.7 \%$ & $94.3 \%$ \\
\hline 2002 & 8,633 & $12.4 \%$ & $74.7 \%$ & $2.7 \%$ & $51.8 \%$ & $94.9 \%$ \\
\hline 2003 & 8,938 & $13.7 \%$ & $72.4 \%$ & $2.9 \%$ & $54.2 \%$ & $95.0 \%$ \\
\hline 2004 & 9,254 & $13.5 \%$ & $70.1 \%$ & $2.8 \%$ & $55.3 \%$ & $94.3 \%$ \\
\hline 2005 & 9,114 & $13.3 \%$ & $68.2 \%$ & $2.7 \%$ & $54.3 \%$ & $93.1 \%$ \\
\hline 2006 & 8,574 & $15.7 \%$ & $63.9 \%$ & $2.8 \%$ & $52.5 \%$ & $92.3 \%$ \\
\hline 2007 & 8,305 & $16.7 \%$ & $61.9 \%$ & $3.2 \%$ & $52.3 \%$ & $93.3 \%$ \\
\hline 2008 & 6,246 & $17.6 \%$ & $61.2 \%$ & $3.4 \%$ & $48.0 \%$ & $92.9 \%$ \\
\hline 2009 & 4,834 & $18.3 \%$ & $57.8 \%$ & $4.2 \%$ & $47.2 \%$ & $93.0 \%$ \\
\hline 2010 & 5,758 & $15.6 \%$ & $57.6 \%$ & $4.9 \%$ & $50.5 \%$ & $93.8 \%$ \\
\hline 2011 & 6,449 & $15.2 \%$ & $59.4 \%$ & $5.4 \%$ & $51.4 \%$ & $92.8 \%$ \\
\hline 2012 & 6,975 & $15.2 \%$ & $57.7 \%$ & $5.5 \%$ & $49.1 \%$ & $92.5 \%$ \\
\hline 2013 & 7,693 & $16.1 \%$ & $57.3 \%$ & $5.3 \%$ & $50.3 \%$ & $92.7 \%$ \\
\hline \multicolumn{7}{|c|}{ Average annual percentage change } \\
\hline $1970-2013$ & $3.9 \%$ & & & & & \\
\hline 2003-2013 & $-1.5 \%$ & & & & & \\
\hline
\end{tabular}

\section{Source:}

Ward's Communications, Ward's Automotive Yearbook, Detroit, MI, 2013, and updates at www.wardsauto.com. (Additional resources: www.wardsauto.com)

${ }^{\text {a }}$ Includes all trucks of 10,000 pounds gross vehicle weight and less sold in the United States.

${ }^{\mathrm{b}}$ Excluding transplants.

${ }^{\mathrm{c}}$ Big 3 includes Chrysler, Ford and General Motors.

${ }^{\mathrm{d}}$ Based on model year factory installations.

${ }^{\mathrm{e}}$ Light-duty vehicles include cars and light trucks.

${ }^{\mathrm{f}}$ Indicates less than 1 percent. 
The production-weighted fuel economy of cars increased dramatically from 1975 (13.5 mpg) to 1985 (23.0 mpg), but rose only $0.5 \mathrm{mpg}$ from 1985 to 2005. Since 2005, fuel economy rose $4.5 \mathrm{mpg}$-from $23.5 \mathrm{mpg}$ in 2005 to 28.0 mpg in 2013. The fuel economy values have been adjusted to provide the best estimate of real world performance.

Table 4.7

Production Sales, Production Shares, and Production-Weighted Fuel Economies of New Domestic and Import Cars, Model Years 1975-2013

\begin{tabular}{|c|c|c|c|c|c|c|}
\hline \multirow[b]{2}{*}{ Model year } & \multicolumn{3}{|c|}{ Car } & \multicolumn{3}{|c|}{ Car SUV } \\
\hline & $\begin{array}{l}\text { Production } \\
\text { (thousands) }\end{array}$ & $\begin{array}{l}\text { Production } \\
\text { share }(\%)^{\mathrm{b}}\end{array}$ & $\begin{array}{c}\text { Fuel } \\
\text { economy } \\
(\mathrm{mpg})\end{array}$ & $\begin{array}{l}\text { Production } \\
\text { (thousands) }\end{array}$ & $\begin{array}{l}\text { Production } \\
\text { share }(\%)^{\mathrm{b}}\end{array}$ & $\begin{array}{c}\text { Fuel } \\
\text { economy } \\
\text { (mpg) }\end{array}$ \\
\hline 1975 & 8,237 & $99.9 \%$ & 13.5 & 10 & $0.1 \%$ & 11.1 \\
\hline 1980 & 9,443 & $100.0 \%$ & 20.0 & 0 & $0.0 \%$ & 14.6 \\
\hline 1981 & 8,733 & $100.0 \%$ & 21.4 & 0 & $0.0 \%$ & 14.7 \\
\hline 1982 & 7,819 & $99.8 \%$ & 22.2 & 13 & $0.2 \%$ & 19.8 \\
\hline 1983 & 8,002 & $99.6 \%$ & 22.1 & 34 & $0.4 \%$ & 20.7 \\
\hline 1984 & 10,675 & $99.5 \%$ & 22.4 & 55 & $0.5 \%$ & 19.3 \\
\hline 1985 & 10,791 & $99.2 \%$ & 23.0 & 88 & $0.8 \%$ & 20.1 \\
\hline 1986 & 11,015 & $99.5 \%$ & 23.7 & 59 & $0.5 \%$ & 18.9 \\
\hline 1987 & 10,731 & $99.1 \%$ & 23.8 & 95 & $0.9 \%$ & 19.4 \\
\hline 1988 & 10,736 & $99.0 \%$ & 24.1 & 109 & $1.0 \%$ & 19.2 \\
\hline 1989 & 10,018 & $98.9 \%$ & 23.7 & 108 & $1.1 \%$ & 19.1 \\
\hline 1990 & 8,810 & $99.3 \%$ & 23.3 & 65 & $0.7 \%$ & 18.8 \\
\hline 1991 & 8,524 & $97.4 \%$ & 23.4 & 224 & $2.6 \%$ & 18.2 \\
\hline 1992 & 8,108 & $97.1 \%$ & 23.1 & 243 & $2.9 \%$ & 17.8 \\
\hline 1993 & 8,456 & $94.7 \%$ & 23.5 & 473 & $5.3 \%$ & 17.0 \\
\hline 1994 & 8,415 & $96.2 \%$ & 23.3 & 332 & $3.8 \%$ & 18.0 \\
\hline 1995 & 9,396 & $97.7 \%$ & 23.4 & 220 & $2.3 \%$ & 17.8 \\
\hline 1996 & 7,890 & $96.5 \%$ & 23.3 & 287 & $3.5 \%$ & 18.4 \\
\hline 1997 & 8,334 & $95.8 \%$ & 23.4 & 361 & $4.2 \%$ & 19.2 \\
\hline 1998 & 7,971 & $94.6 \%$ & 23.4 & 454 & $5.4 \%$ & 18.2 \\
\hline 1999 & 8,376 & $94.5 \%$ & 23.0 & 488 & $5.5 \%$ & 18.5 \\
\hline 2000 & 9,125 & $93.7 \%$ & 22.9 & 617 & $6.3 \%$ & 17.9 \\
\hline 2001 & 8,405 & $91.9 \%$ & 23.0 & 743 & $8.1 \%$ & 18.8 \\
\hline 2002 & 8,301 & $93.2 \%$ & 23.1 & 603 & $6.8 \%$ & 19.3 \\
\hline 2003 & 7,921 & $93.2 \%$ & 23.3 & 575 & $6.8 \%$ & 19.9 \\
\hline 2004 & 7,537 & $92.2 \%$ & 23.1 & 639 & $7.8 \%$ & 20.0 \\
\hline 2005 & 8,027 & $90.8 \%$ & 23.5 & 813 & $9.2 \%$ & 20.2 \\
\hline 2006 & 7,993 & $91.4 \%$ & 23.3 & 751 & $8.6 \%$ & 20.5 \\
\hline 2007 & 8,082 & $89.8 \%$ & 24.1 & 919 & $10.2 \%$ & 20.6 \\
\hline 2008 & 7,319 & $88.8 \%$ & 24.3 & 924 & $11.2 \%$ & 21.2 \\
\hline 2009 & 5,636 & $90.3 \%$ & 25.3 & 608 & $9.7 \%$ & 22.0 \\
\hline 2010 & 6,055 & $86.9 \%$ & 26.2 & 915 & $13.1 \%$ & 23.0 \\
\hline 2011 & 5,728 & $82.6 \%$ & 26.0 & 1,207 & $17.4 \%$ & 23.6 \\
\hline 2012 & 7,379 & $85.3 \%$ & 27.8 & 1,269 & $14.7 \%$ & 23.4 \\
\hline 2013 & 7,731 & $83.5 \%$ & 28.0 & 1,526 & $16.5 \%$ & 24.5 \\
\hline
\end{tabular}

Source:

U.S. Environmental Protection Agency, Light-Duty Automotive Technology, Carbon Dioxide Emissions, and Fuel Economy Trends: 1975 Through 2013, December 2013. (Additional resources: www.epa.gov/fueleconomy/fetrends/1975-2013/420r13011.pdf)

a The fuel economy data on this table are adjusted to provide the best estimate of real world performance. See section 10 of the source document for details on adjustment methodology.

${ }^{\mathrm{b}}$ Production share is based on total of cars plus car SUVs. Percentages may not sum to totals due to rounding. 
A new vehicle classification was created to match the Corporate Average Fuel Economy (CAFE) methodology. Under CAFE, small, two-wheel drive sport utility vehicles will be held to the same standards as cars. The Environmental Protection Agency has defined these vehicles as "car SUVs." The vehicles below make up this category.

Table 4.8

Definition of Car Sport Utility Vehicles in Model Year 2013

\begin{tabular}{ll}
\hline Acura RDX 2WD & Jeep Compass 2WD \\
Buick Encore & Jeep Patriot 2WD \\
Cadillac SRX 2WD & Kia Sorento 2WD \\
Cadillac SRX AWD & Kia Sorento 4WD \\
Chevrolet Captiva AWD & Kia Sportage 2WD \\
Chevrolet Captiva FWD & Lexus RX 350 \\
Chevrolet Equinox AWD & Lexus RX 450h \\
Chevrolet Equinox FWD & Mazda CX-5 2WD \\
Dodge Journey FWD & Mercedes-Benz GLK 350 \\
Ford Edge FWD & Mitsubishi Outlander 2WD \\
Ford Escape FWD & Mitsubishi Outlander Sport 2WD \\
Ford Transit Connect Wagon FWD & Nissan Murano FWD \\
GMC Terrain AWD & Nissan Rogue FWD \\
GMC Terrain FWD & Nissan XTERRA 2WD \\
Honda Crosstour 2WD & Suzuki Grand Vitara \\
Honda Crosstour 4WD & Toyota FJ Cruiser 2WD \\
Honda CR-V 2WD & Toyota RAV4 \\
Hyundai Santa Fe 2WD & Toyota Venza \\
Hyundai Santa Fe Sport 2WD & Volvo XC60 FWD \\
Hyundai Tucson 2WD & Volvo XC70 FWD \\
Infiniti JX35 FWD & VW Tiguan \\
\hline
\end{tabular}

Note: $2 \mathrm{WD}=$ Two-wheel drive. $4 \mathrm{WD}=$ Four-wheel drive. $\mathrm{FWD}=$ Front-wheel drive.

\section{Source:}

U.S. Environmental Protection Agency, Light-Duty Automotive Technology, Carbon Dioxide Emissions, and Fuel Economy Trends: 1975 Through 2013, December 2013. (Additional resources: www.epa.gov/fueleconomy/fetrends/1975-2013/420r13011.pdf) 
Production of sport utility vehicles (SUVs) has grown substantially since 1975. The production-weighted fuel economy of SUVs was more than 20 mpg in 2013. Nearly 60\% of all light trucks produced in 2013 were SUVs.

Table 4.9

Production, Production Shares, and Production-Weighted Fuel Economies of New Domestic and Import Light Trucks, Model Years 1975-2013 ${ }^{\mathrm{a}}$

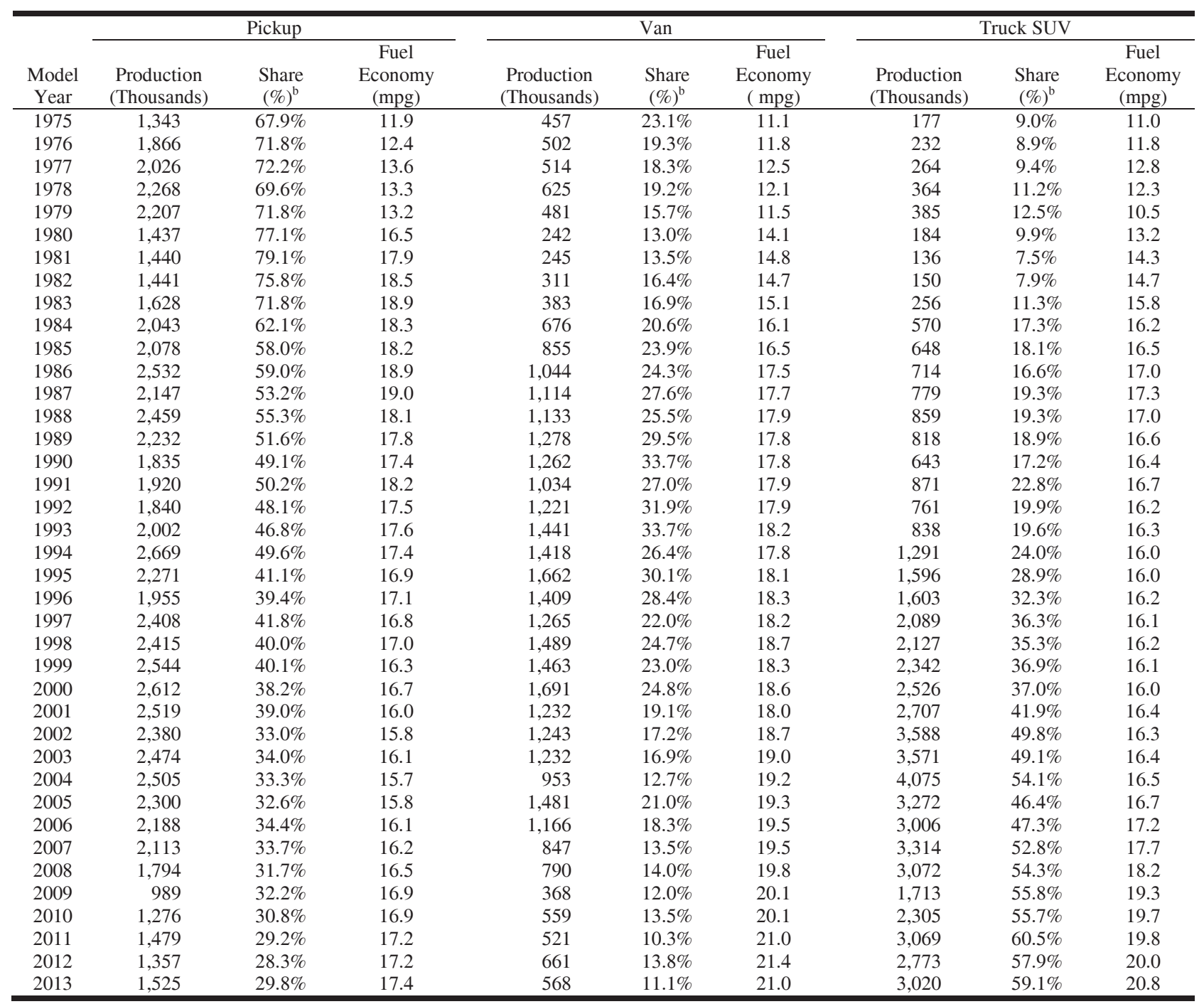

Note: Includes light trucks of $8,500 \mathrm{lbs}$. or less.

Source:

U.S. Environmental Protection Agency, Light-Duty Automotive Technology, Carbon Dioxide Emissions, and Fuel Economy Trends: 1975 Through 2013, December 2013. (Additional resources: www.epa.gov/fueleconomy/fetrends/1975-2013/420r13011.pdf)

${ }^{a}$ The fuel economy data on this table are adjusted to provide the best estimate of real world performance. See section 10 of the source document for details on adjustment methodology.

${ }^{\mathrm{b}}$ Production share is based on the total of pickups, plus vans and truck SUVs. Percentages may not sum to totals due to rounding. 
The average fuel economy of cars more than doubled from 1975 to 2013 while the average fuel economy of light trucks grew by 70\% in that same time period. This was not steady annual growth, but growth in the 1970's and early 1980's followed by a long period with little improvement. Growth resumed around 2008-2009.

Table 4.10

Production and Production-Weighted Fuel Economies of New Domestic and Import Cars, Light Trucks and Light Vehicles, Model Years 1975-2013 ${ }^{a}$

\begin{tabular}{|c|c|c|c|c|c|c|}
\hline \multirow[b]{2}{*}{$\begin{array}{c}\text { Model } \\
\text { Year }\end{array}$} & \multicolumn{2}{|c|}{ All Cars } & \multicolumn{2}{|c|}{ All Light Trucks } & \multicolumn{2}{|c|}{ All Light Vehicles } \\
\hline & $\begin{array}{l}\text { Production } \\
\text { (Thousands) }\end{array}$ & $\begin{array}{c}\text { Fuel } \\
\text { Economy (mpg) }\end{array}$ & $\begin{array}{l}\text { Production } \\
\text { (Thousands) }\end{array}$ & $\begin{array}{c}\text { Fuel } \\
\text { Economy (mpg) }\end{array}$ & $\begin{array}{l}\text { Production } \\
\text { (Thousands) }\end{array}$ & $\begin{array}{c}\text { Fuel } \\
\text { Economy (mpg) } \\
\end{array}$ \\
\hline 1975 & 8,247 & 13.5 & 1,977 & 11.6 & 10,224 & 13.1 \\
\hline 1976 & 9,734 & 14.9 & 2,600 & 12.2 & 12,334 & 14.2 \\
\hline 1977 & 11,318 & 15.6 & 2,805 & 13.3 & 14,123 & 15.1 \\
\hline 1978 & 11,191 & 16.9 & 3,257 & 12.9 & 14,448 & 15.8 \\
\hline 1979 & 10,810 & 17.2 & 3,072 & 12.5 & 13,882 & 15.9 \\
\hline 1980 & 9,444 & 20.0 & 1,863 & 15.8 & 11,307 & 19.2 \\
\hline 1981 & 8,734 & 21.4 & 1,821 & 17.1 & 10,555 & 20.5 \\
\hline 1982 & 7,832 & 22.2 & 1,901 & 17.4 & 9,733 & 21.1 \\
\hline 1983 & 8,035 & 22.1 & 2,267 & 17.7 & 10,302 & 21.0 \\
\hline 1984 & 10,730 & 22.4 & 3,289 & 17.4 & 14,019 & 21.0 \\
\hline 1985 & 10,879 & 23.0 & 3,581 & 17.5 & 14,460 & 21.3 \\
\hline 1986 & 11,074 & 23.7 & 4,291 & 18.2 & 15,365 & 21.8 \\
\hline 1987 & 10,826 & 23.8 & 4,039 & 18.3 & 14,865 & 22.0 \\
\hline 1988 & 10,845 & 24.1 & 4,450 & 17.8 & 15,295 & 21.9 \\
\hline 1989 & 10,126 & 23.6 & 4,327 & 17.6 & 14,453 & 21.4 \\
\hline 1990 & 8,875 & 23.3 & 3,740 & 17.4 & 12,615 & 21.2 \\
\hline 1991 & 8,748 & 23.3 & 3,825 & 17.8 & 12,573 & 21.3 \\
\hline 1992 & 8,350 & 22.9 & 3,822 & 17.3 & 12,172 & 20.8 \\
\hline 1993 & 8,929 & 23.0 & 4,281 & 17.5 & 13,210 & 20.9 \\
\hline 1994 & 8,747 & 23.0 & 5,378 & 17.2 & 14,125 & 20.4 \\
\hline 1995 & 9,616 & 23.3 & 5,529 & 17.0 & 15,145 & 20.5 \\
\hline 1996 & 8,177 & 23.1 & 4,967 & 17.2 & 13,144 & 20.4 \\
\hline 1997 & 8,695 & 23.2 & 5,762 & 16.8 & 14,457 & 20.2 \\
\hline 1998 & 8,425 & 23.0 & 6,030 & 17.1 & 14,455 & 20.1 \\
\hline 1999 & 8,865 & 22.7 & 6,350 & 16.6 & 15,215 & 19.7 \\
\hline 2000 & 9,742 & 22.5 & 6,829 & 16.8 & 16,571 & 19.8 \\
\hline 2001 & 9,148 & 22.6 & 6,458 & 16.5 & 15,606 & 19.6 \\
\hline 2002 & 8,904 & 22.8 & 7,211 & 16.5 & 16,115 & 19.5 \\
\hline 2003 & 8,496 & 23.0 & 7,277 & 16.7 & 15,773 & 19.6 \\
\hline 2004 & 8,176 & 22.9 & 7,533 & 16.5 & 15,709 & 19.3 \\
\hline 2005 & 8,839 & 23.1 & 7,053 & 16.9 & 15,892 & 19.9 \\
\hline 2006 & 8,744 & 23.0 & 6,360 & 17.2 & 15,104 & 20.1 \\
\hline 2007 & 9,001 & 23.7 & 6,275 & 17.4 & 15,276 & 20.6 \\
\hline 2008 & 8,243 & 23.9 & 5,656 & 17.8 & 13,899 & 21.0 \\
\hline 2009 & 6,244 & 25.0 & 3,071 & 18.5 & 9,315 & 22.4 \\
\hline 2010 & 6,969 & 25.7 & 4,141 & 18.8 & 11,110 & 22.6 \\
\hline 2011 & 6,934 & 25.6 & 5,069 & 19.1 & 12,003 & 22.4 \\
\hline 2012 & 8,648 & 27.0 & 4,791 & 19.3 & 13,439 & 23.6 \\
\hline 2013 & 9,257 & 27.4 & 5,114 & 19.7 & 14,371 & 24.0 \\
\hline
\end{tabular}

Source:

U.S. Environmental Protection Agency, Light-Duty Automotive Technology, Carbon Dioxide Emissions, and Fuel Economy Trends: 1975 Through 2013, December 2013. (Additional resources: www.epa.gov/fueleconomy/fetrends/1975-2013/420r13011.pdf)

${ }^{a}$ The fuel economy data on this table are adjusted to provide the best estimate of real world performance. See section 10 of the source document for details on adjustment methodology. 
Back in 1975 only $19.3 \%$ of new light vehicles produced were light trucks. Because of the boom in production of minivans, sport utility vehicles, and pick-up trucks, that number rose to over $40 \%$ from 1998 to 2008. Cars made a comeback to account for $64.4 \%$ in 2013.

Table 4.11

Light Vehicle Production Shares ${ }^{\mathrm{a}}$, Model Years 1975-2013

\begin{tabular}{|c|c|c|c|c|c|c|c|c|}
\hline \multirow[b]{2}{*}{$\begin{array}{l}\text { Model } \\
\text { Year }\end{array}$} & \multirow[b]{2}{*}{ Car } & \multirow[b]{2}{*}{$\begin{array}{l}\text { Car } \\
\text { SUV }\end{array}$} & \multirow[b]{2}{*}{ Pickup } & \multirow[b]{2}{*}{ Van } & \multirow[b]{2}{*}{$\begin{array}{l}\text { Truck } \\
\text { SUV }\end{array}$} & \multirow{2}{*}{$\begin{array}{l}\text { Total Light } \\
\text { Vehicles } \\
\text { Produced } \\
\text { (thousands) }\end{array}$} & \multicolumn{2}{|c|}{ Production Share } \\
\hline & & & & & & & Cars & $\begin{array}{c}\text { Light } \\
\text { Trucks }\end{array}$ \\
\hline 1975 & $80.6 \%$ & $0.1 \%$ & $13.1 \%$ & $4.5 \%$ & $1.7 \%$ & 10,224 & $80.7 \%$ & $19.3 \%$ \\
\hline 1976 & $78.8 \%$ & $0.1 \%$ & $15.1 \%$ & $4.1 \%$ & $1.9 \%$ & 12,334 & $78.9 \%$ & $21.1 \%$ \\
\hline 1977 & $80.0 \%$ & $0.1 \%$ & $14.3 \%$ & $3.6 \%$ & $1.9 \%$ & 14,123 & $80.1 \%$ & $19.9 \%$ \\
\hline 1978 & $77.3 \%$ & $0.1 \%$ & $15.7 \%$ & $4.3 \%$ & $2.5 \%$ & 14,448 & $77.5 \%$ & $22.5 \%$ \\
\hline 1979 & $77.8 \%$ & $0.1 \%$ & $15.9 \%$ & $3.5 \%$ & $2.8 \%$ & 13,882 & $77.9 \%$ & $22.1 \%$ \\
\hline 1980 & $83.5 \%$ & $0.0 \%$ & $12.7 \%$ & $2.1 \%$ & $1.6 \%$ & 11,306 & $83.5 \%$ & $16.5 \%$ \\
\hline 1981 & $82.7 \%$ & $0.0 \%$ & $13.6 \%$ & $2.3 \%$ & $1.3 \%$ & 10,554 & $82.8 \%$ & $17.3 \%$ \\
\hline 1982 & $80.3 \%$ & $0.1 \%$ & $14.8 \%$ & $3.2 \%$ & $1.5 \%$ & 9,732 & $80.5 \%$ & $19.5 \%$ \\
\hline 1983 & $77.7 \%$ & $0.3 \%$ & $15.8 \%$ & $3.7 \%$ & $2.5 \%$ & 10,302 & $78.0 \%$ & $22.0 \%$ \\
\hline 1984 & $76.1 \%$ & $0.4 \%$ & $14.6 \%$ & $4.8 \%$ & $4.1 \%$ & 14,020 & $76.5 \%$ & $23.5 \%$ \\
\hline 1985 & $74.6 \%$ & $0.6 \%$ & $14.4 \%$ & $5.9 \%$ & $4.5 \%$ & 14,460 & $75.2 \%$ & $24.8 \%$ \\
\hline 1986 & $71.7 \%$ & $0.4 \%$ & $16.5 \%$ & $6.8 \%$ & $4.6 \%$ & 15,365 & $72.1 \%$ & $27.9 \%$ \\
\hline 1987 & $72.2 \%$ & $0.6 \%$ & $14.4 \%$ & $7.5 \%$ & $5.2 \%$ & 14,865 & $72.8 \%$ & $27.2 \%$ \\
\hline 1988 & $70.2 \%$ & $0.7 \%$ & $16.1 \%$ & $7.4 \%$ & $5.6 \%$ & 15,295 & $70.9 \%$ & $29.1 \%$ \\
\hline 1989 & $69.3 \%$ & $0.7 \%$ & $15.4 \%$ & $8.8 \%$ & $5.7 \%$ & 14,453 & $70.1 \%$ & $29.9 \%$ \\
\hline 1990 & $69.8 \%$ & $0.5 \%$ & $14.5 \%$ & $10.0 \%$ & $5.1 \%$ & 12,615 & $70.4 \%$ & $29.6 \%$ \\
\hline 1991 & $67.8 \%$ & $1.8 \%$ & $15.3 \%$ & $8.2 \%$ & $6.9 \%$ & 12,573 & $69.6 \%$ & $30.4 \%$ \\
\hline 1992 & $66.6 \%$ & $2.0 \%$ & $15.1 \%$ & $10.0 \%$ & $6.2 \%$ & 12,172 & $68.6 \%$ & $31.4 \%$ \\
\hline 1993 & $64.0 \%$ & $3.6 \%$ & $15.2 \%$ & $10.9 \%$ & $6.3 \%$ & 13,211 & $67.6 \%$ & $32.4 \%$ \\
\hline 1994 & $59.6 \%$ & $2.3 \%$ & $18.9 \%$ & $10.0 \%$ & $9.1 \%$ & 14,125 & $61.9 \%$ & $38.1 \%$ \\
\hline 1995 & $62.0 \%$ & $1.5 \%$ & $15.0 \%$ & $11.0 \%$ & $10.5 \%$ & 15,145 & $63.5 \%$ & $36.5 \%$ \\
\hline 1996 & $60.0 \%$ & $2.2 \%$ & $14.9 \%$ & $10.7 \%$ & $12.2 \%$ & 13,144 & $62.2 \%$ & $37.8 \%$ \\
\hline 1997 & $57.6 \%$ & $2.5 \%$ & $16.7 \%$ & $8.8 \%$ & $14.5 \%$ & 14,458 & $60.1 \%$ & $39.9 \%$ \\
\hline 1998 & $55.1 \%$ & $3.1 \%$ & $16.7 \%$ & $10.3 \%$ & $14.7 \%$ & 14,456 & $58.3 \%$ & $41.7 \%$ \\
\hline 1999 & $55.1 \%$ & $3.2 \%$ & $16.7 \%$ & $9.6 \%$ & $15.4 \%$ & 15,215 & $58.3 \%$ & $41.7 \%$ \\
\hline 2000 & $55.1 \%$ & $3.7 \%$ & $15.8 \%$ & $10.2 \%$ & $15.2 \%$ & 16,571 & $58.8 \%$ & $41.2 \%$ \\
\hline 2001 & $53.9 \%$ & $4.8 \%$ & $16.1 \%$ & $7.9 \%$ & $17.3 \%$ & 15,605 & $58.6 \%$ & $41.4 \%$ \\
\hline 2002 & $51.5 \%$ & $3.7 \%$ & $14.8 \%$ & $7.7 \%$ & $22.3 \%$ & 16,115 & $55.3 \%$ & $44.7 \%$ \\
\hline 2003 & $50.2 \%$ & $3.6 \%$ & $15.7 \%$ & $7.8 \%$ & $22.6 \%$ & 15,773 & $53.9 \%$ & $46.1 \%$ \\
\hline 2004 & $48.0 \%$ & $4.1 \%$ & $15.9 \%$ & $6.1 \%$ & $25.9 \%$ & 15,709 & $52.0 \%$ & $48.0 \%$ \\
\hline 2005 & $50.5 \%$ & $5.1 \%$ & $14.5 \%$ & $9.3 \%$ & $20.6 \%$ & 15,892 & $55.6 \%$ & $44.4 \%$ \\
\hline 2006 & $52.9 \%$ & $5.0 \%$ & $14.5 \%$ & $7.7 \%$ & $19.9 \%$ & 15,104 & $57.9 \%$ & $42.1 \%$ \\
\hline 2007 & $52.9 \%$ & $6.0 \%$ & $13.8 \%$ & $5.5 \%$ & $21.7 \%$ & 15,276 & $58.9 \%$ & $41.1 \%$ \\
\hline 2008 & $52.7 \%$ & $6.6 \%$ & $12.9 \%$ & $5.7 \%$ & $22.1 \%$ & 13,898 & $59.3 \%$ & $40.7 \%$ \\
\hline 2009 & $60.5 \%$ & $6.5 \%$ & $10.6 \%$ & $4.0 \%$ & $18.4 \%$ & 9,315 & $67.0 \%$ & $33.0 \%$ \\
\hline 2010 & $54.5 \%$ & $8.2 \%$ & $11.5 \%$ & $5.0 \%$ & $20.8 \%$ & 11,110 & $62.7 \%$ & $37.3 \%$ \\
\hline 2011 & $47.7 \%$ & $10.1 \%$ & $12.3 \%$ & $4.3 \%$ & $25.6 \%$ & 12,003 & $57.8 \%$ & $42.2 \%$ \\
\hline 2012 & $54.9 \%$ & $9.4 \%$ & $10.1 \%$ & $4.9 \%$ & $20.6 \%$ & 13,438 & $64.4 \%$ & $35.7 \%$ \\
\hline 2013 & $53.8 \%$ & $10.6 \%$ & $10.6 \%$ & $4.0 \%$ & $21.0 \%$ & 14,370 & $64.4 \%$ & $35.6 \%$ \\
\hline
\end{tabular}

Note: Includes light trucks of $8,500 \mathrm{lbs}$. or less.

\section{Source:}

U.S. Environmental Protection Agency, Light-Duty Automotive Technology, Carbon Dioxide Emissions, and Fuel Economy Trends: 1975 Through 2013, December 2013. (Additional resources: www.epa.gov/fueleconomy/fetrends/1975-2013/420r13011.pdf)

\footnotetext{
${ }^{a}$ Percentages may not sum to totals due to rounding.
} 
The effects of the Japanese earthquake/tsunami in 2011 are apparent in the large decline in car production for that year. Light trucks were gaining market share from the early 1980s until 2004, mainly due to increases in the market share of sport utility vehicles (SUVs) and pickup trucks. A new category of SUVs has been added to the vehicle classification-car SUVs. The car SUVs are two-wheel drive SUVs that will be counted as cars in the Corporate Average Fuel Economy Standards for model years 2011-on. A listing of the makes/models of car SUVs is in Table 4.8 .

Figure 4.1. Light Vehicle Production Shares, Model Years 1975-2013

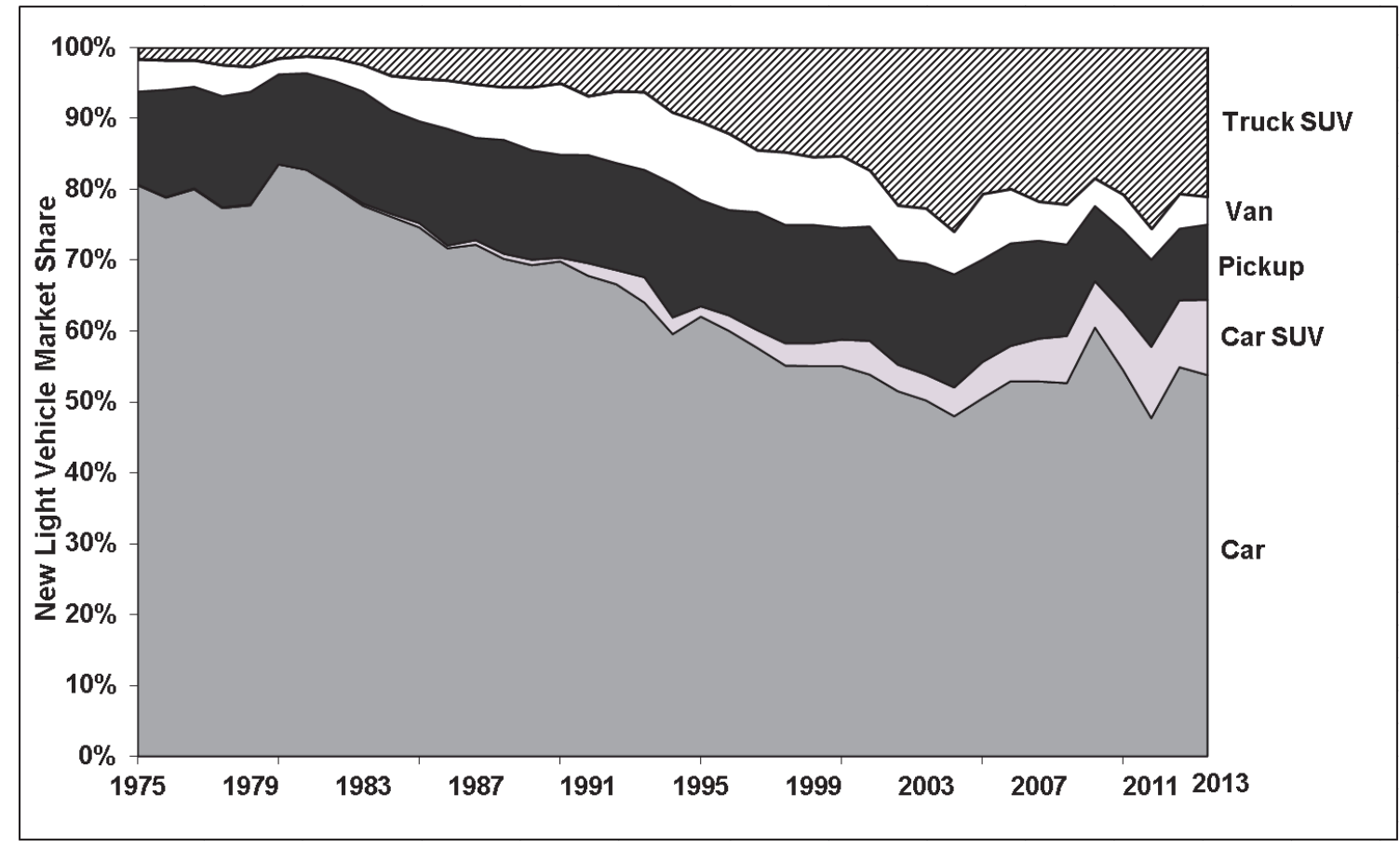

\section{Source:}

See Table 4.11. 
The production-weighted average engine displacement of cars in 1975 was 4.73 liters, but had declined to 2.43 liters by 2013. Car SUVs also experienced a decline in engine displacement. For a list of car SUVs, see Table 4.8.

Table 4.12

Production-Weighted Engine Size of New Domestic and Import Cars Model Years 1975-2013 $\left(\right.$ liters ${ }^{\mathrm{a}}$ )

\begin{tabular}{|c|c|c|}
\hline Model Year & Car & Car SUV \\
\hline 1975 & 4.73 & 4.29 \\
\hline 1976 & 4.70 & 4.32 \\
\hline 1977 & 4.57 & 4.28 \\
\hline 1978 & 4.12 & 4.30 \\
\hline 1979 & 3.91 & 3.03 \\
\hline 1980 & 3.08 & 4.59 \\
\hline 1981 & 2.98 & 5.21 \\
\hline 1982 & 2.87 & 2.60 \\
\hline 1983 & 2.98 & 2.67 \\
\hline 1984 & 2.93 & 2.94 \\
\hline 1985 & 2.90 & 2.80 \\
\hline 1986 & 2.74 & 2.78 \\
\hline 1987 & 2.65 & 2.93 \\
\hline 1988 & 2.63 & 3.26 \\
\hline 1989 & 2.67 & 3.70 \\
\hline 1990 & 2.67 & 3.42 \\
\hline 1991 & 2.66 & 3.52 \\
\hline 1992 & 2.78 & 3.44 \\
\hline 1993 & 2.73 & 3.91 \\
\hline 1994 & 2.75 & 3.42 \\
\hline 1995 & 2.74 & 3.51 \\
\hline 1996 & 2.71 & 3.52 \\
\hline 1997 & 2.68 & 3.11 \\
\hline 1998 & 2.68 & 3.58 \\
\hline 1999 & 2.72 & 3.45 \\
\hline 2000 & 2.71 & 3.47 \\
\hline 2001 & 2.70 & 3.17 \\
\hline 2002 & 2.71 & 3.00 \\
\hline 2003 & 2.71 & 2.97 \\
\hline 2004 & 2.76 & 3.13 \\
\hline 2005 & 2.72 & 3.05 \\
\hline 2006 & 2.82 & 3.01 \\
\hline 2007 & 2.71 & 3.04 \\
\hline 2008 & 2.70 & 2.93 \\
\hline 2009 & 2.54 & 2.87 \\
\hline 2010 & 2.56 & 2.81 \\
\hline 2011 & 2.61 & 2.72 \\
\hline 2012 & 2.42 & 2.74 \\
\hline 2013 & 2.43 & 2.61 \\
\hline \multicolumn{3}{|c|}{ Annual average percentage change } \\
\hline 1975-2013 & $-1.7 \%$ & $-1.3 \%$ \\
\hline 2003-2013 & $-1.1 \%$ & $-1.3 \%$ \\
\hline
\end{tabular}

\section{Source:}

U.S. Environmental Protection Agency, Light-Duty Automotive Technology, Carbon Dioxide Emissions, and Fuel Economy Trends: 1975 Through 2013, December 2013. (Additional resources: www.epa.gov/fueleconomy/fetrends/1975-2013/420r13011.pdf)

${ }^{\mathrm{a}} 1$ liter $=61.02$ cubic inches. 
The engine size of truck sport utility vehicles (SUVs) declined an average of 1.7\% per year from 2003 to 2013.

Table 4.13

Production-Weighted Engine Size of New Domestic and Import Light Trucks Model Years 1975-2013

$\left(\right.$ liters ${ }^{\mathrm{a}}$ )

\begin{tabular}{|c|c|c|c|}
\hline Model Year & Pickup & Van & Truck SUV \\
\hline 1975 & 5.02 & 5.20 & 5.44 \\
\hline 1976 & 5.18 & 5.30 & 5.55 \\
\hline 1977 & 5.12 & 5.44 & 5.52 \\
\hline 1978 & 5.02 & 5.40 & 5.61 \\
\hline 1979 & 4.70 & 5.28 & 5.52 \\
\hline 1980 & 3.86 & 4.72 & 4.83 \\
\hline 1981 & 3.88 & 4.66 & 4.75 \\
\hline 1982 & 3.74 & 4.70 & 5.00 \\
\hline 1983 & 3.47 & 4.82 & 4.35 \\
\hline 1984 & 3.53 & 4.08 & 3.79 \\
\hline 1985 & 3.63 & 3.87 & 3.63 \\
\hline 1986 & 3.38 & 3.73 & 3.44 \\
\hline 1987 & 3.31 & 3.70 & 3.48 \\
\hline 1988 & 3.76 & 3.75 & 3.63 \\
\hline 1989 & 3.86 & 3.69 & 3.99 \\
\hline 1990 & 4.04 & 3.69 & 3.85 \\
\hline 1991 & 3.80 & 3.60 & 3.82 \\
\hline 1992 & 4.01 & 3.64 & 3.85 \\
\hline 1993 & 4.00 & 3.57 & 4.00 \\
\hline 1994 & 4.06 & 3.70 & 4.01 \\
\hline 1995 & 4.20 & 3.79 & 4.01 \\
\hline 1996 & 4.12 & 3.61 & 4.24 \\
\hline 1997 & 4.33 & 3.61 & 4.19 \\
\hline 1998 & 4.13 & 3.56 & 4.14 \\
\hline 1999 & 4.38 & 3.65 & 4.14 \\
\hline 2000 & 4.18 & 3.55 & 4.15 \\
\hline 2001 & 4.41 & 3.75 & 3.92 \\
\hline 2002 & 4.45 & 3.57 & 4.01 \\
\hline 2003 & 4.33 & 3.59 & 4.05 \\
\hline 2004 & 4.61 & 3.58 & 4.13 \\
\hline 2005 & 4.65 & 3.53 & 4.00 \\
\hline 2006 & 4.55 & 3.54 & 3.87 \\
\hline 2007 & 4.69 & 3.59 & 3.94 \\
\hline 2008 & 4.69 & 3.60 & 3.76 \\
\hline 2009 & 4.70 & 3.53 & 3.46 \\
\hline 2010 & 4.80 & 3.51 & 3.48 \\
\hline 2011 & 4.63 & 3.47 & 3.56 \\
\hline 2012 & 4.69 & 3.44 & 3.51 \\
\hline 2013 & 4.70 & & 3.42 \\
\hline & \multicolumn{3}{|c|}{ Annual average percentage change } \\
\hline $1975-2013$ & $-0.2 \%$ & $-1.0 \%$ & $-1.2 \%$ \\
\hline 2003-2013 & $0.8 \%$ & $-0.2 \%$ & $-1.7 \%$ \\
\hline
\end{tabular}

Note: Includes light trucks of 8,500 lbs. or less.

Source:

U.S. Environmental Protection Agency, Light-Duty Automotive Technology, Carbon Dioxide Emissions, and Fuel Economy Trends: 1975 Through 2013, December 2013. (Additional resources:

www.epa.gov/fueleconomy/fetrends/1975-2013/420r13011.pdf)

${ }^{\mathrm{a}} 1$ liter $=61.02$ cubic inches. 
The production-weighted average curb weight of cars and car SUVs has changed very little since 1975.

Table 4.14

Production-Weighted Curb Weight of New Domestic and Import Cars

Model Years 1975-2013

(pounds)

\begin{tabular}{|c|c|c|}
\hline Model Year & Car & Car SUV \\
\hline 1975 & 4,058 & 4,000 \\
\hline 1976 & 4,059 & 3,986 \\
\hline 1977 & 3,944 & 4,000 \\
\hline 1978 & 3,588 & 4,000 \\
\hline 1979 & 3,485 & 3,271 \\
\hline 1980 & 3,101 & 4,000 \\
\hline 1981 & 3,076 & 4,000 \\
\hline 1982 & 3,054 & 2,630 \\
\hline 1983 & 3,112 & 3,124 \\
\hline 1984 & 3,099 & 3,487 \\
\hline 1985 & 3,093 & 3,469 \\
\hline 1986 & 3,041 & 3,479 \\
\hline 1987 & 3,031 & 3,492 \\
\hline 1988 & 3,047 & 3,495 \\
\hline 1989 & 3,099 & 3,497 \\
\hline 1990 & 3,176 & 3,518 \\
\hline 1991 & 3,154 & 3,733 \\
\hline 1992 & 3,240 & 3,713 \\
\hline 1993 & 3,207 & 3,848 \\
\hline 1994 & 3,250 & 3,735 \\
\hline 1995 & 3,263 & 3,763 \\
\hline 1996 & 3,282 & 3,710 \\
\hline 1997 & 3,274 & 3,549 \\
\hline 1998 & 3,306 & 3,824 \\
\hline 1999 & 3,365 & 3,831 \\
\hline 2000 & 3,369 & 3,870 \\
\hline 2001 & 3,380 & 3,765 \\
\hline 2002 & 3,391 & 3,747 \\
\hline 2003 & 3,417 & 3,716 \\
\hline 2004 & 3,462 & 3,854 \\
\hline 2005 & 3,463 & 3,848 \\
\hline 2006 & 3,534 & 3,876 \\
\hline 2007 & 3,507 & 3,935 \\
\hline 2008 & 3,527 & 3,902 \\
\hline 2009 & 3,464 & 3,846 \\
\hline 2010 & 3,474 & 3,949 \\
\hline 2011 & 3,559 & 3,890 \\
\hline 2012 & 3,448 & 3,913 \\
\hline 2013 & 3,504 & 3,951 \\
\hline \multicolumn{3}{|c|}{ Annual average percentage change } \\
\hline $1975-2013$ & $-0.4 \%$ & $0.0 \%$ \\
\hline $2003-2013$ & $0.3 \%$ & $0.6 \%$ \\
\hline
\end{tabular}

\section{Source:}

U.S. Environmental Protection Agency, Light-Duty Automotive Technology, Carbon Dioxide Emissions, and Fuel Economy Trends: 1975 Through 2013, December 2013. (Additional resources:

www.epa.gov/fueleconomy/fetrends/1975-2013/420r13011.pdf) 
The average light vehicle in 2012 contained more than 2,000 pounds of steel, most of it conventional steel. High and medium strength steel, however, made up more than 15\% of the vehicle. The use of aluminum grew from 1995 to 2012, while the use of iron castings declined.

Table 4.15

Average Material Consumption for a Domestic Light Vehicle ${ }^{a}$, Model Years 1995, 2000, and 2012

\begin{tabular}{|c|c|c|c|c|c|c|}
\hline \multirow[b]{2}{*}{ Material } & \multicolumn{2}{|c|}{1995} & \multicolumn{2}{|c|}{2000} & \multicolumn{2}{|c|}{2012} \\
\hline & Pounds & Percentage & Pounds & Percentage & Pounds & Percentage \\
\hline Regular steel & $1,630.0$ & $44.1 \%$ & $1,655.0$ & $42.4 \%$ & $1,346.0$ & $34.3 \%$ \\
\hline High and medium strength steel & 324.0 & $8.8 \%$ & 408.0 & $10.5 \%$ & 606.0 & $15.5 \%$ \\
\hline Stainless steel & 51.0 & $1.4 \%$ & 62.0 & $1.6 \%$ & 68.0 & $1.7 \%$ \\
\hline Other steels & 46.0 & $1.2 \%$ & 26.0 & $0.7 \%$ & 30.0 & $0.8 \%$ \\
\hline Iron castings & 466.0 & $12.6 \%$ & 432.0 & $11.1 \%$ & 280.0 & $7.1 \%$ \\
\hline Aluminum & 231.0 & $6.3 \%$ & 268.0 & $6.9 \%$ & 364.0 & $9.3 \%$ \\
\hline Magnesium castings & 4.0 & $0.1 \%$ & 8.0 & $0.2 \%$ & 11.0 & $0.3 \%$ \\
\hline Copper and brass & 50.0 & $1.4 \%$ & 52.0 & $1.3 \%$ & 72.0 & $1.8 \%$ \\
\hline Lead & 33.0 & $0.9 \%$ & 36.0 & $0.9 \%$ & 37.0 & $0.9 \%$ \\
\hline Zinc castings & 19.0 & $0.5 \%$ & 13.0 & $0.3 \%$ & 8.0 & $0.2 \%$ \\
\hline Powder metal parts & 29.0 & $0.8 \%$ & 36.0 & $0.9 \%$ & 44.0 & $1.1 \%$ \\
\hline Other metals & 4.0 & $0.1 \%$ & 4.0 & $0.1 \%$ & 5.0 & $0.1 \%$ \\
\hline Plastics and plastic composites & 240.0 & $6.5 \%$ & 286.0 & $7.3 \%$ & 355.0 & $9.1 \%$ \\
\hline Rubber & 149.0 & $4.0 \%$ & 166.0 & $4.3 \%$ & 210.0 & $5.4 \%$ \\
\hline Coatings & 23.0 & $0.6 \%$ & 25.0 & $0.6 \%$ & 33.0 & $0.8 \%$ \\
\hline Textiles & 42.0 & $1.1 \%$ & 44.0 & $1.1 \%$ & 49.0 & $1.3 \%$ \\
\hline Fluids and lubricants & 192.0 & $5.2 \%$ & 207.0 & $5.3 \%$ & 217.0 & $5.5 \%$ \\
\hline Glass & 97.0 & $2.6 \%$ & 103.0 & $2.6 \%$ & 95.0 & $2.4 \%$ \\
\hline Other materials & 64.0 & $1.7 \%$ & 71.0 & $1.8 \%$ & 90.0 & $2.3 \%$ \\
\hline Total & $3,694.0$ & $100.0 \%$ & $3,902.0$ & $100.0 \%$ & $3,920.0$ & $100.0 \%$ \\
\hline
\end{tabular}

Source:

Ward's Communications, Ward's Motor Vehicle Facts and Figures, 2013, Detroit, MI, 2013, p. 52 and updates.

${ }^{\text {a }}$ Percentages may not sum to totals due to rounding. 
The number of franchised dealerships which sell new light-duty vehicles (cars and light trucks) has declined 43\% since 1970. This decline, along with increasing light vehicle sales, caused the average number of vehicles sold per dealer to be at an all-time high of 811 vehicles per dealer.

Table 4.16

New Light Vehicle Dealerships and Sales, 1970-2012

\begin{tabular}{|c|c|c|c|}
\hline Calendar year & $\begin{array}{l}\text { Number of franchised new } \\
\text { light vehicle dealerships }{ }^{\mathrm{a}}\end{array}$ & $\begin{array}{c}\text { New light vehicle sales } \\
\text { (thousands) }\end{array}$ & $\begin{array}{c}\text { Light vehicle sales per } \\
\text { dealer }\end{array}$ \\
\hline 1970 & 30,800 & 9,856 & 320 \\
\hline 1975 & 29,600 & 10,677 & 361 \\
\hline 1976 & 29,300 & 12,829 & 438 \\
\hline 1977 & 29,100 & 14,292 & 491 \\
\hline 1978 & 29,000 & 14,788 & 510 \\
\hline 1979 & 28,500 & 13,518 & 474 \\
\hline 1980 & 27,900 & 10,909 & 391 \\
\hline 1981 & 26,350 & 10,235 & 388 \\
\hline 1982 & 25,700 & 10,019 & 390 \\
\hline 1983 & 24,725 & 11,669 & 472 \\
\hline 1984 & 24,725 & 13,579 & 549 \\
\hline 1985 & 24,725 & 14,667 & 593 \\
\hline 1986 & 24,825 & 15,998 & 644 \\
\hline 1987 & 25,150 & 14,802 & 589 \\
\hline 1988 & 25,025 & 15,347 & 613 \\
\hline 1989 & 25,000 & 14,389 & 576 \\
\hline 1990 & 24,825 & 13,851 & 558 \\
\hline 1991 & 24,200 & 12,307 & 509 \\
\hline 1992 & 23,500 & 12,842 & 546 \\
\hline 1993 & 22,950 & 13,869 & 604 \\
\hline 1994 & 22,850 & 15,024 & 657 \\
\hline 1995 & 22,800 & 14,673 & 644 \\
\hline 1996 & 22,750 & 14,998 & 659 \\
\hline 1997 & 22,700 & 15,014 & 661 \\
\hline 1998 & 22,600 & 15,384 & 681 \\
\hline 1999 & 22,400 & 16,711 & 746 \\
\hline 2000 & 22,250 & 17,164 & 771 \\
\hline 2001 & 22,150 & 16,950 & 765 \\
\hline 2002 & 21,800 & 16,675 & 765 \\
\hline 2003 & 21,725 & 16,494 & 759 \\
\hline 2004 & 21,650 & 16,737 & 773 \\
\hline 2005 & 21,640 & 16,774 & 775 \\
\hline 2006 & 21,495 & 16,336 & 760 \\
\hline 2007 & 21,200 & 15,867 & 748 \\
\hline 2008 & 20,770 & 13,015 & 627 \\
\hline 2009 & 20,010 & 10,236 & 512 \\
\hline 2010 & 18,460 & 11,394 & 617 \\
\hline 2011 & 17,700 & 12,539 & 708 \\
\hline 2012 & 17,540 & 14,218 & 811 \\
\hline \multicolumn{4}{|c|}{ Average annual percentage change } \\
\hline 1970-2012 & $-1.3 \%$ & $0.9 \%$ & $2.2 \%$ \\
\hline 2002-2012 & $-2.2 \%$ & $-1.6 \%$ & $0.6 \%$ \\
\hline
\end{tabular}

\section{Source:}

Number of dealers - National Automobile Dealers Association website, www.nada.org. (Additional resources: www.nada.org/Publications/NADADATA/) Light-duty vehicle sales - See tables 4.5 and 4.6.

${ }^{a}$ As of the beginning of the year. 
In 2010 the number of conventional refueling stations fell below 160,000 for the first time in the series history and continued to decline through 2012. The number of vehicles fueling at those stations fell in 2009 for the first time in several years but began rising slowly in 2011. In 2012, there were 0.62 fueling stations per thousand vehicles or 1.61 thousand vehicles per station.

Table 4.17

Conventional Refueling Stations, 1993-2012

\begin{tabular}{|c|c|c|c|c|}
\hline & $\begin{array}{c}\text { Number of retail } \\
\text { outlets }\end{array}$ & $\begin{array}{l}\text { Vehicles in } \\
\text { operation } \\
\text { (thousands) }\end{array}$ & $\begin{array}{l}\text { Stations per } \\
\text { thousand vehicles }\end{array}$ & $\begin{array}{l}\text { Thousand } \\
\text { vehicles per } \\
\text { station }\end{array}$ \\
\hline Year & \multicolumn{3}{|c|}{ Conventional fuels } & \\
\hline 1993 & 207,416 & 186,315 & 1.11 & 0.90 \\
\hline 1994 & 202,878 & 188,714 & 1.08 & 0.93 \\
\hline 1995 & 195,455 & 193,441 & 1.01 & 0.99 \\
\hline 1996 & 190,246 & 198,294 & 0.96 & 1.04 \\
\hline 1997 & 187,892 & 201,071 & 0.93 & 1.07 \\
\hline 1998 & 182,596 & 205,043 & 0.89 & 1.12 \\
\hline 1999 & 180,567 & 209,509 & 0.86 & 1.16 \\
\hline 2000 & 175,941 & 213,299 & 0.82 & 1.21 \\
\hline 2001 & 172,169 & 216,683 & 0.79 & 1.26 \\
\hline 2002 & 170,018 & 221,027 & 0.77 & 1.30 \\
\hline 2003 & 167,571 & 225,882 & 0.74 & 1.35 \\
\hline 2004 & 167,346 & 232,167 & 0.72 & 1.39 \\
\hline 2005 & 168,987 & 238,384 & 0.71 & 1.41 \\
\hline 2006 & 167,476 & 244,643 & 0.69 & 1.46 \\
\hline 2007 & 164,292 & 248,701 & 0.66 & 1.51 \\
\hline 2008 & 161,068 & 249,813 & 0.64 & 1.55 \\
\hline 2009 & 162,350 & 248,972 & 0.65 & 1.53 \\
\hline 2010 & 159,006 & 248,231 & 0.64 & 1.56 \\
\hline 2011 & 157,393 & 248,932 & 0.63 & 1.58 \\
\hline 2012 & 156,065 & 251,497 & 0.62 & 1.61 \\
\hline
\end{tabular}

Notes: The County Business Patterns (CBP) data published by the Bureau of the Census tells the number of establishments by North American Industry Classification System (NAICS). NAICS is an industry classification system that groups establishments into industries based on the activities in which they are primarily engaged. NAICS 447 represents gasoline stations. However, the CBP gasoline station data differ from the National Petroleum News Survey data by as much as 30\% (117,189 stations in 2005); the CBP may not include every gasoline retail outlet due to the classification of the primary activity of the business.

Alternative Fuel Refueling Stations are listed in Chapter 6.

Sources:

Conventional refueling stations: National Petroleum News Survey, 2012.

Conventional vehicles: The Polk Company, Detroit, MI, FURTHER REPRODUCTION PROHIBITED. 
The National Highway Traffic Safety Administration and the Environmental Protection Agency issued joint rulemaking to establish a new National Program to regulate fuel economy and greenhouse gas emissions for model year 2012-2025 cars and light trucks.

Table 4.18

Fuel Economy and Carbon Dioxide Emissions Standards, MY 2012-2025

\begin{tabular}{|c|c|c|c|}
\hline Year & Cars & Light trucks & $\begin{array}{l}\text { Combined cars and light } \\
\text { trucks }\end{array}$ \\
\hline \multicolumn{4}{|c|}{$\begin{array}{l}\text { Average required fuel economy } \\
\text { (miles per gallon) }\end{array}$} \\
\hline 2012 & 33.3 & 25.4 & 29.7 \\
\hline 2013 & 34.2 & 26.0 & 30.5 \\
\hline 2014 & 34.9 & 26.6 & 31.3 \\
\hline 2015 & 36.2 & 27.5 & 32.6 \\
\hline 2016 & 37.8 & 28.8 & 34.1 \\
\hline 2017 & 40.1 & 29.4 & 35.4 \\
\hline 2018 & 41.6 & 30.0 & 36.5 \\
\hline 2019 & 43.1 & 30.6 & 37.7 \\
\hline 2020 & 44.8 & 31.2 & 38.9 \\
\hline 2021 & 46.8 & 33.3 & 41.0 \\
\hline 2022 & 49.0 & 34.9 & 43.0 \\
\hline 2023 & 51.2 & 36.6 & 45.1 \\
\hline 2024 & 53.6 & 38.5 & 47.4 \\
\hline 2025 & 56.2 & 40.3 & 49.7 \\
\hline \multicolumn{4}{|c|}{$\begin{array}{l}\text { Average projected emissions compliance levels under } \\
\text { the footprint-based carbon dioxide standards } \\
\text { (grams per mile) }\end{array}$} \\
\hline 2012 & 263 & 346 & 295 \\
\hline 2013 & 256 & 337 & 286 \\
\hline 2014 & 247 & 326 & 276 \\
\hline 2015 & 236 & 312 & 263 \\
\hline 2016 & 225 & 298 & 250 \\
\hline 2017 & 212 & 295 & 243 \\
\hline 2018 & 202 & 285 & 232 \\
\hline 2019 & 191 & 277 & 222 \\
\hline 2020 & 182 & 269 & 213 \\
\hline 2021 & 172 & 249 & 199 \\
\hline 2022 & 164 & 237 & 190 \\
\hline 2023 & 157 & 225 & 180 \\
\hline 2024 & 150 & 214 & 171 \\
\hline 2025 & 143 & 203 & 163 \\
\hline
\end{tabular}

Note: The required fuel economy, along with projections of $\mathrm{CO}_{2}$ emissions, shown here use a model year 2008 baseline. The presented rates of increase in stringency for NHTSA CAFE standards are lower than the Environmental Protection Agency (EPA) rates of increase in stringency for greenhouse gas (GHG) standards. One major difference is that NHTSA's standards, unlike EPA's, do not reflect the inclusion of air conditioning system refrigerant and leakage improvements, but EPA's standards would allow consideration of such improvements which reduce GHGs but generally do not affect fuel economy. The 2025 EPA GHG standard of 163 grams/mile would be equivalent to $54.5 \mathrm{mpg}$, if the vehicles were to meet this level all through fuel economy improvements. The agencies expect, however, that a portion of these improvements will be made through reductions in air conditioning leakage, which would not contribute to fuel economy.

\section{Source:}

Federal Register, Vol. 77, No. 199, October 15, 2012. (Additional resources: www.nhtsa.dot.gov/fuel-economy) 
The target levels for the fuel economy and carbon dioxide emission standards for vehicles manufactured in model years 2012-on are assigned based on a vehicle's "footprint." Each footprint has a different target. The vehicle footprint is calculated as:

$$
\begin{gathered}
\text { footprint }=\text { track width } \times \text { wheelbase, } \\
\text { where }
\end{gathered}
$$

track width = lateral distance between the centerlines of the base tires at ground, and wheelbase = longitudinal distance between the front and rear wheel centerlines.

Table 4.19

Fuel Economy and Carbon Dioxide Targets for Model Year 2025

\begin{tabular}{llccc}
\hline Vehicle type & Example models & $\begin{array}{c}\text { Example } \\
\text { model footprint } \\
\text { (square feet) }\end{array}$ & $\begin{array}{c}\mathrm{CO}_{2} \text { emissions } \\
\text { target } \\
\text { (grams per mile) }\end{array}$ & $\begin{array}{c}\text { Fuel economy target } \\
\text { (miles per gallon) }\end{array}$ \\
\hline Compact car & Honda Fit & Example Passenger Cars & \\
Midsize car & Ford Fusion & 40 & 131 & 61.1 \\
Fullsize car & Chrysler 300 & 46 & 147 & 54.9 \\
\hline \multicolumn{5}{c}{ Example Light-Duty Trucks } \\
\hline Small SUV & 4WD Ford Escape & 44 & 170 & 48.0 \\
Midsize crossover & Nissan Murano & 49 & 170 & 47.5 \\
Minivan & Toyota Sienna & 55 & 209 & 43.4 \\
Large pickup truck & Chevy Silverado & 67 & 252 & 39.2 \\
\hline
\end{tabular}

Note: Examples use model year 2012 vehicle specifications. The fuel economy from this table will not match the fuel economy listed on the window sticker of a new vehicle. Window sticker fuel economy is calculated by a different methodology than the Corporate Average Fuel Economy.

\section{Source:}

Federal Register, Vol. 77, No. 199, October 15, 2012. (Additional resources: www.nhtsa.gov/fuel-economy) 
The Corporate Average Fuel Economy standards were established by the U.S. Energy Policy and Conservation Act of 1975 (PL94-163). These standards must be met at the manufacturer level. Some manufacturers fall short of meeting the standards while others exceed them. Legislation passed in December 2007 changed the CAFE standards beginning in the 2011 model year (MY). Some two-wheel drive sport utility vehicles are classified as cars under the final standards for MY 2011-2021.

Table 4.20

Car Corporate Average Fuel Economy (CAFE) Standards versus Sales-Weighted Fuel Economy Estimates, 1978-2013 ${ }^{\mathrm{a}}$ (miles per gallon)

\begin{tabular}{|c|c|c|c|c|c|}
\hline \multirow{3}{*}{$\begin{array}{l}\text { Model } \\
\text { year }^{\mathrm{b}}\end{array}$} & \multicolumn{4}{|c|}{ Cars } & \multirow{3}{*}{$\begin{array}{l}\text { CAFE estimates } \\
\text { Cars and light } \\
\text { trucks combined }\end{array}$} \\
\hline & \multirow{2}{*}{$\begin{array}{c}\text { CAFE } \\
\text { standards }\end{array}$} & \multicolumn{3}{|c|}{ CAFE estimates $^{c}$} & \\
\hline & & Domestic & Import & Combined & \\
\hline 1978 & 18.0 & 18.7 & 27.3 & 19.9 & 19.9 \\
\hline 1980 & 20.0 & 22.6 & 29.6 & 24.3 & 23.1 \\
\hline 1985 & 27.5 & 26.3 & $31.5^{-}$ & 27.6 & 25.4 \\
\hline 1986 & 26.0 & 26.9 & 31.6 & 28.2 & 25.9 \\
\hline 1987 & 26.0 & 27.0 & 31.2 & 28.5 & 26.2 \\
\hline 1988 & 26.0 & 27.4 & 31.5 & 28.8 & 26.0 \\
\hline 1989 & 26.5 & 27.2 & 30.8 & 28.4 & 25.6 \\
\hline 1990 & 27.5 & 26.9 & 29.9 & 28.0 & 25.4 \\
\hline 1991 & 27.5 & 27.3 & 30.1 & 28.4 & 25.6 \\
\hline 1992 & 27.5 & 27.0 & 29.2 & 27.9 & 25.1 \\
\hline 1993 & 27.5 & 27.8 & 29.6 & 28.4 & 25.2 \\
\hline 1994 & 27.5 & 27.5 & 29.6 & 28.3 & 24.7 \\
\hline 1995 & 27.5 & 27.7 & 30.3 & 28.6 & 24.9 \\
\hline 1996 & 27.5 & 28.1 & 29.6 & 28.5 & 24.9 \\
\hline 1997 & 27.5 & 27.8 & 30.1 & 28.7 & 24.6 \\
\hline 1998 & 27.5 & 28.6 & 29.2 & 28.8 & 24.7 \\
\hline 1999 & 27.5 & 28.0 & 29.0 & 28.3 & 24.5 \\
\hline 2000 & 27.5 & 28.7 & 28.3 & 28.5 & 24.8 \\
\hline 2001 & 27.5 & 28.7 & 29.0 & 28.8 & 24.5 \\
\hline 2002 & 27.5 & 29.1 & 28.8 & 29.0 & 24.7 \\
\hline 2003 & 27.5 & 29.1 & 29.9 & 29.5 & 25.1 \\
\hline 2004 & 27.5 & 29.9 & 28.7 & 29.5 & 24.6 \\
\hline 2005 & 27.5 & 30.5 & 29.9 & 30.3 & 25.4 \\
\hline 2006 & 27.5 & 30.3 & 29.7 & 30.1 & 25.8 \\
\hline 2007 & 27.5 & 30.6 & 32.2 & 31.2 & 26.6 \\
\hline 2008 & $27.5^{\mathrm{d}}$ & 31.2 & 31.8 & 31.5 & 27.1 \\
\hline 2009 & $27.5^{\mathrm{d}}$ & 32.1 & 33.8 & 32.9 & 29.0 \\
\hline 2010 & $27.5^{\mathrm{d}}$ & 33.1 & 35.2 & 33.9 & 29.3 \\
\hline 2011 & $30.2^{\mathrm{e}}$ & 32.7 & 33.7 & 33.1 & 29.0 \\
\hline 2012 & $32.7^{\mathrm{e}}$ & 34.5 & 36.4 & 35.2 & 30.8 \\
\hline 2013 & $33.5^{\mathrm{e}}$ & 35.5 & 37.1 & 36.0 & 30.8 \\
\hline
\end{tabular}

Source:

U.S. Department of Transportation, NHTSA, "Summary of Fuel Economy Performance," Washington, DC, April 2013. (Additional resources: www.nhtsa.dot.gov)

${ }^{a}$ Only vehicles with at least 75 percent domestic content can be counted in the average domestic fuel economy for a manufacturer.

${ }^{\mathrm{b}}$ Model year as determined by the manufacturer on a vehicle by vehicle basis.

${ }^{c}$ All CAFE calculations are sales-weighted.

${ }^{\mathrm{d}}$ Unreformed standards, which were an option from 2008-2010. See Table 4.18 for reformed standards.

${ }^{\mathrm{e}}$ Projected required average fuel economy standards value based on pre-model year reports. 
The Corporate Average Fuel Economy standards for light trucks are lower than the car standards. Light trucks include pickups, minivans, sport utility vehicles and vans. New legislation passed in December 2007 changed the CAFE standards beginning in the 2011 model year (MY). Some two-wheel drive sport utility vehicles are classified as cars under the final standards for MY 2011-2021.

Table 4.21

\section{Light Truck Corporate Average Fuel Economy (CAFE) Standards versus Sales-Weighted Fuel Economy Estimates, 1978-2013 ${ }^{\mathrm{a}}$ (miles per gallon)}

\begin{tabular}{|c|c|c|c|c|c|}
\hline \multirow{3}{*}{$\begin{array}{c}\text { Model } \\
\text { year }^{c}\end{array}$} & \multicolumn{4}{|c|}{ Light trucks ${ }^{\mathrm{b}}$} & \multirow{3}{*}{$\begin{array}{c}\text { CAFE estimates } \\
\text { Cars and light } \\
\text { trucks combined }\end{array}$} \\
\hline & \multirow{2}{*}{$\begin{array}{c}\text { CAFE } \\
\text { standards }\end{array}$} & \multicolumn{3}{|c|}{ CAFE estimates $^{\mathrm{d}}$} & \\
\hline & & Domestic & Import & Combined & \\
\hline 1978 & $\mathrm{e}$ & $\mathrm{f}$ & $\mathrm{f}$ & $\mathrm{f}$ & 19.9 \\
\hline 1980 & $-\mathrm{e}^{-}$ & 16.8 & 24.3 & 18.5 & 23.1 \\
\hline $1985^{-}$ & 19.5 & 19.6 & 26.5 & 20.7 & 25.4 \\
\hline 1986 & 20.0 & 20.0 & 25.9 & 21.5 & 25.9 \\
\hline 1987 & 20.5 & 20.5 & 25.2 & 21.7 & 26.2 \\
\hline 1988 & 20.5 & 20.6 & 24.6 & 21.3 & 26.0 \\
\hline 1989 & 20.5 & 20.4 & 23.5 & 21.0 & 25.6 \\
\hline 1990 & 20.0 & 20.3 & 23.0 & 20.8 & 25.4 \\
\hline 1991 & 20.2 & 20.9 & 23.0 & 21.3 & 25.6 \\
\hline 1992 & 20.2 & 20.5 & 22.7 & 20.8 & 25.1 \\
\hline 1993 & 20.4 & 20.7 & 22.8 & 21.0 & 25.2 \\
\hline 1994 & 20.5 & 20.5 & 22.1 & 20.8 & 24.7 \\
\hline 1995 & 20.6 & 20.3 & 21.5 & 20.5 & 24.9 \\
\hline 1996 & 20.7 & 20.5 & 22.2 & 20.8 & 24.9 \\
\hline 1997 & 20.7 & 20.1 & 22.1 & 20.6 & 24.6 \\
\hline 1998 & 20.7 & 20.5 & 23.0 & 21.0 & 24.7 \\
\hline 1999 & 20.7 & 20.4 & 22.5 & 20.9 & 24.5 \\
\hline 2000 & 20.7 & 21.1 & 19.7 & 21.3 & 24.8 \\
\hline 2001 & 20.7 & 20.6 & 21.8 & 20.9 & 24.5 \\
\hline 2002 & 20.7 & 20.6 & 21.9 & 21.4 & 24.7 \\
\hline 2003 & 20.7 & 21.8 & 22.4 & 21.8 & 25.1 \\
\hline 2004 & 20.7 & 20.7 & 22.3 & 21.5 & 24.6 \\
\hline 2005 & 21.0 & $\mathrm{f}$ & $\mathrm{f}$ & 22.1 & 25.4 \\
\hline 2006 & 21.6 & f & f & 22.5 & 25.8 \\
\hline 2007 & 22.2 & f & f & 23.1 & 26.6 \\
\hline 2008 & $22.5^{\mathrm{g}}$ & f & f & 23.6 & 27.1 \\
\hline 2009 & $23.1^{\mathrm{g}}$ & f & f & 24.8 & 29.0 \\
\hline 2010 & $23.5^{\mathrm{g}}$ & f & $\mathrm{f}$ & 25.2 & 29.3 \\
\hline 2011 & $24.3^{\mathrm{h}}$ & f & f & 24.7 & 29.0 \\
\hline 2012 & $25.2^{\mathrm{h}}$ & f & $\mathrm{f}$ & 25.0 & 30.8 \\
\hline 2013 & $25.7^{\mathrm{h}}$ & f & $\mathrm{f}$ & 25.3 & 30.8 \\
\hline
\end{tabular}

\section{Source:}

U.S. Department of Transportation, NHTSA, "Summary of Fuel Economy Performance," Washington, DC, April 2013. (Additional resources: www.nhtsa.dot.gov)

${ }^{a}$ Only vehicles with at least $75 \%$ domestic content can be counted in the average domestic fuel economy for a manufacturer.

${ }^{\mathrm{b}}$ Represents two- and four-wheel drive trucks combined. Gross vehicle weight of 0-6,000 pounds for model year 1978-1979 and 0-8,500 pounds for subsequent years.

${ }^{\mathrm{c}}$ Model year as determined by the manufacturer on a vehicle by vehicle basis.

d All CAFE calculations are sales-weighted.

${ }^{\mathrm{e}}$ Standards were set for two-wheel drive and four-wheel drive light trucks, but no combined standard was set in this year.

${ }^{\mathrm{f}}$ Data are not available.

${ }^{\mathrm{g}}$ Unreformed standards, which were an option from 2008-2010. See Table 4.18 for reformed standards.

${ }^{\mathrm{h}}$ Projected required average fuel economy standards value based on pre-model year reports. 
Manufacturers of cars and light trucks whose vehicles do not meet the CAFE standards are fined. Data from the National Highway Traffic Safety Administration show CAFE fine in the year in which the money was collected, which may not be the same year in which it was assessed. A manufacturer can also use CAFE credits to offset fines. Fines for recent model years are still being collected.

Table 4.22

Corporate Average Fuel Economy (CAFE) Fines Collected, 1983-2012 ${ }^{\mathrm{a}}$

\begin{tabular}{|c|c|c|}
\hline Model year & $\begin{array}{l}\text { Current } \\
\text { dollars }\end{array}$ & $\begin{array}{l}2012 \text { constant } \\
\text { dollars }^{\mathrm{b}}\end{array}$ \\
\hline 1983 & $\$ 57,970$ & $\$ 133,630$ \\
\hline 1984 & $\$ 5,958,020$ & $\$ 13,165,791$ \\
\hline 1985 & $\$ 15,564,540$ & $\$ 33,211,199$ \\
\hline 1986 & $\$ 29,871,815$ & $\$ 62,576,546$ \\
\hline 1987 & $\$ 31,260,530$ & $\$ 63,179,843$ \\
\hline 1988 & $\$ 43,470,545$ & $\$ 84,366,664$ \\
\hline 1989 & $\$ 48,549,420$ & $\$ 89,892,383$ \\
\hline 1990 & $\$ 48,308,615$ & $\$ 84,861,271$ \\
\hline 1991 & $\$ 42,243,030$ & $\$ 71,209,591$ \\
\hline 1992 & $\$ 38,286,565$ & $\$ 62,654,067$ \\
\hline 1993 & $\$ 28,688,380$ & $\$ 45,582,560$ \\
\hline 1994 & $\$ 31,498,570$ & $\$ 48,798,129$ \\
\hline 1995 & $\$ 40,787,498$ & $\$ 61,447,276$ \\
\hline 1996 & $\$ 19,301,930$ & $\$ 28,244,789$ \\
\hline 1997 & $\$ 36,211,850$ & $\$ 51,800,769$ \\
\hline 1998 & $\$ 21,739,774$ & $\$ 30,621,605$ \\
\hline 1999 & $\$ 27,516,451$ & $\$ 37,920,840$ \\
\hline 2000 & $\$ 51,067,038$ & $\$ 68,087,605$ \\
\hline 2001 & $\$ 35,507,412$ & $\$ 46,032,121$ \\
\hline 2002 & $\$ 20,041,533$ & $\$ 25,577,630$ \\
\hline 2003 & $\$ 15,225,419$ & $\$ 18,998,179$ \\
\hline 2004 & $\$ 30,411,986$ & $\$ 36,963,523$ \\
\hline 2005 & $\$ 25,057,126$ & $\$ 29,457,069$ \\
\hline 2006 & $\$ 40,933,954$ & $\$ 46,618,007$ \\
\hline 2007 & $\$ 37,385,941$ & $\$ 41,398,210$ \\
\hline 2008 & $\$ 11,619,696$ & $\$ 12,390,968$ \\
\hline 2009 & $\$ 9,148,425$ & $\$ 9,790,495$ \\
\hline 2010 & $\$ 23,803,412$ & $\$ 25,062,922$ \\
\hline 2011 & $\$ 25,573,845$ & $\$ 26,103,083$ \\
\hline 2012 & $\$ 9,819,001$ & $\$ 9,819,001$ \\
\hline
\end{tabular}

\section{Source:}

U.S. Department of Transportation, National Highway Traffic Safety Administration, Office of Vehicle Safety Compliance, Washington, DC, April 2014. (Additional resources: www.nhtsa.dot.gov)

\footnotetext{
collected in that year.

${ }^{\mathrm{b}}$ Adjusted using the Consumer Price Inflation Index.
}

${ }^{a}$ These are fines which are actually collected. Fines which are assessed in certain year may not have been 
Consumers must pay the Gas Guzzler Tax when purchasing a car that has an Environmental Protection Agency (EPA) fuel economy rating (combined city and highway) less than that stipulated in the table below. The Gas Guzzler Tax doubled in 1991 after remaining constant from 1986 to 1990. The tax has not changed since 1991.

This tax does not apply to light trucks such as pickups, minivans, sport utility vehicles, and vans.

Table 4.23

The Gas Guzzler Tax on New Cars (dollars per vehicle)

\begin{tabular}{crrrrrrrr}
\hline $\begin{array}{c}\text { Vehicle fuel } \\
\text { economy (mpg) }\end{array}$ & 1980 & 1981 & 1982 & 1983 & 1984 & 1985 & $1986-90$ & 1991 - on \\
\hline Over 22.5 & 0 & 0 & 0 & 0 & 0 & 0 & 0 & 0 \\
$22.0-22.5$ & 0 & 0 & 0 & 0 & 0 & 0 & 500 & 1,000 \\
$21.5-22.0$ & 0 & 0 & 0 & 0 & 0 & 0 & 500 & 1,000 \\
$21.0-21.5$ & 0 & 0 & 0 & 0 & 0 & 0 & 650 & 1,300 \\
$20.5-21.0$ & 0 & 0 & 0 & 0 & 0 & 500 & 650 & 1,300 \\
$20.0-20.5$ & 0 & 0 & 0 & 0 & 0 & 500 & 850 & 1,700 \\
$19.5-20.0$ & 0 & 0 & 0 & 0 & 0 & 600 & 850 & 1,700 \\
$19.0-19.5$ & 0 & 0 & 0 & 0 & 450 & 600 & 1,050 & 2,100 \\
$18.5-19.0$ & 0 & 0 & 0 & 350 & 450 & 800 & 1,050 & 2,100 \\
$18.0-18.5$ & 0 & 0 & 200 & 350 & 600 & 800 & 1,300 & 2,600 \\
$17.5-18.0$ & 0 & 0 & 200 & 500 & 600 & 1,000 & 1,300 & 2,600 \\
$17.0-17.5$ & 0 & 0 & 350 & 500 & 750 & 1,000 & 1,500 & 3,000 \\
$16.5-17.0$ & 0 & 200 & 350 & 650 & 750 & 1,200 & 1,500 & 3,000 \\
$16.0-16.5$ & 0 & 200 & 450 & 650 & 950 & 1,200 & 1,850 & 3,700 \\
$15.5-16.0$ & 0 & 350 & 450 & 800 & 950 & 1,500 & 1,850 & 3,700 \\
$15.0-15.5$ & 0 & 350 & 600 & 800 & 1,150 & 1,500 & 2,250 & 4,500 \\
$14.5-15.0$ & 200 & 450 & 600 & 1,000 & 1,150 & 1,800 & 2,250 & 4,500 \\
$14.0-14.5$ & 200 & 450 & 750 & 1,000 & 1,450 & 1,800 & 2,700 & 5,400 \\
$13.5-14.0$ & 300 & 550 & 750 & 1,250 & 1,450 & 2,200 & 2,700 & 5,400 \\
$13.0-13.5$ & 300 & 550 & 950 & 1,250 & 1,750 & 2,200 & 3,200 & 6,400 \\
$12.5-13.0$ & 550 & 650 & 950 & 1,550 & 1,750 & 2,650 & 3,200 & 6,400 \\
Under 12.5 & 550 & 650 & 1,200 & 1,550 & 2,150 & 2,650 & 3,850 & 7,700 \\
\hline
\end{tabular}

Source:

Internal Revenue Service, Form 6197, (Rev. 10-05), "Gas Guzzler Tax." (Additional resources: www.irs.ustreas.gov) 
Table 4.24

List of Model Year 2013 Cars with Gas Guzzler Taxes ${ }^{a}$

\begin{tabular}{|c|c|c|c|c|}
\hline Make & Model(s) & Size class & $\begin{array}{c}\text { Combined } \\
\text { unadjusted } \\
\text { city/highway } \\
\text { fuel } \\
\text { economy } \\
\end{array}$ & $\begin{array}{c}\text { Combined } \\
\text { city/highway } \\
\text { fuel } \\
\text { economy }\end{array}$ \\
\hline Aston Martin & DB9 & Minicompact Cars & 19 & 15 \\
\hline Aston Martin & V8 Vantage & Two Seaters & 19 & 15 \\
\hline Aston Martin & V8 Vantage & Two Seaters & 21 & 16 \\
\hline Aston Martin & V8 Vantage $\mathrm{S}$ & Two Seaters & 19 & 15 \\
\hline Aston Martin & V8 Vantage S & Two Seaters & 21 & 16 \\
\hline Audi & $\mathrm{A} 8 \mathrm{~L}$ & Large Cars & 19 & 16 \\
\hline Bentley & Continental Flying Spur & Midsize Cars & 17 & 14 \\
\hline Bentley & Continental GT & Compact Cars & 17 & 14 \\
\hline Bentley & Continental GT & Compact Cars & 19 & 15 \\
\hline Bentley & Continental GTC & Subcompact Cars & 17 & 14 \\
\hline Bentley & Continental GTC & Subcompact Cars & 18 & 15 \\
\hline Bentley & Continental GTC & Subcompact Cars & 22 & 17 \\
\hline Bentley & Continental Supersports Convt & Subcompact Cars & 17 & 14 \\
\hline Bentley & Mulsanne & Midsize Cars & 16 & 13 \\
\hline BMW & $550 \mathrm{i}$ & Midsize Cars & 22 & 17 \\
\hline BMW & $760 \mathrm{Li}$ & Large Cars & 19 & 15 \\
\hline BMW & M3 Convertible & Subcompact Cars & 20 & 16 \\
\hline BMW & M3 Coupe & Subcompact Cars & 21 & 16 \\
\hline BMW & M5 & Midsize Cars & 21 & 16 \\
\hline BMW & M5 & Midsize Cars & 22 & 17 \\
\hline BMW & M6 Convertible & Subcompact Cars & 21 & 16 \\
\hline BMW & M6 Convertible & Subcompact Cars & 22 & 17 \\
\hline BMW & M6 Coupe & Subcompact Cars & 21 & 16 \\
\hline BMW & M6 Coupe & Subcompact Cars & 22 & 17 \\
\hline BMW & M6 Gran Coupe & Compact Cars & 21 & 16 \\
\hline BMW & M6 Gran Coupe & Compact Cars & 22 & 17 \\
\hline Bugatti & Veyron & Two Seaters & 12 & 10 \\
\hline Chrysler & 300 SRT8 & Large Cars & 22 & 17 \\
\hline Chrysler & Challenger SRT8 & Midsize Cars & 22 & 17 \\
\hline Chrysler & Charger SRT8 & Large Cars & 22 & 17 \\
\hline Chrysler & Viper & Two Seaters & 18 & 15 \\
\hline Ferrari & 458 Italia & Two Seaters & 18 & 14 \\
\hline Ferrari & 458 Italia & Two Seaters & 19 & 15 \\
\hline Ferrari & 458 Spider & Two Seaters & 18 & 14 \\
\hline Ferrari & 458 Spider & Two Seaters & 19 & 15 \\
\hline Ferrari & F12 & Two Seaters & 17 & 13 \\
\hline Ferrari & Ferrari California & Minicompact Cars & 20 & 15 \\
\hline Ferrari & Ferrari California & Minicompact Cars & 20 & 16 \\
\hline Ferrari & FF & Midsize Cars & 16 & 13 \\
\hline Ferrari & FF & Midsize Cars & 17 & 13 \\
\hline General Motors & Camaro & Compact Cars & 18 & 14 \\
\hline General Motors & Camaro & Compact Cars & 21 & 16 \\
\hline General Motors & Corvette & Two Seaters & 21 & 17 \\
\hline General Motors & CTS & Midsize Cars & 18 & 14 \\
\hline General Motors & CTS & Midsize Cars & 21 & 16 \\
\hline
\end{tabular}


Table 4.24 (Continued)

List of Model Year 2013 Cars with Gas Guzzler Taxes ${ }^{a}$

\begin{tabular}{|c|c|c|c|c|}
\hline Make & Model(s) & Size class & $\begin{array}{c}\text { Combined } \\
\text { unadjusted } \\
\text { city/highway } \\
\text { fuel } \\
\text { economy } \\
\end{array}$ & $\begin{array}{c}\text { Combined } \\
\text { city/highway } \\
\text { fuel } \\
\text { economy }\end{array}$ \\
\hline General Motors & CTS Wagon & Small Station Wagons & 18 & 14 \\
\hline General Motors & CTS Wagon & Small Station Wagons & 21 & 16 \\
\hline General Motors & XTS Hearse & Special Purpose Vehicle 2WD & 22 & 17 \\
\hline General Motors & XTS Limo & Special Purpose Vehicle 2WD & 22 & 17 \\
\hline Lamborghini & Aventador Coupe & Two Seaters & 16 & 13 \\
\hline Lamborghini & Aventador Roadster & Two Seaters & 14 & 12 \\
\hline Lamborghini & Gallardo Coupe & Two Seaters & 17 & 15 \\
\hline Lamborghini & Gallardo Coupe & Two Seaters & 19 & 16 \\
\hline Lamborghini & Gallardo Spyder & Two Seaters & 16 & 14 \\
\hline Lamborghini & Gallardo Spyder & Two Seaters & 19 & 16 \\
\hline Maserati & Granturismo & Subcompact Cars & 20 & 16 \\
\hline Maserati & Granturismo Convertible & Subcompact Cars & 20 & 15 \\
\hline Maserati & Quattroporte & Large Cars & 18 & 14 \\
\hline Mercedes-Benz & C 63 AMG & Compact Cars & 20 & 15 \\
\hline Mercedes-Benz & C 63 AMG Black Series Coupe & Subcompact Cars & 20 & 15 \\
\hline Mercedes-Benz & C 63 AMG Coupe & Subcompact Cars & 20 & 15 \\
\hline Mercedes-Benz & CL 600 & Compact Cars & 18 & 14 \\
\hline Mercedes-Benz & CL 65 AMG & Compact Cars & 18 & 14 \\
\hline Mercedes-Benz & S 600 & Large Cars & 18 & 14 \\
\hline Mercedes-Benz & S 65 AMG & Large Cars & 18 & 14 \\
\hline Mercedes-Benz & SL 65 AMG & Two Seaters & 21 & 17 \\
\hline Mercedes-Benz & SLS AMG Coupe & Two Seaters & 19 & 15 \\
\hline Mercedes-Benz & SLS AMG GT Coupe & Two Seaters & 19 & 15 \\
\hline Mercedes-Benz & SLS AMG GT Roadster & Two Seaters & 19 & 15 \\
\hline Mercedes-Benz & SLS AMG Roadster & Two Seaters & 19 & 15 \\
\hline Rolls-Royce & Ghost & Large Cars & 19 & 15 \\
\hline Rolls-Royce & Ghost EWB & Large Cars & 19 & 15 \\
\hline Rolls-Royce & Phantom & Large Cars & 17 & 14 \\
\hline Rolls-Royce & Phantom Coupe & Compact Cars & 17 & 14 \\
\hline Rolls-Royce & Phantom Drophead Coupe & Compact Cars & 17 & 14 \\
\hline Rolls-Royce & Phantom EWB & Large Cars & 17 & 14 \\
\hline Roush & Roush Stage 3 Mustang & Subcompact Cars & 21 & 17 \\
\hline
\end{tabular}

Source:

U.S. Department of Energy and U.S. Environmental Protection Agency, Fuel Economy Guide database, www.fueleconomy.gov

${ }^{a}$ Tax is based on unadjusted combined fuel economy; adjusted combined fuel economy is used on window stickers. 
The IRS collected \$73.5 million in 2012 from those buying cars with combined city/highway fuel economy less than 22.5 miles per gallon. This tax does not apply to light trucks such as pickups, minivans, sport utility vehicles, and vans. It is worthy to note that total revenue from fines paid by consumers to purchase gas-guzzling vehicles greatly exceeds the overall fines paid by manufacturers whose vehicles fail to meet CAFE standards (see 4.20).

Table 4.25

Tax Receipts from the Sale of Gas Guzzlers, 1980-2012 (thousands)

\begin{tabular}{|c|c|c|}
\hline Model year & Current dollars & $\begin{array}{c}2012 \\
\text { constant dollars }^{\mathrm{a}}\end{array}$ \\
\hline 1980 & 740 & 2,062 \\
\hline 1981 & 780 & 1,970 \\
\hline 1982 & 1,720 & 4,092 \\
\hline 1983 & 4,020 & 9,267 \\
\hline 1984 & 8,820 & 19,490 \\
\hline 1985 & 39,790 & 84,903 \\
\hline 1986 & 147,660 & 309,323 \\
\hline 1987 & 145,900 & 294,875 \\
\hline 1988 & 116,780 & 226,644 \\
\hline 1989 & 109,640 & 203,006 \\
\hline 1990 & 103,200 & 181,286 \\
\hline 1991 & 118,400 & 199,588 \\
\hline 1992 & 144,200 & 235,976 \\
\hline 1993 & 111,600 & 177,320 \\
\hline 1994 & 64,100 & 99,305 \\
\hline 1995 & 73,500 & 110,729 \\
\hline 1996 & 52,600 & 76,970 \\
\hline 1997 & 48,200 & 68,950 \\
\hline 1998 & 47,700 & 67,188 \\
\hline 1999 & 68,300 & 94,125 \\
\hline 2000 & 70,800 & 94,398 \\
\hline 2001 & 78,200 & 101,379 \\
\hline 2002 & 79,700 & 101,716 \\
\hline 2003 & 126,700 & 158,095 \\
\hline 2004 & 140,800 & 171,132 \\
\hline 2005 & 163,800 & 192,563 \\
\hline 2006 & 201,700 & 229,708 \\
\hline 2007 & 178,700 & 197,878 \\
\hline 2008 & 172,428 & 183,873 \\
\hline 2009 & 99,300 & 106,269 \\
\hline 2010 & 85,226 & 89,736 \\
\hline 2011 & 68,905 & 70,331 \\
\hline 2012 & 73,500 & 73,500 \\
\hline
\end{tabular}

\section{Source:}

Ward's Communications, Detroit, MI, 2014. Original data source: Internal Revenue Service. (Additional resources: www.epa.gov/fueleconomy/guzzler)

\footnotetext{
${ }^{a}$ Adjusted using the Consumer Price Inflation Index.
} 
The Powertrain System Analysis Toolkit (PSAT) provides vehicle simulations for a variety of research purposes. It is used by the Department of Energy to evaluate the fuel efficiency potential of advanced powertrain configurations for different driving conditions. Recently, PSAT was used to develop data on the relationship between steady-state vehicle speed and fuel economy.

Table 4.26

Fuel Economy by Speed, PSAT Model Results

\begin{tabular}{|c|c|c|c|c|c|c|c|c|c|c|}
\hline \multirow[b]{2}{*}{ Speed (mph) } & \multicolumn{3}{|c|}{ Gasoline conventional } & \multicolumn{3}{|c|}{ Diesel conventional } & \multicolumn{4}{|c|}{ Hybrid vehicles } \\
\hline & $\begin{array}{c}\text { Midsize } \\
\text { car }\end{array}$ & $\begin{array}{l}\text { Small } \\
\text { SUV }\end{array}$ & $\begin{array}{l}\text { Large } \\
\text { SUV }\end{array}$ & $\begin{array}{c}\text { Midsize } \\
\text { car }\end{array}$ & $\begin{array}{l}\text { Small } \\
\text { SUV }\end{array}$ & $\begin{array}{l}\text { Large } \\
\text { SUV }\end{array}$ & $\begin{array}{c}2000 \\
\text { Insight }^{\mathrm{a}} \\
\end{array}$ & $\begin{array}{l}2004 \\
\text { Prius } \\
\end{array}$ & $\begin{array}{c}2007 \\
\text { Camry }^{a} \\
\end{array}$ & $\begin{array}{r}2008 \\
\text { Tahoe }^{\mathrm{a}} \\
\end{array}$ \\
\hline 45 & 39.1 & 32.5 & 29.5 & 56.4 & 47.7 & 43.6 & 101.3 & 72.0 & 52.2 & 32.2 \\
\hline 55 & 41.7 & 34.3 & 30.0 & 57.0 & 46.0 & 39.9 & 94.3 & 66.0 & 46.8 & 27.1 \\
\hline 65 & 36.9 & 29.1 & 23.0 & 47.9 & 37.6 & 32.5 & 80.0 & 57.0 & 40.9 & 23.7 \\
\hline 75 & 31.9 & 24.5 & 19.8 & 40.2 & 30.8 & 26.9 & 60.6 & 42.0 & 35.0 & 21.1 \\
\hline \multicolumn{11}{|c|}{ Fuel economy loss } \\
\hline $55-65 \mathrm{mph}$ & $11.5 \%$ & $15.2 \%$ & $23.5 \%$ & $16.0 \%$ & $18.3 \%$ & $18.5 \%$ & $15.2 \%$ & $13.6 \%$ & $12.6 \%$ & $12.4 \%$ \\
\hline $65-75 \mathrm{mph}$ & $13.6 \%$ & $15.8 \%$ & $13.8 \%$ & $16.2 \%$ & $18.1 \%$ & $17.2 \%$ & $24.3 \%$ & $26.3 \%$ & $14.5 \%$ & $11.1 \%$ \\
\hline $55-75 \mathrm{mph}$ & $23.5 \%$ & $28.6 \%$ & $34.0 \%$ & $29.6 \%$ & $33.1 \%$ & $32.6 \%$ & $35.8 \%$ & $36.4 \%$ & $25.3 \%$ & $22.1 \%$ \\
\hline
\end{tabular}

\section{Source:}

Argonne National Laboratory, Powertrain System Analysis Toolkit, July 16, 2009,

www.transportation.anl.gov/modeling_simulation/PSAT/. (Additional resources: www.transportation.anl.gov)

${ }^{a}$ From Argonne National Laboratory Advanced Powertrain Research Facility (Vehicle Test Data). 
The latest study of vehicle fuel economy by speed indicated higher fuel economy around 40 miles per hour, as did the 1973 and 1984 studies. Engineers at Oak Ridge National Laboratory believe that the lowest speed in the vehicle's highest gear is where the best fuel economy is typically obtained. That speed will be different for individual vehicles.

Table 4.27

Fuel Economy by Speed, 1973, 1984, 1997, and 2012 Studies (miles per gallon)

\begin{tabular}{ccccc}
\hline $\begin{array}{c}\text { Speed } \\
\text { (miles per hour })\end{array}$ & $\begin{array}{c}1973^{\mathrm{a}} \\
(13 \text { vehicles })\end{array}$ & $\begin{array}{c}1984^{\mathrm{b}} \\
(15 \text { vehicles })\end{array}$ & $\begin{array}{c}1997^{\mathrm{c}} \\
(9 \text { vehicles })\end{array}$ & $\begin{array}{c}2012^{\mathrm{d}} \\
(74 \text { vehicles })\end{array}$ \\
\hline 15 & $\mathrm{e}$ & 21.1 & 24.4 & $\mathrm{e}$ \\
20 & $\mathrm{e}$ & 25.5 & 27.9 & $\mathrm{e}$ \\
25 & $\mathrm{e}$ & 30.0 & 30.5 & $\mathrm{e}$ \\
30 & 21.1 & 31.8 & 31.7 & $\mathrm{e}$ \\
35 & 21.1 & 33.6 & 31.2 & $\mathrm{e}$ \\
40 & 21.1 & 33.6 & 31.0 & 33.2 \\
45 & 20.3 & 33.5 & 31.6 & $\mathrm{e}$ \\
50 & 19.5 & 31.9 & 32.4 & 31.9 \\
55 & 18.5 & 30.3 & 32.4 & $\mathrm{e}$ \\
60 & 17.5 & 27.6 & 31.4 & 27.9 \\
65 & 16.2 & 24.9 & 29.2 & $\mathrm{e}$ \\
70 & 14.9 & 22.5 & 26.8 & 24.1 \\
75 & $\mathrm{e}$ & 20.0 & 24.8 & $\mathrm{e}$ \\
80 & $\mathrm{e}$ & & $\mathrm{e}$ & 20.5 \\
& & Fuel economy loss & \\
$50-60 \mathrm{mph}$ & $13.3 \%$ & $13.5 \%$ & $3.1 \%$ & $12.5 \%$ \\
$60-70 \mathrm{mph}$ & $14.9 \%$ & $18.5 \%$ & $14.6 \%$ & $13.6 \%$ \\
$50-70 \mathrm{mph}$ & $23.6 \%$ & $29.5 \%$ & $17.3 \%$ & $24.5 \%$ \\
\hline
\end{tabular}

\section{Sources:}

1973- U.S. Department of Transportation, Federal Highway Administration, Office of Highway Planning, The Effect of Speed on Automobile Gasoline Consumption Rates, Washington, DC, October 1973.

1984 - U.S. Department of Transportation, Federal Highway Administration, Fuel Consumption and Emission Values for Traffic Models, Washington, DC, May 1985.

1997 - West, B.H., R.N. McGill, J.W. Hodgson, S.S. Sluder, and D.E. Smith, Development and Verification of Light-Duty Modal Emissions and Fuel Consumption Values for Traffic Models, FHWA-RD-99-068, U.S. Department of Transportation, Federal Highway Administration, Washington, DC, March 1999.

2012 - U.S. Department of Energy and U.S. Environmental Protection Agency, Fuel Economy Guide website: www.fueleconomy.gov. The Green Car Congress, "ORNL researchers quantify the effect of increasing highway speed on fuel economy." February 8, 2013.

${ }^{\mathrm{a}}$ Model years 1970 and earlier cars.

${ }^{\mathrm{b}}$ Model years 1981-84 cars and light trucks.

${ }^{\mathrm{c}}$ Model years 1988-97 cars and light trucks as shown in Table 4.28.

${ }^{\mathrm{d}}$ Model years 2003-2012 cars and light trucks.

${ }^{\mathrm{e}}$ Data are not available. 
Figure 4.2. Fuel Economy by Speed, 1973, 1984, 1997, and 2012 Studies

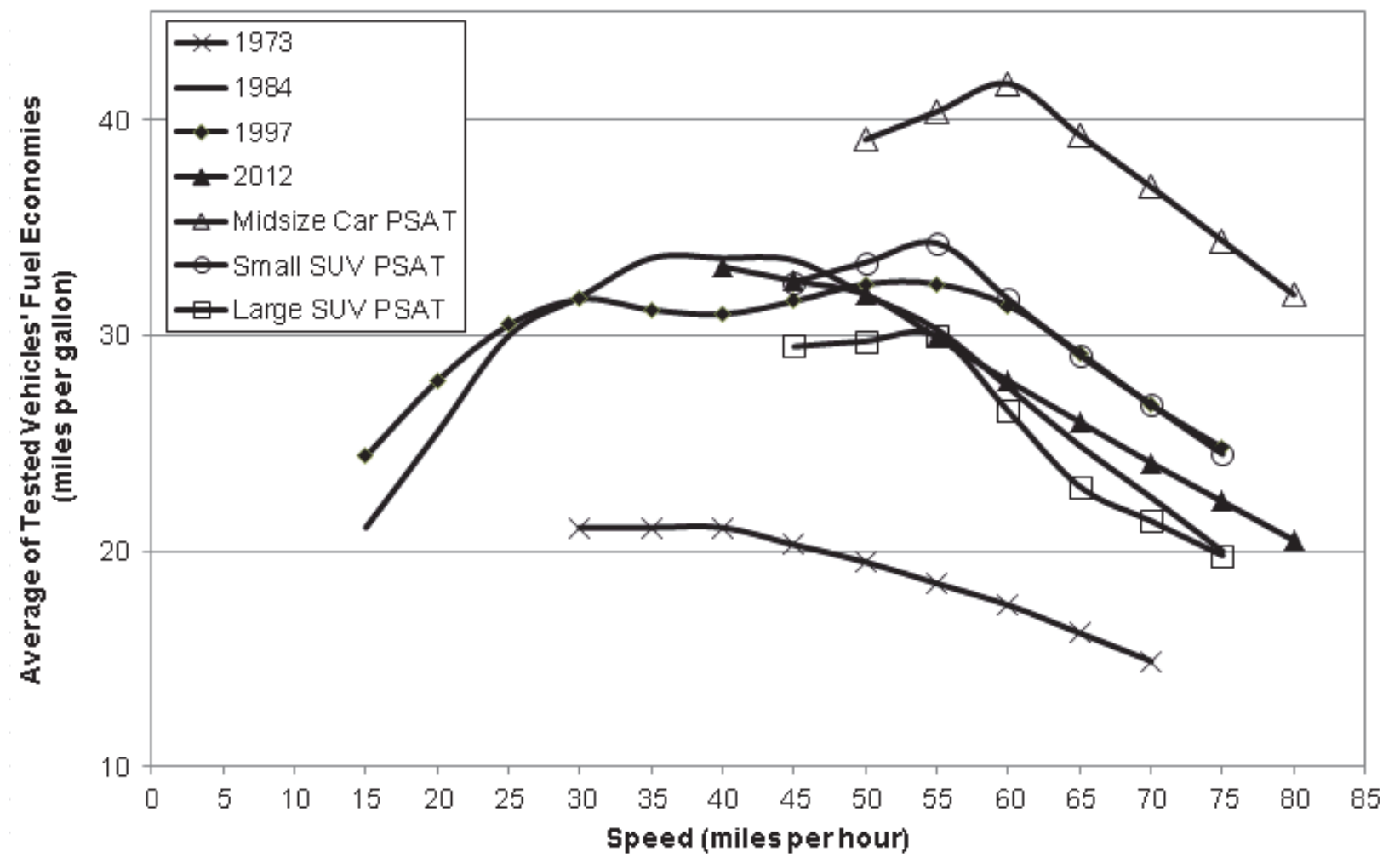

Source:

See Tables 4.26 and 4.27 . 
This table shows the driving cycles for the new methodology that the Environmental Protection Agency (EPA) used to determine fuel economy ratings for new vehicles beginning in model year 2008. In addition to the Urban Driving Cycle and the Highway Driving cycle, the EPA will also use three additional tests to adjust fuel economy ratings to account for higher speeds, air conditioner use, and colder temperatures. Though the EPA uses a complex combination of these five cycles to determine the fuel economy that will be posted on a new vehicle window sticker, the manufacturer's Corporate Average Fuel Economy is still calculated using only the city and highway driving cycles. To know more about new vehicle fuel economy ratings, visit www.fueleconomy.gov.

\section{Table 4.28}

\section{Driving Cycle Attributes}

\begin{tabular}{|c|c|c|c|c|c|}
\hline & \multicolumn{5}{|c|}{ Test schedule } \\
\hline & City & Highway & High speed & $\mathrm{AC}$ & Cold temp \\
\hline Trip type & $\begin{array}{l}\text { Low speeds in } \\
\text { stop-and-go } \\
\text { urban traffic } \\
\end{array}$ & $\begin{array}{c}\text { Free-flow traffic } \\
\text { at highway } \\
\text { speeds } \\
\end{array}$ & $\begin{array}{c}\text { Higher speeds; harder } \\
\text { acceleration \& } \\
\text { braking } \\
\end{array}$ & $\begin{array}{l}\text { AC use under } \\
\text { hot ambient } \\
\text { conditions } \\
\end{array}$ & $\begin{array}{c}\text { City test } \\
\text { w/colder outside } \\
\text { temperature } \\
\end{array}$ \\
\hline Top speed & $56 \mathrm{mph}$ & $60 \mathrm{mph}$ & $80 \mathrm{mph}$ & $54.8 \mathrm{mph}$ & $56 \mathrm{mph}$ \\
\hline Average speed & $20 \mathrm{mph}$ & $48 \mathrm{mph}$ & $48 \mathrm{mph}$ & $22 \mathrm{mph}$ & $20 \mathrm{mph}$ \\
\hline Max. acceleration & $3.3 \mathrm{mph} / \mathrm{sec}$ & $3.2 \mathrm{mph} / \mathrm{sec}$ & $8.46 \mathrm{mph} / \mathrm{sec}$ & $5.1 \mathrm{mph} / \mathrm{sec}$ & $3.3 \mathrm{mph} / \mathrm{sec}$ \\
\hline Simulated distance & $11 \mathrm{mi}$. & $10 \mathrm{mi}$. & $8 \mathrm{mi}$. & $3.6 \mathrm{mi}$. & $11 \mathrm{mi}$. \\
\hline Time & $31 \mathrm{~min}$. & $12.5 \mathrm{~min}$. & $10 \mathrm{~min}$. & $9.9 \mathrm{~min}$. & $31 \mathrm{~min}$. \\
\hline Stops & 23 & None & 4 & 5 & 23 \\
\hline Idling time & $18 \%$ of time & None & $7 \%$ of time & $19 \%$ of time & $18 \%$ of time \\
\hline Engine startup $^{a}$ & Cold & Warm & Warm & Warm & Cold \\
\hline Lab temperature & $68-86^{\circ} \mathrm{F}$ & $68-86^{\circ} \mathrm{F}$ & $68-86^{\circ} \mathrm{F}$ & $95^{\circ} \mathrm{F}$ & $20^{\circ} \mathrm{F}$ \\
\hline Vehicle air conditioning & Off & Off & Off & On & Off \\
\hline
\end{tabular}

\section{Source:}

U.S. Department of Energy and U.S. Environmental Protection Agency, Fuel Economy website, www.fueleconomy.gov.

\footnotetext{
a A vehicle's engine doesn't reach maximum fuel efficiency until it is warm.
} 
These driving cycles simulate the performance of an engine while driving in the city and on the highway. Once the city cycle is completed, the engine is stopped, and then started again for the 8.5 minute hot start cycle. Three additional cycles also influence new vehicle fuel economy ratings beginning with the 2008 model year.

Figure 4.3. City Driving Cycle

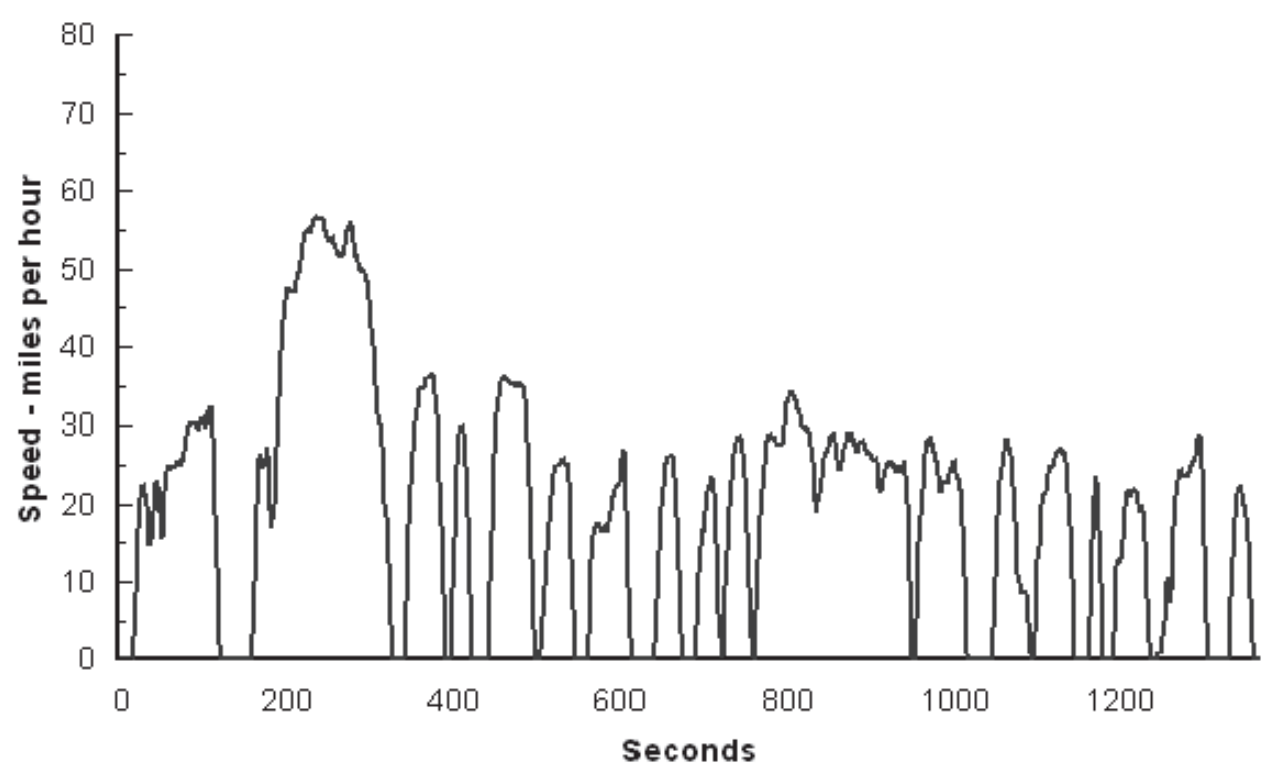

Figure 4.4. Highway Driving Cycle

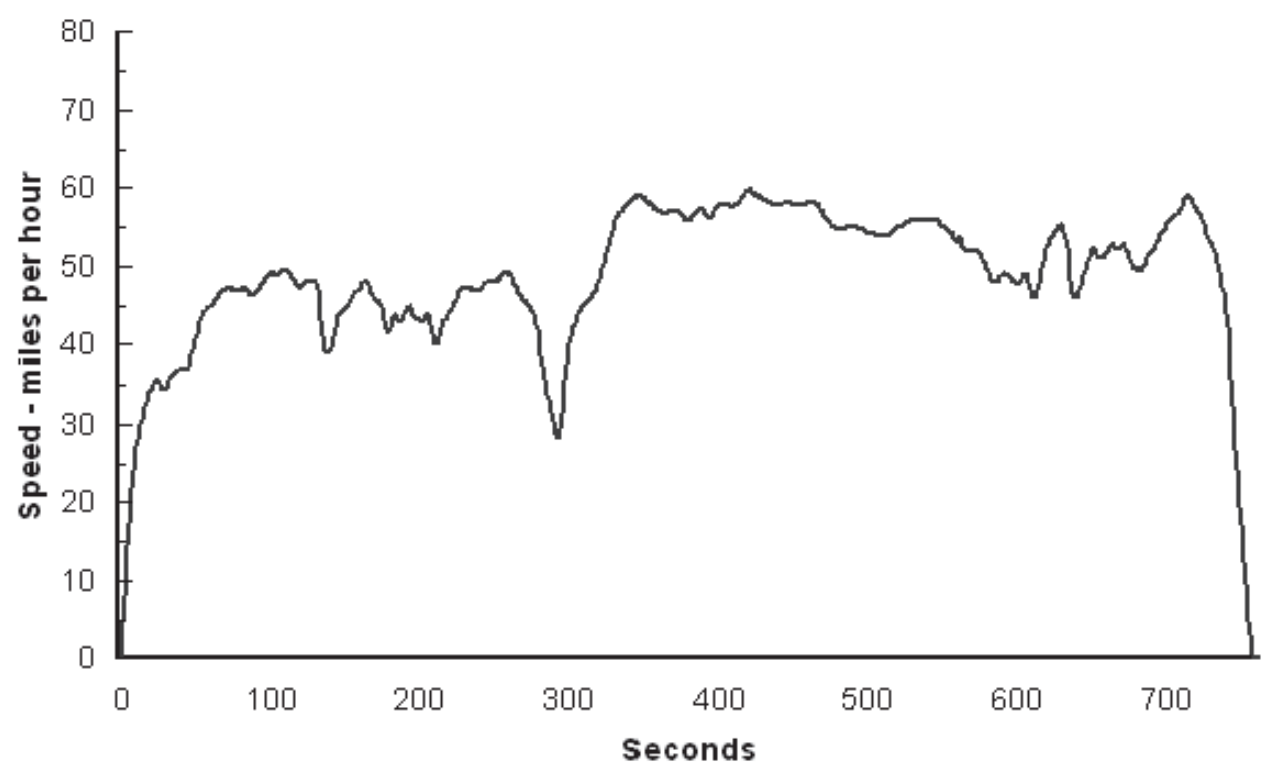

Source:

Code of Federal Regulations, 40CFR, "Subpart B - Fuel Economy Regulations for 1978 and Later Model Year Automobiles - Test Procedures," July 1, 1988 edition, p. 676. 
Beginning with the 2008 model year, these cycles influence the new vehicle fuel economy ratings.

Figure 4.5. Air Conditioning (SC03) Driving Cycle

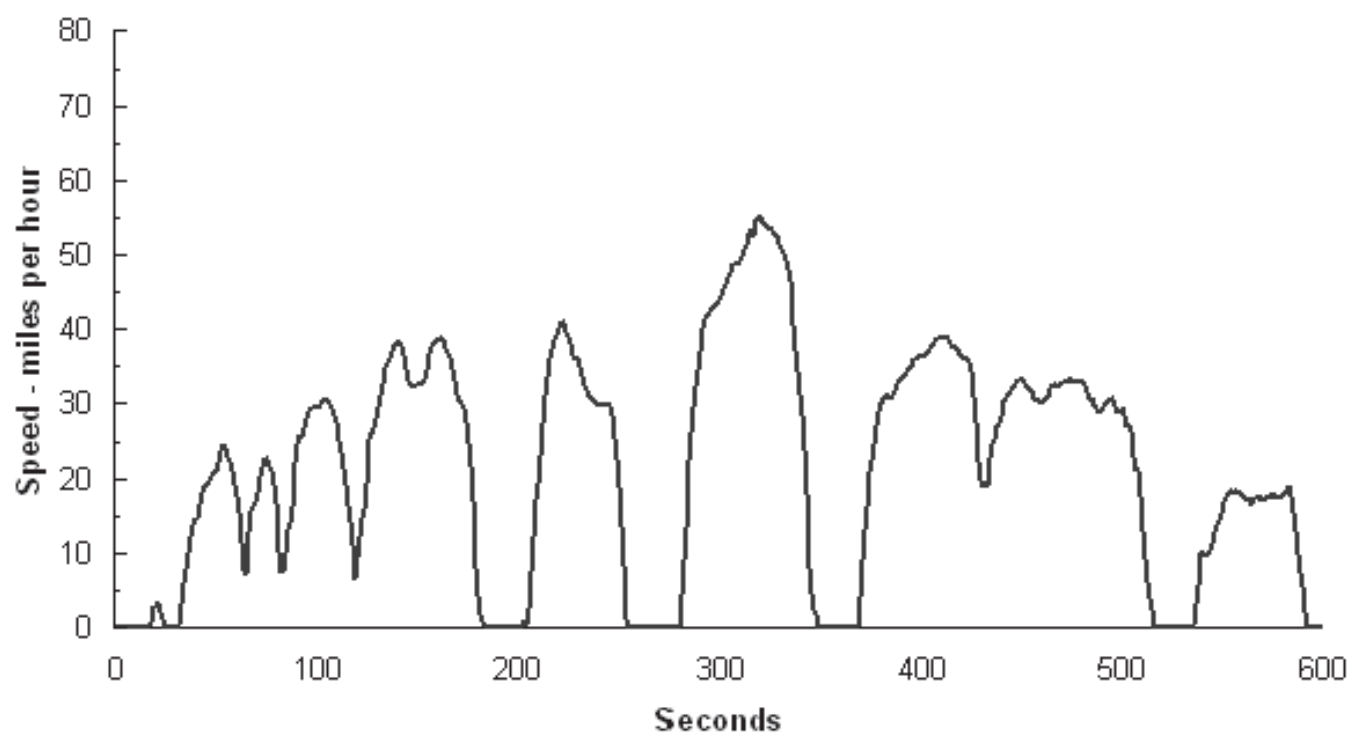

Source:

U.S. Department of Energy and Environmental Protection Agency, Fuel Economy website, www.fueleconomy.gov.

Figure 4.6. Cold Temperature (Cold FTP) Driving Cycle

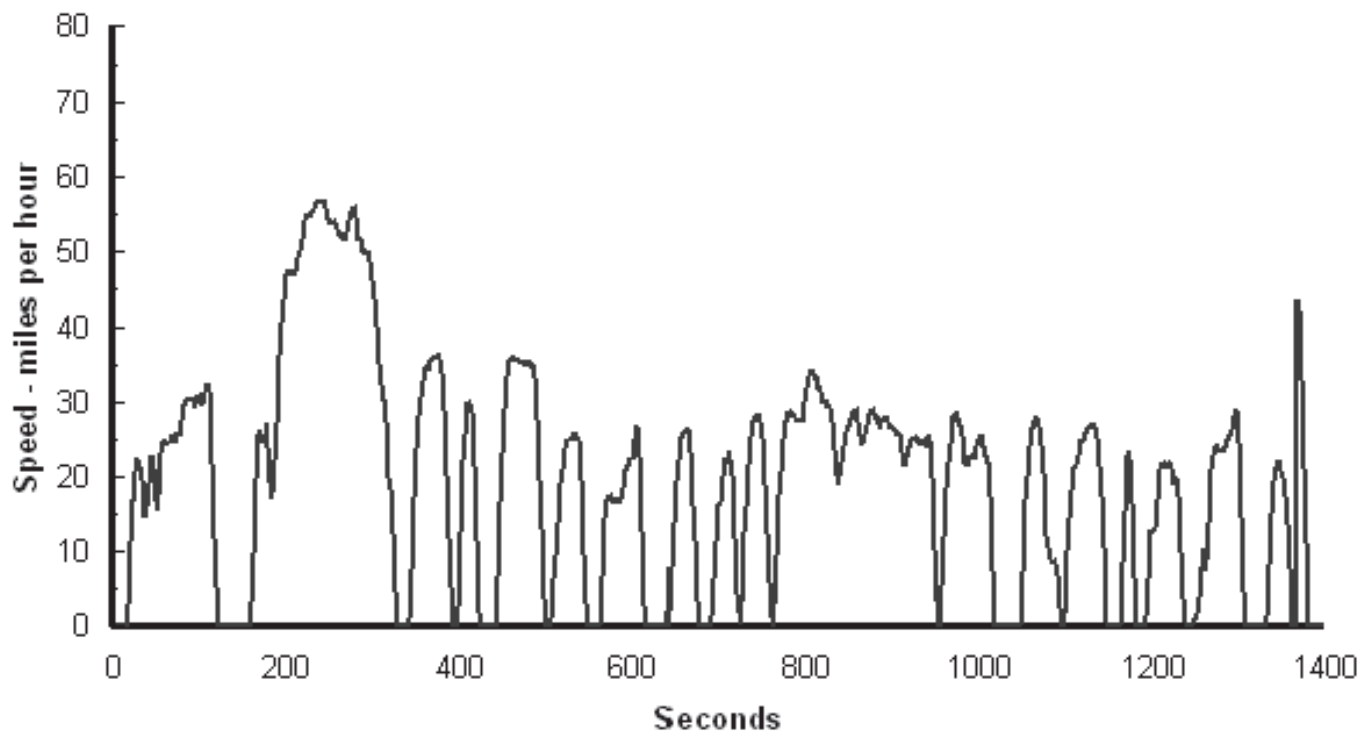

Source:

U.S. Department of Energy and Environmental Protection Agency, Fuel Economy website, www.fueleconomy.gov. 
Beginning with the 2008 model year, this cycle influences the new vehicle fuel economy ratings. The USO6 driving cycle was originally developed as a supplement to the Federal Test Procedure. It is a short-duration cycle (600 seconds) which represents hard-acceleration driving.

Figure 4.7. High-Speed (US06) Driving Cycle

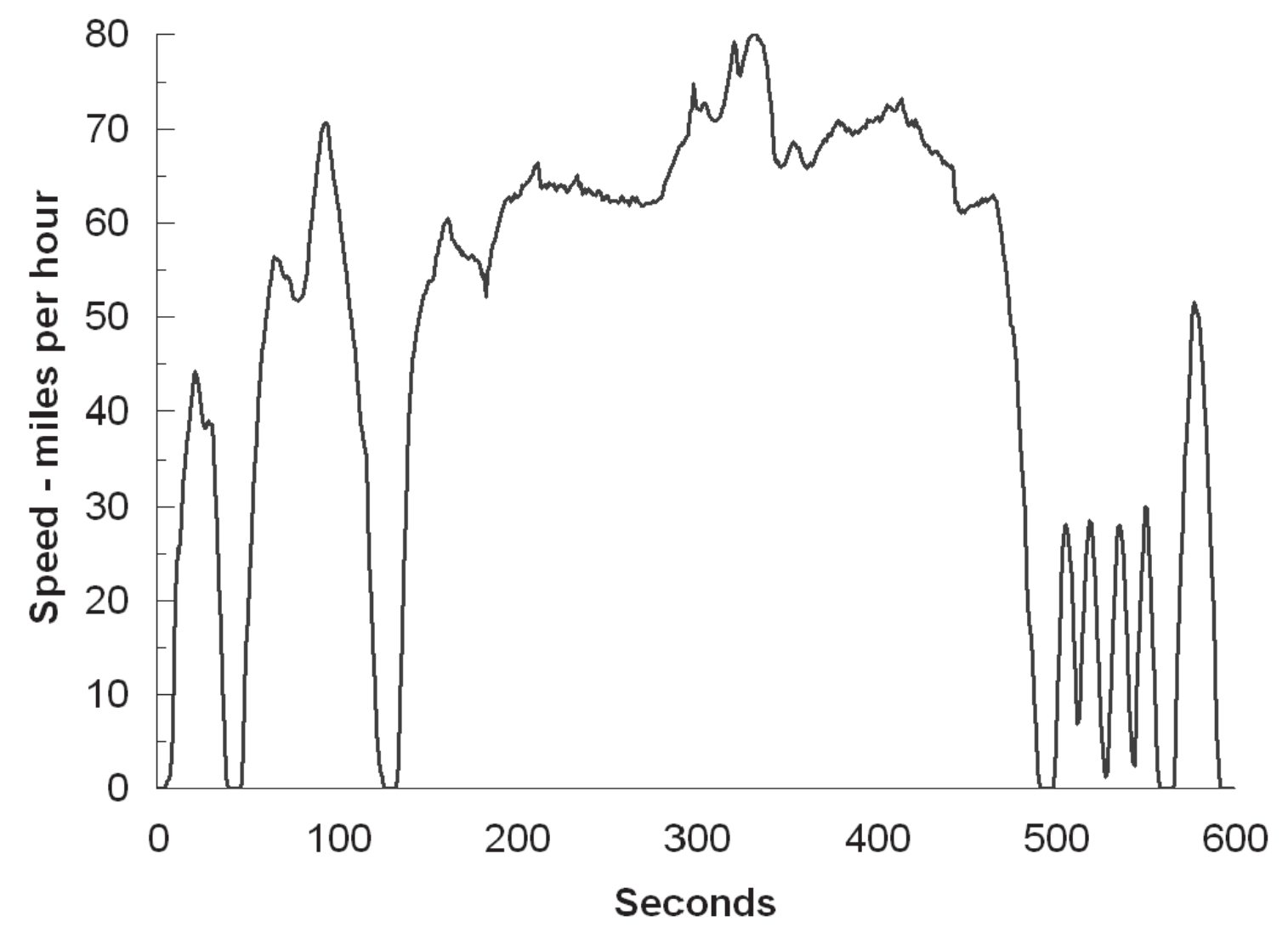

Source:

U.S. Department of Energy and Environmental Protection Agency, Fuel Economy website, www.fueleconomy.gov. 
The Environmental Protection Agency also uses other driving cycles to test new vehicles (although these do not affect the fuel economy ratings). The New York Test Cycle was developed in the 1970's in order to simulate driving in downtown congested areas. The Representative Number Five Test Cycle was developed in the 1990's

to better represent actual on-road driving by combining modern city and freeway driving.

Figure 4.8. New York City Driving Cycle

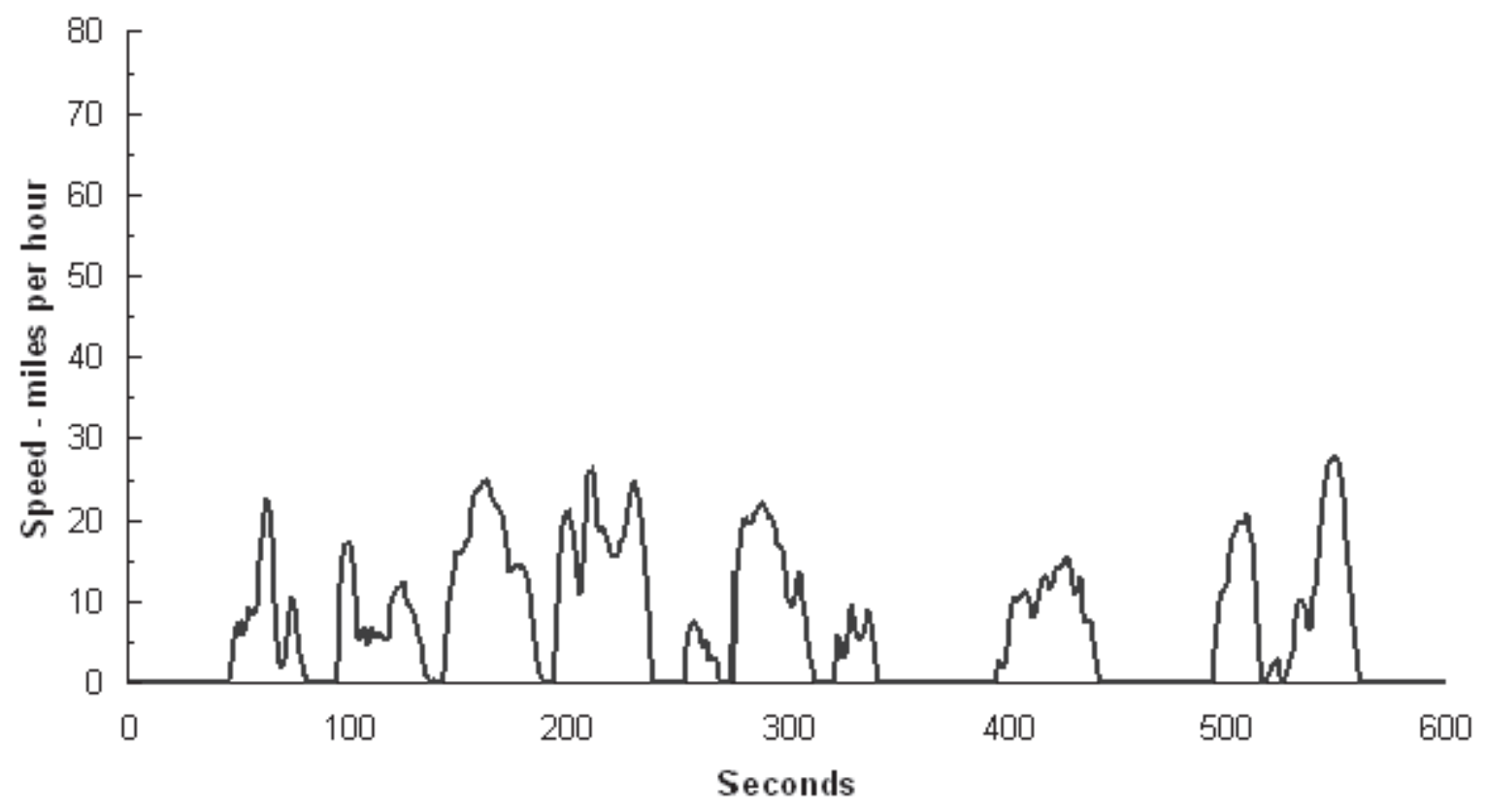

Figure 4.9. Representative Number Five Driving Cycle

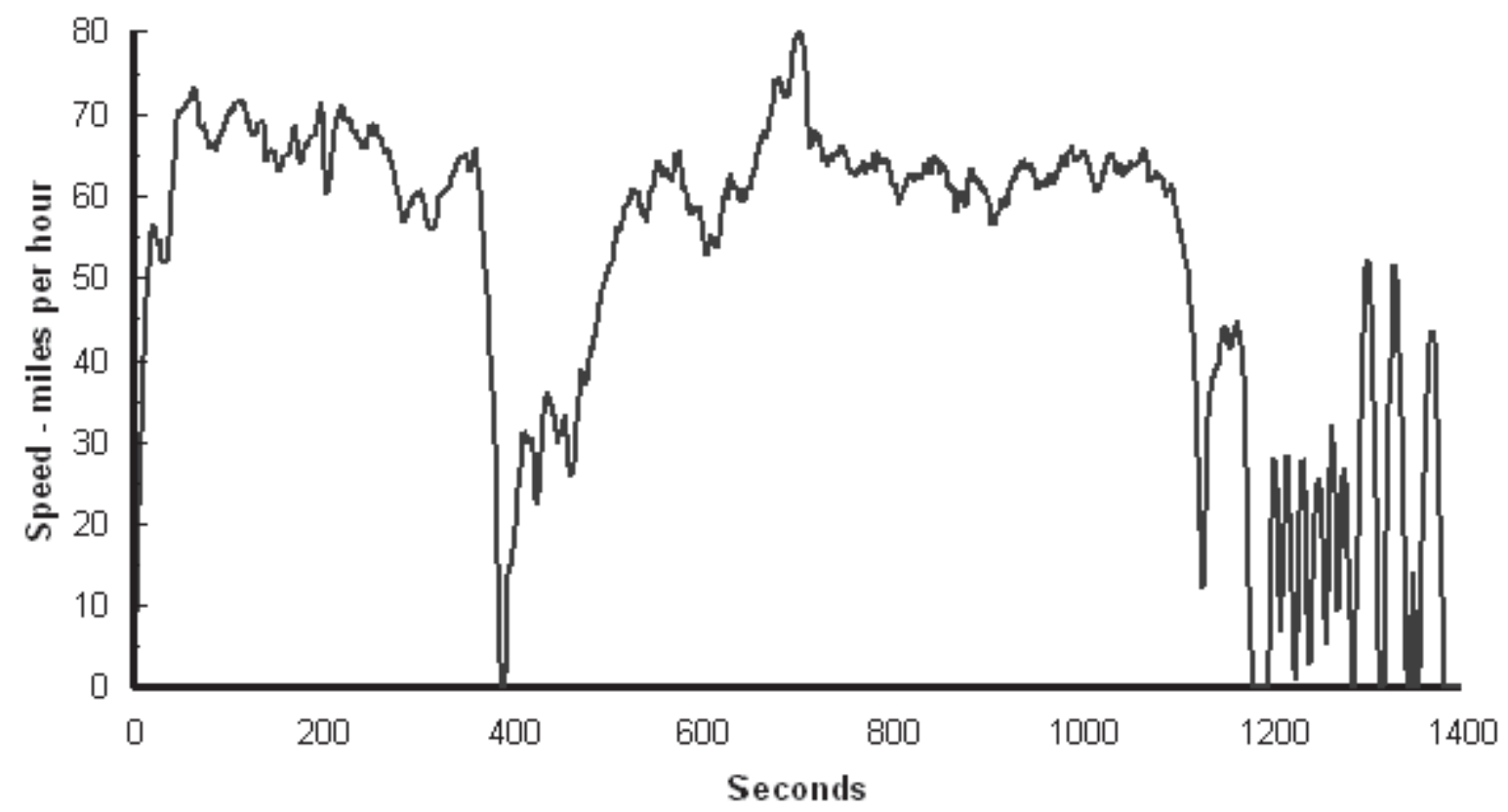

Source:

Data obtained from Michael Wang, Argonne National Laboratory, Argonne, IL, 1997. 
Testing cycles to determine vehicle fuel economy and emissions vary by country and therefore it is difficult to make a direct comparison. Statistics on the driving cycles from Europe, Japan and the U.S. are listed in below. In addition, Table 4.30 displays the cycle results of these countries by vehicle type. Note that the differences in these cycle results vary with each individual vehicle tested.

Table 4.29

Comparison of U.S., European, and Japanese Driving Cycles Attributes

\begin{tabular}{lcccc}
\hline & $\begin{array}{c}\text { Time } \\
(\text { seconds })\end{array}$ & $\begin{array}{c}\text { Average } \\
\text { speed } \\
(\mathrm{mph})\end{array}$ & $\begin{array}{c}\text { Maximum } \\
\text { speed } \\
(\mathrm{mph})\end{array}$ & $\begin{array}{c}\text { Maximum } \\
\text { acceleration } \\
(\mathrm{mph} / \mathrm{s})\end{array}$ \\
\hline Japanese JC08 test cycle & 631 & 14.8 & 43.5 & 1.8 \\
New European Driving Cycle (NEDC) $_{\text {U.S. EPA city cycle (LA4) }}^{\mathrm{a}}$ & 1,181 & 20.9 & 74.6 & 2.4 \\
U.S. EPA highway cycle & 1,375 & 19.5 & 56.7 & 3.3 \\
U.S. Corporate Average Fuel Economy (CAFE) cycle & 766 & 48.2 & 59.9 & 3.3 \\
\hline
\end{tabular}

Note: China uses the NEDC. India uses a modified version of the NEDC called The Modified Indian Driving Cycle which accounts for lower maximum speeds that better represent driving conditions in India.

\section{Source:}

The International Council on Clean Transportation, Passenger Vehicle Greenhouse Gas and Fuel Economy Standards: A Global Update, July 2009.

Table 4.30

Example of Differing Results Using the U.S., European, and Japanese Driving Cycles

\begin{tabular}{lcccccc}
\hline & \multicolumn{3}{c}{ Miles per gallon } & & \multicolumn{2}{c}{ Percentage difference from } \\
\cline { 2 - 3 } \cline { 6 - 7 } Vehicle type & CAFE cycle & NEDC & JC08 cycle & & CAFE to NEDC & CAFE to JC08 \\
\hline Small car & 34.8 & 32.4 & 27.6 & & $-7 \%$ & $-21 \%$ \\
Large car & 26.6 & 24.7 & 21.5 & & $-7 \%$ & $-19 \%$ \\
Minivan & 23.9 & 20.5 & 17.2 & & $-14 \%$ & $-28 \%$ \\
Sport-utility vehicle & 20.2 & 17.6 & 14.6 & & $-13 \%$ & $-28 \%$ \\
Pickup & 18.8 & 15.9 & 13.5 & & $-15 \%$ & $-28 \%$ \\
\hline
\end{tabular}

Note: Simulation results for identical gasoline vehicles (i.e., results for the same small car on each of the three cycles).

\section{Source:}

The International Council on Clean Transportation, Passenger Vehicle Greenhouse Gas and Fuel Economy Standards: A Global Update, July 2009.

${ }^{\text {a }}$ Data are not available.

${ }^{\mathrm{b}}$ The actual Federal Procedure (FTP), which is also the test for emissions certification, repeats the first 505 seconds of the Federal Urban Driving Simulation cycle, hot started, after a 10 minute hot soak. Starting with Model Year 2001, the emissions test-but not the fuel economy test-incorporates a supplemental cycle that simulates aggressive urban driving, coupled with an added air conditioning load. 
Demand response vehicles (also called paratransit or dial-a-ride) are widely used by transit agencies. The vehicles do not operate over a fixed route or on a fixed schedule. The vehicle may be dispatched to pick up several passengers at different pick-up points before taking them to their respective destinations and may even be interrupted en route to these destinations to pick up other passengers. Demand response service is provided primarily by vans. In 2007, the data changed substantially due to improved estimation methodologies. Unfortunately, those data are no longer comparable to the rest of the historical series.

Table 4.31

Summary Statistics on Demand Response Vehicles, 1994-2012

\begin{tabular}{cccccccc}
\hline & $\begin{array}{c}\text { Number of } \\
\text { agencies }\end{array}$ & $\begin{array}{c}\text { Number of } \\
\text { active vehicles }\end{array}$ & $\begin{array}{c}\text { Vehicle-miles } \\
\text { (millions) }\end{array}$ & $\begin{array}{c}\text { Average } \\
\text { miles per } \\
\text { vehicle }\end{array}$ & $\begin{array}{c}\text { Passenger- } \\
\text { miles } \\
\text { (millions) }\end{array}$ & $\begin{array}{c}\text { Average } \\
\text { load } \\
\text { factor }\end{array}$ & $\begin{array}{c}\text { Energy use } \\
\text { trillion Btu) }\end{array}$ \\
\hline 1994 & 5,214 & 28,729 & 463.7 & 16.14 & 577 & & 9.5 \\
1995 & 5,214 & 29,352 & 506.5 & 17.26 & 607 & 1.41 & 9.2 \\
1996 & 5,214 & 30,804 & 548.3 & 17.80 & 656 & 1.21 & 9.9 \\
1997 & 5,214 & 32,509 & 585.3 & 18.00 & 754 & 1.36 & 9.8 \\
1998 & 5,214 & 29,646 & 670.9 & 22.63 & 735 & 1.21 & 10.4 \\
1999 & 5,252 & 31,884 & 718.4 & 22.53 & 813 & 1.34 & 10.6 \\
2000 & 5,252 & 33,080 & 758.9 & 22.94 & 839 & 1.30 & 10.8 \\
2001 & 5,251 & 34,661 & 789.3 & 22.77 & 855 & 1.28 & 11.3 \\
2002 & 5,251 & 34,699 & 802.6 & 23.13 & 853 & 1.24 & 11.6 \\
2003 & 5,346 & 35,954 & 864.0 & 24.03 & 930 & 1.27 & 12.9 \\
2004 & 5,960 & 37,078 & 889.5 & 23.99 & 962 & 1.25 & 13.3 \\
2005 & 5,960 & 41,958 & 978.3 & 23.32 & 1,058 & 1.25 & 14.8 \\
2006 & 5,960 & 43,509 & $1,013.0$ & 23.28 & 1,078 & 1.24 & $\mathrm{~b}$ \\
\hline 2007 & 7,300 & 64,865 & $1,471.4$ & 22.68 & 1,502 & 1.18 & 15.5 \\
2008 & 7,200 & 65,799 & $1,495.2$ & 22.72 & 1,412 & 1.09 & 24.7 \\
2009 & 6,700 & 68,957 & $1,529.2$ & 22.18 & 1,477 & 1.12 & 24.7 \\
2010 & 6,741 & 68,621 & $1,693.6$ & 24.68 & 1,494 & 1.03 & 23.1 \\
2011 & 6,600 & 65,336 & $1,611.8$ & 24.67 & 1,580 & 1.13 & 22.8 \\
2012 & 6,511 & 68,632 & $1,618.1$ & 23.58 & 1,756 & 1.24 & 24.1 \\
\hline
\end{tabular}

Note: See Glossary for a detailed definition of demand response.

Source:

American Public Transportation Association, 2014 Public Transportation Fact Book, Washington, DC, May 2014. (Additional resources: www.apta.com)

a Data are not available.

b Data are not continuous between 2006 and 2007 due to changes in estimation methodology. See source document for details. 


\section{Chapter 5 \\ Heavy Vehicles and Characteristics}

Summary Statistics from Tables in this Chapter

\section{Source}

Table 5.1 Class 3-8 single-unit trucks, 2012

Registration (thousands)

8,190

Vehicle miles (millions)

104,960

Fuel economy (miles per gallon)

Table 5.2 Class 7-8 combination trucks, 2012

Registration (thousands)

Vehicle miles (millions)

Fuel economy (miles per gallon)

Tables 5.14- Freight Shipments, 2012 Commodity Flow Survey

Table 5.17

Value (billion dollars)

13,625

Tons (millions)

11,696

Ton-miles (billions) 
Class 3-8 single-unit trucks include trucks over 10,000 lbs. gross vehicle weight with the cab/engine and cargo space together as one unit. Most of these trucks would be used for business or for individuals with heavy hauling or towing needs. Very heavy single-units, such as concrete mixers and dump trucks, are also in this category. The data series was recently changed by the FHWA back to 2007.

Table 5.1

Summary Statistics for Class 3-8 Single-Unit Trucks, 1970-2012

\begin{tabular}{|c|c|c|c|c|c|}
\hline Year & $\begin{array}{l}\text { Registrations } \\
\text { (thousands) }\end{array}$ & $\begin{array}{l}\text { Vehicle travel } \\
\text { (million miles) }\end{array}$ & $\begin{array}{l}\text { Average annual } \\
\text { miles per vehicle }\end{array}$ & $\begin{array}{c}\text { Fuel use } \\
\text { (million gallons) }\end{array}$ & $\begin{array}{c}\text { Fuel economy } \\
\text { (miles per gallon) }\end{array}$ \\
\hline 1970 & 3,681 & 27,081 & 7,357 & 3,968 & 6.8 \\
\hline 1975 & 4,232 & 34,606 & 8,177 & 5,420 & 6.4 \\
\hline 1976 & 4,350 & 36,390 & 8,366 & 5,706 & 6.4 \\
\hline 1977 & 4,450 & 39,339 & 8,840 & 6,268 & 6.3 \\
\hline 1978 & 4,518 & 42,747 & 9,461 & 6,955 & 6.1 \\
\hline 1979 & 4,505 & 42,012 & 9,326 & 7,050 & 6.0 \\
\hline 1980 & 4,374 & 39,813 & 9,102 & 6,923 & 5.8 \\
\hline 1981 & 4,455 & 39,568 & 8,882 & 6,867 & 5.8 \\
\hline 1982 & 4,325 & 40,658 & 9,401 & 6,803 & 6.0 \\
\hline 1983 & 4,204 & 42,546 & 10,120 & 6,965 & 6.1 \\
\hline 1984 & 4,061 & 44,419 & 10,938 & 7,240 & 6.1 \\
\hline 1985 & 4,593 & 45,441 & 9,894 & 7,399 & 6.1 \\
\hline 1986 & 4,313 & 45,637 & 10,581 & 7,386 & 6.2 \\
\hline 1987 & 4,188 & 48,022 & 11,467 & 7,523 & 6.4 \\
\hline 1988 & 4,470 & 49,434 & 11,059 & 7,701 & 6.4 \\
\hline 1989 & 4,519 & 50,870 & 11,257 & 7,779 & 6.5 \\
\hline 1990 & 4,487 & 51,901 & 11,567 & 8,357 & 6.2 \\
\hline 1991 & 4,481 & 52,898 & 11,805 & 8,172 & 6.5 \\
\hline 1992 & 4,370 & 53,874 & 12,328 & 8,237 & 6.5 \\
\hline 1993 & 4,408 & 56,772 & 12,879 & 8,488 & 6.7 \\
\hline 1994 & 4,906 & 61,284 & 12,492 & 9,032 & 6.8 \\
\hline 1995 & 5,024 & 62,705 & 12,481 & 9,216 & 6.8 \\
\hline 1996 & 5,266 & 64,072 & 12,167 & 9,409 & 6.8 \\
\hline 1997 & 5,293 & 66,893 & 12,638 & 9,576 & 7.0 \\
\hline 1998 & 5,414 & 67,894 & 12,540 & 9,741 & 7.0 \\
\hline 1999 & 5,763 & 70,304 & 12,199 & 9,372 & 7.5 \\
\hline 2000 & 5,926 & 70,500 & 11,897 & 9,563 & 7.4 \\
\hline 2001 & 5,704 & 72,448 & 12,701 & 9,667 & 7.5 \\
\hline 2002 & 5,651 & 75,866 & 13,425 & 10,321 & 7.4 \\
\hline 2003 & 5,849 & 77,757 & 13,294 & 8,881 & 8.8 \\
\hline 2004 & 6,161 & 78,441 & 12,732 & 8,959 & 8.8 \\
\hline 2005 & 6,395 & 78,496 & 12,275 & 9,501 & 8.3 \\
\hline 2006 & 6,649 & 80,344 & 12,084 & 9,852 & 8.2 \\
\hline 2007 & 8,117 & 119,979 & 14,781 & 16,314 & 7.3 \\
\hline 2008 & 8,228 & 126,855 & 15,417 & 17,144 & 7.4 \\
\hline 2009 & 8,356 & 120,207 & 14,386 & 16,253 & 7.4 \\
\hline 2010 & 8,217 & 110,738 & 13,477 & 15,097 & 7.3 \\
\hline 2011 & 7,819 & 103,803 & 13,276 & 14,214 & 7.3 \\
\hline \multirow[t]{2}{*}{2012} & 8,190 & 104,960 & 12,816 & 14,287 & 7.3 \\
\hline & \multicolumn{5}{|c|}{ Average annual percentage change } \\
\hline 1970-2012 & $1.9 \%$ & $3.3 \%$ & $1.3 \%$ & $3.1 \%$ & $0.2 \%$ \\
\hline 2002-2012 & $3.8 \%$ & $3.3 \%$ & $-0.5 \%$ & $3.3 \%$ & $-0.1 \%$ \\
\hline
\end{tabular}

\section{Source:}

U. S. Department of Transportation, Federal Highway Administration, Highway Statistics 2012, Washington, DC, 2014, Table VM-1 and annual. (Additional resources: www.fhwa.dot.gov)

\footnotetext{
${ }^{\text {a }}$ Due to FHWA methodology changes, data from 2007-on are not comparable with previous data.
} 
Class 7-8 combination trucks include all trucks designed to be used in combination with one or more trailers with a gross vehicle weight rating over 26,000 lbs. The average vehicle travel of these trucks (on a per truck basis) far surpasses the travel of other trucks due to long-haul freight movement. The data series was recently changed by the FHWA back to 2007.

Table 5.2

\section{Summary Statistics for Class 7-8 Combination Trucks, 1970-2012}

\begin{tabular}{|c|c|c|c|c|c|}
\hline Year & $\begin{array}{l}\text { Registrations } \\
\text { (thousands) }\end{array}$ & $\begin{array}{l}\text { Vehicle travel } \\
\text { (million miles) }\end{array}$ & $\begin{array}{l}\text { Average annual } \\
\text { miles per vehicle }\end{array}$ & $\begin{array}{c}\text { Fuel use } \\
\text { (million gallons) }\end{array}$ & $\begin{array}{c}\text { Fuel economy } \\
\text { (miles per gallon) }\end{array}$ \\
\hline 1970 & 905 & 35,134 & 38,822 & 7,348 & 4.8 \\
\hline 1975 & 1,131 & 46,724 & 41,312 & 9,177 & 5.1 \\
\hline 1980 & 1,417 & 68,678 & 48,467 & 13,037 & 5.3 \\
\hline 1981 & 1,261 & 69,134 & 54,825 & 13,509 & 5.1 \\
\hline 1982 & 1,265 & 70,765 & 55,941 & 13,583 & 5.2 \\
\hline 1983 & 1,304 & 73,586 & 56,431 & 13,796 & 5.3 \\
\hline 1984 & 1,340 & 77,377 & 57,744 & 14,188 & 5.5 \\
\hline 1985 & 1,403 & 78,063 & 55,640 & 14,005 & 5.6 \\
\hline 1986 & 1,408 & 81,038 & 57,555 & 14,475 & 5.6 \\
\hline 1987 & 1,530 & 85,495 & 55,879 & 14,990 & 5.7 \\
\hline 1988 & 1,667 & 88,551 & 53,120 & 15,224 & 5.8 \\
\hline 1989 & 1,707 & 91,879 & 53,825 & 15,733 & 5.8 \\
\hline 1990 & 1,709 & 94,341 & 55,202 & 16,133 & 5.8 \\
\hline 1991 & 1,691 & 96,645 & 57,153 & 16,809 & 5.7 \\
\hline 1992 & 1,675 & 99,510 & 59,409 & 17,216 & 5.8 \\
\hline 1993 & 1,680 & 103,116 & 61,379 & 17,748 & 5.8 \\
\hline 1994 & 1,681 & 108,932 & 64,802 & 18,653 & 5.8 \\
\hline 1995 & 1,696 & 115,451 & 68,073 & 19,777 & 5.8 \\
\hline 1996 & 1,747 & 118,899 & 68,059 & 20,192 & 5.9 \\
\hline 1997 & 1,790 & 124,584 & 69,600 & 20,302 & 6.1 \\
\hline 1998 & 1,831 & 128,159 & 69,994 & 21,100 & 6.1 \\
\hline 1999 & 2,029 & 132,384 & 65,246 & 24,537 & 5.4 \\
\hline 2000 & 2,097 & 135,020 & 64,387 & 25,666 & 5.3 \\
\hline 2001 & 2,154 & 136,584 & 63,409 & 25,512 & 5.4 \\
\hline 2002 & 2,277 & 138,737 & 60,930 & 26,480 & 5.2 \\
\hline 2003 & 1,908 & 140,160 & 73,459 & 23,815 & 5.9 \\
\hline 2004 & 2,010 & 142,370 & 70,831 & 24,191 & 5.9 \\
\hline 2005 & 2,087 & 144,028 & 69,012 & 27,689 & 5.2 \\
\hline 2006 & 2,170 & 142,169 & 65,516 & 28,107 & 5.1 \\
\hline 2007 & 2,635 & 184,199 & 69,905 & 30,904 & 6.0 \\
\hline 2008 & 2,585 & 183,826 & 71,113 & 30,561 & 6.0 \\
\hline 2009 & 2,617 & 168,100 & 64,234 & 28,050 & 6.0 \\
\hline 2010 & 2,553 & 175,789 & 68,856 & 29,927 & 5.9 \\
\hline 2011 & 2,452 & 163,791 & 66,809 & 28,181 & 5.8 \\
\hline 2012 & 2,469 & 163,358 & 66,161 & 27,926 & 5.8 \\
\hline & \multicolumn{5}{|c|}{ Average annual percentage change } \\
\hline 1970-2012 & $2.4 \%$ & $3.7 \%$ & $1.3 \%$ & $3.2 \%$ & $0.5 \%$ \\
\hline 2002-2012 & $0.8 \%$ & $1.6 \%$ & $0.8 \%$ & $0.5 \%$ & $1.1 \%$ \\
\hline
\end{tabular}

\section{Source:}

U. S. Department of Transportation, Federal Highway Administration, Highway Statistics 2012, Washington, DC, 2014, Table VM-1 and annual. (Additional resources: www.fhwa.dot.gov)

${ }^{a}$ The Federal Highway Administration changed the combination truck travel methodology in 1993.

${ }^{\mathrm{b}}$ Due to FHWA methodology changes, data from 2007-on are not comparable with previous data. 
Truck sales rose in 2010 for the first time since the sales peak in 2004 and have continued to rise since then Trucks under 10,000 lbs. continue to dominate truck sales.

Table 5.3

New Retail Truck Sales by Gross Vehicle Weight, 1970-2013 (thousands)

\begin{tabular}{|c|c|c|c|c|c|c|c|c|c|}
\hline $\begin{array}{c}\text { Calendar } \\
\text { year }\end{array}$ & $\begin{array}{c}\text { Class } 1 \\
6,000 \text { lbs. } \\
\text { or less }\end{array}$ & $\begin{array}{c}\text { Class } 2 \\
6,001- \\
10,000 \mathrm{lbs} .\end{array}$ & $\begin{array}{c}\text { Class } 3 \\
10,001- \\
14,000 \text { lbs. }\end{array}$ & $\begin{array}{c}\text { Class } 4 \\
14,001- \\
16,000 \text { lbs. }\end{array}$ & $\begin{array}{c}\text { Class } 5 \\
16,001- \\
19,500 \text { lbs. }\end{array}$ & $\begin{array}{c}\text { Class } 6 \\
19,501- \\
26,000 \text { lbs. }\end{array}$ & $\begin{array}{c}\text { Class } 7 \\
26,001- \\
33,000 \mathrm{lbs} .\end{array}$ & $\begin{array}{c}\text { Class } 8 \\
33,001 \text { lbs. } \\
\text { and over }\end{array}$ & Total \\
\hline \multicolumn{10}{|c|}{ Domestic sales (import data are not available) } \\
\hline $1970^{\mathrm{b}}$ & 1,049 & 408 & 6 & 12 & 58 & 133 & 36 & 89 & 1,791 \\
\hline 1975 & 1,101 & 952 & 23 & 1 & 9 & 159 & 23 & 83 & 2,351 \\
\hline 1980 & $98 \overline{5}$ & 975 & 4 & $\overline{\mathrm{c}}$ & 2 & 90 & 58 & $1 \overline{1} \overline{7}$ & 2,231 \\
\hline 1981 & 896 & 850 & 1 & $\mathrm{c}$ & 2 & 72 & 51 & 100 & 1,972 \\
\hline 1983 & 1,314 & 1,207 & $\mathrm{c}$ & $\mathrm{c}$ & 1 & 47 & 59 & 82 & 2,710 \\
\hline 1984 & 2,031 & 1,224 & 6 & $\mathrm{c}$ & 5 & 55 & 78 & 138 & 3,538 \\
\hline 1985 & 2,408 & 1,280 & 11 & $\mathrm{c}$ & 5 & 48 & 97 & 134 & 3,983 \\
\hline \multicolumn{10}{|c|}{ Domestic and import sales } \\
\hline 1986 & 3,380 & 1,214 & 12 & $\mathrm{c}$ & 6 & 45 & 101 & 113 & 4,870 \\
\hline 1987 & 3,435 & 1,175 & 14 & 2 & 8 & 44 & 103 & 131 & 4,912 \\
\hline 1988 & 3,467 & 1,333 & 14 & 21 & 8 & 54 & 103 & 148 & 5,149 \\
\hline 1989 & 3,313 & 1,297 & 19 & 27 & 7 & 39 & 93 & 145 & 4,942 \\
\hline 1991 & 3,246 & 876 & 21 & 24 & 3 & 22 & 73 & 99 & 4,365 \\
\hline 1992 & 3,608 & 1,021 & 26 & 26 & 4 & 28 & 73 & 119 & 4,903 \\
\hline 1993 & 4,119 & 1,232 & 27 & 33 & 4 & 27 & 81 & 158 & 5,681 \\
\hline 1994 & 4,527 & 1,506 & 35 & 44 & 4 & 20 & 98 & 186 & 6,421 \\
\hline 1995 & 4,422 & 1,631 & 40 & 53 & 4 & 23 & 107 & 201 & 6,481 \\
\hline 1996 & 4,829 & 1,690 & 52 & 59 & 7 & 19 & 104 & 170 & 6,930 \\
\hline 1997 & 5,085 & 1,712 & 53 & 57 & 9 & 18 & 114 & 179 & 7,226 \\
\hline 1998 & 5,263 & 2,036 & 102 & 43 & 25 & 32 & 115 & 209 & 7,826 \\
\hline 1999 & 5,707 & 2,366 & 122 & 49 & 30 & 48 & 130 & 262 & 8,716 \\
\hline 2000 & 5,965 & 2,421 & 117 & 47 & 29 & 51 & 123 & 212 & 8,965 \\
\hline 2001 & 6,073 & 2,525 & 102 & 52 & 24 & 42 & 92 & 140 & 9,050 \\
\hline 2002 & 6,068 & 2,565 & 80 & 38 & 24 & 45 & 69 & 146 & 9,035 \\
\hline 2003 & 6,267 & 2,671 & 91 & 40 & 29 & 51 & 67 & 142 & 9,357 \\
\hline 2004 & 6,458 & 2,796 & 107 & 47 & 36 & 70 & 75 & 203 & 9,793 \\
\hline 2005 & 6,586 & 2,528 & 167 & 49 & 46 & 60 & 89 & 253 & 9,777 \\
\hline 2006 & 6,136 & 2,438 & 150 & 50 & 49 & 70 & 91 & 284 & 9,268 \\
\hline 2009 & 3,528 & 1,306 & 112 & 20 & 24 & 22 & 39 & 95 & 5,145 \\
\hline 2010 & 4,245 & 1,513 & 161 & 12 & 31 & 29 & 38 & 107 & 6,137 \\
\hline 2011 & 4,714 & 1,735 & 195 & 10 & 42 & 41 & 41 & 171 & 6,951 \\
\hline 2012 & 5,164 & 1,811 & 223 & 9 & 55 & 40 & 47 & 195 & 7,544 \\
\hline 2013 & 5,615 & 2,077 & 254 & 12 & 60 & 47 & 48 & 185 & 8,298 \\
\hline \multicolumn{10}{|c|}{ Average annual percentage change } \\
\hline $1970-1985$ & $5.7 \%$ & $7.9 \%$ & $4.1 \%$ & -1.9 & $-15.1 \%$ & $-6.6 \%$ & $6.8 \%$ & $2.8 \%$ & $5.5 \%$ \\
\hline 1986-2013 & $1.9 \%$ & $2.0 \%$ & $12.0 \%$ & $7.1 \%{ }^{\mathrm{d}}$ & $8.9 \%$ & $-0.2 \%$ & $-2.7 \%$ & $1.8 \%$ & $2.0 \%$ \\
\hline 2003-2013 & $-1.1 \%$ & $-2.5 \%$ & $10.8 \%$ & $-11.3 \%$ & $7.5 \%$ & $-0.8 \%$ & $-3.3 \%$ & $2.7 \%$ & $-1.2 \%$ \\
\hline
\end{tabular}

\section{Source:}

Ward's Communication's, Motor Vehicle Facts and Figures 2013, Southfield, MI, 2013, p. 22, and annual; 2013: Ward's Communications, www.wardsauto.com. (Additional resources: www.wardsauto.com)

\footnotetext{
${ }^{a}$ Sales include domestic-sponsored imports.

${ }^{\mathrm{b}}$ Data for 1970 is based on new truck registrations.

${ }^{\mathrm{c}}$ Data are not available.

d 1987-2013.
} 
The Census Bureau has discontinued the Vehicle Inventory and Use Survey; it was not conducted in 2007. The 2002 data remain the latest available.

\section{Vehicle Inventory and Use Survey}

The Vehicle Inventory and Use Survey (VIUS), which was formerly the Truck Inventory and Use Survey (TIUS), provides data on the physical and operational characteristics of the Nation's truck population. It is based on a probability sample of private and commercial trucks registered (or licensed) in each state. In 1997, the survey was changed to the Vehicle Inventory and Use Survey due to future possibilities of including additional vehicle types. The 2002 VIUS, however, only includes trucks. Copies of the 2002 VIUS report or CD may be obtained by contacting the U.S. Bureau of the Census, Transportation Characteristics Surveys Branch (301) 457-2797. Internet site:

\section{www.census.gov/svsd/www/tiusview.html}

Since 1987, the survey has included minivans, vans, station wagons on truck chassis, and sport utility vehicles in addition to the bigger trucks. The 1977 and 1982 surveys did not include those vehicle types. The estimated number of trucks that were within the scope of the 2002 VIUS and registered in the United States as of July 1, 2002 was 85.2 million. These trucks were estimated to have been driven a total of 1,115 billion miles during 2002, an increase of $6.8 \%$ from 1997 . The average annual miles traveled per truck was estimated at 13,100 miles.

In the 2002 VIUS, there are several ways to classify a truck by weight. The survey respondent was asked the average weight of the vehicle or vehicle-trailer combination when carrying a typical payload; the empty weight (truck minus cargo) of the vehicle as it was usually operated; and the maximum gross weight at which the vehicle or vehicle-trailer combination was operated. The Census Bureau also collected information on the Gross Vehicle Weight Class of the vehicles (decoded from the vehicle identification number) and the registered weight of the vehicles from the State registration files. Some of these weights are only provided in categories, while others are exact weights. Since all these weights could be quite different for a single truck, the tabulations by weight can be quite confusing. In the tables presented here, the Gross Vehicle Weight Class was used. 
Table 5.4

Truck Statistics by Gross Vehicle Weight Class, 2002

\begin{tabular}{|c|c|c|c|c|c|c|}
\hline \multicolumn{2}{|c|}{$\begin{array}{l}\text { Manufacturer's gross vehicle } \\
\text { weight class }\end{array}$} & \multirow{2}{*}{$\begin{array}{c}\begin{array}{c}\text { Number of } \\
\text { trucks }\end{array} \\
51,941,389\end{array}$} & \multirow{2}{*}{$\begin{array}{r}\text { Percentage } \\
\text { of trucks }\end{array}$} & \multirow{2}{*}{$\begin{array}{c}\text { Average } \\
\text { annual miles } \\
\text { per truck } \\
11,882\end{array}$} & \multirow{2}{*}{$\begin{array}{l}\text { Harmonic } \\
\text { mean fuel } \\
\text { economy }\end{array}$} & \multirow{2}{*}{$\begin{array}{c}\begin{array}{c}\text { Percentage } \\
\text { of fuel use }\end{array} \\
42.7 \%\end{array}$} \\
\hline 1) & $6,000 \mathrm{lbs}$ and less & & & & & \\
\hline \multirow[t]{2}{*}{ 2) } & $6,001-10,000 \mathrm{lbs}$ & $28,041,234$ & $32.9 \%$ & 12,684 & 14.3 & $30.5 \%$ \\
\hline & Light truck subtotal & $79,982,623$ & $93.9 \%$ & 12,163 & 16.2 & $73.2 \%$ \\
\hline 3) & $10,001-14,000 \mathrm{lbs}$ & 691,342 & $0.8 \%$ & 14,094 & 10.5 & $1.1 \%$ \\
\hline 4) & $14,001-16,000 \mathrm{lbs}$ & 290,980 & $0.3 \%$ & 15,441 & 8.5 & $0.5 \%$ \\
\hline 5) & $16,001-19,500 \mathrm{lbs}$ & 166,472 & $0.2 \%$ & 11,645 & 7.9 & $0.3 \%$ \\
\hline \multirow[t]{2}{*}{ 6) } & $19,501-26,000 \mathrm{lbs}$ & $1,709,574$ & $2.0 \%$ & 12,671 & 7.0 & $3.2 \%$ \\
\hline & Medium truck subtotal & $2,858,368$ & $3.4 \%$ & 13,237 & 8.0 & $5.2 \%$ \\
\hline 7) & $26,001-33,000 \mathrm{lbs}$ & 179,790 & $0.2 \%$ & 30,708 & 6.4 & $0.9 \%$ \\
\hline \multirow[t]{2}{*}{ 8) } & $33,001 \mathrm{lbs}$ and up & $2,153,996$ & $2.5 \%$ & 45,739 & 5.7 & $20.7 \%$ \\
\hline & Heavy truck subtotal & $2,333,786$ & $2.7 \%$ & 44,581 & 5.8 & $21.6 \%$ \\
\hline \multicolumn{2}{|c|}{ Total } & $85,174,777$ & $100.0 \%$ & 13,088 & 13.5 & $100.0 \%$ \\
\hline
\end{tabular}

Source:

U.S. Department of Commerce, Bureau of the Census, 2002 Vehicle Inventory and Use Survey, Microdata File on $\mathrm{CD}, 2005$. (Additional resources: www.census.gov/svsd/www.tiusview.html)

Table 5.5

Truck Harmonic Mean Fuel Economy by Size Class, 1992, 1997, and 2002 (miles per gallon)

\begin{tabular}{lrcc}
\hline Manufacturer's gross vehicle & 1992 & 1997 & 2002 \\
\multicolumn{1}{c}{ weight class } & TIUS & VIUS & VIUS \\
\hline 1) 6,000 lbs and less & 17.2 & 17.1 & 17.6 \\
2) 6,001-10,000 lbs & 13.0 & 13.6 & 14.3 \\
Light truck subtotal & 15.7 & 15.8 & 16.2 \\
3) 10,000-14,000 lbs & 8.8 & 9.4 & 10.5 \\
4) $14,001-16,000 \mathrm{lbs}$ & 8.8 & 9.3 & 8.5 \\
5) $16,001-19,500 \mathrm{lbs}$ & 7.4 & 8.7 & 7.9 \\
6) $19,501-26,000 \mathrm{lbs}$ & 6.9 & 7.3 & 7.0 \\
Medium truck subtotal & 7.3 & 8.6 & 8.0 \\
7) 26,001-33,000 lbs & 6.5 & 6.4 & 6.4 \\
8) 33,001 lbs and over & 5.5 & 5.7 & 5.7 \\
Large truck subtotal & 5.6 & 6.1 & 5.8 \\
\hline
\end{tabular}

Note: Based on average fuel economy as reported by respondent.

\section{Sources:}

Estimates are based on data provided on the following public use files: U.S. Department of Commerce, Bureau of the Census, Census of Transportation, Washington, DC, 1992 Truck Inventory and Use Survey, 1995; 1997 Vehicle Inventory and Use Survey, 2000, and 2002 Vehicle Inventory and Use Survey, 2005. (Additional resources: www.census.gov/svsd/www/tiusview.html) 
As expected, most light trucks travel within 50 miles of their home base and refuel at public stations. About sixty percent of heavy trucks travel over 50 miles from their home base and 36\% of them refuel at central companyowned refueling stations.

Table 5.6

Truck Statistics by Size, 2002

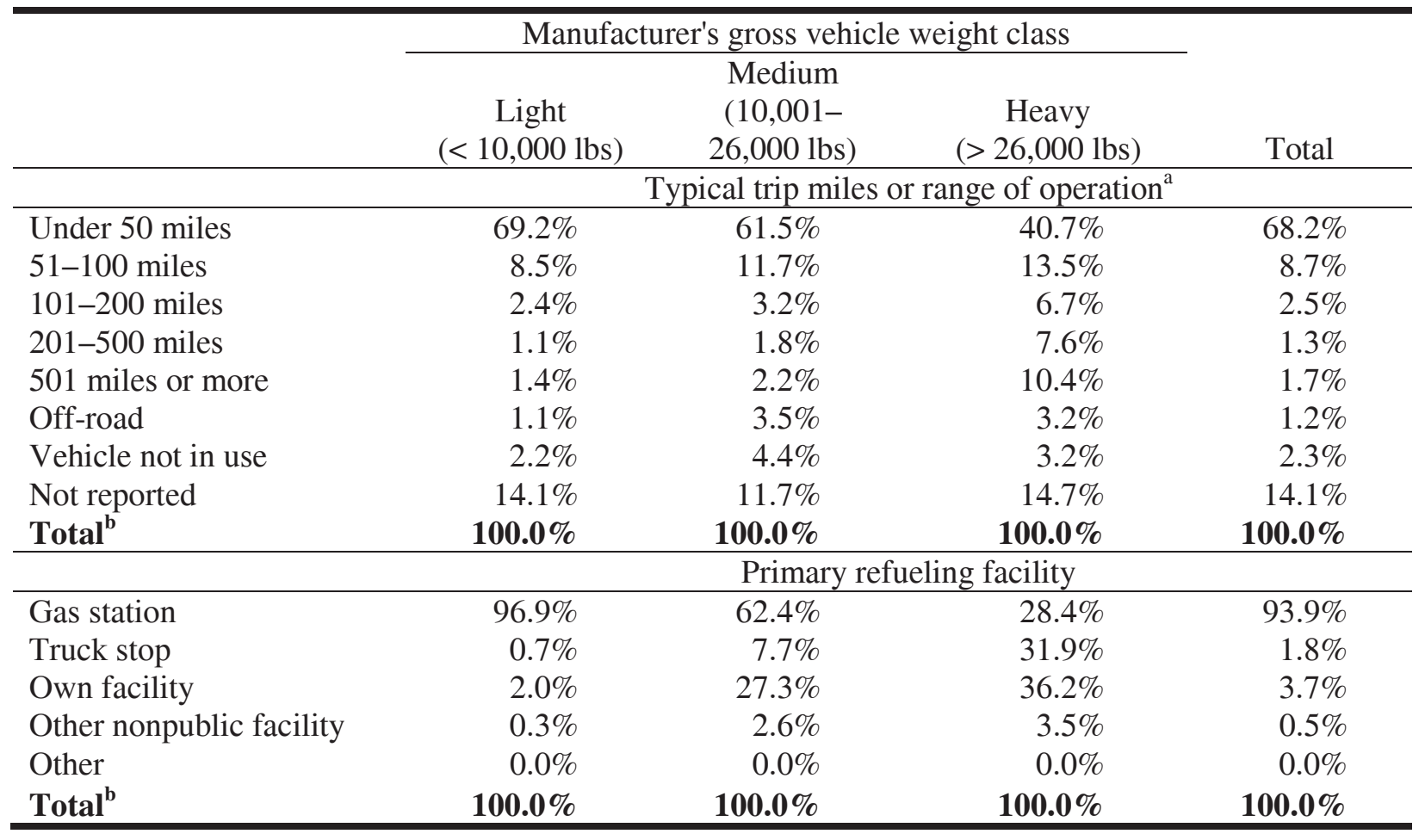

Source:

U.S. Department of Commerce, Bureau of the Census, 2002 Vehicle Inventory and Use Survey, Microdata. File on CD, 2005. (Additional resources: www.census.gov/svsd/www/tiusview.html)

\footnotetext{
${ }^{\text {a }}$ The respondent was asked to choose the category which best described the trips made by the vehicle.

${ }^{\mathrm{b}}$ Percentages may not sum to totals due to rounding.
} 
More medium truck owners listed construction as the truck's major use than any other major use category.

Construction was the second highest major use for light trucks and heavy trucks.

Table 5.7

Percentage of Trucks by Size Ranked by Major Use, 2002

\begin{tabular}{|c|c|c|c|}
\hline Rank & $\begin{array}{c}\text { Light } \\
(<10,000 \mathrm{lbs} \\
\text { average weight })\end{array}$ & $\begin{array}{c}\text { Medium } \\
(10,001-26,000 \mathrm{lbs} \\
\text { average weight })\end{array}$ & $\begin{array}{c}\text { Heavy } \\
(>26,000 \text { lbs average } \\
\text { weight })\end{array}$ \\
\hline 1 & $\begin{array}{c}\text { Personal } \\
81.5 \%\end{array}$ & $\begin{array}{c}\text { Construction } \\
18.4 \%\end{array}$ & $\begin{array}{c}\text { For hire } \\
30.1 \%\end{array}$ \\
\hline 2 & $\begin{array}{c}\text { Construction } \\
4.6 \%\end{array}$ & $\begin{array}{c}\text { Agriculture } \\
16.2 \%\end{array}$ & $\begin{array}{c}\text { Construction } \\
15.9 \%\end{array}$ \\
\hline 3 & $\begin{array}{c}\text { Other services }^{\text {a }} \\
2.5 \%\end{array}$ & $\begin{array}{c}\text { For hire } \\
9.6 \%\end{array}$ & $\begin{array}{c}\text { Agriculture } \\
12.2 \%\end{array}$ \\
\hline 4 & $\begin{array}{c}\text { Not in use } \\
2.2 \%\end{array}$ & $\begin{array}{l}\text { Retail } \\
7.1 \%\end{array}$ & $\begin{array}{l}\text { Retail } \\
5.4 \%\end{array}$ \\
\hline 5 & $\begin{array}{c}\text { Agriculture } \\
1.9 \%\end{array}$ & $\begin{array}{c}\text { Not in use } \\
6.4 \%\end{array}$ & $\begin{array}{c}\text { Not in use } \\
5.1 \%\end{array}$ \\
\hline 6 & $\begin{array}{c}\text { Retail } \\
1.5 \%\end{array}$ & $\begin{array}{c}\text { Leasing } \\
6.2 \%\end{array}$ & $\begin{array}{c}\text { Waste management } \\
5.0 \%\end{array}$ \\
\hline 7 & $\begin{array}{c}\text { Unknown } \\
1.3 \%\end{array}$ & $\begin{array}{c}\text { Wholesale } \\
5.5 \%\end{array}$ & $\begin{array}{c}\text { Manufacturing } \\
4.9 \%\end{array}$ \\
\hline 8 & $\begin{array}{c}\text { Leasing } \\
0.7 \%\end{array}$ & $\begin{array}{c}\text { Waste management } \\
5.4 \%\end{array}$ & $\begin{array}{c}\text { Wholesale } \\
4.8 \%\end{array}$ \\
\hline 9 & $\begin{array}{c}\text { Manufacturing } \\
0.7 \%\end{array}$ & $\begin{array}{c}\text { Utilities } \\
5.0 \%\end{array}$ & $\begin{array}{c}\text { Leasing } \\
4.6 \%\end{array}$ \\
\hline 10 & $\begin{array}{c}\text { Utilities } \\
0.6 \%\end{array}$ & $\begin{array}{c}\text { Personal } \\
4.8 \%\end{array}$ & $\begin{array}{c}\text { Unknown } \\
3.2 \%\end{array}$ \\
\hline 11 & $\begin{array}{c}\text { Waste management } \\
0.6 \%\end{array}$ & $\begin{array}{c}\text { Unknown } \\
4.4 \%\end{array}$ & $\begin{array}{c}\text { Personal } \\
2.5 \%\end{array}$ \\
\hline 12 & $\begin{array}{c}\text { Wholesale } \\
0.6 \%\end{array}$ & $\begin{array}{c}\text { Manufacturing } \\
3.3 \%\end{array}$ & $\begin{array}{c}\text { Mining } \\
2.4 \%\end{array}$ \\
\hline 13 & $\begin{array}{c}\text { Information services } \\
0.4 \%\end{array}$ & $\begin{array}{c}\text { Other services }^{\mathrm{a}} \\
3.2 \%\end{array}$ & $\begin{array}{c}\text { Other services }^{\mathrm{a}} \\
1.3 \%\end{array}$ \\
\hline 14 & $\begin{array}{c}\text { For hire } \\
0.4 \%\end{array}$ & $\begin{array}{c}\text { Food services } \\
1.6 \%\end{array}$ & $\begin{array}{c}\text { Utilities } \\
1.1 \%\end{array}$ \\
\hline 15 & $\begin{array}{c}\text { Food services } \\
0.3 \%\end{array}$ & $\begin{array}{c}\text { Information services } \\
1.3 \%\end{array}$ & $\begin{array}{c}\text { Food services } \\
1.1 \%\end{array}$ \\
\hline 16 & $\begin{array}{l}\text { Arts } \\
0.2 \%\end{array}$ & $\begin{array}{c}\text { Mining } \\
1.1 \%\end{array}$ & $\begin{array}{l}\text { Arts } \\
0.3 \%\end{array}$ \\
\hline 17 & $\begin{array}{c}\text { Mining } \\
0.1 \%\end{array}$ & $\begin{array}{l}\text { Arts } \\
0.5 \%\end{array}$ & $\begin{array}{c}\text { Information services } \\
0.1 \%\end{array}$ \\
\hline
\end{tabular}

Source:

U.S. Department of Commerce, Bureau of the Census, 2002 Vehicle Inventory and Use Survey, Micro data File on CD, 2005. (Additional resources: www.census.gov/svsd/www/tiusview.html)

${ }^{\mathrm{a}}$ Business and personal services. 
Nearly half of trucks in fleets of 11-20 and 21-50 vehicles use company-owned facilities. Most trucks in smaller fleets use public gas stations for fueling.

Table 5.8

Percentage of Trucks by Fleet Size and Primary Fueling Facility, 2002

\begin{tabular}{lccccc}
\hline \multirow{2}{*}{ Truck fleet size } & \multicolumn{4}{c}{ Primary refueling facility } & \\
\cline { 2 - 5 } & Gas station & Truck stop & Own facility & Other's facility $^{\text {Total }^{\mathrm{a}}}$ \\
\hline $1-5$ & $73.8 \%$ & $6.1 \%$ & $18.2 \%$ & $1.9 \%$ & $100.0 \%$ \\
$6-10$ & $55.3 \%$ & $5.7 \%$ & $35.5 \%$ & $3.4 \%$ & $100.0 \%$ \\
$11-20$ & $41.1 \%$ & $5.1 \%$ & $48.9 \%$ & $4.9 \%$ & $100.0 \%$ \\
$21-50$ & $42.9 \%$ & $3.7 \%$ & $49.8 \%$ & $3.6 \%$ & $100.0 \%$ \\
51 or more & $48.3 \%$ & $6.3 \%$ & $44.4 \%$ & $1.0 \%$ & $100.0 \%$ \\
\hline Fleets of 6 or more & & & & & \\
vehicles & $47.6 \%$ & $5.2 \%$ & $43.9 \%$ & $3.4 \%$ & $100.0 \%$ \\
\hline No fleet & $\mathbf{9 6 . 4 \%}$ & $\mathbf{1 . 6 \%}$ & $\mathbf{1 . 7 \%}$ & $\mathbf{0 . 3 \%}$ & $\mathbf{1 0 0 . 0 \%}$ \\
\hline
\end{tabular}

Source:

U.S. Department of Commerce, Bureau of the Census, 2002 Vehicle Inventory and Use Survey, Microdata File on CD, 2005. (Additional resources: www.census.gov/svsd/www/tiusview.html)

${ }^{a}$ Percentages may not sum to totals due to rounding. 
Most trucks are fueled at gas stations but for-hire or warehousing trucks are more often fueled at truck stops. Mining trucks and vehicle leasing or rental trucks fuel at the companies' own facility more than $30 \%$ of the time.

Table 5.9

Share of Trucks by Major Use and Primary Fueling Facility, 2002

\begin{tabular}{|c|c|c|c|c|c|c|}
\hline Major use & $\begin{array}{c}\text { Gas } \\
\text { station }\end{array}$ & $\begin{array}{c}\text { Truck } \\
\text { stop }\end{array}$ & $\begin{array}{c}\text { Own } \\
\text { facility }\end{array}$ & $\begin{array}{l}\text { Others } \\
\text { facility }\end{array}$ & Other & $\mathrm{All}^{\mathrm{a}}$ \\
\hline Personal & $98.6 \%$ & $0.6 \%$ & $0.7 \%$ & $0.1 \%$ & $0.1 \%$ & $100.0 \%$ \\
\hline Other services & $96.0 \%$ & $1.4 \%$ & $1.6 \%$ & $0.9 \%$ & $0.1 \%$ & $100.0 \%$ \\
\hline All & $93.9 \%$ & $1.8 \%$ & $3.7 \%$ & $0.5 \%$ & $0.0 \%$ & $100.0 \%$ \\
\hline Information services & $92.3 \%$ & $0.4 \%$ & $7.2 \%$ & $0.1 \%$ & $0.0 \%$ & $100.0 \%$ \\
\hline Retail trade & $86.6 \%$ & $3.5 \%$ & $8.6 \%$ & $1.2 \%$ & $0.0 \%$ & $100.0 \%$ \\
\hline Construction & $84.7 \%$ & $3.3 \%$ & $9.8 \%$ & $2.2 \%$ & $0.0 \%$ & $100.0 \%$ \\
\hline Accommodation or food services & $82.4 \%$ & $7.5 \%$ & $8.8 \%$ & $1.3 \%$ & $0.0 \%$ & $100.0 \%$ \\
\hline Manufacturing & $81.5 \%$ & $5.1 \%$ & $11.9 \%$ & $1.5 \%$ & $0.0 \%$ & $100.0 \%$ \\
\hline Arts, entertainment, recreation services & $81.1 \%$ & $4.3 \%$ & $14.2 \%$ & $0.3 \%$ & $0.0 \%$ & $100.0 \%$ \\
\hline Waste mgmt, landscaping, admin/support services & $78.2 \%$ & $3.0 \%$ & $17.1 \%$ & $1.6 \%$ & $0.0 \%$ & $100.0 \%$ \\
\hline Wholesale trade & $76.2 \%$ & $6.6 \%$ & $12.0 \%$ & $5.1 \%$ & $0.0 \%$ & $100.0 \%$ \\
\hline Utilities & $72.6 \%$ & $1.8 \%$ & $24.3 \%$ & $1.3 \%$ & $0.0 \%$ & $100.0 \%$ \\
\hline Agriculture, forestry, fishing, hunting & $62.7 \%$ & $6.7 \%$ & $29.4 \%$ & $1.0 \%$ & $0.1 \%$ & $100.0 \%$ \\
\hline Vehicle leasing or rental & $60.2 \%$ & $1.3 \%$ & $31.8 \%$ & $6.8 \%$ & $0.0 \%$ & $100.0 \%$ \\
\hline Mining & $48.7 \%$ & $8.5 \%$ & $34.3 \%$ & $8.5 \%$ & $0.0 \%$ & $100.0 \%$ \\
\hline For-hire or warehousing & $33.3 \%$ & $38.7 \%$ & $25.8 \%$ & $2.3 \%$ & $0.0 \%$ & $100.0 \%$ \\
\hline Overall & $93.9 \%$ & $1.8 \%$ & $3.7 \%$ & $0.5 \%$ & $0.0 \%$ & $100.0 \%$ \\
\hline
\end{tabular}

Source:

U.S. Department of Commerce, Bureau of the Census, 2002 Vehicle Inventory and Use Survey, Microdata File on CD, 2005. (Additional resources: www.census.gov/svsd/www/tiusview.html)

${ }^{a}$ Percentages may not sum to totals due to rounding. 
The figure below shows the distribution of annual travel the two types of Class 7 and 8 vehicles-combination units (separate tractor and trailer) and single units (tractor and trailer on a single chassis). This information is for all trucks and trucks two years old or less. Combination trucks, dominated by box-type trailers, display the greatest amount of annual travel of all heavy vehicle types, as is evidenced both by the range of annual use. Most of the single-unit trucks in the survey travel 40,000 miles per year or less.

Figure 5.1. Distribution of Trucks over 26,000 lbs. by Vehicle-Miles Traveled

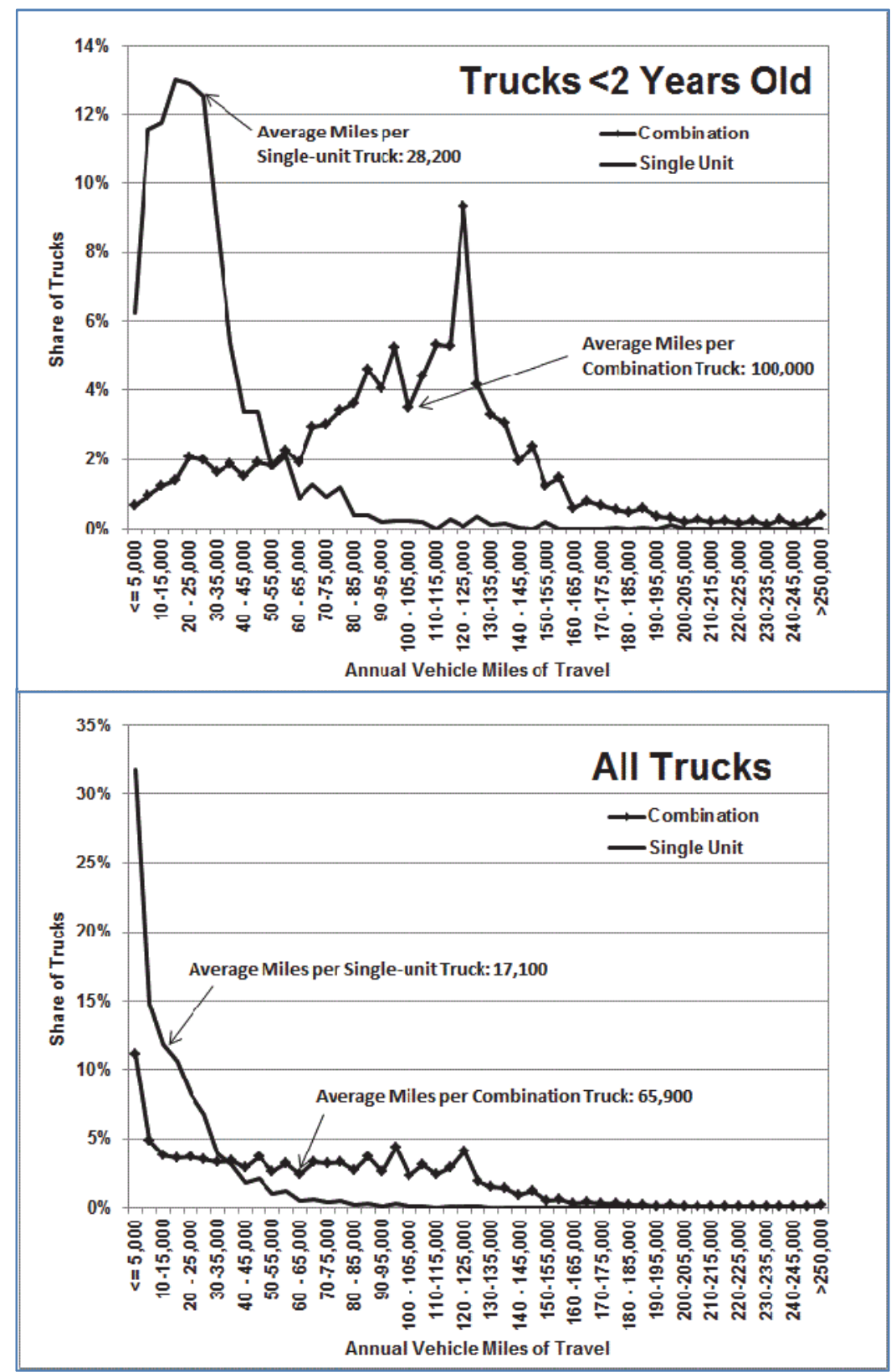

Note: Heavy trucks (class $7 \& 8$ ) are greater than 26,000 pounds gross vehicle weight based on the manufacturer's rating.

\section{Source:}

U.S. Department of Commerce, Bureau of the Census, 2002 Vehicle Inventory and Use Survey, Microdata File on CD, 2005. (Additional resources: www.census.gov/svsd/www/tiusview.html) 
The latest Vehicle Inventory and Use Survey asked truck owners if the truck had certain features as permanent equipment on the truck. Some of the features asked about were onboard computers, idle-reduction devices, navigational systems, and Internet access. Of the 2.3 million heavy trucks (class 7 \& 8) in the United States, nearly $10 \%$ were equipped with onboard computers that had communication capabilities and another $5 \%$ had onboard computers without communication capabilities. Six percent of heavy trucks were equipped with idlereducing technology. Navigational systems and Internet access were available in less than one percent of heavy trucks.

Figure 5.2. Share of Heavy Trucks with Selected Electronic Features, 2002

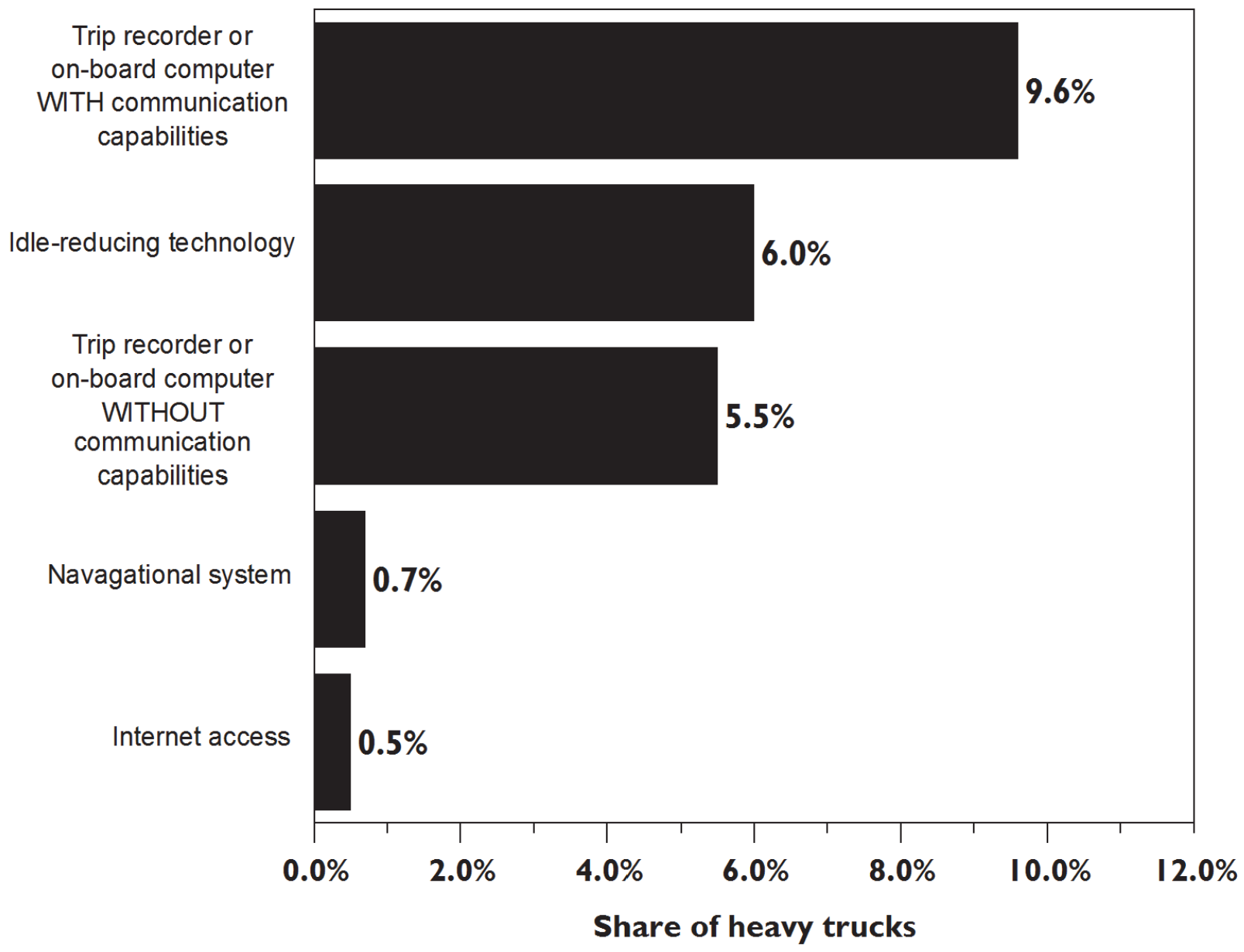

Note: Heavy trucks (class $7 \& 8$ ) are greater than 26,000 pounds gross vehicle weight based on the manufacturer's rating.

\section{Source:}

U.S. Department of Commerce, Bureau of the Census, 2002 Vehicle Inventory and User Survey, Microdata File on $\mathrm{CD}, 2005$. 


\section{Fuel Economy Study for Class 8 Trucks}

As part of a long-term study sponsored by the U.S. Department of Energy (DOE) Office of Vehicle Technologies (OVT), the Oak Ridge National Laboratory (ORNL) in conjunction with several industry partners has collected data and information related to heavy-truck operation in real-world highway environments. The primary objective of the project was to collect real-world performance and spatial data for long-haul operations of Class 8 tractor-trailers from a fleet engaged in normal freight operations. Six model year 2005 Class 8 trucks from the selected fleet, which operates within a large area of the country extending from the east coast to Mountain Time Zone and from Canada to the US-Mexican border, were instrumented and 60 channels of data were collected for over a year at a rate of $5 \mathrm{~Hz}$ (or 5 readings per second). Those channels included information such as instantaneous fuel rate, engine speed, gear ratio, vehicle speed, and other information read from the vehicle's databus; weather information (wind speed, precipitation, air temperature, etc.) gathered from an on-board weather station; spatial information (latitude, longitude, altitude) acquired from a GPS (Global Positioning System) device; and instantaneous tractor and trailer weight obtained from devices mounted on the six participating tractors and ten trailers. Three of the six instrumented tractors and five of the ten instrumented trailers were mounted with New Generation Single Wide-Based Tires and the others with regular dual tires. Over the duration of this phase of the project (just over a year) the six tractors traveled nearly 700,000 miles.

To find out more about this project, contact Oscar Franzese, franzeseo@ornl.gov, 865-946-1304. The final report on this project is available on-line at: cta.ornl.gov/cta/Publications/Reports/ORNL_TM_2008-122.pdf. 
The type of terrain a truck is traveling on can cause significant differences in fuel efficiency. This study (see page 5-13 for project description) shows fuel economy on severe upslopes is less than half that on flat terrain. On severe downslopes, the fuel economy was two times higher than on flat terrain.

Table 5.10

Effect of Terrain on Class 8 Truck Fuel Economy

\begin{tabular}{lccccc}
\hline & & \multicolumn{3}{c}{ Average fuel efficiency (mpg) } \\
\cline { 3 - 6 } & & & Tractors & $\begin{array}{c}\text { Tractors } \\
\text { with single }\end{array}$ & $\begin{array}{c}\text { Difference } \\
\text { between dual } \\
\text { and single }\end{array}$ \\
Type of terrain & $\begin{array}{c}\text { Share of data } \\
\text { records }\end{array}$ & $\begin{array}{c}\text { All } \\
\text { trucks }\end{array}$ & $\begin{array}{c}\text { with dual } \\
\text { tires }\end{array}$ & $\begin{array}{c}\text { (wide) tires } \\
\text { tires (percent) }\end{array}$ \\
\hline Severe upslope (>4\%) & $0.7 \%$ & 2.90 & 2.86 & 2.94 & $2.91 \%$ \\
Mild upslope (1\% to 4\%) & $13.2 \%$ & 4.35 & 4.25 & 4.44 & $4.35 \%$ \\
Flat terrain (1\% to 1\%) & $72.4 \%$ & 7.33 & 7.08 & 7.58 & $7.13 \%$ \\
Mild downslope (-4\% to -1\%) & $12.6 \%$ & 15.11 & 14.64 & 15.57 & $6.36 \%$ \\
Severe downslope (<-4\%) & $1.1 \%$ & 23.5 & 21.82 & 25.3 & $15.97 \%$ \\
\hline
\end{tabular}

Source:

Capps, Gary, Oscar Franzese, Bill Knee, M.B. Lascurain, and Pedro Otaduy. Class-8 Heavy Truck Duty Cycle Project Final Report, ORNL/TM-2008/122, Oak Ridge National Laboratory, Oak Ridge, TN, December 2008. (Additional resources: cta.ornl.gov/cta/Publications/Reports/ORNL_TM_2008-122.pdf) 
This table presents a distribution of distance traveled, fuel consumed, and fuel economy by speed and by type of tires for the vehicles participating in the project (see page 5-13 for project description). The speed bins are divided into 5-mile intervals, going from 0+ mph (i.e., speed $>0.00 \mathrm{mph}$ ) to $85 \mathrm{mph}$, while the four main columns of the table are organized by the type of tires that were mounted on the tractor and trailers. The first row of the table contains information about fuel consumed while the vehicle was idling (i.e., the vehicle was static with the engine on) with the following rows presenting information about the distance traveled, fuel consumed, and fuel economy for each one of the speed intervals. The next-to-the-last row shows the totals for both traveled distances and fuel consumed as well as the overall fuel economy for each tire-combination category. The latter are then used to compute the percentage difference in terms of fuel economy from dual tire tractors and trailers, which is the most common tire setup for large trucks at the present time.

Table 5.11

Fuel Economy for Class 8 Trucks as Function of Speed and Tractor-Trailer Tire Combination

\begin{tabular}{|c|c|c|c|c|c|c|c|c|c|c|c|c|}
\hline \multirow[b]{2}{*}{$\begin{array}{l}\text { Speed } \\
(\mathrm{mph})\end{array}$} & \multicolumn{3}{|c|}{$\begin{array}{l}\text { Dual tire tractor - } \\
\text { dual tire trailer }\end{array}$} & \multicolumn{3}{|c|}{$\begin{array}{c}\text { Dual tire tractor - } \\
\text { single (wide) tire trailer }\end{array}$} & \multicolumn{3}{|c|}{$\begin{array}{c}\text { Single (wide) tire tractor - } \\
\text { dual tire trailer }\end{array}$} & \multicolumn{3}{|c|}{$\begin{array}{l}\text { Single (wide) tire tractor - } \\
\text { single (wide) tire trailer }\end{array}$} \\
\hline & $\begin{array}{c}\text { Distance } \\
\text { traveled } \\
\text { (miles) }\end{array}$ & $\begin{array}{l}\text { Fuel } \\
\text { cons. } \\
\text { (gal) }\end{array}$ & $\begin{array}{c}\text { Fuel } \\
\text { econ. } \\
\text { (MPG) }\end{array}$ & $\begin{array}{c}\text { Distance } \\
\text { traveled } \\
\text { (miles) }\end{array}$ & $\begin{array}{l}\text { Fuel } \\
\text { cons. } \\
\text { (gal) }\end{array}$ & $\begin{array}{c}\text { Fuel } \\
\text { econ. } \\
\text { (MPG) }\end{array}$ & $\begin{array}{c}\text { Distance } \\
\text { traveled } \\
\text { (miles) }\end{array}$ & $\begin{array}{l}\text { Fuel } \\
\text { cons. } \\
\text { (gal) }\end{array}$ & $\begin{array}{c}\text { Fuel } \\
\text { econ. } \\
\text { (MPG) }\end{array}$ & $\begin{array}{c}\text { Distance } \\
\text { traveled } \\
\text { (miles) }\end{array}$ & $\begin{array}{l}\text { Fuel } \\
\text { cons. } \\
\text { (gal) }\end{array}$ & $\begin{array}{l}\text { Fuel } \\
\text { econ. } \\
\text { (MPG) }\end{array}$ \\
\hline Idling & N/A & $1,858.5$ & N/A & N/A & 967.9 & N/A & N/A & $1,676.4$ & N/A & N/A & 706.0 & N/A \\
\hline $0+$ to 5 & 281 & 101.8 & 2.76 & 148 & 50.4 & 2.93 & 368.0 & 124.2 & 3.0 & 156 & 52.8 & 2.96 \\
\hline $5+$ to 10 & 674 & 198.8 & 3.39 & 368 & 103.2 & 3.56 & 808.0 & 245.4 & 3.3 & 331 & 98.8 & 3.35 \\
\hline $10+$ to 15 & 723 & 192.0 & 3.77 & 396 & 98.3 & 4.03 & 848.0 & 216.5 & 3.9 & 343 & 87.0 & 3.95 \\
\hline $15+$ to 20 & 744 & 199.1 & 3.73 & 404 & 100.9 & 4.00 & 882.0 & 221.6 & 4.0 & 361 & 90.5 & 3.98 \\
\hline $20+$ to 25 & 938 & 228.4 & 4.11 & 489 & 113.6 & 4.31 & $1,111.0$ & 244.2 & 4.6 & 462 & 101.1 & 4.57 \\
\hline $25+$ to 30 & 1,178 & 266.9 & 4.41 & 609 & 131.5 & 4.63 & $1,420.0$ & 286.9 & 5.0 & 580 & 117.6 & 4.93 \\
\hline $30+$ to 35 & 1,481 & 336.8 & 4.40 & 753 & 154.2 & 4.88 & $1,774.0$ & 341.1 & 5.2 & 708 & 141.1 & 5.02 \\
\hline $35+$ to 40 & 1,917 & 403.5 & 4.75 & 1,000 & 193.6 & 5.17 & $2,284.0$ & 433.6 & 5.3 & 941 & 184.3 & 5.10 \\
\hline $40+$ to 45 & 2,955 & 584.1 & 5.06 & 1,543 & 285.9 & 5.40 & $3,380.0$ & 603.6 & 5.6 & 1,350 & 254.4 & 5.31 \\
\hline $45+$ to 50 & 4,935 & 907.9 & 5.43 & 2,573 & 447.7 & 5.75 & $5,410.0$ & 872.8 & 6.2 & 2,177 & 360.4 & 6.04 \\
\hline $50+$ to 55 & 9,397 & $1,629.8$ & 5.77 & 4,962 & 811.5 & 6.11 & $10,046.0$ & $1,622.7$ & 6.2 & 3,877 & 625.5 & 6.20 \\
\hline $55+$ to 60 & 20,656 & $3,297.2$ & 6.26 & 11,707 & $1,721.9$ & 6.80 & $22,373.0$ & $3,257.8$ & 6.9 & 8,710 & $1,246.9$ & 6.99 \\
\hline $60+$ to 65 & 38,964 & $5,879.6$ & 6.63 & 21,472 & $2,980.8$ & 7.20 & $34,517.0$ & $4,840.0$ & 7.1 & 14,944 & $2,049.4$ & 7.29 \\
\hline \multicolumn{13}{|c|}{ NOT ADJUSTED FOR TERRAIN: See note below. } \\
\hline $65+$ to 70 & 58,304 & $8,313.2$ & 7.01 & 27,931 & $3,652.2$ & 7.65 & $65,063.0$ & $9,256.4$ & 7.0 & 27,144 & $3,880.1$ & 7.00 \\
\hline $70+$ to 75 & 56,378 & $7,483.2$ & 7.53 & 21,751 & $2,745.5$ & 7.92 & $66,882.0$ & $8,435.6$ & 7.9 & 32,887 & $4,056.1$ & 8.11 \\
\hline $75+$ to 85 & 7,849 & 808.2 & 9.71 & 3,610 & 403.2 & 8.95 & $11,513.0$ & 911.1 & 12.6 & 6,817 & 512.2 & 13.31 \\
\hline Total $^{\mathrm{a}}$ & 207,374 & $30,831.0$ & 6.73 & 99,714 & $13,994.0$ & 7.13 & $228,680.0$ & $31,913.0$ & 7.2 & 101,790 & $13,858.0$ & 7.35 \\
\hline $\begin{array}{l}\text { Percent } \\
\text { increase in } \\
\text { fuel } \\
\text { economy } \\
\text { from dual } \\
\text { tire } \\
\text { trac/trail }\end{array}$ & & & $0.00 \%$ & & & $5.93 \%$ & & & $6.53 \%$ & & & $9.20 \%$ \\
\hline
\end{tabular}

Note: These data were not adjusted to account for the effects of terrain. The increase in fuel economy for speeds above $70 \mathrm{mph}$ is likely due to the vehicle achieving high speeds while traveling down slope. Therefore, this increase in fuel economy is not expected to be characteristic of all travel at these higher speeds.

\section{Source:}

Capps, Gary, Oscar Franzese, Bill Knee, M.B. Lascurain, and Pedro Otaduy. Class- 8 Heavy Truck Duty Cycle Project Final Report, ORNL/TM-2008/122, Oak Ridge National Laboratory, Oak Ridge, TN, December 2008. (Additional resources: cta.ornl.gov/cta/Publications/Reports/ORNL_TM_2008-122.pdf)

${ }^{\text {a }}$ Total fuel consumed does not include fuel consumed while idling. 
The fuel economy information presented in Table 5.11 is on the upper limits of today's large-truck fleets and is mostly a result of driver training and the extensive vehicle maintenance (including constant tire pressure) to which the fleet company participating in this project adheres. Nevertheless, the results of this extensive test indicate that there are substantial gains in terms of fuel economy for large trucks when single (wide) tires are used in combination with dual tires or alone (best case). Figure 5.3 shows the information from Table 5.11 in a graphical form (bars) and also displays for each speed bin the percentage of the total distance that is traveled at that speed (line). It is possible to observe that above $80 \%$ of the distance traveled by long-haul Class 8 trucks is done at speeds above $55 \mathrm{mph}$. Therefore, any gains in fuel economies at these speeds derived from a given tire combination would have a very large impact on the overall fuel economy of these types of trucks. Figure 5.3 shows that, except for the D-S combination within the $65+$ to $70 \mathrm{mph}$, the combinations with all single (wide) tires perform better and, therefore, obtain the largest overall fuel economy.

Figure 5.3. Class 8 Truck Fuel Economy as a Function of Speed and Tractor-Trailer Tire Combination and Percentage of Total Distance Traveled as a Function of Speed

NOT ADJUSTED FOR TERRAIN: See note below.

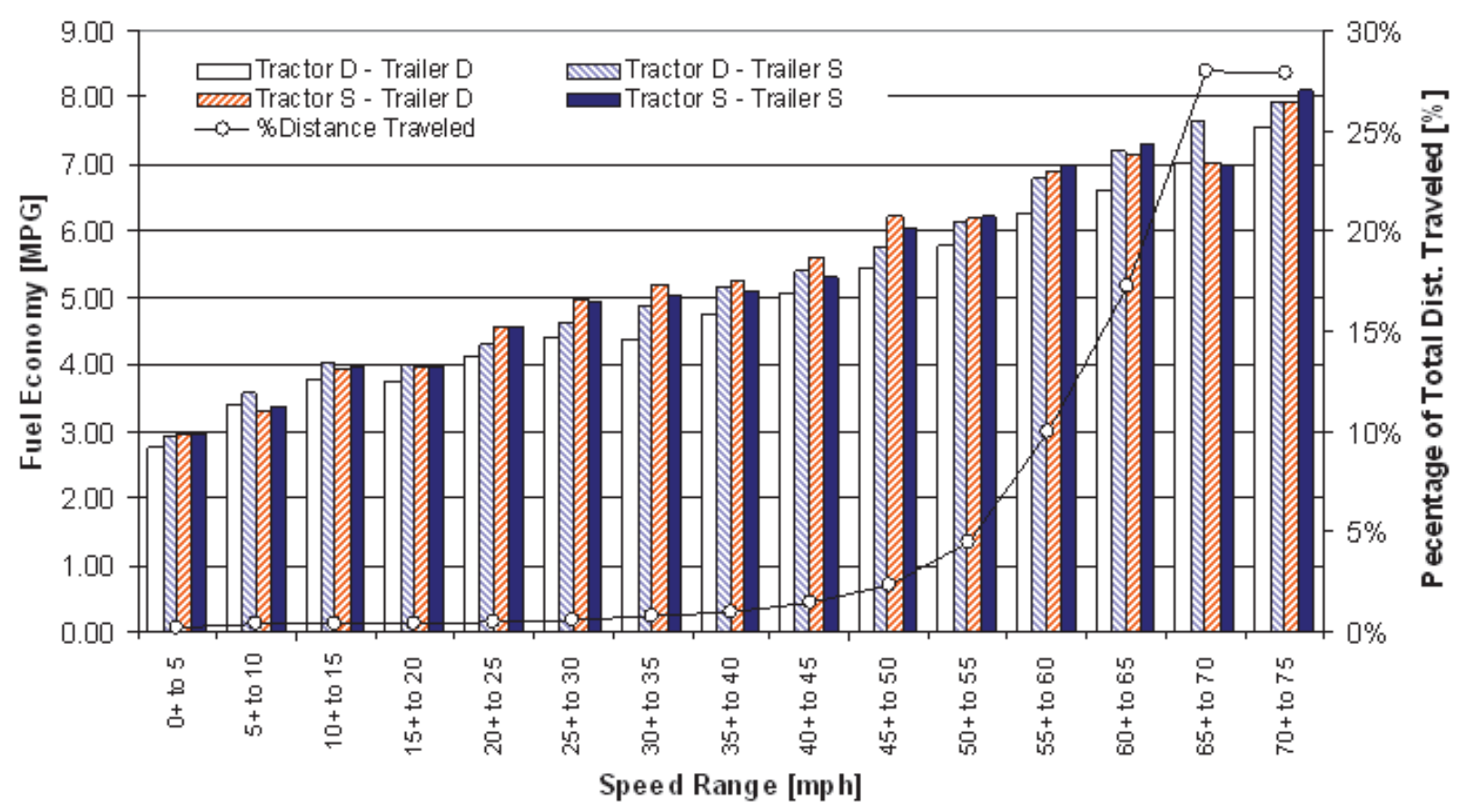

Note: $\mathrm{D}=$ Dual tire. $\mathrm{S}=$ Single (wide) tire.

These data were not adjusted to account for the effects of terrain. The increase in fuel economy for speeds above 70 mph is likely due to the vehicle achieving high speeds while traveling down slope. Therefore, this increase in fuel economy is not expected to be characteristic of all travel at these higher speeds.

Source:

Capps, Gary, Oscar Franzese, Bill Knee, M.B. Lascurain, and Pedro Otaduy. Class-8 Heavy Truck Duty Cycle Project Final Report, ORNL/TM-2008/122, Oak Ridge National Laboratory, Oak Ridge, TN, December 2008. 
This graph presents for each one of the four tire-combination categories the percent of total fuel that is consumed when traveling at different speeds (bars) as well as the average percent of fuel consumed for each speed bin (line). As opposed to Table 5.11, the total fuel consumed on this graph includes the fuel consumed while idling.

\section{Figure 5.4. Class 8 Truck Percent of Total Fuel Consumed as a Function of Speed and Tractor-Trailer Tire Combination}

\section{NOT ADJUSTED FOR TERRAIN: See note below}

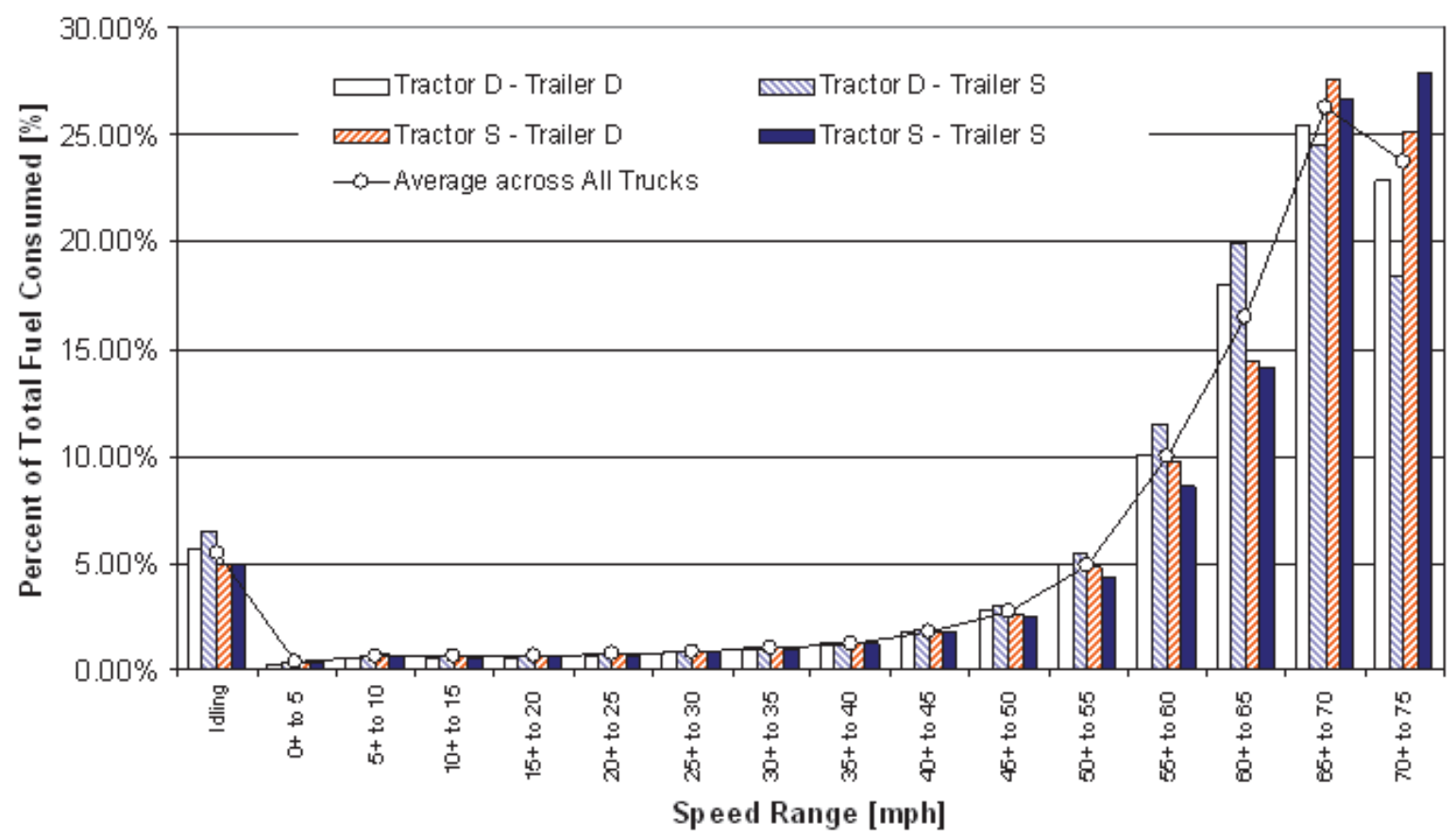

Note: $\mathrm{D}=$ Dual tire. $\mathrm{S}=$ Single (wide) tire.

These data were not adjusted to account for the effects of terrain. The increase in fuel economy for speeds above 70 mph is likely due to the vehicle achieving high speeds while traveling down slope. Therefore, this increase in fuel economy is not expected to be characteristic of all travel at these higher speeds.

\section{Source:}

Capps, Gary, Oscar Franzese, Bill Knee, M.B. Lascurain, and Pedro Otaduy. Class-8 Heavy Truck Duty Cycle Project Final Report, ORNL/TM-2008/122, Oak Ridge National Laboratory, Oak Ridge, TN, December 2008. 
A typical class 8 truck tractor weighs about 17,000 lbs. The powertrain is nearly a quarter of the weight (24\%) while the truck body structure is $19 \%$.

Table 5.12

Class 8 Truck Weight by Component

\begin{tabular}{lrc}
\hline & Pounds & Share of total \\
\hline Wheels and tires & 1,700 & $10 \%$ \\
Chassis/frame & 2,040 & $12 \%$ \\
Drivetrain and suspension & 2,890 & $17 \%$ \\
Misc. accessories/systems & 3,060 & $18 \%$ \\
Truck body structure & 3,230 & $19 \%$ \\
Powertrain & 4,080 & $24 \%$ \\
\hline Total & 17,000 & $100 \%$ \\
\hline
\end{tabular}

Notes:

- Powertrain includes engine and cooling system, transmission and accessories.

- Truck body structure includes cab-in-white, sleeper unit, hood and fairings, interior and glass.

- Miscellaneous accessories/systems include batteries, fuel system, and exhaust hardware.

- Drivetrain and suspension includes drive axles, steer axle, and suspension system.

- Chassis/frame includes frame rails and crossmembers, fifth wheel and brackets. Wheels and tires include a set of 10 aluminum wheels, plus tires.

\section{Source:}

National Academy of Sciences, Technologies and Approaches to Reducing the Fuel Consumption of Medium and Heavy-Duty Vehicles, prepublication copy, March 2010, p. 5-42. 
The gross weight of a vehicle $(G V W)$ is the weight of the empty vehicle plus the weight of the maximum payload that the vehicle was designed to carry. In cars and small light trucks, the difference between the empty weight of the vehicle and the GVW is not significantly different (1,000 to 1,500 lbs). The largest trucks and tractor-trailers, however, have a payload capacity share of $200 \%$, which means they can carry $200 \%$ of their empty weight. The medium-sized trucks (truck classes 3-6) have payload capacity shares between 50\% and 100\%.

Table 5.13

Gross Vehicle Weight vs. Empty Vehicle Weight

\begin{tabular}{|c|c|c|c|c|c|}
\hline Vehicle description & Truck class & $\begin{array}{l}\text { Gross vehicle } \\
\text { weight range } \\
\text { (pounds) }\end{array}$ & $\begin{array}{l}\text { Empty vehicle } \\
\text { weight range } \\
\text { (pounds) }\end{array}$ & $\begin{array}{l}\text { Maximum } \\
\text { payload } \\
\text { capacity } \\
\text { (pounds) }\end{array}$ & $\begin{array}{c}\text { Payload } \\
\text { capacity share } \\
\text { (percent of } \\
\text { empty weight) }\end{array}$ \\
\hline Cars & & $3,200-6,000$ & $2,400-5,000$ & 1,000 & $20 \%$ \\
\hline $\begin{array}{l}\text { Minivans, small SUVs, } \\
\text { small pick-ups }\end{array}$ & 1 & $4,000-2,400$ & $3,200-4,500$ & 1,500 & $33 \%$ \\
\hline $\begin{array}{l}\text { Large SUVs, standard pick- } \\
\text { ups }\end{array}$ & $2 \mathrm{a}$ & $6,001-8,500$ & $4,500-6,000$ & 2,500 & $40 \%$ \\
\hline $\begin{array}{l}\text { Large SUVs, standard pick- } \\
\text { ups }\end{array}$ & $2 b$ & $8,501-10,000$ & $5,000-6,300$ & 3,700 & $60 \%$ \\
\hline $\begin{array}{l}\text { Utility van, multi- purpose, } \\
\text { mini-bus, step van }\end{array}$ & 3 & $10,001-14,000$ & $7,650-8,750$ & 5,250 & $60 \%$ \\
\hline $\begin{array}{l}\text { City delivery, parcel } \\
\text { delivery, large walk-in, } \\
\text { bucket, landscaping }\end{array}$ & 4 & $14,001-16,000$ & $7,650-8,750$ & 7,250 & $80 \%$ \\
\hline $\begin{array}{l}\text { City delivery, parcel } \\
\text { delivery, large walk-in, } \\
\text { bucket }\end{array}$ & 5 & $16,001-19,500$ & $9,500-10,000$ & 8,700 & $80 \%$ \\
\hline $\begin{array}{l}\text { City delivery, school bus, } \\
\text { large walk-in, bucket }\end{array}$ & 6 & $19,501-26,000$ & $11,500-14,500$ & 11,500 & $80 \%$ \\
\hline $\begin{array}{l}\text { City bus, furniture, } \\
\text { refrigerated, refuse, fuel } \\
\text { tanker, dump, tow, concrete, } \\
\text { fire engine, tractor-trailer }\end{array}$ & 7 & $26,001-33,000$ & $11,500-14,500$ & 18,500 & $125 \%$ \\
\hline $\begin{array}{l}\text { Refuse, concrete, furniture, } \\
\text { city bus, tow, fire engine } \\
\text { (straight trucks) }\end{array}$ & $8 a$ & $33,001-80,000$ & $20,000-26,000$ & 54,000 & $200 \%$ \\
\hline $\begin{array}{l}\text { Tractor-trailer: van, } \\
\text { refrigerated, bulk tanker, flat } \\
\text { bed (combination trucks) }\end{array}$ & $8 b$ & $33,001-80,000$ & $20,000-26,000$ & 54,000 & $200 \%$ \\
\hline
\end{tabular}

\section{Source:}

National Academy of Sciences, Technologies and Approaches to Reducing the Fuel Consumption of Medium and Heavy-Duty Vehicles, prepublication copy, March 2010, pp. 2-2 and 5-42. 
According to weigh-in-motion data collected by fifteen states, the majority of 5-axle tractor-trailers on the road weigh between 33,000 and 73,000 lbs. Eleven percent of the tractor-trailers had weight recorded around 72,800 $\mathrm{lbs}$ and $10 \%$ around 68,300 lbs. Another 10\% of tractor-trailers were on the lighter end of the scale - around 37,500 lbs. These data show that only a small percent of trucks on the road are near the maximum roadway gross vehicle weight of $80,000 \mathrm{lbs}$. Thus, most trucks are filling the trailer space to capacity (cubing-out) before they reach the maximum weight limit (weighing-out).

Figure 5.5. Distribution of Class 8 Trucks by On-Road Vehicle Weight, 2008

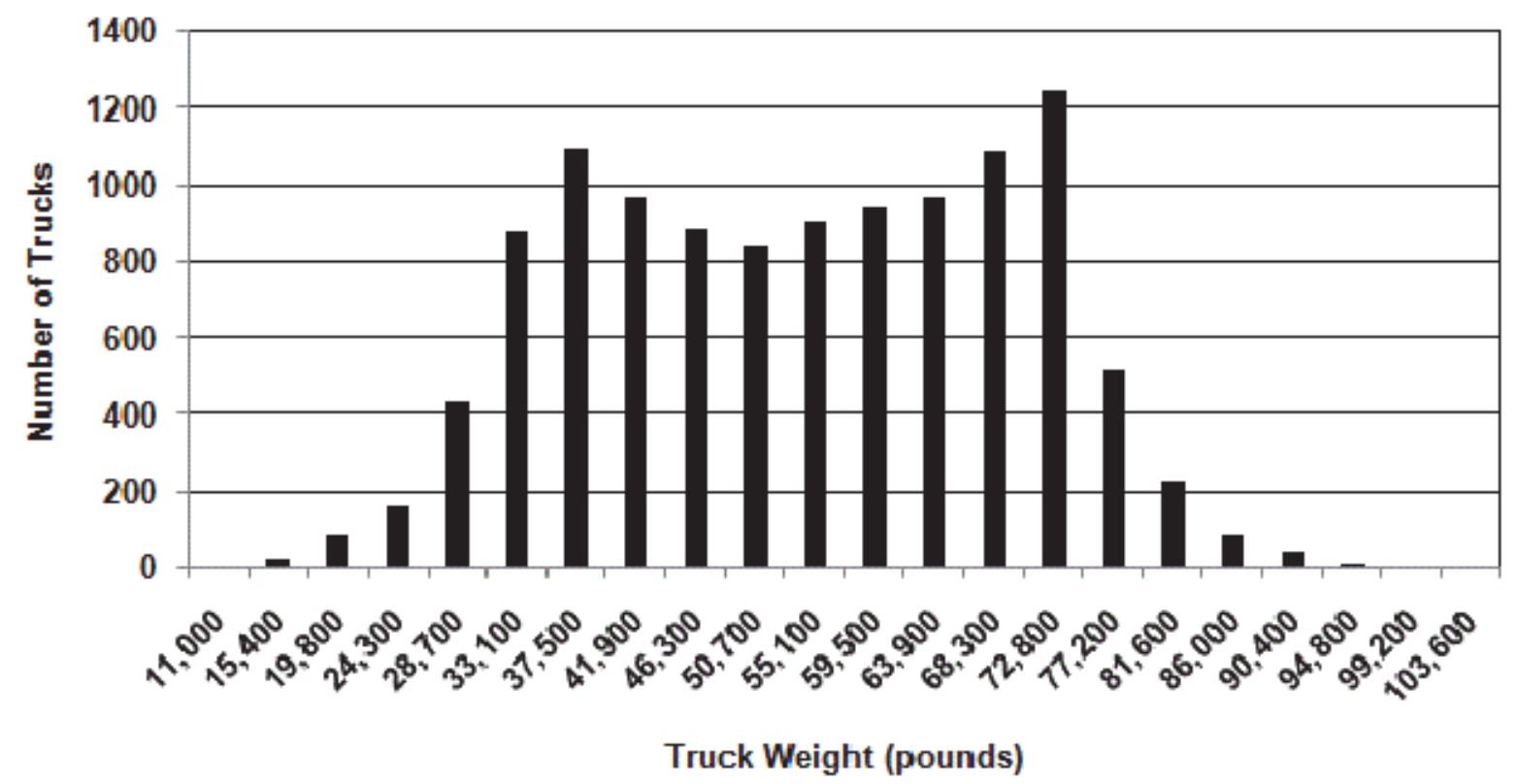

Note: Data are from these 15 States: California, Connecticut, Florida, Georgia, Hawaii, Iowa, Minnesota, Missouri, Montana, North Carolina, Oregon, Pennsylvania, South Dakota, Texas, and Washington.

\section{Source:}

National Academy of Sciences, Technologies and Approaches to Reducing the Fuel Consumption of Medium and Heavy-Duty Vehicles, prepublication copy, March 2010, p. 5-45. Original source: Federal Highway Administration, Vehicle Travel Information System, 2008.

${ }^{\text {a }}$ Study reported data on 5-axle tractor-trailers which are class 8 trucks. Single-unit class 8 trucks were not considered in the study. 


\section{Commodity Flow Survey}

The Commodity Flow Survey (CFS) is designed to provide data on the flow of goods and materials by mode of transport. The 1993, 1997, 2002, 2007 and 2012 CFS are a continuation of statistics collected in the Commodity Transportation Survey from 1963 through 1977, and include major improvements in methodology, sample size, and scope. The 2012 CFS covers business establishments with paid employees that are located in the United States and are classified using the North American Industry Classification System (NAICS) in mining, manufacturing, wholesale trade, and select retail and services establishments. Establishments classified in services, transportation, construction, and most retail and services industries are excluded from the survey. Farms, fisheries, foreign establishments, and most government-owned establishments are also excluded.

The 1993, 1997, 2002, 2007 and 2012 CFS differ from previous surveys in their greatly expanded coverage of intermodalism (i.e., shipments which travel by at least two different modes, such as rail and truck). Earlier surveys reported only the principal mode.

The data can be viewed at: www.bts.gov/publications/commodity_flow_survey. 
Industries covered by the 2012 Commodity Flow Survey (CFS) shipped over 11 billion tons of goods worth over $\$ 13$ trillion. Compared to the 1997 CFS, the value of shipments is up $2.1 \%$ per year and tons shipped are up 0.4\% per year. By value, intermodal shipments increased 2.1\% per year from 1997 to 2012.

Table 5.14

Value of Goods Shipped in the United States: Comparison of the 1997, 2002, 2007 and 2012 Commodity Flow Surveys ${ }^{\mathrm{a}}$

\begin{tabular}{|c|c|c|c|c|c|}
\hline & $\begin{array}{c}1997 \\
\text { (billion } \\
\text { 2012 dollars) }\end{array}$ & $\begin{array}{c}2002 \\
\text { (billion } \\
\text { 2012 dollars) }\end{array}$ & $\begin{array}{c}2007 \\
\text { (billion } \\
\text { 2012 dollars) }\end{array}$ & $\begin{array}{l}2012 \\
\text { (billion } \\
\text { dollars) }\end{array}$ & $\begin{array}{c}\text { Average } \\
\text { annual } \\
\text { percent } \\
\text { change } \\
(1997-2012)\end{array}$ \\
\hline All modes & $9,933.3$ & $10,716.8$ & $12,938.9$ & $13,625.1$ & $2.1 \%$ \\
\hline Single modes & $8,181.8$ & $8,996.6$ & $10,562.8$ & $11,778.8$ & $2.5 \%$ \\
\hline Truck $^{b}$ & $7,126.0$ & $7,957.3$ & $9,230.4$ & $10,038.1$ & $2.3 \%$ \\
\hline For-hire truck & $4,150.4$ & $4,794.9$ & $5,487.5$ & $6,609.3$ & $3.2 \%$ \\
\hline Private truck & $2,913.2$ & $3,120.8$ & $3,742.8$ & $3,428.8$ & $1.1 \%$ \\
\hline Rail & 457.2 & 396.8 & 483.3 & 455.1 & $0.0 \%$ \\
\hline Water & 108.5 & 114.0 & 127.2 & 280.9 & $6.5 \%$ \\
\hline Inland water & 77.1 & 73.3 & 100.8 & 202.2 & $6.6 \%$ \\
\hline Great Lakes & 2.2 & 1.1 & $\mathrm{c}$ & 0.4 & $-10.7 \%$ \\
\hline Deep sea & 29.2 & 39.6 & 25.5 & 55.0 & $4.3 \%$ \\
\hline Multiple waterways & d & d & d & 23.2 & d \\
\hline Air (includes truck and air) & 327.7 & 338.1 & 279.4 & 397.4 & $1.3 \%$ \\
\hline Pipeline $^{\mathrm{e}}$ & 162.4 & 190.4 & 442.5 & 607.2 & $9.7 \%$ \\
\hline Multiple modes & $1,353.1$ & $1,377.3$ & $2,067.1$ & $1,844.2$ & $2.1 \%$ \\
\hline Parcel, U.S.P.S. or courier & $1,224.4$ & $1,260.6$ & $1,729.5$ & $1,578.3$ & $1.7 \%$ \\
\hline Truck and rail & 108.3 & 89.2 & 207.3 & 230.5 & $5.2 \%$ \\
\hline Truck and water & 11.8 & 18.3 & 64.7 & 27.8 & $5.9 \%$ \\
\hline Rail and water & 2.5 & 4.2 & 15.4 & 7.4 & $7.5 \%$ \\
\hline Other multiple modes & 6.1 & 4.9 & 50.2 & $\mathrm{c}$ & d \\
\hline Other and unknown modes & 398.5 & 342.8 & 309.1 & 2.0 & $-29.7 \%$ \\
\hline
\end{tabular}

Source:

U.S. Department of Transportation, Bureau of Transportation Statistics and U.S. Department of Commerce, Bureau of the Census, 2012 Commodity Flow Survey, Table 1a, (1997-2007) and Table 1 (2012-preliminary). (Additional resources: www.rita.dot.gov/bts/sites/rita.dot.gov.bts/files/publications/commodity_flow_survey/ index.html)

${ }^{a}$ Detail may not add to total because of rounding.

b "Truck" as a single mode includes shipments which went by private truck only, for-hire truck only, or a combination of private truck and for-hire truck.

${ }^{c}$ Denotes data do not meet publication standards because of high sampling variability or poor response quality.

${ }^{\mathrm{d}}$ Data are not available.

${ }^{\mathrm{e}}$ CFS data for pipeline exclude most shipments of crude oil. 
Table 5.15

Tons of Freight in the United States: Comparison of the 1997, 2002, 2007 and 2012 Commodity Flow Surveys ${ }^{\mathrm{a}}$

\begin{tabular}{|c|c|c|c|c|c|}
\hline & $\begin{array}{c}1997 \\
\text { (millions) }\end{array}$ & $\begin{array}{c}2002 \\
\text { (millions) }\end{array}$ & $\begin{array}{c}2007 \\
\text { (millions) }\end{array}$ & $\begin{array}{c}2012 \\
\text { (millions) }\end{array}$ & $\begin{array}{c}\text { Average } \\
\text { annual } \\
\text { percent } \\
\text { change } \\
(1997-2012)\end{array}$ \\
\hline All modes & $11,089.7$ & $11,667.9$ & $12,543.4$ & $11,695.5$ & $0.4 \%$ \\
\hline Single modes & $10,436.5$ & $11,086.7$ & $11,698.1$ & $11,276.8$ & $0.5 \%$ \\
\hline Truck $^{\mathrm{b}}$ & $7,700.7$ & $7,842.8$ & $8,778.7$ & $8,190.1$ & $0.4 \%$ \\
\hline For-hire truck & $3,402.6$ & $3,657.3$ & $4,075.1$ & $4,390.8$ & $1.7 \%$ \\
\hline Private truck & $4,137.3$ & $4,149.7$ & $4,703.6$ & $3,799.3$ & $-0.6 \%$ \\
\hline Rail & $1,549.8$ & $1,873.9$ & $1,861.3$ & $1,851.3$ & $1.2 \%$ \\
\hline Water & 563.4 & 681.2 & 403.6 & 510.7 & $-0.7 \%$ \\
\hline Inland water & 414.8 & 458.6 & 343.3 & 374.2 & $-0.7 \%$ \\
\hline Great Lakes & 38.4 & 38.0 & 17.8 & 23.5 & $-3.2 \%$ \\
\hline Deep sea & 110.2 & 184.6 & 42.5 & 63.3 & $-3.6 \%$ \\
\hline Multiple waterways & $\mathrm{c}$ & $\mathrm{c}$ & c & 49.7 & \\
\hline Air (includes truck and air) & 4.5 & 3.8 & 3.6 & 4.7 & $0.3 \%$ \\
\hline Pipeline $^{\mathrm{d}}$ & 618.2 & 685.0 & 650.9 & 720.1 & $1.0 \%$ \\
\hline Multiple modes & 216.7 & 216.7 & $\mathbf{5 7 3 . 7}$ & 347.8 & $3.2 \%$ \\
\hline Parcel, U.S.P.S. or courier & 23.7 & 25.5 & 33.9 & 26.7 & $0.8 \%$ \\
\hline Truck and rail & 54.2 & 43.0 & 225.6 & 224.3 & $9.9 \%$ \\
\hline Truck and water & 33.2 & 23.3 & 145.5 & 48.3 & $2.5 \%$ \\
\hline Rail and water & 79.3 & 105.1 & 54.9 & 48.2 & $-3.3 \%$ \\
\hline Other multiple modes & 26.2 & 19.8 & 113.8 & e & \\
\hline Other and unknown modes & 436.5 & 364.6 & 271.6 & 70.9 & $-11.4 \%$ \\
\hline
\end{tabular}

Source:

U.S. Department of Transportation, Bureau of Transportation Statistics and U.S. Department of Commerce, Bureau of the Census, 2012 Commodity Flow Survey, Table 1a, (1997-2007) and Table 1 (2012-preliminary). (Additional resources: www.rita.dot.gov/bts/sites/rita.dot.gov.bts/files/publications/commodity_flow_survey/ index.html)

${ }^{a}$ Detail may not add to total because of rounding.

b "Truck" as a single mode includes shipments which went by private truck only, for-hire truck only, or a combination of private truck and for-hire truck.

${ }^{\mathrm{c}}$ Data are not available.

${ }^{\mathrm{d}}$ CFS data for pipeline exclude most shipments of crude oil.

${ }^{\mathrm{e}}$ Denotes data do not meet publication standards because of high sampling variability or poor response quality. 
Industries covered by the 2012 Commodity Flow Survey (CFS) accounted for 3.3 trillion ton-miles on the nation's highways, railways, waterways, pipelines, and aviation system. Ton-miles increased an average of $1.5 \%$ per year from 1997 to 2012.

Table 5.16

Growth of Ton-Miles in the United States: Comparison of the 1997, 2002, 2007 and 2012 Commodity Flow Surveys ${ }^{\mathrm{a}}$

\begin{tabular}{|c|c|c|c|c|c|}
\hline & $\begin{array}{c}1997 \\
\text { (billions) }\end{array}$ & $\begin{array}{c}2002 \\
\text { (billions) }\end{array}$ & $\begin{array}{c}2007 \\
\text { (billions) }\end{array}$ & $\begin{array}{c}2012 \\
\text { (billions) } \\
\end{array}$ & $\begin{array}{c}\text { Average } \\
\text { annual } \\
\text { percent } \\
\text { change } \\
(1997-2012)\end{array}$ \\
\hline All modes & $2,661.4$ & $3,137.9$ & $3,344.7$ & $3,319.7$ & $1.5 \%$ \\
\hline Single modes & $2,383.5$ & $2,867.9$ & $2,894.3$ & $3,008.6$ & $1.6 \%$ \\
\hline Truck $^{b}$ & $1,023.5$ & $1,255.9$ & $1,342.1$ & $1,264.3$ & $1.4 \%$ \\
\hline For-hire truck & 741.1 & 959.6 & $1,055.6$ & $1,071.9$ & $2.5 \%$ \\
\hline Private truck & 268.6 & 291.1 & 286.5 & 192.5 & $-2.2 \%$ \\
\hline Rail & $1,022.5$ & $1,261.6$ & $1,344.0$ & $1,477.6$ & $2.5 \%$ \\
\hline Water & 261.7 & 282.7 & 157.3 & 208.3 & $-1.5 \%$ \\
\hline Inland water & 189.3 & 211.5 & 117.5 & 113.6 & $-3.3 \%$ \\
\hline Great Lakes & 13.4 & 13.8 & 6.9 & 9.9 & $-2.0 \%$ \\
\hline Deep sea & 59.0 & 57.4 & 33.0 & 26.8 & $-5.1 \%$ \\
\hline Multiple waterways & c & c & c & 58.0 & \\
\hline Air (includes truck and air) & 6.2 & 5.8 & 4.5 & 6.3 & $0.1 \%$ \\
\hline Pipeline $^{\mathrm{d}}$ & & & & & \\
\hline Multiple modes & 204.5 & 225.7 & 416.6 & 310.8 & $2.8 \%$ \\
\hline Parcel, U.S.P.S. or courier & 18.0 & 19.0 & 28.0 & 20.6 & $0.9 \%$ \\
\hline Truck and rail & 55.6 & 45.5 & 196.8 & 216.3 & $9.5 \%$ \\
\hline Truck and water & 34.8 & 32.4 & 98.4 & 47.9 & $2.2 \%$ \\
\hline Rail and water & 77.6 & 115.0 & 47.1 & 25.4 & $-7.2 \%$ \\
\hline Other multiple modes & 18.6 & 13.8 & 46.4 & e & \\
\hline Other and unknown modes & $\mathbf{7 3 . 4}$ & 44.2 & 33.8 & 0.3 & $-30.7 \%$ \\
\hline
\end{tabular}

Source:

U.S. Department of Transportation, Bureau of Transportation Statistics and U.S. Department of Commerce, Bureau of the Census, 2012 Commodity Flow Survey, Table 1a, (1997-2007) and Table 1 (2012-preliminary). (Additional resources: www.rita.dot.gov/bts/sites/rita.dot.gov.bts/files/publications/commodity_flow_survey/ index.html)

${ }^{a}$ Detail may not add to total because of rounding.

b "Truck" as a single mode includes shipments which went by private truck only, for-hire truck only, or a combination of private truck and for-hire truck.

${ }^{\mathrm{c}}$ Data are not available.

${ }^{\mathrm{d}}$ CFS data for pipeline exclude most shipments of crude oil.

e Denotes data do not meet publication standards because of high sampling variability or poor response quality. 
Table 5.17

Average Miles per Shipment in the United States: Comparison of the 1997, 2002, 2007 and 2012 Commodity Flow Surveys ${ }^{\mathrm{a}}$

\begin{tabular}{|c|c|c|c|c|c|}
\hline & $\begin{array}{c}1997 \\
\text { (miles) }\end{array}$ & $\begin{array}{c}2002 \\
\text { (miles) }\end{array}$ & $\begin{array}{c}2007 \\
\text { (miles) }\end{array}$ & $\begin{array}{c}2012 \\
\text { (miles) }\end{array}$ & $\begin{array}{c}\text { Average } \\
\text { annual } \\
\text { percent } \\
\text { change } \\
(1997-2012)\end{array}$ \\
\hline All modes & 472 & 546 & 619 & 586 & $1.5 \%$ \\
\hline Single modes & 184 & 240 & 234 & 254 & $2.2 \%$ \\
\hline Truck $^{b}$ & 144 & 173 & 206 & 216 & $2.7 \%$ \\
\hline For-hire truck & 485 & 523 & 599 & 489 & $0.1 \%$ \\
\hline Private truck & 53 & 64 & 57 & 46 & $-0.9 \%$ \\
\hline Rail & 769 & 807 & 728 & 811 & $0.4 \%$ \\
\hline Water & 482 & 568 & 520 & 842 & $3.8 \%$ \\
\hline Inland water & 177 & 450 & 144 & 323 & $4.1 \%$ \\
\hline Great Lakes & 204 & 339 & 657 & 385 & $4.3 \%$ \\
\hline Deep sea & 1,024 & 664 & 923 & 976 & $-0.3 \%$ \\
\hline Multiple waterways & c & c & ${ }^{c}$ & 1,301 & c \\
\hline Air (includes truck and air) & 1,380 & 1,919 & 1,304 & 1,229 & $-0.8 \%$ \\
\hline Pipeline $^{\mathrm{d}}$ & & & & & \\
\hline Multiple modes & 813 & 895 & 975 & 880 & $0.5 \%$ \\
\hline Parcel, U.S.P.S. or courier & 813 & 894 & 975 & 879 & $0.5 \%$ \\
\hline Truck and rail & 1,347 & 1,413 & 1,007 & 1,004 & $-1.9 \%$ \\
\hline Truck and water & 1,265 & 1,950 & 1,429 & 1,818 & $2.4 \%$ \\
\hline Rail and water & 1,092 & 957 & 1,928 & 663 & $-3.3 \%$ \\
\hline Other multiple modes & & $\mathrm{e}$ & 1,182 & e & \\
\hline Other and unknown modes & 122 & 130 & 116 & 2 & $-24.0 \%$ \\
\hline
\end{tabular}

Source:

U.S. Department of Transportation, Bureau of Transportation Statistics and U.S. Department of Commerce, Bureau of the Census, 2012 Commodity Flow Survey, Table 1a, (1997-2007) and Table 1 (2012-preliminary). (Additional resources: www.rita.dot.gov/bts/sites/rita.dot.gov.bts/files/publications/commodity_flow_survey/ index.html)

${ }^{a}$ Detail may not add to total because of rounding.

b "Truck" as a single mode includes shipments which went by private truck only, for-hire truck only, or a combination of private truck and for-hire truck.

${ }^{\mathrm{c}}$ Data are not available.

${ }^{\mathrm{d}}$ CFS data for pipeline exclude most shipments of crude oil.

${ }^{\mathrm{e}}$ Denotes data do not meet publication standards because of high sampling variability or poor response quality. 
In 2007, the data changed substantially due to improved estimation methodologies. Unfortunately, those data are no longer comparable to the rest of the historical series.

Table 5.18

Summary Statistics on Transit Buses and Trolleybuses, 1994-2012

\begin{tabular}{cccccc}
\hline Year & $\begin{array}{c}\text { Number of } \\
\text { active buses }\end{array}$ & $\begin{array}{c}\text { Vehicle-miles } \\
\text { (millions) }\end{array}$ & $\begin{array}{c}\text { Passenger- } \\
\text { miles } \\
\text { (millions) }\end{array}$ & $\begin{array}{c}\text { Btu/passenger- } \\
\text { mile }\end{array}$ & $\begin{array}{c}\text { Energy use } \\
\text { (trillion Btu) }\end{array}$ \\
\hline 1994 & 68,766 & 2,176 & 19,019 & 4,262 & 81.1 \\
1995 & 67,802 & 2,198 & 19,005 & 4,307 & 81.9 \\
1996 & 72,353 & 2,234 & 19,280 & 4,340 & 83.7 \\
1997 & 73,425 & 2,259 & 19,793 & 4,434 & 87.8 \\
1998 & 72,788 & 2,188 & 20,542 & 4,399 & 90.4 \\
1999 & 74,885 & 2,290 & 21,391 & 4,344 & 92.9 \\
2000 & 75,665 & 2,329 & 21,433 & 4,531 & 97.1 \\
2001 & 76,675 & 2,389 & 22,209 & 4,146 & 92.1 \\
2002 & 76,806 & 2,425 & 22,029 & 4,133 & 91.1 \\
2003 & 78,000 & 2,435 & 21,438 & 4,213 & 90.3 \\
2004 & 81,630 & 2,484 & 21,550 & 4,364 & 94.0 \\
2005 & 82,642 & 2,498 & 21,998 & 4,250 & 93.5 \\
2006 & 83,689 & 2,507 & 22,985 & 4,316 & 99.2 \\
2007 & 65,808 & 2,314 & 21,132 & 4,372 & 92.4 \\
2008 & 67,096 & 2,388 & 21,918 & 4,348 & 95.3 \\
2009 & 65,363 & 2,345 & 21,645 & 4,242 & 91.8 \\
2010 & 66,810 & 2,425 & 21,172 & 4,118 & 87.2 \\
2011 & 69,654 & 2,425 & 21,574 & 4,240 & 91.5 \\
2012 & 70,757 & 2,417 & 21,251 & 4,030 & 89.7 \\
\hline
\end{tabular}

Source:

American Public Transportation Association, 2014 Public Transportation Fact Book, Washington, DC, May 2014, Appendix A. (Additional resources: www.apta.com)

${ }^{\text {a }}$ Data are not continuous between 2006 and 2007 due to changes in estimation methodology. See source document for details. 


\section{Chapter 6 \\ Alternative Fuel and Advanced Technology Vehicles and Characteristics}

Summary Statistics from Tables in this Chapter

Source

Table 6.1 Alternative fuel vehicles in use, 2011 (latest available)

$1,191,786$

E85

862,837

$L P G$

139,477

CNG

118,214

Electric

67,295

$L N G$

3,436

M85

Table 6.8 Number of alternative fuel refuel sites, 2014

40,578

Electric outlets

22,747

$L P G$

2,987

CNG

1,375

Biodiesel

Fuel type abbreviations are used throughout this chapter.

$B 20=20 \%$ biodiesel, $80 \%$ petroleum diesel

CNG $=$ compressed natural gas

E85 $=85 \%$ ethanol, $15 \%$ gasoline

E95 $=95 \%$ ethanol, $5 \%$ gasoline

$\mathrm{H}_{2}=$ hydrogen

$L N G=$ liquefied natural gas

$L P G=$ liquefied petroleum gas

M85 = $85 \%$ methanol, $15 \%$ gasoline

M100 = $100 \%$ methanol 


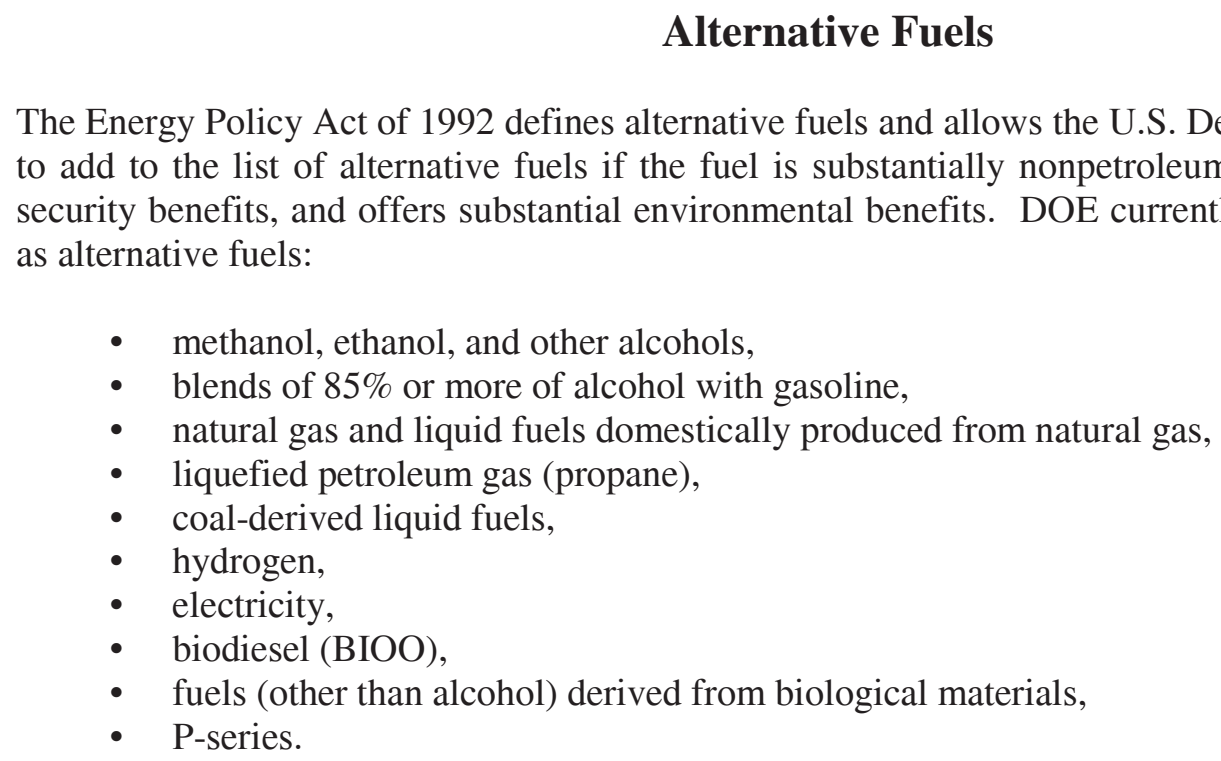
as alternative fuels:

- methanol, ethanol, and other alcohols,

- blends of $85 \%$ or more of alcohol with gasoline,

- natural gas and liquid fuels domestically produced from natural gas,

- liquefied petroleum gas (propane),

- coal-derived liquid fuels,

- hydrogen,

- electricity,

- biodiesel (BIOO),

- fuels (other than alcohol) derived from biological materials,

- P-series.

The Energy Policy Act of 1992 defines alternative fuels and allows the U.S. Department of Energy (DOE) to add to the list of alternative fuels if the fuel is substantially nonpetroleum, yields substantial energy security benefits, and offers substantial environmental benefits. DOE currently recognizes the following

\section{Alternative Fuels Data Center}

DOE established the Alternative Fuels Data Center (AFDC) in 1991 to support its work aimed at fulfilling the Alternative Motor Fuels Act directives. Since then, the AFDC has expanded its focus to include all advanced transportation fuels, vehicles, and technologies. The AFDC is operated and managed by the National Renewable Energy Laboratory (NREL) in Golden, Colorado.

The purposes of the AFDC are:

- to gather and analyze information on the fuel consumption, emissions, operation, and durability of alternative fuel vehicles, and

- to provide unbiased, accurate information on alternative fuels and alternative fuel vehicles to government agencies, private industry, research institutions, and other interested organizations.

Much of the AFDC data can be obtained through their website: www.afdc.energy.gov. Several tables and graphs in this chapter contain statistics which were generated by the AFDC. Below are some links to specific areas of the AFDC website.

Alternative \& Advanced Fuels - www.afdc.energy.gov

Alternative Fueling Station Locator - www.afdc.energy.gov/afdc/locator/stations/

Alternative \& Advanced Vehicles - www.afdc.energy.gov/fuels

State \& Federal Incentives \& Laws - www.afdc.energy.gov/afdc/laws/

Data Analysis \& Trends - www.afdc.energy.gov/data/

Tools - www.afdc.energylgov/tools 
There are almost 1.2 million alternative fuel vehicles in the United States, not including flex-fuel E85 vehicles which operate mainly on gasoline. The E85 vehicles in this table are those believed to be regularly fueled with E85 and represent only fleet vehicles covered under the Energy Policy Act.

Table 6.1

Estimates of Alternative Fuel Highway Vehicles in Use ${ }^{\mathrm{a}}$, 1995-2011

\begin{tabular}{|c|c|c|c|c|c|c|c|c|c|c|}
\hline Year & LPG & $\mathrm{CNG}$ & LNG & M85 & M100 & $\mathrm{E} 85^{\mathrm{b}}$ & E95 & Electricity $^{\mathrm{c}}$ & Hydrogen & Total \\
\hline 1995 & 172,806 & 50,218 & 603 & 18,319 & 386 & 1,527 & 136 & 2,860 & 0 & 246,855 \\
\hline 1996 & 175,585 & 60,144 & 663 & 20,265 & 172 & 4,536 & 361 & 3,280 & 0 & 265,006 \\
\hline 1997 & 175,679 & 68,571 & 813 & 21,040 & 172 & 9,130 & 347 & 4,453 & 0 & 280,205 \\
\hline 1998 & 177,183 & 78,782 & 1,172 & 19,648 & 200 & 12,788 & 14 & 5,243 & 0 & 295,030 \\
\hline 1999 & 178,610 & 91,267 & 1,681 & 18,964 & 198 & 24,604 & 14 & 6,964 & 0 & 322,302 \\
\hline 2000 & 181,994 & 100,750 & 2,090 & 10,426 & 0 & 87,570 & 4 & 11,830 & 0 & 394,664 \\
\hline 2001 & 185,053 & 111,851 & 2,576 & 7,827 & 0 & 100,303 & 0 & 17,847 & 0 & 425,457 \\
\hline 2002 & 187,680 & 120,839 & 2,708 & 5,873 & 0 & 120,951 & 0 & 33,047 & 0 & 471,098 \\
\hline 2003 & 190,369 & 114,406 & 2,640 & 0 & 0 & 176,799 & 0 & 47,485 & 9 & 531,708 \\
\hline 2004 & 182,864 & 118,532 & 2,717 & 0 & 0 & 211,800 & 0 & 49,536 & 43 & 565,492 \\
\hline 2005 & 173,795 & 117,699 & 2,748 & 0 & 0 & 246,363 & 0 & 51,398 & 119 & 592,122 \\
\hline 2006 & 164,846 & 116,131 & 2,798 & 0 & 0 & 297,099 & 0 & 53,526 & 159 & 634,559 \\
\hline 2007 & 158,254 & 114,391 & 2,781 & 0 & 0 & 364,384 & 0 & 55,730 & 223 & 695,763 \\
\hline 2008 & 151,049 & 113,973 & 3,101 & 0 & 0 & 450,327 & 0 & 56,901 & 313 & 775,664 \\
\hline 2009 & 147,030 & 114,270 & 3,176 & 0 & 0 & 504,297 & 0 & 57,185 & 357 & 826,315 \\
\hline 2010 & 143,037 & 115,863 & 3,354 & 0 & 0 & 618,506 & 0 & 57,462 & 421 & 938,643 \\
\hline 2011 & 139,477 & 118,214 & 3,436 & 0 & 0 & 862,837 & 0 & 67,295 & 527 & $1,191,786$ \\
\hline \multicolumn{11}{|c|}{ Average annual percentage change } \\
\hline $1995-2011$ & $-1.3 \%$ & $5.5 \%$ & $11.5 \%$ & $-100.0 \%$ & $-100.0 \%$ & $48.6 \%$ & $-100.0 \%$ & $21.8 \%$ & & $10.3 \%$ \\
\hline
\end{tabular}

Note: These are the latest available data from the Energy Information Administration.

\section{Source:}

U. S. Department of Energy, Energy Information Administration, Alternative Fuel Vehicle Data, Washington, DC, May 2013, website: www.eia.gov/renewable/afv/. 1995-2006, Annual Energy Review, Table 10.4. Estimated Number of Alternative-Fueled Vehicles in Use and Replacement Fuel Consumption.

${ }^{a}$ Vehicles in Use represent accumulated acquisitions, less retirements, as of the end of each calendar year. They do not include concept and demonstration vehicles.

${ }^{\mathrm{b}}$ Includes only those E85 vehicles believed to be used as alternative-fuels vehicles (AFVs), primarily fleetoperated vehicles; excludes other vehicles with E85-fueling capability. In 1997, some vehicle manufacturers began including E85-fueling capability in certain model lines of vehicles. For 2007, the Energy Information Administration (EIA) estimates that the number of E85 vehicles that are capable of operating on E85, motor gasoline, or both, is about 7.1 million. Many of these AFVs are sold and used as traditional gasoline-powered vehicles.

${ }^{\mathrm{c}}$ Excludes HEVs. 
Trollybus, heavy rail, and light rail use nearly all alternative fuels. However, the $40.4 \%$ of buses using alternative fuels replace a lot of traditional fuel use. Ferry boats have the highest average age.

Table 6.2

Alternative Fuel Transit Vehicles, 2013

\begin{tabular}{lccc}
\hline & Average & $\begin{array}{c}\text { Percent } \\
\text { powered by } \\
\text { age }\end{array}$ & $\begin{array}{c}\text { Number } \\
\text { ofternative fuels }\end{array}$ \\
\hline Bus & 7.8 & $40.4 \%$ & vehicles \\
Commuter rail & 19.2 & $16.6 \%$ & 70,187 \\
Ferry boat & 21.7 & $52.6 \%$ & 7,059 \\
Heavy rail & 20.5 & $100.0 \%$ & 186 \\
Light rail & 17.8 & $98.4 \%$ & 10,469 \\
Paratransit & 4.2 & $8.3 \%$ & 1,986 \\
Trolleybus & 11.4 & $100.0 \%$ & 68,632 \\
Vanpool & a & a & 570 \\
\hline
\end{tabular}

Note: See Glossary for definition of modes, such as paratransit and vanpool. The number of vehicles is a 2012 estimate.

\section{Source:}

American Public Transportation Association, 2014 Public Transportation Fact Book, Washington, DC, May 2014, Appendix A. (Additional resources: www.apta.com)

\footnotetext{
${ }^{\mathrm{a}}$ Not available.
} 
Table 6.3

E85 Flex-Fuel Vehicles Available by Manufacturer, Model Year 2014

\begin{tabular}{|c|c|c|}
\hline Model & Type & Emission class \\
\hline \multicolumn{3}{|l|}{ Audi: 1-800-822-AUDI; www.audiusa.com } \\
\hline A4 quattro & Sedan & Tier II Bin 5LEV II ULEV \\
\hline A5 Cabriolet quattro & Sedan & Tier II Bin 5 LEV II ULEV \\
\hline A5 quattro & Sedan & Tier II Bin 5 LEV II ULEV \\
\hline Allroad quattro & Wagon & Tier II Bin 5 LEV II ULEV \\
\hline Q5 AWD & SUV & Tier II Bin 5 LEV II ULEV \\
\hline \multicolumn{3}{|l|}{ Bentley: 1-800-777-6923; www.bentleymotors.com } \\
\hline Continental GT & Sedan & Tier II Bin 5 LEV II LEV \\
\hline Continental GTC & Sedan & Tier II Bin 5 LEV II LEV \\
\hline Continental Flying Spur & Sedan & Tier II Bin 5 LEV II LEV \\
\hline Continental Supersports & Sedan & Tier II Bin 5 LEV II LEV \\
\hline \multicolumn{3}{|l|}{ Chrysler: 1-800-999-FLEET; www.fleet.chrysler.com } \\
\hline Chrysler 200 & Sedan & Tier II Bin 4 LEV II ULEV \\
\hline Chrysler $300 \mathrm{FWD} / \mathrm{AWD}$ & Sedan & Tier II Bin 4 LEV II ULEV \\
\hline Chrysler Town \& Country & Minivan & Tier II Bin 4 \\
\hline Chrysler Avenger & Sedan & Tier II Bin 4 \\
\hline Dodge Charger FWD/AWD & Sedan & Tier II Bin 4 LEV II ULEV \\
\hline Dodge Charger Police Pursuit & Sedan & N/A \\
\hline Dodge Durango Police Special Service Vehicle & SUV & N/A \\
\hline Dodge Durango 2WD/AWD & SUV & Tier II Bin 4 \\
\hline Dodge Grand Caravan & Minivan & Tier II Bin 4 \\
\hline Dodge Ram Journey FWD & SUV & Tier II Bin 4 \\
\hline Dodge Ram 1500 2WD/4WD & Pickup & Tier II Bin 4 \\
\hline Dodge Ram CV & Minivan & Tier II Bin 4 \\
\hline Jeep Grand Cherokee 2WD/FWD & SUV & Tier II Bin 4 \\
\hline \multicolumn{3}{|c|}{ Ford: 1-800-34-FLEET; www.fleet.ford.com; 1-313-322-3000; www.ford.com } \\
\hline Ford E150/250/350 & Van/Wagon & Tier II Bin 8 \\
\hline Ford E150/250/350 & Van/Wagon & Tier II Bin 8 \\
\hline Ford E350/450 Cutaway & Chassis Cab & N/A \\
\hline Ford Expedition 2WD/AWD & SUV & Tier II Bin 4 \\
\hline Ford Explorer 2WD/AWD & SUV & Tier II Bin 5 \\
\hline Ford F150 & Pickup & Tier II Bin 4 \\
\hline Ford F150 & Pickup & Tier II Bin 4 \\
\hline Ford Focus & Sedan & Tier II Bin 4 \\
\hline Ford Police Interceptor & Sedan & N/A \\
\hline Ford Police Interceptor Utility & SUV & N/A \\
\hline Ford Super Duty F250/350 & Pickup & Tier II Bin 8 \\
\hline Ford Super Duty F350 & Chassis Cab & N/A \\
\hline Ford Taurus FWD/AWD & Sedan & Tier II Bin 5 \\
\hline Ford Transit 150/250/350 & Van/Wagon & N/A \\
\hline Ford Transit 250/350 & Cassis Cab & N/A \\
\hline Lincoln Navigator 2WD/AWD & SUV & Tier II Bin 4 \\
\hline \multicolumn{3}{|c|}{ General Motors Corporation: 1-800-462-8782 or 1-888-988-7267; www.gm.com/vehicles } \\
\hline Buick LaCrosse FWD/AWD & Sedan & Tier II Bin 4 \\
\hline Buick Verano & Sedan & N/A \\
\hline Cadillac ATS RWD/AWD & Sedan & N/A \\
\hline Cadillac Escalade ESV 2WD/4WD & SUV & Tier II Bin 4 \\
\hline Cadillac Escalade 2WD/4WD & SUV & Tier II Bin 4 \\
\hline Chevrolet Caprice Police Patrol Vehicle & Sedan & N/A \\
\hline
\end{tabular}


Table 6.3 (continued)

E85 Flex-Fuel Vehicles Available by Manufacturer, Model Year 2014

\begin{tabular}{|c|c|c|}
\hline Model & Type & Emission class \\
\hline \multicolumn{3}{|l|}{ General Motors Corporation (continued) } \\
\hline Chevrolet Captiva FWD/AWD & SUV & Tier II Bin 4 \\
\hline Chevrolet Equinox FWD/AWD & SUV & Tier II Bin 4 \\
\hline Chevrolet Express 1500 2WD/AWD & Van & Tier II Bin 4 \\
\hline Chevrolet Express 2500 2WD & Van & Tier II Bin 8 \\
\hline Chevrolet Express 3500 2WD & Van & Tier II Bin 8 \\
\hline Chevrolet Impala & Sedan & Tier II Bin 4 \\
\hline Chevrolet Impala Limited Police & Sedan & Tier II Bin 4 \\
\hline Chevrolet Silverado 1500 2WD/4WD & Pickup & Tier II Bin 4 \\
\hline Chevrolet Suburban 2WD/4WD & SUV & Tier II Bin 4 \\
\hline Chevrolet Tahoe Police 2WD/4WD & SUV & Tier II Bin 4 \\
\hline Chevrolet Tahoe 2WD/4WD & SUV & Tier II Bin 4 \\
\hline GMC Savana 1500 2WD/AWD & Van & Tier II Bin 4 \\
\hline GMC Savana 2500 2WD & Van & Tier II Bin 8 \\
\hline GMC Savana 3500 2WD & Van & Tier II Bin 8 \\
\hline GMC Sierra 1500 2WD/4WD & Pickup & Tier II Bin 4 \\
\hline GMC Terrain AWD & SUV & Tier II Bin 4 \\
\hline GMC Terrain FWD/AWD & SUV & Tier II Bin 4 \\
\hline GMC Yukon Denali 4WD & SUV & Tier II Bin 4 \\
\hline GMC Yukon XL 2WD/4WD & SUV & Tier II Bin 4 \\
\hline GMC Yukon 2WD & SUV & Tier II Bin 4 \\
\hline GMC Yukon 2WD/4WD & SUV & Tier II Bin 4 \\
\hline \multicolumn{3}{|c|}{ Jaguar: 1-800-4-JAGUAR; www.jaguarusa.com } \\
\hline XF E85 flex fuel & Sedan & Tier II Bin 5 LEV II ULEV \\
\hline XJ E85 flex fuel & Sedan & Tier II Bin 5 LEV II ULEV \\
\hline XJL E85 flex fuel & Sedan & Tier II Bin 5 LEV II ULEV \\
\hline \multicolumn{3}{|c|}{ Land Rover: 1-800-FIND-4WD or 1-800-637-6837; www.landrover.com/us/en/lr/ } \\
\hline Range Rover & SUV & Tier II Bin 5 LEV II ULEV \\
\hline Range Rover Sport & SUV & Tier II Bin 5 LEV II ULEV \\
\hline \multicolumn{3}{|c|}{ Mercedes-Benz: 1-800-FOR-MERCEDES; www.mbusa.com } \\
\hline C300 4Matic & Sedan & Tier II Bin 5 LEV II ULEV \\
\hline C350 Sedan/Coupe & Sedan & Tier II Bin 5 LEV II ULEV \\
\hline E350 4Matic Sedan/Coupe & Sedan & Tier II Bin 5 LEV II ULEV \\
\hline E350 Sedan/Coupe & Sedan & Tier II Bin 5 LEV II ULEV \\
\hline ML350 4Matic & SUV & Tier II Bin 5 LEV II ULEV \\
\hline \multicolumn{3}{|c|}{ Nissan: 1-800-NISSAN-1; www.nissanusa.com } \\
\hline Armada 2WD/4WD & SUV & Tier II Bin 5 \\
\hline Titan 2WD/4WD & Pickup & Tier II Bin 5 \\
\hline \multicolumn{3}{|l|}{ Toyota: 1-800-331-4331; www.toyota.com } \\
\hline Sequoia 4WD & SUV & Tier II Bin 5 \\
\hline Toyota Tundra 4WD & Pickup & Tier II Bin 5 \\
\hline
\end{tabular}

Note: LEV=low emission vehicle. ILEV=inherently low emission vehicle. ULEV=ultra low emission vehicle. $\mathrm{ZEV}=$ zero emission vehicle. TLEV=transitional low emission vehicle. SULEV=super ultra low emission vehicle. See Chapter 12 for details on emissions.

\section{Source:}

U.S. Department of Energy, National Alternative Fuels Data Center, website, www.afdc.energy.gov/afdc/vehicles/index.html, May 2014. (Additional resources: www.eere.energy.gov/afdc/) 
Table 6.4

Non-E85 Alternative Fuel Vehicles Available by Manufacturer, Model Year 2014

\begin{tabular}{|c|c|c|}
\hline Model & Type & Emission class \\
\hline \multicolumn{3}{|c|}{ Chrysler: 1-800-999-FLEET; www.fleet.chrysler.com } \\
\hline $\begin{array}{l}\text { Dodge Ram } 2500 \\
\text { Dodge Ram } 2500 / 3500 \mathrm{HD} \\
\text { Dodge Ram } 3500 \text { Chassis Cab }\end{array}$ & $\begin{array}{c}\text { Bi-Fuel/CNG } \\
\text { B20 } \\
\text { B20 } \\
\end{array}$ & $\begin{array}{l}\text { Pickup } \\
\text { Pickup } \\
\text { Pickup }\end{array}$ \\
\hline \multicolumn{3}{|c|}{ Ford: 1-800-34-FLEET; www.fleet.ford.com; 1-313-322-3000; www.ford.com } \\
\hline $\begin{array}{l}\text { Ford E150/250/350 } \\
\text { Ford E150/250/350 } \\
\text { Ford E350/450 Cutaway } \\
\text { Ford E350/450 Cutaway } \\
\text { Ford F150 } \\
\text { Ford Super Duty F250/350/450 } \\
\text { Ford Super Duty F350/450/550 } \\
\text { Ford Super Duty F350/450/550 } \\
\text { Ford Super Duty F650/750 } \\
\text { Ford Transit Connect } \\
\text { Ford Transit 150/250/350 } \\
\text { Ford Transit 250/350 } \\
\text { Ford Super Duty F250/350/450 } \\
\text { Ford Super Duty F650/750 } \\
\text { Ford Transit } \\
\text { Ford E150/250/350 } \\
\text { Ford E150/250/350 } \\
\text { Ford E350/450 Cutaway } \\
\text { Ford E350/450 Cutaway } \\
\text { Ford F150 } \\
\text { Ford Super Duty F250/350/450 } \\
\text { Ford Super Duty F350/450/550 } \\
\text { Ford Super Duty F350/450/550 } \\
\text { Ford Super Duty F650/750 } \\
\text { Ford Transit Connect } \\
\text { Ford Transit 150/250/350 } \\
\text { Ford Transit 250/350 }\end{array}$ & $\begin{array}{l}\text { CNG Capable w/Prep Package } \\
\text { CNG Capable w/Prep Package } \\
\text { CNG Capable w/Prep Package } \\
\text { CNG Capable w/Prep Package } \\
\text { CNG Capable w/Prep Package } \\
\text { CNG Capable w/Prep Package } \\
\text { CNG Capable w/Prep Package } \\
\text { CNG Capable w/Prep Package } \\
\text { CNG Capable w/Prep Package } \\
\text { CNG Capable w/Prep Package } \\
\text { CNG Capable w/Prep Package } \\
\text { CNG Capable w/Prep Package } \\
\text { B20 } \\
\text { B20 } \\
\text { B20 } \\
\text { LPG Capable w/Prep Package } \\
\text { LPG Capable w/Prep Package } \\
\text { LPG Capable w/Prep Package } \\
\text { LPG Capable w/Prep Package } \\
\text { LPG Capable w/Prep Package } \\
\text { LPG Capable w/Prep Package } \\
\text { LPG Capable w/Prep Package } \\
\text { LPG Capable w/Prep Package } \\
\text { LPG Capable w/Prep Package } \\
\text { LPG Capable w/Prep Package } \\
\text { LPG Capable w/Prep Package } \\
\text { LPG Capable w/Prep Package }\end{array}$ & $\begin{array}{l}\text { Van/Wagon } \\
\text { Van/Wagon } \\
\text { Chassis Cab } \\
\text { Chassis Cab } \\
\text { Pickup } \\
\text { Pickup } \\
\text { Chassis Cab } \\
\text { Chassis Cab } \\
\text { Chassis Cab } \\
\text { Van } \\
\text { Van/Wagon } \\
\text { Chassis Cab } \\
\text { Pickup } \\
\text { Pickup } \\
\text { Van } \\
\text { Van/Wagon } \\
\text { Van/Wagon } \\
\text { Chassis Cab } \\
\text { Chassis Cab } \\
\text { Pickup } \\
\text { Pickup } \\
\text { Chassis Cab } \\
\text { Chassis Cab } \\
\text { Chassis Cab } \\
\text { Van } \\
\text { Van/Wagon } \\
\text { Chassis Cab }\end{array}$ \\
\hline \multicolumn{3}{|c|}{ General Motors Corporation: 1-800-462-8782 or 1-888-988-7267; www.gm.com/vehicles } \\
\hline $\begin{array}{l}\text { Chevrolet Impala } \\
\text { Chevrolet Silverado } 2500 \mathrm{HD} \\
\text { GMC Sierra } 2500 \mathrm{HD} \\
\text { Chevrolet Express 2500/3500 2WD } \\
\text { GMC Savana 2500/3500 2WD } \\
\text { GMC Savana Cutaway 3500/4500 } \\
\text { Chevrolet Cruze } \\
\text { Chevrolet Express 2500/3500 2WD } \\
\text { Chevrolet Silverado 2500/3500 HD WD/4WD } \\
\text { GMC Savana 2500/3500 2WD } \\
\text { GMC Sierra 2500/3500 HD 2WD/4WD } \\
\text { GMC Sierra Denali 2500/3500 HD WD/4WD } \\
\text { Chevrolet Express Cutaway } 4500 \\
\text { GMC Savana Cutaway 4500 }\end{array}$ & $\begin{array}{l}\text { Bi-Fuel/CNG } \\
\text { Bi-Fuel/CNG } \\
\text { Bi-Fuel/CNG } \\
\text { CNG } \\
\text { CNG } \\
\text { CNG } \\
\text { B20 } \\
\text { B20 } \\
\text { B20 } \\
\text { B20 } \\
\text { B20 } \\
\text { B20 } \\
\text { LPG } \\
\text { LPG }\end{array}$ & $\begin{array}{l}\text { Sedan } \\
\text { Pickup } \\
\text { Pickup } \\
\text { Van } \\
\text { Van } \\
\text { Van } \\
\text { Sedan } \\
\text { Van } \\
\text { Pickup } \\
\text { Van } \\
\text { Pickup } \\
\text { Pickup } \\
\text { Van } \\
\text { Van }\end{array}$ \\
\hline \multicolumn{3}{|l|}{ Honda: 1-888-CC-HONDA; www.honda.com } \\
\hline Civic & CNG & Sedan \\
\hline
\end{tabular}


Table 6.4 (continued)

Non-E85 Alternative Fuel Vehicles Available by Manufacturer, Model Year 2014

Note: LEV=low emission vehicle. ILEV=inherently low emission vehicle. ULEV=ultra low emission vehicle. $Z E V=z e r o$ emission vehicle. TLEV=transitional low emission vehicle. SULEV=super ultra low emission vehicle. See Chapter 12 for details on emissions.

Source:

U.S. Department of Energy, National Alternative Fuels Data Center, website, www.afdc.energy.gov/afdc/vehicles/index.html, May 2014. (Additional resources: www.eere.energy.gov/afdc/) 
The hybrid share of all light vehicles reached $3.2 \%$ of all light vehicle sales in 2013. Plug-in vehicles certified for highway use began selling in 2010 and were 0.6\% of the light vehicle market in 2013.

Table 6.5

Hybrid and Plug-In Vehicle Sales, 1999-2013

\begin{tabular}{cccccc}
\hline $\begin{array}{c}\text { Calendar } \\
\text { year }\end{array}$ & $\begin{array}{c}\text { Hybrid vehicle } \\
\text { sales } \\
\text { (thousands) }\end{array}$ & $\begin{array}{c}\text { Plug-in vehicle } \\
\text { sales } \\
\text { (thousands) }\end{array}$ & $\begin{array}{c}\text { All light } \\
\text { vehicle sales } \\
\text { (thousands) }\end{array}$ & $\begin{array}{c}\text { Hybrid share } \\
\text { of all light } \\
\text { vehicles }\end{array}$ & $\begin{array}{c}\text { Plug-in share } \\
\text { of all light } \\
\text { vehicles }\end{array}$ \\
\hline 1999 & 0.0 & 0.0 & 16,711 & $0.0 \%$ & $0.0 \%$ \\
2000 & 9.4 & 0.0 & 17,164 & $0.1 \%$ & $0.0 \%$ \\
2001 & 20.3 & 0.0 & 16,950 & $0.1 \%$ & $0.0 \%$ \\
2002 & 36.0 & 0.0 & 16,675 & $0.2 \%$ & $0.0 \%$ \\
2003 & 47.6 & 0.0 & 16,494 & $0.3 \%$ & $0.0 \%$ \\
2004 & 84.2 & 0.0 & 16,737 & $0.5 \%$ & $0.0 \%$ \\
2005 & 205.9 & 0.0 & 16,774 & $1.2 \%$ & $0.0 \%$ \\
2006 & 251.9 & 0.0 & 16,336 & $1.5 \%$ & $0.0 \%$ \\
2007 & 351.1 & 0.0 & 15,867 & $2.2 \%$ & $0.0 \%$ \\
2008 & 315.8 & 0.0 & 13,015 & $2.4 \%$ & $0.0 \%$ \\
2009 & 290.3 & 0.0 & 10,236 & $2.8 \%$ & $0.0 \%$ \\
2010 & 274.6 & 0.3 & 11,394 & $2.4 \%$ & $0.0 \%$ \\
2011 & 266.5 & 17.8 & 12,539 & $2.1 \%$ & $0.1 \%$ \\
2012 & 434.6 & 53.2 & 14,218 & $3.1 \%$ & $0.4 \%$ \\
2013 & 495.5 & 97.1 & 15,278 & $3.2 \%$ & $0.6 \%$ \\
\hline
\end{tabular}

Note: Plug-in vehicle sales include only those vehicles certified for highway use. Small electric carts and neighborhood electric vehicles are excluded.

\section{Sources:}

Hybrid and Electric Vehicle Sales - Compiled by the Transportation Research Center at Argonne National Laboratory, 2014.

All Light Vehicle Sales - Table 3.11. 
Table 6.6

Electric Drive Vehicles Available by Manufacturer, Model Year 2014

\begin{tabular}{|c|c|c|c|}
\hline Model & Drive Type & Vehicle Type & Emissions Class \\
\hline \multicolumn{4}{|c|}{ Acura: 1-800-999-1009; www.acura.com/ } \\
\hline ILX & $\mathrm{HEV}$ & Sedan & Tier II Bin 2 LEV II PZEV \\
\hline RLX Sport Hybrid SH-AWD & $\mathrm{HEV}$ & Sedan & N/A \\
\hline \multicolumn{4}{|c|}{ Audi: 1-800-822-AUDI; www.audiusa.com } \\
\hline Q5 Hybrid AWD & $\mathrm{HEV}$ & SUV & Tier II Bin 5 LEV II ULEV \\
\hline \multicolumn{4}{|c|}{ BMW: 1-800-831-1117; www.bmwusa.com } \\
\hline ActiveHybrid 3 & $\mathrm{HEV}$ & Sedan & Tier II Bin 5 LEV II ULEV \\
\hline ActiveHybrid 5 & $\mathrm{HEV}$ & Sedan & Tier II Bin 5 LEV II ULEV \\
\hline ActiveHybrid 7 & $\mathrm{HEV}$ & Sedan & Tier II Bin 5 LEV II ULEV \\
\hline i3 & EV & Sedan & N/A \\
\hline i3 w/Range Extender & PHEV & Sedan & N/A \\
\hline i8 Plug-In Hybrid & PHEV & Sedan & N/A \\
\hline \multicolumn{4}{|c|}{ Chrysler: 1-800-999-FLEET; www.fleet.chrysler.com } \\
\hline Fiat 500e & EV & Sedan & Tier II Bin 1 LEV II ZEV \\
\hline \multicolumn{4}{|c|}{ Ford: 1-800-34-FLEET; www.fleet.ford.com; 1-313-322-3000; www.fordvehicles.com } \\
\hline Ford C-MAX Energi & PHEV & Sedan & N/A \\
\hline Ford C-MAX Hybrid & $\mathrm{HEV}$ & Sedan & N/A \\
\hline Ford Focus Electric & EV & Sedan & Tier II Bin 1 LEV II ZEV \\
\hline Ford Fusion Energi & PHEV & Sedan & Tier II Bin 3 LEV II PZEV \\
\hline Ford Fusion Hybrid & $\mathrm{HEV}$ & Sedan & Tier II Bin 3 LEV II PZEV \\
\hline Lincoln MKZ & HEV & Sedan & Tier II Bin 3 LEV II PZEV \\
\hline \multicolumn{4}{|c|}{ General Motors: 1-800-462-8782 or 1-888-988-7267; www.gm.com/vehicles } \\
\hline Cadillac ELR & PHEV & Sedan & N/A \\
\hline Chevrolet Spark & EV & Sedan & Tier II Bin 1 LEV II ZEV \\
\hline Chevrolet Volt & PHEV & Sedan & Tier II Bin 4 LEV II PZEV \\
\hline \multicolumn{4}{|c|}{ Honda: 1-800-999-1009; www.honda.com } \\
\hline Accord & $\mathrm{HEV}$ & Sedan & Tier II Bin 3 LEV III SULEV30 \\
\hline Accord Plug-In Hybrid & PHEV & Sedan & Tier II 2 Bin 2 LEV III SULEV \\
\hline Civic Hybrid & $\mathrm{HEV}$ & Sedan & N/A \\
\hline Clarity & FCV & Sedan & Tier II Bin 1 LEVII ZEV \\
\hline CRZ & $\mathrm{HEV}$ & 2 Seater & Tier II Bin 2 LEV II PZEV \\
\hline Fit EV & $\mathrm{EV}$ & Sedan & Tier II Bin 1 LEV II ZEV \\
\hline Insight & HEV & Sedan & Tier II Bin 2 LEV II PZEV \\
\hline \multicolumn{4}{|c|}{ Hyundai: 1-800-633-5151; www.hyundaiusa.com } \\
\hline Sonata & $\mathrm{HEV}$ & Sedan & Tier II Bin 2 LEV II PZEV \\
\hline Tucson Fuel Cell Vehicle & $\mathrm{FCV}$ & SUV & LEV II ZEV \\
\hline \multicolumn{4}{|c|}{ Infiniti: 1-800-662-6200; www.infinitiusa.com } \\
\hline Q50 Hybrid FWD/AWD & $\mathrm{HEV}$ & Sedan & Tier II Bin 5 LEV II ULEV \\
\hline Q50S Hybrid FWD/AWD & $\mathrm{HEV}$ & Sedan & Tier II Bin 5 LEV II ULEV \\
\hline Q70 Hybrid & $\mathrm{HEV}$ & Sedan & N/A \\
\hline QX60 Hybrid & $\mathrm{HEV}$ & SUV & Tier II Bin 5 LEV II ULEV \\
\hline \multicolumn{4}{|c|}{ Kia: 1-800-333-4KIA; www.kia.com } \\
\hline Optima & $\mathrm{HEV}$ & Sedan & Tier II Bin 2 LEV II PZEV \\
\hline Soul EV & $\mathrm{EV}$ & Sedan & N/A \\
\hline \multicolumn{4}{|c|}{ Lexus: 1-800-255-3987; www.lexus.com } \\
\hline CT 200h & $\mathrm{HEV}$ & Sedan & N/A \\
\hline ES $300 \mathrm{~h}$ & $\mathrm{HEV}$ & Sedan & Tier II Bin 3 LEV II SULEV \\
\hline GS 450h & $\mathrm{HEV}$ & Sedan & Tier II Bin 3 LEV II SULEV \\
\hline LS 600h L & $\mathrm{HEV}$ & Sedan & Tier II Bin 3 LEV II SULEV \\
\hline RX 450h FWD/AWD & $\mathrm{HEV}$ & SUV & Tier II Bin 3 LEV II SULEV \\
\hline
\end{tabular}


Table 6.6 (continued)

Electric Drive Vehicles Available by Manufacturer, Model Year 2014

\begin{tabular}{|c|c|c|c|}
\hline Model & Drive Type & Vehicle Type & Emissions Class \\
\hline \multicolumn{4}{|c|}{ McLaren: +44 (0) 1483 261900; www.cars.mclaren.com } \\
\hline $\mathrm{P} 1$ & PHEV & Sedan & N/A \\
\hline \multicolumn{4}{|c|}{ Mercedes-Benz: 1-800-FOR MERCEDES; www.mbusa.com } \\
\hline B-Class Electric & $\mathrm{EV}$ & Sedan & N/A \\
\hline B-Class F-CELL & FCV & Sedan & N/A \\
\hline E400 Hybrid & $\mathrm{HEV}$ & Sedan & Tier II Bin 4 LEV II PZEV \\
\hline \multicolumn{4}{|c|}{ Mitsubishi: 1-888-MITSU2014; www.mitsubishicars.com } \\
\hline i-MiEV & EV & Sedan & Tier II Bin 1 LEV II ZEV \\
\hline \multicolumn{4}{|c|}{ Nissan: 1-800-NISSAN-1; www.nissanusa.com } \\
\hline Leaf & EV & Sedan & Tier II Bin 1 LEV II ZEV \\
\hline Pathfinder Hybrid 2WD/AWD & $\mathrm{HEV}$ & SUV & Tier II Bin 5 LEV II ULEV \\
\hline \multicolumn{4}{|c|}{ Porsche: 1-800-PORSCHE; www.porsche.com/usa/ } \\
\hline Cayenne S Hybrid & HEV & SUV & Tier II Bin 5 LEV II ULEV \\
\hline Panamera S E-Hybrid & PHEV & Sedan & \\
\hline \multicolumn{4}{|c|}{ Scion: 1-866-70-SCION; www.scion.com } \\
\hline iQ EV & EV & Sedan & Tier II Bin 1 LEV II ZEV \\
\hline \multicolumn{4}{|c|}{ Smart: 1-800-762-7887; www.smartusa.com } \\
\hline fortwo electric drive & EV & Sedan & Tier II Bin 1 LEV II ZEV \\
\hline \multicolumn{4}{|c|}{ Subaru: 1-800-782-2783; www.subaru.com } \\
\hline XV Crosstrek Hybrid & HEV & SUV & N/A \\
\hline \multicolumn{4}{|c|}{ Tesla: 1-877-798-3752; www.teslamotors.com } \\
\hline Model S & EV & Sedan & Tier II Bin 1 LEV II ZEV \\
\hline \multicolumn{4}{|c|}{ Toyota: 1-800-331-4331; www.toyota.com } \\
\hline Avalon & $\mathrm{HEV}$ & Sedan & Tier II Bin 3 LEV II SULEV \\
\hline Camry & $\mathrm{HEV}$ & Sedan & Tier II Bin 3 LEV II PZEV \\
\hline Highlander & $\mathrm{HEV}$ & SUV & N/A \\
\hline Prius & $\mathrm{HEV}$ & Sedan & Tier II Bin 3 LEV II PZEV \\
\hline Prius $c$ & $\mathrm{HEV}$ & Sedan & N/A \\
\hline Prius Plug-in & PHEV & Sedan & Tier II Bin 3 LEV II PZEV \\
\hline Prius $v$ & $\mathrm{HEV}$ & Wagon & Tier II Bin 3 LEV II SULEV \\
\hline RAV4 EV & $\mathrm{EV}$ & SUV & Tier II Bin 1 \\
\hline \multicolumn{4}{|c|}{ Volkswagen: 1-800-DRIVE-VW; www.vw.com } \\
\hline Jetta Hybrid & $\mathrm{HEV}$ & Sedan & Tier II Bin 3 LEV II PZEV \\
\hline Touareg Hybrid & $\mathrm{HEV}$ & SUV & Tier II Bin 5 LEV II ULEV \\
\hline
\end{tabular}

Note: $\mathrm{HEV}=$ hybrid-electric vehicle; $\mathrm{PHEV}=$ plug-in hybrid-electric vehicle; $\mathrm{EV}=$ electric vehicle; FCV $=$ fuel cell vehicle; LEV = low emission vehicle; ILEV = inherently low emission vehicle; ULEV $=$ ultra-low emission vehicle; $\mathrm{ZEV} \mathrm{=} \mathrm{zero} \mathrm{emission} \mathrm{vehicle;} \mathrm{TLEV} \mathrm{=} \mathrm{transitional} \mathrm{low} \mathrm{emission} \mathrm{vehicle;} \mathrm{SULEV} \mathrm{=} \mathrm{super} \mathrm{ultra-low}$ emission vehicle; AT-PZEV = advanced technology - partial zero emissions vehicle. See Chapter 12 for details on emissions.

\section{Source:}

U.S. Department of Energy, National Alternative Fuels Data Center, website, www.afdc.energy.gov/afdc/vehicles/index.html, May 2014. (Additional resources: www.eere.energy.gov/afdc/) 
Table 6.7

Electric-Drive Medium/Heavy Trucks and Buses Available by Manufacturer, 2014

\begin{tabular}{|c|c|c|}
\hline Manufacturer - Model & Drive type & Truck type \\
\hline Autocar - E3 Hybrid & Hybrid diesel-electric & Refuse hauler \\
\hline Balqon - XE-20 & Electric vehicle & Tractor \\
\hline Balqon - XE-30 & Electric vehicle & Tractor \\
\hline Balqon - Mule M150 & Electric vehicle & Vocational truck \\
\hline Boulder Electric vehicle - DV-500 Delivery Truck & Electric vehicle & Van \\
\hline Capacity Trucks - HETT & Electric vehicle & Tractor \\
\hline Champion Bus Inc. - Defende & Hybrid gasoline-electric & Shuttle bus \\
\hline DesignLine Corp. - EcoSaver IV & Hybrid diesel-electric & Transit bus \\
\hline DesignLine Corp. - Eco-Smart 1 & Electric vehicle & Transit bus \\
\hline DesignLine Corp. - EcoSaver IV & Hybrid CNG-electric & Transit bus \\
\hline Ebus - EBUS22FC & Fuel cell vehicle & Shuttle bus \\
\hline Ebus - EBUS22FC & Hybrid gasoline-electric & Shuttle bus \\
\hline ElDorado National - Axess & Hybrid diesel-electric & Transit bus \\
\hline ElDorado National - E-Z Rider II BRT & Hybrid diesel-electric & Transit bus \\
\hline ElDorado National - Axess & Fuel cell vehicle & Transit bus \\
\hline Electric vehicles International - EVI-MD & Electric vehicle & Vocational truck \\
\hline Electric vehicles International - WI EVI & Electric vehicle & Van \\
\hline Enova Systems - Enova Ze step van & Electric vehicle & Van \\
\hline Freightliner - M2 106 Hybrid & Hybrid diesel-electric & Tractor, vocational truck \\
\hline Gillig Corp. - Diesel-Electric Hybrid Bus and CNG Bus & Hybrid diesel-electric & Transit bus \\
\hline Hino - $195 \mathrm{~h}$ & Hybrid diesel-electric & Vocational truck \\
\hline IC Bus - HC Hybrid Series & Hybrid diesel-electric & Shuttle bus \\
\hline Kenworth - T270 hybrid & Hybrid diesel-electric & Tractor, vocational truck \\
\hline Kenworth - T370 diesel electric tractor & Hybrid diesel-electric & Tractor \\
\hline Kenworth - T370 hybrid truck & Hybrid diesel-electric & Vocational truck \\
\hline Motor Coach Industries - D4500 CT Hybrid Commuter Coach & Hybrid diesel-electric & Transit bus \\
\hline New Flyer - Xcelsior & Electric vehicle & Transit bus \\
\hline New Flyer - Xcelsior & Hybrid diesel-electric & Transit bus \\
\hline New Flyer - Xcelsior & Fuel cell vehicle & Transit bus \\
\hline North American Bus Industries - 31LFW / 35LFW / 40LFW & Hybrid diesel-electric & Transit bus \\
\hline North American Bus Industries - 42BRT & Hybrid diesel-electric & Transit bus \\
\hline North American Bus Industries - 60BRT & Hybrid diesel-electric & Transit bus \\
\hline North American Bus Industries - CompoBus & Hybrid diesel-electric & Transit bus \\
\hline Nova Bus - LFS Artic HEV & Hybrid diesel-electric & Transit bus \\
\hline Nova Bus - LFS HEV & Hybrid diesel-electric & Transit bus \\
\hline Nova Bus - LFX & Hybrid diesel-electric & Transit bus \\
\hline Proterra - EcoRide BE35 & Electric vehicle & Transit bus \\
\hline Smith Electric vehicles - Newton & Electric vehicle & Vocational truck \\
\hline Smith Electric vehicles - Newton Step Van & Electric vehicle & Van \\
\hline Trans Tech - ETrans & Electric vehicle & School bus \\
\hline Van Hool - A300L Fuel Cell & Fuel cell vehicle & Transit bus \\
\hline Vision Motor Corp. - Tyrano & Fuel cell vehicle & Tractor \\
\hline Vision Motor Corp. - ZETT Zero Emission & Fuel cell vehicle & Tractor \\
\hline ZeroTruck - ZeroTruck & Electric vehicle & Vocational truck \\
\hline
\end{tabular}

\section{Source:}

U.S. Department of Energy, National Alternative Fuels Data Center, website, www.afdc.energy.gov/afdc/vehicles/index.html, May 2014. (Additional resources: www.eere.energy.gov/afdc/) 
This list includes public and private refuel sites; therefore, not all of these sites are available to the public.

Table 6.8

Number of Alternative Refuel Sites by State and Fuel Type, 2014

\begin{tabular}{|c|c|c|c|c|c|c|c|c|c|}
\hline State & $\begin{array}{l}\mathrm{B} 20 \\
\text { sites }\end{array}$ & $\begin{array}{c}\mathrm{CNG} \\
\text { sites }\end{array}$ & $\begin{array}{l}\mathrm{E} 85 \\
\text { sites }\end{array}$ & $\begin{array}{l}\text { Electric } \\
\text { stations }\end{array}$ & $\begin{array}{c}\text { Electric charging } \\
\text { outlets }\end{array}$ & $\begin{array}{l}\text { Hydrogen } \\
\text { sites }\end{array}$ & $\begin{array}{l}\text { LNG } \\
\text { sites }\end{array}$ & $\begin{array}{l}\text { LPG } \\
\text { sites } \\
\end{array}$ & $\begin{array}{c}\text { Totals by } \\
\text { State }^{\mathrm{a}}\end{array}$ \\
\hline Alabama & 10 & 23 & 33 & 62 & 82 & 0 & 2 & 115 & 327 \\
\hline Alaska & 0 & 1 & 0 & 0 & 0 & 0 & 0 & 6 & 7 \\
\hline Arizona & 79 & 34 & 30 & 302 & 749 & 1 & 6 & 64 & 1,265 \\
\hline Arkansas & 5 & 10 & 39 & 32 & 44 & 0 & 1 & 37 & 168 \\
\hline California & 82 & 275 & 93 & 2,100 & 6,028 & 24 & 44 & 230 & 8,876 \\
\hline Colorado & 17 & 36 & 89 & 170 & 351 & 1 & 0 & 47 & 711 \\
\hline Connecticut & 2 & 15 & 1 & 161 & 278 & 1 & 1 & 15 & 474 \\
\hline Delaware & 1 & 1 & 2 & 13 & 20 & 1 & 0 & 6 & 44 \\
\hline Dist. of Columbia & 7 & 2 & 3 & 61 & 139 & 0 & 0 & 0 & 212 \\
\hline Florida & 17 & 34 & 55 & 544 & 1,115 & 0 & 1 & 69 & 1,835 \\
\hline Georgia & 25 & 27 & 60 & 249 & 467 & 0 & 1 & 60 & 889 \\
\hline Hawaii & 9 & 1 & 3 & 184 & 414 & 3 & 0 & 3 & 617 \\
\hline Idaho & 4 & 10 & 9 & 14 & 21 & 0 & 6 & 25 & 89 \\
\hline Illinois & 12 & 44 & 225 & 360 & 696 & 1 & 1 & 104 & 1,443 \\
\hline Indiana & 7 & 21 & 178 & 123 & 207 & 0 & 1 & 175 & 712 \\
\hline Iowa & 9 & 5 & 184 & 53 & 93 & 0 & 0 & 18 & 362 \\
\hline Kansas & 6 & 8 & 25 & 75 & 127 & 0 & 0 & 37 & 278 \\
\hline Kentucky & 4 & 4 & 60 & 39 & 100 & 0 & 0 & 48 & 255 \\
\hline Louisiana & 2 & 23 & 5 & 45 & 65 & 0 & 1 & 34 & 175 \\
\hline Maine & 3 & 1 & 0 & 20 & 25 & 0 & 0 & 10 & 59 \\
\hline Maryland & 9 & 9 & 34 & 263 & 598 & 0 & 0 & 19 & 932 \\
\hline Massachusetts & 13 & 23 & 8 & 243 & 582 & 1 & 0 & 17 & 887 \\
\hline Michigan & 16 & 22 & 196 & 291 & 736 & 4 & 0 & 74 & 1,339 \\
\hline Minnesota & 11 & 12 & 282 & 167 & 355 & 0 & 0 & 30 & 857 \\
\hline Mississippi & 4 & 6 & 2 & 35 & 38 & 0 & 0 & 117 & 202 \\
\hline Missouri & 4 & 14 & 108 & 100 & 181 & 1 & 0 & 65 & 473 \\
\hline Montana & 6 & 2 & 3 & 4 & 2 & 0 & 0 & 43 & 60 \\
\hline Nebraska & 4 & 9 & 78 & 20 & 33 & 0 & 0 & 22 & 166 \\
\hline Nevada & 5 & 8 & 20 & 83 & 216 & 1 & 2 & 38 & 373 \\
\hline New Hampshire & 4 & 3 & 0 & 38 & 53 & 0 & 0 & 11 & 109 \\
\hline New Jersey & 5 & 30 & 5 & 130 & 233 & 0 & 0 & 9 & 412 \\
\hline New Mexico & 8 & 11 & 15 & 22 & 54 & 0 & 0 & 45 & 155 \\
\hline New York & 36 & 110 & 79 & 435 & 916 & 9 & 0 & 62 & 1,647 \\
\hline North Carolina & 134 & 36 & 22 & 276 & 618 & 0 & 0 & 98 & 1,184 \\
\hline North Dakota & 3 & 1 & 58 & 5 & 6 & 0 & 0 & 19 & 92 \\
\hline Ohio & 18 & 32 & 113 & 153 & 224 & 2 & 3 & 65 & 610 \\
\hline Oklahoma & 5 & 94 & 28 & 30 & 41 & 0 & 0 & 153 & 351 \\
\hline Oregon & 24 & 14 & 8 & 403 & 957 & 0 & 1 & 31 & 1,438 \\
\hline Pennsylvania & 7 & 55 & 35 & 197 & 308 & 2 & 0 & 73 & 677 \\
\hline Rhode Island & 6 & 6 & 0 & 62 & 147 & 0 & 0 & 6 & 227 \\
\hline South Carolina & 60 & 10 & 83 & 141 & 230 & 2 & 1 & 45 & 572 \\
\hline South Dakota & 2 & 0 & 87 & 11 & 23 & 0 & 0 & 18 & 141 \\
\hline Tennessee & 44 & 11 & 58 & 375 & 893 & 0 & 0 & 103 & 1,484 \\
\hline Texas & 19 & 76 & 100 & 692 & 1,774 & 1 & 12 & 479 & 3,153 \\
\hline Utah & 3 & 89 & 4 & 59 & 102 & 0 & 7 & 27 & 291 \\
\hline Vermont & 2 & 3 & 1 & 33 & 68 & 0 & 0 & 2 & 109 \\
\hline Virginia & 12 & 20 & 19 & 211 & 527 & 1 & 0 & 60 & 850 \\
\hline Washington & 33 & 25 & 24 & 523 & 1,426 & 0 & 1 & 68 & 2,100 \\
\hline West Virginia & 2 & 4 & 5 & 26 & 98 & 0 & 0 & 13 & 148 \\
\hline Wisconsin & 4 & 53 & 131 & 153 & 274 & 0 & 1 & 55 & 671 \\
\hline Wyoming & 13 & 12 & 9 & 6 & 13 & 0 & 0 & 17 & 70 \\
\hline Totals by Fuel: & 817 & 1,375 & 2,709 & 9,794 & 22,747 & 56 & 93 & 2,987 & 40,578 \\
\hline
\end{tabular}

Source:

U.S. Department of Energy, Alternative Fuels Data Center website,

www.afdc.energy.gov/afdc/fuels/stations_counts.html, May 2014.

${ }^{\text {a }}$ Totals by State is the total number of fuel types available at stations. Stations are counted once for each type of fuel available. 
Clean Cities is a locally-based government/industry partnership, coordinated by the U.S. Department of Energy to expand the use of alternatives to gasoline and diesel fuel. By combining the decision-making with voluntary action by partners, the "grass-roots" approach of Clean Cities departs from traditional "top-down" Federal programs.

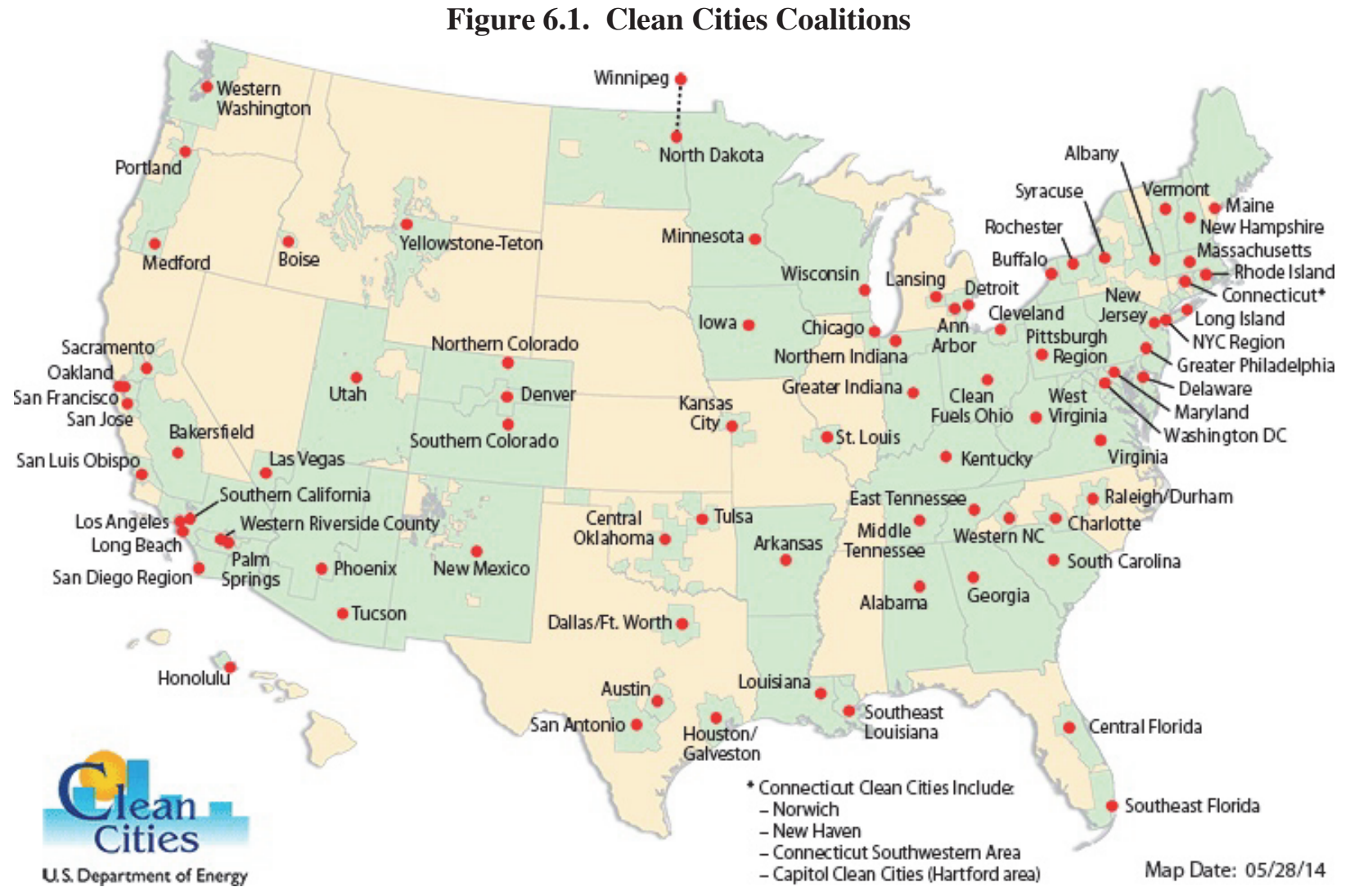

\section{Source:}

U.S. Department of Energy, Alternative Fuel Data Center, May 2014. (Additional resources: www.eere.energy.gov/cleancities/progs/coalition_locations.php) 
In 2012, the United States and Russia were by far the top natural gas producing countries with more than double that of any other country. Although the United States produced more than Russia, Russia has five times more reserves.

Figure 6.2. Natural Gas Production and Reserves for the Top Ten Natural Gas Producing Countries, 2012

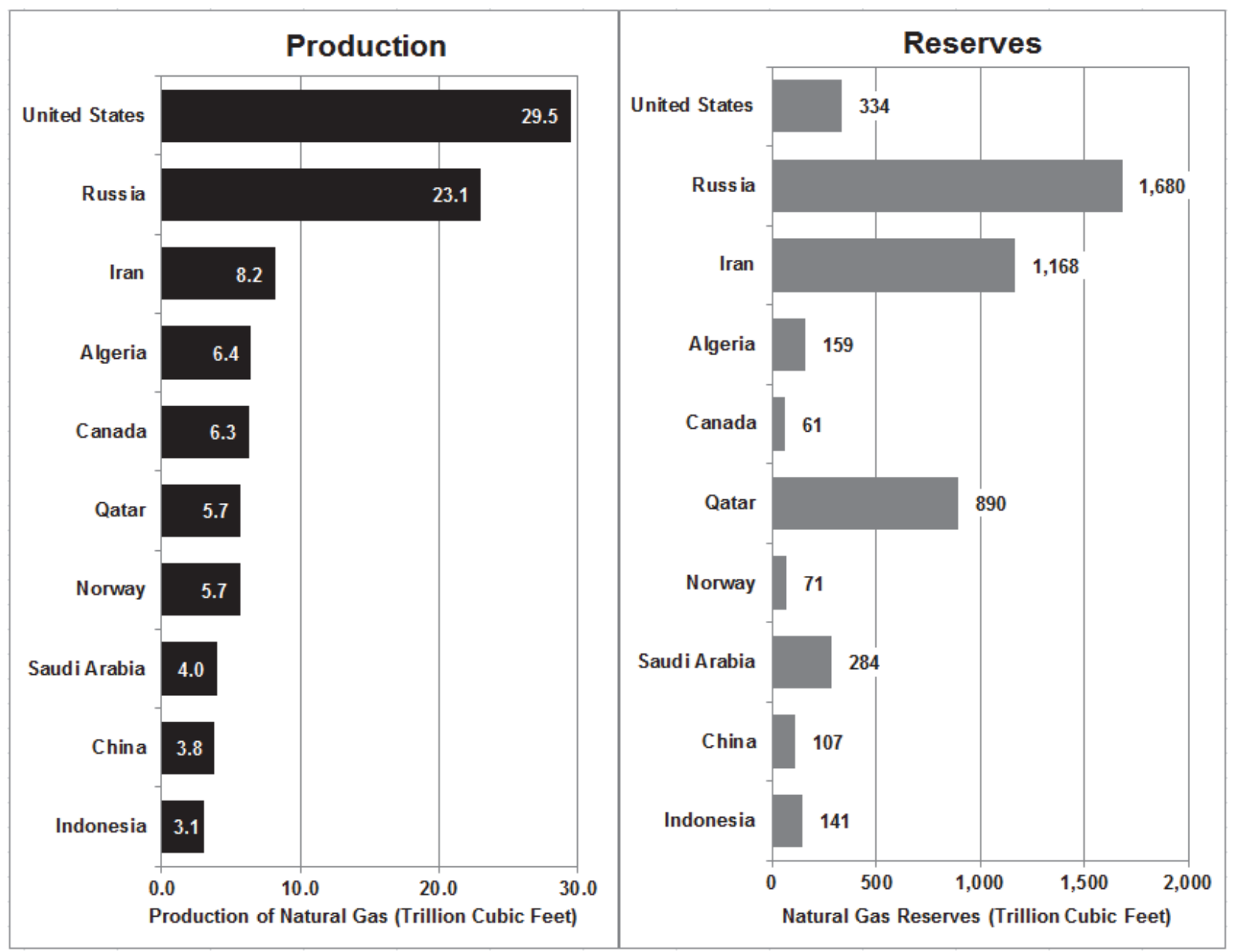

\section{Source:}

U.S. Department of Energy, Energy Information Administration, International Energy Statistics, May 2014. (Additional resources: www.eia.gov/countries/data.cfm) 
Table 6.9

Properties of Conventional and Alternative Liquid Fuels

\begin{tabular}{|c|c|c|c|c|}
\hline \multirow[b]{2}{*}{ Property } & \multicolumn{4}{|c|}{ Liquid Fuels } \\
\hline & Gasoline & Low-sulfur diesel & Methanol & Ethanol \\
\hline Standard chemical formula ${ }^{a}$ & $\mathrm{C}_{4}$ to $\mathrm{C}_{12}$ & $\mathrm{C}_{8}$ to $\mathrm{C}_{25}$ & $\mathrm{CH}_{3} \mathrm{OH}$ & $\mathrm{CH}_{3} \mathrm{CH}_{2} \mathrm{OH}$ \\
\hline Physical state & Liquid & Liquid & Liquid & Liquid \\
\hline Molecular weight & $100-105$ & $\sim 200$ & 32.04 & 46.07 \\
\hline \multicolumn{5}{|l|}{ Composition (weight \%) } \\
\hline Carbon & $85-88$ & 87 & 37.5 & 52.2 \\
\hline Hydrogen & $12-15$ & 13 & 12.6 & 13.1 \\
\hline Oxygen & 0 & 0 & 49.9 & 34.7 \\
\hline Main fuel source(s) & Crude oil & Crude oil & $\begin{array}{l}\text { Natural gas, coal, or } \\
\text { woody biomass }\end{array}$ & $\begin{array}{l}\text { Corn, grains, or } \\
\text { agricultural waste }\end{array}$ \\
\hline $\begin{array}{l}\text { Gasoline gallon equivalent } \\
(\mathrm{GGE})^{\mathrm{b}} \text { (Fuel unit } \\
\text { measured/GGE) }\end{array}$ & $\begin{array}{c}1.0(\mathrm{EO} \\
\text { gasoline })\end{array}$ & $\begin{array}{l}0.889 \text { (Diesel } \\
\text { gal/GGE) }\end{array}$ & $\begin{array}{l}\text { 2.04 Methanol } \\
\text { gal/GGE) }\end{array}$ & $\begin{array}{c}1.20-1.37\left(\mathrm{E} 85^{\mathrm{c}}\right. \\
\mathrm{gal} / \mathrm{GGE}) 1.03(\mathrm{E} 10 \\
\mathrm{gal} / \mathrm{GGE})\end{array}$ \\
\hline Specific gravity $\left(60^{\circ} \mathrm{F} / 60^{\circ} \mathrm{F}\right)$ & $0.72-0.78$ & 0.85 & 0.796 & 0.794 \\
\hline Density (lb./gal @ 60 F) & $6.0-6.5$ & 7.079 & 6.63 & 6.61 \\
\hline Boiling temperature $\left(\mathrm{F}^{\circ}\right)$ & $80-437$ & $356-644$ & 149 & 172 \\
\hline Freezing point $\left(\mathrm{F}^{\circ}\right)$ & -40 & $-40-30$ & -143.5 & -173.2 \\
\hline Autoiginition temperature $\left(\mathrm{F}^{\circ}\right)$ & 495 & $\sim 600$ & 867 & 793 \\
\hline Reid vapor pressure (psi) & $8-15$ & $<0.2$ & 4.6 & 2.3 \\
\hline
\end{tabular}

Source:

Alternative Fuels Data Center, "Properties of Fuel," www.eere.energy.gov/afdc/pdfs/fueltable.pdf , July 2014.

${ }^{\text {a }}$ Standard Chemical Formulas represent idealized fuels. Some table values are expressed in ranges to represent typical fuel variations that are encountered in the field.

${ }^{\mathrm{b}}$ GGE table values above reflect BTU range for common gasoline baseline references (E0, E10, and indolene certification fuel).

${ }^{c} 1$ gallon of E85 has $73 \%$ to $83 \%$ of the energy of one gallon gasoline (variation due to ethanol content in E85) 
Table 6.10

Properties of Conventional and Alternative Gaseous Fuels

\begin{tabular}{|c|c|c|c|}
\hline \multirow[b]{2}{*}{ Property } & \multicolumn{3}{|c|}{ Gaseous Fuels } \\
\hline & Propane (LPG) & $\mathrm{CNG}$ & Hydrogen \\
\hline Standard chemical formula ${ }^{a}$ & $\mathrm{C}_{3} \mathrm{H}_{8}$ & $\mathrm{CH}_{4}$ & $\mathrm{H}_{2}$ \\
\hline Physical state & Compressed gas & Compressed gas & Compressed gas or liquid \\
\hline Molecular weight & 44.1 & 16.04 & 2.02 \\
\hline \multicolumn{4}{|l|}{ Composition (weight \%) } \\
\hline Carbon & 82 & 75 & 0 \\
\hline Hydrogen & 18 & 25 & 100 \\
\hline Oxygen & $\mathrm{n} / \mathrm{a}$ & $\mathrm{n} / \mathrm{a}$ & 0 \\
\hline Main fuel source(s) & Underground reserves & $\begin{array}{l}\text { Underground reserves } \\
\text { and renewable Bio-gas }\end{array}$ & $\begin{array}{l}\text { Natural gas, methanol, } \\
\text { electrolysis, and other energy } \\
\text { sources }\end{array}$ \\
\hline $\begin{array}{l}\text { Gasoline gallon equivalent } \\
(\mathrm{GGE})^{\mathrm{b}} \text { (Fuel unit } \\
\text { measured/GGE) }\end{array}$ & 1.34-1.38 (LPG gal/GGE) & $\begin{array}{l}5.56-5.71(\mathrm{lb} . \\
\text { mass/GGE })^{\mathrm{c}}\end{array}$ & 0.991-1.017 (kg mass/GGE) \\
\hline $\begin{array}{l}\text { Diesel gallon equivalent (DGE) } \\
\text { (Fuel unit measured/DGE) }\end{array}$ & 1.54 (LPG gal/DGE) & 6.38 (lb. mass/DGE) & $\mathrm{n} / \mathrm{a}$ \\
\hline Specific Gravity $\left(60^{\circ} \mathrm{F} / 60^{\circ} \mathrm{F}\right)$ & 1.55 & 0.60 & 0.069 \\
\hline Density (lb./cu ft @60ㅜㄱ) & 0.124 & 0.0458 & 0.0056 \\
\hline Freezing point $\left(\mathrm{F}^{\circ}\right)$ & -305.8 & -296 & -435 \\
\hline Boiling Point $\left({ }^{\circ} \mathrm{F}\right)$ & -44 & -260 & -423 \\
\hline Autoiginition temperature $\left(\mathrm{F}^{\circ}\right)$ & 842 & $900-1,170$ & 932 \\
\hline Reid vapor pressure (psi) & 208 & $\mathrm{n} / \mathrm{a}$ & $\mathrm{n} / \mathrm{a}$ \\
\hline
\end{tabular}

Note: $\mathrm{n} / \mathrm{a}=$ not applicable.

Source:

Alternative Fuels Data Center, "Properties of Fuel," www.eere.energy.gov/afdc/pdfs/fueltable.pdf , July 2014.

\footnotetext{
${ }^{a}$ Standard Chemical Formulas represent idealized fuels. Some table values are expressed in ranges to represent typical fuel variations that are encountered in the field.

${ }^{\mathrm{b}}$ GGE table values above reflect BTU range for common gasoline baseline references (E0, E10, and indolene certification fuel).

${ }^{\mathrm{c}}$ CNG: 1 Gasoline Gallon Equivalent $=5.66 \mathrm{lb}$. (as referenced by NIST Special Publication 854; Report of the 78th NCWM (1993); p. 326; NG data derived from field sampling of pipeline natural gas by IGT/GRI).
} 


\section{Chapter 7 \\ Fleet Vehicles and Characteristics}

Summary Statistics from Tables in this Chapter

\begin{tabular}{|c|c|c|}
\hline Source & & \\
\hline Table 7.1 & Fleet cars, 2013 & $4,438,330$ \\
\hline Figure 7.1 & Fleet trucks $\leq 19,500$ lbs. GVW, 2013 & $4,278,390$ \\
\hline \multirow[t]{4}{*}{ Table 7.3} & Average annual miles per business fleet vehicle & \\
\hline & $S U V s$ & 27,816 \\
\hline & Intermediate cars & 25,068 \\
\hline & Pickup trucks & 25,824 \\
\hline \multirow[t]{8}{*}{ Figure 7.2} & $\begin{array}{l}\text { Average annual miles per Federal Government } \\
\text { fleet vehicle, } 2012\end{array}$ & \\
\hline & $S U V s$ & 10,641 \\
\hline & Sedans & 10,436 \\
\hline & Buses & 9,618 \\
\hline & Heavy trucks & 7,633 \\
\hline & Medium trucks & 6,993 \\
\hline & Light trucks & 6,508 \\
\hline & Ambulances & 5,125 \\
\hline \multirow[t]{6}{*}{ Table 7.4} & Federal government vehicles, FY 2012 & 650,061 \\
\hline & Light trucks $(<8,500 \mathrm{lbs} . G V W)$ & 282,319 \\
\hline & Cars and other passenger vehicles & 245,800 \\
\hline & Medium trucks $(8,500-26,000 \mathrm{lbs} . G V W)$ & 78,630 \\
\hline & Heavy trucks (>26,000 lbs. GVW) & 33,642 \\
\hline & Buses and ambulances & 9,670 \\
\hline
\end{tabular}


Vehicles in fleets of 15 or more are counted as fleet vehicles, as well as vehicles in fleets where five or more vehicles are purchased annually. Historical data on fleets are not available due to definitional changes of what constitutes a fleet.

Figure 7.1. Fleet Vehicles in Service as of January 1, 2013

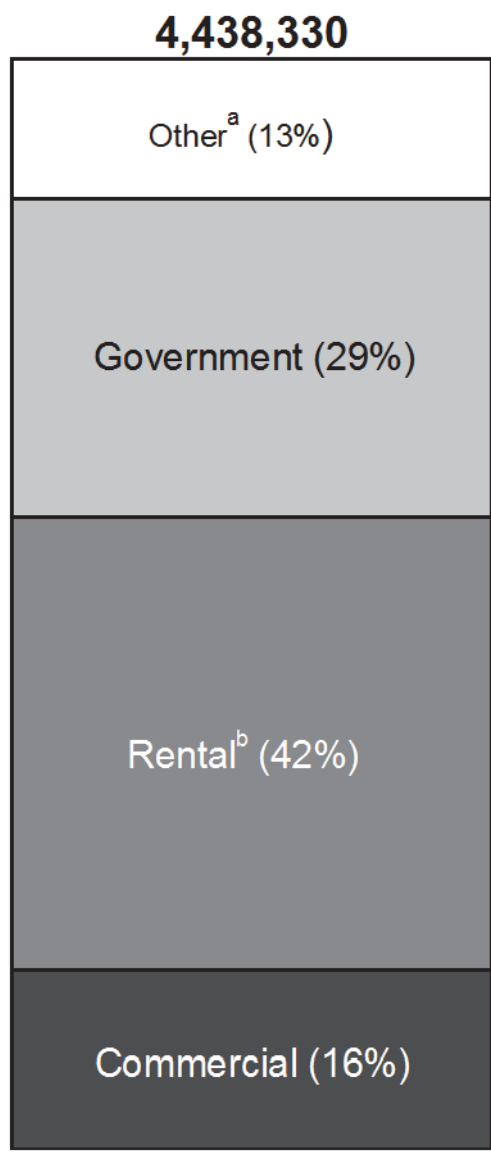

Cars $^{\mathrm{c}}$

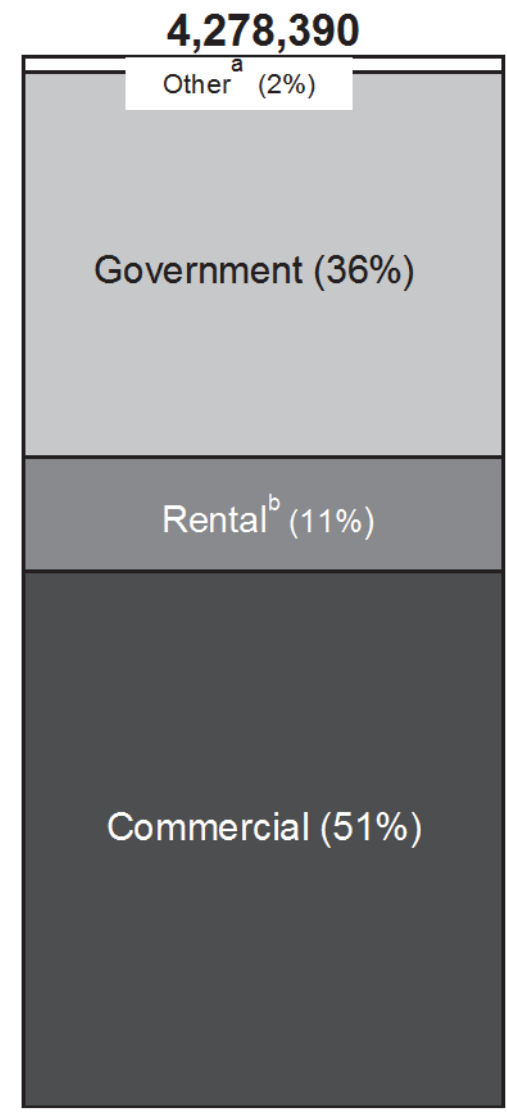

Trucks $^{c}<19,50 I$ lbs. gross vehicle weight

Source:

Bobit Publishing Company, Automotive Fleet Research Department, Automotive Fleet Factbook 2013-2014, Redondo Beach, CA, 2013. (Additional resources: www.fleet-central.com)

${ }^{\mathrm{a}}$ Taxi category includes vans.

${ }^{\mathrm{b}}$ Rental category includes vans and sports utility vehicles under cars, not trucks.

${ }^{c}$ Fleets of 15 or more in operation or 5 or more fleet vehicles purchased annually. 
Rental companies had the largest light fleet vehicle registrations in 2012 with over 1.8 million new vehicles, most of them cars (60.3\%). Only $27.8 \%$ of the new commercial fleet registrations were cars.

Table 7.1

New Light Fleet Vehicle Registrations by Vehicle Type, Model Year 2012

\begin{tabular}{|c|c|c|c|c|}
\hline & Commercial & Rental & Government & Total \\
\hline Cars & $27.8 \%$ & $60.3 \%$ & $33.9 \%$ & $51.1 \%$ \\
\hline Pickup trucks & $33.3 \%$ & $5.5 \%$ & $30.2 \%$ & $13.5 \%$ \\
\hline Vans & $19.3 \%$ & $10.9 \%$ & $15.9 \%$ & $13.2 \%$ \\
\hline Sport utility vehicles & $19.6 \%$ & $23.3 \%$ & $20.1 \%$ & $22.2 \%$ \\
\hline Total & 597,492 & $1,812,036$ & 158,309 & $2,567,837$ \\
\hline
\end{tabular}

Source:

Bobit Publishing Company, Automotive Fleet Factbook 2013-2014, www.automotive-fleet.com/statistics. (Additional resources: www.fleet-central.com)

Table 7.2

Average Length of Time Commercial Fleet Vehicles are in Service, 2012

\begin{tabular}{lc}
\hline Vehicle type & $\begin{array}{c}\text { Average months } \\
\text { in service }\end{array}$ \\
\hline Compact cars & 44 \\
Intermediate cars & 34 \\
Pickup trucks & 49 \\
Minivans & 41 \\
Sport utility vehicles & 33 \\
Full-size vans & 55 \\
\hline
\end{tabular}

Note: Based on data collected from four leading Fleet Management companies.

Source:

Bobit Publishing Company, Automotive Fleet Factbook 2013-2014, www.automotive-fleet.com. (Additional resources: www.fleet-central.com)

Table 7.3

Average Annual Vehicle-Miles of Travel

for Commerical Fleet Vehicles, 2012

\begin{tabular}{lc}
\hline Business fleet vehicles & $\begin{array}{c}\text { Average annual miles of } \\
\text { travel }\end{array}$ \\
\hline Compact cars & 24,876 \\
Intermediate cars & 25,068 \\
Pickup trucks & 25,824 \\
Minivans & 27,864 \\
Sport utility vehicles & 27,816 \\
Full-size vans & 30,204 \\
\hline
\end{tabular}

Source:

Bobit Publishing Company, Automotive Fleet Factbook 2013-2014, www.automotive-fleet.com. (Additional resources: www.fleet-central.com) 
These data, which apply to domestic Federal fleet vehicles, indicate that sport utility vehicles (SUVs) have the highest average annual miles per vehicle, followed closely by sedans and buses.

Figure 7.2. Average Miles per Domestic Federal Vehicle by Vehicle Type, 2012

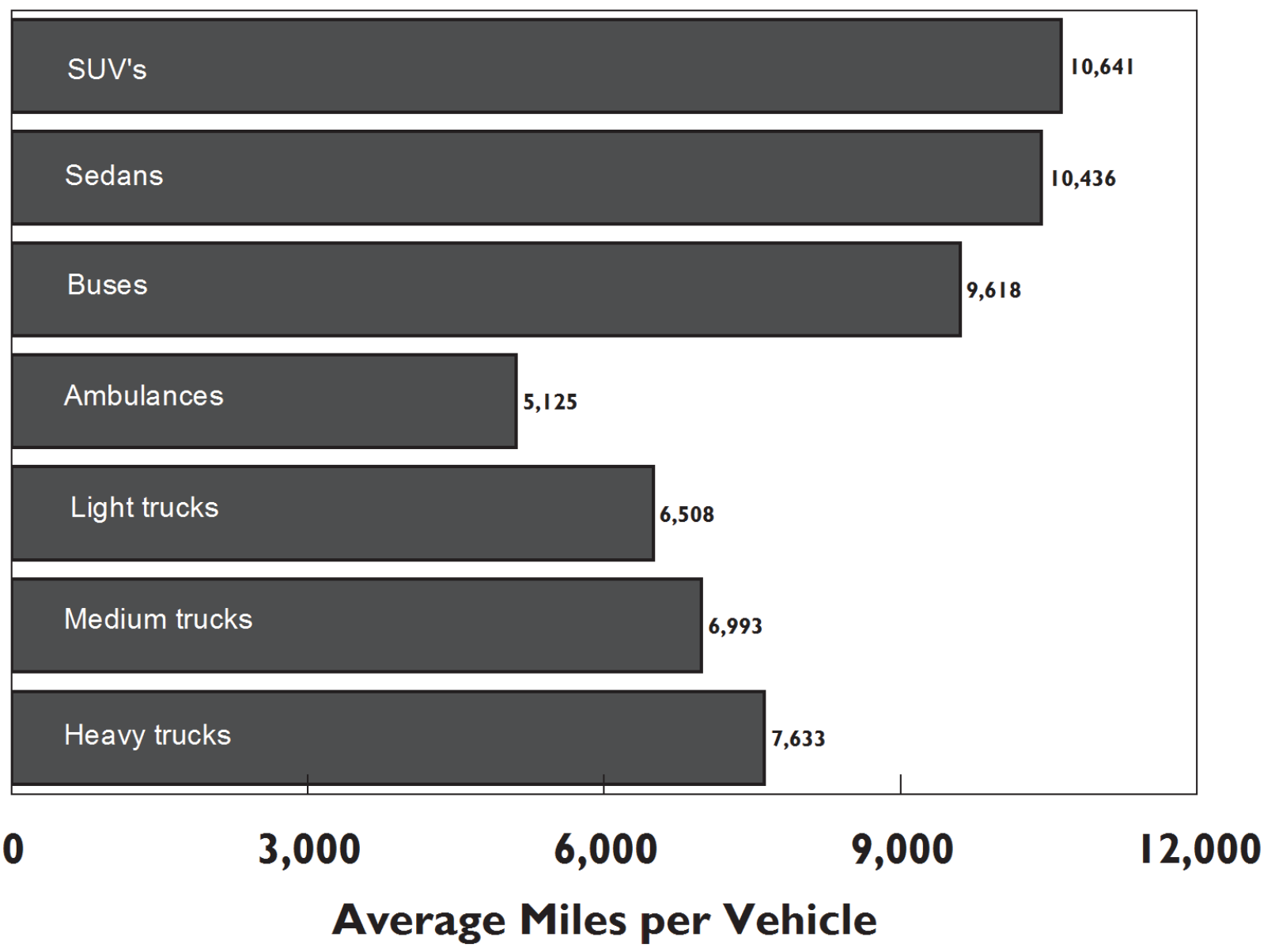

Note: Light trucks $=$ less than 8,500 pounds gross vehicle weight ratio (GVWR).

Medium trucks $=8,501-23,999$ pounds GVWR.

Heavy trucks $=24,000$ pounds GVWR or more.

Source:

U.S. General Services Administrations, Federal Vehicle Policy Division, FY 2012 Federal Fleet Report,

Washington, DC, 2013, Table 4-2. (Additional resources: www.gsa.gov) 
The Federal Government vehicle inventory includes more light trucks than passenger vehicles.

Table 7.4

Federal Government Vehicles, 2001-2012

\begin{tabular}{|c|c|c|c|c|c|c|c|c|c|}
\hline Vehicle Type & 2001 & 2005 & 2006 & 2007 & 2008 & 2009 & 2010 & 2011 & 2012 \\
\hline \multicolumn{10}{|l|}{ Passenger vehicles } \\
\hline Low-speed vehicle & 0 & 0 & 0 & 0 & 0 & 0 & 3,029 & 3,869 & 3,893 \\
\hline Subcompact & 5,462 & 2,401 & 2,181 & 1,968 & 3,058 & 5,935 & 6,797 & 10,658 & 13,867 \\
\hline Compact & 60,938 & 58,284 & 56,220 & 48,495 & 41,482 & 36,662 & 46,489 & 49,657 & 47,999 \\
\hline Midsize & 36,921 & 36,656 & 39,762 & 48,622 & 55,157 & 57,284 & 48,242 & 38,057 & 33,321 \\
\hline Large & 11,107 & 15,966 & 11,783 & 11,907 & 10,679 & 10,230 & 10,063 & 9,146 & 8,571 \\
\hline Limousines & 116 & 191 & 318 & 217 & 238 & 349 & 412 & 158 & 130 \\
\hline Light duty passenger vans & 56,563 & 42,109 & 41,911 & 43,203 & 43,131 & 41,855 & 41,676 & 40,964 & 39,518 \\
\hline Medium duty passenger vans & 727 & 13,252 & 15,657 & 15,231 & 15,696 & 15,362 & 15,218 & 16,633 & 15,740 \\
\hline Light duty SUVs & 40,842 & 50,445 & 52,393 & 53,837 & 56,329 & 64,793 & 66,316 & 68,807 & 73,356 \\
\hline Medium duty SUVs & 0 & 6,096 & 7,192 & 7,733 & 10,837 & 7,344 & 11,117 & 11,448 & 9,405 \\
\hline Total passenger vehicles & 212,676 & 225,400 & 227,417 & 231,213 & 236,607 & 239,814 & 249,359 & 249,397 & 245,800 \\
\hline \multicolumn{10}{|l|}{ Trucks and other vehicles } \\
\hline Light trucks $4 \times 2$ & 227,937 & 243,477 & 241,847 & 243,720 & 243,143 & 244,022 & 241,011 & 238,261 & 233,629 \\
\hline Light trucks $4 \times 4$ & 29,975 & 35,417 & 37,019 & 40,115 & 34,962 & 36,713 & 40,105 & 47,035 & 48,690 \\
\hline Medium trucks & 88,993 & 83,747 & 81,721 & 84,414 & 88,509 & 89,052 & 89,253 & 81,791 & 78,630 \\
\hline Heavy trucks & 27,988 & 35,230 & 33,383 & 32,492 & 32,752 & 32,629 & 32,760 & 33,951 & 33,642 \\
\hline Ambulances & 1,819 & 1,580 & 1,601 & 1,982 & 1,474 & 1,433 & 1,480 & 1,445 & 1,401 \\
\hline Buses & 6,726 & 7,837 & 7,752 & 8,297 & 8,044 & 8,040 & 8,186 & 7,978 & 8,269 \\
\hline $\begin{array}{l}\text { Total trucks and other } \\
\text { vehicles }\end{array}$ & 383,438 & 407,288 & 403,323 & 411,020 & 408,884 & 411,889 & 412,795 & 410,461 & 404,261 \\
\hline $\begin{array}{l}\text { GRAND TOTAL ALL } \\
\text { VEHICLES }\end{array}$ & 596,114 & 632,688 & 630,740 & 642,233 & 645,491 & 651,703 & 662,154 & 659,858 & 650,061 \\
\hline
\end{tabular}

Note: Light trucks $=$ less than 8,500 pounds gross vehicle weight rating (GVWR).

Medium trucks $=8,501-23,999$ pounds GVWR.

Heavy trucks $=24,000$ pounds GVWR or more.

\section{Source:}

U.S. General Services Administration, Federal Supply Service, FY 2012 Federal Fleet Report, Washington, DC, 2013, Tables 2-5 and 2-6. (Additional resources: www.gsa.gov) 
Table 7.5

Federal Fleet Vehicle Acquisitions

by Fuel Type, FY 2002-2012

\begin{tabular}{lrrrrrrrr}
\hline & \multicolumn{10}{c}{ Acquisitions by year } \\
\cline { 2 - 9 } Fuel type & 2002 & \multicolumn{1}{c}{2005} & 2007 & 2008 & 2009 & 2010 & 2011 & 2012 \\
\hline Gasoline & 44,850 & 41,247 & 32,089 & 30,376 & 31,782 & 26,547 & 20,785 & 15,660 \\
Gasoline hybrid & $\mathrm{a}$ & 222 & 458 & 531 & 3,959 & 4,853 & 3,787 & 1,254 \\
Gasoline LGHG $^{\mathrm{b}}$ & 0 & 0 & 0 & 0 & 0 & 0 & 601 & 745 \\
Gasoline plug-in hybrid & 0 & 0 & 0 & 0 & 0 & 0 & 6 & 144 \\
Diesel & 8,107 & 6,049 & 5,809 & 5,897 & 4,742 & 4,136 & 4,422 & 4,383 \\
Diesel hybrid & $\mathrm{c}$ & 1 & 4 & 0 & 4 & 27 & 50 & 36 \\
Diesel LGHG & 0 & 0 & 0 & 0 & 0 & 0 & 14 & 10 \\
CNG & 1,267 & 188 & 129 & 123 & 77 & 60 & 84 & 106 \\
E-85 & 8,054 & 16,892 & 26,581 & 27,792 & 27,850 & 26,789 & 24,785 & 24,214 \\
Electric & 7 & 13 & 7 & 6 & 7 & 1,376 & 450 & 258 \\
LNG & 3 & 0 & 0 & 0 & 0 & 0 & 0 & 0 \\
LPG & 59 & 1 & 4 & 3 & 23 & 2 & 11 & 15 \\
M-85 & 25 & 0 & 0 & 0 & 0 & 0 & 0 & 0 \\
Hydrogen & 0 & 0 & 0 & 1 & 1 & 4 & 4 & 0 \\
\hline Grand total & 62,372 & 64,613 & 65,081 & 64,729 & 68,445 & 63,794 & 54,999 & 46,825 \\
\hline
\end{tabular}

Source:

U.S. General Services Administrations, Federal Vehicle Policy Division, FY 2012 Federal Fleet Report, Washington, DC, 2013, Table 5-4. (Additional resources: www.gsa.gov)

Table 7.6

Fuel Consumed by Federal Government Fleets, FY 2000-2012 (thousand gasoline equivalent gallons)

\begin{tabular}{lrrrrrrrr}
\hline & \multicolumn{1}{c}{ FY00 } & \multicolumn{1}{c}{ FY05 } & \multicolumn{1}{c}{ FY07 } & \multicolumn{1}{c}{ FY08 } & FY09 & FY10 & FY11 & \multicolumn{1}{c}{ FY12 } \\
\hline Gasoline & 284,480 & 300,261 & 293,848 & 299,673 & 301,437 & 322,023 & 321,066 & 302,089 \\
Diesel & 70,181 & 53,363 & 74,806 & 72,262 & 76,456 & 75,329 & 78,252 & 73,228 \\
CNG & 865 & 1,245 & 889 & 731 & 499 & 504 & 436 & 421 \\
Electricity & 1 & 6 & 5 & 4 & 4 & 36 & 90 & 85 \\
Biodiesel (B20) & 569 & 8,052 & 9,515 & 6,991 & 7,393 & 8,258 & 7,696 & 6,353 \\
Biodiesel (B100) & 0 & 0 & 0 & 1 & 5 & 0 & 61 & 344 \\
Methanol/M-85 & 14 & 0 & 0 & 0 & 0 & 0 & 0 & 0 \\
LPG & 34 & 231 & 322 & 399 & 208 & 195 & 187 & 211 \\
Ethanol/E-85 & 347 & 3,060 & 3,854 & 6,293 & 7,923 & 8,201 & 9,521 & 12,261 \\
LNG & 0 & 102 & 95 & 59 & 35 & 0 & 0 & 0 \\
Hydrogen & 0 & 0 & 0 & 0 & 0 & 1 & 0 & 1 \\
\hline Total & 356,491 & 366,320 & 383,334 & 386,415 & 393,961 & 414,548 & 417,308 & 394,994 \\
\hline
\end{tabular}

Source:

U.S. General Services Administrations, Federal Vehicle Policy Division, FY 2012 Federal Fleet Report, Washington, DC, 2013, Table 5-1. (Additional resources: www.gsa.gov)

${ }^{\mathrm{a}}$ Combined with gasoline.

${ }^{\mathrm{b}}$ Low greenhouse gas emissions.

${ }^{\mathrm{c}}$ Combined with diesel.

${ }^{\mathrm{d}}$ B100 cannot be separated from B20 from 2000-2007. 
In FY2000, the General Services Administration owned 143,948 vehicles which they leased to other agencies. In

FY2012, they owned 1,163 vehicles.

Table 7.7

Federal Government Vehicles by Agency, FY 2012

\begin{tabular}{|c|c|c|c|c|c|c|}
\hline Department or agency & Cars & Buses & $\begin{array}{l}\text { Light } \\
\text { trucks }\end{array}$ & $\begin{array}{c}\text { Medium } \\
\text { trucks }\end{array}$ & $\begin{array}{l}\text { Heavy } \\
\text { trucks }\end{array}$ & Total \\
\hline \multicolumn{7}{|l|}{ CIVILIAN } \\
\hline American Battle Monuments Commission & 28 & 0 & 11 & 8 & 0 & 47 \\
\hline Broadcasting Board of Governors & 4 & 10 & 115 & 25 & 19 & 173 \\
\hline Consumer Product Safety Commission & 71 & 0 & 33 & 0 & 0 & 104 \\
\hline Court Services and Offender Supervision Agency & 54 & 0 & 20 & 0 & 0 & 74 \\
\hline Department of Agriculture & 5,046 & 73 & 27,042 & 7,856 & 2,024 & 42,041 \\
\hline Department of Commerce & 301 & 7 & 1,425 & 315 & 52 & 2,100 \\
\hline Department of Education & 74 & 1 & 31 & 0 & 0 & 106 \\
\hline Department of Energy & 750 & 174 & 7,517 & 3,742 & 2,179 & 14,362 \\
\hline Department of Health and Human Services & 2,049 & 17 & 2,403 & 276 & 124 & 4,869 \\
\hline Department of Homeland Security & 12,374 & 451 & 39,418 & 2,373 & 1,428 & 56,044 \\
\hline Department of Housing and Urban Development & 329 & 0 & 73 & 0 & 0 & 402 \\
\hline Department of Justice & 18,383 & 336 & 19,613 & 1,437 & 960 & 40,729 \\
\hline Department of Labor & 1,250 & 302 & 2,479 & 204 & 80 & 4,315 \\
\hline Department of State & 2,663 & 162 & 9,403 & 1,561 & 709 & 14,498 \\
\hline Department of the Interior & 2,848 & 506 & 17,851 & 9,495 & 3,120 & 33,820 \\
\hline Department of Transportation & 1,634 & 3 & 3,666 & 1,007 & 159 & 6,469 \\
\hline Department of Treasury & 2,489 & 1 & 1,149 & 8 & 9 & 3,656 \\
\hline Department of Veterans Affairs & 7,046 & 718 & 8,040 & 855 & 645 & 17,304 \\
\hline Environmental Protection Agency & 268 & 7 & 652 & 125 & 28 & 1,080 \\
\hline Equal Employment Opportunity Commission & 70 & 0 & 11 & 0 & 0 & 81 \\
\hline Federal Communications Commission & 0 & 0 & 103 & 0 & 0 & 103 \\
\hline Federal Housing Finance Agency & 6 & 0 & 0 & 0 & 0 & 6 \\
\hline Federal Maritime Commission & 7 & 0 & 2 & 0 & 0 & 9 \\
\hline Federal Trade Commission & 1 & 0 & 2 & 1 & 0 & 4 \\
\hline General Services Administration & 662 & 3 & 459 & 34 & 5 & 1,163 \\
\hline Government Printing Office & 8 & 0 & 23 & 6 & 5 & 42 \\
\hline Library of Congress & 6 & 2 & 7 & 0 & 3 & 18 \\
\hline National Aeronautics and Space Administration & 523 & 92 & 1,555 & 737 & 369 & 3,276 \\
\hline National Archives \& Records Administration & 12 & 0 & 43 & 10 & 8 & 73 \\
\hline National Gallery of Art & 0 & 0 & 7 & 2 & 1 & 10 \\
\hline National Labor Relations Board & 34 & 0 & 3 & 0 & 0 & 37 \\
\hline National Science Foundation & 34 & 13 & 258 & 180 & 102 & 587 \\
\hline National Transportation Safety Board & 0 & 0 & 6 & 0 & 0 & 6 \\
\hline Nuclear Regulatory Commission & 11 & 0 & 25 & 0 & 5 & 41 \\
\hline Office of Personnel Management & 1,523 & 0 & 139 & 2 & 0 & 1,664 \\
\hline Peace Corps & 43 & 17 & 629 & 0 & 0 & 689 \\
\hline Small Business Administration & 108 & 0 & 57 & 2 & 0 & 167 \\
\hline Smithsonian Institution & 10 & 8 & 345 & 62 & 37 & 462 \\
\hline Social Security Administration & 316 & 6 & 149 & 5 & 26 & 502 \\
\hline Tennessee Valley Authority & 524 & 0 & 1,236 & 1,020 & 43 & 2,823 \\
\hline US Agency for International Development & 89 & 6 & 694 & 24 & 16 & 829 \\
\hline TOTAL CIVILIAN AGENCIES & 61,648 & 2,915 & 146,694 & 31,372 & 12,156 & 254,785 \\
\hline \multicolumn{7}{|l|}{ MILITARY } \\
\hline Corps of Engineers, Civil Works & 835 & 2 & 4,751 & 1,873 & 605 & 8,066 \\
\hline Defense Agencies & 2,220 & 140 & 2,758 & 606 & 430 & 6,154 \\
\hline Department of Air Force & 4,609 & 1,548 & 20,189 & 14,860 & 6,157 & 47,363 \\
\hline Department of Army & 16,104 & 2,423 & 33,172 & 12,524 & 5,622 & 69,845 \\
\hline Department of Navy & 7,793 & 746 & 18,515 & 8,320 & 2,992 & 38,366 \\
\hline United States Marine Corps & 3,549 & 486 & 5,536 & 1,860 & 1,281 & 12,712 \\
\hline TOTAL MILITARY AGENCIES & 35,110 & 5,345 & 84,921 & 40,043 & 17,087 & 182,506 \\
\hline U. S. POSTAL SERVICE & 7,130 & 9 & 190,124 & 7,215 & 4,399 & 208,877 \\
\hline TOTAL ALL FLEETS & 103,888 & 8,269 & 421,739 & 78,630 & 33,642 & 646,168 \\
\hline
\end{tabular}

Note: Light trucks - Less than 8,500 pounds gross vehicle weight ratio (GVWR) (Includes ambulances). Medium trucks $-8,501-23,999$ pounds GVWR. Heavy trucks - 24,000 pounds GVWR or more. Does not include lowspeed vehicles.

\section{Source:}

U.S. General Services Administration, Federal Supply Service, FY 2012 Federal Fleet Report, Washington, DC, 2013, Table 2-1. (Additional resources: www.gsa.gov) 


\section{Chapter 8 \\ Household Vehicles and Characteristics}

Summary Statistics from Tables/Figures in this Chapter

\begin{tabular}{|c|c|c|}
\hline Source & & \\
\hline \multirow[t]{3}{*}{ Table 8.2} & Vehicles per capita, 2012 & 0.801 \\
\hline & Vehicles per licensed driver, 2012 & 1.19 \\
\hline & Vehicles per household, 2012 & 2.08 \\
\hline Table 8.3 & Average household transportation expense, 2012 & $17.5 \%$ \\
\hline \multirow[t]{8}{*}{ Table 8.5} & Share of households owning 3 or more vehicles & \\
\hline & 1960 & $2.5 \%$ \\
\hline & 1970 & $5.5 \%$ \\
\hline & 1980 & $17.5 \%$ \\
\hline & 1990 & $17.3 \%$ \\
\hline & 2000 & $18.3 \%$ \\
\hline & 2010 & $19.5 \%$ \\
\hline & 2012 & $19.3 \%$ \\
\hline \multirow[t]{5}{*}{ Figure 8.1} & Average occupancy rates by vehicle type, 2009 & \\
\hline & Van & 2.35 \\
\hline & Sports Utility & 1.90 \\
\hline & Car & 1.55 \\
\hline & Pickup Truck & 1.49 \\
\hline Table 8.10 & Average annual miles per household vehicle, 2009 & 11,300 \\
\hline Table 8.16 & Share of workers who car pooled, 2012 & $10.0 \%$ \\
\hline \multirow[t]{3}{*}{ Table 8.21} & Long-distance trips in the United States, 2001 & \\
\hline & Person-trips & 2,554 million \\
\hline & Person-miles & 1,138 billion \\
\hline
\end{tabular}


The number of vehicles in the United States is growing faster than the population. The growth in vehicle-miles has slowed in recent years. See Table 8.2 for vehicles per capita and vehicle-miles per capita.

Table 8.1

Population and Vehicle Profile, 1950-2012

\begin{tabular}{|c|c|c|c|c|c|c|}
\hline Year & $\begin{array}{l}\text { Resident } \\
\text { population }^{\text {a }} \\
\text { (thousands) }\end{array}$ & $\begin{array}{c}\text { Total } \\
\text { households } \\
\text { (thousands) }\end{array}$ & $\begin{array}{l}\text { Number of } \\
\text { vehicles in } \\
\text { operation } \\
\text { (thousands) }\end{array}$ & $\begin{array}{c}\text { Total vehicle- } \\
\text { miles } \\
\text { (millions) }\end{array}$ & $\begin{array}{c}\text { Number of } \\
\text { licensed drivers } \\
\text { (thousands) }\end{array}$ & $\begin{array}{l}\text { Number of } \\
\text { civilian } \\
\text { employed } \\
\text { persons } \\
\text { (thousands) }\end{array}$ \\
\hline 1950 & 151,868 & 43,554 & 43,501 & 458,246 & 62,194 & 58,920 \\
\hline 1955 & 165,069 & 47,874 & 56,540 & 605,646 & 74,686 & 62,171 \\
\hline 1960 & 179,979 & 52,799 & 67,906 & 718,762 & 87,253 & 65,778 \\
\hline 1965 & 193,526 & 57,436 & 82,066 & 887,812 & 98,502 & 71,088 \\
\hline 1970 & 205,052 & 63,401 & 98,136 & $1,109,724$ & 111,543 & 78,628 \\
\hline 1975 & 215,973 & 71,120 & 120,054 & $1,327,664$ & 129,791 & 85,846 \\
\hline 1980 & 227,226 & 80,776 & 139,831 & $1,527,295$ & 145,295 & 99,303 \\
\hline 1985 & 238,466 & 86,789 & 157,048 & $1,774,826$ & 156,868 & 107,150 \\
\hline 1986 & 240,651 & 88,458 & 162,094 & $1,834,872$ & 159,487 & 109,597 \\
\hline 1987 & 242,804 & 89,479 & 167,193 & $1,921,204$ & 161,975 & 112,440 \\
\hline 1988 & 245,021 & 91,066 & 171,741 & $2,025,962$ & 162,853 & 114,968 \\
\hline 1989 & 247,342 & 92,830 & 175,960 & $2,096,487$ & 165,555 & 117,342 \\
\hline 1990 & 250,132 & 93,347 & 179,299 & $2,144,362$ & 167,015 & 118,793 \\
\hline 1991 & 253,493 & 94,312 & 181,438 & $2,172,050$ & 168,995 & 117,718 \\
\hline 1992 & 256,894 & 95,669 & 181,519 & $2,247,151$ & 173,125 & 118,492 \\
\hline 1993 & 260,255 & 96,391 & 186,315 & $2,296,378$ & 173,149 & 120,259 \\
\hline 1994 & 263,436 & 97,107 & 188,714 & $2,357,588$ & 175,403 & 123,060 \\
\hline 1995 & 266,557 & 98,990 & 193,441 & $2,422,696$ & 176,628 & 124,900 \\
\hline 1996 & 269,667 & 99,627 & 198,294 & $2,485,848$ & 179,539 & 126,708 \\
\hline 1997 & 272,912 & 101,018 & 201,071 & $2,561,695$ & 182,709 & 129,558 \\
\hline 1998 & 276,115 & 102,528 & 205,043 & $2,631,522$ & 184,980 & 131,463 \\
\hline 1999 & 279,295 & 103,874 & 209,509 & $2,691,056$ & 187,170 & 133,488 \\
\hline 2000 & 282,385 & 104,705 & 213,300 & $2,746,925$ & 190,625 & 136,891 \\
\hline 2001 & 285,309 & 108,209 & 216,683 & $2,797,287$ & 191,276 & 136,933 \\
\hline 2002 & 288,105 & 109,297 & 221,027 & $2,855,508$ & 194,296 & 136,485 \\
\hline 2003 & 290,820 & 111,278 & 225,882 & $2,890,450$ & 196,166 & 137,736 \\
\hline 2004 & 293,463 & 112,000 & 232,167 & $2,964,788$ & 198,889 & 139,252 \\
\hline 2005 & 296,186 & 113,343 & 238,384 & $2,989,430$ & 200,549 & 141,730 \\
\hline 2006 & 298,996 & 114,384 & 244,643 & $3,014,371$ & 202,810 & 144,427 \\
\hline 2007 & 302,004 & 116,011 & 248,701 & $3,031,124$ & 205,742 & 146,047 \\
\hline 2008 & 304,798 & 116,783 & 249,813 & $2,976,528$ & 208,321 & 145,362 \\
\hline 2009 & 307,439 & 117,181 & 248,972 & $2,956,764$ & 209,618 & 139,877 \\
\hline 2010 & 309,326 & 117,538 & 248,231 & $2,967,266$ & 210,115 & 139,064 \\
\hline 2011 & 311,583 & 118,682 & 248,932 & $2,945,402$ & 211,875 & 139,869 \\
\hline 2012 & 313,874 & 121,084 & 251,497 & $2,968,815$ & 211,815 & 142,469 \\
\hline \multicolumn{7}{|c|}{ Average annual percentage change } \\
\hline 1950-2012 & $1.2 \%$ & $1.7 \%$ & $2.9 \%$ & $3.1 \%$ & $2.0 \%$ & $1.4 \%$ \\
\hline 2002-2012 & $0.9 \%$ & $1.0 \%$ & $1.3 \%$ & $0.4 \%$ & $0.9 \%$ & $0.4 \%$ \\
\hline
\end{tabular}

\section{Sources:}

Resident population and civilian employed persons - U.S. Department of Commerce, Bureau of the Census, Online Data Retrieval, Washington, DC, 2014. (Additional resources: www.census.gov)

Vehicles in operation - The Polk Company. FURTHER REPRODUCTION PROHIBITED. (Additional resources: www.polk.com)

Licensed drivers and vehicle-miles - U.S. Department of Transportation, Federal Highway Administration, Highway Statistics 2012, Tables DL-20 and VM-1, and annual. (Additional resources: www.fhwa.dot.gov)

${ }^{a}$ Estimates as of July 1. Includes Armed Forces in the United States. 
Vehicle-miles per capita reached 10,000 miles in 2004 but have declined since that time. There were 1.75 vehicles for every employed civilian in the United States in 2012.

Table 8.2

Vehicles and Vehicle-Miles per Capita, 1950-2012

\begin{tabular}{|c|c|c|c|c|c|c|}
\hline Year & $\begin{array}{l}\text { Vehicles } \\
\text { per capita }\end{array}$ & $\begin{array}{c}\text { Vehicle-miles } \\
\text { per capita }\end{array}$ & $\begin{array}{l}\text { Licensed } \\
\text { drivers per } \\
\text { household }\end{array}$ & $\begin{array}{c}\text { Vehicles per } \\
\text { household }\end{array}$ & $\begin{array}{c}\text { Vehicles } \\
\text { per licensed } \\
\text { driver }\end{array}$ & $\begin{array}{c}\text { Vehicles per } \\
\text { civilian } \\
\text { employed } \\
\text { persons }\end{array}$ \\
\hline 1950 & 0.286 & 3,017 & 1.43 & 1.00 & 0.70 & 0.74 \\
\hline 1955 & 0.343 & 3,669 & 1.56 & 1.18 & 0.76 & 0.91 \\
\hline 1960 & $0.377^{-}$ & 3,994 & 1.65 & 1.29 & 0.78 & 1.03 \\
\hline 1965 & 0.424 & 4,588 & 1.71 & 1.43 & 0.83 & 1.15 \\
\hline 1970 & 0.479 & 5,412 & 1.76 & $1.55^{\circ}$ & 0.88 & 1.25 \\
\hline 1975 & 0.556 & 6,147 & 1.82 & 1.69 & 0.92 & 1.40 \\
\hline 1980 & 0.614 & 6,707 & 1.80 & $1.73^{\circ}$ & 0.96 & 1.41 \\
\hline $1985^{-}$ & 0.659 & 7,443 & 1.81 & $1.81^{\circ}$ & 1.00 & 1.47 \\
\hline 1986 & 0.674 & 7,625 & 1.80 & 1.83 & 1.02 & 1.48 \\
\hline 1987 & 0.689 & 7,913 & 1.81 & 1.87 & 1.03 & 1.49 \\
\hline 1988 & 0.701 & 8,269 & 1.79 & 1.89 & 1.05 & 1.49 \\
\hline 1989 & 0.711 & 8,476 & 1.78 & 1.90 & 1.06 & 1.50 \\
\hline 1990 & 0.717 & 8,573 & 1.79 & 1.92 & 1.07 & 1.51 \\
\hline 1991 & 0.716 & 8,568 & 1.79 & 1.92 & 1.07 & 1.54 \\
\hline 1992 & 0.707 & 8,747 & 1.81 & 1.90 & 1.05 & 1.53 \\
\hline 1993 & 0.716 & 8,824 & 1.80 & 1.93 & 1.08 & 1.55 \\
\hline 1994 & 0.716 & 8,949 & 1.81 & 1.94 & 1.08 & 1.53 \\
\hline 1995 & 0.726 & 9,089 & 1.78 & 1.95 & 1.10 & 1.55 \\
\hline 1996 & 0.735 & 9,218 & 1.80 & 1.99 & 1.10 & 1.56 \\
\hline 1997 & 0.737 & 9,387 & 1.81 & 1.99 & 1.10 & 1.55 \\
\hline 1998 & 0.743 & 9,531 & 1.80 & 2.00 & 1.11 & 1.56 \\
\hline 1999 & 0.750 & 9,635 & 1.80 & 2.02 & 1.12 & 1.57 \\
\hline 2000 & 0.755 & 9,728 & 1.82 & 2.04 & 1.12 & 1.56 \\
\hline 2001 & 0.759 & 9,804 & 1.77 & 2.00 & 1.13 & 1.58 \\
\hline 2002 & 0.767 & 9,911 & 1.78 & 2.02 & 1.14 & 1.62 \\
\hline 2003 & 0.777 & 9,939 & 1.76 & 2.03 & 1.15 & 1.64 \\
\hline 2004 & 0.791 & 10,103 & 1.78 & 2.07 & 1.17 & 1.67 \\
\hline 2005 & 0.805 & 10,093 & 1.77 & 2.10 & 1.19 & 1.68 \\
\hline 2006 & 0.818 & 10,082 & 1.77 & 2.14 & 1.21 & 1.69 \\
\hline 2007 & 0.824 & 10,037 & 1.77 & 2.14 & 1.21 & 1.70 \\
\hline 2008 & 0.820 & 9,766 & 1.78 & 2.14 & 1.20 & 1.72 \\
\hline 2009 & 0.810 & 9,617 & 1.79 & 2.12 & 1.19 & 1.78 \\
\hline 2010 & 0.802 & 9,593 & 1.79 & 2.11 & 1.18 & 1.79 \\
\hline 2011 & 0.799 & 9,469 & 1.79 & 2.10 & 1.17 & 1.78 \\
\hline 2012 & 0.801 & 9,459 & 1.75 & 2.08 & 1.19 & 1.77 \\
\hline \multicolumn{7}{|c|}{ Average annual percentage change } \\
\hline 1950-2012 & $1.7 \%$ & $1.9 \%$ & $0.3 \%$ & $1.2 \%$ & $0.9 \%$ & $1.4 \%$ \\
\hline 2002-2012 & $0.4 \%$ & $-0.5 \%$ & $-0.2 \%$ & $0.3 \%$ & $0.4 \%$ & $0.9 \%$ \\
\hline
\end{tabular}

\section{Sources:}

Resident population and civilian employed persons - U.S. Department of Commerce, Bureau of the Census, Online Data Retrieval, Washington, DC, 2014. (Additional resources: www.census.gov)

Vehicles in operation - The Polk Company. FURTHER REPRODUCTION PROHIBITED. (Additional resources: www.polk.com)

Vehicle-miles - U.S. Department of Transportation, Federal Highway Administration, Highway Statistics 2012, Table VM-1 and annual. (Additional resources: www.fhwa.dot.gov)

\footnotetext{
${ }^{a}$ Includes all vehicles (light and heavy).
} 
Table 8.3

Average Annual Expenditures of Households by Income, 2012

\begin{tabular}{|c|c|c|c|c|c|}
\hline & \multirow[b]{2}{*}{$\begin{array}{c}\text { All } \\
\text { households }\end{array}$} & \multicolumn{4}{|c|}{ Income before taxes } \\
\hline & & $\begin{array}{r}\text { Less than } \\
\$ 5,000 \\
\end{array}$ & $\begin{array}{r}\$ 5,000- \\
\$ 9,999 \\
\end{array}$ & $\begin{array}{r}\$ 10,000- \\
\$ 14,999 \\
\end{array}$ & $\begin{array}{r}\$ 15,000- \\
\$ 19,999 \\
\end{array}$ \\
\hline \multirow[t]{2}{*}{ Total expenditures } & $\$ 51,442$ & $\$ 23,462$ & $\$ 19,783$ & $\$ 20,188$ & $\$ 25,691$ \\
\hline & \multicolumn{5}{|c|}{ Percentage of total expenditures ${ }^{b}$} \\
\hline Food $^{\mathrm{c}}$ & $12.8 \%$ & $16.0 \%$ & $15.3 \%$ & $15.9 \%$ & $15.4 \%$ \\
\hline Housing & $32.8 \%$ & $38.9 \%$ & $41.7 \%$ & $40.5 \%$ & $39.4 \%$ \\
\hline Apparel and services & $3.4 \%$ & $4.0 \%$ & $3.9 \%$ & $2.9 \%$ & $3.7 \%$ \\
\hline Transportation & $17.5 \%$ & $16.7 \%$ & $13.9 \%$ & $14.8 \%$ & $16.1 \%$ \\
\hline Vehicle purchases (net outlay) & $6.2 \%$ & $6.7 \%$ & $3.9 \%$ & $3.5 \%$ & $4.3 \%$ \\
\hline Gasoline and motor oil & $5.4 \%$ & $4.9 \%$ & $5.3 \%$ & $5.6 \%$ & $6.1 \%$ \\
\hline Other vehicle expenditures & $4.8 \%$ & $3.9 \%$ & $3.7 \%$ & $5.1 \%$ & $5.0 \%$ \\
\hline Public transportation & $1.1 \%$ & $1.1 \%$ & $1.0 \%$ & $0.7 \%$ & $0.7 \%$ \\
\hline Health care & $6.9 \%$ & $5.8 \%$ & $6.7 \%$ & $8.6 \%$ & $8.5 \%$ \\
\hline Entertainment & $5.1 \%$ & $4.3 \%$ & $4.1 \%$ & $5.1 \%$ & $4.2 \%$ \\
\hline Personal Insurance \& pensions & $10.9 \%$ & $0.9 \%$ & $1.7 \%$ & $2.3 \%$ & $3.2 \%$ \\
\hline Others ${ }^{\mathrm{d}}$ & $9.6 \%$ & $12.9 \%$ & $12.0 \%$ & $9.2 \%$ & $8.8 \%$ \\
\hline Households $^{\mathrm{e}}$ (thousands) & 124,416 & 4,906 & 5,171 & 8,094 & 8,006 \\
\hline Percentage of households & $100.0 \%$ & $3.9 \%$ & $4.2 \%$ & $6.5 \%$ & $6.4 \%$ \\
\hline \multirow[t]{3}{*}{ Average number of vehicles in $\mathrm{HH}$} & 1.9 & 0.9 & 0.8 & 0.9 & 1.2 \\
\hline & \multicolumn{5}{|c|}{ Income before taxes } \\
\hline & $\begin{array}{r}\$ 20,000- \\
\$ 29,999 \\
\end{array}$ & $\begin{array}{r}\$ 30,000- \\
\$ 39,999 \\
\end{array}$ & $\begin{array}{r}\$ 40,000- \\
\$ 49,999\end{array}$ & $\begin{array}{r}\$ 50,000- \\
\$ 69,999 \\
\end{array}$ & $\begin{array}{l}\$ 70,000 \\
\text { and over }\end{array}$ \\
\hline \multirow[t]{2}{*}{ Total expenditures } & $\$ 30,869$ & $\$ 36,229$ & $\$ 41,567$ & $\$ 49,982$ & $\$ 85,410$ \\
\hline & \multicolumn{5}{|c|}{ Percentage of total expenditures ${ }^{b}$} \\
\hline Food $^{\mathrm{c}}$ & $13.8 \%$ & $13.7 \%$ & $13.7 \%$ & $13.0 \%$ & $11.9 \%$ \\
\hline Housing & $38.0 \%$ & $36.5 \%$ & $35.2 \%$ & $32.6 \%$ & $30.2 \%$ \\
\hline Apparel and services & $3.4 \%$ & $3.2 \%$ & $3.4 \%$ & $3.1 \%$ & $3.4 \%$ \\
\hline Transportation & $16.2 \%$ & $17.4 \%$ & $18.1 \%$ & $19.9 \%$ & $17.3 \%$ \\
\hline Vehicle purchases (net outlay) & $4.6 \%$ & $4.8 \%$ & $5.3 \%$ & $7.7 \%$ & $6.7 \%$ \\
\hline Gasoline and motor oil & $6.1 \%$ & $6.5 \%$ & $6.4 \%$ & $6.2 \%$ & $4.7 \%$ \\
\hline Other vehicle expenditures & $4.7 \%$ & $5.4 \%$ & $5.7 \%$ & $5.1 \%$ & $4.6 \%$ \\
\hline Public transportation & $0.7 \%$ & $0.8 \%$ & $0.8 \%$ & $0.9 \%$ & $1.3 \%$ \\
\hline Health care & $9.2 \%$ & $8.0 \%$ & $7.8 \%$ & $7.4 \%$ & $6.1 \%$ \\
\hline Entertainment & $4.8 \%$ & $5.0 \%$ & $4.7 \%$ & $4.9 \%$ & $5.3 \%$ \\
\hline Personal Insurance \& pensions & $4.1 \%$ & $6.5 \%$ & $8.2 \%$ & $9.8 \%$ & $14.4 \%$ \\
\hline Others ${ }^{\mathrm{d}}$ & $9.7 \%$ & $8.7 \%$ & $8.2 \%$ & $8.5 \%$ & $10.2 \%$ \\
\hline Households $^{\mathrm{e}}$ (thousands) & 14,515 & 13,526 & 11,010 & 17,972 & 41,216 \\
\hline Percentage of households & $11.7 \%$ & $10.9 \%$ & $8.8 \%$ & $14.4 \%$ & $33.1 \%$ \\
\hline Average number of vehicles in $\mathrm{HH}$ & 1.4 & 1.6 & 1.9 & 2.1 & 2.7 \\
\hline
\end{tabular}

\section{Source:}

U.S. Department of Labor, Bureau of Labor Statistics, website: www.bls.gov/cex/, March 2014. (Additional resources: www.bls.gov)

${ }^{a}$ Public assistance monies are included in reported income. Data for those reporting income.

${ }^{\mathrm{b}}$ Percentages may not sum to totals due to rounding.

${ }^{\mathrm{c}}$ Includes alcoholic beverages.

d Includes personal care, reading, education, tobacco and smoking supplies, cash contributions, and miscellaneous items.

e The term household refers to a "consumer unit," which is defined differently than households on Table 8.1. 
The average amount of money that a household spends in a year has changed very little between 1985 and 2012 in constant dollar terms. Expenditures on transportation were 19.4\% of the total in 1985, but were $17.5 \%$ in 2012. Vehicle purchases made up more than one-third of transportation expenditures in 2012, while gas and oil were $31 \%$.

Table 8.4

Annual Household Expenditures for Transportation, 1985-2012 (constant 2012 dollars)

\begin{tabular}{|c|c|c|c|c|c|c|c|}
\hline \multirow[b]{2}{*}{ Year } & \multicolumn{5}{|c|}{ Transportation expenditures } & \multirow{2}{*}{$\begin{array}{c}\text { Average } \\
\text { annual } \\
\text { household } \\
\text { expenditures }\end{array}$} & \multirow{2}{*}{$\begin{array}{l}\text { Transportation } \\
\text { share of } \\
\text { annual } \\
\text { expenditures }\end{array}$} \\
\hline & $\begin{array}{l}\text { Vehicle } \\
\text { purchases }\end{array}$ & $\begin{array}{c}\text { Gas \& } \\
\text { Oil }\end{array}$ & $\begin{array}{c}\text { Other } \\
\text { vehicle } \\
\text { expenses }\end{array}$ & $\begin{array}{c}\text { Public } \\
\text { transportation }\end{array}$ & $\begin{array}{c}\text { Total } \\
\text { transportation }\end{array}$ & & \\
\hline 1985 & 4,402 & 2,232 & 2,723 & 565 & 9,920 & 51,159 & $19.4 \%$ \\
\hline 1986 & 4,898 & 1,934 & 2,878 & 524 & 10,233 & 51,196 & $20.0 \%$ \\
\hline 1987 & 4,074 & 1,781 & 2,892 & 523 & 9,273 & 50,074 & $18.5 \%$ \\
\hline 1988 & 4,635 & 1,813 & 3,014 & 516 & 9,976 & 51,215 & $19.5 \%$ \\
\hline 1989 & 4,347 & 1,827 & 3,081 & 515 & 9,771 & 52,442 & $18.6 \%$ \\
\hline 1990 & 3,814 & 1,852 & 2,930 & 532 & 9,128 & 51,052 & $17.9 \%$ \\
\hline 1991 & 3,631 & 1,682 & 2,994 & 519 & 8,825 & 51,392 & $17.2 \%$ \\
\hline 1992 & 3,546 & 1,592 & 2,955 & 470 & 8,562 & 49,956 & $17.1 \%$ \\
\hline 1993 & 3,677 & 1,552 & 3,000 & 505 & 8,733 & 49,948 & $17.5 \%$ \\
\hline 1994 & 4,188 & 1,534 & 3,081 & 609 & 9,413 & 50,721 & $18.6 \%$ \\
\hline 1995 & 4,031 & 1,528 & 3,108 & 553 & 9,221 & 50,615 & $18.2 \%$ \\
\hline 1996 & 4,276 & 1,618 & 3,142 & 626 & 9,661 & 52,081 & $18.5 \%$ \\
\hline 1997 & 4,085 & 1,588 & 3,307 & 558 & 9,540 & 51,707 & $18.5 \%$ \\
\hline 1998 & 4,286 & 1,451 & 3,219 & 601 & 9,557 & 52,483 & $18.2 \%$ \\
\hline 1999 & 4,695 & 1,476 & 3,218 & 562 & 9,953 & 53,944 & $18.5 \%$ \\
\hline 2000 & 4,621 & 1,755 & 3,127 & 588 & 10,090 & 53,649 & $18.8 \%$ \\
\hline 2001 & 4,898 & 1,672 & 3,172 & 525 & 10,266 & 53,665 & $19.1 \%$ \\
\hline 2002 & 4,822 & 1,598 & 3,254 & 516 & 10,189 & 54,313 & $18.8 \%$ \\
\hline 2003 & 4,830 & 1,688 & 3,015 & 499 & 10,034 & 53,333 & $18.8 \%$ \\
\hline 2004 & 4,129 & 1,942 & 2,874 & 536 & 9,482 & 52,743 & $18.0 \%$ \\
\hline 2005 & 4,166 & 2,366 & 2,750 & 527 & 9,809 & 54,558 & $18.0 \%$ \\
\hline 2006 & 3,896 & 2,536 & 2,682 & 575 & 9,689 & 55,119 & $17.6 \%$ \\
\hline 2007 & 3,592 & 2,640 & 2,870 & 596 & 9,698 & 54,965 & $17.6 \%$ \\
\hline 2008 & 2,938 & 2,895 & 2,795 & 547 & 9,175 & 53,837 & $17.0 \%$ \\
\hline 2009 & 2,843 & 2,125 & 2,714 & 513 & 8,195 & 52,511 & $15.6 \%$ \\
\hline 2010 & 2,725 & 2,245 & 2,594 & 519 & 8,083 & 50,655 & $16.0 \%$ \\
\hline 2011 & 2,724 & 2,710 & 2,505 & 527 & 8,465 & 50,734 & $16.7 \%$ \\
\hline 2012 & 3,210 & 2,756 & 2,490 & 542 & 8,998 & 51,442 & $17.5 \%$ \\
\hline
\end{tabular}

Source:

U.S. Department of Labor, Bureau of Labor Statistics, Consumer Expenditure Survey, www.bls.gov/cex, March 2014. (Additional resources: www.bls.gov)

${ }^{\text {a }}$ Other vehicle expenses include vehicle finance charges, maintenance and repairs, insurance, licenses, and other vehicle charges. 
Household vehicle ownership shows a dramatic increase from 1960 to 1990. In 1960, nearly $79 \%$ of households owned less than two vehicles; by 1990, it declined to 45\%. Census data prior to 1990 indicated that the majority of households owned one vehicle; in 1990 that changed to two vehicles. Since 2000, less than 10\% of households had no vehicles. The American Community Survey now collects these data on an annual basis, thus 2011 and 2012 data are available.

Table 8.5

Household Vehicle Ownership, 1960-2012 Census (percentage)

\begin{tabular}{ccccc}
\hline & $\begin{array}{c}\text { No } \\
\text { vehicles }\end{array}$ & $\begin{array}{c}\text { One } \\
\text { vehicle }\end{array}$ & $\begin{array}{c}\text { Two } \\
\text { vehicles }\end{array}$ & $\begin{array}{c}\text { Three or } \\
\text { more } \\
\text { vehicles }\end{array}$ \\
\hline 1960 & $21.5 \%$ & $56.9 \%$ & $19.0 \%$ & $2.5 \%$ \\
1970 & $17.5 \%$ & $47.7 \%$ & $29.3 \%$ & $5.5 \%$ \\
1980 & $12.9 \%$ & $35.5 \%$ & $34.0 \%$ & $17.5 \%$ \\
1990 & $11.5 \%$ & $33.7 \%$ & $37.4 \%$ & $17.3 \%$ \\
2000 & $9.4 \%$ & $33.8 \%$ & $38.6 \%$ & $18.3 \%$ \\
2010 & $9.1 \%$ & $33.8 \%$ & $37.6 \%$ & $19.5 \%$ \\
2011 & $9.3 \%$ & $34.1 \%$ & $37.5 \%$ & $19.1 \%$ \\
2012 & $9.2 \%$ & $34.1 \%$ & $37.3 \%$ & $19.3 \%$ \\
\hline
\end{tabular}

\section{Source:}

U. S. Department of Transportation, Volpe National Transportation Systems Center, Journey-to-Work Trends in the United States and its Major Metropolitan Area, 1960-1990, Cambridge, MA, 1994, p. 2-2.

2000 data - U.S. Bureau of the Census, American Fact Finder, factfinder.census.gov, Table QT-04, August 2001. (Additional resources: www.census.gov)

2010-2012 data - U.S. Bureau of the Census, American Community Survey, Table CP04, 2012. 


\section{National Household Travel Survey Daily Trip Data}

The Department of Transportation (DOT) collected data on daily trips in 1969, 1977, 1983, 1990 and 1995 via the Nationwide Personal Transportation Survey (NPTS). For 2001, the DOT combined the collection of long trip and daily trip data into one survey - the 2001 National Household Travel Survey (NHTS). The long trip data were not included in the 2009 NHTS.

The NHTS is the nation's inventory of daily travel. The survey includes demographic characteristics of households, people, vehicles, and detailed information on daily travel for all purposes by all modes. NHTS survey data are collected from a sample of U.S. households and expanded to provide national estimates of trips and miles by travel mode, trip purpose, and a host of household attributes.

The NHTS was designed to continue the NPTS series, but as with all data surveys, caution should be used when comparing statistics from one survey to another due to changes in terminology, survey procedures, and target population. The 2001 and 2009 surveys collected data on trips of children under 5 years of age, while the previous NPTS did not. Improved methodologies first used in the collection of trip information in the 1995 NPTS make it difficult to compare these data with past NPTS survey data. Thus, the 1990 NPTS trip data have been adjusted to make it comparable with the later surveys.

The next NHTS will be conducted in 2015.

Table 8.6

Demographic Statistics from the 1969, 1977, 1983, 1990, 1995 NPTS and 2001, 2009 NHTS

\begin{tabular}{lcccccccc}
\hline & & & & & & & & $\begin{array}{c}\text { Percent } \\
\text { change } \\
\end{array}$ \\
& 1969 & 1977 & 1983 & 1990 & 1995 & 2001 & 2009 & $1969-2009$ \\
\hline Persons per household & 3.16 & 2.83 & 2.69 & 2.56 & 2.63 & 2.58 & 2.50 & $-21 \%$ \\
Vehicles per household & 1.16 & 1.59 & 1.68 & 1.77 & 1.78 & 1.89 & 1.87 & $61 \%$ \\
Workers per household & 1.21 & 1.23 & 1.21 & 1.27 & 1.33 & 1.35 & 1.34 & $11 \%$ \\
Licensed drivers per household & 1.65 & 1.69 & 1.72 & 1.75 & 1.78 & 1.77 & 1.88 & $14 \%$ \\
Vehicles per worker & 0.96 & 1.29 & 1.39 & 1.40 & 1.34 & 1.39 & 1.40 & $46 \%$ \\
Vehicles per licensed driver & 0.70 & 0.94 & 0.98 & 1.01 & 1.00 & 1.06 & 1.00 & $42 \%$ \\
Average vehicle trip length (miles) & 8.89 & 8.34 & 7.90 & 8.98 & 9.06 & 9.87 & 9.72 & $9 \%$ \\
\hline
\end{tabular}

Note: Average vehicle trip length for 1990 and 1995 is calculated using only those records with trip mileage information present. The 1969 survey does not include pickups and other light trucks as household vehicles. Data on vehicles per household and licensed drivers per household will not match Table 8.2.

Sources:

U.S. Department of Transportation, Federal Highway Administration, 1990 Nationwide Personal Transportation Survey: Summary of Travel Trends, FHWA-PL-92-027, Washington, DC, March 1992, Table 2. Data for 1995, 2001 and 2009 were generated from the website nhts.ornl.gov. (Additional resources: www.fhwa.dot.gov) 
Due to methodology improvements in collecting trip information, the 2001 and 1995 data should be compared only to the 1990 adjusted data. The original 1990 data are comparable to all previous surveys; however, comparisons should always be made with caution because of differing survey methodologies.

Table 8.7

\section{Average Annual Vehicle-Miles, Vehicle Trips and Trip Length per Household} 1969, 1977, 1983, 1990, 1995 NPTS and 2001, 2009 NHTS

\begin{tabular}{|c|c|c|}
\hline & ey-to-v & All trips \\
\hline \multicolumn{3}{|c|}{ Average annual vehicle-miles per household } \\
\hline 1969 & 4,183 & 12,423 \\
\hline 1977 & 3,815 & 12,036 \\
\hline 1983 & 3,538 & 11,739 \\
\hline 1990 original & 4,853 & 15,100 \\
\hline 1990 adjusted & 4,853 & 18,161 \\
\hline 1995 & 6,492 & 20,895 \\
\hline 2001 & 5,724 & 21,171 \\
\hline 2009 & 5,513 & 19,850 \\
\hline \multicolumn{3}{|c|}{ Average annual vehicle trips per household } \\
\hline 1969 & 445 & 1,396 \\
\hline 1977 & 423 & 1,442 \\
\hline 1983 & 414 & 1,486 \\
\hline 1990 original & 448 & 1,702 \\
\hline 1990 adjusted & 448 & 2,077 \\
\hline 1995 & 553 & 2,321 \\
\hline 2001 & 479 & 2,171 \\
\hline 2009 & 457 & 2,068 \\
\hline \multicolumn{3}{|c|}{ Average vehicle trip length (miles) } \\
\hline 1969 & 9.4 & 8.9 \\
\hline 1977 & 9.0 & 8.4 \\
\hline 1983 & 8.5 & 7.9 \\
\hline 1990 original & 11.0 & 9.0 \\
\hline 1990 adjusted & 11.0 & 8.9 \\
\hline 1995 & 11.8 & 9.1 \\
\hline 2001 & 12.2 & 9.9 \\
\hline 2009 & 12.2 & 9.7 \\
\hline
\end{tabular}

Sources:

U.S. Department of Transportation, Federal Highway Administration, 1990 Nationwide Personal Transportation Survey: Summary of Travel Trends, FHWA-PL-92-027, Washington, DC, March 1992, Table 7. 1990 adjusted data - Oak Ridge National Laboratory, Oak Ridge, TN, August 1998. 1995 NPTS, 2001, 2009 NHTS data were generated from the website nhts.ornl.gov. (Additional resources: www.fhwa.dot.gov, nhts.ornl.gov)

${ }^{a}$ It is believed that the methodology changes in the 1995 NPTS did not affect journey-to-work trips; therefore, no adjustment is necessary. 
In 2001 and 2009 vehicle-miles traveled (vmt) for a three-person household is around 28,000 miles. The number of drivers in a household makes a big difference in vmt, as does the presence of children in the household. Households with children have more than double the vmt of households without children.

Table 8.8

Average Number of Vehicles and Vehicle Travel per Household, 1990 NPTS and 2001 and 2009 NHTS

\begin{tabular}{lccc||rrr}
\hline \multicolumn{1}{l||}{} & \multicolumn{3}{c||}{$\begin{array}{c}\text { Average } \\
\text { number of vehicles } \\
\text { per household }\end{array}$} & \multicolumn{3}{c}{$\begin{array}{c}\text { Average } \\
\text { vehicle-miles traveled } \\
\text { per household }\end{array}$} \\
\hline $\begin{array}{l}\text { Number of licensed } \\
\text { drivers }\end{array}$ & $\mathbf{1 9 9 0}$ & $\mathbf{2 0 0 1}$ & $\mathbf{2 0 0 9}$ & $\mathbf{1 9 9 0}$ & $\mathbf{2 0 0 1}$ & $\mathbf{2 0 0 9}$ \\
\hline 1 & 1.5 & 1.2 & 1.1 & 15,200 & 9,700 & 8,800 \\
2 & 2.1 & 2.2 & 2.2 & 22,900 & 25,800 & 23,500 \\
3 & 2.9 & 3.0 & 3.0 & 29,400 & 37,900 & 37,700 \\
4 or more & 3.8 & 3.8 & 3.9 & 40,500 & 47,200 & 55,200 \\
\hline Household size & & & & & & \\
\hline 1 person & 1.2 & 1.0 & 1.0 & 11,400 & 7,500 & 7,100 \\
2 persons & 1.9 & 2.0 & 2.0 & 19,300 & 21,200 & 17,500 \\
3 persons & 2.2 & 2.3 & 2.3 & 23,700 & 28,400 & 27,900 \\
4 persons & 2.4 & 2.4 & 2.4 & 25,300 & 28,600 & 33,200 \\
5 persons & 2.4 & 2.4 & 2.4 & 24,900 & 33,200 & 33,700 \\
6 or more persons & 2.7 & 2.5 & 2.4 & 29,200 & 33,800 & 33,600 \\
\hline Household urban status & & & & & & \\
\hline Urban & 1.9 & 1.8 & 1.7 & 19,000 & 19,300 & 17,600 \\
Rural & 2.1 & 2.3 & 2.4 & 22,200 & 28,400 & 27,700 \\
\hline Household composition & & & & & & \\
\hline With children & 2.2 & 2.2 & 2.2 & 24,100 & 28,300 & 30,400 \\
Without children & 1.8 & 1.7 & 1.7 & 17,600 & 16,700 & 14,400 \\
\hline All households & $\mathbf{1 . 8}$ & $\mathbf{1 . 9}$ & $\mathbf{1 . 9}$ & $\mathbf{1 8 , 3 0 0}$ & $\mathbf{2 1 , 2 0 0}$ & $\mathbf{1 9 , 9 0 0}$ \\
\hline
\end{tabular}

Source:

Generated from the Department of Transportation, Federal Highway Administration, Nationwide Personal Transportation Survey Public Use Files, Washington, DC, 2000 and the National Household Travel Survey website: nhts.ornl.gov. (Additional resources: nhts.ornl.gov) 
In 2009, $22 \%$ of vehicle trips were traveling to and from work. Another $22 \%$ of trips were for shopping.

Shopping is done close to home, as the average trip length for shopping was only 6.5 miles.

Table 8.9

Trip Statistics ${ }^{a}$ by Trip Purpose, 2001 and 2009 NHTS

\begin{tabular}{|c|c|c|c|c|c|c|c|c|}
\hline \multirow[b]{2}{*}{ Trip purpose } & \multicolumn{2}{|c|}{ Share of trips } & \multicolumn{2}{|c|}{$\begin{array}{l}\text { Share of vehicle- } \\
\text { miles traveled }\end{array}$} & \multicolumn{2}{|c|}{$\begin{array}{l}\text { Trip length } \\
\text { (miles) }\end{array}$} & \multicolumn{2}{|c|}{$\begin{array}{l}\text { Trip length } \\
\text { (minutes) }\end{array}$} \\
\hline & 2001 & 2009 & 2001 & 2009 & 2001 & 2009 & 2001 & 2009 \\
\hline To/from work & $22.1 \%$ & $22.3 \%$ & $27.0 \%$ & $28.7 \%$ & 12.1 & 12.2 & 22.3 & 22.9 \\
\hline Work-related business & $4.1 \%$ & $3.9 \%$ & $8.4 \%$ & $7.2 \%$ & 20.3 & 17.2 & 30.9 & 27.5 \\
\hline Shopping & $21.1 \%$ & $22.8 \%$ & $14.5 \%$ & $15.5 \%$ & 6.7 & 6.5 & 14.4 & 14.4 \\
\hline Other family/personal business & $24.7 \%$ & $21.9 \%$ & $18.7 \%$ & $15.7 \%$ & 7.5 & 6.8 & 15.2 & 14.8 \\
\hline School/church & $4.9 \%$ & $5.0 \%$ & $3.7 \%$ & $4.6 \%$ & 7.5 & 8.8 & 15.8 & 17.5 \\
\hline Medical/dental & $2.2 \%$ & $2.6 \%$ & $2.2 \%$ & $2.6 \%$ & 9.9 & 9.9 & 20.7 & 21.2 \\
\hline Vacation & $0.4 \%$ & $0.7 \%$ & $1.8 \%$ & $2.3 \%$ & 47.4 & 31.4 & 59.6 & 41.3 \\
\hline Visit friends/relatives & $6.3 \%$ & $5.7 \%$ & $9.4 \%$ & $9.4 \%$ & 14.9 & 15.7 & 24.4 & 24.6 \\
\hline Other social/recreational & $13.7 \%$ & $14.9 \%$ & $13.2 \%$ & $13.5 \%$ & 9.6 & 8.6 & 18.2 & 17.2 \\
\hline Other & $0.5 \%$ & $0.3 \%$ & $1.0 \%$ & $0.6 \%$ & 18.1 & 19.0 & 31.4 & 29.7 \\
\hline All & $100.0 \%$ & $100.0 \%$ & $100.0 \%$ & $100.0 \%$ & 9.9 & 9.7 & 18.7 & 18.6 \\
\hline
\end{tabular}

Note: The "All" category for average trip length and duration includes records for which trip purpose was not identified.

\section{Source:}

Generated from the National Household Travel Survey website: nhts.ornl.gov.

${ }^{a}$ Percentages may not sum to totals due to rounding. 
While car occupancy stayed nearly constant from 1995 to 2009, most other vehicle types showed increased occupancy. Vans and sport utility vehicles have higher vehicle occupancies than cars.

Figure 8.1. Average Vehicle Occupancy by Vehicle Type, 1995 NPTS and 2009 NHTS

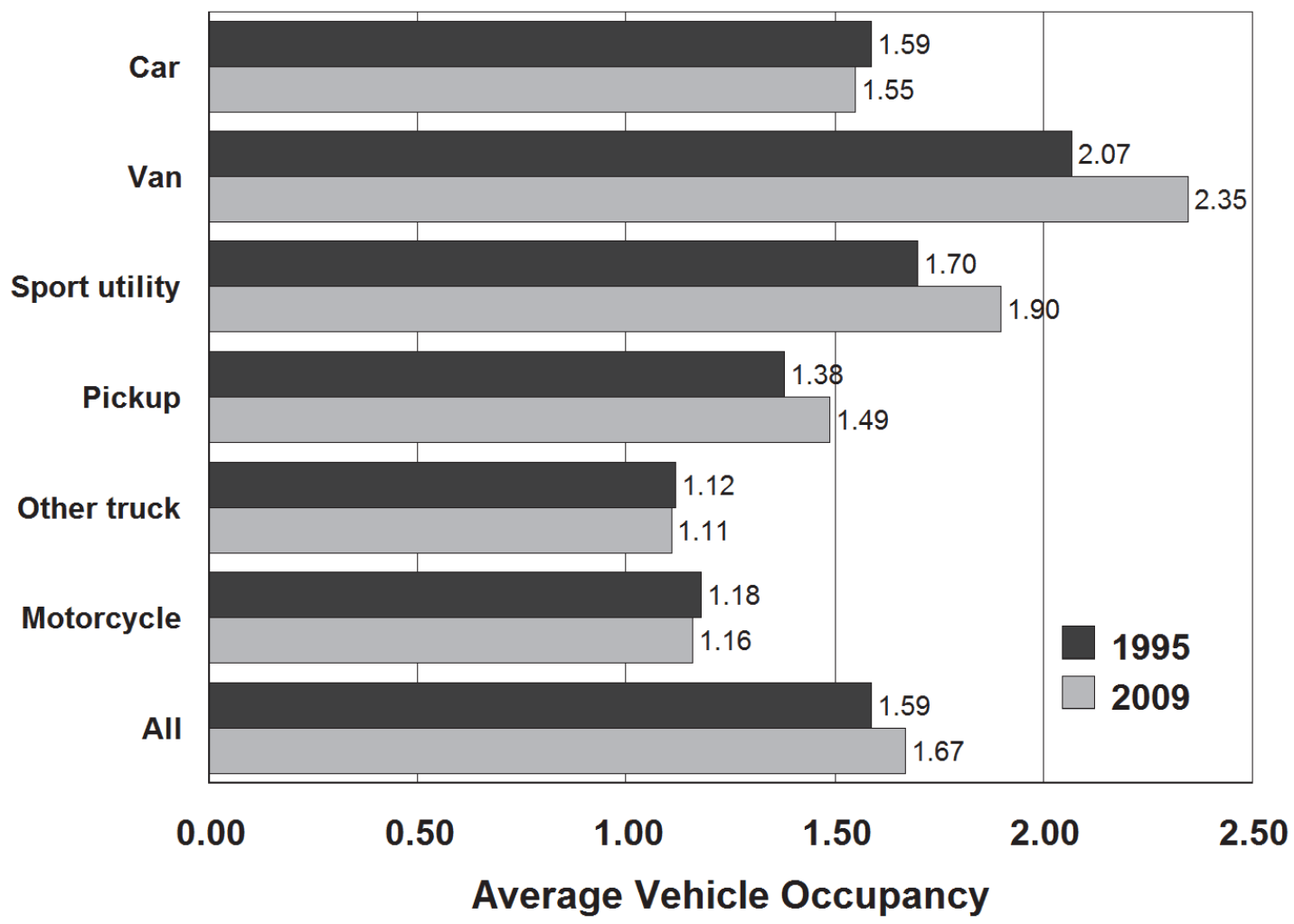

Sources:

U.S. Department of Transportation, Federal Highway Administration, 1995 Nationwide Personal Transportation Survey, Washington, DC, 1997, and 2009 National Household Travel Survey, Washington, DC. (Additional resources: www.fhwa.dot.gov, website: nhts.ornl.gov) 
The average vehicle occupancy, calculated as person-miles per vehicle-mile, is highest for social and recreational purposes. The highest vehicle occupancy levels for all purposes were in 1977. The increase in number of vehicles per household and the decrease in average household size could have contributed to the decline since then.

Figure 8.2. Average Vehicle Occupancy by Trip Purpose 1977 NPTS and 2009 NHTS

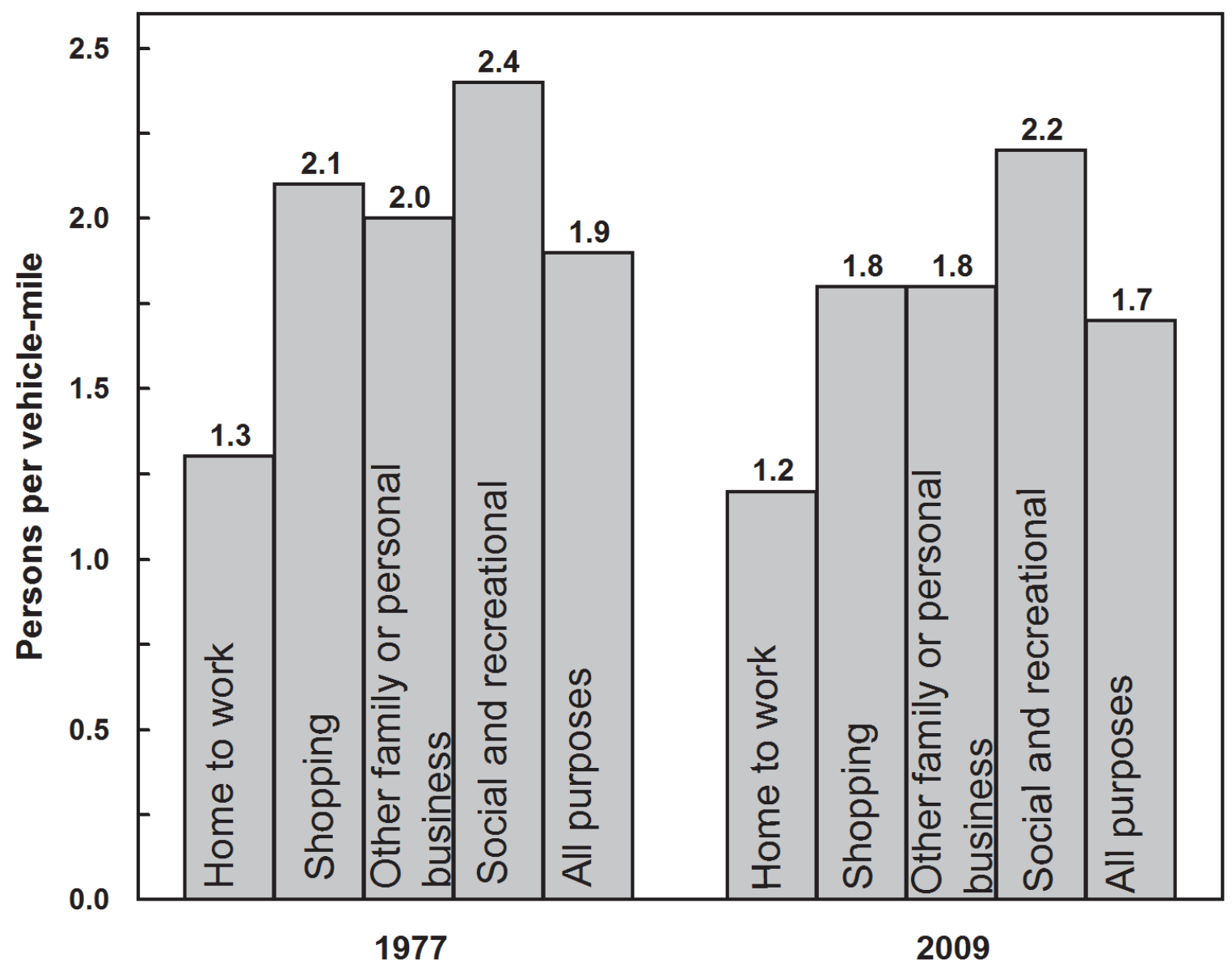

Sources:

U.S. Department of Transportation, Federal Highway Administration, 1990 Nationwide Personal Transportation Survey: Summary of Travel Trends, FHWA-PL-92027, Washington, DC, March 1992, Figure 6. Data from 2009 NHTS were generated from the website nhts.ornl.gov, March 2011. (Additional resources: www.fhwa.dot.gov, nhts.ornl.gov) 
The 1990 household survey reports the highest average annual miles per vehicle and the 1983 survey reports the lowest. These data show that younger vehicles are typically driven more miles than older vehicles.

Table 8.10

Average Annual Miles per Household Vehicle by Vehicle Age

\begin{tabular}{cccccc}
\hline $\begin{array}{c}\text { Vehicle age } \\
\text { (years) }\end{array}$ & $\begin{array}{c}1983 \\
\text { self-reported }\end{array}$ & $\begin{array}{c}1990 \\
\text { self-reported }\end{array}$ & $\begin{array}{c}1995 \\
\text { self-reported }\end{array}$ & $\begin{array}{c}2001 \\
\text { self-reported }\end{array}$ & $\begin{array}{c}2009 \\
\text { self-reported }\end{array}$ \\
\hline Under 1 & 8,200 & 19,600 & 15,900 & 15,500 & 13,200 \\
1 & 15,200 & 16,800 & 16,800 & 14,300 & 14,600 \\
2 & 16,800 & 16,600 & 15,500 & 14,000 & 13,900 \\
3 & 14,500 & 14,700 & 14,400 & 13,100 & 12,700 \\
4 & 13,000 & 13,600 & 14,100 & 12,500 & 12,600 \\
5 & 12,100 & 12,900 & 13,500 & 12,000 & 12,800 \\
6 & 11,300 & 13,200 & 13,200 & 11,800 & 12,100 \\
7 & 10,000 & 12,400 & 12,800 & 11,600 & 11,900 \\
8 & 9,800 & 12,600 & 12,200 & 10,900 & 11,500 \\
9 & 9,000 & 11,500 & 12,200 & 10,800 & 11,300 \\
10 and older & 7,300 & 9,200 & 8,900 & 7,400 & 9,300 \\
\hline All household & 10,400 & 12,500 & 12,200 & 11,100 & 11,300 \\
vehicles & \multicolumn{5}{c}{}
\end{tabular}

Note: Data include all household vehicles, and have been rounded to the nearest hundred.

\section{Sources:}

Nationwide Personal Transportation Study-1983: D. Klinger and J. Richard Kuzmyak, COMSIS Corporation, Personal Travel in the United States, Volume 1: 1983-84 Nationwide Personal Travel Study, prepared for the U.S. Department of Transportation, Washington, DC, August 1986, Table 4-22, p. 4-21. 1990: Generated from the 1990 Nationwide Personal Transportation Study Public Use Tape, March 1992. 1995, 2001 and 2009: Generated from the 2009 NHTS datasets, version 2, February 2011. (Additional resources: nhts.ornl.gov) 
Historically, the data from the Nationwide Personal Transportation Survey (NPTS) are based on estimates reported by survey respondents. For the 1995 NPTS and the 2001 National Household Travel Survey (NHTS), odometer data were also collected. The 1995 data indicate that respondents overestimate the number of miles they drive in a year, but the 2001 data do not show that same trend.

Table 8.11

Self-Reported vs. Odometer Average Annual Miles, 1995 NPTS and 2001 NHTS

\begin{tabular}{ccccc}
\hline $\begin{array}{c}\text { Vehicle age } \\
\text { (years) }\end{array}$ & $\begin{array}{c}1995 \\
\text { self-reported }\end{array}$ & $\begin{array}{c}1995 \\
\text { odometer }\end{array}$ & $\begin{array}{c}2001 \\
\text { self-reported }\end{array}$ & $\begin{array}{c}2001 \\
\text { odometer }\end{array}$ \\
\hline Under 1 & 15,900 & 15,600 & 15,500 & 14,500 \\
1 & 16,800 & 14,500 & 14,300 & 14,200 \\
2 & 15,500 & 14,800 & 14,000 & 13,700 \\
3 & 14,400 & 13,800 & 13,100 & 14,100 \\
4 & 14,100 & 12,900 & 12,500 & 13,400 \\
5 & 13,500 & 12,700 & 12,000 & 12,900 \\
6 & 13,200 & 12,400 & 11,800 & 12,400 \\
7 & 12,800 & 11,600 & 11,600 & 12,100 \\
8 & 12,200 & 11,300 & 10,900 & 11,300 \\
9 & 12,200 & 11,200 & 10,800 & 10,500 \\
10 and older & 8,900 & 9,000 & 7,400 & 8,100 \\
\hline All household & & & & \\
vehicles & 12,200 & 11,800 & 11,000 & 11,800 \\
\hline
\end{tabular}

Note: The 2009 NHTS did not collect similar data. Survey methodology on odometer reading data differs from 1995 to 2001 data.

Source:

Generated from the website: nhts.ornl.gov and 2001 NHTS public use file. 
Figure 8.3. Share of Vehicle Trips by Trip Distance, 2009 NHTS

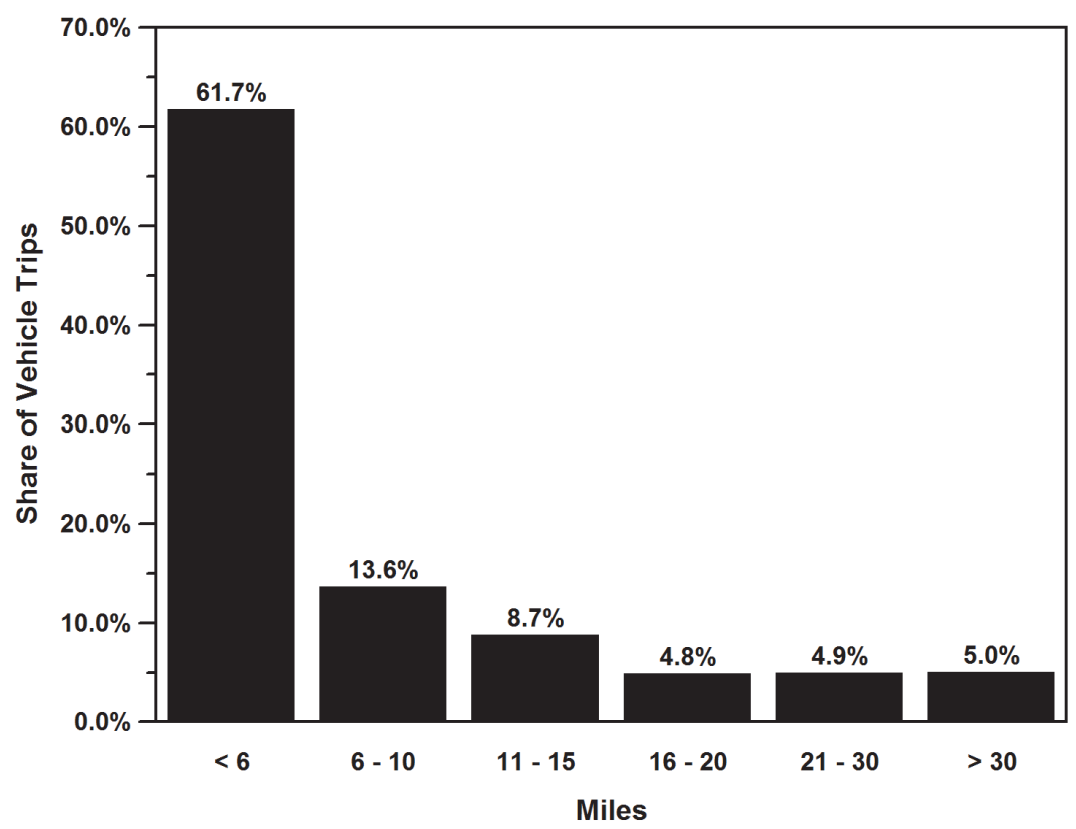

Source:

National Household Travel Survey, website nhts.ornl.gov.

Figure 8.4. Share of Vehicle Trips to Work by Trip Distance, 2009 NHTS

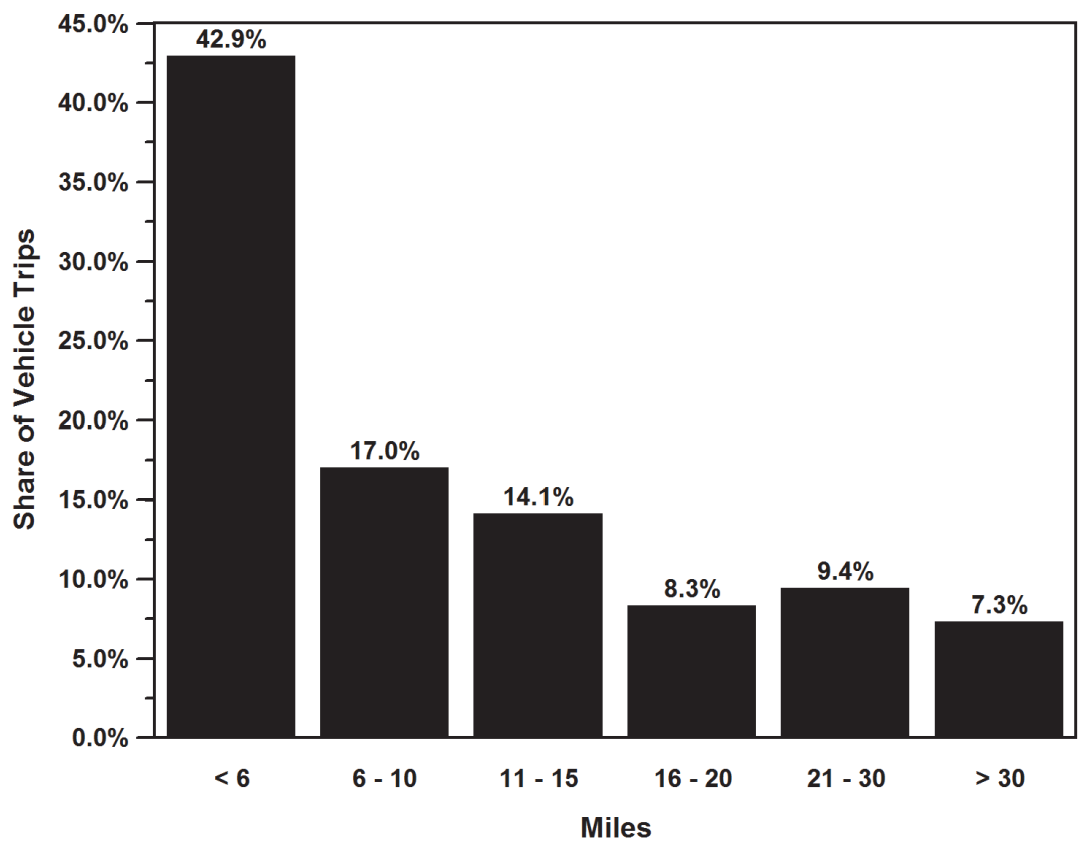

Source:

National Household Travel Survey, website: nhts.ornl.gov. 
Nineteen percent of new vehicles ( 1 year old and under) travel over 20,000 miles per year. Almost half of the vehicles over 20 years old travel less than 4,000 miles in a year.

Table 8.12

Share of Vehicles by Annual Miles of Travel and Vehicle Age, 2009 NHTS

\begin{tabular}{|c|c|c|c|c|c|c|c|}
\hline \multirow[b]{2}{*}{$\begin{array}{l}\text { Annual vehicle miles } \\
\text { of travel }\end{array}$} & \multicolumn{7}{|c|}{ Vehicle age (years) } \\
\hline & $\begin{array}{l}1 \text { and } \\
\text { under }\end{array}$ & 2 & 3 & 4 & 5 & 6 & 7 \\
\hline$<1,000$ miles & $2 \%$ & $3 \%$ & $3 \%$ & $3 \%$ & $3 \%$ & $4 \%$ & $3 \%$ \\
\hline $1-2,000$ miles & $2 \%$ & $3 \%$ & $2 \%$ & $3 \%$ & $3 \%$ & $3 \%$ & $3 \%$ \\
\hline $2-4,000$ miles & $5 \%$ & $6 \%$ & $7 \%$ & $7 \%$ & $6 \%$ & $7 \%$ & $9 \%$ \\
\hline $4-6,000$ miles & $7 \%$ & $10 \%$ & $9 \%$ & $8 \%$ & $8 \%$ & $10 \%$ & $10 \%$ \\
\hline $6-8,000$ miles & $10 \%$ & $10 \%$ & $11 \%$ & $11 \%$ & $10 \%$ & $12 \%$ & $12 \%$ \\
\hline $8-10,000$ miles & $11 \%$ & $11 \%$ & $11 \%$ & $11 \%$ & $11 \%$ & $12 \%$ & $12 \%$ \\
\hline $10-12,000$ miles & $9 \%$ & $11 \%$ & $11 \%$ & $11 \%$ & $12 \%$ & $11 \%$ & $11 \%$ \\
\hline $12-15,000$ miles & $16 \%$ & $15 \%$ & $14 \%$ & $15 \%$ & $15 \%$ & $14 \%$ & $13 \%$ \\
\hline 15 - 20,000 miles & $18 \%$ & $15 \%$ & $17 \%$ & $17 \%$ & $16 \%$ & $14 \%$ & $14 \%$ \\
\hline 20 - 30,000 miles & $13 \%$ & $11 \%$ & $12 \%$ & $11 \%$ & $11 \%$ & $10 \%$ & $9 \%$ \\
\hline$>30,000$ miles & $6 \%$ & $5 \%$ & $4 \%$ & $3 \%$ & $4 \%$ & $4 \%$ & $3 \%$ \\
\hline \multirow[t]{3}{*}{ All } & $100 \%$ & $100 \%$ & $100 \%$ & $100 \%$ & $100 \%$ & $100 \%$ & $100 \%$ \\
\hline & \multicolumn{7}{|c|}{ Vehicle age (years) } \\
\hline & 8 & 9 & 10 & $11-15$ & $16-20$ & Over 20 & \\
\hline$<1,000$ miles & $4 \%$ & $4 \%$ & $4 \%$ & $6 \%$ & $9 \%$ & $19 \%$ & \\
\hline $1-2,000$ miles & $4 \%$ & $4 \%$ & $4 \%$ & $5 \%$ & $7 \%$ & $8 \%$ & \\
\hline $2-4,000$ miles & $9 \%$ & $9 \%$ & $10 \%$ & $11 \%$ & $16 \%$ & $19 \%$ & \\
\hline $4-6,000$ miles & $11 \%$ & $12 \%$ & $12 \%$ & $14 \%$ & $14 \%$ & $14 \%$ & \\
\hline $6-8,000$ miles & $12 \%$ & $12 \%$ & $11 \%$ & $14 \%$ & $13 \%$ & $12 \%$ & \\
\hline $8-10,000$ miles & $13 \%$ & $11 \%$ & $12 \%$ & $12 \%$ & $10 \%$ & $7 \%$ & \\
\hline $10-12,000$ miles & $11 \%$ & $11 \%$ & $11 \%$ & $10 \%$ & $8 \%$ & $6 \%$ & \\
\hline 12 - 15,000 miles & $13 \%$ & $13 \%$ & $12 \%$ & $10 \%$ & $8 \%$ & $5 \%$ & \\
\hline 15 - 20,000 miles & $12 \%$ & $13 \%$ & $14 \%$ & $9 \%$ & $7 \%$ & $5 \%$ & \\
\hline 20 - 30,000 miles & $9 \%$ & $8 \%$ & $7 \%$ & $7 \%$ & $4 \%$ & $3 \%$ & \\
\hline$>30,000$ miles & $3 \%$ & $3 \%$ & $3 \%$ & $3 \%$ & $2 \%$ & $2 \%$ & \\
\hline All & $100 \%$ & $100 \%$ & $100 \%$ & $100 \%$ & $100 \%$ & $100 \%$ & \\
\hline
\end{tabular}

\section{Source:}

Generated from the Department of Transportation, Federal Highway Administration, 2009 National Household Travel Survey website: nhts.ornl.gov. (Additional resources: nhts.ornl.gov) 
The average driver makes three trips per day with an average of 9.7 miles for each trip.

Table 8.13

Household Vehicle Trips, 2009 NHTS

\begin{tabular}{cccc}
\hline & $\begin{array}{c}\text { Number of daily } \\
\text { vehicle trips } \\
\text { (per driver) }\end{array}$ & $\begin{array}{c}\text { Average } \\
\text { vehicle trip } \\
\text { length (miles) }\end{array}$ & $\begin{array}{c}\text { Daily vehicle } \\
\text { miles of travel } \\
\text { (per driver) }\end{array}$ \\
\hline 1990 & 3.3 & 8.9 & 28.5 \\
1995 & 3.6 & 9.1 & 32.1 \\
2001 & 3.4 & 9.9 & 32.7 \\
2009 & 3.0 & 9.7 & 29.0 \\
\hline
\end{tabular}

Source:

National Household Travel Survey website: nhts.ornl.gov.

Figure 8.5. Average Daily Miles Driven (per Driver), 2009 NHTS

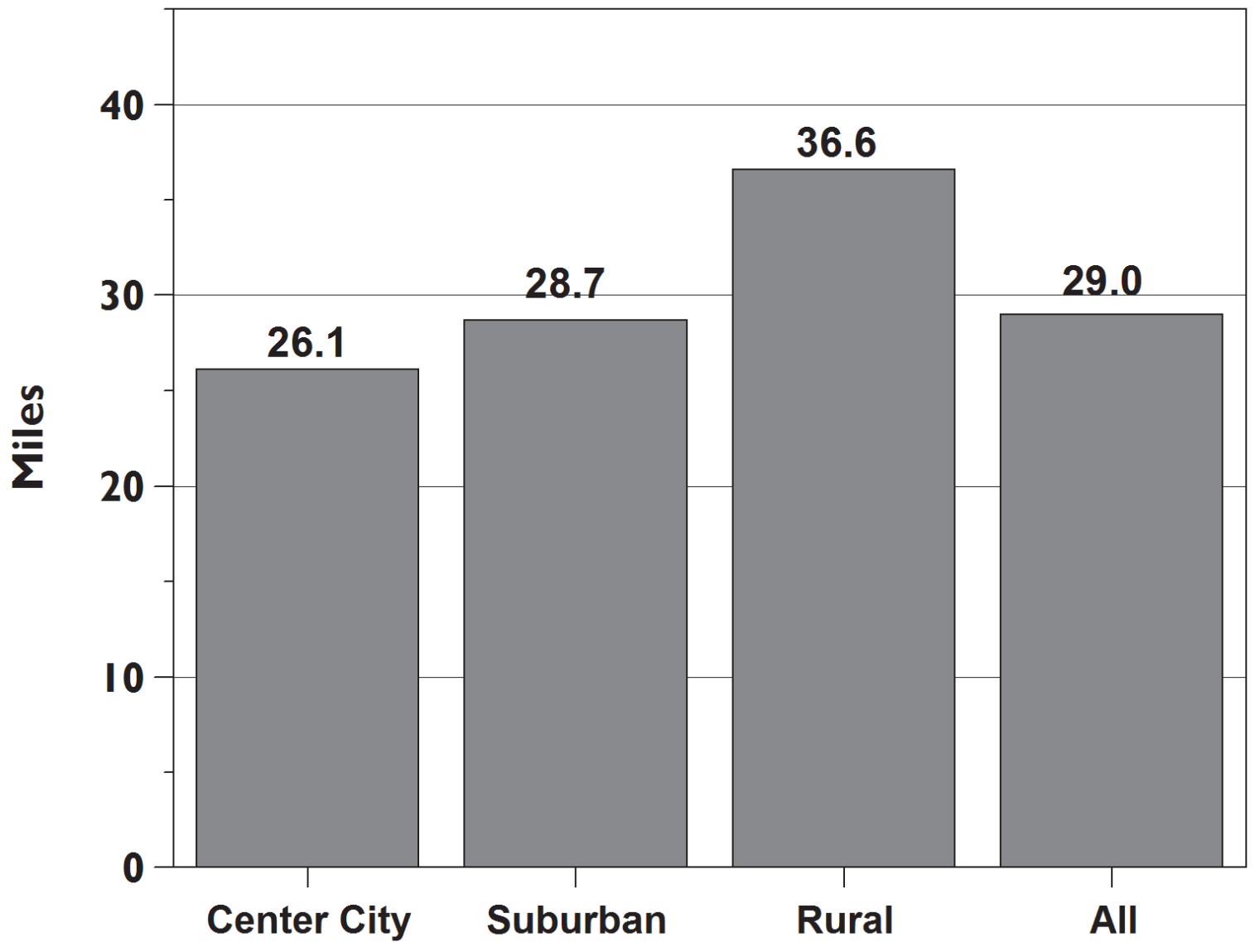

Source:

National Household Travel Survey website: nhts.ornl.gov. 
Table 8.14

Daily Vehicle Miles of Travel (per Vehicle) by Number of Vehicles in the Household, 2009 NHTS

\begin{tabular}{ccc}
\hline & \multicolumn{2}{c}{ Daily miles per vehicle } \\
\cline { 2 - 3 } Number of household vehicles & 2001 & 2009 \\
\hline 1 & 25.6 & 29.1 \\
2 & 27.5 & 32.7 \\
3 & 24.2 & 31.3 \\
4 & 23.0 & 30.2 \\
5 & 21.1 & 27.6 \\
More than 5 & 18.4 & 27.2 \\
\hline All & 25.2 & 31.1 \\
\hline
\end{tabular}

Source:

2009 National Household Travel Survey, website: nhts.ornl.gov.

Table 8.15

Daily and Annual Vehicle Miles of Travel and Average Age for Each Vehicle in a Household, 2009 NHTS

\begin{tabular}{cccc}
\hline $\begin{array}{c}\text { Vehicle number } \\
\text { One-vehicle household }\end{array}$ & $\begin{array}{c}\text { Average } \\
\text { daily miles }\end{array}$ & $\begin{array}{c}\text { Average } \\
\text { annual miles }\end{array}$ & $\begin{array}{c}\text { Average age } \\
\text { (years) }\end{array}$ \\
$\begin{array}{c}\text { Two-vehicle household } \\
1\end{array}$ & 29.0 & 10,600 & 9.0 \\
2 & 43.6 & 15,900 & 7.6 \\
Three-vehicle household & 21.4 & 7,800 & 9.0 \\
1 & & & \\
2 & 50.7 & 18,500 & 7.9 \\
3 & 28.2 & 10,300 & 9.1 \\
Four-vehicle household & 14.0 & 5,100 & 11.8 \\
1 & & & \\
2 & 56.2 & 20,500 & 8.5 \\
3 & 33.2 & 12,100 & 8.8 \\
4 & 20.3 & 7,400 & 11.4 \\
1 & 9.9 & 3,600 & 13.2 \\
2 & & & \\
3 & 57.8 & 21,100 & 8.5 \\
4 & 34.0 & 12,400 & 9.4 \\
5 & 22.7 & 8,300 & 12.3 \\
Five-vehicle household & 14.2 & 5,200 & 12.7 \\
1 & 6.3 & 2,300 & 16.8 \\
2 & & & \\
3 & 61.4 & 22,400 & 10.2 \\
4 & 38.1 & 13,900 & 9.8 \\
5 & 26.3 & 9,600 & 12.2 \\
6 & 17.5 & 6,400 & 12.5 \\
Six-vehicle household & 10.4 & 3,800 & 14.5 \\
& 4.4 & 1,600 & 17.9 \\
\hline & & &
\end{tabular}

Source:

2009 National Household Travel Survey, website: nhts.ornl.gov. 
Figure 8.6. Daily Vehicle Miles of Travel for Each Vehicle in a Household, 2009 NHTS

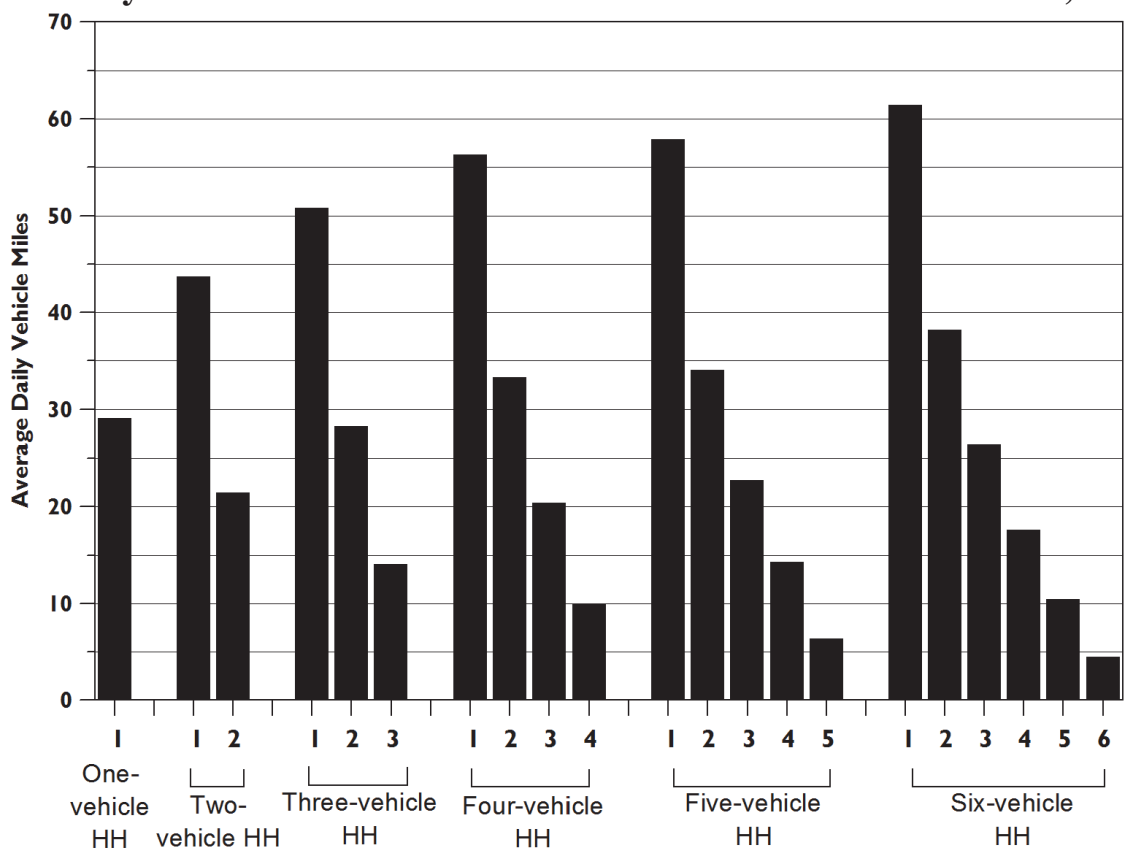

Source:

2009 National Household Travel Survey, website: nhts.ornl.gov.

Figure 8.7. Annual Vehicle Miles of Travel for Each Vehicle in a Household, 2009 NHTS

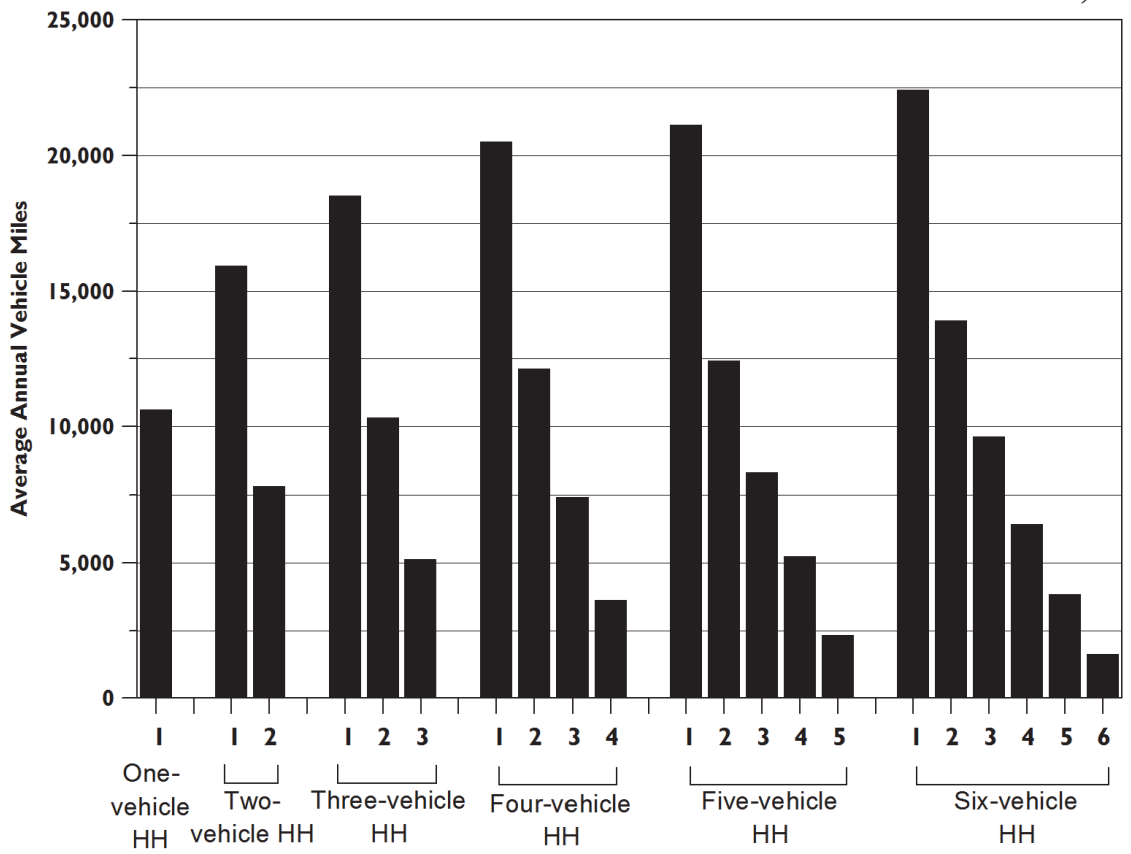

Source:

2009 National Household Travel Survey, website: nhts.ornl.gov. 
According to the U.S. Census data, the percentage of workers who car pooled has dropped from $19.7 \%$ in 1980 to $10.0 \%$ in 2012. The percent of workers using public transit declined from $6.4 \%$ to $5.3 \%$ in the ten-year period between 1980 and 1990, but stayed relatively the same from 1990 to 2012 ( 5.0\%). The average travel time increased by 3.7 minutes from 1980 to 2012. The American Community Survey (ACS) now collects journey-towork data on an annual basis. It shows the average commute time as 25.4 minutes in 2012.

Table 8.16

Means of Transportation to Work, 1980, 1990, 2000, and 2012

\begin{tabular}{|c|c|c|c|c|c|c|c|c|}
\hline \multirow[b]{2}{*}{ Means of transportation } & \multicolumn{2}{|c|}{1980 Census } & \multicolumn{2}{|c|}{1990 Census } & \multicolumn{2}{|c|}{2000 Census } & \multicolumn{2}{|c|}{2012 ACS } \\
\hline & $\begin{array}{c}\text { Number of } \\
\text { workers } \\
\text { (thousands) }\end{array}$ & Share & $\begin{array}{c}\text { Number of } \\
\text { workers } \\
\text { (thousands) }\end{array}$ & Share & $\begin{array}{c}\text { Number of } \\
\text { workers } \\
\text { (thousands) }\end{array}$ & Share & $\begin{array}{c}\text { Number of } \\
\text { workers } \\
\text { (thousands) }\end{array}$ & Share \\
\hline Private vehicle & 81,258 & $84.1 \%$ & 99,593 & $86.5 \%$ & 112,737 & $87.9 \%$ & 120,552 & $86.2 \%$ \\
\hline Drove alone & 62,193 & $64.4 \%$ & 84,215 & $73.2 \%$ & 97,102 & $75.7 \%$ & 106,520 & $76.1 \%$ \\
\hline Car pooled & 19,065 & $19.7 \%$ & 15,378 & $13.4 \%$ & 15,635 & $12.2 \%$ & 14,032 & $10.0 \%$ \\
\hline Public transportation & 6,175 & $6.4 \%$ & 6,068 & $5.3 \%$ & 6,068 & $4.7 \%$ & 7,127 & $5.1 \%$ \\
\hline Bus or trolley bus ${ }^{a}$ & 3,925 & $4.1 \%$ & 3,445 & $3.0 \%$ & 3,207 & $2.5 \%$ & 3,695 & $2.6 \%$ \\
\hline Streetcar or trolley car ${ }^{a}$ & $b$ & $b$ & 78 & $0.1 \%$ & 73 & $0.1 \%$ & 88 & $0.1 \%$ \\
\hline Subway or elevated & 1,529 & $1.6 \%$ & 1,755 & $1.5 \%$ & 1,886 & $1.5 \%$ & 2,389 & $1.7 \%$ \\
\hline Railroad & 554 & $0.6 \%$ & 574 & $0.5 \%$ & 658 & $0.5 \%$ & 757 & $0.5 \%$ \\
\hline Ferryboat & $b$ & $b$ & 37 & $0.0 \%$ & 44 & $0.0 \%$ & 39 & $0.0 \%$ \\
\hline Taxicab & 167 & $0.2 \%$ & 179 & $0.2 \%$ & 200 & $0.2 \%$ & 159 & $0.1 \%$ \\
\hline Motorcycle & 419 & $0.4 \%$ & 237 & $0.2 \%$ & 142 & $0.1 \%$ & 317 & $0.2 \%$ \\
\hline Bicycle & 468 & $0.5 \%$ & 467 & $0.4 \%$ & 488 & $0.4 \%$ & 786 & $0.6 \%$ \\
\hline Walked only & 5,413 & $5.6 \%$ & 4,489 & $3.9 \%$ & 3,759 & $2.9 \%$ & 3,938 & $2.8 \%$ \\
\hline Other means & 703 & $0.7 \%$ & 809 & $0.7 \%$ & 901 & $0.7 \%$ & 1,196 & $0.9 \%$ \\
\hline Worked at home & 2,180 & $2.3 \%$ & 3,406 & $3.0 \%$ & 4,184 & $3.3 \%$ & 5,978 & $4.3 \%$ \\
\hline Total workers & 96,616 & $100.0 \%$ & 115,069 & $100.0 \%$ & 128,279 & $100.0 \%$ & 139,894 & $100.0 \%$ \\
\hline Average travel time (minutes) & 21.7 & & 22.4 & & 25.5 & & 25.4 & \\
\hline
\end{tabular}

Sources:

1980-1990 data - Provided by the Journey-to-Work and Migration Statistics Branch, Population Division, U.S. Bureau of the Census.

2000 data - U.S. Bureau of the Census, Journey to Work: 2000, Tables 1 and 2, 1990-2000, March 2004 (www.census.gov/population/www/socdemo/journey.html).

2012 data - U.S. Bureau of the Census, 2008-2012 American Community Survey Five-Year Estimates, Tables B08301 and S0802. (Additional resources: www.census.gov)

\footnotetext{
a This category was "Bus or streetcar" in 1980.

${ }^{\mathrm{b}}$ Data are not available.
} 
Table 8.17

Characteristics of U.S. Daily per Vehicle Driving vs. Dwelling Unit Type and Density

\begin{tabular}{lcccc}
\hline & $\begin{array}{c}\text { Share of } \\
\text { vehicles in } \\
\text { density type }\end{array}$ & $\begin{array}{c}\text { Hours per } \\
\text { vehicle } \\
\text { per day }\end{array}$ & $\begin{array}{c}\text { Average vehicle } \\
\text { speed } \\
\text { (miles/hour) }\end{array}$ & $\begin{array}{c}\text { Miles } \\
\text { per vehicle } \\
\text { per day }\end{array}$ \\
\hline All classes detached single & $77.0 \%$ & 0.92 & 32.0 & 29.6 \\
All classes other & $23.0 \%$ & 0.99 & 27.7 & 27.4 \\
$<1,000 /$ sq. mile detached single & $81.6 \%$ & 0.91 & 34.7 & 31.6 \\
<1,000/sq. mile all other & $18.4 \%$ & 0.91 & 32.5 & 29.5 \\
1,000-4,000/sq. mile detached single & $75.5 \%$ & 0.94 & 27.5 & 26.0 \\
1,000-4,000/sq. mile all other & $24.5 \%$ & 1.03 & 25.1 & 25.9 \\
4,000-10,000/sq. mile detached single & $42.5 \%$ & 0.96 & 26.1 & 25.1 \\
4,000-10,000/sq. mile all other & $57.5 \%$ & 1.15 & 21.5 & 24.6 \\
10,000-25,000/sq. mile detached single & $17.8 \%$ & 1.02 & 18.2 & 18.5 \\
10,000-25,000/sq. mile all other & $82.2 \%$ & 1.05 & 21.3 & 22.3 \\
$>25,000 /$ sq. mile detached single & $9.8 \%$ & 0.72 & 20.5 & 14.8 \\
$>25,000 /$ sq. mile all other & $90.2 \%$ & 1.23 & 21.9 & 26.9 \\
\hline
\end{tabular}

Source:

Generated from the 2009 National Household Survey website: nhts.ornl.gov.

Table 8.18

Housing Unit Characteristics, 2010

\begin{tabular}{lcc}
\hline & $\begin{array}{c}\text { Share of occupied } \\
\text { housing units }\end{array}$ & $\begin{array}{c}\% \text { with garage or } \\
\text { carport }\end{array}$ \\
\hline Type of housing unit & & \\
$\quad$ New construction $(<=4$ years) & $2.2 \%$ & $75.7 \%$ \\
$\quad$ Manufactured/mobile homes & $6.3 \%$ & $39.1 \%$ \\
\hline Geographic location (Census Region) & $18.3 \%$ & $50.5 \%$ \\
$\quad$ Northeast & $22.4 \%$ & $74.0 \%$ \\
Midwest & $37.1 \%$ & $60.0 \%$ \\
South & $22.3 \%$ & $79.2 \%$ \\
$\quad$ West & & \\
\hline Tenure & $66.2 \%$ & $79.4 \%$ \\
$\quad$ Owner & $33.8 \%$ & $38.8 \%$ \\
$\quad$ Renter & 114,907 units & $65.6 \%$ \\
\hline All occupied units & & \\
\hline
\end{tabular}

Source:

U.S. Bureau of the Census, 2011 American Housing Survey, Table C-02-AO. (Additional information: www.census.gov/housing/ahs) 
The average commute time was 25.4 minutes in 2012. Two thirds of workers travel less than 30 minutes to work in 2012. In 1990, 32.5\% of workers commuted less than 15 minutes; in 2012 that number dropped to $28.1 \%$.

Table 8.19

Workers by Commute Time, 1990, 2000 and 2012

\begin{tabular}{llrr}
\hline \multicolumn{1}{c}{ Commute time } & 1990 & 2000 & 2012 \\
\hline Less than 15 minutes & $32.5 \%$ & $29.4 \%$ & $28.1 \%$ \\
15-29 minutes & $37.0 \%$ & $36.1 \%$ & $36.4 \%$ \\
30-39 minutes & $15.2 \%$ & $15.8 \%$ & $16.3 \%$ \\
40-59 minutes & $9.2 \%$ & $10.7 \%$ & $11.2 \%$ \\
60 minutes or more & $6.1 \%$ & $8.0 \%$ & $8.1 \%$ \\
\hline Average travel time (minutes) & 22.4 & 25.5 & 25.4 \\
\hline
\end{tabular}

\section{Sources:}

1990-2000 - U.S. Bureau of the Census, Journey to Work: 2000, Tables 1 and 2, 1990-2000, March 2004.

2012 - U.S. Bureau of the Census, 2008-2012 American Community Survey, 5-Year Estimates, Tables S0802 and B08303. (Additional resources: www.census.gov) 
Sales of bicycles with wheel sizes of 20 inches and over have grown at an average annual rate of $1.2 \%$ from 1981 to 2012. Bicycle sales experienced a large decline in 2009, which brought total sales to 14.9 million-a new low in the 18-year series. Sales in 2012 were 18.7 million.

Table 8.20

Bicycle Sales, 1981-2012

(millions)

\begin{tabular}{|c|c|c|c|}
\hline & $\begin{array}{c}\text { Wheel } \\
\text { sizes under } \\
20 \text { inches }\end{array}$ & $\begin{array}{l}\text { Wheel sizes } \\
\text { of } 20 \text { inches } \\
\text { and over }\end{array}$ & $\begin{array}{c}\text { All } \\
\text { wheel } \\
\text { sizes }\end{array}$ \\
\hline 1981 & a & 8.9 & $\mathrm{a}$ \\
\hline 1982 & $\mathrm{a}$ & 6.8 & a \\
\hline 1983 & a & 9.0 & a \\
\hline 1984 & a & 10.1 & a \\
\hline 1985 & $\mathrm{a}$ & 11.4 & a \\
\hline 1986 & a & 12.3 & a \\
\hline 1987 & a & 12.6 & a \\
\hline 1988 & $\mathrm{a}$ & 9.9 & $\mathrm{a}$ \\
\hline 1989 & a & 10.7 & a \\
\hline 1990 & a & 10.8 & a \\
\hline 1991 & a & 11.6 & a \\
\hline 1992 & 3.7 & 11.6 & 15.3 \\
\hline 1993 & 3.8 & 13.0 & 16.8 \\
\hline 1994 & 4.2 & 12.5 & 16.7 \\
\hline 1995 & 4.1 & 12.0 & 16.1 \\
\hline 1996 & 4.5 & 10.9 & 15.4 \\
\hline 1997 & 4.2 & 11.0 & 15.2 \\
\hline 1998 & 4.7 & 11.1 & 15.8 \\
\hline 1999 & 5.9 & 11.6 & 17.5 \\
\hline 2000 & 9.0 & 11.9 & 20.9 \\
\hline 2001 & 5.4 & 11.3 & 16.7 \\
\hline 2002 & 5.9 & 13.6 & 19.5 \\
\hline 2003 & 5.6 & 12.9 & 18.5 \\
\hline 2004 & 5.3 & 13.0 & 18.3 \\
\hline 2005 & 5.8 & 14.0 & 19.8 \\
\hline 2006 & 5.5 & 12.7 & 18.2 \\
\hline 2007 & 5.4 & 12.8 & 18.2 \\
\hline 2008 & 5.1 & 13.4 & 18.5 \\
\hline 2009 & 4.7 & 10.2 & 14.9 \\
\hline 2010 & 6.3 & 13.5 & 19.8 \\
\hline 2011 & 4.7 & 11.0 & 15.7 \\
\hline 2012 & 5.7 & 13.0 & 18.7 \\
\hline \multicolumn{4}{|c|}{ Average annual percentage change } \\
\hline 1981-2012 & a & $1.2 \%$ & a \\
\hline 2002-2012 & $-0.3 \%$ & $-0.5 \%$ & $-0.4 \%$ \\
\hline
\end{tabular}

\section{Source:}

1981-1996: Bicycle Manufacturers Association. 1997-on: National Bicycle Dealers Association. (Additional resources: www.nbda.com)

${ }^{a}$ Data are not available. 
In 2009, 4.5\% of walk trips and 10.9\% of bike trips were to/from work. Forty-seven percent of all bike trips were for social/recreational purposes. Nearly $15 \%$ of walk trips were shopping trips.

Figure 8.8. Walk and Bike Trips by Trip Purpose, 2009 NHTS

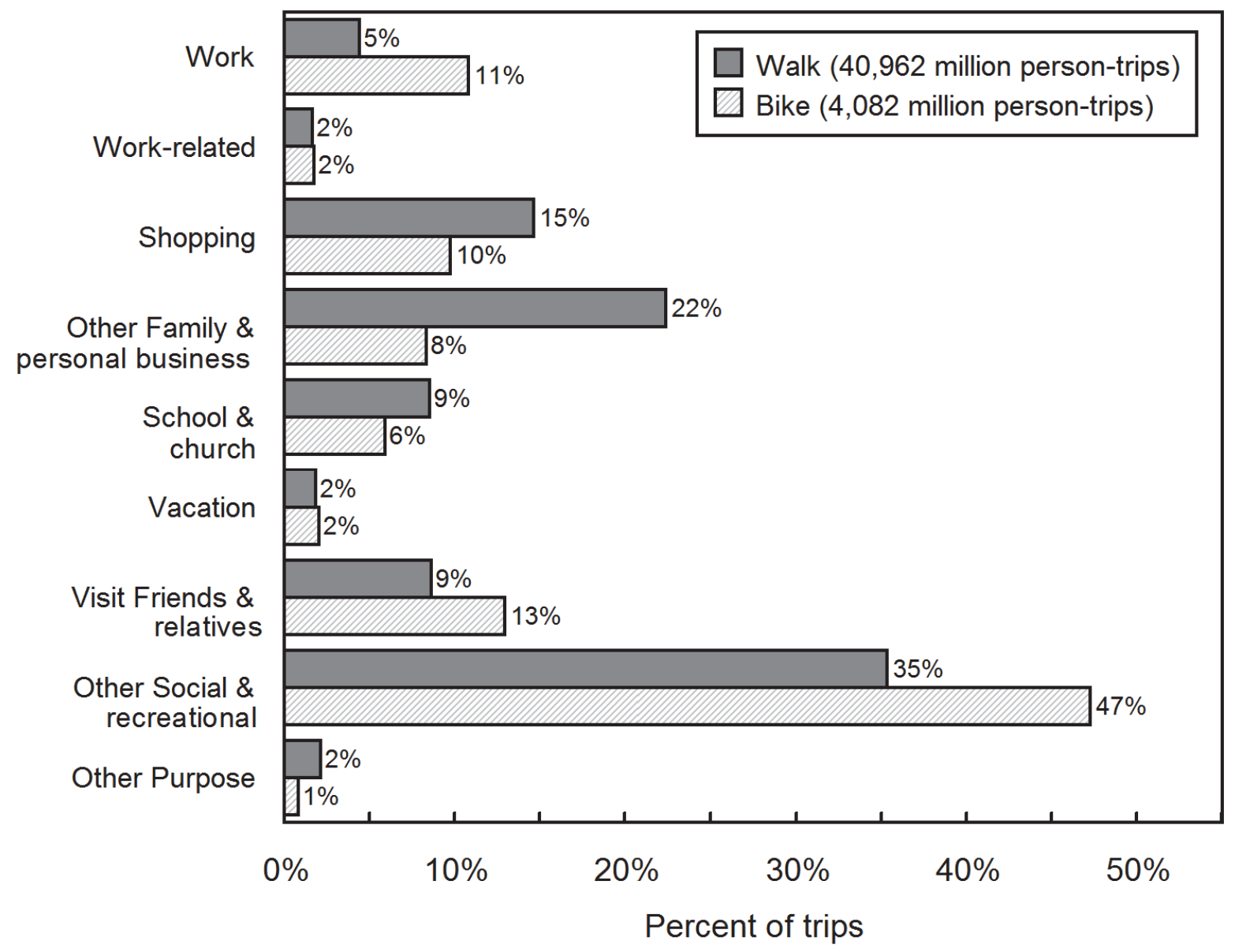

Note: Percentages may not sum to totals due to rounding.

\section{Source:}

U.S. Department of Transportation, Federal Highway Administration, 2009 National Household Travel Survey website: nhts.ornl.gov. 
In 2009 only data on daily trips were collected in the NHTS. The 2001 data are still the latest available on longdistance trips.

\section{Long Distance Trips - 2001 National Household Travel Survey}

The 2001 National Household Travel Survey (NHTS) collected data on long-distance trips as well as everyday travel. The everyday travel data is a continuation of the Nationwide Personal Transportation Survey (NPTS), while the long-distance travel data is a continuation of the American Travel Survey (ATS) which was collected in 1977 and 1985. The survey collected trip-related data such as mode of transportation, duration, distance and purpose of trip. It also gathered demographic, geographic, and economic data for analysis purposes.

A long-distance trip is defined as a trip of 50 miles or more, one-way. Long-trip data from the 2001 NHTS were released in the summer of 2004. For additional information about the 2001 NHTS data, contact the Bureau of Transportation Statistics at 202-366-3282 or visit the following website: www.bts.gov/programs/national_household_travel_survey. 
Table 8.21

Long-Distance Trip ${ }^{a}$ Characteristics, 2001 NHTS

\begin{tabular}{|c|c|c|c|c|}
\hline \multirow[b]{2}{*}{ Trip characteristic } & \multicolumn{2}{|c|}{ Person trips } & \multicolumn{2}{|c|}{ Person miles } \\
\hline & (thousands) & (percent) & (thousands) & (percent) \\
\hline Total & $2,554,068$ & 100.0 & $1,138,322,697$ & 100.0 \\
\hline \multicolumn{5}{|l|}{ Principal means of transportation: } \\
\hline Personal use vehicles & $2,310,376$ & 90.5 & $735,882,255$ & 64.7 \\
\hline Airplane & 165,039 & 6.5 & $367,888,741$ & 32.3 \\
\hline Commercial airplane & 158,880 & 6.2 & $361,717,015$ & 31.8 \\
\hline Bus $^{\text {b }}$ & 52,962 & 2.1 & $23,747,433$ & 2.1 \\
\hline Intercity bus & 3,456 & 0.1 & $1,765,696$ & 0.2 \\
\hline Charter, tour, or school bus & 45,952 & 1.8 & $21,019,942$ & 1.9 \\
\hline Train & 20,672 & 0.8 & $9,266,373$ & 0.8 \\
\hline \multicolumn{5}{|l|}{ Round trip distance: } \\
\hline 100 to 300 miles & $1,688,358$ & 66.1 & $284,586,370$ & 25.0 \\
\hline 300 to 499 miles & 373,550 & 14.6 & $143,571,597$ & 12.6 \\
\hline 500 to 999 miles & 261,802 & 10.3 & $180,669,482$ & 15.9 \\
\hline 1,000 to 1,999 miles & 125,665 & 4.9 & $178,629,838$ & 15.7 \\
\hline 2,000 miles or more & 104,694 & 4.1 & $350,865,409$ & 30.8 \\
\hline Mean (miles) & 446 & c & $\mathrm{c}^{\prime}$ & c \\
\hline Median (miles) & 206 & $\mathrm{c}$ & $\mathrm{c}$ & c \\
\hline \multicolumn{5}{|l|}{ Calendar quarter: } \\
\hline 1 st quarter & 566,502 & 22.2 & $246,556,190$ & 21.7 \\
\hline 2nd quarter & 653,310 & 25.6 & $298,154,812$ & 26.2 \\
\hline 3rd quarter & 734,878 & 28.8 & $341,021,290$ & 30.0 \\
\hline 4 th quarter & 599,378 & 23.5 & $252,590,405$ & 22.2 \\
\hline \multicolumn{5}{|l|}{ Main purpose of trip: } \\
\hline Commuting & 329,395 & 12.9 & $65,877,968$ & 5.8 \\
\hline Other business & 405,866 & 15.9 & $242,353,212$ & 21.3 \\
\hline Personal/leisure & $1,406,411$ & 55.1 & $667,471,358$ & 58.7 \\
\hline Personal business & 322,645 & 12.6 & $130,020,982$ & 11.4 \\
\hline Other & 88,230 & 3.5 & $32,031,679$ & 2.8 \\
\hline \multicolumn{5}{|l|}{ Nights away from home: } \\
\hline None & $1,454,847$ & 57.0 & $304,469,524$ & 26.8 \\
\hline 1 to 3 nights & 808,281 & 31.7 & $414,219,147$ & 36.4 \\
\hline 4 to 7 nights & 214,464 & 8.4 & $269,265,597$ & 23.7 \\
\hline 8 or more nights & 76,475 & 3.0 & $150,368,429$ & 13.2 \\
\hline \multicolumn{5}{|l|}{ Destination: } \\
\hline Within Census division & $2,077,810$ & 81.4 & $549,651,116$ & 48.3 \\
\hline Across Census division, within Census & 196,890 & 7.7 & $134,930,113$ & 11.9 \\
\hline Across Census region & 279,367 & 10.9 & $453,741,468$ & 39.9 \\
\hline
\end{tabular}

Note: Long-distance trips were not included in the 2009 NHTS.

Source:

U.S. Bureau of Transportation Statistics and the U.S. Federal Highway Administration, 2001 National Household Transportation Survey. (Additional resources: www.bts.gov/programs/national_household_travel_survey)

\footnotetext{
${ }^{\text {a }}$ A long-distance trip is defined as a trip of 50 miles or more, one-way.

${ }^{\mathrm{b}}$ Includes other types of buses.

${ }^{\mathrm{c}}$ Not applicable.
} 


\section{Chapter 9 \\ Nonhighway Modes}

Summary Statistics from Tables in this Chapter

\begin{tabular}{crr}
\hline Source & & \\
\hline & Passenger-miles & (millions) \\
Table 9.2 & Domestic and international air carrier, 2013 & 847,971 \\
Table 9.10 & Amtrak, 2012 & 6,804 \\
Table 9.11 & Commuter rail, 2012 & 11,181 \\
Table 9.12 & Transit rail, 2012 & 19,835 \\
& Freight ton-miles & (millions) \\
Table 9.5 & Domestic waterborne commerce, 2012 & 475,000 \\
Table 9.8 & Class I railroad, 2012 & $1,712,567$ \\
Table 9.2 & Passenger energy use & (trillion Btus) \\
Table 9.3 & Domestic and international air carrier, 2013 & $2,279.1$ \\
Table 9.6 & General aviation, 2012 & 228.8 \\
Table 9.10 & Recreational boats, 2012 & 244.8 \\
Table 9.11 & Amtrak, 2012 & 14.4 \\
Table 9.12 & Commuter rail, 2012 & 31.7 \\
& Transit rail, 2012 & 47.6 \\
\hline
\end{tabular}


Nonhighway transportation modes accounted for 18.6\% of total transportation energy use in 2012.

Table 9.1

Nonhighway Energy Use Shares, 1970-2012

\begin{tabular}{|c|c|c|c|c|c|c|}
\hline \multirow[b]{2}{*}{ Year } & \multicolumn{6}{|c|}{ Share of transportation energy use } \\
\hline & Air & Water & Pipeline & Rail & $\begin{array}{c}\text { Nonhighway } \\
\text { total }\end{array}$ & $\begin{array}{c}\text { Transportation } \\
\text { total (trillion Btu) }\end{array}$ \\
\hline 1970 & $8.5 \%$ & $5.4 \%$ & $6.4 \%$ & $3.6 \%$ & $24.0 \%$ & 15,395 \\
\hline 1971 & $8.1 \%$ & $4.8 \%$ & $6.3 \%$ & $3.5 \%$ & $22.8 \%$ & 16,015 \\
\hline 1972 & $7.7 \%$ & $4.6 \%$ & $6.1 \%$ & $3.4 \%$ & $21.9 \%$ & 17,036 \\
\hline 1973 & $7.7 \%$ & $5.0 \%$ & $5.6 \%$ & $3.4 \%$ & $21.7 \%$ & 17,874 \\
\hline 1974 & $7.3 \%$ & $5.1 \%$ & $5.4 \%$ & $3.6 \%$ & $21.5 \%$ & 17,174 \\
\hline 1975 & $7.3 \%$ & $5.3 \%$ & $4.8 \%$ & $3.2 \%$ & $20.7 \%$ & 17,424 \\
\hline 1976 & $7.2 \%$ & $5.9 \%$ & $4.3 \%$ & $3.1 \%$ & $20.6 \%$ & 18,492 \\
\hline 1977 & $7.1 \%$ & $6.2 \%$ & $4.1 \%$ & $3.1 \%$ & $20.4 \%$ & 19,126 \\
\hline 1978 & $7.1 \%$ & $6.9 \%$ & $3.9 \%$ & $2.9 \%$ & $20.8 \%$ & 20,097 \\
\hline 1979 & $7.6 \%$ & $5.8 \%$ & $4.4 \%$ & $3.1 \%$ & $20.9 \%$ & 19,652 \\
\hline 1980 & $7.6 \%$ & $7.4 \%$ & $4.7 \%$ & $3.1 \%$ & $22.8 \%$ & 18,940 \\
\hline 1981 & $7.8 \%$ & $6.8 \%$ & $4.8 \%$ & $3.0 \%$ & $22.4 \%$ & 18,741 \\
\hline 1982 & $7.9 \%$ & $5.8 \%$ & $4.7 \%$ & $2.6 \%$ & $21.1 \%$ & 18,237 \\
\hline 1983 & $7.8 \%$ & $5.3 \%$ & $4.0 \%$ & $2.6 \%$ & $19.8 \%$ & 18,368 \\
\hline 1984 & $8.5 \%$ & $5.1 \%$ & $4.1 \%$ & $2.8 \%$ & $20.5 \%$ & 18,962 \\
\hline 1985 & $8.7 \%$ & $4.5 \%$ & $3.9 \%$ & $2.6 \%$ & $19.8 \%$ & 19,205 \\
\hline 1986 & $9.0 \%$ & $6.5 \%$ & $3.6 \%$ & $2.4 \%$ & $21.5 \%$ & 20,276 \\
\hline 1987 & $9.1 \%$ & $6.6 \%$ & $3.7 \%$ & $2.4 \%$ & $21.9 \%$ & 20,771 \\
\hline 1988 & $9.3 \%$ & $6.6 \%$ & $4.1 \%$ & $2.4 \%$ & $22.4 \%$ & 21,327 \\
\hline 1989 & $9.1 \%$ & $7.0 \%$ & $4.1 \%$ & $2.4 \%$ & $22.6 \%$ & 21,685 \\
\hline 1990 & $9.5 \%$ & $6.7 \%$ & $4.3 \%$ & $2.3 \%$ & $22.8 \%$ & 21,581 \\
\hline 1991 & $9.0 \%$ & $7.2 \%$ & $4.1 \%$ & $2.3 \%$ & $22.6 \%$ & 21,182 \\
\hline 1992 & $8.9 \%$ & $7.3 \%$ & $3.9 \%$ & $2.2 \%$ & $22.3 \%$ & 21,841 \\
\hline 1993 & $8.9 \%$ & $6.4 \%$ & $4.0 \%$ & $2.3 \%$ & $21.6 \%$ & 22,322 \\
\hline 1994 & $9.0 \%$ & $6.1 \%$ & $4.1 \%$ & $2.3 \%$ & $21.6 \%$ & 22,930 \\
\hline 1995 & $9.1 \%$ & $6.3 \%$ & $4.1 \%$ & $2.4 \%$ & $21.9 \%$ & 23,465 \\
\hline 1996 & $9.2 \%$ & $5.9 \%$ & $4.1 \%$ & $2.4 \%$ & $21.6 \%$ & 23,974 \\
\hline 1997 & $9.5 \%$ & $5.1 \%$ & $4.2 \%$ & $2.4 \%$ & $21.2 \%$ & 24,327 \\
\hline 1998 & $9.2 \%$ & $5.0 \%$ & $3.6 \%$ & $2.3 \%$ & $20.2 \%$ & 24,662 \\
\hline 1999 & $9.6 \%$ & $5.3 \%$ & $3.5 \%$ & $2.3 \%$ & $20.6 \%$ & 25,960 \\
\hline 2000 & $9.7 \%$ & $5.5 \%$ & $3.4 \%$ & $2.3 \%$ & $21.0 \%$ & 26,273 \\
\hline 2001 & $9.2 \%$ & $4.6 \%$ & $3.4 \%$ & $2.3 \%$ & $19.6 \%$ & 25,945 \\
\hline 2002 & $8.4 \%$ & $4.7 \%$ & $3.5 \%$ & $2.3 \%$ & $18.9 \%$ & 26,536 \\
\hline 2003 & $8.5 \%$ & $4.0 \%$ & $3.2 \%$ & $2.3 \%$ & $18.0 \%$ & 26,715 \\
\hline 2004 & $9.0 \%$ & $4.8 \%$ & $3.0 \%$ & $2.4 \%$ & $19.2 \%$ & 27,173 \\
\hline 2005 & $9.2 \%$ & $5.0 \%$ & $3.1 \%$ & $2.4 \%$ & $19.6 \%$ & 27,582 \\
\hline 2006 & $9.0 \%$ & $5.2 \%$ & $3.0 \%$ & $2.4 \%$ & $19.7 \%$ & 27,760 \\
\hline 2007 & $8.6 \%$ & $5.3 \%$ & $3.0 \%$ & $2.2 \%$ & $19.2 \%$ & 29,223 \\
\hline 2008 & $8.4 \%$ & $5.1 \%$ & $3.2 \%$ & $2.2 \%$ & $18.9 \%$ & 28,592 \\
\hline 2009 & $7.8 \%$ & $4.9 \%$ & $3.4 \%$ & $2.0 \%$ & $18.2 \%$ & 27,107 \\
\hline 2010 & $7.9 \%$ & $5.5 \%$ & $3.5 \%$ & $2.1 \%$ & $19.0 \%$ & 27,185 \\
\hline 2011 & $8.1 \%$ & $5.2 \%$ & $3.6 \%$ & $2.3 \%$ & $19.2 \%$ & 26,600 \\
\hline 2012 & $8.0 \%$ & $4.5 \%$ & $3.8 \%$ & $2.3 \%$ & $18.6 \%$ & 26,211 \\
\hline
\end{tabular}

Source:

See Appendix A for Nonhighway Energy Use. 
These data include ALL international and domestic certificated route air carrier statistics; therefore, the data are different than those in Chapter 2. Revenue aircraft-miles, passenger-miles, and seat-miles began to rise in 2010 Passenger load factor was $82.7 \%$ in 2013.

Table 9.2

Summary Statistics for U.S. Domestic and International Certificated Route Air Carriers (Combined Totals), 1970-2013

\begin{tabular}{|c|c|c|c|c|c|c|c|}
\hline Year & $\begin{array}{l}\text { Revenue } \\
\text { aircraft- } \\
\text { miles } \\
\text { (millions) }\end{array}$ & $\begin{array}{c}\text { Revenue } \\
\text { passenger-miles } \\
\text { (millions) }\end{array}$ & $\begin{array}{l}\text { Available } \\
\text { seat-miles } \\
\text { (millions) }\end{array}$ & $\begin{array}{l}\text { Available } \\
\text { seats per } \\
\text { aircraft }^{\text {b }} \\
\end{array}$ & $\begin{array}{l}\text { Passenger load } \\
\text { factor } \\
\text { (percentage) }^{\mathrm{c}}\end{array}$ & $\begin{array}{c}\text { Revenue cargo } \\
\text { ton-miles } \\
\text { (millions) }\end{array}$ & $\begin{array}{c}\text { Energy use } \\
\text { (trillion } \\
\text { Btu })^{\mathrm{d}} \\
\end{array}$ \\
\hline 1970 & 2,542 & 148,137 & 264,904 & 104 & $55.9 \%$ & 3,755 & $1,363.4$ \\
\hline 1975 & 2,241 & 173,324 & 315,823 & 141 & $54.9 \%$ & 5,062 & $1,283.4$ \\
\hline 1980 & $\begin{array}{r}2 \\
-\end{array}$ & 267,722 & 448,479 & 153 & $59.7 \%$ & 7,885 & $1,386.0$ \\
\hline 1985 & 3,462 & 351,073 & 565,677 & 163 & $62.1 \%$ & 9,048 & $1,701.4$ \\
\hline 1986 & 3,873 & 378,923 & 623,075 & 161 & $60.8 \%$ & 10,987 & $1,847.1$ \\
\hline 1987 & 4,182 & 417,808 & 670,825 & 160 & $62.3 \%$ & 13,137 & $1,954.9$ \\
\hline 1988 & 4,354 & 437,649 & 696,337 & 160 & $62.9 \%$ & 14,632 & $2,049.4$ \\
\hline 1989 & 4,442 & 447,480 & 703,888 & 158 & $63.6 \%$ & 16,347 & $2,087.4$ \\
\hline 1990 & 4,724 & 472,236 & 753,211 & 159 & $62.7 \%$ & 16,403 & $2,180.2$ \\
\hline 1991 & 4,661 & 463,296 & 738,030 & 158 & $62.8 \%$ & 16,149 & $2,085.2$ \\
\hline 1992 & 4,899 & 493,715 & 772,869 & 158 & $63.9 \%$ & 17,306 & $2,116.4$ \\
\hline 1993 & 5,118 & 505,996 & 793,959 & 155 & $63.7 \%$ & 19,083 & $2,169.7$ \\
\hline 1994 & 5,360 & 537,518 & 809,259 & 151 & $66.4 \%$ & 21,773 & $2,271.5$ \\
\hline 1995 & 5,627 & 558,794 & 832,081 & 148 & $67.2 \%$ & 23,375 & $2,338.6$ \\
\hline 1996 & 5,855 & 596,164 & 859,721 & 147 & $69.3 \%$ & 24,892 & $2,409.1$ \\
\hline 1997 & 6,025 & 620,029 & 880,715 & 146 & $70.4 \%$ & 27,610 & $2,513.6$ \\
\hline 1998 & 6,220 & 634,933 & 899,029 & 145 & $70.6 \%$ & 28,015 & $2,459.5$ \\
\hline 1999 & 6,558 & 668,626 & 942,311 & 144 & $71.0 \%$ & 25,147 & $2,665.0$ \\
\hline 2000 & 6,946 & 708,926 & 981,080 & 141 & $72.3 \%$ & 30,221 & $2,750.4$ \\
\hline 2001 & 6,814 & 664,849 & 950,519 & 139 & $69.9 \%$ & 27,882 & $2,592.5$ \\
\hline 2002 & 6,834 & 655,215 & 913,898 & 134 & $71.7 \%$ & 30,507 & $2,430.1$ \\
\hline 2003 & 7,367 & 674,160 & 922,440 & 125 & $73.1 \%$ & 32,446 & $2,470.6$ \\
\hline 2004 & 7,479 & 752,341 & $1,000,193$ & 134 & $75.2 \%$ & 37,958 & $2,657.2$ \\
\hline 2005 & 7,716 & 795,117 & $1,029,316$ & 133 & $77.2 \%$ & 39,286 & $2,693.3$ \\
\hline 2006 & 8,220 & 810,086 & $1,027,526$ & 125 & $78.8 \%$ & 38,251 & $2,661.1$ \\
\hline 2007 & 8,415 & 842,007 & $1,060,093$ & 126 & $79.4 \%$ & 38,433 & $2,684.6$ \\
\hline 2008 & 8,142 & 823,783 & $1,040,840$ & 128 & $79.1 \%$ & 35,227 & $2,547.8$ \\
\hline 2009 & 7,534 & 779,997 & 975,307 & 129 & $80.0 \%$ & 30,317 & $2,303.2$ \\
\hline 2010 & 7,666 & 809,051 & 991,934 & 129 & $81.6 \%$ & 35,209 & $2,335.3$ \\
\hline 2011 & 7,783 & 825,916 & $1,012,597$ & 130 & $81.6 \%$ & 35,713 & $2,370.3$ \\
\hline 2012 & 7,727 & 832,733 & $1,012,261$ & 131 & $82.3 \%$ & 34,937 & $2,307.3$ \\
\hline 2013 & 7,724 & 847,971 & $1,025,569$ & 133 & $82.7 \%$ & 33,561 & $2,279.1$ \\
\hline \multicolumn{8}{|c|}{ Average annual percentage change } \\
\hline 1970-2013 & $2.6 \%$ & $4.1 \%$ & $3.2 \%$ & $0.6 \%$ & & $5.2 \%$ & $1.2 \%$ \\
\hline 2003-2013 & $0.5 \%$ & $2.3 \%$ & $1.1 \%$ & $0.6 \%$ & & $0.3 \%$ & $-0.8 \%$ \\
\hline
\end{tabular}

Sources:

U.S. Department of Transportation, Bureau of Transportation Statistics, www.transtats.bts.gov. (Additional resources: www.transtats.bts.gov and www.rita.dot.gov)

1970-76 Energy Use - Department of Transportation, Civil Aeronautics Board, Fuel Cost and Consumption, Washington, DC, 1981, and annual.

${ }^{a}$ Data are for all U.S. air carriers reporting on Form 41.

${ }^{\mathrm{b}}$ Available seats per aircraft is calculated as the ratio of available seat-miles to revenue aircraft-miles.

${ }^{c}$ Passenger load factor is calculated as the ratio of revenue passenger-miles to available seat-miles for scheduled and nonscheduled services.

${ }^{\mathrm{d}}$ Energy use includes fuel purchased abroad for international flights. 
General aviation includes: (1) aircraft operating under general operating and flight rules; (2) not-for-hire airplanes with a seating capacity of 20 or more or a maximum payload capacity of 6,000 lbs. or more; (3) rotorcraft external load operations; (4) on-demand and commuter operations not covered under Federal Aviation Regulations Part 121; and (5) agricultural aircraft operations.

Table 9.3

Summary Statistics for General Aviation, 1970-2012

\begin{tabular}{|c|c|c|c|}
\hline Calendar year & Total number of aircraft & $\begin{array}{l}\text { Aircraft hours flown } \\
\text { (thousands) }\end{array}$ & Energy use (trillion btu) \\
\hline 1970 & $131,700^{\mathrm{a}}$ & $26,030^{b}$ & 94.4 \\
\hline 1975 & 168,475 & 30,298 & 110.7 \\
\hline 1980 & 211,045 & 41,016 & 165.9 \\
\hline 1981 & 213,226 & 40,704 & 161.2 \\
\hline 1982 & 209,779 & 36,457 & 173.6 \\
\hline 1983 & 213,293 & 35,249 & 134.2 \\
\hline 1984 & 220,943 & 36,119 & 155.3 \\
\hline 1985 & 196,500 & 31,456 & 143.9 \\
\hline 1986 & 205,300 & 31,782 & 147.9 \\
\hline 1987 & 202,700 & 30,883 & 139.1 \\
\hline 1988 & 196,200 & 31,114 & 148.5 \\
\hline 1989 & 205,000 & 32,332 & 134.1 \\
\hline 1990 & 198,000 & 32,096 & 131.8 \\
\hline 1991 & 196,874 & 29,862 & 120.0 \\
\hline 1992 & 185,650 & 26,747 & 103.7 \\
\hline 1993 & 177,120 & 24,455 & 93.6 \\
\hline 1994 & 172,935 & 24,092 & 95.3 \\
\hline 1995 & 188,089 & 26,612 & 106.6 \\
\hline 1996 & 191,129 & 26,909 & 111.0 \\
\hline 1997 & 192,414 & 27,713 & 121.1 \\
\hline 1998 & 204,710 & 28,100 & 147.4 \\
\hline 1999 & 219,464 & 31,231 & 172.1 \\
\hline 2000 & 217,533 & 29,960 & 175.2 \\
\hline 2001 & 211,446 & 27,017 & 165.1 \\
\hline 2002 & 211,244 & 27,040 & 141.5 \\
\hline 2003 & 209,708 & 27,329 & 141.4 \\
\hline 2004 & 219,426 & 28,126 & 175.9 \\
\hline 2005 & 224,352 & 26,982 & 242.4 \\
\hline 2006 & 221,943 & 27,705 & 256.3 \\
\hline 2007 & 231,607 & 27,852 & 243.6 \\
\hline 2008 & 228,663 & 26,009 & 265.7 \\
\hline 2009 & 223,877 & 23,763 & 210.3 \\
\hline 2010 & 223,370 & 24,802 & 221.2 \\
\hline 2011 & 220,770 & 24,570 & 227.1 \\
\hline 2012 & 209,034 & 24,403 & 228.8 \\
\hline \multicolumn{4}{|c|}{ Average annual percentage change } \\
\hline 1970-2012 & $1.1 \%$ & $-0.2 \%$ & $2.1 \%$ \\
\hline 2002-2012 & $-0.1 \%$ & $-1.0 \%$ & $4.9 \%$ \\
\hline
\end{tabular}

Sources:

U.S. Department of Transportation, Federal Aviation Administration, General Aviation Activity and Avionics Survey: Calendar Year 2012, Tables 1.1, 1.4, 5.1, and annual. 2011 Data: Aviation Forecasts, Tables 28 and 29, May 2013. (Additional resources: www.faa.gov/data-research/aviation_data_statistics/general_aviation)

${ }^{a}$ Active fixed-wing general aviation aircraft only.

${ }^{\mathrm{b}}$ Includes rotorcraft. 
In the early seventies, domestic waterborne commerce accounted for over 60\% of total tonnage, but by 1994 foreign tonnage grew to more than half of all waterborne tonnage. Total foreign and domestic tons shipped were about 2.31 billion tons in 2012, down from a peak of 2.59 billion tons in 2006.

Table 9.4

Tonnage Statistics for Domestic and

International Waterborne Commerce, 1970-2012

(million tons shipped)

\begin{tabular}{|c|c|c|c|c|}
\hline Year & $\begin{array}{l}\text { Foreign and domestic } \\
\text { total }\end{array}$ & Foreign total $^{\mathrm{a}}$ & Domestic total $^{\mathrm{b}}$ & Percent domestic of total \\
\hline 1970 & 1,532 & 581 & 951 & $62.1 \%$ \\
\hline 1975 & 1,695 & 749 & 946 & $55.8 \%$ \\
\hline 1976 & 1,835 & 856 & 979 & $53.4 \%$ \\
\hline 1977 & 1,908 & 935 & 973 & $51.0 \%$ \\
\hline 1978 & 2,021 & 946 & 1,075 & $53.2 \%$ \\
\hline 1979 & 2,073 & 993 & 1,080 & $52.1 \%$ \\
\hline 1980 & 1,999 & 921 & 1,077 & $53.9 \%$ \\
\hline 1981 & 1,942 & 887 & 1,054 & $54.3 \%$ \\
\hline 1982 & 1,777 & 820 & 957 & $53.9 \%$ \\
\hline 1983 & 1,708 & 751 & 957 & $56.0 \%$ \\
\hline 1984 & 1,836 & 803 & 1,033 & $56.3 \%$ \\
\hline 1985 & 1,788 & 774 & 1,014 & $56.7 \%$ \\
\hline 1986 & 1,874 & 837 & 1,037 & $55.3 \%$ \\
\hline 1987 & 1,967 & 891 & 1,076 & $54.7 \%$ \\
\hline 1988 & 2,088 & 976 & 1,112 & $53.3 \%$ \\
\hline 1989 & 2,140 & 1,038 & 1,103 & $51.5 \%$ \\
\hline 1990 & 2,164 & 1,042 & 1,122 & $51.8 \%$ \\
\hline 1991 & 2,092 & 1,014 & 1,079 & $51.6 \%$ \\
\hline 1992 & 2,132 & 1,037 & 1,095 & $51.4 \%$ \\
\hline 1993 & 2,128 & 1,060 & 1,068 & $50.2 \%$ \\
\hline 1994 & 2,215 & 1,116 & 1,099 & $49.6 \%$ \\
\hline 1995 & 2,240 & 1,147 & 1,093 & $48.8 \%$ \\
\hline 1996 & 2,284 & 1,183 & 1,101 & $48.2 \%$ \\
\hline 1997 & 2,333 & 1,221 & 1,113 & $47.7 \%$ \\
\hline 1998 & 2,340 & 1,245 & 1,094 & $46.8 \%$ \\
\hline 1999 & 2,323 & 1,261 & 1,062 & $45.7 \%$ \\
\hline 2000 & 2,425 & 1,355 & 1,070 & $44.1 \%$ \\
\hline 2001 & 2,393 & 1,351 & 1,042 & $43.5 \%$ \\
\hline 2002 & 2,340 & 1,319 & 1,021 & $43.6 \%$ \\
\hline 2003 & 2,394 & 1,378 & 1,016 & $42.4 \%$ \\
\hline 2004 & 2,552 & 1,505 & 1,047 & $41.0 \%$ \\
\hline 2005 & 2,527 & 1,499 & 1,029 & $40.7 \%$ \\
\hline 2006 & 2,588 & 1,565 & 1,023 & $39.5 \%$ \\
\hline 2007 & 2,564 & 1,543 & 1,022 & $39.9 \%$ \\
\hline 2008 & 2,477 & 1,521 & 956 & $38.6 \%$ \\
\hline 2009 & 2,211 & 1,354 & 857 & $38.8 \%$ \\
\hline 2010 & 2,335 & 1,441 & 894 & $38.3 \%$ \\
\hline 2011 & 2,368 & 1,480 & 888 & $37.5 \%$ \\
\hline 2012 & 2,307 & 1,422 & 885 & $38.4 \%$ \\
\hline \multicolumn{5}{|c|}{ Average annual percentage change } \\
\hline 1970-2012 & $1.0 \%$ & $2.2 \%$ & $-0.2 \%$ & \\
\hline $2002-2012$ & $-0.1 \%$ & $0.8 \%$ & $-1.4 \%$ & \\
\hline
\end{tabular}

\section{Source:}

U.S. Department of the Army, Corps of Engineers, The U.S. Waterway System, Transportation Facts and Information, November 2013. (Additional resources: www.navigationdatacenter.us/index.htm)

a All movements between the United States and foreign countries and between Puerto Rico and the Virgin Islands and foreign countries are classified as foreign trade.

b All movements between U.S. ports, continental and noncontiguous, and on the inland rivers, canals, and connecting channels of the United States, Puerto Rico, and the Virgin Islands, excluding the Panama Canal. Beginning in 1996, fish was excluded for internal and intra port domestic traffic. 
The U.S. Army Corps of Engineers Navigation Data Center collects a wealth of waterborne commerce data. Energy use data, however, have never been collected as part of this effort. The energy use data collected by the Energy Information Administration (EIA) on vessel bunkering was formerly displayed on this table. The EIA data include different uses of fuel, not just fuel for domestic waterborne commerce; therefore it was misleading to display those data together.

Table 9.5 Summary Statistics for Domestic Waterborne Commerce, 1970-2012

\begin{tabular}{|c|c|c|c|c|}
\hline Year & Number of vessels ${ }^{\mathrm{a}}$ & $\begin{array}{c}\text { Ton-miles } \\
\text { (billions) }\end{array}$ & Tons shipped $^{\mathrm{b}}$ (millions) & $\begin{array}{c}\text { Average length of hau } \\
\text { (miles) }\end{array}$ \\
\hline 1970 & 25,832 & 596 & 949 & 628.2 \\
\hline 1975 & 31,666 & 566 & 944 & 599.9 \\
\hline 1980 & 38,792 & 922 & 1,074 & 856.4 \\
\hline 1985 & 41,672 & 893 & 1,011 & 883.5 \\
\hline 1990 & 41,119 & 834 & 1,118 & 745.7 \\
\hline 1991 & 39,233 & 848 & 1,074 & 789.9 \\
\hline 1992 & 39,210 & 857 & 1,090 & 785.7 \\
\hline 1993 & 39,064 & 790 & 1,063 & 742.7 \\
\hline 1994 & 39,064 & 815 & 1,093 & 745.5 \\
\hline 1995 & 39,445 & 808 & 1,086 & 743.6 \\
\hline 1996 & 41,104 & 765 & 1,093 & 699.4 \\
\hline 1997 & 41,419 & 707 & 1,106 & 639.5 \\
\hline 1998 & 42,032 & 673 & 1,087 & 619.0 \\
\hline 1999 & 41,766 & 656 & 1,056 & 621.1 \\
\hline 2000 & 39,641 & 646 & 1,064 & 606.8 \\
\hline 2001 & 41,588 & 622 & 1,037 & 599.7 \\
\hline 2002 & 41,002 & 612 & 1,016 & 602.5 \\
\hline 2003 & 39,983 & 606 & 1,010 & 600.3 \\
\hline 2004 & 40,290 & 621 & 1,042 & 596.4 \\
\hline 2005 & 41,354 & 591 & 1,024 & 577.4 \\
\hline 2006 & 41,109 & 562 & 1,018 & 548.7 \\
\hline 2007 & 40,695 & 553 & 1,016 & 544.2 \\
\hline 2008 & 40,301 & 521 & 952 & 546.7 \\
\hline 2009 & 40,109 & 477 & 852 & 559.7 \\
\hline 2010 & 40,512 & 503 & 894 & 562.8 \\
\hline 2011 & 40,521 & 500 & 888 & 563.5 \\
\hline 2012 & 40,530 & 475 & 885 & 536.5 \\
\hline \multicolumn{5}{|c|}{ Average annual percentage change } \\
\hline 1970-2012 & $1.1 \%$ & $-0.5 \%$ & $-0.2 \%$ & $-0.4 \%$ \\
\hline $2002-2012$ & $-0.1 \%$ & $-2.5 \%$ & $-1.4 \%$ & $-1.2 \%$ \\
\hline
\end{tabular}

Sources:

Number of vessels 1970-92, 1995-2010 - U.S. Department of the Army, Corps of Engineers, Waterborne Transportation Lines of the United States, 2011, New Orleans, LA, 2012, Table 2, p. 6, and annual. 1993-94 - U.S. Department of the Army, Corps of Engineers, The U.S. Waterway System-Facts, Navigation Data Center, New Orleans, Louisiana, January 1996.

Ton-miles, tons shipped, average length of haul - U.S. Department of the Army, Corps of Engineers, Waterborne Commerce of the United States, Calendar Year 2011, Part 5: National Summaries, New Orleans, LA, 2012, Table 1-4, pp. 1-6, 1-7, and annual. (Additional resources: www.navigationdatacenter.us/index.htm)

Number of vessels, ton-miles, tons shipped and average length of haul, 2011-2012 - U.S. Department of the Army, Corps of Engineers, The U.S. Waterway System, Transportation Facts and Information Fact Card.

${ }^{\text {a }}$ Grand total for self-propelled and non-self-propelled.

${ }^{\mathrm{b}}$ These figures are not consistent with the figures on Table 9.4 because intra-territory tons are not included in this table. Intra-territory traffic is traffic between ports in Puerto Rico and the Virgin Islands. 
The data displayed in this table come from the Environmental Protection Agency's NONROAD2008a model.

Table 9.6

Recreational Boat Energy Use, 1970-2012

\begin{tabular}{|c|c|c|c|c|}
\hline & Number of boats & Diesel fuel & Gasoline & Total energy use \\
\hline Year & (thousands) & & (trillion Btu) & \\
\hline 1970 & 10,087 & 5.5 & 151.7 & 157.2 \\
\hline 1971 & 10,137 & 6.5 & 152.6 & 159.2 \\
\hline 1972 & 10,187 & 7.6 & 153.6 & 161.2 \\
\hline 1973 & 10,237 & 8.6 & 154.5 & 163.2 \\
\hline 1974 & 10,287 & 9.7 & 155.5 & 165.1 \\
\hline 1975 & 10,337 & 10.7 & 156.4 & 167.1 \\
\hline 1976 & 10,387 & 11.8 & 157.4 & 169.1 \\
\hline 1977 & 10,437 & 12.8 & 158.3 & 171.1 \\
\hline 1978 & 10,487 & 13.9 & 159.3 & 173.1 \\
\hline 1979 & 10,537 & 14.9 & 160.2 & 175.1 \\
\hline 1980 & 10,587 & 16.0 & 161.2 & 177.1 \\
\hline 1981 & 10,637 & 17.0 & 162.1 & 179.1 \\
\hline 1982 & 10,687 & 18.0 & 163.1 & 181.1 \\
\hline 1983 & 10,737 & 19.1 & 164.0 & 183.1 \\
\hline 1984 & 10,787 & 20.1 & 165.0 & 185.1 \\
\hline 1985 & 10,837 & 21.2 & 165.9 & 187.1 \\
\hline 1986 & 10,887 & 22.2 & 166.9 & 189.1 \\
\hline 1987 & 10,937 & 23.3 & 167.8 & 191.1 \\
\hline 1988 & 11,030 & 24.3 & 170.4 & 194.7 \\
\hline 1989 & 11,122 & 25.4 & 172.9 & 198.3 \\
\hline 1990 & 11,215 & 26.4 & 175.4 & 201.8 \\
\hline 1991 & 11,327 & 27.5 & 178.7 & 206.2 \\
\hline 1992 & 11,440 & 28.5 & 182.0 & 210.5 \\
\hline 1993 & 11,553 & 29.5 & 185.3 & 214.8 \\
\hline 1994 & 11,770 & 30.6 & 192.5 & 223.1 \\
\hline 1995 & 11,988 & 31.6 & 199.7 & 231.3 \\
\hline 1996 & 12,206 & 32.7 & 206.8 & 239.5 \\
\hline 1997 & 12,244 & 33.7 & 207.2 & 240.9 \\
\hline 1998 & 12,283 & 34.8 & 207.4 & 242.2 \\
\hline 1999 & 12,321 & 35.8 & 207.1 & 243.0 \\
\hline 2000 & 12,359 & 36.8 & 206.6 & 243.4 \\
\hline 2001 & 12,464 & 37.9 & 206.9 & 244.9 \\
\hline 2002 & 12,568 & 39.0 & 206.7 & 245.7 \\
\hline 2003 & 12,673 & 40.2 & 206.0 & 246.2 \\
\hline 2004 & 12,777 & 41.3 & 205.0 & 246.2 \\
\hline 2005 & 12,882 & 42.4 & 203.7 & 246.1 \\
\hline 2006 & 12,984 & 43.5 & 202.5 & 245.9 \\
\hline 2007 & 13,086 & 44.6 & 201.2 & 245.8 \\
\hline 2008 & 13,189 & 45.7 & 200.0 & 245.7 \\
\hline 2009 & 13,291 & 46.8 & 198.8 & 245.6 \\
\hline 2010 & 13,393 & 47.9 & 197.3 & 245.2 \\
\hline 2011 & 13,497 & 49.0 & 195.9 & 244.9 \\
\hline 2012 & 13,602 & 50.1 & 194.7 & 244.8 \\
\hline \multicolumn{5}{|c|}{ Average annual percentage change } \\
\hline 1970-2012 & $0.7 \%$ & $5.4 \%$ & $0.6 \%$ & $1.1 \%$ \\
\hline $2002-2012$ & $0.8 \%$ & $2.5 \%$ & $-0.6 \%$ & $0.0 \%$ \\
\hline
\end{tabular}

\section{Source:}

U.S. Environmental Protection Agency, NONROAD2008a model, downloadable file from www.epa.gov/otaq/nonrdmdl.htm. 
The Interstate Commerce Commission designates Class I railroads on the basis of annual gross revenues. In 2012, seven railroads were given this designation. The number of railroads designated as Class I has changed considerably in the last 30 years; in 1976 there were 52 railroads given Class I designation.

Table 9.7

Class I Railroad Freight Systems in the United States Ranked by Revenue Ton-Miles, 2012

\begin{tabular}{lcr}
\hline \multicolumn{1}{c}{ Railroad } & $\begin{array}{c}\text { Revenue ton-miles } \\
\text { (billions) }\end{array}$ & Percent \\
\hline Burlington Northern and Santa Fe Railway Company & 658 & $38.4 \%$ \\
Union Pacific Railroad Company & 521 & $30.4 \%$ \\
CSX Transportation & 226 & $13.2 \%$ \\
Norfolk Southern Railway & 186 & $10.9 \%$ \\
Canadian National, Grand Trunk Corporation & 56 & $3.3 \%$ \\
Canadian Pacific Soo Railway & 37 & $2.2 \%$ \\
Kansas City Southern Railway Company & 30 & $1.8 \%$ \\
\hline Total & $\mathbf{1 , 7 1 4}$ & $\mathbf{1 0 0 . 0 \%}$ \\
\hline
\end{tabular}

\section{Source:}

Association of American Railroads, Railroad Facts, 2013 Edition, Washington, DC, December 2013, p. 66. (Additional resources: www.aar.org) 
Revenue ton-miles for Class I freight railroads was over 1.7 trillion in 2012. Though there are many regional and local freight railroads, the Class I freight railroads accounted for $94 \%$ of the railroad industry's freight revenue in 2012 and 69\% of the industry's mileage operated. The energy intensity of Class I railroads hit an all-time low of 289 btu/ton-mile in 2010 and continued to be below 300 btu/ton-mile in 2012.

Table 9.8

Summary Statistics for Class I Freight Railroads, 1970-2012

\begin{tabular}{|c|c|c|c|c|c|c|c|c|c|}
\hline Year & $\begin{array}{l}\text { Number of } \\
\text { locomotives } \\
\text { in service }^{\mathrm{a}}\end{array}$ & $\begin{array}{l}\text { Number of } \\
\text { freight cars } \\
\text { (thousands) }^{\mathrm{b}}\end{array}$ & $\begin{array}{c}\text { Train- } \\
\text { miles } \\
\text { (millions) }\end{array}$ & $\begin{array}{l}\text { Car-miles } \\
\text { (millions) }\end{array}$ & $\begin{array}{c}\text { Tons } \\
\text { originated }^{\mathrm{c}} \\
\text { (millions) }^{\text {millons }}\end{array}$ & $\begin{array}{c}\text { Average } \\
\text { length of } \\
\text { haul } \\
\text { (miles) }\end{array}$ & $\begin{array}{l}\text { Revenue } \\
\text { ton-miles } \\
\text { (millions) }\end{array}$ & $\begin{array}{c}\text { Energy } \\
\text { intensity } \\
\text { (Btu/ton- } \\
\text { mile) }\end{array}$ & $\begin{array}{c}\text { Energy } \\
\text { use } \\
\text { (trillion } \\
\text { Btu) }\end{array}$ \\
\hline 1970 & $27,077^{\mathrm{d}}$ & 1,424 & 427 & 29,890 & 1,485 & 515 & 764,809 & 691 & 528.1 \\
\hline 1975 & 27,846 & 1,359 & 403 & 27,656 & 1,395 & 541 & 754,252 & 687 & 518.3 \\
\hline 1980 & 28,094 & 1,168 & 428 & 29,277 & 1,492 & 616 & 918,958 & 597 & 548.7 \\
\hline 1981 & 27,421 & 1,111 & 408 & 27,968 & 1,453 & 626 & 910,169 & 572 & 521.0 \\
\hline 1983 & 25,448 & 1,007 & 346 & 24,358 & 1,293 & 641 & 828,275 & 525 & 435.1 \\
\hline 1984 & 24,117 & 948 & 369 & 26,409 & 1,429 & 645 & 921,542 & 510 & 469.9 \\
\hline 1985 & 22,548 & 867 & 347 & 24,920 & 1,320 & 665 & 876,984 & 497 & 436.1 \\
\hline 1986 & 20,790 & 799 & 347 & 24,414 & 1,306 & 664 & 867,722 & 486 & 421.5 \\
\hline 1987 & 19,647 & 749 & 361 & 25,627 & 1,372 & 688 & 943,747 & 456 & 430.3 \\
\hline 1988 & 19,364 & 725 & 379 & 26,339 & 1,430 & 697 & 996,182 & 443 & 441.4 \\
\hline 1989 & 19,015 & 682 & 383 & 26,196 & 1,403 & 723 & $1,013,841$ & 437 & 442.6 \\
\hline 1990 & 18,835 & 659 & 380 & 26,159 & 1,425 & 726 & $1,033,969$ & 420 & 434.7 \\
\hline 1992 & 18,004 & 605 & 390 & 26,128 & 1,399 & 763 & $1,066,781$ & 393 & 419.2 \\
\hline 1993 & 18,161 & 587 & 405 & 26,883 & 1,397 & 794 & $1,109,309$ & 389 & 431.6 \\
\hline 1994 & 18,505 & 591 & 441 & 28,485 & 1,470 & 817 & $1,200,701$ & 388 & 465.4 \\
\hline 1995 & 18,812 & 583 & 458 & 30,383 & 1,550 & 843 & $1,305,688$ & 372 & 485.9 \\
\hline 1996 & 19,269 & 571 & 469 & 31,715 & 1,611 & 842 & $1,355,975$ & 368 & 499.4 \\
\hline 1997 & 19,684 & 568 & 475 & 31,660 & 1,585 & 851 & $1,348,926$ & 370 & 499.7 \\
\hline 1998 & 20,261 & 576 & 475 & 32,657 & 1,649 & 835 & $1,376,802$ & 365 & 502.0 \\
\hline 1999 & 20,256 & 579 & 490 & 33,851 & 1,717 & 835 & $1,433,461$ & 363 & 520.0 \\
\hline 2000 & 20,028 & 560 & 504 & 34,590 & 1,738 & 843 & $1,465,960$ & 352 & 516.0 \\
\hline 2001 & 19,745 & 500 & 500 & 34,243 & 1,742 & 859 & $1,495,472$ & 346 & 517.3 \\
\hline 2002 & 20,506 & 478 & 500 & 34,680 & 1,767 & 853 & $1,507,011$ & 345 & 520.3 \\
\hline 2003 & 20,774 & 467 & 516 & 35,555 & 1,799 & 862 & $1,551,438$ & 344 & 533.9 \\
\hline 2004 & 22,015 & 474 & 535 & 37,071 & 1,844 & 902 & $1,662,598$ & 341 & 566.2 \\
\hline 2005 & 22,779 & 475 & 548 & 37,712 & 1,899 & 894 & $1,696,425$ & 337 & 571.4 \\
\hline 2006 & 23,732 & 475 & 563 & 38,995 & 1,957 & 906 & $1,771,897$ & 330 & 584.5 \\
\hline 2007 & 24,143 & 460 & 543 & 38,186 & 1,940 & 913 & $1,770,545$ & 320 & 566.9 \\
\hline 2010 & 23,893 & 398 & 476 & 35,541 & 1,851 & 914 & $1,691,004$ & 289 & 488.1 \\
\hline 2011 & 24,250 & 381 & 493 & 36,649 & 1,885 & 917 & $1,729,256$ & 298 & 514.6 \\
\hline 2012 & 24,707 & 381 & 500 & 36,525 & 1,760 & 973 & $1,712,567$ & 294 & 504.0 \\
\hline \multicolumn{10}{|c|}{ Average annual percentage change } \\
\hline 1970-2012 & $-0.2 \%$ & $-3.1 \%$ & $0.4 \%$ & $0.5 \%$ & $0.4 \%$ & $1.5 \%$ & $1.9 \%$ & $-2.0 \%$ & $-0.1 \%$ \\
\hline 2002-2012 & $1.9 \%$ & $-2.2 \%$ & $0.0 \%$ & $0.5 \%$ & $0.0 \%$ & $1.3 \%$ & $1.3 \%$ & $-1.6 \%$ & $-0.3 \%$ \\
\hline
\end{tabular}

\section{Source:}

Association of American Railroads, Railroad Facts, 2013 Edition, Washington, DC, December 2013, pp. 27, 28, 33, 34, 36, 50 and 52. (Additional resources: www.aar.org)

${ }^{\text {a }}$ Does not include self-powered units.

${ }^{\mathrm{b}}$ Does not include private or shipper-owned cars. Beginning in 2001, Canadian-owned U.S. railroads are excluded.

${ }^{c}$ Tons originated is a more accurate representation of total tonnage than revenue tons. Revenue tons often produces double-counting of loads switched between rail companies.

${ }^{\mathrm{d}}$ Data represent total locomotives used in freight and passenger service. Separate estimates are not available. 
According to the 2012 Commodity Flow Survey, 7\% of all freight ton-miles are rail intermodal shipments (truck/rail or rail/water). See Table 5.16 for details. The number of trailers and containers moved by railroads has increased more than seven-fold from 1965 to 2012. Containerization has increased in the last two decades, evidenced by the $367 \%$ increase in the number of containers from 1988 to 2012. The number of trailers moved by rail, however, fell to an all-time low in 2009, but has continued to increase since that time.

Table 9.9

Intermodal Rail Traffic, 1965-2012

\begin{tabular}{|c|c|c|c|}
\hline Year & Trailers \& containers & Trailers & Containers \\
\hline 1965 & $1,664,929$ & $\mathrm{~b}$ & $\mathrm{~b}$ \\
\hline 1970 & $2,363,200$ & $\mathrm{~b}^{-}$ & $\mathrm{b}^{-}$ \\
\hline 1975 & $2,238,117$ & b & $\mathrm{b}$ \\
\hline 1980 & $3,059,402$ & $\mathrm{~b}^{-}$ & -5 \\
\hline 1981 & $3,150,522$ & $\mathrm{~b}$ & $\mathrm{~b}$ \\
\hline 1982 & $3,396,973$ & $\mathrm{~b}$ & $\mathrm{~b}$ \\
\hline 1983 & $4,090,078$ & $\mathrm{~b}$ & $\mathrm{~b}$ \\
\hline 1984 & $4,565,743$ & $\mathrm{~b}$ & $\mathrm{~b}$ \\
\hline 1985 & $4,590,952$ & $\mathrm{~b}$ & b \\
\hline 1986 & $4,997,229$ & $\mathrm{~b}$ & $\mathrm{~b}$ \\
\hline 1987 & $5,503,819$ & $\mathrm{~b}$ & $\mathrm{~b}$ \\
\hline 1988 & $5,779,547$ & $3,481,020$ & $2,298,527$ \\
\hline 1989 & $5,987,355$ & $3,496,262$ & $2,491,093$ \\
\hline 1990 & $6,206,782$ & $3,451,953$ & $2,754,829$ \\
\hline 1991 & $6,246,134$ & $3,201,560$ & $3,044,574$ \\
\hline 1992 & $6,627,841$ & $3,264,597$ & $3,363,244$ \\
\hline 1993 & $7,156,628$ & $3,464,126$ & $3,692,502$ \\
\hline 1994 & $8,128,228$ & $3,752,502$ & $4,375,726$ \\
\hline 1995 & $7,936,172$ & $3,492,463$ & $4,443,709$ \\
\hline 1996 & $8,143,258$ & $3,302,128$ & $4,841,130$ \\
\hline 1997 & $8,698,308$ & $3,453,907$ & $5,244,401$ \\
\hline 1998 & $8,772,663$ & $3,353,032$ & $5,419,631$ \\
\hline 1999 & $8,907,626$ & $3,207,407$ & $5,700,219$ \\
\hline 2000 & $9,176,890$ & $2,888,630$ & $6,288,260$ \\
\hline 2001 & $8,935,444$ & $2,603,423$ & $6,332,021$ \\
\hline 2002 & $9,312,360$ & $2,531,338$ & $6,781,022$ \\
\hline 2003 & $9,955,605$ & $2,625,837$ & $7,329,768$ \\
\hline 2004 & $10,993,662$ & $2,928,123$ & $8,065,539$ \\
\hline 2005 & $11,693,512$ & $2,979,906$ & $8,713,606$ \\
\hline 2006 & $12,282,221$ & $2,882,699$ & $9,399,522$ \\
\hline 2007 & $12,026,631$ & $2,600,635$ & $9,425,996$ \\
\hline 2008 & $11,499,978$ & $2,478,890$ & $9,021,088$ \\
\hline 2009 & $9,875,967$ & $1,639,603$ & $8,236,364$ \\
\hline 2010 & $11,283,151$ & $1,684,684$ & $9,598,467$ \\
\hline 2011 & $11,892,418$ & $1,698,615$ & $10,193,803$ \\
\hline 2012 & $12,267,416$ & $1,518,323$ & $10,749,093$ \\
\hline \multicolumn{4}{|c|}{ Average annual percentage change } \\
\hline $1965-2012$ & $4.3 \%$ & $\mathrm{~b}$ & $\mathrm{~b}$ \\
\hline 2002-2012 & $2.8 \%$ & $-5.0 \%$ & $4.7 \%$ \\
\hline
\end{tabular}

Source:

Association of American Railroads, Railroad Facts, 2013 Edition, Washington, DC, December 2013, p. 26. (Additional resources: www.aar.org)

${ }^{\text {a }}$ Beginning in 1995, the Grand Trunk Western Railroad and the Soo Line Railroad Company are excluded. Beginning in 1999, the Illinois Central data are excluded. Beginning in 2002, the Wisconsin Central data are excluded.

${ }^{\mathrm{b}}$ Data are not available. 
The National Railroad Passenger Corporation, known as Amtrak, began operation in 1971. Amtrak revenue passenger-miles have grown at an average annual rate of $3.0 \%$ from 1971 to 2012.

Table 9.10

Summary Statistics for the National Railroad Passenger Corporation (Amtrak), 1971-2012

\begin{tabular}{|c|c|c|c|c|c|c|c|c|}
\hline Year & $\begin{array}{l}\text { Number of } \\
\text { locomotives } \\
\text { in service }\end{array}$ & $\begin{array}{c}\text { Number of } \\
\text { passenger } \\
\text { cars }\end{array}$ & $\begin{array}{l}\text { Train-miles } \\
\text { (thousands) }\end{array}$ & $\begin{array}{l}\text { Car-miles } \\
\text { (thousands) }\end{array}$ & $\begin{array}{c}\text { Revenue } \\
\text { passenger- } \\
\text { miles } \\
\text { (millions) }\end{array}$ & $\begin{array}{l}\text { Average } \\
\text { trip length } \\
\text { (miles) }\end{array}$ & $\begin{array}{l}\text { Energy intensity } \\
\text { (Btu per revenue } \\
\text { passenger-mile) }\end{array}$ & $\begin{array}{l}\text { Energy } \\
\text { use } \\
\text { (trillion } \\
\text { Btu) }\end{array}$ \\
\hline 1971 & $\mathrm{a}$ & 1,165 & 16,537 & 140,147 & 1,993 & 188 & $\mathrm{a}$ & $\mathrm{a}$ \\
\hline 1975 & 355 & 1,913 & 30,166 & 253,898 & 3,753 & 224 & 3,548 & 13.3 \\
\hline 1980 & 448 & 2,128 & 29,487 & 235,235 & 4,503 & 217 & 3,065 & 13.8 \\
\hline 1985 & 382 & 1,818 & 30,038 & 250,642 & 4,785 & 238 & 2,703 & 12.9 \\
\hline 1987 & 381 & 1,850 & 29,515 & 261,054 & 5,361 & 259 & 2,450 & 13.1 \\
\hline 1988 & 391 & 1,845 & 30,221 & 277,774 & 5,686 & 265 & 2,379 & 13.5 \\
\hline 1989 & 312 & 1,742 & 31,000 & 285,255 & 5,859 & 274 & 2,614 & 15.3 \\
\hline 1990 & 318 & 1,863 & 33,000 & 300,996 & 6,057 & 273 & 2,505 & 15.2 \\
\hline 1991 & 316 & 1,786 & 34,000 & 312,484 & 6,273 & 285 & 2,417 & 15.2 \\
\hline 1992 & 336 & 1,796 & 34,000 & 307,282 & 6,091 & 286 & 2,534 & 15.4 \\
\hline 1993 & 360 & 1,853 & 34,936 & 302,739 & 6,199 & 280 & 2,565 & 15.9 \\
\hline 1994 & 411 & 1,874 & 34,940 & 305,600 & 5,869 & 276 & 2,282 & 13.4 \\
\hline 1996 & 348 & 1,501 & 30,542 & 277,750 & 5,066 & 257 & 2,690 & 13.6 \\
\hline 1997 & 292 & 1,572 & 32,000 & 287,760 & 5,166 & 255 & 2,811 & 14.5 \\
\hline 1998 & 362 & 1,347 & 32,926 & 315,823 & 5,325 & 251 & 2,788 & 14.8 \\
\hline 1999 & 385 & 1,285 & 34,080 & 349,337 & 5,289 & 245 & 2,943 & 15.6 \\
\hline 2000 & 385 & 1,891 & 35,404 & 371,215 & 5,574 & 243 & 3,235 & 18.0 \\
\hline 2001 & 401 & 2,084 & 36,512 & 377,705 & 5,571 & 238 & 3,257 & 18.1 \\
\hline 2002 & 372 & 2,896 & 37,624 & 378,542 & 5,314 & 228 & 3,212 & 17.1 \\
\hline 2003 & 442 & 1,623 & 37,459 & 331,864 & 5,680 & 231 & 2,800 & 15.9 \\
\hline 2004 & 276 & 1,211 & 37,159 & 308,437 & 5,511 & 219 & 2,760 & 15.2 \\
\hline 2005 & 258 & 1,186 & 36,199 & 264,796 & 5,381 & 215 & 2,709 & 14.6 \\
\hline 2006 & 319 & 1,191 & 36,083 & 263,908 & 5,410 & 220 & 2,650 & 14.3 \\
\hline 2007 & 270 & 1,164 & 37,484 & 266,545 & 5,784 & 218 & 2,516 & 14.5 \\
\hline 2008 & 278 & 1,177 & 37,736 & 271,762 & 6,179 & 215 & 2,398 & 14.8 \\
\hline 2009 & 274 & 1,214 & 38,300 & 282,764 & 5,914 & 217 & 2,435 & 14.4 \\
\hline 2010 & 282 & 1,274 & 37,453 & 294,820 & 6,420 & 220 & 2,271 & 14.6 \\
\hline 2011 & 287 & 1,301 & 37,090 & 296,315 & 6,670 & 213 & 2,214 & 14.5 \\
\hline \multicolumn{9}{|c|}{ Average annual percentage change } \\
\hline 1971-2012 & $\mathrm{a}$ & $1.4 \%$ & $2.0 \%$ & $2.0 \%$ & $3.0 \%$ & $0.4 \%$ & a & a \\
\hline 2002-2012 & $2.7 \%$ & $-3.2 \%$ & $0.0 \%$ & $-1.7 \%$ & $2.5 \%$ & $-0.4 \%$ & $-3.7 \%$ & $-1.6 \%$ \\
\hline
\end{tabular}

\section{Sources:}

1971-83 - Association of American Railroads, Economics and Finance Department, Statistics of Class I Railroads, Washington, DC, and annual.

1984-88 - Association of American Railroads, Railroad Facts, 1988 Edition, Washington, DC, December 1989, p. 61, and annual.

1989-93 - Personal communication with the Corporate Accounting Office of Amtrak, Washington, D.C.

1994-2012 - Number of locomotives in service, number of passenger cars, train-miles, car-miles, revenue passenger-miles, and average trip length - Association of American Railroads, Railroad Facts, 2013 Edition, Washington, DC, 2013, p. 77.

Energy use - Personal communication with the Amtrak, Washington, DC. (Additional resources: www.amtrak.com, www.aar.org)

${ }^{\mathrm{a}}$ Data are not available.

${ }^{b}$ Energy use for 1994 on is not directly comparable to earlier years. Some commuter rail energy use may have been inadvertently included in earlier years. 
Commuter rail, which is also known as regional rail or suburban rail, is long-haul rail passenger service operating between metropolitan and suburban areas, whether within or across state lines. Commuter rail lines usually have reduced fares for multiple rides and commutation tickets for regular, recurring riders.

Table 9.11

Summary Statistics for Commuter Rail Operations, 1984-2012

\begin{tabular}{|c|c|c|c|c|c|c|c|}
\hline Year & $\begin{array}{l}\text { Number of } \\
\text { passenger } \\
\text { vehicles }\end{array}$ & $\begin{array}{l}\text { Vehicle- } \\
\text { miles } \\
\text { (millions) }\end{array}$ & $\begin{array}{l}\text { Passenger } \\
\text { trips } \\
\text { (millions) }\end{array}$ & $\begin{array}{l}\text { Passenger- } \\
\text { miles } \\
\text { (millions) }\end{array}$ & $\begin{array}{l}\text { Average } \\
\text { trip length } \\
\text { (miles) }\end{array}$ & $\begin{array}{c}\text { Energy intensity } \\
\text { (Btu/passenger- } \\
\text { mile) }\end{array}$ & $\begin{array}{c}\text { Energy } \\
\text { use } \\
\text { (trillion } \\
\text { Btu) }\end{array}$ \\
\hline 1984 & 4,075 & 167.9 & 267 & 6,207 & 23.2 & 2,804 & 17.4 \\
\hline 1985 & 4,035 & 182.7 & 275 & 6,534 & 23.8 & 2,826 & 18.5 \\
\hline 1986 & 4,440 & 188.6 & 306 & 6,723 & 22.0 & 2,926 & 19.7 \\
\hline 1987 & 4,686 & 188.9 & 311 & 6,818 & 21.9 & 2,801 & 19.1 \\
\hline 1988 & 4,649 & 202.2 & 325 & 6,964 & 21.4 & 2,872 & 19.7 \\
\hline 1989 & 4,472 & 209.6 & 330 & 7,211 & 21.9 & 2,864 & 20.7 \\
\hline 1990 & 4,982 & 212.7 & 328 & 7,082 & 21.6 & 2,822 & 20.0 \\
\hline 1991 & 5,126 & 214.9 & 318 & 7,344 & 23.1 & 2,770 & 20.3 \\
\hline 1992 & 5,164 & 218.8 & 314 & 7,320 & 23.3 & 2,629 & 19.2 \\
\hline 1993 & 4,982 & 223.9 & 322 & 6,940 & 21.6 & 2,976 & 20.7 \\
\hline 1994 & 5,126 & 230.8 & 339 & 7,996 & 23.6 & 2,682 & 21.4 \\
\hline 1995 & 5,164 & 237.7 & 344 & 8,244 & 24.0 & 2,632 & 21.7 \\
\hline 1996 & 5,240 & 241.9 & 352 & 8,351 & 23.7 & 2,582 & 21.6 \\
\hline 1997 & 5,426 & 250.7 & 357 & 8,038 & 22.5 & 2,724 & 21.9 \\
\hline 1998 & 5,536 & 259.5 & 381 & 8,704 & 22.8 & 2,646 & 23.0 \\
\hline 1999 & 5,550 & 265.9 & 396 & 8,766 & 22.1 & 2,714 & 23.8 \\
\hline 2000 & 5,498 & 270.9 & 413 & 9,402 & 22.8 & 2,551 & 24.0 \\
\hline 2001 & 5,572 & 277.3 & 419 & 9,548 & 22.8 & 2,515 & 24.0 \\
\hline 2002 & 5,724 & 283.7 & 414 & 9,504 & 22.9 & 2,514 & 23.9 \\
\hline 2003 & 5,959 & 286.0 & 410 & 9,559 & 23.3 & 2,545 & 24.3 \\
\hline 2004 & 6,228 & 294.7 & 414 & 9,719 & 23.5 & 2,569 & 25.0 \\
\hline 2005 & 6,392 & 303.4 & 423 & 9,473 & 22.4 & 2,743 & 26.0 \\
\hline 2006 & 6,403 & 314.7 & 441 & 10,361 & 23.5 & 2,527 & 26.2 \\
\hline 2007 & 6,391 & 325.7 & 459 & 11,153 & 24.3 & 2,638 & 29.4 \\
\hline 2008 & 6,617 & 310.2 & 472 & 11,049 & 23.4 & 2,656 & 29.3 \\
\hline 2009 & 6,941 & 343.5 & 468 & 11,232 & 24.0 & 2,812 & 31.6 \\
\hline 2010 & 6,927 & 345.3 & 464 & 10,874 & 23.4 & 2,897 & 31.5 \\
\hline 2011 & 7,193 & 345.2 & 466 & 11,427 & 24.5 & 2,794 & 31.9 \\
\hline 2012 & 7,059 & 346.4 & 471 & 11,181 & 23.7 & 2,838 & 31.7 \\
\hline \multicolumn{8}{|c|}{ Average annual percentage change } \\
\hline 1984-2012 & $2.0 \%$ & $2.6 \%$ & $2.0 \%$ & $2.1 \%$ & $0.1 \%$ & & \\
\hline 2002-2012 & $2.1 \%$ & $2.0 \%$ & $1.3 \%$ & $1.6 \%$ & $0.3 \%$ & & \\
\hline
\end{tabular}

Source:

American Public Transportation Association, 2014 Public Transportation Fact Book, Washington, DC, May 2014, Appendix A. (Additional resources: www.apta.com) 
This table on transit rail operations includes data on light rail and heavy rail systems. Light rail vehicles are usually single vehicles driven electrically with power drawn from overhead wires. Heavy rail is characterized by high speed and rapid acceleration of rail cars operating on a separate right-of-way.

Table 9.12

Summary Statistics for Rail Transit Operations, 1970-2012

\begin{tabular}{|c|c|c|c|c|c|c|c|}
\hline Year & $\begin{array}{c}\text { Number of } \\
\text { passenger } \\
\text { vehicles }\end{array}$ & $\begin{array}{l}\text { Vehicle- } \\
\text { miles } \\
\text { (millions) }\end{array}$ & $\begin{array}{l}\text { Passenger } \\
\text { trips } \\
\text { (millions) }^{\mathrm{b}}\end{array}$ & $\begin{array}{c}\text { Passenger- } \\
\text { miles } \\
\text { (millions) }^{\mathrm{c}}\end{array}$ & $\begin{array}{c}\text { Average trip } \\
\text { length } \\
\text { (miles) }^{\mathrm{d}}\end{array}$ & $\begin{array}{l}\text { Energy intensity } \\
\text { (Btu/passenger- } \\
\text { mile })^{\mathrm{e}}\end{array}$ & $\begin{array}{c}\text { Energy use } \\
\text { (trillion Btu) }\end{array}$ \\
\hline 1970 & 10,548 & 440.8 & 2,116 & 12,273 & $\mathrm{f}$ & 2,157 & 26.5 \\
\hline $1980^{-}$ & 10,654 & 402.2 & 2,241 & 10,939 & 4.9 & 2,312 & 25.3 \\
\hline 1985 & 11,109 & 467.8 & 2,422 & 10,777 & 4.4 & 2,809 & 30.3 \\
\hline 1986 & 11,083 & 492.8 & 2,467 & 11,018 & 4.5 & 3,042 & 33.5 \\
\hline 1988 & 11,370 & 538.3 & 2,462 & 11,836 & 4.8 & 3,072 & 36.2 \\
\hline 1989 & 11,261 & 553.4 & 2,704 & 12,539 & 4.6 & 2,909 & 36.5 \\
\hline 1990 & 11,332 & 560.9 & 2,521 & 12,046 & 4.8 & 3,024 & 36.4 \\
\hline 1991 & 11,426 & 554.8 & 2,356 & 11,190 & 4.7 & 3,254 & 36.4 \\
\hline 1992 & 11,303 & 554.0 & 2,395 & 11,438 & 4.8 & 3,155 & 36.1 \\
\hline 1993 & 11,286 & 549.8 & 2,234 & 10,936 & 4.9 & 3,373 & 36.9 \\
\hline 1994 & 11,192 & 565.8 & 2,453 & 11,501 & 4.7 & 3,338 & 38.4 \\
\hline 1995 & 11,156 & 571.8 & 2,284 & 11,419 & 5.0 & 3,340 & 38.1 \\
\hline 1997 & 11,471 & 598.9 & 2,692 & 13,091 & 4.9 & 2,856 & 37.4 \\
\hline 1998 & 11,521 & 609.5 & 2,669 & 13,412 & 5.0 & 2,823 & 37.9 \\
\hline 1999 & 11,603 & 626.4 & 2,813 & 14,108 & 5.0 & 2,785 & 39.3 \\
\hline 2000 & 12,168 & 648.0 & 2,952 & 15,200 & 5.1 & 2,797 & 42.5 \\
\hline 2001 & 12,084 & 662.4 & 3,064 & 15,615 & 5.1 & 2,803 & 43.8 \\
\hline 2002 & 12,479 & 681.9 & 3,025 & 15,095 & 5.0 & 2,872 & 43.3 \\
\hline 2003 & 12,236 & 694.2 & 3,005 & 15,082 & 5.0 & 2,837 & 42.8 \\
\hline 2004 & 12,480 & 709.7 & 3,098 & 15,930 & 5.1 & 2,750 & 43.8 \\
\hline 2005 & 12,755 & 715.4 & 3,189 & 16,118 & 5.1 & 2,783 & 44.9 \\
\hline 2006 & 12,853 & 726.4 & 3,334 & 16,587 & 5.0 & 2,707 & 44.9 \\
\hline 2007 & 13,032 & 741.2 & 3,879 & 18,070 & 4.7 & 2,577 & 46.6 \\
\hline 2008 & 13,346 & 762.8 & 4,001 & 18,941 & 4.7 & 2,521 & 47.8 \\
\hline 2009 & 13,529 & 775.3 & 3,955 & 19,004 & 4.8 & 2,516 & 47.8 \\
\hline 2010 & 13,614 & 759.6 & 4,007 & 18,580 & 4.6 & 2,520 & 46.8 \\
\hline 2011 & 13,328 & 744.1 & 4,083 & 19,520 & 4.8 & 2,459 & 48.0 \\
\hline 2012 & 12,455 & 749.5 & 4,192 & 19,835 & 4.7 & 2,398 & 47.6 \\
\hline 2002-2012 & $0.0 \%$ & $0.9 \%$ & $3.3 \%$ & $2.8 \%$ & $-0.6 \%$ & $-1.8 \%$ & $1.0 \%$ \\
\hline
\end{tabular}

Sources:

American Public Transportation Association, 2014 Public Transportation Fact Book, Washington, DC, May 2014, Appendix A. (Additional resources: www.apta.com)

Energy use - See Appendix A for Rail Transit Energy Use.

${ }^{a}$ Heavy rail and light rail. Series not continuous between 1983 and 1984 because of a change in data source by the American Public Transit Association (APTA). Beginning in 1984, data provided by APTA are taken from mandatory reports filed with the Urban Mass Transit Administration (UMTA). Data for prior years were provided on a voluntary basis by APTA members and expanded statistically.

${ }^{\mathrm{b}}$ 1970-79 data represents total passenger rides; after 1979, data represents unlinked passenger trips.

${ }^{\mathrm{c}}$ Estimated for years $1970-76$ based on an average trip length of 5.8 miles.

${ }^{\mathrm{d}}$ Calculated as the ratio of passenger-miles to passenger trips.

${ }^{\mathrm{e}}$ Large system-to-system variations exist within this category.

${ }^{\mathrm{f}}$ Data are not available.

${ }^{g}$ Average annual percentage change is calculated for years 1980-2012. 


\section{Chapter 10 Transportation and the Economy}

Summary Statistics from Tables/Figures in this Chapter

Source

Figure 10.2 Share of gasoline cost attributed to taxes, 2013

$\begin{array}{lr}\text { Canada } & 31 \% \\ \text { France } & 56 \% \\ \text { Germany } & 57 \% \\ \text { Japan } & 41 \% \\ \text { United Kingdom } & 60 \% \\ \text { United States } & 14 \% \\ \text { erage price of a new car, 2012 (current dollars) } & 25,517 \\ \text { Domestic } & 24,025 \\ \text { Import } & 29,191\end{array}$

Table 10.12 Car operating costs, 2013

Variable costs (constant 2013 dollars per 10,000 miles) 2,042

Fixed costs (constant 2013 dollars per 10,000 miles) 5,792

Table 10.16 Transportation sector share of total employment

2000

$8.3 \%$

2013

$7.3 \%$ 
The Transportation Services Index (TSI) was created by the U.S. Department of Transportation Bureau of Transportation Statistics (BTS). It is an index that measures the movement of freight and passengers. The Freight TSI consists of:

- for-hire trucking (parcel services are not included);

- freight railroad services (including rail-based intermodal shipments such as containers on flat cars);inland waterway traffic;

- pipeline movements (including principally petroleum and petroleum products and natural gas); and

- air freight.

The index does not include international or coastal steamship movements, private trucking, courier services, or the United States Postal Services.

The index does not include intercity bus, sightseeing services, taxi service, private car usage, or bicycling and other nonmotorized means of transportation.

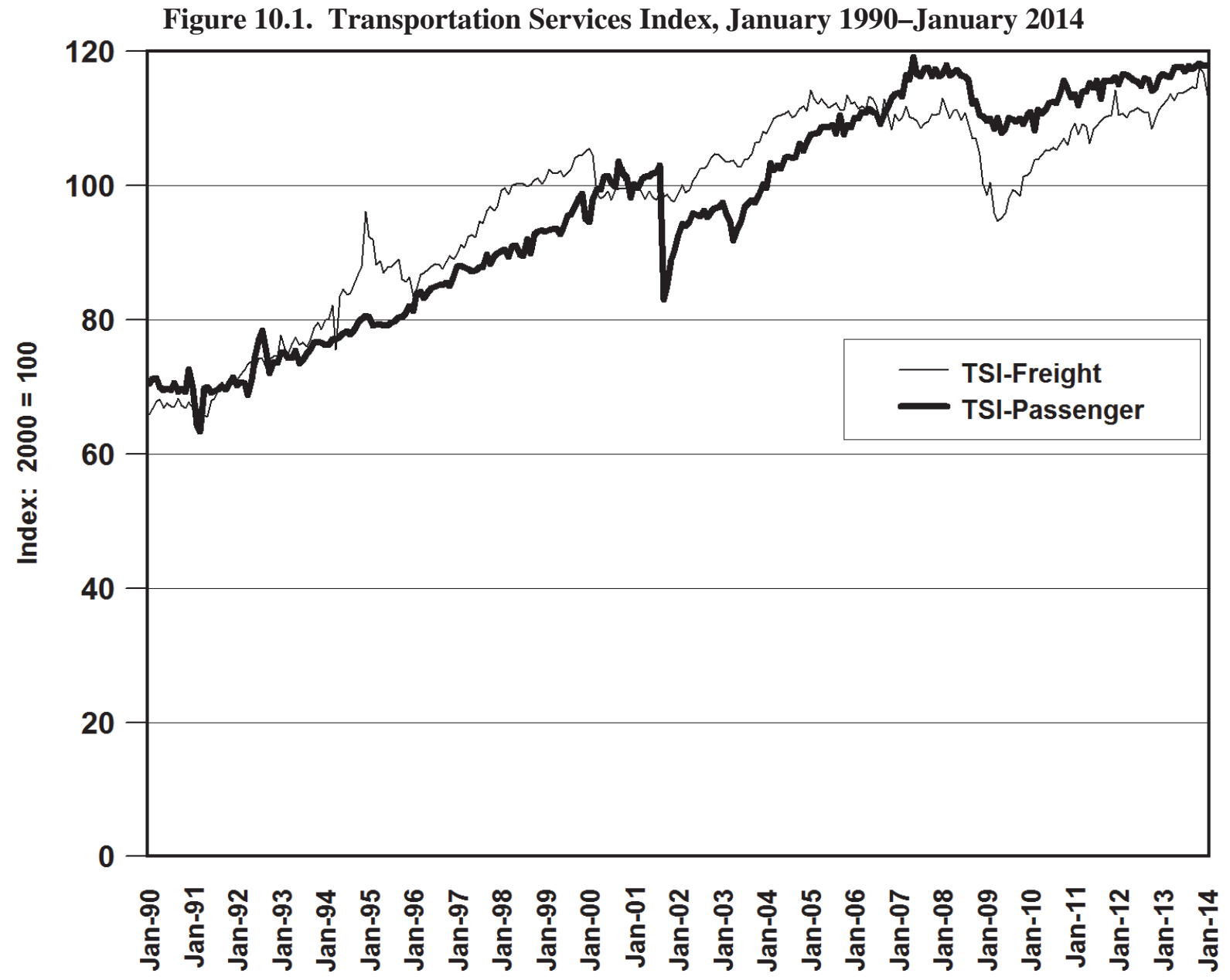

Source:

U.S. Department of Transportation, Bureau of Transportation Statistics, Transportation Services Index website, www.bts.gov/xml/tsi/src/index.html. (Additional resources: www.bts.gov) 
The United States prices are the lowest of these listed countries. Those in France, Japan, Korea, the United Kingdom, and Germany paid, on average, more than six dollars per gallon in 2013. Data for China and India have been discontinued by the International Energy Agency.

Table 10.1

Gasoline Prices ${ }^{\text {a }}$ for Selected Countries, 1990-2013

\begin{tabular}{|c|c|c|c|c|c|c|c|}
\hline & \multicolumn{6}{|c|}{ Current dollars per gallon } & $\begin{array}{c}\text { Average annual } \\
\text { percentage change }\end{array}$ \\
\hline & 1990 & 1995 & 2000 & 2005 & 2010 & $2013^{\mathrm{b}}$ & $1990-2013$ \\
\hline China & c & 1.03 & c & 1.70 & 3.71 & $c$ & $\mathrm{c}$ \\
\hline Japan & 3.16 & 4.43 & 3.65 & 4.28 & 5.73 & 6.00 & $2.8 \%$ \\
\hline India & c & $\mathrm{c}$ & c & 3.71 & 4.29 & c & c \\
\hline Korea & $\mathrm{c}$ & c & c & 5.28 & 5.60 & 6.73 & $\mathrm{c}$ \\
\hline France $^{d}$ & 3.63 & 4.26 & 3.80 & 5.46 & 6.74 & 7.66 & $3.3 \%$ \\
\hline United Kingdom $^{\mathrm{d}}$ & 2.82 & 3.21 & 4.58 & 5.97 & 6.83 & 8.01 & $4.6 \%$ \\
\hline Germany $^{\mathrm{d}}$ & 2.65 & 3.96 & 3.45 & 5.75 & 7.11 & 8.03 & $4.9 \%$ \\
\hline Canada & 1.87 & 1.53 & 1.86 & 2.89 & 3.79 & 4.50 & $3.9 \%$ \\
\hline \multirow[t]{3}{*}{ United States ${ }^{\mathrm{e}}$} & 1.16 & 1.15 & 1.51 & 2.27 & 2.78 & 3.29 & $4.6 \%$ \\
\hline & \multicolumn{6}{|c|}{ Constant 2013 dollars ${ }^{\mathrm{f}}$ per gallon } & $\begin{array}{c}\text { Average annual } \\
\text { percentage change }\end{array}$ \\
\hline & 1990 & 1995 & 2000 & 2005 & 2010 & $2013^{b}$ & 1990-2013 \\
\hline China & c & 1.57 & $\mathrm{c}$ & 2.03 & 3.96 & c & c \\
\hline Japan & 5.63 & 6.77 & 4.94 & 5.10 & 6.13 & 6.00 & $0.3 \%$ \\
\hline India & $\mathrm{c}$ & c & c & 4.42 & 4.59 & c & c \\
\hline Korea & $\mathrm{c}$ & c & c & 6.29 & 5.98 & 6.73 & c \\
\hline France $^{d}$ & 6.47 & 6.51 & 5.14 & 6.51 & 7.20 & 7.66 & $0.7 \%$ \\
\hline United Kingdom $^{\mathrm{d}}$ & 5.03 & 4.91 & 6.20 & 7.12 & 7.30 & 8.01 & $2.0 \%$ \\
\hline Germany $^{\mathrm{d}}$ & 4.72 & 6.05 & 4.67 & 6.86 & 7.59 & 8.03 & $2.3 \%$ \\
\hline Canada & 3.33 & 2.34 & 2.52 & 3.45 & 4.05 & 4.50 & $1.3 \%$ \\
\hline United States ${ }^{\mathrm{e}}$ & 2.07 & 1.76 & 2.04 & 2.71 & 2.97 & 3.29 & $2.0 \%$ \\
\hline
\end{tabular}

Note: Comparisons between prices and price trends in different countries require care. They are of limited validity because of fluctuations in exchange rates; differences in product quality, marketing practices, and market structures; and the extent to which the standard categories of sales are representative of total national sales for a given period.

\section{Source:}

International Energy Agency, Energy Prices and Taxes, Fourth Quarter, 2013, Paris, France, 2014. (Additional resources: www.iea.org)

${ }^{\text {a }}$ Prices represent the retail prices (including taxes) for regular unleaded gasoline, except for France, Germany and the United Kingdom which are premium unleaded gasoline.

b $4^{\text {th }}$ quarter 2013.

${ }^{c}$ Data are not available.

${ }^{\mathrm{d}}$ Premium gasoline.

e These estimates are international comparisons only and do not necessarily correspond to gasoline price estimates in other sections of the book.

${ }^{\mathrm{f}}$ Adjusted by the U.S. Consumer Price Inflation Index. 
Of these selected countries, the United Kingdom had the highest diesel fuel price average in 2013, while the United States had the lowest. In fact, all countries listed except the United States had diesel prices over \$5 per gallon in 2013 .

Table 10.2

Diesel Fuel Prices ${ }^{\text {a }}$ for Selected Countries, 1998-2013

\begin{tabular}{|c|c|c|c|c|c|c|c|}
\hline & \multicolumn{6}{|c|}{ Current dollars per gallon } & $\begin{array}{c}\text { Average annua } \\
\text { percentage } \\
\text { change }\end{array}$ \\
\hline & 1990 & 2000 & 2005 & 2010 & 2012 & $2013^{b}$ & 1990-2013 \\
\hline China & c & $\mathrm{c}$ & 1.69 & 3.65 & $\mathrm{c}$ & $\mathrm{c}$ & $\mathrm{c}$ \\
\hline Japan & 1.75 & 2.85 & 3.44 & 4.86 & 6.03 & 5.21 & $4.9 \%$ \\
\hline Korea & $\mathrm{c}$ & 2.05 & 3.98 & 4.92 & 6.07 & 6.06 & $\mathrm{c}$ \\
\hline France & 1.78 & 2.95 & 4.81 & 5.74 & 6.79 & 6.82 & $6.0 \%$ \\
\hline United Kingdom & 2.04 & 4.66 & 6.25 & 6.97 & 8.51 & 8.48 & $6.4 \%$ \\
\hline Germany & 2.72 & 2.79 & 5.01 & 6.15 & 7.25 & 7.29 & $4.4 \%$ \\
\hline \multirow[t]{3}{*}{ United States $^{\mathrm{d}}$} & 0.99 & 1.50 & 2.40 & 2.99 & 3.97 & 3.87 & $6.1 \%$ \\
\hline & \multicolumn{6}{|c|}{ Constant 2013 dollars ${ }^{\mathrm{e}}$ per gallon } & $\begin{array}{c}\text { Average annua } \\
\text { percentage } \\
\text { change }\end{array}$ \\
\hline & 1990 & 2000 & 2005 & 2010 & 2012 & $2013^{b}$ & 1990-2013 \\
\hline China & $\mathrm{c}$ & $\mathrm{c}$ & 2.02 & 3.90 & $\mathrm{c}$ & $\mathrm{c}$ & $\mathrm{c}$ \\
\hline Japan & 3.12 & 3.86 & 4.11 & 5.20 & 6.12 & 5.21 & $2.3 \%$ \\
\hline Korea & $\mathrm{c}$ & 2.78 & 4.75 & 5.26 & 6.16 & 6.06 & $\mathrm{c}$ \\
\hline France & 3.17 & 3.99 & 5.74 & 6.13 & 6.89 & 6.82 & $3.4 \%$ \\
\hline United Kingdom & 3.64 & 6.30 & 7.46 & 7.45 & 8.63 & 8.48 & $3.7 \%$ \\
\hline Germany & 4.85 & 3.78 & 5.97 & 6.57 & 7.36 & 7.29 & $1.8 \%$ \\
\hline United States $^{\mathrm{d}}$ & 1.76 & 2.02 & 2.86 & 3.20 & 4.03 & 3.87 & $3.5 \%$ \\
\hline
\end{tabular}

Note: Comparisons between prices and price trends in different countries require care. They are of limited validity because of fluctuations in exchange rates; differences in product quality, marketing practices, and market structures; and the extent to which the standard categories of sales are representative of total national sales for a given period.

\section{Source:}

International Energy Agency, Energy Prices and Taxes, Fourth Quarter, 2013, Paris, France, 2014. (Additional resources: www.iea.org)

${ }^{\text {a }}$ Prices represent the retail prices (including taxes) for car diesel fuel for non-commercial (household) use.

${ }^{\mathrm{b}} 4^{\text {th }}$ quarter 2013.

${ }^{\mathrm{c}}$ Data are not available.

${ }^{\mathrm{d}}$ These estimates are for international comparisons only and do not necessarily correspond to gasoline price estimates in other sections of the book.

${ }^{\mathrm{e}}$ Adjusted by the U.S. Consumer Price Inflation Index. 
In 2013 close to sixty percent of the cost of gasoline in France, Germany, and the United Kingdom went for taxes. Of the listed countries, the United States has the lowest percentage of taxes.

Figure 10.2. Gasoline Prices for Selected Countries, 1990 and 2013

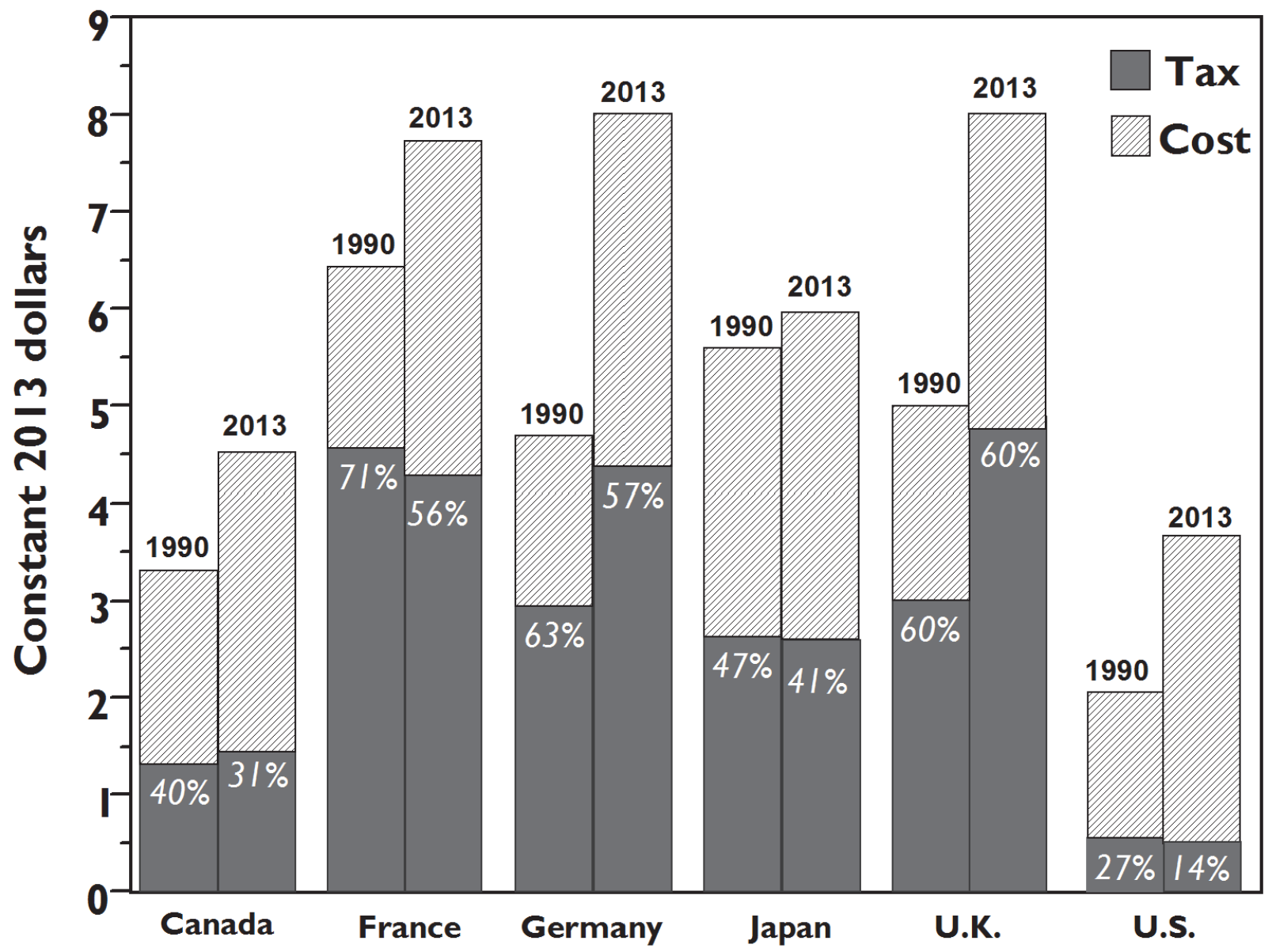

Source:

Table 10.1 and International Energy Agency, Energy Prices \& Taxes, Fourth Quarter, 2013, Paris, France, 2014. (Additional resources: www.iea.org) 
Diesel fuel is taxed heavily in the European countries shown here. The U.S. diesel fuel tax share is the lowest of the listed countries.

Figure 10.3. Diesel Prices for Selected Countries, 1990 and 2013

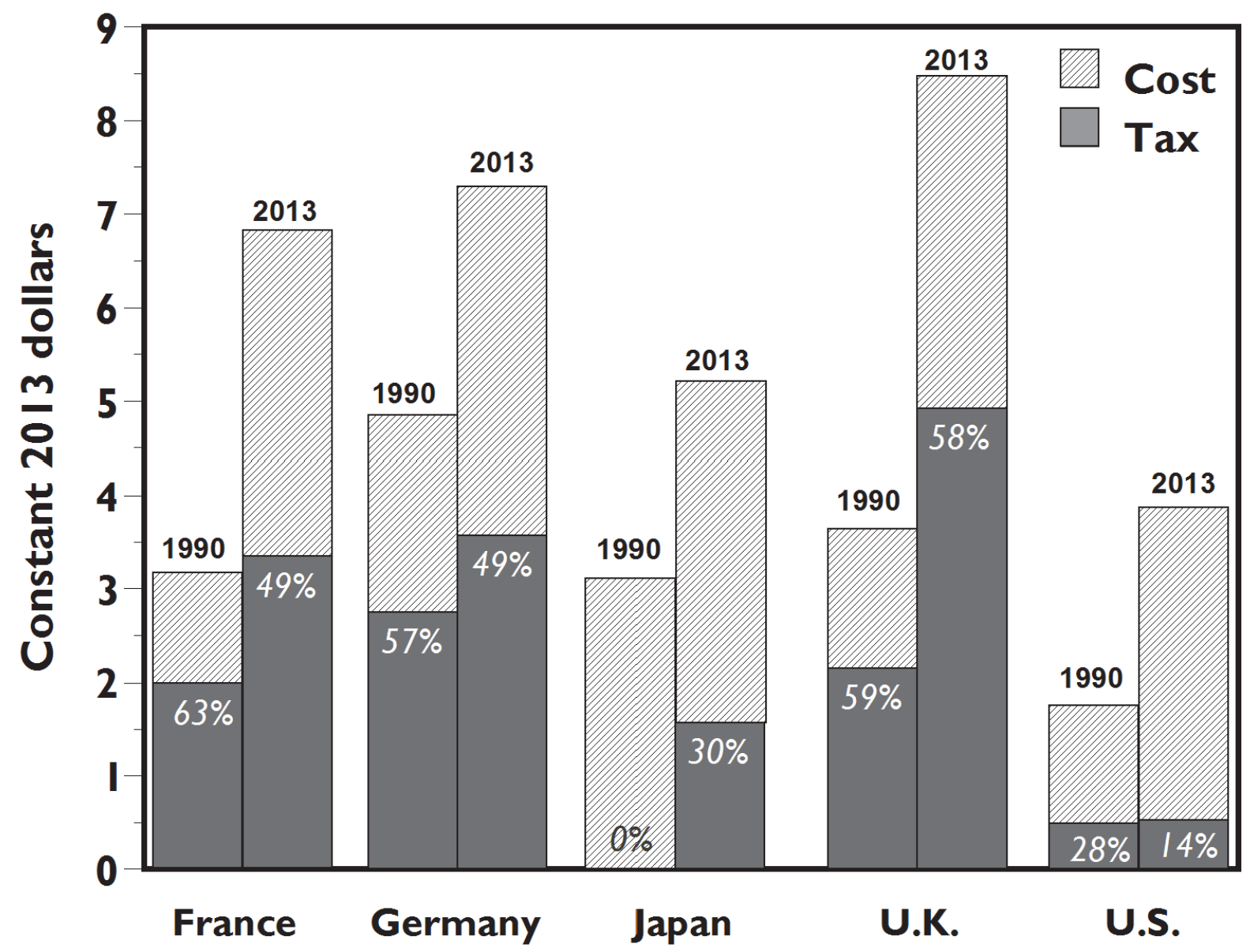

Note: Data for Canada are not available.

\section{Source:}

Table 10.2 and International Energy Agency, Energy Prices \& Taxes, Fourth Quarter, 2013, Paris, France, 2014. (Additional resources: www.iea.org) 
Though the cost of crude oil certainly influences the price of gasoline, it is not the only factor which determines the price at the pump. Processing cost, transportation cost, and taxes also play a major part of the cost of a gallon of gasoline. The average price of a barrel of crude oil (in constant 2013 dollars) increased by $163 \%$ from 2000 to 2013, while the average price of a gallon of gasoline increased 77\% in this same time period.

Table 10.3

Prices for a Barrel of Crude Oil and a Gallon of Gasoline, 1978-2013

\begin{tabular}{|c|c|c|c|c|c|}
\hline \multirow[b]{2}{*}{ Year } & \multicolumn{2}{|c|}{$\begin{array}{c}\text { Crude oil }^{\mathrm{a}} \\
\text { (dollars per barrel) }\end{array}$} & \multicolumn{2}{|c|}{$\begin{array}{c}\text { Gasoline }^{\mathrm{b}} \\
\text { (cents per gallon) }\end{array}$} & \multirow{2}{*}{$\begin{array}{l}\text { Ratio of } \\
\text { gasoline to } \\
\text { crude oil }\end{array}$} \\
\hline & Current & Constant $2013^{\mathrm{c}}$ & Current & Constant $2013^{\mathrm{c}}$ & \\
\hline 1978 & 12.5 & 44.5 & 65.2 & 233.0 & 219.8 \\
\hline $1980^{\circ}$ & 28.1 & 79.4 & 122.1 & 345.2 & 182.7 \\
\hline 1985 & 26.8 & 57.9 & 119.6 & 258.9 & 187.8 \\
\hline 1986 & 14.6 & 30.9 & 93.1 & 197.9 & 268.7 \\
\hline 1987 & 17.9 & 36.7 & 95.7 & 196.2 & 224.5 \\
\hline 1988 & 14.7 & 28.9 & 96.3 & 189.6 & 275.7 \\
\hline 1989 & 18.0 & 33.8 & 106.0 & 199.1 & 247.7 \\
\hline 1990 & 22.2 & 39.6 & 121.7 & 216.9 & 230.0 \\
\hline 1991 & 19.1 & 32.6 & 119.6 & 204.6 & 263.5 \\
\hline 1992 & 18.4 & 30.6 & 119.0 & 197.6 & 271.2 \\
\hline 1993 & 16.4 & 26.5 & 117.3 & 189.1 & 300.2 \\
\hline 1994 & 15.6 & 24.5 & 117.4 & 184.5 & 316.3 \\
\hline 1995 & 17.2 & 26.3 & 120.5 & 184.2 & 293.7 \\
\hline 1996 & 20.7 & 30.7 & 128.8 & 191.2 & 261.2 \\
\hline 1997 & 19.0 & 27.6 & 129.1 & 187.4 & 284.8 \\
\hline 1998 & 12.5 & 17.9 & 111.5 & 159.4 & 374.0 \\
\hline 1999 & 17.5 & 24.5 & 122.1 & 170.7 & 292.9 \\
\hline 2000 & 28.3 & 38.2 & 156.3 & 211.4 & 232.3 \\
\hline 2001 & 23.0 & 30.2 & 153.1 & 201.4 & 280.2 \\
\hline 2002 & 24.1 & 31.2 & 144.1 & 186.6 & 251.1 \\
\hline 2003 & 28.5 & 36.1 & 163.8 & 207.4 & 241.1 \\
\hline 2004 & 37.0 & 45.6 & 192.3 & 237.1 & 218.4 \\
\hline 2005 & 50.2 & 59.9 & 233.8 & 278.9 & 195.5 \\
\hline 2006 & 60.2 & 69.6 & 263.5 & 304.5 & 183.7 \\
\hline 2007 & 67.9 & 76.3 & 284.9 & 320.1 & 176.1 \\
\hline 2008 & 94.7 & 102.5 & 331.7 & 358.9 & 147.0 \\
\hline 2009 & 59.3 & 64.4 & 240.1 & 260.7 & 170.1 \\
\hline 2010 & 76.7 & 81.9 & 283.6 & 303.0 & 155.3 \\
\hline 2011 & 101.9 & 105.5 & 357.7 & 370.5 & 147.5 \\
\hline 2012 & 100.9 & 102.4 & 369.5 & 374.9 & 153.8 \\
\hline 2013 & 100.5 & 100.5 & 358.4 & 358.4 & 149.8 \\
\hline \multicolumn{6}{|c|}{ Average annual percentage change } \\
\hline 1978-2013 & $6.1 \%$ & $2.4 \%$ & $5.0 \%$ & $1.2 \%$ & \\
\hline 2003-2013 & $13.4 \%$ & $10.8 \%$ & $8.1 \%$ & $5.6 \%$ & \\
\hline
\end{tabular}

Sources:

Crude oil - U.S. Department of Energy, Energy Information Administration, Monthly Energy Review, March 2014, Washington, DC, Table 9.1.

Gasoline - U.S. Department of Energy, Energy Information Administration, Monthly Energy Review, March 2014, Washington, DC, Table 9.4. (Additional resources: www.eia.doe.gov)

${ }^{a}$ Refiner acquisition cost of composite (domestic and imported) crude oil.

${ }^{b}$ Average for all types. These prices were collected from a sample of service stations in 85 urban areas selected to represent all urban consumers. Urban consumers make up about $80 \%$ of the total U.S. population.

${ }^{c}$ Adjusted by the Consumer Price Inflation Index. 
The price of diesel fuel was lower than gasoline in constant dollars prior to 2005 but since that time the price of diesel fuel has increased to become higher than gasoline.

Table 10.4

Retail Prices for Motor Fuel, 1978-2013 (cents per gallon, including tax)

\begin{tabular}{|c|c|c|c|c|}
\hline \multirow[b]{2}{*}{ Year } & \multicolumn{2}{|c|}{ Diesel fuel $^{\mathrm{a}}$} & \multicolumn{2}{|c|}{ Average for all gasoline types } \\
\hline & Current & $\begin{array}{c}\text { Constant } \\
2013^{\mathrm{c}}\end{array}$ & Current & $\begin{array}{c}\text { Constant } \\
2013^{c}\end{array}$ \\
\hline 1978 & $\mathrm{~d}$ & d & 65 & 233 \\
\hline 1979 & d & d & 88 & 283 \\
\hline 1980 & 101 & 286 & 122 & 345 \\
\hline 1981 & 118 & 302 & 135 & 347 \\
\hline 1982 & 116 & 280 & 128 & 309 \\
\hline 1983 & 120 & 281 & 123 & 287 \\
\hline 1984 & 122 & 274 & 120 & 269 \\
\hline 1985 & 122 & 264 & 120 & 259 \\
\hline 1986 & 94 & 200 & 93 & 198 \\
\hline 1987 & 96 & 197 & 96 & 196 \\
\hline 1988 & 95 & 187 & 96 & 190 \\
\hline 1989 & 102 & 192 & 106 & 199 \\
\hline 1990 & 107 & 191 & 122 & 217 \\
\hline 1991 & 91 & 156 & 120 & 205 \\
\hline 1992 & 106 & 176 & 119 & 198 \\
\hline 1993 & 98 & 158 & 117 & 189 \\
\hline 1994 & 111 & 175 & 117 & 185 \\
\hline 1995 & 111 & 170 & 121 & 184 \\
\hline 1996 & 124 & 183 & 129 & 191 \\
\hline 1997 & 120 & 174 & 129 & 187 \\
\hline 1998 & 104 & 149 & 112 & 159 \\
\hline 1999 & 112 & 157 & 122 & 171 \\
\hline 2000 & 149 & 202 & 156 & 211 \\
\hline 2001 & 140 & 184 & 153 & 201 \\
\hline 2002 & 132 & 171 & 144 & 187 \\
\hline 2003 & 151 & 191 & 164 & 207 \\
\hline 2004 & 181 & 223 & 192 & 237 \\
\hline 2005 & 240 & 287 & 234 & 279 \\
\hline 2006 & 271 & 313 & 264 & 304 \\
\hline 2007 & 289 & 324 & 285 & 320 \\
\hline 2008 & 380 & 411 & 332 & 359 \\
\hline 2009 & 247 & 268 & 240 & 261 \\
\hline 2010 & 299 & 319 & 284 & 303 \\
\hline 2011 & 384 & 398 & 358 & 371 \\
\hline 2012 & 397 & 403 & 370 & 375 \\
\hline 2013 & 392 & 392 & 358 & 358 \\
\hline \multicolumn{5}{|c|}{ Average annual percentage change } \\
\hline 1978-2013 & $4.2 \% \mathrm{e}^{\mathrm{e}}-\mathrm{r}-\mathrm{e}$ & $1.0 \% \mathrm{e}^{\mathrm{e}}$ & $5.0 \%$ & $1.3 \%$ \\
\hline 2003-2013 & $10.0 \%$ & $7.6 \%$ & $8.1 \%$ & $5.8 \%$ \\
\hline
\end{tabular}

Sources:

Gasoline - U.S. Department of Energy, Energy Information Administration, Monthly Energy Review, March 2014, Washington, DC, Table 9.4.

Diesel - U.S. Department of Energy, Energy Information Administration, International Energy Annual 2004, Washington, DC, June 2004, Table 7.2. 2005-2013 data from EIA website. (Additional resources: www.eia.doe.gov)

${ }^{a}$ 1980-1993: Collected from a survey of prices on January 1 of the current year. 1994-on: Annual average.

${ }^{b}$ These prices were collected from a sample of service stations in 85 urban areas selected to represent all urban consumers. Urban consumers make up about 80 percent of the total U.S. population.

${ }^{\mathrm{c}}$ Adjusted by the Consumer Price Inflation Index.

${ }^{\mathrm{d}}$ Data are not available.

${ }^{\mathrm{e}}$ Average annual percentage change is from the earliest year possible to 2013. 
The fuel prices shown here are refiner sales prices of transportation fuels to end users, excluding tax. Sales to end users are those made directly to the ultimate consumer, including bulk consumers. Bulk sales to utility, industrial, and commercial accounts previously included in the wholesale category are now counted as sales to end users.

Table 10.5

Refiner Sales Prices for Propane and No. 2 Diesel, 1978-2013 (cents per gallon, excluding tax)

\begin{tabular}{|c|c|c|c|c|}
\hline \multirow[b]{3}{*}{ Year } & \multicolumn{2}{|c|}{ Propane $^{a}$} & \multicolumn{2}{|c|}{ No 2. diesel fuel } \\
\hline & & Constant & & Constant \\
\hline & Current & $2013^{\mathrm{b}}$ & Current & $2013^{\mathrm{b}}$ \\
\hline 1978 & 33.5 & 119.7 & 37.7 & 134.7 \\
\hline 1979 & 35.7 & 114.6 & 58.5 & 187.7 \\
\hline 1980 & 48.2 & 136.3 & 81.8 & 231.2 \\
\hline 1981 & 56.5 & 144.8 & 99.5 & 255.0 \\
\hline 1982 & 59.2 & 142.9 & 94.2 & 227.4 \\
\hline 1983 & 70.9 & 165.8 & 82.6 & 193.2 \\
\hline 1984 & 73.7 & 165.2 & 82.3 & 184.5 \\
\hline 1985 & 71.7 & 155.2 & 78.9 & 170.8 \\
\hline 1986 & 74.5 & 158.4 & 47.8 & 101.6 \\
\hline 1987 & 70.1 & 143.8 & 55.1 & 113.0 \\
\hline 1988 & 71.4 & 140.6 & 50.0 & 98.5 \\
\hline 1989 & 61.5 & 115.6 & 58.5 & 109.9 \\
\hline 1990 & 74.5 & 132.8 & 72.5 & 129.2 \\
\hline 1991 & 73.0 & 124.8 & 64.8 & 110.8 \\
\hline 1992 & 64.3 & 106.7 & 61.9 & 102.8 \\
\hline 1993 & 67.3 & 108.5 & 60.2 & 97.0 \\
\hline 1994 & 53.0 & 83.3 & 55.4 & 87.1 \\
\hline 1995 & 49.2 & 75.2 & 56.0 & 85.6 \\
\hline 1996 & 60.5 & 89.8 & 68.1 & 101.1 \\
\hline 1997 & 55.2 & 80.1 & 64.2 & 93.2 \\
\hline 1998 & 40.5 & 57.9 & 49.4 & 70.6 \\
\hline 1999 & 45.8 & 64.0 & 58.4 & 81.6 \\
\hline 2000 & 60.3 & 81.6 & 93.5 & 126.5 \\
\hline 2001 & 50.6 & 66.5 & 84.2 & 110.7 \\
\hline 2002 & 41.9 & 54.3 & 76.2 & 98.7 \\
\hline 2003 & 57.7 & 73.0 & 94.4 & 119.5 \\
\hline 2004 & 83.9 & 103.4 & 124.3 & 153.3 \\
\hline 2005 & 108.9 & 129.9 & 178.6 & 213.1 \\
\hline 2006 & 135.8 & 157.0 & 209.6 & 242.3 \\
\hline 2007 & 148.9 & 167.4 & 226.7 & 254.8 \\
\hline 2008 & 189.2 & 204.7 & 315.0 & 340.8 \\
\hline 2009 & 122.0 & 132.5 & 183.4 & 199.2 \\
\hline 2010 & 148.1 & 158.2 & 213.4 & 227.9 \\
\hline 2011 & 170.9 & 177.1 & 311.7 & 322.9 \\
\hline 2012 & 113.9 & 115.6 & 320.2 & 325.0 \\
\hline 2013 & 102.8 & 102.8 & 312.2 & 312.2 \\
\hline \multicolumn{5}{|c|}{ Average annual percentage change } \\
\hline 1978-2013 & $3.3 \%$ & $-0.4 \%$ & $6.2 \%$ & $2.4 \%$ \\
\hline 2003-2013 & $5.9 \%$ & $3.5 \%$ & $12.7 \%$ & $10.1 \%$ \\
\hline
\end{tabular}

\section{Source:}

U.S. Department of Energy, Energy Information Administration, Petroleum Data Analysis Tools, Refiner Petroleum Product Prices by Sales Type, April 2014, Washington, DC. (Additional resources: www.eia.doe.gov)

${ }^{\mathrm{a}}$ Consumer grade.

${ }^{\mathrm{b}}$ Adjusted by the Consumer Price Inflation Index. 
Prices of finished aviation gasoline (current dollars) dropped in 2009 but then began to climb. In 2012 both finished aviation gasoline and kerosene-type jet fuel reached their all-time high.

Table 10.6

Refiner Sales Prices for Aviation Gasoline and Jet Fuel, 1978-2013 (cents per gallon, excluding tax)

\begin{tabular}{|c|c|c|c|c|}
\hline \multirow[b]{2}{*}{ Year } & \multicolumn{2}{|c|}{ Finished aviation gasoline } & \multicolumn{2}{|c|}{ Kerosene-type jet fuel } \\
\hline & Current & Constant $2013^{\mathrm{a}}$ & Current & Constant $2013^{\mathrm{a}}$ \\
\hline 1978 & 51.6 & 184.4 & 38.6 & 137.9 \\
\hline 1979 & 68.9 & 221.1 & 66.0 & 211.8 \\
\hline 1980 & 108.4 & 306.5 & 86.6 & 244.8 \\
\hline 1981 & 130.3 & 333.9 & 101.2 & 259.4 \\
\hline 1982 & 131.2 & 316.7 & 95.3 & 230.1 \\
\hline 1983 & 125.5 & 293.5 & 85.4 & 199.7 \\
\hline 1984 & 123.4 & 276.7 & 83.0 & 186.1 \\
\hline 1985 & 120.1 & 260.0 & 79.4 & 171.9 \\
\hline 1986 & 101.1 & 214.9 & 49.5 & 105.2 \\
\hline 1987 & 90.7 & 186.0 & 53.8 & 110.3 \\
\hline 1988 & 89.1 & 175.5 & 49.5 & 97.5 \\
\hline 1989 & 99.5 & 186.9 & 58.3 & 109.5 \\
\hline 1990 & 112.0 & 199.6 & 77.3 & 137.8 \\
\hline 1991 & 104.7 & 179.1 & 65.0 & 111.2 \\
\hline 1992 & 102.7 & 170.5 & 60.0 & 99.6 \\
\hline 1993 & 99.0 & 159.6 & 57.7 & 93.0 \\
\hline 1994 & 95.7 & 150.4 & 53.4 & 83.9 \\
\hline 1995 & 100.5 & 153.6 & 53.9 & 82.4 \\
\hline 1996 & 111.6 & 165.7 & 64.6 & 95.9 \\
\hline 1997 & 112.8 & 163.7 & 61.3 & 89.0 \\
\hline 1998 & 95.7 & 136.8 & 45.0 & 64.3 \\
\hline 1999 & 105.9 & 148.1 & 53.3 & 74.5 \\
\hline 2000 & 130.6 & 176.7 & 88.0 & 119.0 \\
\hline 2001 & 132.3 & 174.0 & 76.3 & 100.4 \\
\hline 2002 & 128.8 & 166.8 & 71.6 & 92.7 \\
\hline 2003 & 149.3 & 189.0 & 87.1 & 110.3 \\
\hline 2004 & 181.9 & 224.3 & 120.8 & 149.0 \\
\hline 2005 & 223.1 & 266.1 & 172.3 & 205.5 \\
\hline 2006 & 268.2 & 309.9 & 196.1 & 226.6 \\
\hline 2007 & 284.9 & 320.1 & 217.1 & 243.9 \\
\hline 2008 & 327.3 & 354.1 & 302.0 & 326.8 \\
\hline 2009 & 244.2 & 265.2 & 171.9 & 186.7 \\
\hline 2010 & 302.8 & 323.5 & 218.5 & 233.4 \\
\hline 2011 & 380.3 & 393.9 & 301.4 & 312.1 \\
\hline 2012 & 397.1 & 402.9 & 308.0 & 312.5 \\
\hline 2013 & 393.2 & 393.2 & 295.3 & 295.3 \\
\hline & & Average annual $p$ & & \\
\hline $1978-2013$ & $6.0 \%$ & $2.2 \%$ & $6.0 \%$ & $2.2 \%$ \\
\hline 2003-2013 & $10.2 \%$ & $7.6 \%$ & $13.0 \%$ & $10.3 \%$ \\
\hline
\end{tabular}

\section{Source:}

U.S. Department of Energy, Energy Information Administration, Petroleum Data Analysis Tools, Refiner Petroleum Product Prices by Sales Type, April 2014, Washington, DC. (Additional resources: www.eia.doe.gov)

${ }^{a}$ Adjusted by the Consumer Price Inflation Index. 
The federal government taxes highway motor fuel and uses the money to pay for roadway upkeep and improvement, as well as other related expenditures. Compressed natural gas $(C N G)$ and liquefied petroleum gas $(L P G)$ have the lowest taxes, while diesel fuel and liquefied natural gas $(L N G)$ have the highest.

Table 10.7

Federal Excise Taxes on Motor Fuels, 2011

\begin{tabular}{lcc}
\hline Fuel & Cents per gallon & Effective Date \\
\hline Gasoline & 18.4 & October 1, 1997 \\
Diesel and kerosene & 24.4 & October 1, 1997 \\
Gasohol $^{\mathrm{a}}$ & 18.4 & January 1, 2005 \\
Other special fuels $^{\mathrm{b}}$ & 18.4 & October 1, 1997 \\
CNG & $18.3^{\mathrm{c}}$ & October 1, 2006 \\
LNG & 24.3 & October 1, 2006 \\
LPG & 18.3 & October 1, 2006 \\
\hline
\end{tabular}

\section{Source:}

U.S. Department of Transportation, Federal Highway Administration, Highway Statistics 2012, Washington, DC, 2014, Table FE-21B. (Additional resources: www.fhwa.dot.gov)

${ }^{a}$ All gasohol blends are taxed at the same rate.

${ }^{\mathrm{b}}$ Includes benzol, benzene, naphtha, and other liquids used as a motor fuel.

${ }^{\mathrm{c}}$ Compressed natural gas is 18.3 cents per energy equivalent of a gallon of gasoline. 
These states have laws and incentives for alternative fuels production and/or use.

Table 10.8

Federal and State Alternative Fuel Incentives, 2014

\begin{tabular}{|c|c|c|c|c|c|c|c|c|}
\hline State & Biodiesel & Ethanol & $\begin{array}{c}\text { Natural } \\
\text { Gas }\end{array}$ & $\begin{array}{l}\text { Liquefied } \\
\text { petroleum } \\
\text { gas (LPG) }\end{array}$ & $\begin{array}{c}\text { Electric } \\
\text { vehicles } \\
\text { (EVs) }\end{array}$ & $\begin{array}{c}\text { Neighborhood } \\
\text { electric } \\
\text { vehicles } \\
(\mathrm{NEVs}) \\
\end{array}$ & $\begin{array}{l}\text { Hydrogen } \\
\text { fuel cells }\end{array}$ & $\begin{array}{l}\text { Aftermarket } \\
\text { conversions }\end{array}$ \\
\hline Federal & 35 & 29 & 28 & 27 & 23 & 2 & 25 & 5 \\
\hline Alabama & 4 & 3 & 3 & 3 & 3 & 0 & 2 & 0 \\
\hline Alaska & 2 & 2 & 3 & 2 & 1 & 1 & 1 & 1 \\
\hline Arizona & 6 & 5 & 14 & 14 & 14 & 1 & 11 & 0 \\
\hline Arkansas & 5 & 4 & 8 & 5 & 2 & 0 & 3 & 3 \\
\hline California & 16 & 14 & 27 & 18 & 41 & 3 & 25 & 7 \\
\hline Colorado & 6 & 7 & 10 & 8 & 8 & 1 & 5 & 3 \\
\hline Connecticut & 4 & 4 & 5 & 4 & 5 & 0 & 6 & 4 \\
\hline Delaware & 3 & 3 & 3 & 5 & 3 & 1 & 2 & 0 \\
\hline Dist. of Columbia & 1 & 2 & 3 & 3 & 3 & 0 & 3 & 0 \\
\hline Florida & 8 & 7 & 5 & 5 & 8 & 1 & 2 & 1 \\
\hline Georgia & 6 & 6 & 5 & 3 & 6 & 0 & 3 & 2 \\
\hline Hawaii & 8 & 10 & 4 & 4 & 8 & 2 & 5 & 0 \\
\hline Idaho & 3 & 1 & 2 & 3 & 1 & 1 & 2 & 0 \\
\hline Illinois & 15 & 16 & 7 & 6 & 13 & 1 & 6 & 4 \\
\hline Indiana & 14 & 15 & 17 & 12 & 10 & 2 & 7 & 7 \\
\hline Iowa & 9 & 11 & 4 & 3 & 4 & 1 & 4 & 1 \\
\hline Kansas & 7 & 12 & 5 & 4 & 1 & 1 & 1 & 2 \\
\hline Kentucky & 10 & 11 & 8 & 6 & 4 & 1 & 2 & 1 \\
\hline Louisiana & 6 & 7 & 8 & 6 & 4 & 1 & 1 & 3 \\
\hline Maine & 5 & 5 & 3 & 3 & 5 & 3 & 2 & 0 \\
\hline Maryland & 2 & 3 & 1 & 1 & 8 & 2 & 1 & 1 \\
\hline Massachusetts & 5 & 4 & 4 & 2 & 3 & 1 & 3 & 0 \\
\hline Michigan & 3 & 2 & 3 & 2 & 5 & 0 & 2 & 0 \\
\hline Minnesota & 7 & 12 & 2 & 4 & 6 & 3 & 3 & 0 \\
\hline Mississippi & 5 & 5 & 7 & 6 & 3 & 0 & 2 & 2 \\
\hline Missouri & 5 & 5 & 4 & 5 & 4 & 1 & 4 & 0 \\
\hline Montana & 6 & 6 & 3 & 3 & 1 & 2 & 1 & 1 \\
\hline Nebraska & 3 & 4 & 5 & 3 & 2 & 1 & 1 & 1 \\
\hline Nevada & 6 & 4 & 9 & 9 & 9 & 1 & 8 & 0 \\
\hline New Hampshire & 6 & 3 & 3 & 3 & 3 & 3 & 3 & 1 \\
\hline New Jersey & 3 & 3 & 4 & 3 & 4 & 2 & 2 & 1 \\
\hline New Mexico & 10 & 7 & 5 & 5 & 6 & 1 & 7 & 1 \\
\hline New York & 6 & 7 & 8 & 4 & 6 & 1 & 7 & 2 \\
\hline North Carolina & 11 & 10 & 6 & 6 & 11 & 0 & 5 & 1 \\
\hline North Dakota & 11 & 8 & 3 & 2 & 1 & 1 & 2 & 0 \\
\hline Ohio & 5 & 6 & 7 & 5 & 4 & 0 & 4 & 1 \\
\hline Oklahoma & 10 & 12 & 14 & 9 & 7 & 1 & 7 & 7 \\
\hline Oregon & 10 & 10 & 9 & 8 & 13 & 2 & 7 & 3 \\
\hline Pennsylvania & 4 & 4 & 3 & 3 & 4 & 0 & 2 & 2 \\
\hline Rhode Island & 3 & 2 & 2 & 1 & 4 & 1 & 3 & 0 \\
\hline South Carolina & 7 & 5 & 2 & 3 & 2 & 1 & 6 & 0 \\
\hline South Dakota & 6 & 7 & 1 & 2 & 0 & 0 & 0 & 0 \\
\hline Tennessee & 8 & 8 & 6 & 4 & 3 & 1 & 2 & 0 \\
\hline Texas & 7 & 6 & 15 & 10 & 7 & 1 & 4 & 3 \\
\hline Utah & 1 & 1 & 10 & 6 & 6 & 0 & 2 & 3 \\
\hline Vermont & 7 & 6 & 6 & 6 & 7 & 3 & 7 & 1 \\
\hline Virginia & 18 & 14 & 16 & 12 & 14 & 1 & 12 & 5 \\
\hline Washington & 18 & 15 & 9 & 8 & 20 & 1 & 6 & 4 \\
\hline West Virginia & 5 & 4 & 8 & 6 & 4 & 1 & 4 & 2 \\
\hline Wisconsin & 12 & 9 & 7 & 8 & 6 & 1 & 7 & 0 \\
\hline Wyoming & 0 & 1 & 4 & 0 & 0 & 0 & 0 & 1 \\
\hline Totals & 383 & 367 & 358 & 293 & 340 & 56 & 242 & 87 \\
\hline
\end{tabular}

Source:

U.S. Department of Energy, Energy Efficiency and Renewable Energy, Alternative Fuels Data Center. Data downloaded March 2014. (Additional resources: www.eere.energy.gov/afdc/laws/matrix/tech) 
Table 10.9

Federal and State Advanced Technology Incentives, 2014

\begin{tabular}{|c|c|c|c|c|}
\hline State & $\begin{array}{l}\text { Hybrid electric vehicles (HEV) or } \\
\text { plug-in hybrid vehicles (PHEVs) }\end{array}$ & $\begin{array}{l}\text { Fuel economy or } \\
\text { efficiency }\end{array}$ & Idle reduction & Other $^{\mathrm{a}}$ \\
\hline Federal & 8 & 13 & 7 & 8 \\
\hline Alabama & 1 & 1 & 2 & 0 \\
\hline Alaska & 0 & 1 & 0 & 0 \\
\hline Arizona & 6 & 0 & 2 & 1 \\
\hline Arkansas & 0 & 1 & 2 & 0 \\
\hline California & 32 & 5 & 4 & 10 \\
\hline Colorado & 6 & 0 & 1 & 0 \\
\hline Connecticut & 4 & 2 & 3 & 3 \\
\hline Delaware & 2 & 3 & 3 & 0 \\
\hline Dist. of Columbia & 1 & 3 & 1 & 1 \\
\hline Florida & 5 & 1 & 1 & 0 \\
\hline Georgia & 1 & 1 & 2 & 1 \\
\hline Hawaii & 8 & 1 & 1 & 0 \\
\hline Idaho & 2 & 1 & 0 & 0 \\
\hline Illinois & 10 & 3 & 5 & 0 \\
\hline Indiana & 9 & 3 & 4 & 4 \\
\hline Iowa & 0 & 0 & 0 & 0 \\
\hline Kansas & 0 & 1 & 1 & 0 \\
\hline Kentucky & 3 & 1 & 0 & 0 \\
\hline Louisiana & 2 & 1 & 0 & 0 \\
\hline Maine & 3 & 2 & 3 & 1 \\
\hline Maryland & 6 & 0 & 1 & 1 \\
\hline Massachusetts & 3 & 0 & 1 & 1 \\
\hline Michigan & 3 & 0 & 0 & 0 \\
\hline Minnesota & 4 & 1 & 3 & 2 \\
\hline Mississippi & 2 & 1 & 1 & 0 \\
\hline Missouri & 0 & 0 & 1 & 0 \\
\hline Montana & 0 & 1 & 0 & 0 \\
\hline Nebraska & 0 & 0 & 1 & 0 \\
\hline Nevada & 5 & 0 & 1 & 0 \\
\hline New Hampshire & 3 & 2 & 5 & 1 \\
\hline New Jersey & 4 & 3 & 1 & 2 \\
\hline New Mexico & 2 & 1 & 1 & 1 \\
\hline New York & 6 & 2 & 4 & 3 \\
\hline North Carolina & 8 & 1 & 4 & 1 \\
\hline North Dakota & 0 & 0 & 0 & 0 \\
\hline Ohio & 0 & 0 & 2 & 0 \\
\hline Oklahoma & 2 & 0 & 2 & 2 \\
\hline Oregon & 7 & 1 & 4 & 4 \\
\hline Pennsylvania & 2 & 1 & 4 & 1 \\
\hline Rhode Island & 3 & 1 & 2 & 4 \\
\hline South Carolina & 4 & 0 & 2 & 0 \\
\hline South Dakota & 0 & 0 & 0 & 1 \\
\hline Tennessee & 3 & 2 & 0 & 0 \\
\hline Texas & 4 & 0 & 3 & 0 \\
\hline Utah & 2 & 4 & 3 & 1 \\
\hline Vermont & 5 & 3 & 4 & 3 \\
\hline Virginia & 5 & 3 & 2 & 1 \\
\hline Washington & 7 & 4 & 3 & 3 \\
\hline West Virginia & 2 & 0 & 2 & 1 \\
\hline Wisconsin & 4 & 0 & 2 & 0 \\
\hline Wyoming & 0 & 0 & 1 & 0 \\
\hline Totals & 199 & 75 & 102 & 62 \\
\hline
\end{tabular}

Source:

U.S. Department of Energy, Energy Efficiency and Renewable Energy, Alternative Fuels Data Center. Data downloaded March 2014. (Additional resources: www.eere.energy.gov/afdc/laws/matrix/tech)

a Includes Clean Fuel Initiatives and Pollution Prevention. 
The average price of a new car in $2012(\$ 25,517)$ was very close to the average price in $1916(\$ 22,765)$ when adjusted for inflation. Average new car prices were at their lowest in 1940 (\$12,733). Since 1914 the highest average price was in the year $1998(\$ 28,684)$.

Table 10.10

Average Price of a New Car, 1913-2012

\begin{tabular}{cc|cc|cc|cc}
\hline Year & $\begin{array}{c}2012 \\
\text { Constant } \\
\text { dollars }\end{array}$ & Year & $\begin{array}{c}2012 \\
\text { Constant } \\
\text { dollars }\end{array}$ & Year & $\begin{array}{c}2012 \\
\text { Constant } \\
\text { dollars }\end{array}$ & $\begin{array}{c}2012 \\
\text { Year }\end{array}$ & $\begin{array}{c}\text { donstant } \\
\text { dollars }\end{array}$ \\
\hline 1913 & $\$ 33,184$ & 1938 & $\$ 14,662$ & 1963 & $\$ 20,422$ & 1988 & $\$ 27,039$ \\
1914 & $\$ 34,341$ & 1939 & $\$ 13,698$ & 1964 & $\$ 20,524$ & 1989 & $\$ 26,609$ \\
1915 & $\$ 28,553$ & 1940 & $\$ 12,733$ & 1965 & $\$ 20,135$ & 1990 & $\$ 26,424$ \\
1916 & $\$ 22,765$ & 1941 & $\$ 12,898$ & 1966 & $\$ 20,119$ & 1991 & $\$ 26,086$ \\
1917 & $\$ 21,029$ & 1942 & $\$ 13,063$ & 1967 & $\$ 22,107$ & 1992 & $\$ 26,733$ \\
1918 & $\$ 19,293$ & 1943 & $\$ 13,229$ & 1968 & $\$ 20,831$ & 1993 & $\$ 26,806$ \\
1919 & $\$ 19,100$ & 1944 & $\$ 13,394$ & 1969 & $\$ 20,831$ & 1994 & $\$ 27,736$ \\
1920 & $\$ 18,907$ & 1945 & $\$ 13,559$ & 1970 & $\$ 20,959$ & 1995 & $\$ 27,056$ \\
1921 & $\$ 20,064$ & 1946 & $\$ 13,724$ & 1971 & $\$ 21,213$ & 1996 & $\$ 27,477$ \\
1922 & $\$ 21,222$ & 1947 & $\$ 13,889$ & 1972 & $\$ 21,306$ & 1997 & $\$ 27,517$ \\
1923 & $\$ 19,293$ & 1948 & $\$ 14,546$ & 1973 & $\$ 20,953$ & 1998 & $\$ 28,684$ \\
1924 & $\$ 17,363$ & 1949 & $\$ 16,950$ & 1974 & $\$ 20,677$ & 1999 & $\$ 28,541$ \\
1925 & $\$ 17,171$ & 1950 & $\$ 17,371$ & 1975 & $\$ 21,124$ & 2000 & $\$ 27,466$ \\
1926 & $\$ 16,978$ & 1951 & $\$ 17,667$ & 1976 & $\$ 21,862$ & 2001 & $\$ 27,839$ \\
1927 & $\$ 16,785$ & 1952 & $\$ 19,137$ & 1977 & $\$ 22,027$ & 2002 & $\$ 27,119$ \\
1928 & $\$ 16,592$ & 1953 & $\$ 19,161$ & 1978 & $\$ 22,463$ & 2003 & $\$ 27,010$ \\
1929 & $\$ 16,399$ & 1954 & $\$ 18,813$ & 1979 & $\$ 21,653$ & 2004 & $\$ 26,832$ \\
1930 & $\$ 16,206$ & 1955 & $\$ 18,710$ & 1980 & $\$ 21,104$ & 2005 & $\$ 27,059$ \\
1931 & $\$ 18,135$ & 1956 & $\$ 19,250$ & 1981 & $\$ 22,505$ & 2006 & $\$ 26,916$ \\
1932 & $\$ 20,064$ & 1957 & $\$ 21,384$ & 1982 & $\$ 23,530$ & 2007 & $\$ 26,456$ \\
1933 & $\$ 18,907$ & 1958 & $\$ 22,622$ & 1983 & $\$ 24,449$ & 2008 & $\$ 24,998$ \\
1934 & $\$ 17,749$ & 1959 & $\$ 22,670$ & 1984 & $\$ 25,136$ & 2009 & $\$ 24,798$ \\
1935 & $\$ 15,820$ & 1960 & $\$ 21,815$ & 1985 & $\$ 25,260$ & 2010 & $\$ 25,666$ \\
1936 & $\$ 13,891$ & 1961 & $\$ 20,772$ & 1986 & $\$ 26,504$ & 2011 & $\$ 25,561$ \\
1937 & $\$ 14,277$ & 1962 & $\$ 20,650$ & 1987 & $\$ 27,054$ & 2012 & $\$ 25,517$ \\
\hline
\end{tabular}

Note: These data are based on an average car and do not include prices for pickups, vans, or sport utility vehicles. Estimations were used for years 1941-1946.

\section{Sources:}

Compiled by Jacob Ward, Vehicle Technologies Program, U.S. Department of Energy, from the following sources. Raff, D.M.G. \& Trajtenberg, M. (1995), "Quality-Adjusted Prices for the American Automobile Industry: 1906-1940," National Bureau of Economic Research, Inc.; Gordon, R.J. (1990), The Measurement of Durable Goods Prices, National Bureau of Economic Research, Inc.; and U.S. Department of Commerce, Bureau of Economic Analysis (2013), National Income and Product Accounts. 
In current dollars, import cars, on average, were less expensive than domestic cars until 1982. Since then, import prices have almost tripled, while domestic prices have more than doubled (current dollars).

Table 10.11

Average Price of a New Car (Domestic and Import), 1970-2012

\begin{tabular}{|c|c|c|c|c|c|c|}
\hline \multirow[b]{2}{*}{ Year } & \multicolumn{2}{|c|}{ Domestic $^{a}$} & \multicolumn{2}{|c|}{ Import } & \multicolumn{2}{|c|}{ Total } \\
\hline & $\begin{array}{l}\text { Current } \\
\text { dollars }\end{array}$ & $\begin{array}{c}\text { Constant } 2012 \\
\text { dollars }\end{array}$ & $\begin{array}{l}\text { Current } \\
\text { dollars }\end{array}$ & $\begin{array}{c}\text { Constant } 2012 \\
\text { dollars }\end{array}$ & $\begin{array}{l}\text { Current } \\
\text { dollars }\end{array}$ & $\begin{array}{c}\text { Constant } 2012 \\
\text { dollars }\end{array}$ \\
\hline 1970 & 3,708 & 21,942 & 2,648 & 15,669 & 3,542 & 20,959 \\
\hline $1975^{\circ}$ & 5,084 & 21,696 & 4,384 & $18,709^{\circ}$ & 4,950 & 21,124 \\
\hline 1980 & 7,609 & 21,201 & 7,482 & 20,847 & 7,574 & 21,104 \\
\hline 1981 & 8,912 & 22,510 & 8,896 & 22,469 & 8,910 & 22,505 \\
\hline 1982 & 9,865 & 23,471 & 9,957 & 23,690 & 9,890 & 23,530 \\
\hline 1983 & 10,516 & 24,241 & 10,868 & 25,052 & 10,606 & 24,449 \\
\hline 1984 & 11,079 & 24,482 & 12,336 & 27,260 & 11,375 & 25,136 \\
\hline 1985 & 11,589 & 24,728 & 12,853 & 27,425 & 11,838 & 25,260 \\
\hline 1986 & 12,319 & 25,806 & 13,670 & 28,636 & 12,652 & 26,504 \\
\hline 1987 & 12,922 & 26,116 & 14,470 & 29,245 & 13,386 & 27,054 \\
\hline 1988 & 13,418 & 26,041 & 15,221 & 29,541 & 13,932 & 27,039 \\
\hline 1989 & 13,936 & 25,803 & 15,510 & 28,718 & 14,371 & 26,609 \\
\hline 1990 & 14,489 & 25,452 & 16,640 & 29,231 & 15,042 & 26,424 \\
\hline 1991 & 15,192 & 25,609 & 16,327 & 27,523 & 15,475 & 26,086 \\
\hline 1992 & 15,644 & 25,601 & 18,593 & 30,427 & 16,336 & 26,733 \\
\hline 1993 & 15,976 & 25,384 & 20,261 & 32,192 & 16,871 & 26,806 \\
\hline 1994 & 16,930 & 26,228 & 21,989 & 34,066 & 17,903 & 27,736 \\
\hline 1995 & 16,864 & 25,406 & 23,202 & 34,954 & 17,959 & 27,056 \\
\hline 1996 & 17,468 & 25,561 & 26,205 & 38,346 & 18,777 & 27,477 \\
\hline 1997 & 17,600 & 25,177 & 27,509 & 39,351 & 19,236 & 27,517 \\
\hline 1998 & 18,479 & 26,029 & 29,614 & 41,713 & 20,364 & 28,684 \\
\hline 1999 & 19,032 & 26,228 & 27,542 & 37,956 & 20,710 & 28,541 \\
\hline 2000 & 19,586 & 26,114 & 25,965 & 34,619 & 21,041 & 28,054 \\
\hline 2001 & 20,042 & 25,983 & 25,787 & 33,430 & 21,474 & 27,839 \\
\hline 2002 & 18,897 & 24,117 & 27,440 & 35,020 & 21,249 & 27,119 \\
\hline 2003 & 19,971 & 24,920 & 26,081 & 32,544 & 21,646 & 27,010 \\
\hline 2004 & 20,536 & 24,960 & 25,941 & 31,529 & 22,076 & 26,832 \\
\hline 2005 & 21,593 & 25,385 & 26,621 & 31,296 & 23,017 & 27,059 \\
\hline 2006 & 22,166 & 25,244 & 27,062 & 30,820 & 23,634 & 26,916 \\
\hline 2007 & 22,284 & 24,676 & 27,465 & 30,413 & 23,892 & 26,456 \\
\hline 2008 & 22,205 & 23,679 & 25,903 & 27,622 & 23,442 & 24,998 \\
\hline 2009 & 22,125 & 23,678 & 25,223 & 26,993 & 23,172 & 24,798 \\
\hline 2010 & 22,986 & 24,202 & 27,297 & 28,741 & 24,376 & 25,666 \\
\hline 2011 & 23,561 & 24,049 & 28,277 & 28,862 & 25,043 & 25,561 \\
\hline 2012 & 24,025 & 24,025 & 29,191 & 29,191 & 25,517 & 25,517 \\
\hline \multicolumn{7}{|c|}{ Average annual percentage change } \\
\hline 1970-2012 & $4.5 \%$ & $0.2 \%$ & $5.9 \%$ & $1.5 \%$ & $4.8 \%$ & $0.5 \%$ \\
\hline $2002-2012$ & $2.4 \%$ & $0.0 \%$ & $0.6 \%$ & $-1.8 \%$ & $1.8 \%$ & $-0.6 \%$ \\
\hline
\end{tabular}

Note: These data are based on an average car and do not include prices for pickups, vans, or sport utility vehicles. Estimations were used for years 1941-1946.

Source:

U.S. Department of Commerce, Bureau of Economic Analysis, Average Transaction Price per New Car, Washington, DC, 2014. (Additional resources: www.bea.gov)

${ }^{\text {a }}$ Includes all vehicles produced in the United States regardless of manufacturer.

${ }^{\mathrm{b}}$ Adjusted by the Consumer Price Inflation Index. 
The total cost of operating a car is the sum of the fixed cost (depreciation, insurance, finance charge, and license fee) and the variable cost (gas and oil, tires, and maintenance), which is related to the amount of travel. The gas and oil share of total cost was $18.4 \%$ in 2013 which is one of the highest shares in the series history.

Table 10.12

Car Operating Cost per Mile, 1985-2013

\begin{tabular}{|c|c|c|c|c|c|}
\hline \multirow[b]{2}{*}{ Model year } & \multicolumn{3}{|c|}{ Constant 2013 dollars per 10,000 miles $^{\mathrm{a}}$} & \multirow{2}{*}{$\begin{array}{l}\text { Total cost per } \\
\text { mile }^{\mathrm{b}}(\text { constant } \\
\left.2013 \text { cents }^{\mathrm{a}}\right)\end{array}$} & \multirow{2}{*}{$\begin{array}{l}\text { Percentage gas } \\
\text { and oil of total } \\
\text { cost }\end{array}$} \\
\hline & Variable cost & Fixed cost & Total cost & & \\
\hline 1985 & 1,606 & 4,462 & 6,069 & 60.69 & $19.9 \%$ \\
\hline 1986 & 1,386 & 4,904 & 6,289 & 62.89 & $15.1 \%$ \\
\hline 1987 & 1,374 & 4,774 & 6,148 & 61.48 & $14.7 \%$ \\
\hline 1988 & 1,556 & 5,967 & 7,522 & 75.22 & $13.6 \%$ \\
\hline 1989 & 1,503 & 5,486 & 6,989 & 69.89 & $14.2 \%$ \\
\hline 1990 & 1,497 & 5,803 & 7,301 & 73.01 & $13.2 \%$ \\
\hline 1991 & 1,659 & 6,099 & 7,758 & 77.58 & $14.6 \%$ \\
\hline 1992 & 1,494 & 6,283 & 7,777 & 77.77 & $12.6 \%$ \\
\hline 1993 & 1,483 & 6,000 & 7,484 & 74.84 & $12.7 \%$ \\
\hline 1994 & 1,430 & 6,030 & 7,460 & 74.60 & $11.8 \%$ \\
\hline 1995 & 1,467 & 6,122 & 7,589 & 75.89 & $11.7 \%$ \\
\hline 1996 & 1,425 & 6,226 & 7,651 & 76.51 & $10.9 \%$ \\
\hline 1997 & 1,568 & 6,311 & 7,878 & 78.78 & $12.2 \%$ \\
\hline 1998 & 1,529 & 6,471 & 8,001 & 80.01 & $11.1 \%$ \\
\hline 1999 & 1,482 & 6,516 & 7,998 & 79.98 & $9.8 \%$ \\
\hline 2000 & 1,650 & 6,391 & 8,041 & 80.41 & $11.6 \%$ \\
\hline 2001 & 1,789 & 6,078 & 7,867 & 78.67 & $13.2 \%$ \\
\hline 2002 & 1,528 & 6,311 & 7,839 & 78.39 & $9.7 \%$ \\
\hline 2003 & 1,659 & 6,183 & 7,842 & 78.42 & $11.6 \%$ \\
\hline 2004 & 1,554 & 6,947 & 8,501 & 85.01 & $9.4 \%$ \\
\hline 2005 & 1,682 & 6,456 & 8,137 & 81.37 & $12.0 \%$ \\
\hline 2006 & 1,745 & 5,415 & 7,160 & 71.60 & $15.3 \%$ \\
\hline 2007 & 1,629 & 5,354 & 6,983 & 69.83 & $14.3 \%$ \\
\hline 2008 & 1,835 & 5,842 & 7,677 & 76.77 & $16.4 \%$ \\
\hline 2009 & 1,674 & 6,000 & 7,675 & 76.75 & $14.3 \%$ \\
\hline 2010 & 1,787 & 6,110 & 7,897 & 78.97 & $15.4 \%$ \\
\hline 2011 & 1,837 & 6,066 & 7,903 & 79.03 & $16.2 \%$ \\
\hline 2012 & 1,993 & 5,830 & 7,823 & 78.23 & $18.4 \%$ \\
\hline 2013 & 2,042 & 5.792 & 7,834 & 78.34 & $18.4 \%$ \\
\hline \multicolumn{6}{|c|}{ Average annual percentage change } \\
\hline $1985-2013$ & $0.9 \%$ & $0.9 \%$ & $0.9 \%$ & $0.9 \%$ & \\
\hline
\end{tabular}

\section{Source:}

Ward's Communications, Motor Vehicle Facts and Figures 2013, Southfield, Michigan, 2013, p. 55, and annual. Original data from AAA "Your Driving Costs." (Additional resources: newsroom.aaa.com)

\footnotetext{
${ }^{a}$ Adjusted by the Consumer Price Inflation Index.

${ }^{\mathrm{b}}$ Based on 10,000 miles per year.
} 
While the previous table shows costs per mile, this table presents costs per year for fixed costs associated with car operation. For 2013 model year cars, the fixed cost is over $\$ 16$ per day.

Table 10.13

Fixed Car Operating Costs per Year, 1975-2013 (constant 2013 dollars) $^{a}$

\begin{tabular}{|c|c|c|c|c|c|c|}
\hline Model year & Insurance $^{\mathrm{b}}$ & $\begin{array}{l}\text { License, } \\
\text { registration } \\
\text { \& taxes }\end{array}$ & Depreciation & $\begin{array}{c}\text { Finance } \\
\text { charge }\end{array}$ & Total & $\begin{array}{l}\text { Average } \\
\text { fixed cost } \\
\text { per day }\end{array}$ \\
\hline 1975 & 1,658 & 130 & 3,347 & $\mathrm{c}$ & 5,135 & 14.07 \\
\hline 1977 & 1,991 & 284 & 3,256 & $\mathrm{c}$ & 5,532 & 15.15 \\
\hline 1978 & 1,515 & 264 & 3,194 & $\mathrm{c}$ & 4,974 & 13.61 \\
\hline 1979 & 1,550 & 289 & 3,023 & $\mathrm{c}$ & 5,811 & 15.92 \\
\hline 1980 & 1,385 & 232 & 2,935 & $\mathrm{c}$ & 5,748 & 15.75 \\
\hline 1981 & 1,307 & 226 & 3,298 & c & 6,087 & 16.68 \\
\hline 1982 & 1,084 & 130 & 3,273 & $\mathrm{c}$ & 5,789 & 15.86 \\
\hline 1983 & 1,099 & 227 & 3,036 & $\mathrm{c}$ & 5,599 & 15.34 \\
\hline 1984 & 1,132 & 238 & 2,706 & $\mathrm{c}$ & 5,260 & 14.42 \\
\hline 1985 & 1,007 & 238 & 2,732 & 1,156 & 5,133 & 14.07 \\
\hline 1986 & 1,082 & 276 & 2,806 & 1,354 & 5,518 & 15.11 \\
\hline 1987 & 1,097 & 262 & 3,064 & 1,079 & 5,502 & 15.07 \\
\hline 1988 & 1,128 & 274 & 3,513 & 1,113 & 6,028 & 16.52 \\
\hline 1989 & 1,212 & 271 & 3,791 & 1,105 & 6,378 & 17.47 \\
\hline 1990 & 1,200 & 294 & 4,201 & 1,212 & 6,907 & 18.93 \\
\hline 1991 & 1,211 & 287 & 4,283 & 455 & 6,236 & 17.09 \\
\hline 1992 & 1,307 & 289 & 4,511 & 1,322 & 7,429 & 20.36 \\
\hline 1993 & 1,199 & 287 & 4,562 & 1,080 & 7,129 & 19.54 \\
\hline 1994 & 1,209 & 305 & 4,621 & 1,019 & 7,154 & 19.60 \\
\hline 1995 & 1,197 & 310 & 4,697 & 1,049 & 7,253 & 19.87 \\
\hline 1996 & 1,255 & 319 & 4,707 & 1,066 & 7,347 & 20.13 \\
\hline 1997 & 1,229 & 314 & 4,749 & 1,115 & 7,407 & 20.29 \\
\hline 1998 & 1,286 & 323 & 4,808 & 1,162 & 7,579 & 20.77 \\
\hline 1999 & 1,356 & 316 & 4,805 & 1,158 & 7,635 & 20.92 \\
\hline 2000 & 1,312 & 302 & 4,724 & 1,149 & 7,487 & 20.51 \\
\hline 2001 & 1,304 & 274 & 4,667 & 1,139 & 7,383 & 20.23 \\
\hline 2002 & 1,313 & 260 & 4,818 & 1,072 & 7,464 & 20.45 \\
\hline 2003 & 1,395 & 260 & 4,733 & 942 & 7,329 & 20.08 \\
\hline 2004 & 1,977 & 512 & 4,664 & 914 & 8,067 & 22.10 \\
\hline 2005 & 1,536 & 464 & 4,627 & 881 & 7,509 & 20.58 \\
\hline 2006 & 1,070 & 618 & 3,920 & 827 & 6,435 & 17.63 \\
\hline 2007 & 1,107 & 604 & 3,811 & 824 & 6,346 & 17.38 \\
\hline 2008 & 1,020 & 599 & 3,593 & 820 & 6,033 & 16.53 \\
\hline 2009 & 1,060 & 616 & 3,758 & 846 & 6,280 & 17.20 \\
\hline 2010 & 1,101 & 625 & 3,797 & 861 & 6,384 & 17.49 \\
\hline 2011 & 1,003 & 616 & 3,861 & 852 & 6,332 & 17.35 \\
\hline 2012 & 1,016 & 619 & 3,596 & 858 & 6,089 & 16.68 \\
\hline 2013 & 1,029 & 611 & 3,571 & 848 & 6,059 & 16.60 \\
\hline \multicolumn{7}{|c|}{ Average annual percentage change } \\
\hline 1975-2013 & $-1.2 \%$ & $4.2 \%$ & $0.2 \%$ & $\mathrm{c}$ & $0.4 \%$ & $0.4 \%$ \\
\hline 2003-2013 & $-3.0 \%$ & $8.9 \%$ & $-2.8 \%$ & $-1.0 \%$ & $-1.9 \%$ & $-1.9 \%$ \\
\hline
\end{tabular}

\section{Source:}

Ward's Communications, Motor Vehicle Facts and Figures 2013, Southfield, Michigan, 2013, p. 55 and annual. Original data from AAA "Your Driving Costs." (Additional resources: newsroom.aaa.com)

${ }^{a}$ Adjusted by the Consumer Price Inflation Index.

${ }^{\mathrm{b}}$ Fire \& Theft: $\$ 50$ deductible 1975 through 1977; $\$ 100$ deductible 1978 through 1992; \$250 deductible for 1993 - 2003; \$100 deductible 2004-2013. Collision: \$100 deductible through 1992; $\$ 500$ deductible for 1993 - on. Property Damage \& Liability: coverage $=\$ 100,000 / \$ 300,000$.

${ }^{\mathrm{c}}$ Data are not available. 
Table 10.14

Personal Consumption Expenditures, 1970-2013

(billion dollars)

\begin{tabular}{|c|c|c|c|c|c|}
\hline \multirow[b]{2}{*}{ Year } & \multicolumn{2}{|c|}{ Personal consumption expenditures } & \multicolumn{2}{|c|}{$\begin{array}{l}\text { Transportation personal } \\
\text { consumption expenditures }\end{array}$} & \multirow[b]{2}{*}{$\begin{array}{l}\text { Transportation PCE } \\
\text { as a percent of PCE }\end{array}$} \\
\hline & Current & $\begin{array}{c}\text { Constant } \\
2013^{\mathrm{a}}\end{array}$ & Current & $\begin{array}{c}\text { Constant } \\
2013^{\mathrm{a}}\end{array}$ & \\
\hline 1970 & 647.70 & $3,036.30$ & 80.80 & 378.80 & $12.5 \%$ \\
\hline 1980 & $1,754.60$ & $4,221.10$ & 241.70 & 581.50 & $13.8 \%$ \\
\hline 1990 & $3,825.60$ & $6,110.90$ & 455.70 & 727.90 & $11.9 \%$ \\
\hline 2000 & $6,801.60$ & $8,865.80$ & 811.20 & $1,057.40$ & $11.9 \%$ \\
\hline 2005 & $8,790.30$ & $10,199.30$ & 983.20 & $1,140.80$ & $11.2 \%$ \\
\hline 2006 & $9,297.50$ & $10,466.00$ & $1,016.80$ & $1,144.60$ & $10.9 \%$ \\
\hline 2007 & $9,744.40$ & $10,685.10$ & $1,058.30$ & $1,160.50$ & $10.9 \%$ \\
\hline 2008 & $10,005.50$ & $10,759.80$ & $1,040.40$ & $1,118.80$ & $10.4 \%$ \\
\hline 2009 & $9,842.90$ & $10,503.40$ & 891.30 & 951.10 & $9.1 \%$ \\
\hline 2010 & $10,201.90$ & $10,744.50$ & 968.30 & $1,019.80$ & $9.5 \%$ \\
\hline 2011 & $10,711.80$ & $11,063.00$ & $1,085.80$ & $1,121.40$ & $10.1 \%$ \\
\hline 2012 & $11,149.60$ & $11,317.60$ & $1,136.80$ & $1,153.90$ & $10.2 \%$ \\
\hline 2013 & $11,496.20$ & $11,496.20$ & $1,157.40$ & $1,157.40$ & $10.1 \%$ \\
\hline
\end{tabular}

Note: Transportation PCE includes the following categories: transportation, motor vehicles and parts, and gasoline and oil.

\section{Source:}

U.S. Department of Commerce, Bureau of Economic Analysis, National Income and Product Accounts, Table 2.3.5, www.bea.gov

Table 10.15

Consumer Price Indices, 1970-2013

$(1970=1.000)$

\begin{tabular}{|c|c|c|c|c|c|}
\hline Year & $\begin{array}{l}\text { Consumer price } \\
\text { index }\end{array}$ & $\begin{array}{c}\text { Transportation } \\
\text { consumer price index }\end{array}$ & $\begin{array}{c}\text { New car } \\
\text { consumer price } \\
\text { index }\end{array}$ & $\begin{array}{c}\text { Used car } \\
\text { consumer price } \\
\text { index }\end{array}$ & $\begin{array}{c}\text { Gross national product } \\
\text { index }\end{array}$ \\
\hline 1970 & 1.000 & 1.000 & 1.000 & 1.000 & 1.000 \\
\hline 1980 & 2.124 & 2.216 & 1.667 & 1.997 & 2.702 \\
\hline 1990 & 3.369 & 3.213 & 2.286 & 3.769 & 5.557 \\
\hline 2000 & 4.438 & 4.088 & 2.689 & 4.994 & 9.542 \\
\hline 2005 & 5.034 & 4.637 & 2.597 & 4.468 & 12.186 \\
\hline 2008 & 5.549 & 5.215 & 2.527 & 4.293 & 13.761 \\
\hline 2009 & 5.529 & 4.780 & 2.554 & 4.070 & 13.458 \\
\hline 2010 & 5.620 & 5.157 & 2.599 & 4.587 & 14.011 \\
\hline 2011 & 5.797 & 5.663 & 2.672 & 4.776 & 14.594 \\
\hline 2012 & 5.917 & 5.796 & 2.716 & 4.818 & 15.243 \\
\hline 2013 & 6.004 & 5.798 & 2.745 & 4.804 & 15.760 \\
\hline
\end{tabular}

\section{Sources:}

Bureau of Labor Statistics, Consumer Price Index Table 1A for 2013, and annual.

(Additional resources: www.bls.gov)

GNP - U.S. Department of Commerce, Bureau of Economic Analysis, National Income and Product Accounts, Table 1.7.5. (Additional resources: www.bea.gov)

${ }^{a}$ Adjusted by the GNP price deflator.

b Transportation Consumer Price Index includes new and used cars, gasoline, car insurance rates, intracity mass transit, intracity bus fare, and airline fares. 
The data below were summarized from the Bureau of Labor Statistics (BLS) Current Employment Statistics Survey data using the North American Industry Classification System (NAICS). Transportation-related employment was $7.3 \%$ of total employment in 2013.

Table 10.16

Transportation-Related Employment, 2000 and $2013^{\mathrm{a}}$ (thousands)

\begin{tabular}{|c|c|c|c|}
\hline & 2000 & 2013 & $\begin{array}{l}\text { Percent } \\
\text { change }\end{array}$ \\
\hline Truck transportation (includes drivers) & $1,405.8$ & $1,380.1$ & $-1.8 \%$ \\
\hline Transit and ground transportation & 372.1 & 445.8 & $19.8 \%$ \\
\hline Air transportation & 614.4 & 449.0 & $-26.9 \%$ \\
\hline Rail transportation & 231.7 & 232.0 & $0.1 \%$ \\
\hline Water transportation & 56.0 & 65.5 & $17.0 \%$ \\
\hline Pipeline transportation & 46.0 & 44.0 & $-4.3 \%$ \\
\hline Motor vehicle and parts - retail & $1,846.9$ & $1,792.0$ & $-3.0 \%$ \\
\hline Motor vehicles and parts - wholesale & 355.7 & 327.0 & $-8.1 \%$ \\
\hline Gasoline stations - retail & 935.7 & 865.2 & $-7.5 \%$ \\
\hline Automotive repair and maintenance & 888.1 & 841.7 & $-5.2 \%$ \\
\hline Automotive equipment rental and leasing & 208.3 & 178.8 & $-14.2 \%$ \\
\hline Manufacturing & $2,143.9$ & $1,557.4$ & $-27.4 \%$ \\
\hline Cars and light trucks & 237.4 & 152.4 & $-35.8 \%$ \\
\hline Heavy-duty trucks & 54.0 & 25.9 & $-52.0 \%$ \\
\hline Motor vehicle bodies and trailers & 182.7 & 134.1 & $-26.6 \%$ \\
\hline Motor vehicle parts & 839.5 & 507.8 & $-39.5 \%$ \\
\hline Aerospace products and parts & 516.7 & 492.5 & $-4.7 \%$ \\
\hline Railroad rolling stock \& other transportation equipment & 72.7 & 57.6 & $-20.8 \%$ \\
\hline Ship \& boat building & 154.1 & 134.2 & $-12.9 \%$ \\
\hline Tires & 86.8 & 52.9 & $-39.1 \%$ \\
\hline Oil and gas pipeline construction & 72.2 & 132.6 & $83.7 \%$ \\
\hline Highway street and bridge construction & 340.1 & 296.0 & $-13.0 \%$ \\
\hline Scenic \& sightseeing & 27.5 & 28.9 & $5.1 \%$ \\
\hline Support activities for transportation & 537.4 & 593.9 & $10.5 \%$ \\
\hline Couriers and messengers & 605.0 & 544.4 & $-10.0 \%$ \\
\hline Travel arrangement and reservation services & 298.6 & 194.8 & $-34.8 \%$ \\
\hline Total transportation-related employment & $10,985.4$ & $9,969.1$ & $-9.3 \%$ \\
\hline Total nonfarm employment & $132,019.0$ & $136,368.0$ & $3.3 \%$ \\
\hline Transportation-related to total employment & $8.3 \%$ & $7.3 \%$ & \\
\hline
\end{tabular}

Source:

Bureau of Labor Statistics website query system: www.bls.gov/data/, (Additional resources: www.bls.gov)

\footnotetext{
${ }^{\mathrm{a}}$ Not seasonally adjusted.
} 
The total number of employees involved in the manufacture of motor vehicles decreased by over $66 \%$ from 1990 to 2013 and by almost 78\% for those involved in the manufacture of motor vehicle parts. Beginning in 2008, the share of production workers fell below $80 \%$ for manufacturers of both vehicles and parts.

Table 10.17 U.S. Employment for Motor Vehicles and Motor Vehicle Parts Manufacturing, 1990-2013 ${ }^{\mathrm{a}}$

\begin{tabular}{|c|c|c|c|}
\hline Year & All employees & Production workers & $\begin{array}{c}\text { Share of production workers } \\
\text { to total employees }\end{array}$ \\
\hline \multicolumn{4}{|c|}{ Motor vehicles } \\
\hline 1990 & 271.4 & 243.4 & $89.7 \%$ \\
\hline 1991 & 258.4 & 234.8 & $90.9 \%$ \\
\hline 1992 & 259.9 & 234.0 & $90.0 \%$ \\
\hline 1993 & 263.7 & 234.8 & $89.0 \%$ \\
\hline 1994 & 281.5 & 250.9 & $89.1 \%$ \\
\hline 1995 & 294.7 & 273.7 & $92.9 \%$ \\
\hline 1996 & 285.3 & 271.2 & $95.1 \%$ \\
\hline 1997 & 286.8 & 273.6 & $95.4 \%$ \\
\hline 1998 & 283.6 & 254.8 & $89.8 \%$ \\
\hline 1999 & 291.3 & 254.3 & $87.3 \%$ \\
\hline 2000 & 291.4 & 251.0 & $86.1 \%$ \\
\hline 2001 & 278.7 & 236.4 & $84.8 \%$ \\
\hline 2002 & 265.4 & 220.8 & $83.2 \%$ \\
\hline 2003 & 264.6 & 217.1 & $82.0 \%$ \\
\hline 2004 & 255.9 & 208.0 & $81.3 \%$ \\
\hline 2005 & 247.6 & 198.6 & $80.2 \%$ \\
\hline 2006 & 236.5 & 191.8 & $81.1 \%$ \\
\hline 2007 & 220.0 & 177.3 & $80.6 \%$ \\
\hline 2008 & 191.6 & 151.1 & $78.9 \%$ \\
\hline 2009 & 146.4 & 114.2 & $78.0 \%$ \\
\hline 2010 & 152.6 & 120.7 & $79.1 \%$ \\
\hline 2011 & 157.9 & 124.7 & $79.0 \%$ \\
\hline 2012 & 167.6 & 134.7 & $80.4 \%$ \\
\hline 2013 & 178.3 & 147.4 & $82.7 \%$ \\
\hline \multicolumn{4}{|c|}{ Motor vehicle parts } \\
\hline 1990 & 653.0 & 527.4 & $80.8 \%$ \\
\hline 1991 & 638.9 & 514.7 & $80.6 \%$ \\
\hline 1992 & 661.2 & 537.0 & $81.2 \%$ \\
\hline 1993 & 677.8 & 554.7 & $81.8 \%$ \\
\hline 1994 & 735.6 & 606.9 & $82.5 \%$ \\
\hline 1995 & 786.9 & 647.7 & $82.3 \%$ \\
\hline 1996 & 799.9 & 657.4 & $82.2 \%$ \\
\hline 1997 & 808.9 & 662.4 & $81.9 \%$ \\
\hline 1998 & 818.2 & 660.3 & $80.7 \%$ \\
\hline 1999 & 837.1 & 674.2 & $80.5 \%$ \\
\hline 2000 & 839.5 & 676.7 & $80.6 \%$ \\
\hline 2001 & 774.7 & 624.9 & $80.7 \%$ \\
\hline 2002 & 733.6 & 590.9 & $80.5 \%$ \\
\hline 2003 & 707.8 & 567.6 & $80.2 \%$ \\
\hline 2004 & 692.1 & 561.6 & $81.1 \%$ \\
\hline 2005 & 678.1 & 553.9 & $81.7 \%$ \\
\hline 2006 & 654.7 & 533.7 & $81.5 \%$ \\
\hline 2007 & 607.9 & 488.9 & $80.4 \%$ \\
\hline 2008 & 543.7 & 430.6 & $79.2 \%$ \\
\hline 2009 & 413.7 & 317.8 & $76.8 \%$ \\
\hline 2010 & 418.9 & 323.3 & $77.2 \%$ \\
\hline 2011 & 445.5 & 345.0 & $77.4 \%$ \\
\hline 2012 & 482.8 & 365.3 & $75.7 \%$ \\
\hline 2013 & 507.8 & 384.4 & $75.7 \%$ \\
\hline
\end{tabular}

Source:

Tabulated from the U.S. Department of Labor, Bureau of Labor Statistics, www.bls.gov, March 2014.

${ }^{a}$ Not seasonally adjusted. 


\section{Chapter 11 \\ Greenhouse Gas Emissions}

Summary Statistics from Tables/Figures in this Chapter

\begin{tabular}{|c|c|c|c|}
\hline \multicolumn{4}{|l|}{ Source } \\
\hline \multirow[t]{8}{*}{ Table 11.1} & Carbon dioxide emissions (million metric tonnes) & 1990 & 2013 \\
\hline & United States & 4,989 & 5,368 \\
\hline & OECD Europe & 4,149 & 4,060 \\
\hline & China & 2,293 & 9,131 \\
\hline & Russia & 2,393 & 1,614 \\
\hline & Japan & 1,054 & 1,261 \\
\hline & Non-OECD Europe & 1,853 & 1,087 \\
\hline & India & 573 & 1,748 \\
\hline \multirow[t]{4}{*}{ Table 11.5} & \multicolumn{3}{|c|}{$\begin{array}{l}\text { Transportation share of U.S. carbon dioxide emissions from fossil fuel } \\
\text { consumption }\end{array}$} \\
\hline & 1990 & & $31.7 \%$ \\
\hline & 2005 & & $33.3 \%$ \\
\hline & 2012 & & $34.7 \%$ \\
\hline Table 11.6 & \multicolumn{2}{|c|}{ Motor gasoline share of transportation carbon dioxide emissions } & $62.9 \%$ \\
\hline \multirow[t]{3}{*}{ Table 11.10} & \multicolumn{3}{|l|}{ Average annual carbon footprint (short tons of $\mathrm{CO}_{2}$ ) } \\
\hline & Cars & & 6.8 \\
\hline & Light trucks & & 9.5 \\
\hline
\end{tabular}


The U.S. accounted for $23.2 \%$ of the World's carbon dioxide emissions in 1990 and $16.5 \%$ in 2013. Nearly half (42\%) of the U.S. carbon emissions are from oil use.

Table 11.1

World Carbon Dioxide Emissions, 1990 and 2013

\begin{tabular}{lrrrrr}
\hline & \multicolumn{2}{c}{1990} & & \multicolumn{2}{c}{2013} \\
\cline { 2 - 3 } \cline { 5 - 6 } & $\begin{array}{c}\text { Million } \\
\text { metric tons }\end{array}$ & $\begin{array}{c}\text { Percent of } \\
\text { emissions } \\
\text { from oil use }\end{array}$ & & $\begin{array}{c}\text { Million } \\
\text { metric tons }\end{array}$ & $\begin{array}{c}\text { Percent of } \\
\text { emissions } \\
\text { from oil use }\end{array}$ \\
\hline United States & 4,989 & $44 \%$ & & 5,368 & $42 \%$ \\
Canada & 471 & $48 \%$ & & 553 & $53 \%$ \\
Mexico & 302 & $77 \%$ & & 523 & $60 \%$ \\
OECD ${ }^{\text {a Europe }}$ & 4,149 & $45 \%$ & & 4,060 & $45 \%$ \\
OECD Asia & 243 & $59 \%$ & & 588 & $38 \%$ \\
Japan & 1,054 & $65 \%$ & & 1,261 & $44 \%$ \\
Australia/New Zealand & 298 & $38 \%$ & & 441 & $36 \%$ \\
Russia & 2,393 & $33 \%$ & & 1,614 & $24 \%$ \\
Non-OECD Europe & 1,853 & $32 \%$ & & 1,087 & $34 \%$ \\
China & 2,293 & $15 \%$ & & 9,131 & $14 \%$ \\
India & 573 & $28 \%$ & & 1,748 & $25 \%$ \\
Non-OECD Asia & 811 & $57 \%$ & & 1,926 & $48 \%$ \\
Middle East & 704 & $70 \%$ & & 1,874 & $57 \%$ \\
Africa & 659 & $46 \%$ & & 1,085 & $45 \%$ \\
Central \& South America & 695 & $76 \%$ & & 1,288 & $70 \%$ \\
Total World & 21,487 & $42 \%$ & & 32,547 & $35 \%$ \\
\hline
\end{tabular}

Source:

U.S. Department of Energy, Energy Information Administration, Annual Outlook 2013, Washington, DC, July 2013. (Additional resources: www.eia.doe.gov) countries.

${ }^{\text {a }}$ OECD is the Organization for Economic Cooperation and Development. See Glossary for included 
Global Warming Potentials (GWP) were developed to allow comparison of the ability of each greenhouse gas to trap heat in the atmosphere relative to carbon dioxide. Extensive research has been performed and it has been discovered that the effects of various gases on global warming are too complex to be precisely summarized by a single number. Further understanding of the subject also causes frequent changes to estimates. Despite that, the scientific community has developed approximations, the latest of which are shown below. Most analysts use the 100-year time horizon.

Table 11.2

Numerical Estimates of Global Warming Potentials Compared with Carbon Dioxide (kilogram of gas per kilogram of carbon dioxide)

\begin{tabular}{|c|c|c|c|}
\hline \multirow[b]{2}{*}{ Gas } & \multirow{2}{*}{$\begin{array}{l}\text { Lifetime } \\
\text { (years) }\end{array}$} & \multicolumn{2}{|c|}{$\begin{array}{l}\text { Global warming potential } \\
\text { direct effect for time horizons of }\end{array}$} \\
\hline & & 20 years & 100 years \\
\hline Carbon Dioxide $\left(\mathrm{CO}_{2}\right)$ & $5-200^{\mathrm{a}}$ & 1 & 1 \\
\hline Methane $\left(\mathrm{CH}_{4}\right)^{\mathrm{b}}$ & 12.4 & 86 & 34 \\
\hline Tetrafluoroethane (HFC-134a) & 13.4 & 3,790 & 1,550 \\
\hline Trichlorofluoromethane (CFC-11) & 45 & 7,020 & 5,350 \\
\hline Nitrous Oxide $\left(\mathrm{N}_{2} \mathrm{O}\right)$ & 121 & 268 & 298 \\
\hline Perfluoromethane $\left(\mathrm{CF}_{4}\right)$ & 50,000 & 4,950 & 7,350 \\
\hline
\end{tabular}

Note: Includes climate-carbon feedbacks.

Source:

Myhre, G., D. Shindell, F.-M. Breon, W. Collins, J. Fuglestvedt, J. Huang, D. Koch, J.-F. Lamarque, D. Lee, B. Mendoza, T. Nakajima, A. Robock, G. Stephens, T. Takemura and H. Zhang, 2013: Anthropogenic and Natural Radiative Forcing. In: Climate Change 2013: The Physical Science Basis. Contribution of Working Group 1 to the Fifth Assessment Report of the Intergovernmental Panel on Climate Change [Stocker, R.F., D. Qin, G.-K. Plattner, M. Tignor, S.K. Allen, J. Boschung, A. Nauels, Y. Zia, V. Bex and P.M. Midgley (eds)]. Cambridge university Press, Cambridge, United Kingdom and New York, NY, USA.

\footnotetext{
${ }^{a}$ No single lifetime can be defined for carbon dioxide due to different rates of uptake by different removal processes.

${ }^{b}$ These values do not include carbon dioxide from methane oxidation. Perturbation lifetime is used in the calculation of metrics.
} 
Carbon dioxide emissions in 2012 were 5\% higher than in 1990. This is a decrease from 2011. Carbon dioxide accounts for the majority of greenhouse gases.

Table 11.3

U.S. Emissions of Greenhouse Gases, based on Global Warming Potential, 1990-2012 (million metric tonnes carbon dioxide equivalent ${ }^{\mathrm{a}}$ )

\begin{tabular}{lccccc}
\hline & $\begin{array}{c}\text { Carbon } \\
\text { dioxide }\end{array}$ & Methane & $\begin{array}{c}\text { Nitrous } \\
\text { oxide }\end{array}$ & $\begin{array}{c}\text { High } \\
\text { GWP gases }\end{array}$ & Total \\
\hline 1990 & $5,075.1$ & 632.0 & 398.5 & 90.2 & $6,195.8$ \\
2005 & $6,054.1$ & 581.7 & 415.5 & 135.1 & $7,186.4$ \\
2008 & $5,887.1$ & 602.0 & 423.1 & 133.3 & $7,045.5$ \\
2009 & $5,458.5$ & 593.1 & 412.1 & 124.7 & $6,588.4$ \\
2010 & $5,664.4$ & 582.4 & 409.1 & 134.8 & $6,790.7$ \\
2011 & $5,530.7$ & 575.3 & 417.1 & 145.5 & $6,668.5$ \\
2012 & $5,319.2$ & 564.5 & 409.9 & 150.5 & $6,444.1$ \\
\hline
\end{tabular}

Note: This greenhouse gas emissions inventory includes two "adjustments to energy consumption" which make the data different from Table 11.5. The adjustments are as follows:

(1) Emissions from U.S. Territories are included.

(2) International bunker fuels and military bunker fuels are excluded from the U.S. total.

Source:

U.S. Environmental Protection Agency, Draft Inventory of U. S. Greenhouse Gas Emissions and Sinks: 1990-2012.

February 2014, www.epa.gov/climatechange/Downloads/ghgemissions/US-GHG-Inventory-2014-Main-

Text.pdf

${ }^{a}$ Carbon dioxide equivalents are computed by multiplying the weight of the gas being measured by its estimated Global Warming Potential (See Table 11.2).

${ }^{\mathrm{b}} \mathrm{GWP}=$ Global warming potential. Includes HFC-hydrofluorocarbons; PFC-perfluorocarbons; and $\mathrm{SF}_{6}{ }^{-}$ sulfur hexaflouride. 
The transportation sector accounts for the largest share of total greenhouse gas emissions, due to the high share of carbon dioxide emissions. The industrial sector accounts for nearly the same amount of total greenhouse gas emissions as the transportation sector.

Table 11.4

Total U.S. Greenhouse Gas Emissions by End-Use Sector, 2012 (million metric tonnes carbon dioxide equivalent ${ }^{\mathrm{a}}$ )

\begin{tabular}{lccrrr}
\hline & & & & \multicolumn{2}{c}{ Total } \\
& $\begin{array}{c}\text { Carbon } \\
\text { dioxide }\end{array}$ & Methane & $\begin{array}{c}\text { Nitrous } \\
\text { oxide }\end{array}$ & $\begin{array}{c}\text { pydroflurocarbons, } \\
\text { perflurocarbons, } \\
\text { sulfur hexafluoride }\end{array}$ & $\begin{array}{c}\text { gas } \\
\text { emissions }\end{array}$ \\
\hline Residential & $1,021.1$ & 3.3 & 8.9 & 29.4 & $1,062.7$ \\
Commercial & 904.3 & 118.2 & 13.6 & 32.4 & $1,068.5$ \\
Agricultural & 120.7 & 217.0 & 338.6 & 0.2 & 676.5 \\
Industrial & $1,525.5$ & 244.6 & 33.9 & 32.9 & $1,816.9$ \\
Transportation & $1,747.6$ & 1.4 & 14.9 & 55.6 & $1,819.5$ \\
Transportation share of total & $32.9 \%$ & $0.2 \%$ & $3.6 \%$ & $36.9 \%$ & $28.2 \%$ \\
\hline Total greenhouse gas emissions & $5,319.2$ & 564.5 & 409.9 & 150.5 & $6,444.1$ \\
\hline
\end{tabular}

Note: Totals may not sum due to rounding.

\section{Source:}

U.S. Environmental Protection Agency, Draft Inventory of U.S. Greenhouse Gas Emissions and Sinks, 1990-2012. February 2014. (Additional resources: www.epa.gov/climatechange/Downloads/ghgemissions/US-GHGInventory-2014-Main-Text.pdf)

${ }^{\text {a }}$ Carbon dioxide equivalents are computed by multiplying the weight of the gas being measured by its estimated Global Warming Potential (See Table 11.2). 
Gases which contain carbon can be measured in terms of the full molecular weight of the gas or just in terms of their carbon content. This table presents carbon dioxide gas. The ratio of the weight of carbon to carbon dioxide is 0.2727 . The transportation sector accounts for approximately one-third of carbon emissions.

Table 11.5

U.S. Carbon Emissions from Fossil Fuel Consumption

by End-Use Sector, 1990-2012 ${ }^{\mathrm{a}}$

(million metric tonnes of carbon dioxide)

\begin{tabular}{|c|c|c|c|c|c|c|}
\hline & \multicolumn{4}{|c|}{ End use sector } & \multirow{2}{*}{$\begin{array}{c}\text { Transportation } \\
\text { percentage }\end{array}$} & \multirow{2}{*}{$\begin{array}{l}\mathrm{CO}_{2} \text { from } \\
\text { all sectors }\end{array}$} \\
\hline & Residential & Commercial & Industrial & Transportation & & \\
\hline 1990 & 931.4 & 757.0 & $1,535.3$ & $1,497.0$ & $31.7 \%$ & $4,720.7$ \\
\hline 2005 & $1,214.7$ & $1,027.2$ & $1,560.4$ & $1,896.5$ & $33.3 \%$ & $5,698.8$ \\
\hline 2007 & $1,205.2$ & $1,047.7$ & $1,559.9$ & $1,909.7$ & $33.4 \%$ & $5,722.5$ \\
\hline 2008 & $1,189.9$ & $1,039.8$ & $1,499.3$ & $1,820.7$ & $32.8 \%$ & $5,549.7$ \\
\hline 2009 & $1,123.5$ & 976.8 & $1,324.6$ & $1,753.7$ & $33.9 \%$ & $5,178.6$ \\
\hline 2010 & $1,175.0$ & 993.9 & $1,421.3$ & $1,768.4$ & $33.0 \%$ & $5,358.6$ \\
\hline 2011 & $1,125.6$ & 960.5 & $1,392.1$ & $1,749.3$ & $33.5 \%$ & $5,227.5$ \\
\hline 2012 & $1,015.3$ & 898.7 & $1,362.9$ & $1,739.3$ & $34.7 \%$ & $5,016.2$ \\
\hline \multicolumn{7}{|c|}{ Average annual percentage change } \\
\hline 1990-2012 & $0.4 \%$ & $0.8 \%$ & $-0.5 \%$ & $0.7 \%$ & & $0.3 \%$ \\
\hline 2005-2012 & $-2.5 \%$ & $-1.9 \%$ & $-1.9 \%$ & $-1.2 \%$ & & $-1.8 \%$ \\
\hline
\end{tabular}

Source:

U.S. Environmental Protection Agency, Draft Inventory of U.S. Greenhouse Gas Emissions and Sinks, $1990-2012$. February 2014. (Additional resources: www.epa.gov/climatechange/Downloads/ghgemissions/US-GHGInventory-2014-Main-Text.pdf)

${ }^{a}$ Includes energy from petroleum, coal, and natural gas. Electric utility emissions are distributed across consumption sectors. 
Most U.S. transportation sector carbon dioxide emissions come from petroleum fuels (97.5\%). Motor gasoline has been responsible for about two-thirds of U.S. carbon dioxide emissions over the last twenty years.

Table 11.6

U.S. Carbon Emissions from Fossil Fuel Combustion in the Transportation End-Use Sector

\begin{tabular}{|c|c|c|c|c|c|c|}
\hline \multirow[b]{2}{*}{ Fuel } & \multicolumn{2}{|c|}{1990} & \multicolumn{2}{|c|}{2005} & \multicolumn{2}{|c|}{2012} \\
\hline & Emissions & Percentage & Emissions & Percentage & Emissions & Percentage \\
\hline & \multicolumn{6}{|c|}{ Petroleum } \\
\hline Motor gasoline & 983.7 & $65.7 \%$ & $1,187.8$ & $62.6 \%$ & $1,102.8$ & $62.9 \%$ \\
\hline $\mathrm{LPG}^{\mathrm{a}}$ & 1.4 & $0.1 \%$ & 1.7 & $0.1 \%$ & 2.0 & $0.1 \%$ \\
\hline Jet fuel & 184.2 & $12.3 \%$ & 189.3 & $10.0 \%$ & 146.6 & $8.4 \%$ \\
\hline Distillate fuel & 262.9 & $17.6 \%$ & 458.1 & $24.2 \%$ & 436.3 & $24.9 \%$ \\
\hline Residual fuel & 22.6 & $1.5 \%$ & 19.3 & $1.0 \%$ & 19.4 & $1.1 \%$ \\
\hline Aviation gas & 3.1 & $0.2 \%$ & 2.4 & $0.1 \%$ & 1.9 & $0.1 \%$ \\
\hline \multirow[t]{2}{*}{ Subtotal } & $1,457.9$ & $97.4 \%$ & $1,858.6$ & $98.0 \%$ & $1,709.0$ & $97.5 \%$ \\
\hline & \multicolumn{6}{|c|}{ Other energy } \\
\hline Natural gas & 36.0 & $2.4 \%$ & 33.1 & $1.7 \%$ & 38.8 & $2.2 \%$ \\
\hline Electricity ${ }^{\mathrm{b}}$ & 3.0 & $0.2 \%$ & 4.7 & $0.2 \%$ & 4.3 & $0.2 \%$ \\
\hline Total $^{\mathrm{c}}$ & $1,496.9$ & $100.0 \%$ & $1,896.4$ & $100.0 \%$ & $1,752.1$ & $100.0 \%$ \\
\hline
\end{tabular}

Source:

U.S. Environmental Protection Agency, Draft Inventory of U.S. Greenhouse Gas Emissions and Sinks, 1990-2012. February 2014. (Additional resources: www.epa.gov/climatechange/Downloads/ghgemissions/US-GHGInventory-2014-Main-Text.pdf)

\footnotetext{
${ }^{\mathrm{a}}$ Liquified petroleum gas.

${ }^{\mathrm{b}}$ Share of total electric utility carbon dioxide emissions weighted by sales to the transportation sector.

${ }^{\mathrm{c}}$ Percentages may not sum to totals due to rounding.
} 
Highway vehicles are responsible for the majority of greenhouse gas emissions in the transportation sector.

Table 11.7

Transportation Greenhouse Gas Emissions by Mode, 1990 and 2012 (Million metric tonnes of carbon dioxide equivalent)

\begin{tabular}{|c|c|c|c|}
\hline & Carbon dioxide & Methane & Nitrous oxide \\
\hline \multicolumn{4}{|c|}{1990} \\
\hline Highway total & $1,190.5$ & 4.2 & 40.4 \\
\hline Cars, light trucks, motorcycles & 952.2 & 4.0 & 39.6 \\
\hline Medium \& heavy trucks and buses & 238.3 & 0.2 & 0.8 \\
\hline Water & 44.5 & 0.0 & 0.6 \\
\hline Air & 187.4 & 0.1 & 1.8 \\
\hline Rail & 38.5 & 0.1 & 0.3 \\
\hline Pipeline & 36.0 & 0.0 & 0.0 \\
\hline Other & 0.0 & 0.2 & 0.9 \\
\hline Total $^{\mathrm{a}}$ & $1,496.9$ & 4.6 & 44.0 \\
\hline \multicolumn{4}{|c|}{2012} \\
\hline Highway total & $1,474.1$ & 1.3 & 12.6 \\
\hline Cars, light trucks, motorcycles & $1,065.3$ & 1.2 & 11.7 \\
\hline Medium \& heavy trucks and buses & 408.8 & 0.1 & 0.9 \\
\hline Water & 36.6 & 0.0 & 0.5 \\
\hline Air & 145.1 & 0.0 & 1.4 \\
\hline Rail & 44.1 & 0.1 & 0.3 \\
\hline Pipeline & 39.4 & 0.0 & 0.0 \\
\hline Other & 0.0 & 0.4 & 1.6 \\
\hline Total $^{\mathrm{a}}$ & $1,739.3$ & 1.8 & 16.4 \\
\hline \multicolumn{4}{|c|}{ Percent change 1990-2012 } \\
\hline Highway total & $23.8 \%$ & $-69.0 \%$ & $-68.8 \%$ \\
\hline Cars, light trucks, motorcycles & $11.9 \%$ & $-70.0 \%$ & $-70.5 \%$ \\
\hline Medium \& heavy trucks and buses & $71.5 \%$ & $-50.0 \%$ & $12.5 \%$ \\
\hline Water & $-17.8 \%$ & $0.0 \%$ & $-16.7 \%$ \\
\hline Air & $-22.6 \%$ & $-100.0 \%$ & $-22.2 \%$ \\
\hline Rail & $14.5 \%$ & $0.0 \%$ & $0.0 \%$ \\
\hline Pipeline & $9.4 \%$ & $0.0 \%$ & $0.0 \%$ \\
\hline Other & $0.0 \%$ & $100.0 \%$ & $77.8 \%$ \\
\hline Total $^{\mathrm{a}}$ & $16.2 \%$ & $-60.9 \%$ & $-62.7 \%$ \\
\hline
\end{tabular}

Note: Emissions from U.S. Territories, International bunker fuels, and military bunker fuels are not included.

\section{Source:}

U.S. Environmental Protection Agency, Draft Inventory of U.S. Greenhouse Gas Emissions and Sinks: 1990-2012, Tables 3-12, 3-13, 3-14, February 2014. (Additional resources:

www.epa.gov/climatechange/Downloads/ghgemissions/US-GHG-Inventory-2014-Main-Text.pdf)

${ }^{a}$ The sums of subcategories may not equal totals due to rounding. 


\section{The Greenhouse Gases, Regulated Emissions, and Energy Use in Transportation (GREET) Model}

\section{greet.es.anl.gov}

Sponsored by the U.S. Department of Energy's Office of Energy Efficiency and Renewable Energy (EERE), Argonne has developed a full life-cycle model called GREET ${ }^{T M}$ (Greenhouse gases, Regulated Emissions, and Energy use in Transportation). It allows researchers and analysts to evaluate energy and emission impacts of various vehicle and fuel combinations on a full fuel-cycle/vehicle-cycle basis. The first version of GREET was released in 1996. Since then, Argonne has continued to update and expand the model. The most recent GREET versions are GREET 12013 version for fuel-cycle analysis and GREET 22013 version for vehicle-cycle analysis.

\section{Figure 11.1. GREET Model}

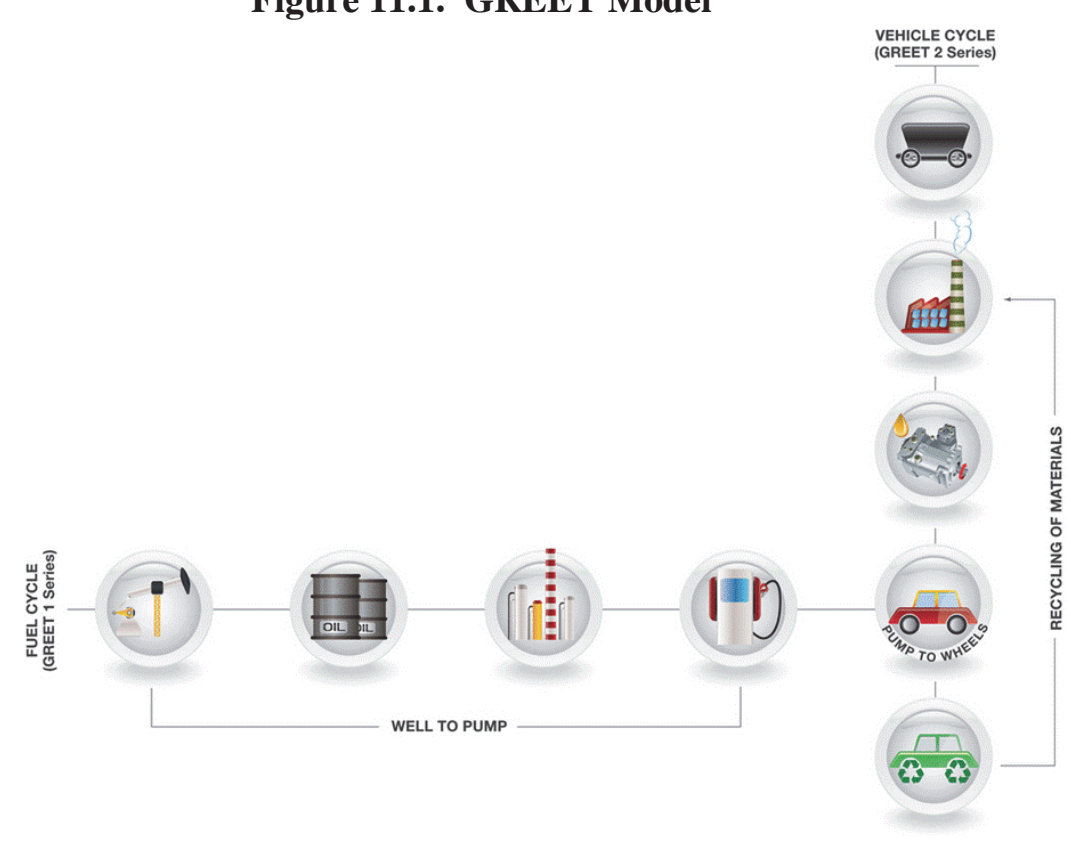

For a given vehicle and fuel system, GREET separately calculates the following:

- Consumption of total energy (energy in non-renewable and renewable sources), fossil fuels (petroleum, natural gas, and coal together), petroleum, coal and natural gas.

- Emissions of $\mathrm{CO}_{2}$-equivalent greenhouse gases - primarily carbon dioxide $\left(\mathrm{CO}_{2}\right)$, methane $\left(\mathrm{CH}_{4}\right)$, and nitrous oxide $\left(\mathrm{N}_{2} \mathrm{O}\right)$.

- Emissions of six criteria pollutants: volatile organic compounds (VOCs), carbon monoxide (CO), nitrogen oxide (NOx), particulate matter with size smaller than 10 micron $\left(\mathrm{PM}_{10}\right)$, particulate matter with size smaller than 2.5 micron $\left(\mathrm{PM}_{2.5}\right)$, and sulfur oxides ( $\left.\mathrm{SOx}\right)$. 
GREET includes more than 100 fuel production pathways and more than 80 vehicle/fuel systems. These vehicle/fuel systems cover all major vehicle technologies in the market and R\&D arena: conventional spark-ignition engine vehicles; spark-ignition, direct-injection engine vehicles; compression-ignition, direct-injection engine vehicles; hybrid electric vehicles; plug-in hybrid electric vehicles; battery-powered electric vehicles; and ffuel-cell vehicles.

Recently, GREET was expanded to include air and marine modes as well.

\section{Figure 11.2. GREET Model Feedstocks and Fuels}

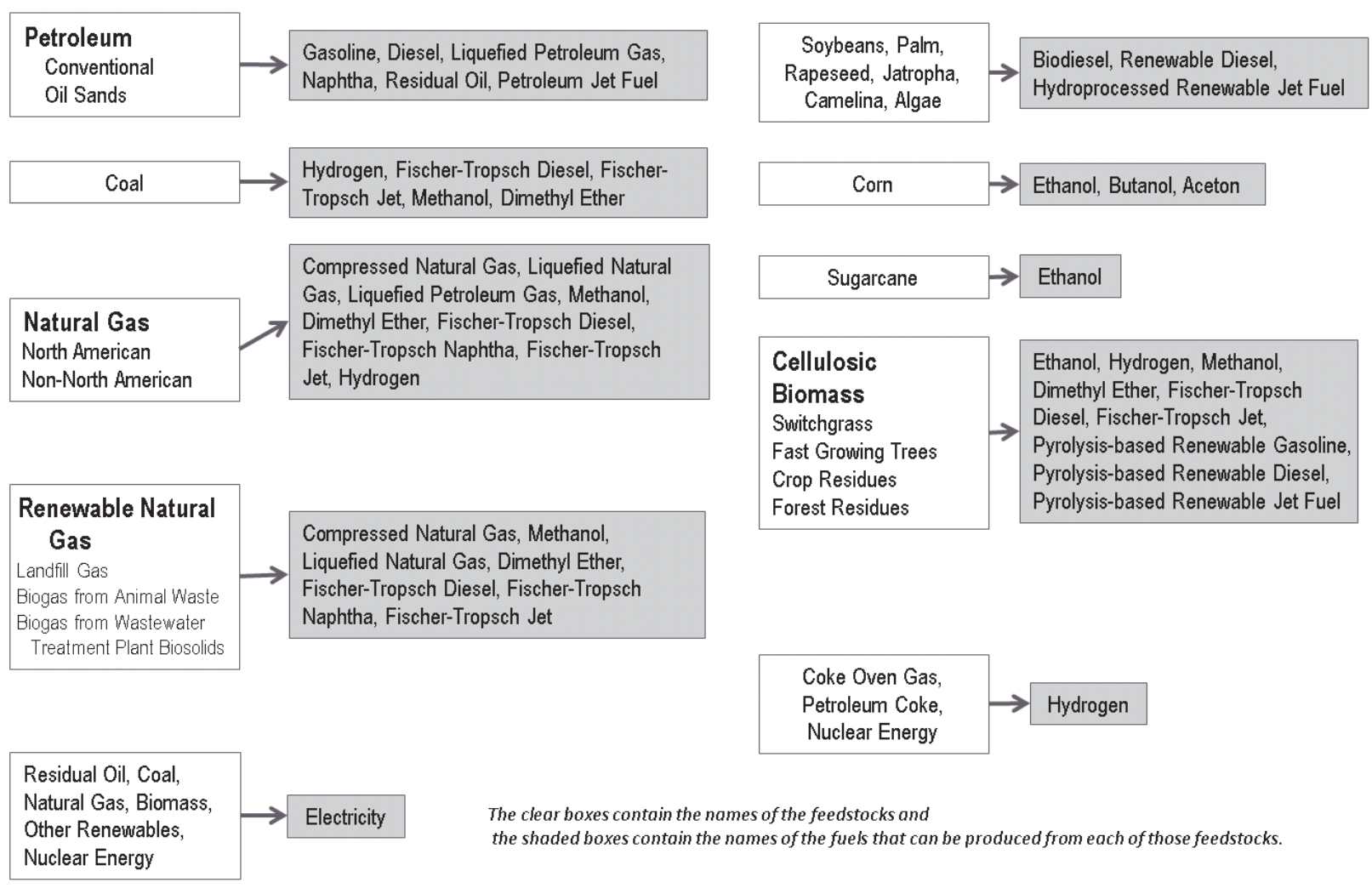

To address technology improvements over time, GREET simulates vehicle/fuel systems over the period from 1990 to 2035, in five-year intervals.

For additional information about the GREET model, see the GREET website, or contact:

Michael Q. Wang

Argonne National Laboratory

9700 South Cass Avenue, ES/362

Argonne, IL 60439-4815

phone: $630-252-2819$

fax: 630-252-3443

email: mqwang@anl.gov 
These are results from the GREET model (see preceding pages for description). For electricity, three different grid mixes are compared - the U.S., California, and RFC West. California's (CA) grid mix was chosen due to the high renewable energy mix in that state. The RFC West is an electricity grid subregion that includes Indiana, Ohio, West Virginia, and parts of Illinois, Pennsylvania, Virginia, Kentucky, and Wisconsin. The RFC West uses a high amount of coal to produce electricity.

Figure 11.3. Well-to-Wheel Emissions for Various Fuels and Vehicle Technologies

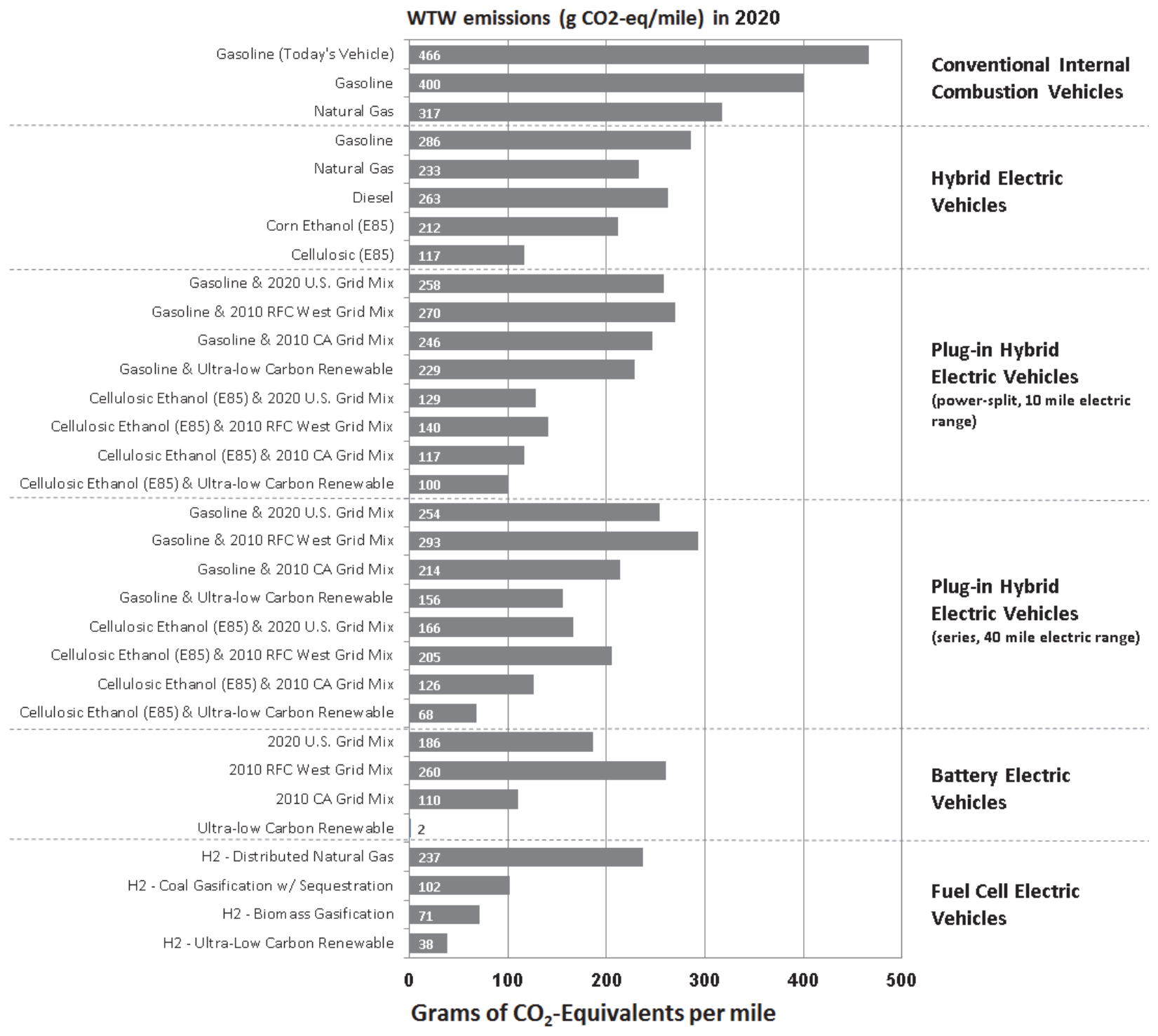

Note: $\mathrm{H} 2=$ hydrogen .

Source: Argonne National Laboratory, GREET 12013 Model. 


\section{Carbon Footprint}

The carbon footprint measures a vehicle's impact on climate change in tons of carbon dioxide $\left(\mathrm{CO}_{2}\right)$ emitted annually. The following three tables show the carbon footprint for various vehicle classes. The sales-weighted average fuel economy rating for each vehicle class, based on $45 \%$ highway and 55\% city driving, is used to determine the average annual carbon footprint for vehicles in the class. An estimate of 15,000 annual miles is used for each vehicle class and for each year in the series. The equation to calculate carbon footprint uses results of the GREET model version 1.8.

CarbonFootprint $=\left(\mathrm{CO}_{2} \times L \mathrm{HV} \times \frac{\text { AnnualMiles }}{\text { CombinedMPG }}\right)+\left(\mathrm{CH}_{4}+\mathrm{N}_{2} \mathrm{O}\right) \times$ AnnualMiles

where:

$\mathrm{CO}_{2}=\left(\right.$ Tailpipe $\mathrm{CO}_{2}+$ Upstream Greenhouse Gases $)$ in grams per million Btu

LHV $=$ Lower (or net) Heating Value in million Btu per gallon

$\mathrm{CH}_{4}=$ Tailpipe ${\underline{\mathrm{CO}_{2}}}_{2}$ equivalent methane in grams per mile

$\mathrm{N}_{2} \mathrm{O}=$ Tailpipe $\underline{\mathrm{CO}}_{2}$ equivalent nitrous oxide in grams per mile

Note: The Environmental Protection Agency publishes tailpipe emissions in the Light-Duty Automotive Technology, Carbon Dioxide Emissions, and Fuel Economy Trends: 1975 through 2013, www.epa.gov/otaq/fetrends.htm. 
The production-weighted average annual carbon footprint for cars and car SUVs declined between 1975 and 2013.

Table 11.8

Production-Weighted Annual Carbon Footprint of New Domestic and Import Cars Model Years 1975-2013 (short tons of $\mathrm{CO}_{2}$ )

\begin{tabular}{|c|c|c|}
\hline Model Year & Car & Car SUV \\
\hline 1975 & 13.8 & 16.7 \\
\hline 1980 & 9.3 & 12.8 \\
\hline 1981 & 8.7 & 12.7 \\
\hline 1982 & 8.4 & 9.4 \\
\hline 1983 & 8.5 & 9.0 \\
\hline 1984 & 8.3 & 9.7 \\
\hline 1985 & 8.1 & 9.3 \\
\hline 1986 & 7.9 & 9.9 \\
\hline 1987 & 7.9 & 9.6 \\
\hline 1988 & 7.7 & 9.7 \\
\hline 1989 & 7.9 & 9.8 \\
\hline 1990 & 8.0 & 9.9 \\
\hline 1991 & 8.0 & 10.2 \\
\hline 1992 & 8.1 & 10.5 \\
\hline 1993 & 8.0 & 10.9 \\
\hline 1994 & 8.0 & 10.3 \\
\hline 1995 & 8.0 & 10.5 \\
\hline 1996 & 8.0 & 10.1 \\
\hline 1997 & 8.0 & 9.7 \\
\hline 1998 & 8.0 & 10.2 \\
\hline 1999 & 8.1 & 10.1 \\
\hline 2000 & 8.2 & 10.4 \\
\hline 2001 & 8.1 & 9.9 \\
\hline 2002 & 8.1 & 9.7 \\
\hline 2003 & 8.0 & 9.4 \\
\hline 2004 & 8.1 & 9.3 \\
\hline 2005 & 8.0 & 9.2 \\
\hline 2006 & 8.0 & 9.1 \\
\hline 2007 & 7.8 & 9.0 \\
\hline 2008 & 7.7 & 8.8 \\
\hline 2009 & 7.4 & 8.5 \\
\hline 2010 & 7.2 & 8.1 \\
\hline 2011 & 7.2 & 7.9 \\
\hline 2012 & 6.7 & 8.0 \\
\hline 2013 & 6.7 & 7.6 \\
\hline \multicolumn{3}{|c|}{ Average annual percentage change } \\
\hline 1975-2013 & $-1.9 \%$ & $-2.1 \%$ \\
\hline 2003-2013 & $-1.8 \%$ & $-2.1 \%$ \\
\hline
\end{tabular}

\section{Source:}

Calculated using fuel economy from the U.S. Environmental Protection Agency, Light-Duty Automotive Technology, Carbon Dioxide Emissions, and Fuel Economy Trends: 1975 Through 2013, December 2013. See page 11-12 for details. (Additional resources: www.epa.gov/fueleconomy/fetrends/1975-2013/420r13011.pdf)

a Annual carbon footprint is based on 15,000 miles of annual driving. Includes tailpipe plus upstream emissions.

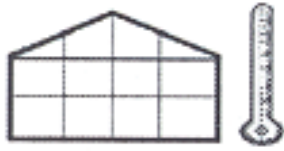


The production-weighted average annual footprint of pickups, vans, and truck SUVs decreased from 1975 to 2013. Truck SUVs experienced the greatest decline.

Table 11.9

Production-Weighted Annual Carbon Footprint of New Domestic and Import Trucks Model Years 1975-2013 ${ }^{\mathrm{a}}$ (short tons of $\mathrm{CO}_{2}$ )

\begin{tabular}{|c|c|c|c|}
\hline Model Year & Pickup & Van & Truck SUV \\
\hline 1975 & 15.6 & 16.8 & 16.9 \\
\hline $1980^{\circ}$ & 11.3 & 13.2 & 14.1 \\
\hline 1981 & 10.4 & 12.6 & 13.0 \\
\hline 1982 & 10.1 & 12.7 & 12.7 \\
\hline 1983 & 9.9 & 12.4 & 11.8 \\
\hline 1984 & 10.2 & 11.6 & 11.5 \\
\hline 1985 & 10.2 & 11.3 & 11.3 \\
\hline 1986 & 9.9 & 10.7 & 10.9 \\
\hline 1987 & 9.8 & 10.6 & 10.8 \\
\hline 1988 & 10.3 & 10.4 & 11.0 \\
\hline 1989 & 10.5 & 10.5 & 11.3 \\
\hline 1990 & 10.7 & 10.5 & 11.3 \\
\hline 1991 & 10.3 & 10.4 & 11.1 \\
\hline 1992 & 10.7 & 10.4 & 11.5 \\
\hline 1993 & 10.6 & 10.2 & 11.4 \\
\hline 1994 & 10.7 & 10.5 & 11.6 \\
\hline 1995 & 11.0 & 10.3 & 11.7 \\
\hline 1996 & 10.9 & 10.2 & 11.5 \\
\hline 1997 & 11.1 & 10.3 & 11.6 \\
\hline 1998 & 11.0 & 10.0 & 11.5 \\
\hline 1999 & 11.4 & 10.2 & 11.6 \\
\hline 2000 & 11.2 & 10.0 & 11.6 \\
\hline 2001 & 11.7 & 10.3 & 11.4 \\
\hline 2002 & 11.8 & 10.0 & 11.4 \\
\hline 2003 & 11.6 & 9.8 & 11.4 \\
\hline 2004 & 11.8 & 9.7 & 11.3 \\
\hline 2005 & 11.8 & 9.7 & 11.1 \\
\hline 2006 & 11.5 & 9.6 & 10.9 \\
\hline 2007 & 11.5 & 9.6 & 10.5 \\
\hline 2008 & 11.3 & 9.4 & 10.3 \\
\hline 2009 & 11.0 & 9.3 & 9.7 \\
\hline 2010 & 11.1 & 9.3 & 9.5 \\
\hline 2011 & 10.9 & 8.9 & 9.4 \\
\hline 2012 & 10.9 & 8.7 & 9.3 \\
\hline 2013 & 10.7 & 8.9 & 9.0 \\
\hline \multicolumn{4}{|c|}{ Average annual percentage change } \\
\hline 1975-2013 & $-1.0 \%$ & $-1.7 \%$ & $-1.6 \%$ \\
\hline 2003-2013 & $-0.8 \%$ & $-1.0 \%$ & $-2.3 \%$ \\
\hline
\end{tabular}

Note: Includes light trucks of 8,500 lbs. or less.

\section{Source:}

Calculated using fuel economy from the U.S. Environmental Protection Agency, Light-Duty Automotive Technology, Carbon Dioxide Emissions, and Fuel Economy Trends: 1975 Through 2013, December 2013. See page 11-12 for details. (Additional resources: www.epa.gov/fueleconomy/fetrends/1975-2013/420r13011.pdf) emissions.

a Annual carbon footprint is based on 15,000 miles of annual driving. Includes tailpipe plus upstream

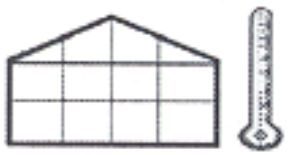


Between 1975 and 2013, the production-weighted average annual carbon footprint for light vehicles dropped dramatically. Cars experienced the greatest decrease at 50.6\% while the carbon footprint for light trucks decreased by $40.7 \%$.

Table 11.10

Average Annual Carbon Footprint by Vehicle Classification, 1975 and $2013^{\mathrm{a}}$ (short tons of $\mathrm{CO}_{2}$ )

\begin{tabular}{|c|c|c|c|c|c|}
\hline \multirow[b]{2}{*}{ Fuel } & \multicolumn{2}{|c|}{ Production share } & \multicolumn{2}{|c|}{ Carbon footprint } & \multirow{2}{*}{$\begin{array}{r}\text { Percent change } \\
1975-2013\end{array}$} \\
\hline & 1975 & 2013 & 1975 & 2013 & \\
\hline \multicolumn{6}{|c|}{ Cars } \\
\hline Car & $80.6 \%$ & $53.8 \%$ & 13.8 & 6.7 & $-51.8 \%$ \\
\hline Car SUV & $0.1 \%$ & $10.6 \%$ & 16.7 & 7.6 & $-54.4 \%$ \\
\hline Total cars & $80.7 \%$ & $64.4 \%$ & 13.8 & 6.8 & $-50.6 \%$ \\
\hline \multicolumn{6}{|c|}{ Light trucks } \\
\hline Van & $4.5 \%$ & $4.0 \%$ & 16.8 & 8.9 & $-46.9 \%$ \\
\hline Truck SUV & $1.7 \%$ & $21.0 \%$ & 16.9 & 9.0 & $-46.9 \%$ \\
\hline Pickup & $13.1 \%$ & $10.6 \%$ & 15.6 & 10.7 & $-31.2 \%$ \\
\hline Total light trucks & $19.3 \%$ & $35.6 \%$ & 16.0 & 9.5 & $-40.7 \%$ \\
\hline
\end{tabular}

Source:

Calculated using fuel economy from the U.S. Environmental Protection Agency, Light-Duty Automotive Technology, Carbon Dioxide Emissions, and Fuel Economy Trends: 1975 Through 2013, December 2013. See page 11-12 for details. (Additional resources: www.epa.gov/fueleconomy/fetrends/1975-2013/420r13011.pdf)

${ }^{\text {a }}$ Annual carbon footprint is based on 15,000 miles of annual driving. Includes tailpipe and upstream emissions. 
The amount of carbon dioxide released into the atmosphere by a vehicle is primarily determined by the carbon content of the fuel. However, there is a small portion of the fuel that is not oxidized into carbon dioxide when the fuel is burned. The Environmental Protection Agency (EPA) has published information on carbon dioxide emissions from gasoline and diesel which takes the oxidation factor into account and is based on the carbon content used in EPA's fuel economy analyses. The other fuels listed come from the Energy Information Administration.

Table 11.11

Direct Carbon Dioxide Emissions from a Gallon of Fuel ${ }^{\mathrm{a}}$

\begin{tabular}{lccc}
\hline & $\begin{array}{c}\text { Grams } \\
\text { per gallon }\end{array}$ & $\begin{array}{c}\text { Kilograms } \\
\text { per gallon }\end{array}$ & $\begin{array}{c}\text { Pounds } \\
\text { per gallon }\end{array}$ \\
\hline Gasoline & 8,887 & 8.9 & 19.6 \\
Diesel & 10,180 & 10.2 & 22.4 \\
E85 & 1,340 & 1.3 & 3.0 \\
B20 & 8,120 & 8.1 & 17.9 \\
LPG & 5,805 & 5.8 & 12.8 \\
Propane & 5,740 & 5.8 & 12.7 \\
Aviation gasoline & 8,320 & 8.3 & 18.3 \\
Jet fuel & 9,751 & 9.6 & 21.5 \\
Kerosene & 9,751 & 9.8 & 21.5 \\
Residual fuel & 11,791 & 11.8 & 26.0 \\
\hline
\end{tabular}

Sources:

Gasoline and Diesel: U.S. Environmental Protection Agency, "Greenhouse Gas Emissions from a Typical Passenger Vehicle," December 2011. (Additional resources: www.epa.gov/otaq)

All others: Energy Information Administration, Voluntary Reporting of Greenhouse Gases Program, Fuel and Energy Source Codes and Emission Coefficients.

a Direct emissions are from the "tank-to-wheels" process. No upstream emissions are included. 


\section{Chapter 12 \\ Criteria Air Pollutants}

Summary Statistics from Tables in this Chapter

\begin{tabular}{|c|c|c|}
\hline Source & & \\
\hline \multirow[t]{7}{*}{ Table 12.1} & Transportation's share of U.S. emissions, 2013 & \\
\hline & $\mathrm{CO}$ & $53.7 \%$ \\
\hline & $N O_{X}$ & $59.0 \%$ \\
\hline & VOC & $23.4 \%$ \\
\hline & $P M-2.5$ & $5.9 \%$ \\
\hline & $P M-10$ & $2.2 \%$ \\
\hline & $\mathrm{SO}_{2}$ & $2.1 \%$ \\
\hline
\end{tabular}


Transportation accounts for the majority of carbon monoxide and nitrogen oxide emissions. Highway vehicles are responsible for the largest share of transportation emissions.

Table 12.1

Total National Emissions of the Criteria Air Pollutants by Sector, 2013 (millions of short tons/percentage)

\begin{tabular}{lrrrrrr}
\hline Sector & \multicolumn{1}{c}{ CO } & NOx & \multicolumn{1}{c}{ VOC } & PM-10 & PM-2.5 & \multicolumn{1}{c}{ SO $_{2}$} \\
\hline Highway vehicles & $\mathbf{2 4 . 8 0}$ & $\mathbf{5 . 0 1}$ & $\mathbf{2 . 1 6}$ & $\mathbf{0 . 2 7}$ & $\mathbf{0 . 1 9}$ & $\mathbf{0 . 0 3}$ \\
& $33.8 \%$ & $38.2 \%$ & $12.2 \%$ & $1.3 \%$ & $3.0 \%$ & $0.6 \%$ \\
Other off-highway & $\mathbf{1 4 . 6 5}$ & $\mathbf{2 . 7 3}$ & $\mathbf{1 . 9 9}$ & $\mathbf{0 . 2 0}$ & $\mathbf{0 . 1 8}$ & $\mathbf{0 . 0 8}$ \\
& $19.9 \%$ & $20.8 \%$ & $11.2 \%$ & $0.9 \%$ & $2.9 \%$ & $1.5 \%$ \\
Transportation total & $\mathbf{3 9 . 4 5}$ & $\mathbf{7 . 7 4}$ & $\mathbf{4 . 1 5}$ & $\mathbf{0 . 4 7}$ & $\mathbf{0 . 3 7}$ & $\mathbf{0 . 1 1}$ \\
& $53.7 \%$ & $59.0 \%$ & $23.4 \%$ & $2.2 \%$ & $5.9 \%$ & $2.1 \%$ \\
Stationary source fuel combustion & $\mathbf{4 . 6 3}$ & $\mathbf{3 . 6 9}$ & $\mathbf{0 . 6 3}$ & $\mathbf{0 . 9 8}$ & $\mathbf{0 . 8 3}$ & $\mathbf{4 . 2 4}$ \\
& $6.3 \%$ & $28.1 \%$ & $3.6 \%$ & $4.7 \%$ & $13.3 \%$ & $82.1 \%$ \\
Industrial processes & $\mathbf{1 . 9 8}$ & $\mathbf{1 . 1 8}$ & $\mathbf{6 . 9 7}$ & $\mathbf{0 . 9 4}$ & $\mathbf{0 . 3 9}$ & $\mathbf{0 . 5 8}$ \\
& $2.7 \%$ & $9.0 \%$ & $39.3 \%$ & $4.5 \%$ & $6.3 \%$ & $11.3 \%$ \\
Waste disposal and recycling total & $\mathbf{1 . 1 1}$ & $\mathbf{0 . 0 8}$ & $\mathbf{0 . 1 3}$ & $\mathbf{0 . 2 0}$ & $\mathbf{0 . 1 7}$ & $\mathbf{0 . 0 2}$ \\
& $1.5 \%$ & $0.6 \%$ & $0.7 \%$ & $1.0 \%$ & $2.7 \%$ & $0.3 \%$ \\
Miscellaneous & $\mathbf{2 6 . 2 6}$ & $\mathbf{0 . 4 4}$ & $\mathbf{5 . 8 7}$ & $\mathbf{1 8 . 2 8}$ & $\mathbf{4 . 4 9}$ & $\mathbf{0 . 2 2}$ \\
& $35.8 \%$ & $3.3 \%$ & $33.0 \%$ & $87.6 \%$ & $71.8 \%$ & $4.2 \%$ \\
Total of all sources & $\mathbf{7 3 . 4 3}$ & $\mathbf{1 3 . 1 3}$ & $\mathbf{1 7 . 7 5}$ & $\mathbf{2 0 . 8 7}$ & $\mathbf{6 . 2 6}$ & $\mathbf{5 . 1 7}$ \\
& $100.0 \%$ & $100.0 \%$ & $100.0 \%$ & $100.0 \%$ & $100.0 \%$ & $100.0 \%$ \\
\hline
\end{tabular}

Note: $\mathrm{CO}=$ Carbon monoxide. $\mathrm{NO}_{\mathrm{x}}=$ Nitrogen oxides. $\mathrm{VOC}=$ Volatile organic compounds. $\mathrm{PM}-10=$ Particulate matter less than 10 microns. PM-2.5 = Particulate matter less than 2.5 microns. $\mathrm{SO}_{2}=$ Sulfur dioxide.

\section{Source:}

U. S. Environmental Protection Agency, National Emission Inventory Air Pollutant Emission Trends website www.epa.gov/ttn/chief/trends. (Additional resources: www.epa.gov/oar/oaqps) 
The transportation sector accounted for almost 54\% of the nation's carbon monoxide (CO) emissions in 2013. Highway vehicles are by far the source of the greatest amount of CO. For details on the highway emissions of CO, see Table 12.3.

Table 12.2

Total National Emissions of Carbon Monoxide, 1970-2013 (million short tons)

\begin{tabular}{lrrrrrrr}
\hline & & & & & & & $\begin{array}{c}\text { Percent } \\
\text { of total, }\end{array}$ \\
Source category & 1970 & \multicolumn{1}{c}{1980} & \multicolumn{1}{c}{1990} & 2000 & 2010 & 2013 & \multicolumn{1}{c}{2013} \\
\hline Highway vehicles & 163.23 & 143.83 & 110.26 & 68.06 & 28.51 & 24.80 & $33.8 \%$ \\
$\quad$ Other off-highway & 11.37 & 16.69 & 21.45 & 24.18 & 15.47 & 14.65 & $19.9 \%$ \\
Transportation total & 174.60 & 160.52 & 131.71 & 92.24 & 43.98 & 39.45 & $53.7 \%$ \\
Stationary fuel combustion total & 4.63 & 7.30 & 5.51 & 4.78 & 4.55 & 4.63 & $6.3 \%$ \\
Industrial processes total & 9.84 & 6.95 & 4.77 & 2.63 & 1.90 & 1.98 & $2.7 \%$ \\
Waste disposal and recycling total & 7.06 & 2.30 & 1.08 & 1.85 & 1.20 & 1.11 & $1.5 \%$ \\
Miscellaneous total & 7.91 & 8.34 & 11.12 & 12.96 & 23.02 & 26.26 & $35.8 \%$ \\
\hline Total of all sources & $\mathbf{2 0 4 . 0 4}$ & $\mathbf{1 8 5 . 4 1}$ & $\mathbf{1 5 4 . 1 9}$ & $\mathbf{1 1 4 . 4 6}$ & $\mathbf{7 4 . 6 5}$ & $\mathbf{7 3 . 4 3}$ & $\mathbf{1 0 0 . 0 \%}$ \\
\hline
\end{tabular}

Source:

U. S. Environmental Protection Agency, National Emission Inventory Air Pollutant Emission Trends website www.epa.gov/ttn/chief/trends. (Additional resources: www.epa.gov/oar/oaqps)

${ }^{a}$ The sums of subcategories may not equal total due to rounding. 
Though gasoline-powered light vehicles continue to be responsible for the majority of carbon monoxide emissions from highway vehicles, the total pollution from light vehicles in 2005 is about a third of what it was in 1970. This is despite the fact that there were many more light vehicles on the road in 2005.

Table 12.3

Emissions of Carbon Monoxide from Highway Vehicles, 1970-2005 ${ }^{\mathrm{a}}$ (million short tons)

\begin{tabular}{|c|c|c|c|c|c|c|c|}
\hline Source category & 1970 & 1980 & 1990 & 1995 & 2000 & 2005 & $\begin{array}{c}\text { Percent of } \\
\text { total, } \\
2005 \\
\end{array}$ \\
\hline \multicolumn{8}{|c|}{ Gasoline powered } \\
\hline $\begin{array}{l}\text { Light vehicles \& } \\
\text { motorcycles }\end{array}$ & 119.14 & 98.21 & 67.24 & 46.54 & 36.40 & 24.19 & $50.2 \%$ \\
\hline Light trucks ${ }^{\mathrm{b}}$ & 22.27 & 28.83 & 32.23 & 29.81 & 27.04 & 21.19 & $43.9 \%$ \\
\hline Heavy vehicles & 21.27 & 15.35 & 8.92 & 5.96 & 3.42 & 1.97 & $4.1 \%$ \\
\hline Total & 162.68 & 142.39 & 108.39 & 82.31 & 66.86 & 47.35 & $98.2 \%$ \\
\hline \multicolumn{8}{|c|}{ Diesel powered } \\
\hline Light vehicles & 0.01 & 0.03 & 0.04 & 0.02 & 0.01 & 0.01 & $0.0 \%$ \\
\hline Light trucks ${ }^{b}$ & 0.06 & 0.05 & 0.03 & 0.02 & 0.01 & 0.01 & $0.0 \%$ \\
\hline Heavy vehicles & 0.49 & 1.36 & 1.81 & 1.53 & 1.19 & 0.85 & $1.8 \%$ \\
\hline Total & 0.56 & 1.43 & 1.87 & 1.57 & 1.20 & 0.87 & $1.8 \%$ \\
\hline \multicolumn{8}{|c|}{ Total } \\
\hline Highway vehicle total & 163.23 & 143.83 & 110.26 & 83.88 & 68.06 & 48.22 & $100.0 \%$ \\
\hline Percent diesel & $0.3 \%$ & $1.0 \%$ & $1.7 \%$ & $1.9 \%$ & $1.8 \%$ & $1.8 \%$ & \\
\hline
\end{tabular}

Note: Data beyond 2005 are not available.

Source:

U. S. Environmental Protection Agency, National Emission Inventory Air Pollutant Emission Trends website www.epa.gov/ttn/chief/trends. (Additional resources: www.epa.gov/oar/oaqps)

\footnotetext{
${ }^{a}$ The sums of subcategories may not equal total due to rounding.

${ }^{\mathrm{b}}$ Less than 8,500 pounds.
} 
The transportation sector accounted for almost $60 \%$ of the nation's nitrogen oxide (NOx) emissions in 2013, with the majority coming from highway vehicles. For details on the highway emissions of NOx, see Table 12.5.

Table 12.4

Total National Emissions of Nitrogen Oxides, 1970-2013 (million short tons)

\begin{tabular}{lrrrrrrr}
\hline & & & & & & & $\begin{array}{c}\text { Percent } \\
\text { of total, }\end{array}$ \\
Source category & 1970 & 1980 & 1990 & 2000 & 2010 & 2013 & 2013 \\
\hline Highway vehicles & 12.62 & 11.49 & 9.59 & 8.39 & 5.73 & 5.01 & $38.2 \%$ \\
$\quad$ Other off-highway & 2.65 & 3.35 & 3.78 & 4.17 & 3.36 & 2.73 & $20.8 \%$ \\
Transportation total & 15.27 & 14.84 & 13.37 & 12.56 & 9.09 & 7.74 & $59.0 \%$ \\
Stationary fuel combustion total & 10.06 & 11.32 & 10.89 & 8.82 & 4.36 & 3.69 & $28.1 \%$ \\
Industrial processes total & 0.78 & 0.56 & 0.80 & 0.81 & 1.12 & 1.18 & $9.0 \%$ \\
Waste disposal and recycling total & 0.44 & 0.11 & 0.09 & 0.13 & 0.09 & 0.08 & $0.6 \%$ \\
Miscellaneous total & 0.33 & 0.25 & 0.37 & 0.28 & 0.31 & 0.44 & $3.3 \%$ \\
\hline Total of all sources & $\mathbf{2 6 . 8 8}$ & $\mathbf{2 7 . 0 8}$ & $\mathbf{2 5 . 5 2}$ & $\mathbf{2 2 . 6 0}$ & $\mathbf{1 4 . 9 7}$ & $\mathbf{1 3 . 1 3}$ & $\mathbf{1 0 0 . 0 \%}$ \\
\hline
\end{tabular}

\section{Source:}

U. S. Environmental Protection Agency, National Emission Inventory Air Pollutant Emission Trends website www.epa.gov/ttn/chief/trends. (Additional resources: www.epa.gov/oar/oaqps)

\footnotetext{
${ }^{\mathrm{a}}$ The sums of subcategories may not equal total due to rounding.
} 
Heavy diesel-powered vehicles were responsible for nearly one-half (44.1\%) of highway vehicle nitrogen oxide emissions in 2005, while light gasoline vehicles were responsible for the rest.

Table 12.5

Emissions of Nitrogen Oxides from Highway Vehicles, 1970-2005 ${ }^{\mathrm{a}}$ (million short tons)

\begin{tabular}{|c|c|c|c|c|c|c|c|}
\hline Source category & 1970 & 1980 & 1990 & 1995 & 2000 & 2005 & $\begin{array}{l}\text { Percent } \\
\text { of total, } \\
2005\end{array}$ \\
\hline \multicolumn{8}{|c|}{ Gasoline powered } \\
\hline Light vehicles \& motorcycles & 8.54 & 6.63 & 4.26 & 3.05 & 2.31 & 1.63 & $25.5 \%$ \\
\hline Light trucks ${ }^{\mathrm{b}}$ & 1.54 & 1.58 & 1.50 & 1.46 & 1.44 & 1.56 & $24.4 \%$ \\
\hline Heavy vehicles & 0.72 & 0.62 & 0.57 & 0.52 & 0.45 & 0.38 & $5.9 \%$ \\
\hline Total & 10.81 & 8.83 & 6.33 & 5.03 & 4.20 & 3.57 & $55.9 \%$ \\
\hline \multicolumn{8}{|c|}{ Diesel powered } \\
\hline Light vehicles & 0.00 & 0.03 & 0.04 & 0.02 & 0.01 & 0.00 & $0.0 \%$ \\
\hline Light trucks ${ }^{\mathrm{b}}$ & 0.07 & 0.05 & 0.02 & 0.01 & 0.01 & 0.01 & $0.2 \%$ \\
\hline Heavy vehicles & 1.76 & 2.59 & 3.19 & 3.82 & 4.18 & 2.81 & $44.0 \%$ \\
\hline Total & 1.83 & 2.66 & 3.26 & 3.85 & 4.19 & 2.82 & $44.1 \%$ \\
\hline \multicolumn{8}{|c|}{ Total } \\
\hline Highway vehicle total & 12.64 & 11.49 & 9.59 & 8.88 & 8.39 & 6.39 & $100.0 \%$ \\
\hline Percent diesel & $14.5 \%$ & $23.1 \%$ & $34.0 \%$ & $43.4 \%$ & $49.9 \%$ & $44.1 \%$ & \\
\hline
\end{tabular}

Note: Data beyond 2005 are not available.

\section{Source:}

U. S. Environmental Protection Agency, National Emission Inventory Air Pollutant Emission Trends website www.epa.gov/ttn/chief/trends. (Additional resources: www.epa.gov/oar/oaqps)

\footnotetext{
a The sums of subcategories may not equal total due to rounding.

${ }^{\mathrm{b}}$ Less than 8,500 pounds.
} 
The transportation sector accounted for over $23 \%$ of the nation's volatile organic compound (VOC) emissions in 2013, with the majority coming from highway vehicles. For details on the highway emissions of VOC, see Table 12.7.

Table 12.6

Total National Emissions of Volatile Organic Compounds, 1970-2013 ${ }^{\text {a }}$ (million short tons)

\begin{tabular}{lrrrrrrr}
\hline & & & & & & & $\begin{array}{c}\text { Percent } \\
\text { of total, }\end{array}$ \\
Source category & 1970 & 1980 & 1990 & 2000 & 2010 & 2013 & 2013 \\
\hline Highway vehicles & 16.91 & 13.87 & 9.39 & 5.33 & 2.78 & 2.16 & $12.2 \%$ \\
$\quad$ Off-highway & 1.62 & 2.19 & 2.66 & 2.64 & 2.32 & 1.99 & $11.2 \%$ \\
Transportation total & 18.53 & 16.06 & 12.05 & 7.97 & 5.10 & 4.15 & $23.4 \%$ \\
Stationary fuel combustion total & 0.72 & 1.05 & 1.01 & 1.18 & 0.61 & 0.63 & $3.6 \%$ \\
Industrial processes total & 12.33 & 12.10 & 9.01 & 7.21 & 6.90 & 6.97 & $39.3 \%$ \\
Waste disposal and recycling total & 1.98 & 0.76 & 0.99 & 0.42 & 0.15 & 0.13 & $0.7 \%$ \\
Miscellaneous total & 1.10 & 1.13 & 1.06 & 0.73 & 5.16 & 5.87 & $33.0 \%$ \\
\hline Total of all sources & $\mathbf{3 4 . 6 6}$ & $\mathbf{3 1 . 1 0}$ & $\mathbf{2 4 . 1 2}$ & $\mathbf{1 7 . 5 1}$ & $\mathbf{1 7 . 9 2}$ & $\mathbf{1 7 . 7 5}$ & $\mathbf{1 0 0 . 0 \%}$ \\
\hline
\end{tabular}

Source:

U. S. Environmental Protection Agency, National Emission Inventory Air Pollutant Emission Trends website www.epa.gov/ttn/chief/trends. (Additional resources: www.epa.gov/oar/oaqps)

${ }^{a}$ The sum of subcategories may not equal total due to rounding. The EPA's definition of volatile organic compounds excludes methane, ethane, and certain other nonphotochemically reactive organic compounds. 
Gasoline-powered vehicles are responsible for over $95 \%$ of highway vehicle emissions of volatile organic compounds. VOC emissions from highway vehicles in 2005 were about one-quarter of the 1990 level.

Table 12.7

Emissions of Volatile Organic Compounds from Highway Vehicles, 1970-2005 (thousand short tons)

\begin{tabular}{|c|c|c|c|c|c|c|c|}
\hline Source category & 1970 & 1980 & 1990 & 1995 & 2000 & 2005 & $\begin{array}{l}\text { Percent of } \\
\text { total, } \\
2005\end{array}$ \\
\hline \multicolumn{8}{|c|}{ Gasoline powered } \\
\hline Light vehicles \& motorcycles & 11,996 & 9,304 & 5,690 & 3,768 & 2,903 & 2,111 & $51.8 \%$ \\
\hline Light trucks ${ }^{\mathrm{b}}$ & 2,776 & 2,864 & 2,617 & 2,225 & 1,929 & 1,629 & $39.9 \%$ \\
\hline Heavy vehicles & 1,679 & 1,198 & 633 & 421 & 256 & 171 & $4.2 \%$ \\
\hline Total & 16,451 & 13,366 & 8,940 & 6,414 & 5,088 & 3,911 & $95.9 \%$ \\
\hline \multicolumn{8}{|c|}{ Diesel powered } \\
\hline Light vehicles & 8 & 16 & 18 & 9 & 3 & 2 & $0.0 \%$ \\
\hline Light trucks ${ }^{b}$ & 41 & 28 & 15 & 10 & 4 & 6 & $0.1 \%$ \\
\hline Heavy vehicles & 411 & 459 & 415 & 315 & 230 & 159 & $3.9 \%$ \\
\hline Total & 460 & 503 & 448 & 335 & 238 & 167 & $4.1 \%$ \\
\hline \multicolumn{8}{|c|}{ Total } \\
\hline Highway vehicle total & 16,911 & 13,869 & $\mathbf{9 , 3 8 8}$ & 6,749 & 5,326 & 4,078 & $100.0 \%$ \\
\hline Percent diesel & $2.7 \%$ & $3.6 \%$ & $4.8 \%$ & $5.0 \%$ & $4.5 \%$ & $4.1 \%$ & \\
\hline
\end{tabular}

Note: Data beyond 2005 are not available.

\section{Source:}

U. S. Environmental Protection Agency, National Emission Inventory Air Pollutant Emission Trends website www.epa.gov/ttn/chief/trends. (Additional resources: www.epa.gov/oar/oaqps)

${ }^{a}$ The sums of subcategories may not equal total due to rounding.

${ }^{\mathrm{b}}$ Less than 8,500 pounds. 
The transportation sector accounted for more than $2 \%$ of the nation's particulate matter (PM-10) emissions in 2013. For details on the highway emissions of PM-10, see Table 12.9.

Table 12.8

Total National Emissions of Particulate Matter (PM-10), 1970-2013 (million short tons)

\begin{tabular}{|c|c|c|c|c|c|c|c|}
\hline Source category & 1970 & 1980 & 1990 & 2000 & 2010 & 2013 & $\begin{array}{l}\text { Percent of } \\
\text { total, } 2013\end{array}$ \\
\hline Highway vehicles & 0.48 & 0.43 & 0.39 & 0.23 & 0.28 & 0.27 & $1.3 \%$ \\
\hline Off-highway & 0.16 & 0.26 & 0.33 & 0.32 & 0.23 & 0.20 & $0.9 \%$ \\
\hline Transportation total & 0.64 & 0.69 & 0.72 & 0.55 & 0.51 & 0.47 & $2.2 \%$ \\
\hline Stationary fuel combustion total & 2.87 & 2.45 & 1.20 & 1.47 & 0.98 & 0.98 & $4.7 \%$ \\
\hline Industrial processes total & 7.67 & 2.75 & 1.04 & 0.71 & 1.05 & 0.94 & $4.5 \%$ \\
\hline Waste disposal and recycling total & 1.00 & 0.27 & 0.27 & 0.36 & 0.21 & 0.20 & $1.0 \%$ \\
\hline Miscellaneous total & 0.84 & 0.85 & 24.54 & 20.65 & 18.14 & 18.28 & $87.6 \%$ \\
\hline Total of all sources & 13.02 & 7.01 & 27.77 & 23.74 & 20.89 & 20.87 & $100.0 \%$ \\
\hline
\end{tabular}

Note: Because PM-10 is fine particle matter less than 10 microns, it also includes PM-2.5. Specific data for PM-2.5 are shown on Tables 12.10 and 12.11 .

\section{Source:}

U. S. Environmental Protection Agency, National Emission Inventory Air Pollutant Emission Trends website www.epa.gov/ttn/chief/trends. (Additional resources: www.epa.gov/oar/oaqps)

\footnotetext{
${ }^{\text {a }}$ Fine particle matter less than 10 microns. The sums of subcategories may not equal total due to rounding.
} 
Since the mid-1980's, diesel-powered vehicles have been responsible for more than half of highway vehicle emissions of particulate matter (PM-10). Heavy vehicles are clearly the main source.

Table 12.9

Emissions of Particulate Matter (PM-10) from Highway Vehicles, 1970-2005 ${ }^{\mathrm{a}}$ (thousand short tons)

\begin{tabular}{|c|c|c|c|c|c|c|c|}
\hline Source category & 1970 & 1980 & 1990 & 1995 & 2000 & 2005 & $\begin{array}{l}\text { Percent of total, } \\
2005\end{array}$ \\
\hline \multicolumn{8}{|c|}{ Gasoline powered } \\
\hline Light vehicles \& motorcycles & 249 & 141 & 56 & 53 & 51 & 46 & $25.1 \%$ \\
\hline Light trucks ${ }^{\mathrm{b}}$ & 74 & 49 & 31 & 32 & 31 & 35 & $19.1 \%$ \\
\hline Heavy vehicles & 44 & 30 & 17 & 13 & 10 & 8 & $4.4 \%$ \\
\hline Total & 367 & 220 & 104 & 98 & 92 & 89 & $48.6 \%$ \\
\hline \multicolumn{8}{|c|}{ Diesel powered } \\
\hline Light vehicles & 2 & 9 & 11 & 4 & 1 & 1 & $0.5 \%$ \\
\hline Light trucks ${ }^{\mathrm{b}}$ & 19 & 12 & 5 & 3 & 1 & 1 & $0.5 \%$ \\
\hline Heavy vehicles & 92 & 191 & 268 & 199 & 135 & 92 & $50.3 \%$ \\
\hline Total & 113 & 212 & 284 & 206 & 137 & 94 & $51.4 \%$ \\
\hline \multicolumn{8}{|c|}{ Total } \\
\hline Highway vehicle total & 480 & 432 & 387 & 304 & 230 & 183 & $100.0 \%$ \\
\hline Percent diesel & $23.5 \%$ & $49.1 \%$ & $73.4 \%$ & $67.8 \%$ & $59.6 \%$ & $51.4 \%$ & \\
\hline
\end{tabular}

Note: Because PM-10 is fine particle matter less than 10 microns, it also includes PM-2.5. Specific data for PM-2.5 are shown on Tables 12.10 and 12.11. Data beyond 2005 are not available.

Source:

U. S. Environmental Protection Agency, National Emission Inventory Air Pollutant Emission Trends website www.epa.gov/ttn/chief/trends. (Additional resources: www.epa.gov/oar/oaqps)

${ }^{a}$ The sums of subcategories may not equal total due to rounding.

${ }^{\mathrm{b}}$ Less than 8,500 pounds. 
The transportation sector accounted for almost 6\% of the nation's particulate matter (PM-2.5) emissions in 2013.

For details on the highway emissions of PM-2.5, see Table 12.11.

Table 12.10

Total National Emissions of Particulate Matter (PM-2.5), 1990-2013 (million short tons)

\begin{tabular}{lccccccc}
\hline & & & & & & & \multicolumn{2}{c}{$\begin{array}{c}\text { Percent } \\
\text { of total, }\end{array}$} \\
Source category & 1990 & 1995 & 2000 & 2005 & 2010 & 2013 & \multicolumn{1}{c}{2013} \\
\hline Highway vehicles & 0.32 & 0.25 & 0.17 & 0.31 & 0.20 & 0.19 & $3.0 \%$ \\
$\quad$ Off-highway & 0.30 & 0.31 & 0.30 & 0.29 & 0.21 & 0.18 & $2.9 \%$ \\
Transportation total & 0.62 & 0.56 & 0.47 & 0.60 & 0.41 & 0.37 & $5.9 \%$ \\
Stationary fuel combustion total & 0.91 & 0.90 & 1.29 & 1.13 & 0.83 & 0.83 & $13.3 \%$ \\
Industrial processes total & 0.56 & 0.50 & 0.50 & 0.53 & 0.42 & 0.39 & $6.3 \%$ \\
Waste disposal and recycling total & 0.23 & 0.25 & 0.33 & 0.27 & 0.18 & 0.17 & $2.7 \%$ \\
Miscellaneous total & 5.23 & 4.73 & 4.69 & 3.07 & 4.15 & 4.49 & $71.8 \%$ \\
\hline Total of all sources & $\mathbf{7 . 5 5}$ & $\mathbf{6 . 9 4}$ & $\mathbf{7 . 2 8}$ & $\mathbf{5 . 6 0}$ & $\mathbf{5 . 9 9}$ & $\mathbf{6 . 2 5}$ & $\mathbf{1 0 0 . 0 \%}$ \\
\hline
\end{tabular}

Source:

U. S. Environmental Protection Agency, National Emission Inventory Air Pollutant Emission Trends website www.epa.gov/ttn/chief/trends. (Additional resources: www.epa.gov/oar/oaqps) 
Diesel vehicles are responsible for the majority of highway vehicle PM-2.5 emissions. Nearly two-thirds of the highway vehicles' PM-2.5 emissions are from heavy diesel trucks.

Table 12.11

Emissions of Particulate Matter (PM-2.5) from Highway Vehicles, 1990-2005 ${ }^{\mathrm{a}}$ (thousand short tons)

\begin{tabular}{|c|c|c|c|c|c|}
\hline Source category & 1990 & 1995 & 2000 & 2005 & $\begin{array}{l}\text { Percent of } \\
\text { total, } 2005 \\
\end{array}$ \\
\hline \multicolumn{6}{|c|}{ Gasoline powered } \\
\hline Light vehicles \& motorcycles & 35 & 30 & 27 & 23 & $18.0 \%$ \\
\hline Light trucks ${ }^{\mathrm{b}}$ & 21 & 20 & 18 & 18 & $14.1 \%$ \\
\hline Heavy vehicles & 11 & 9 & 7 & 6 & $4.7 \%$ \\
\hline Total & 67 & 59 & 52 & 47 & $36.7 \%$ \\
\hline \multicolumn{6}{|c|}{ Diesel powered } \\
\hline Light vehicles & 9 & 4 & 1 & 1 & $0.8 \%$ \\
\hline Light trucks ${ }^{\mathrm{b}}$ & 4 & 2 & 1 & 1 & $0.8 \%$ \\
\hline Heavy vehicles & 243 & 179 & 119 & 79 & $61.7 \%$ \\
\hline Total & 256 & 185 & 121 & 81 & $63.3 \%$ \\
\hline \multicolumn{6}{|c|}{ Total } \\
\hline Highway vehicle total & 323 & 244 & 173 & 128 & $100.0 \%$ \\
\hline Percent diesel & $79.3 \%$ & $75.8 \%$ & $69.9 \%$ & $63.3 \%$ & \\
\hline
\end{tabular}

Note: Data beyond 2005 are not available.

\section{Source:}

U. S. Environmental Protection Agency, National Emission Inventory Air Pollutant Emission Trends website www.epa.gov/ttn/chief/trends. (Additional resources: www.epa.gov/oar/oaqps)

${ }^{\mathrm{a}}$ The sums of subcategories may not equal total due to rounding.

${ }^{\mathrm{b}}$ Less than 8,500 pounds. 


\section{EMISSION STANDARDS}

The U.S. Environmental Protection Agency (EPA) regulates emissions from mobile sources including vehicles, engines, and motorized equipment that produce exhaust and evaporative emissions. Mobile sources contribute to four main air pollutants: carbon monoxide, hydrocarbons, nitrogen oxides, and particulate matter. The EPA not only sets standards for the vehicles, engines, and equipment, but also the fuels that they use. Tables 12.15 through Table 12.29 contain summaries of the current standards.

\begin{tabular}{|ll|}
\hline Acronyms Used on Tables 12.15 through Table 12.29 \\
bhp & Brake horsepower-hour \\
CI & Compression-ignition \\
CO & Carbon Monoxide \\
DE & Diesel engine \\
g & Gram \\
g/kN & Grams per kilonewton \\
g/mi & Grams per mile \\
GVW & Gross vehicle weight \\
HC & Hydrocarbons \\
HCHO & Formaldehyde \\
HLDT & Heavy light-duty truck \\
Hp-hr & Horsepower-hour \\
kW & Kilowatt \\
kW-hr & Kilowatt-hour \\
LDT & Light-duty truck \\
LDV & Light-duty vehicle \\
LEV & Low-emission vehicle \\
LLDT & Light light-duty truck \\
LVW & Loaded vehicle weight \\
MDPV & Medium-duty passenger vehicle \\
& (8,500-10,000 lbs. GVWR) \\
NMHC & Non-methane hydrocarbon \\
NMOG & Non-methane organic gases \\
NOx & Nitrogen oxides \\
PM & Particulate matter \\
ppm & Parts per million \\
rPR & Rated pressure ratio \\
SI & Spark-ignition \\
SULEV & Super-ultra-low-emission vehicle \\
ULEV & Ultra-low-emission vehicle \\
ZEV & Zero-emission vehicle \\
\hline &
\end{tabular}


The Environmental Protection Agency issued final Tier 3 emission standards in 2014. The combined emissions of non-methane organic gases (NMOG) and nitrogen oxides (NOx) that new gasoline engines are allowed to produce from 2017 to 2025 are regulated in these new standards. These standards apply to a corporate average, meaning that some vehicles produced in those model years will emit more than the standard, while others will emit less, so long as the average for each Original Equipment Manufacturer (OEM) product offerings meets the standard.

Table 12.12

Tier 3 Non-Methane Organic Gases and Nitrogen Oxide Standards (milligrams per mile)

\begin{tabular}{ccccc}
\hline Light-duty & $\begin{array}{c}\text { LDT2, 3, 4, and } \\
\text { vehicles and } \\
\text { medium-duty } \\
\text { Model Year }\end{array}$ & passenger vehicles & Class 2b trucks & Class 3 trucks \\
\hline 2016 & $\mathrm{a}$ & 101 & $333^{\mathrm{b}}$ & $548^{\mathrm{b}}$ \\
2017 & 86 & 92 & $310^{\mathrm{b}}$ & $508^{\mathrm{b}}$ \\
2018 & 79 & 83 & 278 & 451 \\
2019 & 72 & 74 & 253 & 400 \\
2020 & 65 & 65 & 228 & 349 \\
2021 & 58 & 56 & 203 & 298 \\
2022 & 51 & 47 & 178 & 247 \\
2023 & 44 & 38 & 178 & 247 \\
2024 & 37 & 30 & 178 & 247 \\
2025 and later & 30 & & 178 & 247 \\
\hline
\end{tabular}

Notes: Standards are for the Federal Test Procedure. Different standards apply for the Supplemental Federal Test Procedure. For vehicles over 6,000 lbs. gross vehicle weight rating (GVWR), the standards apply beginning in MY 2018.

LDT1 = Light trucks less than 6,000 lbs. GVWR and less than 3,750 lbs. loaded vehicle weight (LVW).

LDT2, 3, 4 = Light trucks less than 8,500 lbs. GVWR and more than 3, $750 \mathrm{lbs} . \mathrm{LVW}$.

Class $2 \mathrm{~b}$ trucks $=$ trucks $8,501-10,000 \mathrm{lbs}$. GVWR.

Class 3 trucks $=$ trucks 10,001-14,000 lbs. GVWR.

\section{Source:}

Federal Register Vol. 79, No. 81, Monday, April 28, 2014.

\footnotetext{
${ }^{\mathrm{a}}$ Not applicable.

${ }^{\mathrm{b}}$ Voluntary standard.
} 
Table 12.13

Tier 3 Particulate Matter Emission Standards for Light Gasoline Vehicles, MY 2017 and Beyond (milligrams per mile)

\begin{tabular}{cccc}
\hline Model Year & $\begin{array}{c}\text { Certification standard } \\
\text { (milligrams per mile) }\end{array}$ & $\begin{array}{c}\text { In-use standard } \\
\text { (milligrams per mile) }\end{array}$ & $\begin{array}{c}\text { Phase-in (percent of } \\
\text { U.S. sales) }\end{array}$ \\
\hline 2017 & 3 & 6 & $20^{\mathrm{a}}$ \\
2018 & 3 & 6 & 20 \\
2019 & 3 & 6 & 40 \\
2020 & 3 & 6 & 70 \\
2021 & 3 & 6 & 100 \\
2022 -on & 3 & 3 & 100 \\
\hline
\end{tabular}

Note: Standards are for the Federal Test Procedure. The standards apply to all light-duty vehicles, light-duty trucks, and medium-duty passenger vehicles. For vehicles over 6,000 lbs. gross vehicle weight rating, the standards apply beginning in MY 2018.

Source:

Federal Register Vol. 79, No. 81, Monday, April 28, 2014.

Table 12.14

Tier 3 Evaporative Emission Standards (grams per test)

\begin{tabular}{lc}
\hline \multicolumn{1}{c}{ Vehicle class } & $\begin{array}{c}\text { Highest hot soak + diurnal level } \\
\text { (over both 2-day and 3-day diurnal tests) }\end{array}$ \\
\hline Light-duty vehicles and LDT1 & 0.3 \\
LDT2 & 0.4 \\
LDT3, LT4, and medium-duty passenger vehicles & 0.5 \\
Heavy-duty gasoline vehicles & 0.6 \\
\hline
\end{tabular}

Note: $\mathrm{LDT} 1=$ Light trucks less than 6,000 lbs. gross vehicle weight rating $(\mathrm{GVWR})$ and less than 3,750 lbs. loaded vehicle weight (LVW).

LDT2 $=$ Light trucks less than 6,000 lbs. GVWR and less than 3,750 lbs. LVW.

LDT3, $4=$ Light trucks less than 8,500 lbs. GVWR and more than 3,750 lbs. LVW.

Heavy-duty gasoline vehicles $=$ trucks over 10,000 lbs. GVWR.

\section{Source:}

Federal Register Vol. 79, No. 81, Monday, April 28, 2014.

${ }^{a}$ Manufacturers comply with $20 \%$ of their light-duty truck fleet under 6,000 lbs. gross vehicle weight, alternatively with $10 \%$ of their total light-duty vehicle, light-duty trucks and medium-duty passenger vehicle fleet. 
Table 12.15

Light-Duty Vehicle, Light-Duty Truck, and Medium-Duty Passenger Vehicle - Tier 2 Exhaust Emission Standards

\begin{tabular}{|c|c|c|c|c|c|c|c|c|c|c|c|}
\hline & \multirow{2}{*}{ Standard } & \multicolumn{5}{|c|}{ Emission limits at 50,000 miles } & \multicolumn{5}{|c|}{$\begin{array}{l}\text { Emission limits at full useful life } \\
\qquad(120,000 \text { miles })^{\mathrm{a}}\end{array}$} \\
\hline & & $\begin{array}{l}\text { NOx } \\
(g / m i)\end{array}$ & $\begin{array}{c}\text { NMOG } \\
(\mathrm{g} / \mathrm{mi})\end{array}$ & $\begin{array}{c}\mathrm{CO} \\
(\mathrm{g} / \mathrm{mi})\end{array}$ & $\begin{array}{c}\mathrm{PM} \\
(\mathrm{g} / \mathrm{mi})\end{array}$ & $\begin{array}{r}\mathrm{HCHO} \\
(\mathrm{g} / \mathrm{mi})\end{array}$ & $\begin{array}{l}\text { NOx } \\
(\mathrm{g} / \mathrm{mi})\end{array}$ & $\begin{array}{c}\mathrm{NMOG} \\
(\mathrm{g} / \mathrm{mi})\end{array}$ & $\begin{array}{c}\mathrm{CO} \\
(\mathrm{g} / \mathrm{mi})\end{array}$ & $\begin{array}{c}\mathrm{PM} \\
(\mathrm{g} / \mathrm{mi})\end{array}$ & $\begin{array}{r}\mathrm{HCHO} \\
(\mathrm{g} / \mathrm{mi})\end{array}$ \\
\hline \multirow{11}{*}{ Federal } & Bin 1 & - & - & - & - & - & 0 & 0 & 0 & 0 & 0 \\
\hline & Bin 2 & - & - & - & - & - & 0.02 & 0.01 & 2.1 & 0.01 & 0.004 \\
\hline & Bin 3 & - & - & - & - & - & 0.03 & 0.055 & 2.1 & 0.01 & 0.011 \\
\hline & Bin 4 & - & - & - & - & - & 0.04 & 0.07 & 2.1 & 0.01 & 0.011 \\
\hline & Bin 5 & 0.05 & 0.075 & 3.4 & - & 0.015 & 0.07 & 0.09 & 4.2 & 0.01 & 0.018 \\
\hline & Bin 6 & 0.08 & 0.075 & 3.4 & - & 0.015 & 0.1 & 0.09 & 4.2 & 0.01 & 0.018 \\
\hline & Bin 7 & 0.11 & 0.075 & 3.4 & - & 0.015 & 0.15 & 0.09 & 4.2 & 0.02 & 0.018 \\
\hline & Bin 8 & 0.14 & $\begin{array}{l}0.100 / \\
0.125^{\mathrm{c}}\end{array}$ & 3.4 & - & 0.015 & 0.2 & $\begin{array}{c}0.125 / \\
0.156\end{array}$ & 4.2 & 0.02 & 0.018 \\
\hline & $\operatorname{Bin} 9^{b}$ & 0.2 & $\begin{array}{c}0.075 / \\
0.140\end{array}$ & 3.4 & - & 0.015 & 0.3 & $\begin{array}{c}0.090 / \\
0.180\end{array}$ & 4.2 & 0.06 & 0.018 \\
\hline & $\operatorname{Bin} 10^{\mathrm{b}}$ & 0.4 & $\begin{array}{c}0.125 / \\
0.160\end{array}$ & $\begin{array}{l}3.4 / \\
4.4\end{array}$ & - & $\begin{array}{c}0.015 / \\
0.018\end{array}$ & 0.6 & $\begin{array}{c}0.156 / \\
0.230\end{array}$ & $\begin{array}{c}4.2 / \\
6.4\end{array}$ & 0.08 & $\begin{array}{c}0.018 / \\
0.027\end{array}$ \\
\hline & Bin $11^{b}$ & 0.6 & 0.195 & 5 & - & 0.022 & 0.9 & 0.28 & 7.3 & 0.12 & 0.032 \\
\hline
\end{tabular}

Note: Tests Covered: Federal Test Procedure (FTP), cold carbon monoxide, highway, and idle. Definitions of acronyms are on page 12-13.

Source:

40 CR 86 Subpart S. (Additional resources: www.epa.gov/otaq/standards)

a In lieu of intermediate useful life standards (50,000 miles) or to gain additional nitrogen oxides credit, manufacturers may optionally certify to the Tier 2 exhaust emission standards with a useful life of 150,000 miles.

${ }^{\mathrm{b}}$ Bins 9-11 expired in 2006 for light-duty vehicles and light light-duty trucks and 2008 for heavy light-duty trucks and medium-duty passenger vehicles.

${ }^{\mathrm{c}}$ Pollutants with two numbers have a separate certification standard (1st number) and in-use standard (2nd number). 
Table 12.16

Light-Duty Vehicle, Light-Duty Truck, and Medium-Duty Passenger Vehicle - Tier 2 Evaporative Emission Standards

\begin{tabular}{|c|c|c|c|c|c|}
\hline & Vehicle type & $\begin{array}{c}\text { Model } \\
\text { year }\end{array}$ & $\begin{array}{l}3 \text { Day diurnal } \\
+ \text { hot soak } \\
\text { (g/test) }\end{array}$ & $\begin{array}{c}\text { Supplemental } \\
2 \text { day diurnal } \\
+ \text { hot soak } \\
\text { (g/test) }\end{array}$ & $\begin{array}{c}\text { Running } \\
\text { loss } \\
(\mathrm{g} / \mathrm{mi})\end{array}$ \\
\hline \multirow{7}{*}{ Federal } & $\mathrm{LDV}^{\mathrm{LLDT}} \mathrm{s}^{\mathrm{a}}$ & 2004 & 0.95 & 1.20 & 0.05 \\
\hline & $\mathrm{HLDTs}^{\mathrm{b}}$ & 2004 & 1.20 & 1.50 & 0.05 \\
\hline & $\mathrm{MDPV}^{\mathrm{a}, \mathrm{b}}$ & 2004 & 1.40 & 1.75 & 0.05 \\
\hline & $\mathrm{LDV}^{\mathrm{a}}$ & 2009 & 0.50 & 0.65 & 0.05 \\
\hline & LLDT $^{\mathrm{a}}$ & 2009 & 0.65 & 0.85 & 0.05 \\
\hline & HLDT $^{\mathrm{b}}$ & 2010 & 0.90 & 1.15 & 0.05 \\
\hline & $\mathrm{MDPV}^{\mathrm{a}, \mathrm{b}}$ & 2010 & 1.00 & 1.25 & 0.05 \\
\hline
\end{tabular}

Note: Multi-fuel vehicle phase-in applies. Definitions of acronyms are on page 12-13.

Source:

40 CR 86 Subpart S. (Additional resources: www.epa.gov/otaq/standards)

${ }^{a}$ For liquefied petroleum gas-fueled light-duty vehicles (LDV), light-duty trucks (LDT), and medium-duty passenger vehicles (MDPV): 0.15 grams hydrocarbon per gallon (0.04 grams per liter) of fuel dispensed.

${ }^{\mathrm{b}}$ Refueling standards for heavy light-duty trucks (HLDT) are subject to phase-in requirements. MDPVs must also comply with the phase-in requirement and must be grouped with HLDTs to determine phase-in compliance. 
Table 12.17

Heavy-Duty Highway Compression-Ignition Engines and Urban Buses - Exhaust Emission Standards

\begin{tabular}{|c|c|c|c|c|c|c|c|c|c|c|}
\hline & Year & $\begin{array}{c}\mathrm{HC} \\
(\mathrm{g} / \mathrm{bhp}- \\
\mathrm{hr})\end{array}$ & $\begin{array}{c}\mathrm{NMHC} \\
(\mathrm{g} / \mathrm{bhp}- \\
\mathrm{hr})\end{array}$ & $\begin{array}{c}\mathrm{NMHC}+\mathrm{NOx} \\
(\mathrm{g} / \mathrm{bhp}- \\
\mathrm{hr})\end{array}$ & $\begin{array}{c}\text { NOx } \\
(\mathrm{g} / \mathrm{bhp}- \\
\mathrm{hr})\end{array}$ & $\begin{array}{c}\text { PM } \\
\text { (g/bhp- } \\
\text { hr) }\end{array}$ & $\begin{array}{c}\mathrm{CO} \\
(\mathrm{g} / \mathrm{bhp}- \\
\mathrm{hr})\end{array}$ & $\begin{array}{l}\text { Idle CO } \\
\text { (percent } \\
\text { Exhaust } \\
\text { gas flow) }\end{array}$ & $\begin{array}{c}\text { Smoke }^{\mathrm{a}} \\
\text { (percentage) }\end{array}$ & $\begin{array}{c}\text { Useful life } \\
\text { (hours/years/miles) }\end{array}$ \\
\hline \multirow{9}{*}{ Federal $^{\mathrm{b}}$} & $1974-78$ & - & - & 16 & - & - & 40 & - & $20 / 15 / 50$ & - \\
\hline & $1979-84$ & 1.5 & - & 10 & - & - & 25 & - & $20 / 15 / 50$ & - \\
\hline & $1985-87$ & 1.3 & - & - & 10.7 & - & 15.5 & - & $20 / 15 / 50$ & $\begin{array}{l}\text { LHDDE: - / } 8 \text { / 110,000 } \\
\text { MHDDE: - / } 8 \text { / 185,000 } \\
\text { HHDDE: - / } 8 \text { / 290,000 }\end{array}$ \\
\hline & 1988-89 & $1.3^{\mathrm{d}}$ & - & - & 10.7 & 0.6 & 15.5 & $0.5^{c}$ & $20 / 15 / 50$ & \multirow{5}{*}{$\begin{array}{c}\text { 1990-97 and 1998+ for } \\
\text { HC, CO, and PM: } \\
\text { LHDDE: - / } 8 \text { / 110,000 } \\
\text { MHDDE: - / } 8 \text { / 185,000 } \\
\text { HHDDE: - / } 8 \text { / 290,000 } \\
\text { 1994+ urban buses for PM } \\
\text { only: } \\
\text { LHDDE: - / } 10 \text { / 110,000 } \\
\text { 1998+ for NOx: } \\
\text { LHDDE: - / } 10 \text { / 110,000 } \\
\text { MHDDE: - / } 10 \text { / 185,000 } \\
\text { HHDDE: - / } 10 \text { / 290,000 }\end{array}$} \\
\hline & 1990 & $1.3^{\mathrm{d}}$ & - & - & 6.0 & 0.6 & 15.5 & $0.5^{\circ}$ & $20 / 15 / 50$ & \\
\hline & 1991-93 & 1.3 & - & - & $5.0[\mathrm{ABT}]$ & $\begin{array}{c}0.25[\mathrm{ABT}] \\
0.10^{\mathrm{e}}\end{array}$ & 15.5 & $0.5^{\mathrm{C}}$ & $20 / 15 / 50$ & \\
\hline & $1994-97$ & 1.3 & - & - & $5.0[\mathrm{ABT}]$ & $\begin{array}{c}0.1[\mathrm{ABT}] \\
0.07^{f}, 0.05^{\mathrm{g}}\end{array}$ & 15.5 & $0.5^{\mathrm{c}}$ & $20 / 15 / 50$ & \\
\hline & $1998-2003$ & 1.3 & - & - & $4.0[\mathrm{ABT}]$ & $\begin{array}{c}0.1[\mathrm{ABT}] \\
0.05^{\mathrm{g}}\end{array}$ & 15.5 & $0.5^{\mathrm{c}}$ & $20 / 15 / 50$ & \\
\hline & $\begin{array}{l}2004-2006^{h} \\
2007+h, k, l, m, n\end{array}$ & - & $0.14^{\circ}$ & $\begin{array}{c}2.4 \text { (or } 2.5 \\
\text { with a limit of } \\
0.5 \text { on } \\
\mathrm{NMHC}^{\circ} \\
{\left[^{\circ} \mathrm{ABT}^{\mathrm{i}, j}\right]} \\
\\
2.4 \text { (or } 2.5 \\
\text { with a limit of } \\
0.5 \text { on NMHC) } \\
{[\mathrm{ABT}]}\end{array}$ & $0.2^{\circ}$ & $\begin{array}{c}0.1 \\
0.05^{\mathrm{g}}\end{array}$ & 15.5 & 0.5 & $20 / 15 / 50$ & $\begin{array}{c}\text { For all pollutants: } \\
\text { LHDDE: - / 10 / 110,000 } \\
\text { MHDDE: - / 10 / 185,000 } \\
\text { HHDDE: } 22,000 \text { / } 10 / \\
435,000\end{array}$ \\
\hline
\end{tabular}

Note: The test procedures are the EPA Transient Test Procedure and the EPA Smoke Test Procedure. Definitions of acronyms are on page 12-13.

\section{Sources:}

40 CFR 86.099-11 Emission standards for 1999 and later model year diesel heavy-duty engines and vehicles.

40 CFR 86.004-11 Emission standards for 2004 and later model year diesel heavy-duty engines and vehicles.

40 CFR 86.007-11 Emission standards and supplemental requirements for 2007 and later model year diesel heavyduty engines and vehicles. (Additional resources: www.epa.gov/otaq/standards)

${ }^{a}$ Percentages apply to smoke opacity at acceleration/lug/peak modes.

${ }^{\mathrm{b}}$ Standards for 1990 apply only to diesel-fueled heavy-duty engines (HDE). Standards for 1991+ apply to both diesel- and methanol-fueled HDEs. Standards that apply to urban buses specifically are footnoted.

${ }^{c}$ This standard applies to the following fueled engines for the following model years: methanol - 1990+, natural gas and liquefied petroleum gas (LPG) - 1994+.

${ }^{d}$ For petroleum-fueled engines, the standard is for hydrocarbons (HC). For methanol-fueled engines, the standard is for total hydrocarbon equivalent (THCE).

${ }^{\mathrm{e}}$ Certification standard for urban buses for 1993 .

${ }^{\mathrm{f}}$ Certification standard for urban buses from 1994-95.

${ }^{\mathrm{g}}$ Certification standard for urban buses from 1996 and later. The in-use standard is 0.07 .

${ }^{\mathrm{h}}$ Load Response Test certification data submittal requirements take effect for heavy-duty diesel engines beginning in model year 2004. The following requirements take effect with the 2007 model year: steady-state test requirement and Not-to-Exceed (NTE) test procedures for testing of in-use engines. On-board diagnostic requirements applicable to heavy-duty diesel vehicles and engines up to 14,000 pounds gross vehicle weight rating (GVWR) phase in from the 2005 through 2007 model years. 


\section{Table 12.17 (continued) \\ Heavy-Duty Highway Compression-Ignition Engines and Urban Buses - Exhaust Emission Standards}

${ }^{\mathrm{i}}$ The modified averaging, banking, and trading program for 1998 and later model year engines applies only to diesel cycle engines. Credits generated under the modified program may be used only in 2004 and later model years.

${ }^{\mathrm{j}}$ For heavy-duty diesel engines, there are three options to the measurement procedures currently in place for alternative fueled engines: (1) use a THC measurement in place of an non-methane hydrocarbon (NMHC) measurement; (2) use a measurement procedure specified by the manufacturer with prior approval of the Administrator; or (3) subtract two percent from the measured THC value to obtain an NMHC value. The methodology must be specified at time of certification and will remain the same for the engine family throughout the engines' useful life. For natural gas vehicles, EPA allows the option of measuring NMHC through direct quantification of individual species by gas chromatography.

${ }^{\mathrm{k}}$ Starting in 2006, refiners must begin producing highway diesel fuel that meets a maximum sulfur standard of 15 parts per million (ppm).

${ }^{1}$ Subject to a Supplemental Emission Test (1.0 x Federal Test Procedure [FTP] standard (or Family Emission Limit [FEL]) for nitrogen oxides [NOx], NMHC, and particulate matter [PM]) and a NTE test (1.5 x FTP standard [or FEL] for NOx, NMHC, and PM).

m EPA adopted the lab-testing and field-testing specifications in 40 CFR Part 1065 for heavy-duty highway engines, including both diesel and Otto-cycle engines. These procedures replace those previously published in 40 Code of Federal Regulations (CFR) Part 86, Subpart N. Any new testing for 2010 and later model years must be done using the 40 CFR Part 1065 procedures.

${ }^{n}$ Two-phase in-use NTE testing program for heavy-duty diesel vehicles. The program begins with the 2007 model year for gaseous pollutants and 2008 for PM. The requirements apply to diesel engines certified for use in heavy-duty vehicles (including buses) with GVWRs greater than 8,500 pounds. However, the requirements do not apply to any heavy-duty diesel vehicle that was certified using a chassis dynamometer, including medium-duty passenger vehicles with GVWRs of between 8,500 and 10,000 pounds.

${ }^{\circ}$ NOx and NMHC standards will be phased in together between 2007 and 2010. The phase-in will be on a percent-of-sales basis: 50 percent from 2007 to 2009 and 100 percent in 2010.

$\mathrm{p}$ Note that for an individual engine, if the useful life hours interval is reached before the engine reaches 10 years or 100,000 miles, the useful life shall become 10 years or 100,000 miles, whichever occurs first, as required under Clean Air Act section 202(d). 
Table 12.18

Heavy-Duty Highway Spark-Ignition Engines - Exhaust Emission Standards

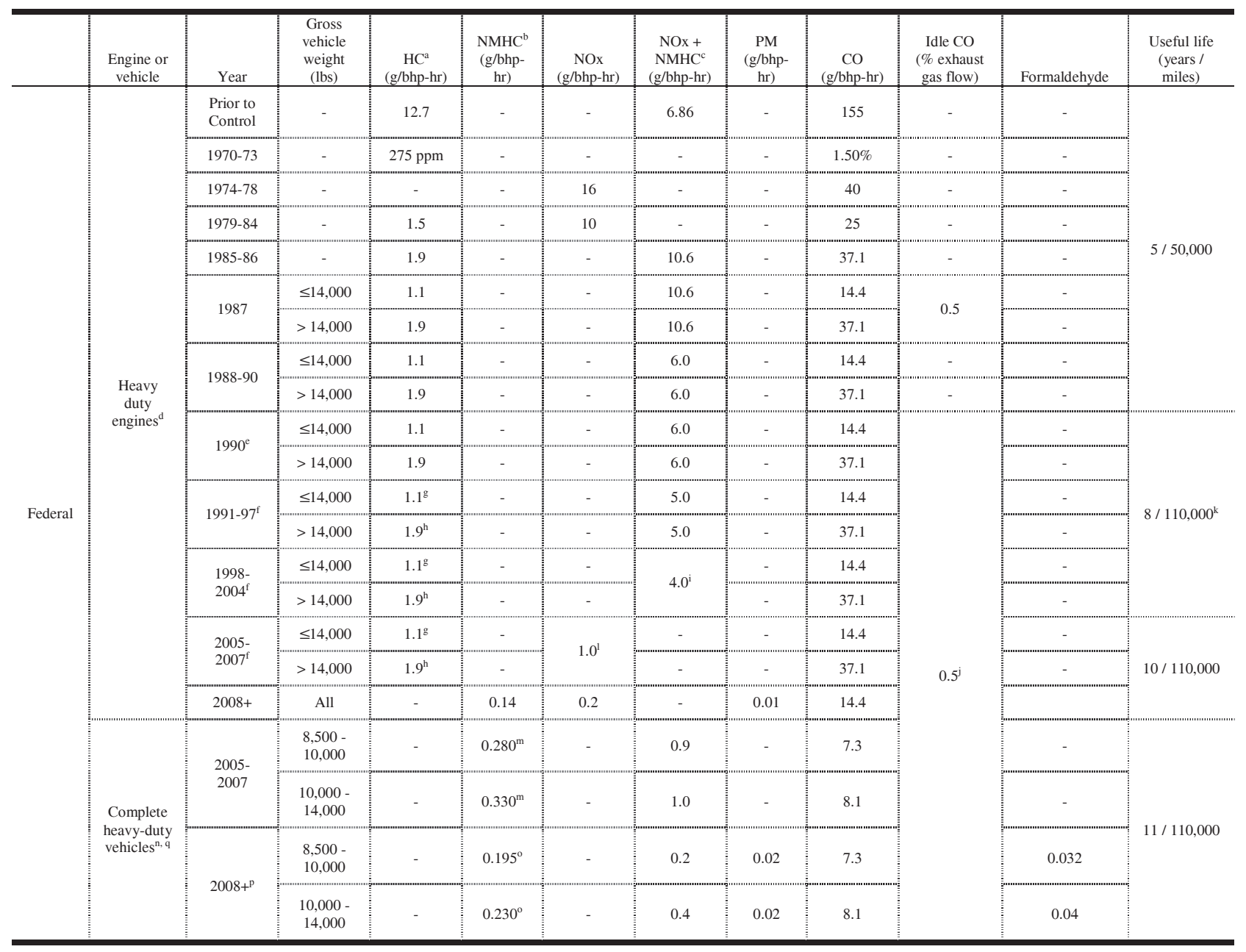

Note: Definitions of acronyms are on page 12-13.

\section{Sources:}

40 CFR 86.1816-05, 86.1816-08 Emission standards for complete heavy-duty vehicles

40 CFR 86.1806-01, 86.1806-04, 86.1806-05 Onboard diagnostics requirements

40 CFR 86.1817-05, 86.1817-08 Complete heavy-duty vehicle averaging, banking, and trading program

40 CFR 86.091-10 Heavy-duty engine averaging, banking, and trading program for 1991 and later - Not available in the e-CFR

40 CFR Part 86 Subpart B Vehicle test procedures (Additional resources: www.epa.gov/otaq/standards)

${ }^{\text {a }}$ For methanol-fueled engines, the standard is for total hydrocarbon equivalent (THCE).

${ }^{b}$ For methanol and alcohol fueled vehicles the standard is for non-methane hydrocarbon equivalent (NMHCE).

${ }^{\mathrm{c}}$ For methanol fueled engines the standard is for nitrogen oxides (NOx) plus NMHCE.

${ }^{\mathrm{d}}$ Standards for heavy-duty engines are expressed in grams per brake horsepower-hour (g/bhp-hr). Starting with the 1998 model year, crankcase emissions are not allowed.

${ }^{\mathrm{e}}$ Standards for 1990 apply to gasoline and methanol-fueled engines.

${ }^{\mathrm{f}}$ Standards for 1991 and later apply to gasoline and methanol engines and are optional for natural gas and Liquefied Petroleum Gas-fueled engines through the 1996 model year.

${ }^{\mathrm{g}}$ For natural gas fueled engines the standard is $0.9 \mathrm{~g} / \mathrm{bhp}-\mathrm{hr}$ non-methane hydrocarbon (NMHC). 
Table 12.18 (continued)

Heavy-Duty Highway Spark-Ignition Engines - Exhaust Emission Standards

${ }^{\mathrm{h}}$ For natural gas fueled engines the standard is $1.7 \mathrm{~g} / \mathrm{bhp}-\mathrm{hr}$ NMHC.

i The NOx standard is 5.0 for all natural gas-fueled engines.

${ }^{\mathrm{j}}$ This standard applies to the following engines utilizing aftertreatment technology (except for methanol) for the following model years: gasoline/1990+; natural gas and LPG/1991+; methanol/1990+. Starting in 2005, engines certified to on-board diagnostics requirements are not required to meet the idle carbon monoxide (CO) standard.

${ }^{\mathrm{k}}$ Useful life is expressed in years or miles, whichever comes first. Useful life for the 1998 and later NOx standard and for all 2004 standards is 10 years or 110,000 miles, whichever comes first.

${ }^{1}$ Manufacturers can choose this standard or one of the following options: (1) a standard of $1.5 \mathrm{~g} / \mathrm{bhp}-\mathrm{hr}$ NMHC+NOX that applies to the 2004 through 2007 model years, with complete heavy-duty vehicle standards taking effect in 2005; or (2) a standard of $1.5 \mathrm{~g} / \mathrm{bhp}-\mathrm{hr}$ NMHC + NOX that would apply to the 2003 through 2007 heavy-duty engines and optionally to 2003 through 2006 complete heavy-duty vehicles.

$\mathrm{m}$ Standard is expressed as non-methane organic gas, but compliance can optionally be shown using measurement of NMHC or total hydrocarbon (THC).

${ }^{\mathrm{n}}$ Complete heavy-duty vehicles have the primary load-carrying container or device attached. Incomplete heavy-duty vehicles are certified to heavy-duty engine standards. Standards for complete heavy-duty vehicles are expressed in grams per mile (g/mi). Starting in 2005 (or 2003 or 2004 depending on the selected phase in option; see footnote 1), complete heavy-duty vehicles under 14,000 lbs gross vehicle weight are tested on chassis-based rather than engine-based procedures and must meet these complete heavy-duty vehicle standards.

${ }^{\circ}$ Although expressed as NMHC, compliance can optionally be shown using measurement of NMOG or THC.

${ }^{\mathrm{p}}$ At least 50 percent of a manufacturer's sales must meet these standards in 2008, with 100 percent required in 2009.

${ }^{\mathrm{q}}$ Gross vehicle weight ranges are more accurately specified as follows: 8,500 $\leq \mathrm{GVW} \leq 10,000$ and 10,000< GVW $<14,000$. 
Table 12.19

Heavy-Duty Highway Compression-Ignition and Spark-Ignition Engines - Evaporative Emission Standards

\begin{tabular}{|c|c|c|c|c|c|c|c|c|c|}
\hline & $\begin{array}{c}\text { Engine } \\
\text { type }\end{array}$ & Year & $\begin{array}{c}\text { Gross vehicle } \\
\text { weight (lbs) }\end{array}$ & $\begin{array}{l}\text { Conventional } \\
\text { diurnal + hot } \\
\text { soak }(\mathrm{g} / \mathrm{test})^{\mathrm{a}}\end{array}$ & $\begin{array}{c}\text { Three-diurnal } \\
\text { test sequence } \\
\left(\mathrm{g} / \text { test }^{\mathrm{b}}\right.\end{array}$ & $\begin{array}{c}\text { Supplemental } \\
\text { two-diurnal } \\
\text { test sequence } \\
(\mathrm{g} / \text { test })^{\mathrm{c}} \\
\end{array}$ & $\begin{array}{c}\text { Running } \\
\text { loss } \\
(\mathrm{gpm})^{\mathrm{c}}\end{array}$ & $\begin{array}{l}\text { Spitback } \\
\left(\mathrm{g} / \text { test }^{\mathrm{c}}\right.\end{array}$ & Useful life $^{\mathrm{d}}$ \\
\hline \multirow{10}{*}{ Federal } & \multirow{6}{*}{ SI } & \multirow{2}{*}{$1991-95$} & $\leq 14,000$ & 3.0 & - & - & - & - & \multirow{2}{*}{$8 / 110,000$} \\
\hline & & & $>14,000^{\mathrm{e}}$ & 4.0 & - & - & - & - & \\
\hline & & \multirow{2}{*}{$\begin{array}{c}\text { 1996-2007 } \\
(\text { Enhanced })^{\mathrm{f}}\end{array}$} & $\leq 14,000$ & - & 3.0 & 3.5 & \multirow{4}{*}{0.05} & 1.0 & \multirow{2}{*}{$10 / 120,000$} \\
\hline & & & $>14,000^{\mathrm{e}}$ & - & 4.0 & 4.5 & & - & \\
\hline & & \multirow{2}{*}{$\begin{array}{c}2008+ \\
\text { (Enhanced) }\end{array}$} & $8500-14,000$ & - & 1.4 & 1.75 & & 1.0 & \multirow{2}{*}{$11 / 110,000$} \\
\hline & & & $>14,000^{\mathrm{e}}$ & - & 1.9 & 2.3 & & - & \\
\hline & \multirow{4}{*}{ CI } & \multirow{2}{*}{ 1996-97 } & $\leq 14,000$ & - & 3.0 & - & - & - & \multirow{4}{*}{$\begin{array}{l}\text { MHDDE: } 8 \text { / 185,000 } \\
\text { HHDDE: } 8 \text { / 290,000 } \\
\text { MHDDE: } 8 \text { / 185,000 } \\
\text { HHDDE: } 8 \text { / 290,000 }\end{array}$} \\
\hline & & & $>14,000^{\mathrm{e}}$ & - & 4.0 & - & - & - & \\
\hline & & \multirow{2}{*}{$\begin{array}{c}1998+ \\
\left(_{\text {Enhanced })^{\mathrm{g}}}\right.\end{array}$} & $\leq 14,000$ & - & 3.0 & 3.5 & \multirow{2}{*}{0.05} & 1.0 & \\
\hline & & & $>14,000^{\mathrm{e}}$ & - & 4.0 & 4.5 & & - & \\
\hline
\end{tabular}

Note: Definitions of acronyms are on page 12-13.

\section{Sources:}

40 CFR 86.099-11 Emission standards for 1999 and later model year diesel heavy-duty engines and vehicles.

40 CFR 86.004-11 Emission standards for 2004 and later model year diesel heavy-duty engines and vehicles.

CFR 86.007-11 Emission standards and supplemental requirements for 2007 and later model year diesel heavy-duty engines and vehicles. (Additional resources: www.epa.gov/otaq/standards)

${ }^{a}$ Applies to gasoline and methanol engines. Standard is hydrocarbon (HC) for gasoline engines, total hydrocarbon equivalent (THCE) for methanol engines.

${ }^{\mathrm{b}}$ For spark-ignition (SI) engines, standard applies to gasoline, methanol, natural gas, and liquefied petroleum gas engines. For compression-ignition (CI) engines, standard applies to methanol, natural gas, and liquefied petroleum gas engines. Standard is THCE for methanol engines, HC for others.

${ }^{c}$ For SI engines, standard applies to gasoline and methanol engines. For CI engines, standard applies to methanol engines. Standard is THCE for methanol engines, HC for others.

${ }^{\mathrm{d}}$ Useful life is expressed in years or miles, whichever comes first.

${ }^{\mathrm{e}}$ Vehicles over 26,000 pounds gross vehicle weight may demonstrate compliance with an engineering design evaluation in lieu of testing.

${ }^{\mathrm{f}}$ A new enhanced evaporative test procedure applies, which is considerably more stringent than the previous test procedure despite the fact that the standard values do not change from prior years. Gasoline and methanol engines are phased in at the following rates of a manufacturer's sales for the specified model year: 1996: 20 percent; 1997: 40 percent; 1998: 90 percent; 1999: 100 percent.

${ }^{g}$ A new enhanced evaporative test procedure applies, which is considerably more stringent than the previous test procedure despite the fact that the standard values do not change from prior years. Methanol-fueled vehicles are phased in at a rate of 90 percent of a manufacturer's production in 1998 and 100 percent in 1999. 
Table 12.20

California Car, Light Truck and Medium Truck Emission Certification Standards

\begin{tabular}{|c|c|c|c|c|c|}
\hline Vehicle type & $\begin{array}{l}\text { Vehicle } \\
\text { emission } \\
\text { category }\end{array}$ & $\begin{array}{l}\text { Non-methane } \\
\text { organic gases } \\
+ \text { nitrogen } \\
\text { oxides }(\mathrm{g} / \mathrm{mi}) \\
\end{array}$ & $\begin{array}{c}\text { Carbon } \\
\text { monoxide } \\
(\mathrm{g} / \mathrm{mi}) \\
\end{array}$ & $\begin{array}{l}\text { Formaldehyde } \\
(\mathrm{mg} / \mathrm{mi})\end{array}$ & $\begin{array}{c}\text { Particulates } \\
(\mathrm{g} / \mathrm{mi})\end{array}$ \\
\hline \multirow{3}{*}{$\begin{array}{l}\text { All passenger cars; } \\
\text { LDTs } 8,500 \mathrm{lbs} . \mathrm{GVW} \text { or } \\
\text { less }\end{array}$} & LEV160 & 0.16 & 4.2 & 4 & 0.01 \\
\hline & ULEV125 & 0.125 & 2.1 & 4 & 0.01 \\
\hline & ULEV70 & 0.07 & 1.7 & 4 & 0.01 \\
\hline \multirow[t]{2}{*}{ All MDPVs } & ULEV50 & 0.05 & 1.7 & 4 & 0.01 \\
\hline & SULEV30 & 0.03 & 1.0 & 4 & 0.01 \\
\hline $\begin{array}{l}\text { Vehicles in this category } \\
\text { are tested at their loaded } \\
\text { vehicle weight }\end{array}$ & SULEV20 & 0.02 & 1.0 & 4 & 0.01 \\
\hline \multirow{2}{*}{$\begin{array}{l}\text { MDVs } \\
8,501-10,000 \mathrm{lbs} \text { GVW }\end{array}$} & LEV395 & 0.395 & 6.4 & 6 & 0.12 \\
\hline & ULEV340 & 0.34 & 6.4 & 6 & 0.06 \\
\hline \multirow{4}{*}{$\begin{array}{l}\text { Vehicles in this category } \\
\text { are tested at their adjusted } \\
\text { loaded vehicle weight }\end{array}$} & ULEV250 & 0.25 & 6.4 & 6 & 0.06 \\
\hline & ULEV200 & 0.2 & 4.2 & 6 & 0.06 \\
\hline & SULEV170 & 0.17 & 4.2 & 6 & 0.06 \\
\hline & SULEV150 & 0.15 & 3.2 & 6 & 0.06 \\
\hline \multirow{2}{*}{$\begin{array}{l}\text { MDVs } \\
10,000-14,000 \mathrm{lbs} \text { GVW }\end{array}$} & LEV630 & 0.63 & 7.3 & 6 & 0.12 \\
\hline & ULEV570 & 0.57 & 7.3 & 6 & 0.06 \\
\hline \multirow{4}{*}{$\begin{array}{l}\text { Vehicles in this category } \\
\text { are tested at their adjusted } \\
\text { loaded vehicle weight }\end{array}$} & ULEV400 & 0.4 & 7.3 & 6 & 0.06 \\
\hline & ULEV270 & 0.27 & 4.2 & 6 & 0.06 \\
\hline & SULEV230 & 0.23 & 4.2 & 6 & 0.06 \\
\hline & SULEV200 & 0.2 & 3.7 & 6 & 0.06 \\
\hline
\end{tabular}

Note: Definitions of acronyms are on page 12-13.

Source:

California LEV III Regulations with amendments effective December 2012. (Additional resources: www.arb.ca.gov) 
Table 12.21

Aircraft - Exhaust Emission Standards

\begin{tabular}{|c|c|c|c|c|c|c|c|}
\hline & Year & $\begin{array}{c}\text { Pressure } \\
\text { ratio }(\mathrm{PR})\end{array}$ & Applicability $^{\mathrm{a}}$ & $\mathrm{HC}(\mathrm{g} / \mathrm{kN})$ & NOx & $\begin{array}{c}\mathrm{CO} \\
(\mathrm{g} / \mathrm{kN})\end{array}$ & Smoke \\
\hline \multirow{16}{*}{ Federal $^{\text {b }}$} & $1974+$ & - & T8 & - & - & - & 30 \\
\hline & $1976+$ & - & TF with $\mathrm{rO}^{\mathrm{c}} \geq 129 \mathrm{kN}$ & - & - & - & $83.6(\mathrm{rO})^{-0.274}$ \\
\hline & $1978+$ & - & $\mathrm{T}^{\mathrm{d}}$ & - & - & - & 25 \\
\hline & $1983+$ & - & $\mathrm{TF}$ with $\mathrm{rO}<26.7 \mathrm{kN}$ & - & - & - & $\begin{array}{c}83.6(\mathrm{rO})^{-0.274} \mathrm{NTE} \\
\max \text { of } \mathrm{SN}=50\end{array}$ \\
\hline & \multirow{4}{*}{$1984+$} & - & $\begin{array}{c}\text { T3, T8, TF with } \mathrm{rO} \geq 26.7 \\
\text { kN }\end{array}$ & 19.6 & - & - & $\begin{array}{c}83.6(\mathrm{rO})^{-0.274} \mathrm{NTE} \\
\text { max of } \mathrm{SN}=50\end{array}$ \\
\hline & & - & TSS & $140(.92)^{\mathrm{rPR}}$ & - & - & $\begin{array}{c}83.6(\mathrm{rO})^{-0.274} \mathrm{NTE} \\
\max \text { of } \mathrm{SN}=50\end{array}$ \\
\hline & & - & TSS with $\mathrm{rO} \geq 26.7 \mathrm{kN}$ & $140(.92)^{\mathrm{rPR}}$ & - & - & $\begin{array}{c}83.6(\mathrm{rO})^{-0.274} \mathrm{NTE} \\
\max \text { of } \mathrm{SN}=50\end{array}$ \\
\hline & & - & $\mathrm{TP}$ with $\mathrm{rO} \geq 1,000 \mathrm{~kW}$ & - & - & - & $187(\mathrm{rO})^{-0.168}$ \\
\hline & & - & $\begin{array}{c}\text { T3, T8, TF with } \mathrm{rO}>26.7 \\
\text { kN }\end{array}$ & 19.6 & $40+2(\mathrm{rPR})$ & 118 & $\begin{array}{c}83.6(\mathrm{rO})^{-0.274} \mathrm{NTE} \\
\text { max of } \mathrm{SN}=50\end{array}$ \\
\hline & $1997+$ & - & $\begin{array}{c}\text { T3, T8, TF newly } \\
\text { certified with rO }>26.7 \\
\mathrm{kN}\end{array}$ & 19.6 & $32+1.6(\mathrm{rPR})$ & 118 & $\begin{array}{c}83.6(\mathrm{rO})^{-0.274} \mathrm{NTE} \\
\max \text { of } \mathrm{SN}=50\end{array}$ \\
\hline & $2000+$ & - & $\begin{array}{c}\mathrm{T} 3, \mathrm{~T} 8, \mathrm{TF} \text { newly } \\
\text { manufactured with } \mathrm{rO}> \\
26.7 \mathrm{kN}\end{array}$ & 19.6 & $32+1.6(\mathrm{rPR})$ & 118 & $\begin{array}{c}83.6(\mathrm{rO})^{-0.274} \mathrm{NTE} \\
\text { max of } \mathrm{SN}=50\end{array}$ \\
\hline & & & $\begin{array}{c}\text { T3, T8, TF newly } \\
\text { certified with } \mathrm{rO}>89 \mathrm{kN}\end{array}$ & - & $19+1.6(\mathrm{rPR})$ & - & - \\
\hline & & $\mathrm{PR} \leq 30$ & $\begin{array}{c}\text { T3, T8, TF newly } \\
\text { certified with } 26.7 \mathrm{kN}< \\
\text { rO } \leq 89 \mathrm{kN}\end{array}$ & - & $\begin{array}{c}37.572+1.6(\mathrm{rPR})- \\
0.2087(\mathrm{rO})\end{array}$ & - & - \\
\hline & $2005+$ & & $\begin{array}{c}\mathrm{T} 3, \mathrm{~T} 8, \mathrm{TF} \text { newly } \\
\text { certified with rO>89 kN }\end{array}$ & - & $7+2.0(\mathrm{rPR})$ & - & - \\
\hline & & $\begin{array}{c}30<\mathrm{PR}< \\
62.5\end{array}$ & $\begin{array}{c}\mathrm{T} 3, \mathrm{~T} 8, \mathrm{TF} \text { newly } \\
\text { certified with } 26.7 \mathrm{kN}<\mathrm{r} 0 \\
\leq 89 \mathrm{kN}\end{array}$ & - & $\begin{array}{c}42.71+1.4286(\mathrm{rPR})- \\
0.4013(\mathrm{rO})+0.00642(\mathrm{rP} \\
\mathrm{R})(\mathrm{rO})\end{array}$ & - & - \\
\hline & & $\mathrm{PR} \leq 62.5$ & $\mathrm{~T} 3, \mathrm{~T} 8, \mathrm{TF}$ & - & $32+1.6(\mathrm{rPR})$ & - & - \\
\hline
\end{tabular}

Note: The test procedures are the International Civil Aviation Organization (ICAO) Smoke Emission Test Procedure and the ICAO Gaseous Emissions Test Procedure. There is no useful life or warranty period for purposes of compliance with aircraft emissions standards. Definitions of acronyms are on page 12-13.

\section{Source:}

40 CFR Part 87, Aircraft emission standards, test procedures, certification requirements (Additional resources: www.epa.gov/otaq/standards)

${ }^{\text {a }}$ T8=all aircraft gas turbine engines of the JT8D model family

$\mathrm{TF}=$ all turbofan and turbojet aircraft engines except engines of Class T3, T8, and TSS

T3=all aircraft gas turbine engines of the JT3D model family

TSS=all aircraft gas turbine engines for aircraft operations at supersonic flight speeds

$\mathrm{TP}=$ all aircraft turboprop engines

b Federal standards apply to planes operating in the United States, regardless of where they were manufactured.

${ }^{\mathrm{c}}$ Rated output (rO) is the maximum power/thrust available for takeoff.

${ }^{\mathrm{d}} \mathrm{T} 3$ engines are no longer manufactured but are in the existing fleet. 
These standards apply to construction and agricultural equipment, such as excavators, paving equipment, tractors, combines, bulldozers, and skidders.

Table 12.22

Nonroad Compression-Ignition Engines - Exhaust Emission Standards

\begin{tabular}{|c|c|c|c|c|c|c|c|c|c|c|}
\hline & $\begin{array}{c}\text { Rated } \\
\text { power } \\
(\mathrm{kW})\end{array}$ & Tier & $\begin{array}{c}\text { Model } \\
\text { year }\end{array}$ & $\begin{array}{c}\text { NMHC } \\
(\mathrm{g} / \mathrm{kW} \\
-\mathrm{hr})\end{array}$ & $\begin{array}{c}\text { NMHC } \\
+ \text { NOx } \\
(\mathrm{g} / \mathrm{kW} \\
-\mathrm{hr})\end{array}$ & $\begin{array}{c}\text { NOx } \\
(\mathrm{g} / \mathrm{kW} \\
-\mathrm{hr})\end{array}$ & $\begin{array}{c}\mathrm{PM} \\
(\mathrm{g} / \mathrm{kW} \\
-\mathrm{hr})\end{array}$ & $\begin{array}{c}\mathrm{CO} \\
(\mathrm{g} / \mathrm{kW} \\
-\mathrm{hr})\end{array}$ & $\begin{array}{c}\text { Smoke }^{\mathrm{a}} \\
\text { percentage }\end{array}$ & $\begin{array}{c}\text { Useful life } \\
\text { (hours/years) }^{\mathrm{b}}\end{array}$ \\
\hline \multirow{45}{*}{ Federal } & \multirow{3}{*}{$\mathrm{kW}<8$} & 1 & $2000-2004$ & -- & 10.5 & -- & 1.0 & 8.0 & \multirow{45}{*}{$20 / 15 / 50$} & \multirow{3}{*}{$3,000 / 5$} \\
\hline & & 2 & $2005-2007$ & -- & 7.5 & -- & 0.80 & 8.0 & & \\
\hline & & 4 & $2008+$ & -- & 7.5 & --" & $0.40^{c}$ & 8.0 & & \\
\hline & \multirow{3}{*}{$8 \leq \mathrm{kW}<19$} & 1 & $2000-2004$ & -- & 9.5 & - & 0.80 & 6.6 & & \multirow{3}{*}{$3,000 / 5$} \\
\hline & & 2 & 2005-2007 & --" & 7.5 & -- & 0.80 & 6.6 & & \\
\hline & & 4 & $2008+$ & -- & 7.5 & -"- & 0.40 & 6.6 & & \\
\hline & \multirow{4}{*}{$19 \leq \mathrm{kW}<37$} & 1 & $1999-2003$ & -- & 9.5 & -- & 0.80 & 5.5 & & \multirow{4}{*}{$5,000 / 7^{d}$} \\
\hline & & 2 & 2004-2007 & -- & 7.5 & -- & 0.60 & 5.5 & & \\
\hline & & \multirow{2}{*}{4} & $2008-2012$ & -- & 7.5 & -- & 0.30 & 5.5 & & \\
\hline & & & $2013+$ & -- & 4.7 & -- & 0.03 & 5.5 & & \\
\hline & \multirow{6}{*}{$37 \leq \mathrm{kW}<56$} & 1 & $1998-2003$ & -- & -- & 9.2 & -- & -- & & \multirow{35}{*}{$8,000 / 10$} \\
\hline & & 2 & 2004-2007 & -- & 7.5 & -- & 0.40 & 5.0 & & \\
\hline & & $3^{\mathrm{e}}$ & 2008-2011 & -- & 4.7 & -"- & 0.40 & 5.0 & & \\
\hline & & $4(\text { Option } 1)^{\mathrm{f}}$ & 2008-2012 & -- & 4.7 & -- & 0.30 & 5.0 & & \\
\hline & & $4(\text { Option } 2)^{\mathrm{f}}$ & 2012 & -- & 4.7 & -- & 0.03 & 5.0 & & \\
\hline & & 4 & $2013+$ & -- & 4.7 & -- & 0.03 & 5.0 & & \\
\hline & \multirow{5}{*}{$56 \leq \mathrm{kW}<75$} & 1 & $1998-2003$ & -- & -- & 9.2 & -- & -- & & \\
\hline & & 2 & 2004-2007 & -- & 7.5 & -- & 0.40 & 5.0 & & \\
\hline & & 3 & 2008-2011 & -- & 4.7 & -"- & 0.40 & 5.0 & & \\
\hline & & \multirow{2}{*}{4} & $2012-2103^{g}$ & -- & 4.7 & -- & 0.02 & 5.0 & & \\
\hline & & & $2014 t^{\mathrm{h}}$ & 0.19 & -- & 0.4 & 0.02 & 5.0 & & \\
\hline & \multirow{5}{*}{$75 \leq \mathrm{kW}<130$} & 1 & 1997-2002 & -- & -- & 9.2 & -- & -- & & \\
\hline & & 2 & $2003-2006$ & -- & 6.6 & -- & 0.3 & 5.0 & & \\
\hline & & 3 & 2007-2011 & -- & 4.0 & -- & 0.3 & 5.0 & & \\
\hline & & \multirow{2}{*}{4} & $2012-2013^{\mathrm{g}}$ & -- & 4.0 & -- & 0.02 & 5.0 & & \\
\hline & & & $2014+$ & 0.19 & -- & 0.4 & 0.02 & 5.0 & & \\
\hline & \multirow{5}{*}{$\begin{array}{c}130 \leq \mathrm{kW}< \\
225\end{array}$} & 1 & 1996-2002 & $1.3^{\mathrm{i}}$ & -- & 9.2 & 0.54 & 11.4 & & \\
\hline & & 2 & 2003-2005 & -- & 6.6 & -- & 0.20 & 3.5 & & \\
\hline & & 3 & $2006-2010$ & -- & 4.0 & -- & 0.20 & 3.5 & & \\
\hline & & \multirow{2}{*}{4} & $2011-2013^{g}$ & -- & 4.0 & -- & 0.02 & 3.5 & & \\
\hline & & & $2014 t^{\text {h" }}$ & 0.19 & -- & 0.4 & 0.02 & 3.5 & & \\
\hline & \multirow{5}{*}{$\begin{array}{c}225 \leq \mathrm{kW}< \\
450\end{array}$} & 1 & 1996-2000 & $1.3^{\mathrm{i}}$ & -- & 9.2 & 0.54 & 11.4 & & \\
\hline & & 2 & $2001-2005$ & -- & 6.4 & -- & 0.20 & 3.5 & & \\
\hline & & 3 & 2006-2010 & -- & 4.0 & -- & 0.20 & 3.5 & & \\
\hline & & \multirow{2}{*}{4} & $2011-2013^{g}$ & -- & 4.0 & -- & 0.02 & 3.5 & & \\
\hline & & & $2014+{ }^{\mathrm{h}}$ & 0.19 & -- & 0.4 & 0.02 & 3.5 & & \\
\hline & \multirow{5}{*}{$\begin{array}{c}450 \leq \mathrm{kW}< \\
560\end{array}$} & 1 & $1996-2001$ & $1.3^{\mathrm{i}}$ & -- & 9.2 & 0.54 & 11.4 & & \\
\hline & & 2 & 2002-2005 & -- & 6.4 & -- & 0.20 & 3.5 & & \\
\hline & & 3 & 2006-2010 & -- & 4.0 & -- & 0.20 & 3.5 & & \\
\hline & & \multirow{2}{*}{4} & $2011-2013^{g}$ & -- & 4.0 & -- & 0.02 & 3.5 & & \\
\hline & & & $2014+^{\mathrm{h}}$ & 0.19 & -- & 0.4 & 0.02 & 3.5 & & \\
\hline & & 1 & $2000-2005$ & $1.3^{\mathrm{i}}$ & -- & 9.2 & 0.54 & 11.4 & & \\
\hline & $560 \leq \mathrm{kW}<$ & 2 & 2006-2010 & -- & 6.4 & -- & 0.20 & 3.5 & & \\
\hline & 900 & 4 & 2011-2014 & 0.4 & -- & 3.5 & 0.10 & 3.5 & & \\
\hline & & 4 & $2015 t^{\mathrm{h}}$ & 0.19 & -- & $3.5^{j}$ & $0.04^{\mathrm{k}}$ & 3.5 & & \\
\hline
\end{tabular}


Table 12.22 (continued)

Nonroad Compression-Ignition Engines - Exhaust Emission Standards

\begin{tabular}{|c|c|c|c|c|c|c|c|c|c|c|}
\hline & $\begin{array}{c}\text { Rated } \\
\text { power } \\
(\mathrm{kW})\end{array}$ & Tier & $\begin{array}{l}\text { Model } \\
\text { year }\end{array}$ & $\begin{array}{c}\text { NMHC } \\
(\mathrm{g} / \mathrm{kW} \\
-\mathrm{hr})\end{array}$ & $\begin{array}{c}\text { NMHC } \\
+\mathrm{NOx} \\
(\mathrm{g} / \mathrm{kW} \\
-\mathrm{hr})\end{array}$ & $\begin{array}{c}\text { NOx } \\
(\mathrm{g} / \mathrm{kW} \\
-\mathrm{hr})\end{array}$ & $\begin{array}{c}\mathrm{PM} \\
(\mathrm{g} / \mathrm{kW} \\
-\mathrm{hr})\end{array}$ & $\begin{array}{c}\mathrm{CO} \\
(\mathrm{g} / \mathrm{kW} \\
-\mathrm{hr})\end{array}$ & $\begin{array}{c}\text { Smoke }^{\mathrm{a}} \\
\text { percentage }\end{array}$ & $\begin{array}{c}\text { Useful life } \\
\text { (hours/years) }\end{array}$ \\
\hline \multirow{4}{*}{ Federal } & \multirow{4}{*}{$\mathrm{kW}>900$} & 1 & $2000-2005$ & $1.3^{\mathrm{i}}$ & -- & 9.2 & 0.54 & 11.4 & \multirow{4}{*}{$20 / 15 / 50$} & \multirow{4}{*}{$8,000 / 10$} \\
\hline & & 2 & $2006-2010$ & -- & 6.4 & -- & 0.20 & 3.5 & & \\
\hline & & \multirow{2}{*}{4} & $2011-2014$ & 0.4 & -- & $3.5^{\mathrm{j}}$ & 0.10 & 3.5 & & \\
\hline & & & $2015+^{\mathrm{h}}$ & 0.19 & -- & $3.5^{\mathrm{j}}$ & $0.04^{\mathrm{k}}$ & 3.5 & & \\
\hline
\end{tabular}

Note: Definitions of acronyms are on page 12-13.

\section{Source:}

40 CFR 98.112 = Exhaust emission standards

40 CFR 1039.101 = Exhaust emission standards for after 2014 model year

40 CFR 1039.102 = Exhaust emission standards for model year 2014 and earlier

40 CFR 1039 Subpart F = Exhaust emissions transient and steady state test procedures

40 CFR Part 86 Subpart I = Smoke emission test procedures

40 CFR Part $1065=$ Test equipment and emissions measurement procedures (Additional resources: www.epa.gov/otaq/standards)

${ }^{\text {a }}$ Smoke emissions may not exceed 20 percent during the acceleration mode, 15 percent during the lugging mode, and 50 percent during the peaks in either mode. Smoke emission standards do not apply to single-cylinder engines, constant-speed engines, or engines certified to a PM emission standard of 0.07 grams per kilowatt-hour $(\mathrm{g} / \mathrm{kW}-\mathrm{hr})$ or lower. Smoke emissions are measured using procedures in $40 \mathrm{CFR}$ Part 86 Subpart I.

${ }^{\mathrm{b}}$ Useful life and warranty period are expressed hours and years, whichever comes first.

${ }^{\mathrm{c}}$ Hand-startable air-cooled direct injection engines may optionally meet a PM standard of $0.60 \mathrm{~g} / \mathrm{kW}$ hr. These engines may optionally meet Tier 2 standards through the 2009 model years. In 2010 these engines are required to meet a PM standard of $0.60 \mathrm{~g} / \mathrm{kW}-\mathrm{hr}$.

${ }^{\mathrm{d}}$ Useful life for constant speed engines with rated speed 3,000 revolutions per minute (rpm) or higher is 5 years or 3,000 hours, whichever comes first.

e These Tier 3 standards apply only to manufacturers selecting Tier 4 Option 2. Manufacturers selecting Tier 4 Option 1 will be meeting those standards in lieu of Tier 3 standards.

${ }^{\mathrm{f}}$ A manufacturer may certify all their engines to either Option 1 or Option 2 sets of standards starting in the indicated model year. Manufacturers selecting Option 2 must meet Tier 3 standards in the 2008-2011 model years.

${ }_{\mathrm{g}}^{\mathrm{g}}$ These standards are phase-out standards. Not more than 50 percent of a manufacturer's engine production is allowed to meet these standards in each model year of the phase out period. Engines not meeting these standards must meet the final Tier 4 standards.

${ }^{\mathrm{h}}$ These standards are phased in during the indicated years. At least 50 percent of a manufacturer's engine production must meet these standards during each year of the phase in. Engines not meeting these standards must meet the applicable phase-out standards.

${ }^{i}$ For Tier 1 engines the standard is for total hydrocarbons.

${ }^{\mathrm{j}}$ The NOx standard for generator sets is $0.67 \mathrm{~g} / \mathrm{kW}-\mathrm{hr}$.

${ }^{\mathrm{k}}$ The PM standard for generator sets is $0.03 \mathrm{~g} / \mathrm{kW}-\mathrm{hr}$. 
These standards apply to gasoline and propane industrial equipment such as forklifts, generators, airport service equipment, compressors and ice-grooming machines.

Table 12.23

Nonroad Large Spark-Ignition Engines - Exhaust and Evaporative Emission Standards

\begin{tabular}{|c|c|c|c|c|c|c|c|c|c|}
\hline & \multirow[b]{2}{*}{ Tier } & \multirow[b]{2}{*}{ Year } & \multicolumn{2}{|c|}{$\begin{array}{l}\text { General duty-cycle } \\
\text { standards }\end{array}$} & \multicolumn{2}{|c|}{$\begin{array}{l}\text { Alternative standards for } \\
\text { severe-duty engines }\end{array}$} & \multicolumn{2}{|c|}{ Field testing standards } & \multirow[b]{2}{*}{$\begin{array}{c}\text { Useful life } \\
\text { (years/hours) }\end{array}$} \\
\hline & & & $\begin{array}{l}\mathrm{HC}+\mathrm{NOx}{ }^{\mathrm{a}} \\
(\mathrm{g} / \mathrm{kW}-\mathrm{hr})\end{array}$ & $\begin{array}{c}\mathrm{CO} \\
(\mathrm{g} / \mathrm{kW}-\mathrm{hr}) \\
\end{array}$ & $\begin{array}{l}\mathrm{HC}+\mathrm{NOx}{ }^{\mathrm{a}} \\
(\mathrm{g} / \mathrm{kW}-\mathrm{hr})\end{array}$ & $\begin{array}{c}\mathrm{CO} \\
(\mathrm{g} / \mathrm{kW}-\mathrm{hr}) \\
\end{array}$ & $\begin{array}{l}\mathrm{HC}+\mathrm{NOx}{ }^{\mathrm{a}} \\
(\mathrm{g} / \mathrm{kW}-\mathrm{hr})\end{array}$ & $\begin{array}{c}\mathrm{CO} \\
(\mathrm{g} / \mathrm{kW}-\mathrm{hr}) \\
\end{array}$ & \\
\hline \multirow{6}{*}{ Federal $^{b}$} & $1^{\mathrm{c}}$ & $2004-2006$ & $4.0^{\mathrm{d}}$ & 50.0 & $4.0^{\mathrm{d}}$ & 130.0 & - & - & $7 / 5,000^{\mathrm{e}}$ \\
\hline & \multirow{5}{*}{$2^{\mathrm{f}}$} & \multirow{5}{*}{$2007+$} & $2.7^{\mathrm{f}}$ & $4.4^{\mathrm{f}}$ & 2.7 & 130.0 & $3.8^{f}$ & $6.5^{f}$ & $7 / 5,000^{\mathrm{e}}$ \\
\hline & & & \multicolumn{7}{|c|}{ Evaporative emission standards (for engines fueled by a volatile liquid fuel) } \\
\hline & & & $\begin{array}{l}\text { Fuel line } \\
\text { permeation }\end{array}$ & \multicolumn{5}{|c|}{$\begin{array}{l}\text { Nonmetallic fuel lines must meet the permeation specifications of SAE } \\
\qquad \mathbf{J} 2260 \text { (November 1996) }\end{array}$} & \multirow{3}{*}{$5 /-$} \\
\hline & & & $\begin{array}{l}\text { Diurnal } \\
\text { emissions }\end{array}$ & \multicolumn{5}{|c|}{$\begin{array}{c}\text { Evaporative HC emissions may not exceed } 0.2 \text { grams per gallon of fuel tank } \\
\text { capacity }\end{array}$} & \\
\hline & & & Running loss & \multicolumn{5}{|c|}{$\begin{array}{l}\text { Liquid fuel in the fuel tank may not reach boiling during continuous engine } \\
\text { operation in the final installation at an ambient temperature of } 30^{\circ} \mathrm{C}\end{array}$} & \\
\hline
\end{tabular}

\section{Sources:}

40 CFR $1048.101=$ Exhaust emission standards

40 CFR 1048.105 = Evaporative emission standards

40 CFR 1048.110 = Engine diagnostic requirements (Additional resources: www.epa.gov/otaq/standards)

a The numerical emission standards for hydrocarbons (HC) must be met based on the following types of hydrocarbon emissions for engines powered by the following fuels: (1) non-methane hydrocarbons (NMHC) for natural gas; (2) total hydrocarbon equivalent (THCE) for alcohol; and (3) total hydrocarbons (THC) for other fuels.

${ }^{\mathrm{b}}$ Voluntary Blue Sky standards for large spark-ignition (SI) engines are available. Engines with displacement at or below 1,000 cubic centimeters (cc) and maximum power at or below 30 kilowatts $(\mathrm{kW})$ may be certified under the program for small SI engines.

${ }^{c}$ Emission standards are based on testing over a steady-state duty-cycle.

${ }^{\mathrm{d}}$ The Tier $1 \mathrm{HC}$ plus nitrogen oxides (NOx) emission standard for in-use testing is 5.4 grams per $\mathrm{kW}$-hour (g/kW-hr).

${ }^{\mathrm{e}}$ Useful life is expressed in years and hours, whichever comes first. These are the minimum useful life requirements. For severe-duty engines, the minimum useful life is seven years or 1,500 hours of operation, whichever comes first. A longer useful life in hours is required if: (a) the engine is designed to operate longer than the minimum useful life based on the recommended rebuild interval; or (b) the basic mechanical warranty is longer than the minimum useful life.

${ }^{\mathrm{f}}$ Optional engine certification is allowed according to the following formula: $(\mathrm{HC}+\mathrm{NOx}) \times \mathrm{CO}^{0.784} \leq 8.57$. The HC+NOx and carbon monoxide (CO) emission levels selected to satisfy this formula, rounded to the nearest 0.1 $\mathrm{g} / \mathrm{kW}-\mathrm{hr}$, become the emission standards that apply for those engines. One may not select an HC+NOx emission standard higher than $2.7 \mathrm{~g} / \mathrm{kW}-\mathrm{hr}$ or a CO emission standard higher than $20.6 \mathrm{~g} / \mathrm{kW}-\mathrm{hr}$. 
Table 12.24

Locomotives - Exhaust Emission Standards

\begin{tabular}{|c|c|c|c|c|c|c|c|c|c|}
\hline & $\begin{array}{l}\text { Duty- } \\
\text { cycle }^{\mathrm{b}}\end{array}$ & Tier & Year $^{\mathrm{c}}$ & $\begin{array}{c}\mathrm{HC}^{\mathrm{i}} \\
(\mathrm{g} / \mathrm{hp}-\mathrm{hr})\end{array}$ & $\begin{array}{c}\text { NOx } \\
\text { (g/bhp-hr) }\end{array}$ & $\begin{array}{c}\text { PM } \\
\text { (g/bhp-hr) }\end{array}$ & $\begin{array}{c}\text { CO } \\
\text { (g/bhp-hr) }\end{array}$ & $\begin{array}{c}\text { Smoke } \\
\left(^{\text {percentage })^{\mathrm{m}}}\right.\end{array}$ & $\begin{array}{l}\text { Minimum useful life } \\
\text { (hours / years / miles) }^{\mathrm{n}}\end{array}$ \\
\hline \multirow{11}{*}{ Federal $^{\mathrm{a}}$} & \multirow{6}{*}{$\begin{array}{l}\text { Line- } \\
\text { haul }\end{array}$} & Tier 0 & $\begin{array}{l}1973- \\
1992^{\mathrm{d}, \mathrm{e}}\end{array}$ & 1.0 & $9.5[\mathrm{ABT}]$ & $0.22[\mathrm{ABT}]$ & 5.0 & $30 / 40 / 50$ & $\begin{array}{c}(7.5 \times \mathrm{hp}) / 10 / \\
750,000^{\circ}\end{array}$ \\
\hline & & \multirow{2}{*}{ Tier 1} & \multirow{2}{*}{$\begin{array}{l}1993- \\
2004^{\mathrm{d}, \mathrm{e}}\end{array}$} & \multirow{2}{*}{0.55} & \multirow{2}{*}{$7.4[\mathrm{ABT}]$} & \multirow[t]{2}{*}{$0.22[\mathrm{ABT}]$} & \multirow[t]{2}{*}{2.2} & \multirow[t]{2}{*}{$25 / 40 / 50$} & $\begin{array}{c}(7.5 \times \mathrm{hp}) / 10 / \\
750,000^{\circ}\end{array}$ \\
\hline & & & & & & & & & $(7.5 \times \mathrm{hp}) / 10 /-$ \\
\hline & & Tier 2 & $\begin{array}{l}2005- \\
2011^{\text {d }}\end{array}$ & 0.30 & $5.5[\mathrm{ABT}]$ & $0.10^{\mathrm{k}}[\mathrm{ABT}]$ & 1.5 & $20 / 40 / 50$ & $(7.5 \times \mathrm{hp}) / 10 /-$ \\
\hline & & Tier 3 & $\begin{array}{l}2012- \\
2014^{f}\end{array}$ & 0.30 & $5.5[\mathrm{ABT}]$ & $0.10[\mathrm{ABT}]$ & 1.5 & $20 / 40 / 50$ & $(7.5 \times \mathrm{hp}) / 10 /-$ \\
\hline & & Tier 4 & $2015+^{g}$ & 0.14 & $1.3[\mathrm{ABT}]$ & $0.03[\mathrm{ABT}]$ & 1.5 & - & $(7.5 \times h p) / 10 /-$ \\
\hline & \multirow{5}{*}{ Switch } & Tier 0 & $\begin{array}{l}1973- \\
2001\end{array}$ & 2.10 & $11.8[\mathrm{ABT}]$ & $0.26[\mathrm{ABT}]$ & 8.0 & $30 / 40 / 50$ & $\begin{array}{c}(7.5 \times \mathrm{hp}) / 10 / \\
750,000^{\circ}\end{array}$ \\
\hline & & Tier 1 & $\begin{array}{l}2002- \\
2004^{h}\end{array}$ & 1.20 & $11.0[\mathrm{ABT}]$ & $0.26[\mathrm{ABT}]$ & 2.5 & $25 / 40 / 50$ & $(7.5 \times \mathrm{hp}) / 10 /-$ \\
\hline & & Tier 2 & $\begin{array}{l}2005- \\
2010^{h}\end{array}$ & 0.60 & $8.1[\mathrm{ABT}]$ & $0.13^{1}[\mathrm{ABT}]$ & 2.4 & $20 / 40 / 50$ & $(7.5 \times \mathrm{hp}) / 10 /-$ \\
\hline & & Tier 3 & $\begin{array}{l}2011- \\
2014\end{array}$ & 0.60 & $5.0[\mathrm{ABT}]$ & $0.10[\mathrm{ABT}]$ & 2.4 & $20 / 40 / 50$ & $(7.5 \times \mathrm{hp}) / 10 /-$ \\
\hline & & Tier 4 & $2015+$ & $0.14^{\mathrm{j}}$ & $1.3^{\mathrm{j}}[\mathrm{ABT}]$ & $0.03[\mathrm{ABT}]$ & 2.4 & - & (7.5 x hp)/ $10 /-$ \\
\hline
\end{tabular}

Sources:

40 CFR 1033.101 = Emission Standards and Useful Life

${ }^{a}$ These standards apply to locomotives that are propelled by engines with total rated horsepower (hp) of 750 kilowatts $(\mathrm{kW})(1006 \mathrm{hp})$ or more, unless the owner chooses to have the equipment certified to meet the requirements of locomotives. This does not include vehicles propelled by engines with total rated horsepower of less than $750 \mathrm{~kW}$ (1006 hp); see the requirements in 40 Code of Federal Regulations (CFR) Parts 86, 89 and 1039. The test procedures specify chassis-based testing of locomotives. These test procedures include certification testing, production line testing, and in-use testing using the Federal Test Procedure (FTP) when the locomotive has reached between 50-70 percent of its useful life.

${ }^{\mathrm{b}}$ Line-haul locomotives are powered by an engine with a maximum rated power (or a combination of engines having a total rated power) greater than $2300 \mathrm{hp}$. Switch locomotives are powered by an engine with a maximum rated power (or a combination of engines having a total rated power) of $2300 \mathrm{hp}$ or less.

${ }^{c}$ The Tier 0 standards apply to locomotives manufactured after 1972 when they are manufactured or remanufactured. Note that interim standards may apply for Tier 0 or Tier 1 locomotives remanufactured in 2008 or 2009, or for Tier 2 locomotives manufactured or remanufactured in 2008-2012.

d Line-haul locomotives subject to the Tier 0 through Tier 2 emission standards must also meet switch standards of the same tier.

e The Tier 0 standards apply for 1993-2001 locomotives not originally manufactured with a separate loop intake air cooling system.

${ }_{\mathrm{f}}^{\mathrm{f}}$ Tier 3 line-haul locomotives must also meet Tier 2 switch standards.

${ }^{\mathrm{g}}$ Manufacturers using credits may elect to meet a combined nitrogen oxides (NOx) plus hydrocarbon (HC) standard of 1.4 grams per brakehorsepower-hour (g/bhp-hr) instead of the otherwise applicable Tier 4 NOx and HC standards.

${ }^{\mathrm{h}}$ Tier 1 and Tier 2 switch locomotives must also meet line-haul standards of the same tier.

${ }^{\mathrm{i}}$ The numerical emission standards for $\mathrm{HC}$ must be met based on the following types of hydrocarbon emissions for locomotives powered by the following fuels: (1) alcohol: total hydrocarbon equivalent (THCE) emissions for Tier 3 and earlier locomotives, and non-methane hydrocarbon equivalent (NMHCE) for Tier 4; (2) natural gas and liquefied petroleum gas: non-methane hydrocarbon (NMHC) emissions; and (3) diesel: total hydrocarbon (THC) emissions for Tier 3 and earlier locomotives, and NMHC for Tier 4. 


\section{Table 12.24 (continued) \\ Locomotives - Exhaust Emission Standards}

\footnotetext{
${ }^{\mathrm{j}}$ Manufacturers may elect to meet a combined NOx+HC standard of $1.4 \mathrm{~g} / \mathrm{bhp}$-hr instead of the otherwise applicable Tier 4 NOx and HC standards.

${ }^{\mathrm{k}}$ The line-haul particulate matter (PM) standard for newly remanufactured Tier 2 locomotives is $0.20 \mathrm{~g} / \mathrm{bhp}-$ hr until January 1, 2013, except as specified in 40 CFR Part 1033.150(a).

${ }^{1}$ The switch PM standard for new Tier 2 locomotives is $0.24 \mathrm{~g} / \mathrm{bhp}-\mathrm{hr}$ until January 1, 2013, except as specified in 40 CFR Part 1033.150(a).

m The smoke opacity standards apply only for locomotives certified to one or more PM standards or Family Emission Limits (FEL) greater than $0.05 \mathrm{~g} / \mathrm{bhp}-\mathrm{hr}$. Percentages apply to smoke opacity at steady state/30-second peak/3-second peak, as measured continuously during testing.

${ }^{\mathrm{n}}$ Useful life and warranty period are expressed in megawatt-hours (mw-hr), years, or miles, whichever comes first. Manufacturers are required to certify to longer useful lives if their locomotives are designed to last longer between overhauls than the minimum useful life value.

${ }^{\mathrm{o}}$ For locomotives originally manufactured before January 1, 2000, and not equipped with mw-hr meters.
} 
These standards apply to auxiliary and propulsion engines used by all types of recreational and commercial vessels, from small fishing boats to ocean-going ships.

Table 12.25

Marine Compression-Ignition (CI) Engines - Exhaust Emission Standards

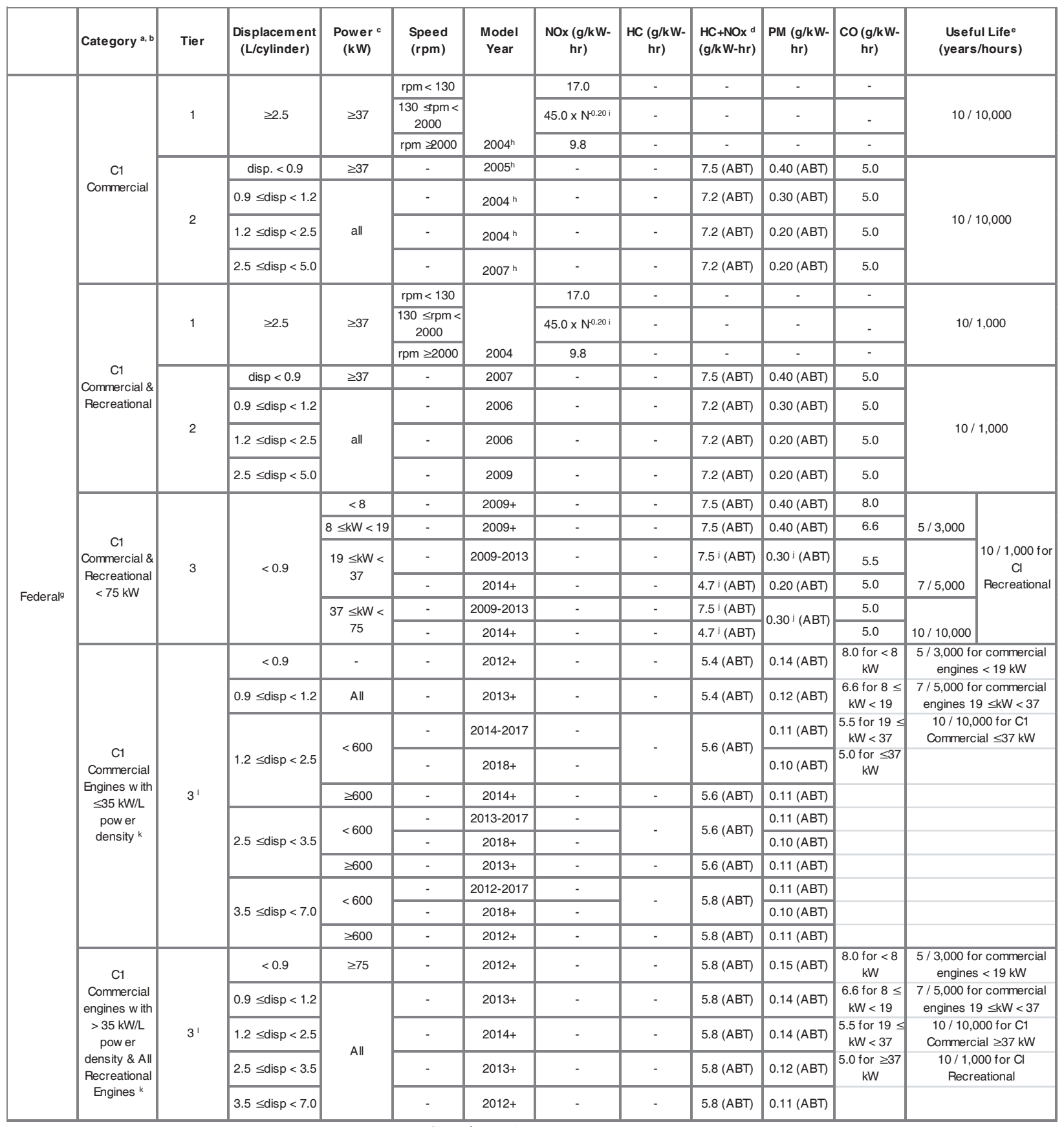

(Continued on next page) 
Table 12.25 (continued)

Marine Compression-Ignition (CI) Engines - Exhaust Emission Standards

\begin{tabular}{|c|c|c|c|c|c|c|c|c|c|c|c|c|}
\hline & Category a, b & Tier & $\begin{array}{l}\text { Displacement } \\
\text { (L/cylinder) }\end{array}$ & $\begin{array}{l}\text { Power }{ }^{\mathrm{c}} \\
(\mathrm{kW})\end{array}$ & $\begin{array}{l}\text { Speed } \\
(\mathrm{rpm})\end{array}$ & $\begin{array}{l}\text { Model } \\
\text { Year }\end{array}$ & $\begin{array}{c}\text { NOx (g/kW- } \\
\mathrm{hr})\end{array}$ & $\begin{array}{c}\mathrm{HC}(\mathrm{g} / \mathrm{kWW}- \\
\mathrm{hr})\end{array}$ & $\begin{array}{l}\text { HC+NOx }{ }^{d} \\
\text { (g/kW-hr) }\end{array}$ & $\begin{array}{c}\text { PM (g/kW- } \\
\mathrm{hr})\end{array}$ & $\begin{array}{c}\mathrm{CO}(\mathrm{g} / \mathrm{kW}- \\
\mathrm{hr})\end{array}$ & $\begin{array}{c}\text { Useful Life } \\
\text { (years/hours) }\end{array}$ \\
\hline \multirow{33}{*}{ Federal $^{9}$} & \multirow{5}{*}{$\begin{array}{c}\text { C1 } \\
\text { Commercial > } \\
600 \mathrm{~kW}\end{array}$} & \multirow{5}{*}{$4^{\mathrm{m}}$} & All & $\begin{array}{c}600 \leq \mathrm{kW}< \\
1,400\end{array}$ & - & $2017+$ & 1.8 (ABT) & - & $0.19 \mathrm{HC}^{n}$ & 0.04 (ABT) & \multirow{5}{*}{5.0} & \multirow{5}{*}{$10 / 10,000$} \\
\hline & & & All & $\begin{array}{c}1,400 \leq \mathrm{kW} \\
<2,000\end{array}$ & - & $2016+$ & 1.8 (ABT) & - & $0.19 \mathrm{HC}^{\mathrm{n}}$ & 0.04 (ABT) & & \\
\hline & & & All & $\begin{array}{c}2,000 \leq \mathrm{kW} \\
<3,700\end{array}$ & - & $2014+$ & $1.8(\mathrm{ABT})$ & - & $0.19 \mathrm{HC}^{n}$ & 0.04 (ABT) & & \\
\hline & & & \multirow{2}{*}{$<7.0$} & \multirow{2}{*}{$\geq 3,700$} & - & 2014-2015 & 1.8 (ABT) & - & $0.19 \mathrm{HC}^{\mathrm{n}}$ & 0.12 (ABT) & & \\
\hline & & & & & - & $2016+$ & 1.8 (ABT) & - & $0.19 \mathrm{HC}^{\mathrm{n}}$ & 0.06 (ABT) & & \\
\hline & \multirow{19}{*}{$\mathrm{C} 2$} & \multirow{3}{*}{1} & \multirow{3}{*}{$\geq 2.5$} & \multirow{3}{*}{$\geq 37$} & $\mathrm{rpm}<130$ & \multirow{3}{*}{2004} & 17.0 & - & - & - & - & \multirow{3}{*}{$10 / 20,000$} \\
\hline & & & & & \begin{tabular}{|c|}
$130 \leq \mathrm{rpm}<$ \\
2,000
\end{tabular} & & $45.0 \times \mathrm{N}^{\mathrm{N} .20 \mathrm{i}}$ & - & - & - & - & \\
\hline & & & & & $\mathrm{rpm} \geq 2,000$ & & 9.8 & - & - & - & - & \\
\hline & & \multirow{5}{*}{2} & $\begin{array}{c}5.0 \leq \text { disp }< \\
15.0\end{array}$ & all & - & \multirow{5}{*}{2007} & - & - & 7.8 (ABT) & 0.27 (ABT) & 5.0 & \multirow{5}{*}{$10 / 20,000$} \\
\hline & & & $\begin{array}{c}15.0 \leq \text { disp }< \\
20.0\end{array}$ & $<3,300$ & - & & - & - & $8.7(\mathrm{ABT})$ & 0.50 (ABT) & 5.0 & \\
\hline & & & $\begin{array}{c}15.0 \leq \text { disp }< \\
20.0\end{array}$ & $\geq 3,300$ & - & & - & - & 9.8 (ABT) & 0.50 (ABT) & 5.0 & \\
\hline & & & $\begin{array}{c}20.0 \leq \text { disp }< \\
25.0\end{array}$ & all & - & & - & - & $9.8(\mathrm{ABT})$ & 0.50 (ABT) & 5.0 & \\
\hline & & & $\begin{array}{c}25.0 \leq \mathrm{disp}< \\
30.0\end{array}$ & all & - & & - & - & 11.0 (ABT) & 0.50 (ABT) & 5.0 & \\
\hline & & \multirow{5}{*}{$3^{o, p}$} & $7.0 \leq$ disp $<$ & $<2,000$ & - & \multirow[b]{2}{*}{$2013+$} & - & - & 6.2 (ABT) & 0.14 (ABT) & 5.0 & \multirow{5}{*}{$10 / 20,000$} \\
\hline & & & 15.0 & $\begin{array}{c}2,000 \leq \mathrm{kW} \\
<3,700\end{array}$ & - & & - & - & 7.8 (ABT) & 0.14 (ABT) & 5.0 & \\
\hline & & & $\begin{array}{c}15.0 \leq \text { disp }< \\
20.0\end{array}$ & $<2,000$ & - & \multirow{3}{*}{$2014+$} & - & - & 7.0 (ABT) & 0.34 (ABT) & 5.0 & \\
\hline & & & $\begin{array}{c}20.0 \leq \text { disp }< \\
25.0\end{array}$ & $<2,000$ & - & & - & - & 9.8 (ABT) & 0.27 (ABT) & 5.0 & \\
\hline & & & $\begin{array}{c}25.0 \leq \text { disp }< \\
30.0\end{array}$ & $<2,000$ & - & & - & - & 11.0 (ABT) & 0.27 (ABT) & 5.0 & \\
\hline & & \multirow{6}{*}{$4^{m, p}$} & All & $\begin{array}{c}600 \leq \mathrm{kW}< \\
1,400\end{array}$ & - & $2017+$ & 1.8 (ABT) & - & $0.19 \mathrm{HC}^{n}$ & 0.04 (ABT) & \multirow[b]{6}{*}{5.0} & \multirow{6}{*}{$10 / 20,000$} \\
\hline & & & All & $\begin{array}{c}1400 \leq \mathrm{kW} \\
<2,000\end{array}$ & - & $2016+$ & 1.8 (ABT) & - & $0.19 \mathrm{HC}^{\mathrm{n}}$ & 0.04 (ABT) & & \\
\hline & & & All & $\begin{array}{c}2,000 \leq \mathrm{kW} \\
<3,700^{9}\end{array}$ & - & $2014+$ & 1.8 (ABT) & - & $0.19 \mathrm{HC}^{\mathrm{n}}$ & 0.04 (ABT) & & \\
\hline & & & $<15.0$ & & - & 2014-2015 & 1.8 (ABT) & - & $0.19 \mathrm{HC}^{n}$ & 0.12 (ABT) & & \\
\hline & & & $\begin{array}{c}15.0 \leq \text { dis } p< \\
30.0\end{array}$ & $\geq 3,700$ & - & 2014-2015 & 1.8 (ABT) & - & $0.19 \mathrm{HC}^{n}$ & 0.25 (ABT) & & \\
\hline & & & All & & - & $2016+$ & 1.8 (ABT) & - & $0.19 \mathrm{HC}^{\mathrm{n}}$ & 0.06 (ABT) & & \\
\hline & \multirow{9}{*}{ C3 } & \multirow{3}{*}{1} & & & $\mathrm{rpm}<130$ & & 17.0 & - & - & - & - & \multirow{3}{*}{$3 / 10,000$} \\
\hline & & & $\$ 0.0$ & All & $\begin{array}{c}130 \leq \mathrm{rpm}< \\
2,000\end{array}$ & 2004 & $45.0 \times \mathrm{N}^{0.20 i}$ & - & - & - & - & \\
\hline & & & & & $\mathrm{rpm} \geq 2,000$ & & 9.8 & - & - & - & - & \\
\hline & & & & & $\mathrm{rpm}<130$ & & 14.4 & & - & - & & \\
\hline & & 2 & $\$ 0.0$ & All & \begin{tabular}{|c|}
$130 \leq \mathrm{rpm}<$ \\
2,000
\end{tabular} & 2011 & $44.0 \times \mathrm{N}^{0.23 i}$ & 2.0 & - & - & 5.0 & $3 / 10,000$ \\
\hline & & & & & $\mathrm{rpm} \geq 2,000$ & & 7.7 & & - & - & & \\
\hline & & & & & $\mathrm{rpm}<130$ & & 3.4 & & - & - & & \\
\hline & & 3 & $\geq 30.0$ & All & \begin{tabular}{|c|}
$130 \leq \mathrm{rpm}<$ \\
2,000
\end{tabular} & 2016 & $9.0 \times \mathrm{N}^{0.201}$ & 2.0 & - & - & 5.0 & $3 / 10,000$ \\
\hline & & & & & $\mathrm{rpm} \geq 2,000$ & & 2.0 & & - & - & & \\
\hline
\end{tabular}

\section{Sources:}

40 CFR $89.104=$ Tiers 1 and 2 useful life \& warranty period for marine CI engines less than $37 \mathrm{~kW}$

40 CFR $89.112=$ Tiers 1 and 2 emission standards for marine CI engines less than $37 \mathrm{~kW}$

$40 \mathrm{CFR} 89$ Subpart $\mathrm{E}=$ Tiers 1 and 2 test procedures for marine $\mathrm{CI}$ engines less than $37 \mathrm{~kW}$

40 CFR $94.8=$ Tiers 1 and 2 emission standards for $\mathrm{C} 1$ (both commercial \& recreational), C2 and C 3 engines

40 CFR 94.9 = Tiers 1 and 2 useful life for C1 (both commercial \& recreational), C2 and C3 engines

40 CFR 94 Subpart B = Tiers 1 and 2 test procedures for $\mathrm{C} 1$ (both commercial \& recreational), $\mathrm{C} 2$ and $\mathrm{C} 3$ engines

40 CFR 1042.101 = Tiers 3 and 4 exhaust emission standards and useful life 


\title{
Table 12.25 (continued) Marine Compression-Ignition (CI) Engines - Exhaust Emission Standards
}

\author{
Sources (continued): \\ 40 CFR 1042.107 = Tiers 3 and 4 evaporative emission standards engines using a volatile liquid fuel (e.g., \\ methanol) \\ 40 CFR $1042.120=$ Tiers 3 and 4 warranty period \\ 40 CFR 1042 Subpart F = Tiers 3 and 4 test procedures (Additional resources: www.epa.gov/otaq/standards)
}

\footnotetext{
${ }^{\mathrm{a}}$ For Tiers 1 and 2, Category 1 marine engines are greater than or equal to 37 kilowatts $(\mathrm{kW})$ and have a displacement less than 5.0 liters per cylinder (L/cylinder); Category 2 marine engines have a displacement greater than or equal to $5.0 \mathrm{~L} /$ cylinder and less than $30 \mathrm{~L} /$ cylinder; and Category 3 marine engines have a displacement greater than or equal to $30.0 \mathrm{~L} /$ cylinder. For Tiers 3 and 4, Category 1 represents engines up to 7 L/cylinder displacement; and Category 2 includes engines from 7 to $30 \mathrm{~L} /$ cylinder. The definition of Category 3 marine engines remains the same.

b Tiers 1 and 2 for marine engines less than $37 \mathrm{~kW}$ are subject to the same emission standards as for landbased engines. See Table 1 in 40 Code of Federal Regulations (CFR) Part 89.112 and 40 CFR Part 89.104.

${ }^{\mathrm{c}}$ For Tiers 1 and 2, this refers to the rated power; for Tiers 3 and 4, this refers to the maximum engine power.

d Total hydrocarbon (THC) plus nitrogen oxides (NOx) for Tier 2 standards.

${ }^{\mathrm{e}}$ Useful life is expressed in hours or years, whichever comes first. For Tiers 3 and 4, a longer useful life in hours for an engine family must be specified if either:1) the engine is designed, advertised, or marketed to operate longer than the minimum useful life; or 2) the basic mechanical warranty is longer than the minimum useful life.

${ }^{\mathrm{f}}$ Warranty period is expressed in years and hours, whichever comes first.

${ }^{\mathrm{g}}$ For Tiers 3 and 4, there are no evaporative emission standards for diesel-fueled engines, or engines using other nonvolatile or nonliquid fuels (e.g., natural gas). If an engine uses a volatile liquid fuel, such as methanol, the engine's fuel system and the vessel in which the engine is installed must meet the evaporative emission requirements of 40 Code of Federal Regulations (CFR) Part 1045 that apply with respect to spark-ignition engines. Manufacturers subject to evaporative emission standards must meet the requirements of 40 CFR 1045.112 as described in 40 CFR 1060.1(a)(2).

${ }^{\mathrm{h}}$ Indicates the model years for which the specified standards start.

${ }^{\mathrm{i}} \mathrm{N}$ is the maximum test speed of the engine in revolutions per minute (rpm).

${ }^{\mathrm{j}}$ Manufacturers of Tier 3 engines greater than or equal to $19 \mathrm{~kW}$ and less than $75 \mathrm{~kW}$ with displacement below $0.9 \mathrm{~L} /$ cylinder may alternatively certify some or all of their engine families to a particulate matter (PM) emission standard of 0.20 grams per kilowatt-hour (g/kW-hr) and a NOx+HC emission standard fo $5.8 \mathrm{~g} / \mathrm{kW}-\mathrm{hr}$ for 2014 and later model years.

${ }^{\mathrm{k}}$ The applicable Tier $2 \mathrm{NOx}+\mathrm{HC}$ standards continue to apply instead of the Tier 3 values for engines at or above $2000 \mathrm{~kW}$.

${ }^{1}$ These Tier 3 standards apply to Category 1 engines below $3700 \mathrm{~kW}$ except for recreational marine engines at or above $3700 \mathrm{~kW}$ (with any displacement), which must meet the Tier 3 standards specified for recreational marine engines with a displacement of 3.5 to $7.0 \mathrm{~L} /$ cylinder.

${ }^{\mathrm{m}}$ The following provisions are optional: 1)Manufacturers may use NOx credits to certify Tier 4 engines to a $\mathrm{NOX}+\mathrm{HC}$ emission standard of $1.9 \mathrm{~g} / \mathrm{kW}-\mathrm{hr}$ instead of the NOX and HC standards. See 40 CFR 1042.101(a)(8)(i) for more details. 2) For engines below $1000 \mathrm{~kW}$, manufacturers may delay complying with the Tier 4 standards until October 1, 2017. 3) For engines at or above $3700 \mathrm{~kW}$, manufacturers may delay complying with the Tier 4 standards until December 31, 2016.

${ }^{\mathrm{n}}$ The Tier 4 standard is for $\mathrm{HC}$ (not HC+NOx) in $\mathrm{g} / \mathrm{kW}-\mathrm{hr}$.

${ }^{\circ}$ These Tier 3 standards apply to Category 2 engines below $3700 \mathrm{~kW}$; no Tier 3 standards apply for Category 2 engines at or above $3700 \mathrm{~kW}$, although there are Tier 4 standards that apply.
} 
Table 12.25 (continued)

Marine Compression-Ignition (CI) Engines - Exhaust Emission Standards

${ }^{\mathrm{p}}$ An alternative set of Tier 3 and Tier 4 standards for PM, NOx, and HC are available for Category 2 engines at or above $1400 \mathrm{~kW}$, but must be applied to all of a manufacturer's engines in a given displacement category in model years 2012 through 2015 .

\begin{tabular}{cccccc}
\hline & $\begin{array}{c}\text { Maximum } \\
\text { engine } \\
\text { power }\end{array}$ & $\begin{array}{c}\text { Model } \\
\text { year }\end{array}$ & $\begin{array}{c}\text { PM } \\
(\mathrm{g} / \mathrm{kW}-\mathrm{hr})\end{array}$ & $\begin{array}{c}\text { NOx } \\
(\mathrm{g} / \mathrm{kW}-\mathrm{hr})\end{array}$ & $\begin{array}{c}\mathrm{HC} \\
(\mathrm{g} / \mathrm{kW}-\mathrm{hr})\end{array}$ \\
\hline 3 & $\mathrm{~kW} \geq 1400$ & $2012-2014$ & 0.14 & \multicolumn{2}{c}{$7.8 \mathrm{NOx}+\mathrm{HC}$} \\
4 & $1400 \leq \mathrm{kW}<3700$ & 2015 & 0.04 & 1.8 & 0.19 \\
& $\mathrm{~kW} \geq 3700$ & 2015 & 0.06 & 1.8 & 0.19 \\
\hline
\end{tabular}

q Interim Tier 4 PM standards apply for 2014 and 2015 model year Category 2 engines with per-cylinder displacement at or above 15.0 liters: $0.34 \mathrm{~g} / \mathrm{kW}$-hr for engines $2000=\mathrm{kW}<3000$, and $0.27 \mathrm{~g} / \mathrm{kW}$-hr for engines $3300=\mathrm{kW}<3700$. 
These standards apply to gasoline boats and personal watercraft, such as pleasure boats, jet-skis, outboard engines and sterndrive/inboard engines.

Table 12.26

Marine Spark-Ignition Engines and Vessels - Exhaust Emission Standards

\begin{tabular}{|c|c|c|c|c|c|c|c|c|}
\hline & & & \multirow[b]{2}{*}{$\begin{array}{c}\text { Model } \\
\text { year }\end{array}$} & \multicolumn{2}{|c|}{$\begin{array}{l}\mathrm{HC}+\mathrm{NOx}^{\mathrm{a}} \\
(\mathrm{g} / \mathrm{KW}-\mathrm{hr})\end{array}$} & \multicolumn{2}{|c|}{$\begin{array}{c}\mathrm{CO}^{\mathrm{c}} \\
(\mathrm{g} / \mathrm{KW}-\mathrm{hr})\end{array}$} & \multirow[b]{2}{*}{$\begin{array}{c}\text { Useful life } \\
\text { (hours/years) }^{\mathrm{d}}\end{array}$} \\
\hline & \multicolumn{2}{|c|}{ Engine type } & & $\mathrm{P} \leq 4.3 \mathrm{~kW}^{\mathrm{b}}$ & $\mathrm{P}>4.3 \mathrm{~kW}^{\mathrm{b}}$ & $\begin{array}{c}\mathrm{P} \leq 4.3 \\
\mathrm{~kW}^{\mathrm{b}}\end{array}$ & $\begin{array}{c}\mathrm{P}>4.3 \\
\mathrm{~kW}^{\mathrm{b}}\end{array}$ & \\
\hline \multirow{14}{*}{ Federal $^{\mathrm{e}}$} & \multirow{10}{*}{\multicolumn{2}{|c|}{$\begin{array}{l}\text { Personal watercraft \& } \\
\text { outboard marine engines }\end{array}$}} & 1998 & $278 \mathrm{ABT}$ & $\begin{array}{c}(0.917 \times(151+ \\
\left.557 / \mathrm{P}^{0.9}+2.44\right) \\
{[\mathrm{ABT}]}\end{array}$ & -- & -- & \multirow{9}{*}{$350 / 5$} \\
\hline & & & 1999 & $253 \mathrm{ABT}$ & $\begin{array}{c}(0.833 \times(151+ \\
\left.557 / \mathrm{P}^{0.9}+2.89\right) \\
{[\mathrm{ABT}]}\end{array}$ & -- & -- & \\
\hline & & & 2000 & $228 \mathrm{ABT}$ & $\begin{array}{c}(0.750 \times(151+ \\
\left.557 / \mathrm{P}^{0.9}\right)+3.33 \\
{[\mathrm{ABT}]}\end{array}$ & -- & -- & \\
\hline & & & 2001 & $204 \mathrm{ABT}$ & $\begin{array}{c}(0.667 \times(151+ \\
\left.557 / \mathrm{P}^{0.9}\right)+3.78 \\
{[\mathrm{ABT}]}\end{array}$ & -- & -- & \\
\hline & & & 2002 & $179 \mathrm{ABT}$ & $\begin{array}{c}(0.583 \times(151+ \\
\left.557 / \mathrm{P}^{0.9}\right)+4.22 \\
{[\mathrm{ABT}]}\end{array}$ & -- & -- & \\
\hline & & & 2003 & $155 \mathrm{ABT}$ & $\begin{array}{c}(0.500 \times(151+ \\
\left.557 / \mathrm{P}^{0.9}\right)+4.67 \\
{[\mathrm{ABT}]}\end{array}$ & -- & -- & \\
\hline & & & 2004 & $130 \mathrm{ABT}$ & $\begin{array}{c}(0.417 \times(151+ \\
\left.557 / \mathrm{P}^{0.9}\right)+5.11 \\
{[\mathrm{ABT}]}\end{array}$ & -- & -- & \\
\hline & & & 2005 & $105 \mathrm{ABT}$ & $\begin{array}{c}(0.333 \times(151+ \\
\left.557 / \mathrm{P}^{0.9}\right)+5.56 \\
{[\mathrm{ABT}]}\end{array}$ & -- & -- & \\
\hline & & & $\begin{array}{l}2006- \\
2009\end{array}$ & $81 \mathrm{ABT}$ & $\begin{array}{c}(0.250 \times(151+ \\
\left.557 / \mathrm{P}^{0.9}\right)+6.00 \\
{[\mathrm{ABT}]}\end{array}$ & -- & -- & \\
\hline & & & $2010+^{\mathrm{g}}$ & $\begin{array}{l}30 \mathrm{ABT} \\
{[\mathrm{ABT}]}\end{array}$ & $\begin{array}{c}2.1+0.09 \mathrm{x} \\
(151+ \\
\left.557 / \mathrm{P}^{0.9}\right) \\
{[\mathrm{ABT}]}\end{array}$ & $\begin{array}{l}500-5.0 \\
\quad x P\end{array}$ & 300 & $\begin{array}{c}\text { Personal } \\
\text { Watercraft: } 350 \\
/ 5^{\mathrm{h}} \\
\text { Outboard: } 350 \\
/ 10^{\mathrm{h}}\end{array}$ \\
\hline & \multirow{4}{*}{$\begin{array}{l}\text { Sterndrive/ } \\
\text { inboard } \\
\text { engines }\end{array}$} & $\begin{array}{c}\text { Conventional } \\
\text { engines }\end{array}$ & $2010+$ & & & {$[\mathrm{A}]$} & & $480 / 10^{\mathrm{i}}$ \\
\hline & & \multirow{3}{*}{$\begin{array}{l}\text { High- } \\
\text { performance } \\
\text { engines }\end{array}$} & & $\mathrm{P} \leq \mathrm{kW}^{\mathrm{b}}$ & $\mathrm{P}>485 \mathrm{~kW}^{\mathrm{b}}$ & \multirow{3}{*}{\multicolumn{2}{|c|}{350}} & $\begin{array}{c}\mathrm{P} \leq 485 \mathrm{~kW}: \\
150 / 3\end{array}$ \\
\hline & & & 2010 & 20.0 & 25.0 & & & $\begin{array}{c}\mathrm{P}>485 \mathrm{~kW}: \\
50 / 1\end{array}$ \\
\hline & & & $2011+$ & 16.0 & 22.0 & & & \\
\hline
\end{tabular}

\section{Sources:}

40 CFR 91.104 = Outboard and personal watercraft (PWC) exhaust emission standards (1998-2009)

40 CFR 91.105 = Outboard and PWC useful life (1998-2009)

40 CFR 1045.103 = Outboard and PWC exhaust emission standards (2010+)

40 CFR 1045.105 = Sterndrive/Inboard exhaust emission standards

40 CFR 1045.107 = Not-to-exceed exhaust emission standards (Additional resources: www.epa.gov/otaq/standards)

${ }^{\mathrm{a}}$ The numerical emission standards for hydrocarbons (HC) must be met based on the following types of HC emissions for engines powered by the following fuels: (1) total hydrocarbon equivalent for alcohol; (2) non-methane hydrocarbon for natural gas; and (3) total hydrocarbons for other fuels. 
Table 12.26 (continued) Marine Spark-Ignition Engines and Vessels - Exhaust Emission Standards

stands for the maximum engine power in kilowatts.

${ }^{c}$ Manufacturers may generate or use emission credits for averaging, but not for banking or trading.

${ }^{\mathrm{d}}$ Useful life and warranty period are expressed hours or years of operation (unless otherwise indicated), whichever comes first.

${ }^{\mathrm{e}}$ The test procedure for federal standards uses the International Organization for Standardization (ISO) 8178 E4 5-Mode Steady-State Test Cycle.

${ }^{\mathrm{f}}$ Also applies to model year (MY) 1997 engine families certified pursuant to 40 Code of Federal Regulations (CFR) 91.205.

${ }^{g}$ Not-to-exceed emission standards specified in 40 CFR 1045.107 also apply.

${ }^{\mathrm{h}}$ A longer useful life in terms of hours must be specified for the engine family if the average service life is longer than the minimum value as described in 40 CFR 1045.103(e)(3).

${ }^{i}$ The useful life may not be shorter than: (1) 150 hours of operation; (2) the recommended overhaul interval; or (3) the engine's mechanical warranty. A longer useful life must be specified in terms of hours if the average service life is longer than the minimum value as described in 40 CFR 1045.105(e)(3). 
These standards apply to land-based recreational vehicles, such as snowmobiles, dirt bikes, all-terrain vehicles and go-karts.

Table 12.27

Nonroad Recreational Engines and Vehicles - Exhaust Emission Standards

\begin{tabular}{|c|c|c|c|c|c|c|c|c|}
\hline & & & & $\mathrm{HC}^{\mathrm{a}}$ & $\begin{array}{l}\mathrm{HC}+ \\
\mathrm{NOx}\end{array}$ & \multicolumn{2}{|c|}{$\mathrm{CO}$} & \multirow{2}{*}{$\begin{array}{l}\text { Minimum useful life } \\
\text { (hours/years/km) }\end{array}$} \\
\hline & Vehicle & Phase & Year & $\mathrm{g} / \mathrm{kW}-\mathrm{hr}$ & $\mathrm{g} / \mathrm{km}$ & $\mathrm{g} / \mathrm{kW}-\mathrm{hr}$ & $\mathrm{g} / \mathrm{km}$ & \\
\hline \multirow{5}{*}{ Federal } & \multirow{3}{*}{ Snowmobiles $^{c}$} & $1^{\mathrm{d}}$ & $2006+$ & $\begin{array}{c}100 \\
{[\mathrm{ABT}]}\end{array}$ & - & $\begin{array}{c}275 \\
{[\mathrm{ABT}]}\end{array}$ & - & \multirow{3}{*}{$400 / 5 / 8,000$} \\
\hline & & 2 & $\begin{array}{r}2010- \\
2011\end{array}$ & 75 [ABT] & - & $\begin{array}{c}275 \\
{[\mathrm{ABT}]}\end{array}$ & - & \\
\hline & & $3^{\mathrm{e}}$ & $2012+$ & $\begin{array}{c}150^{\mathrm{f}} \\
{[\mathrm{ABT}]}\end{array}$ & - & $\begin{array}{c}400^{\mathrm{f}} \\
{[\mathrm{ABT}]}\end{array}$ & - & \\
\hline & $\begin{array}{l}\text { Off-highway } \\
\text { motorcycles }^{\mathrm{g}}\end{array}$ & $1^{\mathrm{d}}$ & $2006+$ & - & $\begin{array}{c}2.0^{\mathrm{h}, \mathrm{i}} \\
{[\mathrm{ABT}]}\end{array}$ & - & $\begin{array}{c}25^{\mathrm{h}, \mathrm{i}} \\
{[\mathrm{ABT}]}\end{array}$ & $\begin{array}{c}>70 \mathrm{cc} \\
\text { Displacement: }- \text { / } 5 \text { / } \\
10,000 \\
\leq 70 \mathrm{cc} \\
\text { Displacement: - / } 5 \text { / } \\
5,000\end{array}$ \\
\hline & $\mathrm{ATVs}^{\mathrm{g}}$ & $1^{\mathrm{d}}$ & $2006+$ & - & $\begin{array}{c}1.5^{\mathrm{j}, \mathrm{k}} \\
{[\mathrm{ABT}]}\end{array}$ & - & $\begin{array}{c}35^{\mathrm{k}} \\
{[\mathrm{ABT}]}\end{array}$ & $\begin{array}{c}\geq 100 \mathrm{cc} \\
\text { Displacement: } 1000 / \\
5 / 10,000 \\
<100 \mathrm{cc} \\
\text { Displacement: } 500 / \\
5 / 5,000\end{array}$ \\
\hline
\end{tabular}

Sources:

40 CFR 1051.101-115 = Emission standards (Additional resources: www.epa.gov/otaq/standards)

${ }^{a}$ The numerical emission standards for hydrocarbons (HC) must be met based on the following types of hydrocarbon emissions for recreational engines and vehicles powered by the following fuels: (1) non-methane hydrocarbons for natural gas; (2) total hydrocarbon equivalent for alcohol; and (3) total hydrocarbons for other fuels.

${ }^{\mathrm{b}}$ Useful life is expressed in hours, years, or kilometers, whichever comes first; warranty period is expressed in hours, months, or kilometers $(\mathrm{km})$, whichever comes first. Nonroad recreational engines and vehicles must meet emission standards over their full useful life. A longer useful life in terms of $\mathrm{km}$ and hours must be specified for the engine family if the average service life is longer than the minimum value as described in 40 Code of Federal Regulations (CFR) 1051 Subpart B.

${ }^{\mathrm{c}}$ Test procedures for snowmobiles use the equipment and procedures for spark-ignition engines in $40 \mathrm{CFR}$ Part 1065.

${ }^{\mathrm{d}}$ Phase 1 standards will be phased in: 50 percent by 2006, 100 percent by 2007.

${ }^{\mathrm{e}}$ Litigation on the November 2002 final rule resulted in a court decision that requires EPA to clarify the evidence and analysis upon which the Phase 3 carbon monoxide (CO) and HC standards were based. EPA will address this in a future rulemaking.

${ }^{\mathrm{f}}$ These are the maximum allowable family emission limits (FEL). The HC and CO standards are defined by a functional relationship as described in 40 CFR 1051.103(a)(2).

${ }^{\mathrm{g}}$ For off-highway motorcycles and ATVs, chassis dynamometer emissions test procedures are specified in 40 CFR Part 86, Subpart F and engine dynamometer emissions test procedures are specified in 40 CFR Part 1065.

${ }^{\mathrm{h}}$ Maximum allowable FEL: 20.0 grams per kilometer $(\mathrm{g} / \mathrm{km}$ ) for $\mathrm{HC}$ plus nitrogen oxides (NOx) and $50 \mathrm{~g} / \mathrm{km}$ for $\mathrm{CO}$. 
Table 12.27 (continued) Nonroad Recreational Engines and Vehicles - Exhaust Emission Standards

${ }^{\mathrm{i}}$ Manufacturers may certify off-highway motorcycles with engines that have total displacement of 70 cubic centimeters (cc) or less to an HC+NOx standard of 16.1 grams per kilowatt-hour (g/kW-hr) (with an FEL cap of $32.2 \mathrm{~g} / \mathrm{kW}$-hr) and a CO standard of $519 \mathrm{~g} / \mathrm{kW}-\mathrm{hr}$.

${ }_{\mathrm{j}}^{\mathrm{j}}$ Maximum allowable FEL for HC+NOx is $20.0 \mathrm{~g} / \mathrm{km}$.

${ }^{\mathrm{k}}$ Manufacturers may certify all-terrain vehicles with engines that have total displacement of less than $100 \mathrm{cc}$ to an $\mathrm{HC}+\mathrm{NOx}$ standard of $25.0 \mathrm{~g} / \mathrm{KW}-\mathrm{hr}$ (with an FEL cap of $40.0 \mathrm{~g} / \mathrm{kW}-\mathrm{hr}$ ) and a CO standard of $500 \mathrm{~g} / \mathrm{kW}-\mathrm{hr}$. 
The latest standards were established by the Environmental Protection Agency in conjunction with the Tier 3 emission standards.

Table 12.28

Gasoline Sulfur Standards

\begin{tabular}{|c|c|c|c|c|c|c|c|c|}
\hline & \multirow[b]{2}{*}{ Regulated entity } & \multicolumn{7}{|c|}{ Refinery average and per-gallon cap by year (ppm) } \\
\hline & & 2004 & 2005 & 2006 & 2007 & $2008-2016$ & $2017-2019$ & 2020 \\
\hline \multirow{4}{*}{ Federal } & $\begin{array}{c}\text { Large refiners / } \\
\text { importers }^{\mathrm{a}}\end{array}$ & $120^{\mathrm{b}} / 300^{\mathrm{c}}$ & $30 / 90^{\mathrm{b}} / 300$ & $30 / 80$ & $30 / 80$ & $30 / 80$ & $10 / 80$ & $10 / 80$ \\
\hline & $\begin{array}{l}\text { GPA } \\
\text { refiners }\end{array}$ & $150 / 300^{\mathrm{c}}$ & $150 / 300$ & $150 / 300$ & $30 / 80$ & $30 / 80$ & $30 / 80$ & $10 / 80$ \\
\hline & $\begin{array}{c}\text { Small } \\
\text { refiners } f, g, h\end{array}$ & $\mathrm{k}$ & $\mathrm{k}$ & $\mathrm{k}$ & $\mathrm{k}$ & $30 / 80$ & $30 / 80$ & $10 / 80$ \\
\hline & $\begin{array}{l}\text { Downstream } \\
\text { standards } \mathrm{i}, \mathrm{j}\end{array}$ & 378 & 326 & 95 & 95 & 95 & 95 & 95 \\
\hline
\end{tabular}

Source:

40 CFR Part 80 Subpart H (Additional resources: www.epa.gov/otaq/standards)

${ }^{\text {a }}$ Standards effective January 1 at the refinery gate.

b No Refinery Average Standard applies in 2004; Corporate Average Standard applies in 2004 (120 ppm) and 2005 (90 ppm).

${ }^{\mathrm{c}}$ Cap exceedances up to 50 ppm in 2004 must be made up in 2005.

${ }^{\mathrm{d}}$ Geographic Phase-in Area (GPA) refiners must also comply with the corporate average standards in 2004 and 2005 if less than 50\% of the refiner's gasoline is designated as GPA gasoline in a given compliance period.

${ }^{\text {e }}$ GPA refiners may receive an additional two years (i.e., through 2008) to comply with the $30 / 80 \mathrm{ppm}$ gasoline sulfur standards in exchange for producing $95 \%$ of their highway diesel fuel at the 15 ppm sulfur standard by June 1, 2006.

${ }^{\mathrm{f}}$ Small refiners may receive an additional two years (i.e., through 2009) to comply with the $30 / 80 \mathrm{ppm}$ gasoline sulfur standards via a hardship demonstration.

${ }^{g}$ Small refiners may receive an additional three years (i.e., through 2010) to comply with the $30 / 80 \mathrm{ppm}$ gasoline sulfur standards in exchange for producing $95 \%$ of their highway diesel fuel at the 15 ppm sulfur standard by June 1, 2006.

${ }^{\mathrm{h}}$ Small refiners may receive a $20 \%$ increase in their annual average and per-gallon cap standards in exchange for producing $95 \%$ of their highway, nonroad, locomotive, and marine diesel fuel at the 15 ppm sulfur standard by June 1, 2006.

${ }^{\mathrm{i}}$ Downstream standards are effective February 1 at any downstream location other than at a retail outlet or wholesale purchaser-consumer (e.g., pipelines and terminals) and March 1 at any downstream location.

${ }^{\mathrm{j}}$ Downstream standards for gasoline that is not blended with small refiner gasoline are shown. Refer to the Code of Federal Regulations (CFR) for the downstream standards that apply when a gasoline blend includes small refiner gasoline.

\begin{tabular}{|c|c|c|}
\hline \multirow{2}{*}{$\begin{array}{c}\text { 1997-98 Refinery } \\
\text { baseline sulfur level } \\
(\mathrm{ppm})\end{array}$} & \multicolumn{2}{|c|}{$\begin{array}{l}\text { Small refiner interim gasoline sulfur standards } \\
\qquad(\mathrm{ppm}) 2004-2007\end{array}$} \\
\hline & Average & Cap \\
\hline 0 to 30 & 30 & 300 \\
\hline 31 to 200 & baseline level & 300 \\
\hline 201 to 400 & 200 & 300 \\
\hline 401 to 600 & $50 \%$ of baseline & $1.5 \mathrm{x}$ avg. standard \\
\hline 601 and above & 300 & 450 \\
\hline
\end{tabular}


Ultra-low sulfur diesel (ULSD) fuel is necessary for new advanced emission control technologies. It also reduces particulate matter in the existing fleet of nonroad engines and equipment.

Table 12.29

Highway, Nonroad, Locomotive, and Marine (NRLM) Diesel Fuel Sulfur Standards

\begin{tabular}{|c|c|c|c|c|c|c|c|c|c|c|c|}
\hline & \multirow[b]{2}{*}{ Regulated entity } & \multirow{2}{*}{$\begin{array}{l}\text { Covered } \\
\text { fuel }\end{array}$} & \multicolumn{9}{|c|}{ Per-gallon maximum sulfur level by year (ppm) } \\
\hline & & & $2006^{\mathrm{a}}$ & $2007^{\mathrm{b}}$ & 2008 & 2009 & $2010^{\mathrm{c}, \mathrm{d}}$ & 2011 & 2012 & 2013 & 2014 \\
\hline \multirow{8}{*}{ Federal } & $\begin{array}{l}\text { Large refiners \& } \\
\text { importers }\end{array}$ & Highway & \multicolumn{4}{|c|}{$\begin{array}{c}80 \% 15 \\
20 \% 500\end{array}$} & \multirow{2}{*}{\multicolumn{5}{|c|}{15}} \\
\hline & Small refiners & Highway & \multicolumn{4}{|c|}{500} & & & & & \\
\hline & \multirow{3}{*}{$\begin{array}{l}\text { Large refiners \& } \\
\text { importers }\end{array}$} & NR & - & 500 & 500 & 500 & 15 & 15 & 15 & 15 & 15 \\
\hline & & $\mathrm{LM}$ & - & 500 & 500 & 500 & 500 & 500 & 15 & 15 & 15 \\
\hline & & $\begin{array}{l}\text { NRLM } \\
\text { with } \\
\text { credits }^{\mathrm{e}}\end{array}$ & - & HS & HS & HS & 500 & 500 & 500 & 500 & 15 \\
\hline & Small refiners & NRLM $^{\mathrm{f}}$ & - & HS & HS & HS & 500 & 500 & 500 & 500 & 15 \\
\hline & \multirow{2}{*}{$\begin{array}{c}\text { Transmix } \\
\text { processor \& in-use }\end{array}$} & $\mathrm{NR}^{\mathrm{e}}$ & - & HS & HS & HS & 500 & 500 & 500 & 500 & 15 \\
\hline & & $\mathrm{LM}^{\mathrm{e}}$ & - & HS & HS & HS & 500 & 500 & 500 & 500 & 500 \\
\hline
\end{tabular}

Source:

40 CFR Part 80 Subpart I (Additional resources: www.epa.gov/otaq/standards)

${ }^{a}$ For highway diesel fuel, standards are effective June 1 for refiners/importers, September 1 for pipelines and terminals, and October 15 for retailers and wholesale purchaser-consumers. Anti-downgrading provisions effective October 16, 2006.

b For Nonroad, Locomotive, and Marine (NRLM) diesel fuel, standards are effective June 1 for refiners; downstream requirements apply for Northeast/Mid-Atlantic area only (August 1 for terminals, October 1 for retailers and wholesale purchaser-consumers, and December 1 for in-use).

${ }^{c}$ For highway diesel fuel, standards are effective June 1 for refiners/importers, October 1 for pipelines and terminals, and December 1 for retailers and wholesale purchaser-consumers.

${ }^{\mathrm{d}}$ For NRLM diesel fuel, standards are effective June 1 for refiners, August 1 for terminals, October 1 for retailers and wholesale purchaser-consumers, and December 1 for in-use.

${ }^{\mathrm{e}}$ Excluding the Northeast and Alaska.

${ }^{\mathrm{f}}$ Excluding the Northeast, with approval in Alaska. 
$12-40$

TRANSPORTATION ENERGY DATA BOOK: EDITION 33-2014 
APPENDIX A

SOURCES \& METHODOLOGIES 
A-2

TRANSPORTATION ENERGY DATA BOOK: EDITION 33-2014 


\section{SOURCES \& METHODOLOGIES}

This appendix contains documentation of the estimation procedures used by ORNL. The reader can examine the methodology behind the estimates and form an opinion as to their utility. The appendix is arranged by subject heading. Only tables which contain ORNL estimations are documented in Appendix A; all other tables have sources listed at the bottom of the table. Since abbreviations are used throughout the appendix, a list of abbreviations is also included.

\section{Contents of Appendix A}

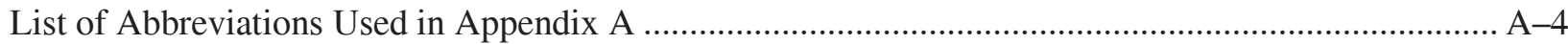

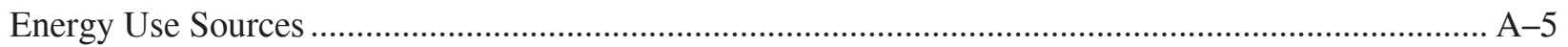

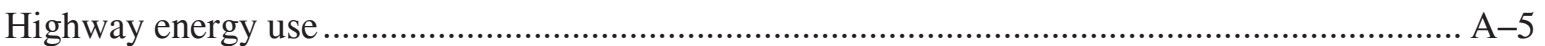

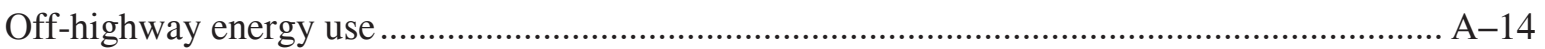

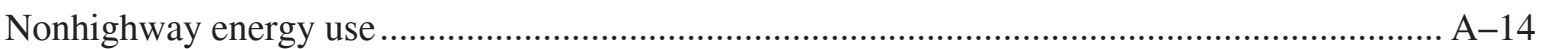

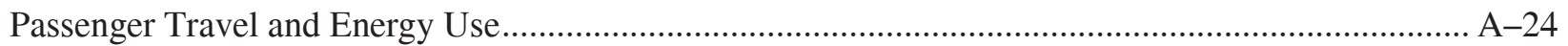

Highway Passenger Mode Energy Intensities ................................................................................. A-28

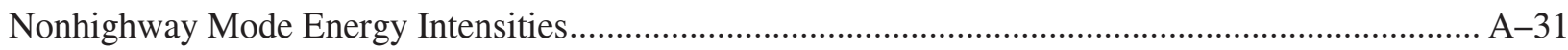

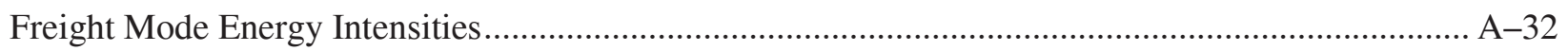

Car/Light Truck Shares ............................................................................................................. A -33 


\section{List of Abbreviations Used in Appendix A}

$\begin{array}{ll}\text { AAMA } & \text { American Automobile Manufacturers Association } \\ \text { AAR } & \text { Association of American Railroads } \\ \text { APTA } & \text { American Public Transportation Association } \\ \text { Amtrak } & \text { National Railroad Passenger Corporation } \\ \text { Btu } & \text { British thermal unit } \\ \text { DOC } & \text { Department of Commerce } \\ \text { DOE } & \text { Department of Energy } \\ \text { DOT } & \text { Department of Transportation } \\ \text { EIA } & \text { Energy Information Administration } \\ \text { EPA } & \text { Environmental Protection Agency } \\ \text { FAA } & \text { Federal Aviation Administration } \\ \text { FHWA } & \text { Federal Highway Administration } \\ \text { GSA } & \text { General Services Administration } \\ \text { gvw } & \text { gross vehicle weight } \\ \text { lpg } & \text { liquefied petroleum gas } \\ \text { mpg } & \text { miles per gallon } \\ \text { NHTS } & \text { National Household Travel Survey } \\ \text { NHTSA } & \text { National Highway Traffic Safety Administration } \\ \text { NPTS } & \text { Nationwide Personal Transportation Survey } \\ \text { NVPP } & \text { National Vehicle Population Profile } \\ \text { ORNL } & \text { Oak Ridge National Laboratory } \\ \text { pmt } & \text { passenger-miles traveled } \\ \text { RECS } & \text { Residential Energy Consumption Survey } \\ \text { RTECS } & \text { Residential Transportation Energy Consumption Survey } \\ \text { TIUS } & \text { Truck Inventory and Use Survey } \\ \text { TSC } & \text { Trans }\end{array}$




\section{Energy Use Sources}

\section{Highway energy use}

\section{Cars}

Fuel use in gallons (1970-2008) - DOT, FHWA, Highway Statistics 2008, Table VM-1 and annual editions back to 1996; DOT, FHWA, Highway Statistics Summary to 1995.

Fuel use in gallons (2009 - 2012) - See Appendix A for Car/Light Truck Shares.

Fuel type distribution - Fuel use was distributed among fuel types using the percentages shown in Table A.1. The FHWA discontinued gasohol data in 2005. Therefore, data from EIA, Alternatives to Traditional Transportation Fuels, 2006-2011, Table C1 were used. 
Table A.1

Car Fuel Use and Fuel Type Shares for Calculation of Energy Use

\begin{tabular}{|c|c|c|c|c|c|c|}
\hline \multirow[b]{2}{*}{ Year } & \multirow{2}{*}{$\begin{array}{c}\text { Fuel use } \\
\text { (million gallons) }\end{array}$} & \multirow{2}{*}{$\begin{array}{c}\text { Source for } \\
\text { Gasohol shares }\end{array}$} & \multirow{2}{*}{$\begin{array}{c}\text { Source for } \\
\text { gasoline/diesel shares }\end{array}$} & \multicolumn{3}{|c|}{ Shares by fuel type ${ }^{b}$} \\
\hline & & & & Gasoline & Gasohol & Diesel \\
\hline 1970 & 67,820 & & 1984 NVPP & $99.8 \%$ & $0.0 \%$ & $0.2 \%$ \\
\hline 1971 & 71,346 & & interpolated & $99.2 \%$ & $0.0 \%$ & $0.8 \%$ \\
\hline 1972 & 75,937 & & interpolated & $98.7 \%$ & $0.0 \%$ & $1.3 \%$ \\
\hline 1973 & 78,233 & & interpolated & $98.1 \%$ & $0.0 \%$ & $1.9 \%$ \\
\hline 1974 & 74,229 & & interpolated & $97.5 \%$ & $0.0 \%$ & $2.5 \%$ \\
\hline 1975 & 74,140 & & interpolated & $97.0 \%$ & $0.0 \%$ & $3.0 \%$ \\
\hline 1976 & 78,297 & & interpolated & $96.4 \%$ & $0.0 \%$ & $3.6 \%$ \\
\hline 1977 & 79,060 & & interpolated & $95.8 \%$ & $0.0 \%$ & $4.2 \%$ \\
\hline 1978 & 80,652 & & interpolated & $95.3 \%$ & $0.0 \%$ & $4.7 \%$ \\
\hline 1979 & 76,588 & & 1979 RTECS & $94.7 \%$ & $0.0 \%$ & $5.3 \%$ \\
\hline 1980 & 69,981 & FHWA, MF-33e & interpolated & $93.9 \%$ & $0.5 \%$ & $5.6 \%$ \\
\hline 1981 & 69,112 & FHWA, MF-33e & 1981 RTECS & $93.4 \%$ & $0.7 \%$ & $5.9 \%$ \\
\hline 1982 & 69,116 & FHWA, MF-33e & interpolated & $93.5 \%$ & $2.3 \%$ & $4.2 \%$ \\
\hline 1983 & 70,322 & FHWA, MF-33e & 1983 RTECS & $93.2 \%$ & $4.3 \%$ & $2.5 \%$ \\
\hline 1984 & 70,663 & FHWA, MF-33e & interpolated & $92.7 \%$ & $5.3 \%$ & $2.0 \%$ \\
\hline 1985 & 71,518 & FHWA, MF-33e & 1985 RTECS & $90.8 \%$ & $7.7 \%$ & $1.5 \%$ \\
\hline 1986 & 73,174 & FHWA, MF-33e & interpolated & $91.0 \%$ & $7.6 \%$ & $1.4 \%$ \\
\hline 1987 & 73,308 & FHWA, MF-33e & interpolated & $92.4 \%$ & $6.3 \%$ & $1.3 \%$ \\
\hline 1988 & 73,345 & FHWA, MF-33e & 1988 RTECS & $91.4 \%$ & $7.4 \%$ & $1.2 \%$ \\
\hline 1989 & 73,913 & FHWA, MF-33e & interpolated & $92.6 \%$ & $6.2 \%$ & $1.2 \%$ \\
\hline 1990 & 69,568 & FHWA, MF-33e & interpolated & $92.0 \%$ & $6.8 \%$ & $1.2 \%$ \\
\hline 1991 & 64,318 & FHWA, MF-33e & 1991 RTECS & $90.8 \%$ & $8.0 \%$ & $1.2 \%$ \\
\hline 1992 & 65,436 & FHWA, MF-33e & interpolated & $90.8 \%$ & $7.9 \%$ & $1.2 \%$ \\
\hline 1993 & 67,047 & FHWA, MF-33e & interpolated & $89.7 \%$ & $9.1 \%$ & $1.3 \%$ \\
\hline 1994 & 67,874 & FHWA, MF-33e & 1994 RTECS & $89.1 \%$ & $9.6 \%$ & $1.3 \%$ \\
\hline 1995 & 68,072 & FHWA, MF-33e & interpolated & $87.6 \%$ & $11.2 \%$ & $1.2 \%$ \\
\hline 1996 & 69,221 & FHWA, MF-33e & interpolated & $88.8 \%$ & $10.1 \%$ & $1.0 \%$ \\
\hline 1997 & 69,892 & FHWA, MF-33e & interpolated & $86.9 \%$ & $12.2 \%$ & $0.9 \%$ \\
\hline 1998 & 71,695 & FHWA, MF-33e & interpolated & $88.0 \%$ & $11.2 \%$ & $0.8 \%$ \\
\hline 1999 & 73,283 & FHWA, MF-33e & interpolated & $88.3 \%$ & $11.0 \%$ & $0.6 \%$ \\
\hline 2000 & 73,065 & FHWA, MF-33e & 2000 NVPP & $86.9 \%$ & $12.6 \%$ & $0.5 \%$ \\
\hline 2001 & 73,559 & FHWA, MF-33e & 2001 NVPP & $86.5 \%$ & $13.0 \%$ & $0.5 \%$ \\
\hline 2002 & 75,471 & FHWA, MF-33e & 2001 NVPP & $83.9 \%$ & $15.6 \%$ & $0.5 \%$ \\
\hline 2003 & 74,590 & FHWA, MF-33e & 2001 NVPP & $75.3 \%$ & $24.2 \%$ & $0.5 \%$ \\
\hline 2004 & 75,402 & FHWA, MF-33e & 2001 NVPP & $67.2 \%$ & $32.3 \%$ & $0.5 \%$ \\
\hline 2005 & 77,418 & FHWA, MF-33e & 2001 NVPP & $66.9 \%$ & $32.6 \%$ & $0.5 \%$ \\
\hline 2006 & 75,009 & EIA, C1 & 2001 NVPP & $78.2 \%$ & $21.3 \%$ & $0.5 \%$ \\
\hline 2007 & 74,377 & EIA, C1 & 2001 NVPP & $72.9 \%$ & $26.6 \%$ & $0.5 \%$ \\
\hline 2008 & 71,497 & EIA, C1 & 2001 NVPP & $61.8 \%$ & $37.7 \%$ & $0.5 \%$ \\
\hline 2009 & 66,587 & EIA, C1 & 2001 NVPP & $55.8 \%$ & $43.7 \%$ & $0.5 \%$ \\
\hline 2010 & 62,245 & EIA, C1 & 2001 NVPP & $49.5 \%$ & $50.0 \%$ & $0.5 \%$ \\
\hline 2011 & 59,899 & EIA, C1 & 2001 NVPP & $48.7 \%$ & $50.8 \%$ & $0.5 \%$ \\
\hline \multirow[t]{2}{*}{2012} & \multirow{2}{*}{\multicolumn{2}{|c|}{ Heat content used for conversion to btu: }} & 2001 NVPP & $48.6 \%$ & $50.9 \%$ & $0.5 \%$ \\
\hline & & & & $\begin{array}{l}125,000 \\
\text { btu/gallon }\end{array}$ & $\begin{array}{l}120,900 \\
\text { btu/gallon }\end{array}$ & $\begin{array}{l}138,700 \\
\text { btu/gallon }\end{array}$ \\
\hline
\end{tabular}

${ }^{a}$ Data are not continuous between 2008 and 2009 due to changes in source.

${ }^{\mathrm{b}}$ Percentages may not sum due to rounding. 
Motorcycles

DOT, FHWA, Highway Statistics 2012, Table VM-1, and annual editions. The FHWA made methodology changes for Highway Statistics 2009-10. At that time, they published historical data back to 2007 which do not match the previous data.

Table A.2

\section{Motorcycle Fuel Use}

\begin{tabular}{lccc}
\hline Year & $\begin{array}{c}\text { Fuel use } \\
\text { (thousand gallons) }\end{array}$ & Year & $\begin{array}{c}\text { Fuel use } \\
\text { (thousand gallons) }\end{array}$ \\
\hline 1970 & 59,580 & 1992 & 191,140 \\
1971 & 72,140 & 1993 & 198,120 \\
1972 & 86,620 & 1994 & 204,800 \\
1973 & 103,880 & 1995 & 198,262 \\
1974 & 108,900 & 1996 & 195,940 \\
1975 & 112,580 & 1997 & 201,620 \\
1976 & 120,060 & 1998 & 205,660 \\
1977 & 126,980 & 1999 & 211,680 \\
1978 & 143,160 & 2000 & 209,380 \\
1979 & 172,740 & 2001 & 192,780 \\
1980 & 204,280 & 2002 & 191,040 \\
1981 & 213,800 & 2003 & 190,780 \\
1982 & 198,200 & 2004 & 202,447 \\
1983 & 175,200 & 2005 & 189,495 \\
1984 & 175,680 & 2006 & 221,030 \\
1985 & 181,720 & 2007 & 474,923 \\
1986 & 187,940 & 2008 & 489,417 \\
1987 & 190,120 & 2009 & 482,290 \\
1988 & 200,480 & 2010 & 426,732 \\
1989 & 207,420 & 2011 & 426,378 \\
1990 & 191,140 & 2012 & 489,115 \\
1991 & 183,560 & & \\
\hline Heat content used for conversion to btu: & 125,000 btu/gallon \\
\hline \multicolumn{4}{|}{} \\
\hline
\end{tabular}

${ }^{a}$ Data are not continuous between 2006 and 2007 due to changes in estimation methodology. See source document for details. 
Buses

Transit:

APTA, 2014 Public Transportation Fact Book, Washington, DC, 2014. Includes motorbus and trolley bus data.

Table A.3

Transit Bus Fuel Use

\begin{tabular}{|c|c|c|c|c|c|c|c|c|}
\hline Year & $\begin{array}{l}\text { LNG } \\
\text { (million } \\
\text { gallons) }\end{array}$ & $\begin{array}{l}\text { LPG } \\
\text { (million } \\
\text { gallons) }\end{array}$ & $\begin{array}{l}\text { CNG } \\
\text { (million } \\
\text { gallons) }\end{array}$ & $\begin{array}{l}\text { Gasoline } \\
\text { (million } \\
\text { gallons) }\end{array}$ & $\begin{array}{l}\text { Diesel } \\
\text { fuel } \\
\text { (million } \\
\text { gallons) }\end{array}$ & $\begin{array}{c}\text { Electricity } \\
\text { (thousand } \\
\text { kilowatt } \\
\text { hours) }\end{array}$ & $\begin{array}{l}\text { Biodiesel } \\
\text { (million } \\
\text { gallons) }\end{array}$ & $\begin{array}{c}\text { Methanol } \\
\text { (million } \\
\text { gallons) }\end{array}$ \\
\hline 1994 & 1.1 & 0.2 & 3.1 & 2.1 & 565.1 & 102.9 & $\mathrm{a}$ & 12.5 \\
\hline 1995 & 1.7 & 0.3 & 10.0 & 2.3 & 563.8 & 100.0 & $\mathrm{a}$ & 12.0 \\
\hline 1996 & 2.3 & 0.6 & 11.5 & 1.8 & 577.7 & 69.0 & $\mathrm{a}$ & 11.6 \\
\hline 1997 & 3.3 & 1.0 & 20.0 & 2.7 & 597.6 & 78.0 & $\mathrm{a}$ & 8.7 \\
\hline 1998 & 3.1 & 0.9 & 32.6 & 2.0 & 606.6 & 74.0 & $\mathrm{a}$ & 5.0 \\
\hline 1999 & 5.3 & 0.8 & 39.9 & 1.4 & 618.0 & 75.0 & $\mathrm{a}$ & 2.7 \\
\hline 2000 & 10.5 & 0.7 & 50.4 & 1.3 & 635.2 & 77.0 & $\mathrm{a}$ & 0.8 \\
\hline 2001 & 11.7 & 1.2 & 60.9 & 1.5 & 587.2 & 74.0 & $\mathrm{a}$ & 0.8 \\
\hline 2002 & 16.8 & 1.8 & 77.8 & 1.3 & 559.0 & 73.0 & $\mathrm{a}$ & 1.8 \\
\hline 2003 & 14.2 & 1.8 & 94.9 & 1.1 & 536.0 & 69.0 & $\mathrm{a}$ & 1.9 \\
\hline 2004 & 16.5 & 1.7 & 106.7 & 1.8 & 550.5 & 68.0 & $\mathrm{a}$ & 4.7 \\
\hline 2005 & 18.3 & 2.0 & 117.2 & 1.0 & 533.8 & 67.0 & $\mathrm{a}$ & 8.1 \\
\hline 2006 & 19.6 & 1.6 & 138.8 & 2.3 & 536.7 & 62.0 & 20.5 & 0.9 \\
\hline 2007 & 18.3 & $\mathrm{a}$ & 129.1 & 2.5 & 494.1 & 61.0 & 25.8 & 1.3 \\
\hline 2008 & 17.9 & $\mathrm{a}$ & 135.5 & 3.8 & 493.3 & 62.2 & 41.8 & 0.9 \\
\hline 2009 & 25.5 & a & 141.6 & 6.7 & 455.5 & 69.5 & 40.6 & 0.0 \\
\hline 2010 & 23.0 & a & 126.2 & 8.1 & 435.4 & 66.0 & 43.5 & 0.0 \\
\hline 2011 & 21.6 & $\mathrm{a}$ & 131.1 & 8.9 & 455.1 & 61.0 & 51.1 & 0.0 \\
\hline 2012 & 19.6 & a & 127.3 & 12.5 & 439.0 & 61.0 & 56.6 & 0.0 \\
\hline $\begin{array}{l}\text { Heat content used } \\
\text { for conversion } \\
\text { to btu: }\end{array}$ & $\begin{array}{l}84,800 \\
\text { btu/gallon }\end{array}$ & $\begin{array}{l}91,300 \\
\text { btu/gallon }\end{array}$ & $\begin{array}{l}138,700 \\
\text { btu/gallon }\end{array}$ & $\begin{array}{l}125,000 \\
\text { btu/gallon }\end{array}$ & $\begin{array}{l}138,700 \\
\text { btu/gallon }\end{array}$ & $\begin{array}{l}64,600 \\
\text { btu/gallon }\end{array}$ & & $\begin{array}{l}10,339 \\
\text { but/kWhr }\end{array}$ \\
\hline
\end{tabular}

Note: CNG is reported in diesel-gallon equivalents.

${ }^{\mathrm{a}}$ Data are not available. 
Intercity and School:

Eno Transportation Foundation, Transportation in America, 2001, Nineteenth Edition, 2003, Washington, DC, pp. 20-23. School bus fuel was assumed to be $90 \%$ diesel fuel and $10 \%$ gasoline based on estimates from the National Association of State Directors of Pupil Transportation Services. Intercity bus fuel was assumed to be $100 \%$ diesel.

Table A.4

Intercity and School Bus Fuel Use

\begin{tabular}{|c|c|c|}
\hline Year & $\begin{array}{c}\text { Intercity } \\
\text { (million gallons) }\end{array}$ & $\begin{array}{c}\text { School } \\
\text { (million gallons) }\end{array}$ \\
\hline 1970 & 305.34 & 299.88 \\
\hline 1971 & 296.73 & 309.75 \\
\hline 1972 & 288.12 & 319.62 \\
\hline 1973 & 252.42 & 327.04 \\
\hline 1974 & 216.72 & 334.46 \\
\hline 1975 & 181.02 & 341.88 \\
\hline 1976 & 182.28 & 389.76 \\
\hline 1977 & 181.86 & 401.52 \\
\hline 1978 & 180.18 & 406.98 \\
\hline 1979 & 205.38 & 404.88 \\
\hline 1980 & 213.78 & 379.68 \\
\hline 1981 & 205.38 & 386.82 \\
\hline 1982 & 227.22 & 398.58 \\
\hline 1983 & 237.30 & 400.68 \\
\hline 1984 & 169.26 & 375.06 \\
\hline 1985 & 165.48 & 425.04 \\
\hline 1986 & 148.68 & 462.42 \\
\hline 1987 & 155.82 & 487.20 \\
\hline 1988 & 160.44 & 511.14 \\
\hline 1989 & 166.74 & 498.12 \\
\hline 1990 & 159.60 & 472.08 \\
\hline 1991 & 160.44 & 533.40 \\
\hline 1992 & 157.08 & 546.00 \\
\hline 1993 & 171.36 & 533.40 \\
\hline 1994 & 195.30 & 546.00 \\
\hline 1995 & 195.30 & 545.16 \\
\hline 1996 & 199.92 & 545.16 \\
\hline 1997 & 212.52 & 544.74 \\
\hline 1998 & 220.08 & 550.20 \\
\hline 1999 & 241.08 & 555.66 \\
\hline 2000 & 233.10 & 577.08 \\
\hline 2001 & $217.35^{*}$ & $538.08 *$ \\
\hline 2002 & $210.22 *$ & $520.44 *$ \\
\hline 2003 & $208.32 *$ & $515.72 *$ \\
\hline 2004 & $208.87 *$ & $517.09 *$ \\
\hline 2005 & $214.37 *$ & $530.70 *$ \\
\hline 2006 & $208.32 *$ & $515.72 *$ \\
\hline 2007 & $214.37 *$ & $530.70^{*}$ \\
\hline 2008 & $218.48 *$ & $540.89 *$ \\
\hline 2009 & $224.58 *$ & $556.00 *$ \\
\hline 2010 & $214.95 *$ & $532.15^{*}$ \\
\hline 2011 & $215.53 *$ & $533.58 *$ \\
\hline 2012 & 230.33 & 570.22 \\
\hline Fuel type shares & $100 \%$ diesel & $\begin{array}{c}90 \% \text { diesel } \\
10 \% \text { gasoline }\end{array}$ \\
\hline $\begin{array}{l}\text { Heat content used for } \\
\text { conversion to btu: }\end{array}$ & $\begin{array}{c}138,700 \\
\text { btu/gallon }\end{array}$ & $\begin{array}{l}138,700 \mathrm{btu} / \text { gallon } \\
125,000 \mathrm{btu} / \text { gallon }\end{array}$ \\
\hline
\end{tabular}

*Estimated using the rate of change of bus vehicle-miles traveled from FHWA Highway Statistics, Table VM-1 (recently revised). 


\section{Trucks}

Light Trucks:

Fuel use in gallons (1970-2007) - DOT, FHWA, Highway Statistics 2008, Table VM-1 and annual editions back to 1996 and DOT, FHWA, Highway Statistics Summary to 1995.

Fuel use in gallons (2008 - 2012) - Results of a model developed by ORNL to estimate data for cars and light trucks since the FHWA discontinued their VM-1 series showing cars and light trucks separately. The model uses data from FHWA Highway Statistics 2012, EPA Light-Duty Automotive Technology, Carbon Dioxide Emissions, and Fuel Economy Trends: 1975 Through 2013, and R.L. Polk to estimate the number of vehicles, vehicle-miles of travel, energy use, and fuel efficiency of cars and light trucks. Documentation of the model will be published in an ORNL report, forthcoming.

Fuel type distribution - Fuel use was distributed among fuel types using the percentages shown in Table A.1. The FHWA discontinued gasohol data in 2005. Therefore, data from EIA, Alternatives to Traditional Transportation Fuels, 2006-2011, Table C.1 were used. 
Table A.5

Light Truck Fuel Use and Fuel Type Shares for Calculation of Energy Use

\begin{tabular}{|c|c|c|c|c|c|c|c|}
\hline \multirow[b]{2}{*}{ Year } & \multirow{2}{*}{$\begin{array}{l}\text { Fuel use } \\
\text { (million } \\
\text { gallons) }\end{array}$} & \multirow{2}{*}{$\begin{array}{c}\text { Source for } \\
\text { gasohol shares }\end{array}$} & \multirow{2}{*}{$\begin{array}{c}\text { Source for } \\
\text { gasoline/diesel } \\
\text { /lpg shares }\end{array}$} & \multicolumn{4}{|c|}{ Shares by fuel type } \\
\hline & & & & Gasoline & Gasohol & Diesel & Lpg \\
\hline 1970 & 12,313 & & 1977 TIUS & $97.6 \%$ & $0.0 \%$ & $1.6 \%$ & $0.8 \%$ \\
\hline 1971 & 13,484 & & 1977 TIUS & $97.6 \%$ & $0.0 \%$ & $1.6 \%$ & $0.8 \%$ \\
\hline 1972 & 15,150 & & 1977 TIUS & $97.6 \%$ & $0.0 \%$ & $1.6 \%$ & $0.8 \%$ \\
\hline 1973 & 16,828 & & 1977 TIUS & $97.6 \%$ & $0.0 \%$ & $1.6 \%$ & $0.8 \%$ \\
\hline 1974 & 16,657 & & 1977 TIUS & $97.6 \%$ & $0.0 \%$ & $1.6 \%$ & $0.8 \%$ \\
\hline 1975 & 19,081 & & 1977 TIUS & $97.6 \%$ & $0.0 \%$ & $1.6 \%$ & $0.8 \%$ \\
\hline 1976 & 20,828 & & 1977 TIUS & $97.6 \%$ & $0.0 \%$ & $1.6 \%$ & $0.8 \%$ \\
\hline 1977 & 22,383 & & 1977 TIUS & $97.6 \%$ & $0.0 \%$ & $1.6 \%$ & $0.8 \%$ \\
\hline 1978 & 24,162 & & Interpolated & $97.1 \%$ & $0.0 \%$ & $2.0 \%$ & $0.9 \%$ \\
\hline 1979 & 24,445 & & Interpolated & $96.7 \%$ & $0.0 \%$ & $2.4 \%$ & $1.0 \%$ \\
\hline 1980 & 23,796 & FHWA, MF-33e & Interpolated & $95.7 \%$ & $0.5 \%$ & $2.7 \%$ & $1.0 \%$ \\
\hline 1981 & 23,697 & FHWA, MF-33e & Interpolated & $95.1 \%$ & $0.7 \%$ & $3.1 \%$ & $1.1 \%$ \\
\hline 1982 & 22,702 & FHWA, MF-33e & 1982 TIUS & $93.0 \%$ & $2.3 \%$ & $3.5 \%$ & $1.2 \%$ \\
\hline 1983 & 23,945 & FHWA, MF-33e & Interpolated & $91.0 \%$ & $4.3 \%$ & $3.5 \%$ & $1.2 \%$ \\
\hline 1984 & 25,604 & FHWA, MF-33e & Interpolated & $90.0 \%$ & $5.3 \%$ & $3.5 \%$ & $1.2 \%$ \\
\hline 1985 & 27,363 & FHWA, MF-33e & Interpolated & $87.6 \%$ & $7.7 \%$ & $3.5 \%$ & $1.2 \%$ \\
\hline 1986 & 29,074 & FHWA, MF-33e & Interpolated & $87.7 \%$ & $7.6 \%$ & $3.5 \%$ & $1.2 \%$ \\
\hline 1987 & 30,598 & FHWA, MF-33e & 1987 TIUS & $89.0 \%$ & $6.3 \%$ & $3.5 \%$ & $1.2 \%$ \\
\hline 1988 & 32,653 & FHWA, MF-33e & Interpolated & $88.2 \%$ & $7.4 \%$ & $3.5 \%$ & $1.0 \%$ \\
\hline 1989 & 33,271 & FHWA, MF-33e & Interpolated & $89.5 \%$ & $6.2 \%$ & $3.4 \%$ & $0.8 \%$ \\
\hline 1990 & 35,611 & FHWA, MF-33e & Interpolated & $89.2 \%$ & $6.8 \%$ & $3.4 \%$ & $0.7 \%$ \\
\hline 1991 & 38,217 & FHWA, MF-33e & Interpolated & $88.1 \%$ & $8.0 \%$ & $3.3 \%$ & $0.5 \%$ \\
\hline 1992 & 40,929 & FHWA, MF-33e & 1992 TIUS & $88.5 \%$ & $7.9 \%$ & $3.3 \%$ & $0.3 \%$ \\
\hline 1993 & 42,851 & FHWA, MF-33e & Interpolated & $87.3 \%$ & $9.1 \%$ & $3.3 \%$ & $0.3 \%$ \\
\hline 1994 & 44,112 & FHWA, MF-33e & Interpolated & $86.8 \%$ & $9.6 \%$ & $3.3 \%$ & $0.3 \%$ \\
\hline 1995 & 45,605 & FHWA, MF-33e & Interpolated & $85.1 \%$ & $11.2 \%$ & $3.4 \%$ & $0.3 \%$ \\
\hline 1996 & 47,354 & FHWA, MF-33e & Interpolated & $86.2 \%$ & $10.1 \%$ & $3.4 \%$ & $0.3 \%$ \\
\hline 1997 & 49,388 & FHWA, MF-33e & 1997 VIUS & $84.2 \%$ & $12.2 \%$ & $3.4 \%$ & $0.2 \%$ \\
\hline 1998 & 50,462 & FHWA, MF-33e & Interpolated & $85.0 \%$ & $11.2 \%$ & $3.5 \%$ & $0.3 \%$ \\
\hline 1999 & 52,859 & FHWA, MF-33e & Interpolated & $84.9 \%$ & $11.0 \%$ & $3.6 \%$ & $0.4 \%$ \\
\hline 2000 & 52,939 & FHWA, MF-33e & Interpolated & $83.1 \%$ & $12.6 \%$ & $3.8 \%$ & $0.6 \%$ \\
\hline 2001 & 53,522 & FHWA, MF-33e & Interpolated & $82.4 \%$ & $13.0 \%$ & $3.9 \%$ & $0.7 \%$ \\
\hline 2002 & 55,220 & FHWA, MF-33e & 2002 VIUS & $79.6 \%$ & $15.6 \%$ & $4.0 \%$ & $0.8 \%$ \\
\hline 2003 & 60,758 & FHWA, MF-33e & 2002 VIUS & $71.0 \%$ & $24.2 \%$ & $4.0 \%$ & $0.8 \%$ \\
\hline 2004 & 63,417 & FHWA, MF-33e & 2002 VIUS & $62.9 \%$ & $32.3 \%$ & $4.0 \%$ & $0.8 \%$ \\
\hline 2005 & 58,869 & FHWA, MF-33e & 2002 VIUS & $62.6 \%$ & $32.6 \%$ & $4.0 \%$ & $0.8 \%$ \\
\hline 2006 & 60,685 & EIA, C1 & 2002 VIUS & $73.9 \%$ & $21.3 \%$ & $4.0 \%$ & $0.8 \%$ \\
\hline 2007 & 61,836 & EIA, C1 & 2002 VIUS & $68.6 \%$ & $26.6 \%$ & $4.0 \%$ & $0.8 \%$ \\
\hline 2008 & 61,199 & EIA, C1 & 2002 VIUS & $57.5 \%$ & $37.7 \%$ & $4.0 \%$ & $0.8 \%$ \\
\hline 2009 & 61,824 & EIA, C1 & 2002 VIUS & $51.5 \%$ & $43.7 \%$ & $4.0 \%$ & $0.8 \%$ \\
\hline 2010 & 64,687 & EIA, C1 & 2002 VIUS & $45.2 \%$ & $50.0 \%$ & $4.0 \%$ & $0.8 \%$ \\
\hline 2011 & 65,786 & EIA, C1 & 2002 VIUS & $44.4 \%$ & $50.8 \%$ & $4.0 \%$ & $0.8 \%$ \\
\hline \multirow[t]{2}{*}{2012} & 66,395 & EIA, C1 & 2002 VIUS & $44.3 \%$ & $50.9 \%$ & $4.0 \%$ & $0.8 \%$ \\
\hline & & Heat content used for & rsion to btu: & $\begin{array}{l}125,000 \\
\text { btu/gallon }\end{array}$ & $\begin{array}{l}\text { 120,900 } \\
\text { btu/gallon }\end{array}$ & $\begin{array}{l}138,700 \\
\text { btu/gallon }\end{array}$ & $\begin{array}{l}90,800 \\
\text { btu/gallon }\end{array}$ \\
\hline
\end{tabular}

${ }^{a}$ Data are not continuous between 2008 and 2009 due to changes in source. 
Medium/Heavy Trucks:

DOT, FHWA, Highway Statistics 2012, Table VM-1 and annual editions back to 1996 and DOT, FHWA, Highway Statistics Summary to 1995. The FHWA made methodology changes for Highway Statistics 2009. At that time, they published historical data back to 2007 which do not match the previous data. Total gallons for medium/heavy trucks are the sum of single-unit trucks and combination trucks.

Table A.6

Medium/Heavy Truck Fuel Use and Fuel Type Shares for Calculation of Energy Use

\begin{tabular}{|c|c|c|c|c|c|}
\hline \multirow[b]{2}{*}{ Year } & \multirow{2}{*}{$\begin{array}{c}\text { Fuel use } \\
\text { (million gallons) }\end{array}$} & \multirow{2}{*}{$\begin{array}{c}\text { Source for } \\
\text { fuel type shares }\end{array}$} & \multicolumn{3}{|c|}{ Shares by fuel type } \\
\hline & & & Gasoline & Diesel & Lpg \\
\hline 1970 & 11,316 & 1977 TIUS & $10.4 \%$ & $89.5 \%$ & $0.1 \%$ \\
\hline $1975^{-}$ & 14,598 & 1977 TIUS & $10.4 \%$ & $89.5 \%$ & $0.1 \%$ \\
\hline 1976 & 15,408 & 1977 TIUS & $10.4 \%$ & $89.5 \%$ & $0.1 \%$ \\
\hline 1977 & 17,082 & 1977 TIUS & $10.4 \%$ & $89.5 \%$ & $0.1 \%$ \\
\hline 1978 & 19,121 & Interpolated & $16.2 \%$ & $83.5 \%$ & $0.3 \%$ \\
\hline 1979 & 19,913 & Interpolated & $22.1 \%$ & $77.5 \%$ & $0.5 \%$ \\
\hline 1980 & 19,960 & Interpolated & $27.9 \%$ & $71.4 \%$ & $0.6 \%$ \\
\hline 1981 & 20,376 & Interpolated & $33.8 \%$ & $65.4 \%$ & $0.8 \%$ \\
\hline 1982 & 20,386 & 1982 TIUS & $39.6 \%$ & $59.4 \%$ & $1.0 \%$ \\
\hline 1983 & 20,761 & Interpolated & $35.6 \%$ & $63.6 \%$ & $0.8 \%$ \\
\hline 1984 & 21,428 & Interpolated & $31.5 \%$ & $67.8 \%$ & $0.7 \%$ \\
\hline 1985 & 21,405 & Interpolated & $27.5 \%$ & $72.0 \%$ & $0.5 \%$ \\
\hline 1986 & 21,861 & Interpolated & $23.4 \%$ & $76.2 \%$ & $0.4 \%$ \\
\hline 1987 & 22,513 & 1987 TIUS & $19.4 \%$ & $80.4 \%$ & $0.2 \%$ \\
\hline 1988 & 22,925 & Interpolated & $18.8 \%$ & $81.0 \%$ & $0.3 \%$ \\
\hline 1989 & 23,512 & Interpolated & $18.1 \%$ & $81.6 \%$ & $0.3 \%$ \\
\hline 1990 & 24,490 & Interpolated & $17.5 \%$ & $82.1 \%$ & $0.4 \%$ \\
\hline 1991 & 24,981 & Interpolated & $16.8 \%$ & $82.7 \%$ & $0.4 \%$ \\
\hline 1992 & 25,453 & 1992 TIUS & $16.2 \%$ & $83.3 \%$ & $0.5 \%$ \\
\hline 1993 & 26,236 & Interpolated & $15.4 \%$ & $84.1 \%$ & $0.5 \%$ \\
\hline 1994 & 27,685 & Interpolated & $14.7 \%$ & $84.8 \%$ & $0.5 \%$ \\
\hline 1995 & 28,828 & Interpolated & $13.9 \%$ & $85.6 \%$ & $0.5 \%$ \\
\hline 1996 & 29,601 & Interpolated & $13.2 \%$ & $86.3 \%$ & $0.5 \%$ \\
\hline 1997 & 29,878 & 1997 VIUS & $12.4 \%$ & $87.1 \%$ & $0.5 \%$ \\
\hline 1998 & 30,841 & Interpolated & $12.1 \%$ & $87.4 \%$ & $0.5 \%$ \\
\hline 1999 & 33,909 & Interpolated & $11.8 \%$ & $87.6 \%$ & $0.5 \%$ \\
\hline 2000 & 35,229 & Interpolated & $11.6 \%$ & $87.9 \%$ & $0.5 \%$ \\
\hline 2001 & 35,179 & Interpolated & $11.3 \%$ & $88.1 \%$ & $0.5 \%$ \\
\hline 2002 & 36,800 & 2002 VIUS & $11.0 \%$ & $88.4 \%$ & $0.5 \%$ \\
\hline 2003 & 35,775 & 2002 VIUS & $11.0 \%$ & $88.4 \%$ & $0.5 \%$ \\
\hline 2004 & 33,150 & 2002 VIUS & $11.0 \%$ & $88.4 \%$ & $0.5 \%$ \\
\hline 2005 & 37,190 & 2002 VIUS & $11.0 \%$ & $88.4 \%$ & $0.5 \%$ \\
\hline 2006 & 37,959 & 2002 VIUS & $11.0 \%$ & $88.4 \%$ & $0.5 \%$ \\
\hline 2007 & 47,218 & 2002 VIUS & $11.0 \%$ & $88.4 \%$ & $0.5 \%$ \\
\hline 2008 & 47,705 & 2002 VIUS & $11.0 \%$ & $88.4 \%$ & $0.5 \%$ \\
\hline 2009 & 44,303 & 2002 VIUS & $11.0 \%$ & $88.4 \%$ & $0.5 \%$ \\
\hline 2010 & 45,024 & 2002 VIUS & $11.0 \%$ & $88.4 \%$ & $0.5 \%$ \\
\hline 2011 & 42,396 & 2002 VIUS & $11.0 \%$ & $88.4 \%$ & $0.5 \%$ \\
\hline 2012 & 42,213 & 2002 VIUS & $11.0 \%$ & $88.4 \%$ & $0.5 \%$ \\
\hline Heat conte & or conversion to btu: & & 125,000 & 138,700 & 90,800 \\
\hline
\end{tabular}

${ }^{a}$ Data are not continuous between 2006 and 2007 due to changes in methodology. See source for details. 
Shares of Class 3-6 and 7-8 energy use by fuel type were calculated from the 2002 Vehicle Inventory and Use Survey (VIUS) and applied to all years 1970-2012.

Table A.7

Share of Medium and Heavy Truck Energy Use

\begin{tabular}{lccc}
\hline & \multicolumn{2}{c}{ Share of energy use } & \\
\cline { 2 - 3 } Fuel type & Class 3-6 & Class 7-8 & Total \\
\hline Gasoline & $92 \%$ & $8 \%$ & $100 \%$ \\
Diesel & $14 \%$ & $86 \%$ & $100 \%$ \\
LPG & $99 \%$ & $1 \%$ & $100 \%$ \\
\hline
\end{tabular}




\section{Off-highway energy use}

U.S. Environmental Protection Agency, NONROAD2008a model, results generated May 2014. Gallons of fuel by fuel type were produced for agricultural equipment, airport equipment, construction and mining equipment, industrial equipment, lawn and garden equipment, logging equipment, railroad maintenance equipment, and recreational equipment. Some non-transportation-related equipment, such as generators, chain saws, compressors, and pumps, were excluded from the data.

\section{Nonhighway energy use}

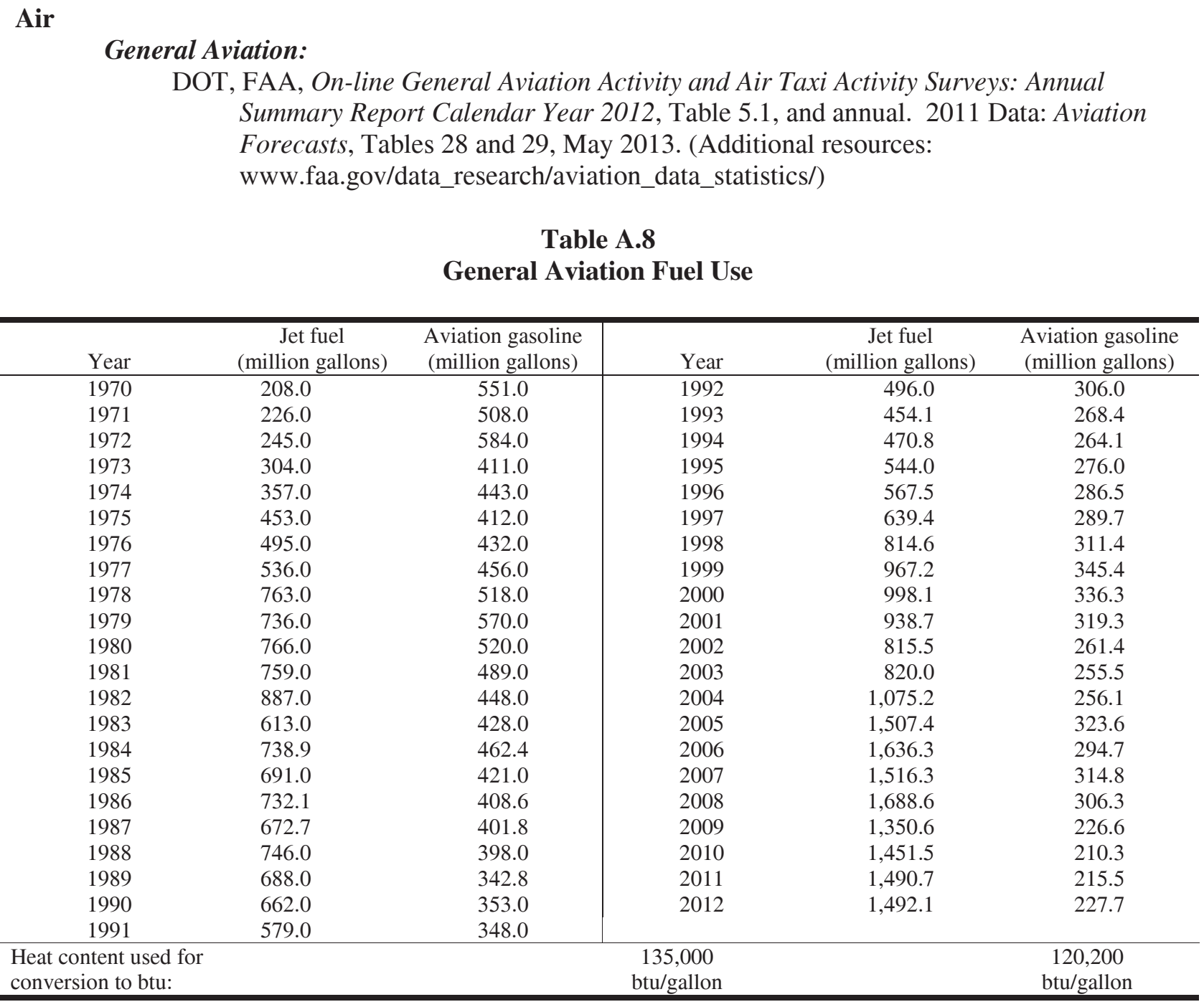




\section{Domestic and International Air Carrier:}

DOT, Bureau of Transportation Statistics, "Fuel Cost and Consumption Tables," www.transtats.bts.gov/fuel.asp. The table below shows all international fuel use. Because the data for international include fuel purchased abroad, for the tables in Chapter 2, the international total was divided in half to estimate domestic fuel use for international flights.

Table A.9

Air Carrier Fuel Use

\begin{tabular}{|c|c|c|c|}
\hline Year & $\begin{array}{c}\text { Domestic } \\
\text { (thousand gallons) }\end{array}$ & $\begin{array}{c}\text { International } \\
\text { (thousand gallons) }\end{array}$ & $\begin{array}{c}\text { Total } \\
\text { (thousand gallons) }\end{array}$ \\
\hline 1970 & \multirow{7}{*}{\multicolumn{2}{|c|}{$\begin{array}{l}\text { Separate estimates for domestic } \\
\text { and international are not available } \\
\text { from 1970-1976. }\end{array}$}} & $10,085,000$ \\
\hline 1971 & & & $10,140,000$ \\
\hline 1972 & & & $10,302,000$ \\
\hline 1973 & & & $10,671,000$ \\
\hline 1974 & & & $10,417,260$ \\
\hline 1975 & & & $10,412,640$ \\
\hline 1976 & & & $10,400,040$ \\
\hline 1977 & $8,202,051$ & $1,708,376$ & $9,910,427$ \\
\hline 1978 & $8,446,117$ & $1,741,918$ & $10,188,035$ \\
\hline 1979 & $8,865,885$ & $1,828,435$ & $10,694,320$ \\
\hline 1980 & $8,519,233$ & $1,747,306$ & $10,266,539$ \\
\hline 1981 & $8,555,249$ & $2,032,520$ & $10,587,769$ \\
\hline 1982 & $8,432,465$ & $1,967,733$ & $10,400,198$ \\
\hline 1983 & $8,672,574$ & $1,998,289$ & $10,670,863$ \\
\hline 1984 & $9,625,958$ & $2,286,407$ & $11,912,365$ \\
\hline 1985 & $10,115,007$ & $2,487,929$ & $12,602,936$ \\
\hline 1986 & $11,137,331$ & $2,544,996$ & $13,682,327$ \\
\hline 1987 & $11,586,838$ & $2,893,617$ & $14,480,455$ \\
\hline 1988 & $11,917,904$ & $3,262,824$ & $15,180,728$ \\
\hline 1989 & $11,905,144$ & $3,557,294$ & $15,462,438$ \\
\hline 1990 & $12,429,305$ & $3,963,081$ & $16,392,386$ \\
\hline 1991 & $11,506,477$ & $3,939,666$ & $15,446,144$ \\
\hline 1992 & $11,762,852$ & $4,120,132$ & $15,882,983$ \\
\hline 1993 & $11,958,663$ & $4,113,321$ & $16,071,984$ \\
\hline 1994 & $12,475,549$ & $4,310,879$ & $16,786,428$ \\
\hline 1995 & $12,811,717$ & $4,511,418$ & $17,323,135$ \\
\hline 1996 & $13,187,305$ & $4,658,093$ & $17,845,398$ \\
\hline 1997 & $13,659,581$ & $4,964,181$ & $18,623,762$ \\
\hline 1998 & $13,876,971$ & $5,185,562$ & $19,062,533$ \\
\hline 1999 & $14,402,127$ & $5,250,492$ & $19,652,619$ \\
\hline 2000 & $14,844,592$ & $5,474,685$ & $20,319,277$ \\
\hline 2001 & $14,017,461$ & $5,237,487$ & $19,254,948$ \\
\hline 2002 & $12,848,329$ & $4,990,798$ & $17,839,127$ \\
\hline 2003 & $12,958,581$ & $4,836,356$ & $17,794,936$ \\
\hline 2004 & $13,622,603$ & $4,931,546$ & $18,554,149$ \\
\hline 2005 & $13,778,869$ & $5,520,889$ & $19,309,758$ \\
\hline 2006 & $13,694,437$ & $6,017,638$ & $19,712,075$ \\
\hline 2007 & $13,681,664$ & $6,204,502$ & $19,886,165$ \\
\hline 2008 & $12,666,911$ & $6,186,747$ & $18,853,658$ \\
\hline 2009 & $11,339,220$ & $5,721,298$ & $17,060,517$ \\
\hline 2010 & $11,256,900$ & $6,041,500$ & $17,288,400$ \\
\hline 2011 & $11,035,400$ & $6,522,600$ & $17,558,000$ \\
\hline 2012 & $10,510,200$ & $6,580,500$ & $17,091,100$ \\
\hline $\begin{array}{l}\text { Heat content used for } \\
\text { conversion to btu: }\end{array}$ & $\begin{array}{c}135,000 \\
\text { btu/gallon }\end{array}$ & $\begin{array}{c}135,000 \\
\text { btu/gallon }\end{array}$ & $\begin{array}{c}135,000 \\
\text { btu/gallon }\end{array}$ \\
\hline
\end{tabular}




\section{Water}

\section{Freight:}

Total - DOE, EIA, Petroleum and Other Liquids Database, May 2014. Adjusted sales of distillate and residual fuel oil for vessel bunkering. (This may include some amounts of bunker fuels used for recreational purposes.)

\section{Table A.10}

Diesel and Residual Fuel Oil for Vessel Bunkering

\begin{tabular}{|c|c|c|}
\hline Year & $\begin{array}{l}\text { Distillate fuel oil } \\
\text { (thousand gallons) }\end{array}$ & $\begin{array}{l}\text { Residual fuel oil } \\
\text { (thousand gallons) }\end{array}$ \\
\hline 1970 & 819,000 & $3,774,120$ \\
\hline 1975 & $1,097,880$ & $4,060,140$ \\
\hline 1976 & $1,220,100$ & $4,977,000$ \\
\hline 1977 & $1,407,420$ & $5,416,740$ \\
\hline 1978 & $1,578,822$ & $6,614,790$ \\
\hline 1979 & $1,630,858$ & $8,002,672$ \\
\hline 1980 & 717,376 & $7,454,242$ \\
\hline 1981 & $1,723,143$ & $7,922,512$ \\
\hline 1982 & $1,423,216$ & $6,408,818$ \\
\hline 1983 & $1,418,890$ & $5,724,115$ \\
\hline 1984 & $1,692,045$ & $5,688,931$ \\
\hline 1985 & $1,894,265$ & $5,269,733$ \\
\hline 1986 & $2,034,215$ & $5,690,250$ \\
\hline 1987 & $2,223,258$ & $5,869,154$ \\
\hline 1988 & $2,310,367$ & $6,025,511$ \\
\hline 1989 & $2,356,444$ & $6,621,100$ \\
\hline 1990 & $2,197,004$ & $6,248,095$ \\
\hline 1991 & $2,167,640$ & $6,786,055$ \\
\hline 1992 & $2,240,170$ & $7,199,078$ \\
\hline 1993 & $2,043,745$ & $6,269,882$ \\
\hline 1994 & $2,026,899$ & $5,944,383$ \\
\hline 1995 & $1,978,105$ & $6,431,238$ \\
\hline 1996 & $2,177,608$ & $5,804,977$ \\
\hline 1997 & $2,107,561$ & $4,789,861$ \\
\hline 1998 & $2,125,568$ & $4,640,153$ \\
\hline 1999 & $2,064,590$ & $5,598,630$ \\
\hline 2000 & $2,041,433$ & $6,192,294$ \\
\hline 2001 & $2,099,011$ & $4,345,284$ \\
\hline 2002 & $2,056,465$ & $4,783,956$ \\
\hline 2003 & $1,863,150$ & $3,801,425$ \\
\hline 2004 & $2,313,448$ & $4,886,978$ \\
\hline 2005 & $2,115,381$ & $5,533,552$ \\
\hline 2006 & $2,206,690$ & $6,000,434$ \\
\hline 2007 & $2,158,930$ & $6,773,950$ \\
\hline 2008 & $1,980,729$ & $6,274,047$ \\
\hline 2009 & $2,138,690$ & $5,331,657$ \\
\hline 2010 & $2,427,051$ & $6,032,367$ \\
\hline 2011 & $2,651,859$ & $5,207,886$ \\
\hline 2012 & $1,844,070$ & $4,588,162$ \\
\hline $\begin{array}{l}\text { Heat content used for } \\
\text { conversion to btu: }\end{array}$ & $\begin{array}{c}138,700 \\
\text { btu/gallon }\end{array}$ & $\begin{array}{c}149,700 \\
\text { btu/gallon }\end{array}$ \\
\hline $\begin{array}{l}\text { Domestic share of total } \\
\text { fuel use }\end{array}$ & $77.5 \%$ & $9.3 \%$ \\
\hline
\end{tabular}




\section{Recreational Boating:}

Fuel use by recreational boating comes from the EPA's NONROAD2008A model.

Table A.11

Recreational Boating Fuel Use

\begin{tabular}{|c|c|c|}
\hline Year & $\begin{array}{l}\text { Diesel use } \\
\text { (gallons) }\end{array}$ & $\begin{array}{l}\text { Gasoline use } \\
\text { (gallons) }\end{array}$ \\
\hline 1970 & $39,589,953$ & $1,213,397,311$ \\
\hline 1971 & $47,130,906$ & $1,220,995,448$ \\
\hline 1972 & $54,671,856$ & $1,228,593,572$ \\
\hline 1973 & $62,212,803$ & $1,236,191,635$ \\
\hline 1974 & $69,753,735$ & $1,243,789,752$ \\
\hline 1975 & $77,294,680$ & $1,251,387,972$ \\
\hline 1976 & $84,835,632$ & $1,258,986,070$ \\
\hline 1977 & $92,376,573$ & $1,266,584,111$ \\
\hline 1978 & $99,917,523$ & $1,274,182,341$ \\
\hline 1979 & $107,458,470$ & $1,281,780,460$ \\
\hline 1980 & $114,999,421$ & $1,289,378,532$ \\
\hline 1981 & $122,540,357$ & $1,296,976,672$ \\
\hline 1982 & $130,081,302$ & $1,304,574,832$ \\
\hline 1983 & $137,622,248$ & $1,312,172,890$ \\
\hline 1984 & $145,163,202$ & $1,319,771,007$ \\
\hline 1985 & $152,704,140$ & $1,327,369,146$ \\
\hline 1986 & $160,245,074$ & $1,334,967,322$ \\
\hline 1987 & $167,786,030$ & $1,342,565,455$ \\
\hline 1988 & $175,326,970$ & $1,362,856,034$ \\
\hline 1989 & $182,867,916$ & $1,383,146,636$ \\
\hline 1990 & $190,408,869$ & $1,403,437,194$ \\
\hline 1991 & $197,949,808$ & $1,429,688,292$ \\
\hline 1992 & $205,490,749$ & $1,455,939,504$ \\
\hline 1993 & $213,031,707$ & $1,482,190,597$ \\
\hline 1994 & $220,572,649$ & $1,539,794,180$ \\
\hline 1995 & $228,113,596$ & $1,597,269,921$ \\
\hline 1996 & $235,654,521$ & $1,654,446,069$ \\
\hline 1997 & $243,195,481$ & $1,657,737,628$ \\
\hline 1998 & $250,736,414$ & $1,659,056,085$ \\
\hline 1999 & $258,159,525$ & $1,657,198,161$ \\
\hline 2000 & $265,582,657$ & $1,652,906,973$ \\
\hline 2001 & $273,547,835$ & $1,655,303,922$ \\
\hline 2002 & $281,512,965$ & $1,653,583,696$ \\
\hline 2003 & $289,478,093$ & $1,648,070,959$ \\
\hline 2004 & $297,443,197$ & $1,639,713,127$ \\
\hline 2005 & $305,408,463$ & $1,629,873,278$ \\
\hline 2006 & $313,420,594$ & $1,619,603,593$ \\
\hline 2007 & $321,432,801$ & $1,609,567,873$ \\
\hline 2008 & $329,445,068$ & $1,599,830,522$ \\
\hline 2009 & $337,457,287$ & $1,590,749,216$ \\
\hline 2010 & $345,469,668$ & $1,578,405,558$ \\
\hline 2011 & $353,434,754$ & $1,566,937,275$ \\
\hline 2012 & $361,399,927$ & $1,557,381,573$ \\
\hline $\begin{array}{l}\text { Heat content used for } \\
\text { conversion to btu: }\end{array}$ & $\begin{array}{c}138,700 \\
\text { btu/gallon }\end{array}$ & $\begin{array}{c}\text { 125,000 } \\
\text { btu/gallon }\end{array}$ \\
\hline
\end{tabular}




\section{Pipeline}

The sum of natural gas, crude petroleum and petroleum product, and coal slurry and water.

\section{Natural Gas:}

The amount of natural gas used to transport natural gas was defined as "pipeline fuel" as reported in DOE, EIA, Natural Gas Annual 2013, Table 1. Cubic feet were converted to Btu using $1,031 \mathrm{Btu} / \mathrm{ft} 3$. Electricity use was estimated using the following procedure as reported on p. 5-110 of J. N. Hooker et al., End Use Energy Consumption DataBase: Transportation Sector. The energy consumption of a natural gas pipeline was taken to be the energy content of the fuel used to drive the pumps. Some $94 \%$ of the installed pumping horsepower was supplied by natural gas. The remaining $6 \%$ of the horsepower was generated more efficiently, mostly by electric motors. The energy consumed by natural gas pipeline pumps that were electrically powered was not known. In order to estimate the electricity consumed, the Btu of natural gas pipeline fuel consumed was multiplied by a factor of 0.015 . From this computed value, electricity efficiency and generation loss must be taken into account. The electricity energy use in Btu must be converted to $\mathrm{kWhr}$, using the conversion factor $29.305 \times 10-5 \mathrm{kWhr} / \mathrm{Btu}$. Electricity generation and distribution efficiency was $29 \%$. When generation and distribution efficiency are taken into account, $1 \mathrm{kWhr}$ equals 10,339 Btu.

\section{Crude petroleum and petroleum product:}

J. N. Hooker, Oil Pipeline Energy Consumption and Efficiency, ORNL-5697, ORNL, Oak Ridge, TN, 1981. (Data held constant; Latest available data.)

\section{Coal slurry and water:}

W. F. Banks, Systems, Science and Software, Energy Consumption in the Pipeline Industry, LaJolla, CA, October 1977. (Data held constant; Latest available data.) 
Table A.12

Pipeline Fuel Use

\begin{tabular}{|c|c|c|c|}
\hline Year & $\begin{array}{c}\text { Natural gas } \\
\text { (million cubic } \\
\text { feet) }\end{array}$ & $\begin{array}{l}\text { Estimated natural } \\
\text { gas pipeline } \\
\text { electricity use } \\
\text { (million kWhr) }\end{array}$ & $\begin{array}{c}\text { Electricity } \\
\text { constant } \\
\text { (trillion btu) }\end{array}$ \\
\hline 1970 & 722,166 & $3,272.9$ & 212.1 \\
\hline 1971 & 742,592 & $3,365.4$ & 212.1 \\
\hline 1972 & 766,156 & $3,472.2$ & 212.1 \\
\hline 1973 & 728,177 & $3,300.1$ & 212.1 \\
\hline 1974 & 668,792 & $3,031.0$ & 212.1 \\
\hline 1975 & 582,963 & $2,642.0$ & 212.1 \\
\hline 1976 & 548,323 & $2,485.0$ & 212.1 \\
\hline 1977 & 532,669 & $2,414.1$ & 212.1 \\
\hline 1978 & 530,451 & $2,404.0$ & 212.1 \\
\hline 1979 & 600,964 & $2,723.6$ & 212.1 \\
\hline 1980 & 634,622 & $2,876.1$ & 212.1 \\
\hline 1981 & 642,325 & $2,911.0$ & 212.1 \\
\hline 1982 & 596,411 & $2,703.0$ & 212.1 \\
\hline 1983 & 490,042 & $2,220.9$ & 212.1 \\
\hline 1984 & 528,754 & $2,396.3$ & 212.1 \\
\hline 1985 & 503,766 & $2,283.1$ & 212.1 \\
\hline 1986 & 485,041 & $2,198.2$ & 212.1 \\
\hline 1987 & 519,170 & $2,352.9$ & 212.1 \\
\hline 1988 & 613,912 & $2,782.3$ & 212.1 \\
\hline 1989 & 629,308 & $2,852.0$ & 212.1 \\
\hline 1990 & 659,816 & $2,990.3$ & 212.1 \\
\hline 1991 & 601,305 & $2,725.1$ & 212.1 \\
\hline 1992 & 587,710 & $2,663.5$ & 212.1 \\
\hline 1993 & 624,308 & $2,829.4$ & 212.1 \\
\hline 1994 & 685,362 & $3,106.1$ & 212.1 \\
\hline 1995 & 700,335 & $3,173.9$ & 212.1 \\
\hline 1996 & 711,446 & $3,224.3$ & 212.1 \\
\hline 1997 & 751,470 & $3,405.7$ & 212.1 \\
\hline 1998 & 635,477 & $2,880.0$ & 212.1 \\
\hline 1999 & 645,319 & $2,924.6$ & 212.1 \\
\hline 2000 & 642,210 & $2,910.5$ & 212.1 \\
\hline 2001 & 624,964 & $2,832.3$ & 212.1 \\
\hline 2002 & 666,920 & $3,022.5$ & 212.1 \\
\hline 2003 & 591,492 & $2,680.7$ & 212.1 \\
\hline 2004 & 566,187 & $2,566.0$ & 212.1 \\
\hline 2005 & 584,026 & $2,646.8$ & 212.1 \\
\hline 2006 & 584,213 & $2,647.7$ & 212.1 \\
\hline 2007 & 621,364 & $2,816.0$ & 212.1 \\
\hline 2008 & 647,956 & $2,936.6$ & 212.1 \\
\hline 2009 & 670,174 & $3,037.2$ & 212.1 \\
\hline 2010 & 674,124 & $3,055.1$ & 212.1 \\
\hline 2011 & 687,784 & $3,117.0$ & 212.1 \\
\hline 2012 & 728,163 & $3,300.0$ & 212.1 \\
\hline $\begin{array}{l}\text { Heat content used for } \\
\text { conversion to btu: }\end{array}$ & $\begin{array}{c}1,031 \\
\text { btu/cubic foot }\end{array}$ & $\begin{array}{c}10,339 \\
\text { Btu/kWhr }\end{array}$ & \\
\hline
\end{tabular}

Note: Formula for estimating electricity use for natural gas pipelines is:

Natural gas use (in million cubic ft) $\times 1,031 \mathrm{btu} /$ cubic $\mathrm{ft} \times 0.015 \times 29.305 \times 10-5 \mathrm{kWhr} / \mathrm{btu}$. 


\section{Rail}

\section{Freight:}

AAR, Railroad Facts, 2013 Edition, Washington, DC, 2014.

Table A.13

Class I Freight Railroad Fuel Use

\begin{tabular}{cc}
\hline Year & $\begin{array}{c}\text { Diesel fuel } \\
\text { (thousand gallons) }\end{array}$ \\
\hline 1970 & $3,807,663$ \\
1971 & $3,822,907$ \\
1972 & $3,996,985$ \\
1973 & $4,160,730$ \\
1974 & $4,175,375$ \\
1975 & $3,736,484$ \\
1976 & $3,895,542$ \\
1977 & $3,985,069$ \\
1978 & $3,968,007$ \\
1979 & $4,072,187$ \\
1980 & $3,955,996$ \\
1981 & $3,756,439$ \\
1982 & $3,178,116$ \\
1983 & $3,137,295$ \\
1984 & $3,388,173$ \\
1985 & $3,144,190$ \\
1986 & $3,039,069$ \\
1987 & $3,102,227$ \\
1988 & $3,182,267$ \\
1989 & $3,190,815$ \\
1990 & $3,134,446$ \\
1991 & $2,925,970$ \\
1992 & $3,022,108$ \\
1993 & $3,111,981$ \\
1994 & $3,355,802$ \\
1995 & $3,503,096$ \\
1996 & $3,600,649$ \\
1997 & $3,602,793$ \\
1998 & $3,619,341$ \\
1999 & $3,749,428$ \\
2000 & $3,720,107$ \\
2001 & $3,729,985$ \\
2002 & $3,751,413$ \\
2003 & $3,849,229$ \\
2004 & $4,082,236$ \\
2005 & $4,119,879$ \\
2006 & $4,214,459$ \\
2007 & $4,087,405$ \\
2008 & $3,911,178$ \\
2009 & $3,220,059$ \\
2010 & $3,519,021$ \\
2011 & $3,710,485$ \\
2012 & $3,634,025$ \\
conversion to btu: & 138,700 \\
& Btulgallon \\
\hline
\end{tabular}




\section{Passenger:}

Commuter - APTA, 2014 Public Transportation Fact Book, Washington, DC, 2014.

Table A.14

Commuter Rail Fuel Use

\begin{tabular}{|c|c|c|}
\hline Year & $\begin{array}{l}\text { Diesel } \\
\text { (thousand gallons) }\end{array}$ & $\begin{array}{l}\text { Electricity } \\
\quad \text { (million kWhr) }\end{array}$ \\
\hline 1984 & 58,320 & 901 \\
\hline 1985 & 55,372 & 1,043 \\
\hline 1986 & 54,608 & 1,170 \\
\hline 1987 & 51,594 & 1,155 \\
\hline 1988 & 53,054 & 1,195 \\
\hline 1989 & 52,516 & 1,293 \\
\hline 1990 & 52,681 & 1,226 \\
\hline 1991 & 54,315 & 1,239 \\
\hline 1992 & 54,951 & 1,124 \\
\hline 1993 & 59,766 & 1,196 \\
\hline 1994 & 61,900 & 1,244 \\
\hline 1995 & 63,064 & 1,253 \\
\hline 1996 & 61,888 & 1,255 \\
\hline 1997 & 63,195 & 1,270 \\
\hline 1998 & 69,200 & 1,299 \\
\hline 1999 & 73,005 & 1,322 \\
\hline 2000 & 70,818 & 1,370 \\
\hline 2001 & 72,204 & 1,354 \\
\hline 2002 & 72,847 & 1,334 \\
\hline 2003 & 72,264 & 1,383 \\
\hline 2004 & 71,999 & 1,449 \\
\hline 2005 & 76,714 & 1,484 \\
\hline 2006 & 78,600 & 1,478 \\
\hline 2007 & 80,700 & 1,763 \\
\hline 2008 & 83,500 & 1,718 \\
\hline 2009 & 95,000 & 1,780 \\
\hline 2010 & 93,200 & 1,797 \\
\hline 2011 & 95,000 & 1,813 \\
\hline 2012 & 94,000 & 1,808 \\
\hline \multirow{2}{*}{$\begin{array}{l}\text { Heat content used for } \\
\text { conversion to btu: }\end{array}$} & 138,700 & 10,339 \\
\hline & Btu/gallon & $\mathrm{Btu} / \mathrm{kWhr}$ \\
\hline
\end{tabular}


Transit - APTA, 2014 Public Transportation Fact Book, Washington, DC, 2014. Includes light rail and heavy rail.

Table A.15

Transit Rail Fuel Use

\begin{tabular}{|c|c|c|c|}
\hline \multirow[b]{2}{*}{ Year } & \multicolumn{3}{|c|}{ Electricity (million kWhr) } \\
\hline & Light rail & Heavy rail & Total \\
\hline 1970 & & & 2,561 \\
\hline 1971 & & & 2,556 \\
\hline 1972 & & & 2,428 \\
\hline 1973 & & & 2,331 \\
\hline 1974 & & & 2,630 \\
\hline 1975 & & & 2,646 \\
\hline 1976 & \multirow{2}{*}{\multicolumn{2}{|c|}{$\begin{array}{l}\text { Light rail and heavy rail data are } \\
\text { not available separately from }\end{array}$}} & 2,576 \\
\hline 1977 & & & 2,303 \\
\hline 1978 & \multicolumn{2}{|c|}{1970 to 1985.} & 2,223 \\
\hline 1979 & & & 2,473 \\
\hline 1980 & & & 2,446 \\
\hline 1981 & & & 2,655 \\
\hline 1982 & & & 2,722 \\
\hline 1983 & & & 2,930 \\
\hline 1984 & & & 3,092 \\
\hline 1985 & & & 2,928 \\
\hline 1986 & 173 & 3,066 & 3,239 \\
\hline 1987 & 191 & 3,219 & 3,410 \\
\hline 1988 & 243 & 3,256 & 3,499 \\
\hline 1989 & 242 & 3,286 & 3,528 \\
\hline 1990 & 239 & 3,284 & 3,523 \\
\hline 1991 & 274 & 3,248 & 3,522 \\
\hline 1992 & 297 & 3,193 & 3,490 \\
\hline 1993 & 281 & 3,287 & 3,568 \\
\hline 1994 & 282 & 3,431 & 3,713 \\
\hline 1995 & 288 & 3,401 & 3,689 \\
\hline 1996 & 321 & 3,322 & 3,643 \\
\hline 1997 & 363 & 3,253 & 3,616 \\
\hline 1998 & 382 & 3,280 & 3,662 \\
\hline 1999 & 416 & 3,385 & 3,801 \\
\hline 2000 & 563 & 3,549 & 4,112 \\
\hline 2001 & 587 & 3,646 & 4,233 \\
\hline 2002 & 510 & 3,683 & 4,193 \\
\hline 2003 & 507 & 3,632 & 4,138 \\
\hline 2004 & 553 & 3,684 & 4,237 \\
\hline 2005 & 571 & 3,769 & 4,339 \\
\hline 2006 & 634 & 3,709 & 4,343 \\
\hline 2007 & 687 & 3,817 & 4,505 \\
\hline 2008 & 721 & 3,898 & 4,619 \\
\hline 2009 & 738 & 3,866 & 4,624 \\
\hline 2010 & 749 & 3,780 & 4,529 \\
\hline 2011 & 789 & 3,854 & 4,643 \\
\hline 2012 & 806 & 3,795 & 4,601 \\
\hline Heat content used for & 10,339 & 10,339 & 10,339 \\
\hline conversion to btu: & Btu/kWhr & Btu/kWhr & Btu/kWhr \\
\hline
\end{tabular}


Intercity - Personal communication with Amtrak, Washington, DC, 2013.

Table A.16

Intercity Rail Fuel Use

\begin{tabular}{ccc}
\hline Year & $\begin{array}{c}\text { Diesel fuel } \\
\text { (thousand } \\
\text { gallons) }\end{array}$ & $\begin{array}{c}\text { Electricity } \\
\text { (thousand } \mathrm{kWhr} \text { ) }\end{array}$ \\
\hline 1994 & 73,516 & 308,948 \\
1995 & 72,371 & 335,818 \\
1996 & 71,226 & 362,689 \\
1997 & 75,656 & 389,559 \\
1998 & 75,999 & 416,429 \\
1999 & 79,173 & 443,300 \\
2000 & 94,968 & 470,170 \\
2001 & 96,846 & 455,703 \\
2002 & 84,432 & 518,306 \\
2003 & 74,621 & 536,950 \\
2004 & 68,605 & 550,695 \\
2005 & 65,477 & 531,377 \\
2006 & 62,463 & 548,856 \\
2007 & 61,824 & 577,864 \\
2008 & 63,428 & 582,022 \\
2009 & 61,704 & 564,968 \\
2010 & 63,474 & 558,662 \\
2011 & 63,450 & 555,425 \\
2012 & 63,058 & 549,201 \\
\hline Heat content used for & 138,700 & 10,339 \\
conversion to Btu & Btu/gallon & Btu/kWhr \\
\hline
\end{tabular}

\section{Calculation of}

Million Barrels per Day

Crude Oil Equivalent

One gallon of gasoline, diesel fuel, or lpg is estimated to be the equivalent of one gallon of crude oil.

Petroleum used for electricity was calculated using the following formula:

$(\{[(\mathrm{BTU} * \mathrm{~S}) / \mathrm{G}] / \mathrm{P}\} / 365) / 1000$

$\mathrm{BTU}=$ Btus of electricity from Table 2.5

$\mathrm{S}=\quad$ Share of petroleum used in making primary electricity (Calculated from Table 2.6 from the EIA, Monthly Energy Review)

$\mathrm{G}=$ Electricity generation and distribution (assumed 29\%)

$\mathrm{P}=$ Btus per barrel of petroleum product (Table A3 from the EIA, Monthly Energy Review). 


\section{Passenger Travel and Energy Use}

\section{Cars}

Number of vehicles - DOT, FHWA, Highway Statistics 2012, Table MV-1 and annual editions back to 2009.

Vehicle-miles - See Appendix A "Car and Light Truck Shares" section.

Passenger-miles - Vehicle-miles multiplied by an average load factor.

Load factor - 2009 NHTS shows car load factor as 1.55 persons per vehicle.

Energy intensities -

Btu per vehicle-mile - Car energy use divided by vehicle-miles.

Btu per passenger-mile - Car energy use divided by passenger-miles.

Energy use - See Energy Use Sources, p. A-5. Data series shown in Table 2.7.

\section{Light Trucks}

Number of vehicles - DOT, FHWA, Highway Statistics 2012, Table MV-9 and annual editions back to 2009. Columns for pickups, vans, sport utility vehicles, and other light trucks.

Vehicle-miles - See Appendix A "Car and Light Truck Shares" section.

Passenger-miles - Vehicle-miles multiplied by an average load factor.

Load factor - 2009 NHTS shows personal light truck load factor as 1.84 persons per vehicle.

Energy intensities -

Btu per vehicle-mile - Personal light truck energy use divided by personal light truck vehicle-miles. Btu per passenger-mile - Personal light truck energy use divided by personal light truck passengermiles.

Energy use - See Energy Use Sources, p. A-10, A-12 (light trucks, medium/heavy trucks). Data by truck type were multiplied by the shares of truck fuel use which are for personal use (Table A.17) which were derived by ORNL from the 2002 VIUS Micro Data File on CD. 
Table A.17

Share of Trucks, Truck Travel, and Fuel Use for Personal Travel

\begin{tabular}{ll}
\hline Personal trucks & \\
$85.6 \%$ & 2-axle, 4-tire trucks \\
$26.9 \%$ & Other single-unit and combination trucks \\
Personal truck travel & \\
$80.9 \%$ & 2-axle, 4-tire trucks \\
$13.1 \%$ & Other single-unit and combination trucks \\
Personal truck fuel use & \\
$78.0 \%$ & 2-axle, 4-tire trucks \\
$6.0 \%$ & Other single-unit and combination trucks \\
\hline
\end{tabular}

Note: Since these shares come from the 2002 VIUS, they may underestimate the amount of personal trucks, truck travel, and energy use for 2012.

\section{Motorcycles}

Number of vehicles, vehicle-miles - DOT, FHWA, Highway Statistics 2012, Table VM-1.

Passenger-miles - Vehicle-miles multiplied by an average load factor.

Load factor - 2009 NHTS shows motorcycle load factor as 1.16 persons per vehicle.

Energy intensities -

Btu per vehicle-mile - Motorcycle energy use divided by vehicle-miles.

Btu per passenger-mile - Motorcycle energy use divided by passenger-miles.

Energy use - See Energy Use Sources, p. A-7. Data series shown in Table 2.7.

\section{Demand Response}

Number of vehicles, vehicle-miles, passenger-miles - APTA, 2014 Public Transportation Fact Book, Washington, DC, 2014.

Load factor - Passenger-miles divided by vehicle-miles.

Energy intensities -

Btu per vehicle-mile - Energy use divided by vehicle-miles.

Btu per passenger-mile - Energy use divided by passenger-miles.

Energy use - APTA, 2014 Public Transportation Fact Book, Washington, DC, 2014.

\section{Buses}

\section{Transit}

Number of vehicles, vehicle-miles, passenger-miles - APTA, 2014 Public Transportation Fact Book, Washington, DC, 2014. Data series shown on Table 5.18.

Load factor - Passenger-miles divided by vehicle-miles.

Energy intensities -

Btu per vehicle-mile - Transit bus energy use divided by transit bus vehicle-miles.

Btu per passenger-mile - Transit bus energy use divided by transit bus passenger-miles.

Energy use - See Energy Use Sources, p. A-8. Data series shown in Table 5.18. 
Intercity

Energy use - See Energy Use Sources, p. A-9. Because the data past 2000 are not available, the rate of change in bus VMT from FHWA, Highway Statistics 2012, was used to estimate the change in energy use.

\section{School}

Number of vehicles - DOT, FHWA, Highway Statistics 2012, Table MV-10.

Energy use - See Energy Use Sources, p. A-9. Because the data past 2000 are not available, the rate of change in bus VMT from FHWA, Highway Statistics 2012, was used to estimate the change in energy use.

\section{$\underline{\text { Air }}$}

\section{Certificated air carriers}

Aircraft-miles, passenger-miles - DOT, BTS, U.S. Air Traffic Statistics Through April 2014, www.bts.gov/xml/air_traffic/src/index.xml, Washington, DC.

Load factor - Passenger-miles divided by aircraft-miles.

Energy intensities Btu per passenger-mile - Certificated air carrier energy use divided by passenger-miles.

Energy use - See Energy Use Sources, p. A-15. All of domestic fuel use and half of international fuel use was considered to be domestic use.

Note: These data differ from the data in Table 9.2 because that table contains data on ALL domestic AND international air carrier energy use and passenger-miles.

\section{General aviation}

Number of vehicles - DOT, FAA, General Aviation and Air Taxi Activity Surveys - CY 2012. 2011 Data: Aviation Forecasts, Tables 28 and 29, May 2014. Data series shown in Table 9.3.

\section{Energy intensities -}

Btu per passenger-mile - General aviation energy use divided by passenger-miles.

Energy use - See Energy Use Sources, p. A-14. Data series shown in Table 9.3.

\section{$\underline{\text { Recreational boating }}$}

Number of vehicles and energy use - U.S. EPA, NONROAD2008a model. 


\section{Rail}

\section{Intercity}

Number of vehicles, vehicle-miles, passenger-miles - AAR, Railroad Facts, 2013 Edition, Washington, DC, 2013.

Load factor - Passenger-miles divided by vehicle-miles.

Energy Intensities -

Btu per vehicle-mile - Intercity rail energy use divided by vehicle-miles.

Btu per passenger-mile - Intercity rail energy use divided by passenger-miles.

Energy use - See Energy Use Sources, p. A-23. Data series shown in Table 9.10.

\section{Transit}

Number of vehicles, vehicle-miles, passenger-miles - APTA, 2014 Public Transportation Fact Book, Washington, DC, 2014. Sum of light and heavy rail transit. Data series shown on Table 9.12.

Load factor - Passenger-miles divided by vehicle-miles.

\section{Energy intensities -}

Btu per vehicle-mile - Light and heavy transit rail energy use divided by vehicle-miles.

Btu per passenger-mile - Light and heavy transit rail energy use divided by passenger-miles.

Energy use - See Energy Use Sources, p. A-22. Data series shown in Table 9.12.

\section{Commuter}

Number of vehicles, vehicle-miles, passenger-miles - APTA, 2014 Public Transportation Fact Book, Washington, DC, 2014. Data series shown on Table 9.11.

Load factor - Passenger-miles divided by vehicle-miles.

Energy intensities -

Btu per vehicle-mile - Commuter rail energy use divided by vehicle-miles.

Btu per passenger-mile - Commuter rail energy use divided by passenger-miles.

Energy use - See Energy Use Sources, p. A-21. Data series shown in Table 9.11. 


\section{Highway Passenger Mode Energy Intensities}

\section{Cars}

Btu per vehicle-mile - Car energy use divided by car vehicle miles of travel.

Energy use - See Energy Use Sources, p. A-5. Data series shown in Table 2.7.

Vehicle-miles - 1970-2008: DOT, FHWA, Highway Statistics 2008, Table VM-1 and annual editions back to 1996 and DOT, FHWA, Highway Statistics Summary to 1995. Data series shown in Table 4.1.

2009-2012: See Appendix A for Car/Light Truck Shares.

Btu per passenger-mile - Car energy use divided by car passenger-miles.

Energy use - See Energy Use Sources, p. A-5. Data series shown in Table 2.7.

Passenger miles - Vehicle miles multiplied by an average load factor.

Vehicle-miles - DOT, FHWA, Highway Statistics 2009, Table VM-1 and annual editions back to 1996 and DOT, FHWA, Highway Statistics Summary to 1995. Data series shown in Table 4.1.

Load factor - NPTS 1969, 1977, 1983/84, 1990, and 1995; NHTS 2001 and 2009. Data series shown in Table A.18. 
Table A.18

Car Load Factor used to Calculate Passenger-Miles

\begin{tabular}{|c|c|c|}
\hline Year & Source & Load Factor \\
\hline 1970 & 1969 NPTS & 1.90 \\
\hline 1971 & Interpolated & 1.90 \\
\hline 1972 & Interpolated & 1.90 \\
\hline 1973 & Interpolated & 1.90 \\
\hline 1974 & Interpolated & 1.90 \\
\hline 1975 & Interpolated & 1.90 \\
\hline 1976 & Interpolated & 1.90 \\
\hline 1977 & 1977 NPTS & 1.90 \\
\hline 1978 & Interpolated & 1.88 \\
\hline 1979 & Interpolated & 1.87 \\
\hline 1980 & Interpolated & 1.85 \\
\hline 1981 & Interpolated & 1.83 \\
\hline 1982 & Interpolated & 1.82 \\
\hline 1983 & $1983 / 84$ NPTS & 1.80 \\
\hline 1984 & Interpolated & 1.77 \\
\hline 1985 & Interpolated & 1.74 \\
\hline 1986 & Interpolated & 1.71 \\
\hline 1987 & Interpolated & 1.69 \\
\hline 1988 & Interpolated & 1.66 \\
\hline 1989 & Interpolated & 1.63 \\
\hline 1990 & 1990 NPTS & 1.60 \\
\hline 1991 & Interpolated & 1.60 \\
\hline 1992 & Interpolated & 1.60 \\
\hline 1993 & Interpolated & 1.60 \\
\hline 1994 & Interpolated & 1.60 \\
\hline 1995 & 1995 NPTS & 1.60 \\
\hline 1996 & Interpolated & 1.60 \\
\hline 1997 & Interpolated & 1.59 \\
\hline 1998 & Interpolated & 1.59 \\
\hline 1999 & Interpolated & 1.58 \\
\hline 2000 & Interpolated & 1.58 \\
\hline 2001 & 2001 NHTS & 1.57 \\
\hline 2002 & 2001 NHTS & 1.57 \\
\hline 2003 & 2001 NHTS & 1.57 \\
\hline 2004 & 2001 NHTS & 1.57 \\
\hline 2005 & 2001 NHTS & 1.57 \\
\hline 2006 & 2001 NHTS & 1.57 \\
\hline 2007 & 2001 NHTS & 1.57 \\
\hline 2008 & 2009 NHTS & 1.55 \\
\hline 2009 & 2009 NHTS & 1.55 \\
\hline 2010 & 2009 NHTS & 1.55 \\
\hline 2011 & 2009 NHTS & 1.55 \\
\hline 2012 & 2009 NHTS & 1.55 \\
\hline
\end{tabular}




\section{Light trucks}

Btu per vehicle-mile - Light truck energy use divided by light truck vehicle miles of travel.

Energy use - See Energy Use Sources, p. A-10. Data series shown in Table 2.7.

Vehicle-miles - 1970-2008: DOT, FHWA, Highway Statistics 2008, Table VM-1 and annual editions back to 1996 and DOT, FHWA, Highway Statistics Summary to 1995. Data series shown in Table 4.2. 2009-2012: See Appendix A for Car/Light Truck Shares.

\section{Buses}

Transit

Btu per vehicle-mile - Transit bus energy use divided by transit bus vehicle-miles.

Energy use - See Energy Use Sources, p. A-8. Data series shown in Table 5.18.

Vehicle-miles - APTA, 2014 Public Transportation Fact Book, Washington, DC, 2014. Data series shown on Table 5.18.

Btu per passenger-mile - Transit bus energy use divided by transit bus passenger-miles.

Energy use - See Energy Use Sources, p. A-8. Data series shown in Table 5.18.

Passenger-miles - APTA, 2014 Public Transportation Fact Book, Washington, DC, 2014. Data series shown on Table 5.18.

\section{Intercity}

Btu per passenger-mile - Data are not available.

Energy use - See Energy Use Sources, p. A-9. Because the data past 2000 are not available, the rate of change in bus VMT from FHWA, Highway Statistics 2012, was used to estimate the change in energy use.

Passenger-miles - Data are not available. 


\section{Nonhighway Mode Energy Intensities}

\section{$\underline{\text { Air }}$}

\section{Certificated air carriers}

Btu per passenger-mile - Certificated air carrier energy use divided by passenger-miles.

Energy use - See Energy Use Sources, p. A-15. All of domestic fuel use and half of international fuel use was considered to be domestic use.

Passenger-miles - DOT, BTS, Air Carrier Traffic Statistics, Washington, DC, www.apps.bts.gov/xml/air_traffic/src/index.html. Pre-1994 data are from various editions of the FAA Statistical Handbook of Aviation (no longer published). Scheduled service passenger-miles of domestic air carriers and half of international air carriers were used to coincide with fuel use.

Note: These data differ from the data in Table 9.2 because that table contains data on ALL domestic AND international air carrier energy use and passenger-miles.

\section{General aviation}

Btu per passenger-mile - Data are not available.

Energy use - See Energy Use Sources, p. A-14. Data series shown in Table 9.3.

Passenger-miles - Data are not available.

\section{Rail}

\section{Intercity}

Btu per passenger-mile - Intercity rail energy use divided by passenger-miles.

Energy use - See Energy Use Sources, p. A-23. Data series shown in Table 9.10.

Passenger-miles - AAR, Railroad Facts, 2013 Edition, and previous annual editions.

\section{Transit}

Btu per passenger-mile - Transit rail energy use divided by passenger-miles.

Energy use - See Energy Use Sources, p. A-22. Data series shown in Table 9.12.

Passenger-miles - APTA, 2014 Public Transportation Fact Book, Washington, DC, 2014. Data series shown on Table 9.12.

\section{Commuter}

Btu per passenger-mile - Commuter rail energy use divided by passenger-miles.

Energy use - See Energy Use Sources, p. A-21. Data series shown in Table 9.11.

Passenger-miles - APTA, 2014 Public Transportation Fact Book, Washington, DC, 2014. Data series shown on Table 9.11. 


\section{Freight Mode Energy Intensities}

\section{Truck}

Btu per vehicle-mile - Heavy single-unit and combination truck energy use divided by vehicle miles Energy use - See Energy Use Sources (medium/heavy trucks), p. A-12.

Vehicle-miles - DOT, FHWA, Highway Statistics 2012, Table VM-1 and annual editions back to 1996 and DOT, FHWA, Highway Statistics Summary to 1995. Data series is the total of vehicle travel data on Tables 5.1 and 5.2.

\section{$\underline{\text { Rail }}$}

Btu per freight car-mile - Class I rail energy use divided by freight car-miles.

Energy use - See Energy Use Sources, p. A-20. Data series shown in Table 9.8.

Freight car miles - AAR, Railroad Facts, 2013 Edition, Washington, DC, 2013. Data series shown in Table 9.8 .

Btu per ton-mile - Class I rail energy use divided by ton-miles.

Energy use - See Energy Use Sources, p. A-20. Data series shown in Table 9.8.

Ton-miles - AAR, Railroad Facts, 2013 Edition, Washington, DC, 2013. Data series shown in

Table 9.8.

\section{Water}

Btu per ton-mile - Domestic waterborne commerce energy use on taxable waterways divided by tonmiles on taxable waterways.

Energy use - Modeled by Chrisman A. Dager, University of Tennessee, Knoxville, using Waterborne Commerce Statistics Center detail records and annual IRS reports on the Inland Waterway Trust Fund tax on diesel fuel used on the inland waterway.

Ton-miles - Based on detailed records from the U.S. Department of the Army, Army Corps of Engineers, Waterborne Commerce Statistics Center. Includes only ton-miles on taxable waterways. 


\section{Car and Light Truck Shares}

In 2011, the Federal Highway Administration (FHWA) changed the methodology for producing the data on the VM-1 Table in the annual Highway Statistics publication. Historically, VM-1 included individual categories for passenger cars and 2-axle, 4-tire trucks. VM-1 included the vehicle miles of travel (VMT), registrations, fuel use, and fuel economy of passenger cars and 2-axle, 4-tire trucks. After the methodology change, the categories of light vehicles on VM-1 changed to Light-Duty Vehicles with Short wheelbase (less than or equal to 121 inches) and Light-Duty Vehicles with Long Wheelbase (over 121 inches). As some passenger cars have long wheelbases and some 2-axle, 4-tire trucks have short wheelbases, the categories of cars and 2-axle, 4-tire trucks are no longer available. Despite these changes, there are many transportation analysts who require information on cars and 2-axle, 4-tire trucks. Thus, a new methodology to estimate the data in these categories was developed for years 2009 through 2012.

\section{Cars}

Registrations - DOT, FHWA, Highway Statistics 2012, Table MV-1 and annual editions back to 2009.

Vehicle travel -

Total for all light vehicles - DOT, FHWA, Highway Statistics 2012, Table VM-1 and annual editions back to 2009; sum of light-duty short wheelbase and light-duty long wheelbase VMT.

Cars - Using historical shares of passenger cars/2-axle, 4-tire trucks from the Highway Statistics, the percent of light vehicle travel attributable to cars was estimated for 2009-2012, keeping in mind the economic conditions present in those years and the general trend in total light vehicle VMT. The estimated share was applied to total VMT as shown in Table A.19.

Table A.19

Estimated Car VMT

\begin{tabular}{llll}
\hline & $\begin{array}{l}\text { Total Light Vehicle } \\
\text { VMT (billions) }\end{array}$ & $\begin{array}{l}\text { Share Attributable to } \\
\text { Cars }\end{array}$ & $\begin{array}{l}\text { Total Car } \\
\text { VMT (billions) }\end{array}$ \\
\hline 2009 & $2,633.3$ & $59.5 \%$ & $1,566.8$ \\
2010 & $2,648.5$ & $56.5 \%$ & $1,496.4$ \\
2011 & $2,650.5$ & $55.0 \%$ & $1,457.8$ \\
2012 & $2,664.5$ & $54.0 \%$ & $1,438.8$ \\
\hline
\end{tabular}

Miles per Vehicle - Vehicle travel divided by registrations.

Fuel Use - Vehicle travel divided by fuel economy.

Fuel Economy - DOE, EIA, Annual Energy Outlook 2014, April 2014 and annual editions back to 2012.

\section{2-axle, 4-tire Trucks}

Registrations - DOT, FHWA, Highway Statistics 2012, Table MV-1 and annual editions back to 2009.

Vehicle travel -

Total for all light vehicles - DOT, FHWA, Highway Statistics 2012, Table VM-1 and annual editions back to 2009; sum of light-duty short wheelbase and light-duty long wheelbase VMT.

2-axle, 4-tire truck VMT - Using historical shares of passenger cars/2-axle, 4-tire trucks from the Highway Statistics, the percent of light vehicle travel attributable to cars was estimated for 20092012, keeping in mind the economic conditions present in those years and the general trend in total light vehicle VMT. The estimated share was applied to total VMT as shown in Table A.20. 
Table A.20

Estimated 2-axle, 4-tire Truck VMT

\begin{tabular}{cccc}
\hline Year & $\begin{array}{c}\text { Total Light Vehicle } \\
\text { VMT (billions) }\end{array}$ & $\begin{array}{c}\text { Share Attributable to } \\
\text { 2-axle, 4-tire Trucks }\end{array}$ & $\begin{array}{c}\text { Total 2-axle, 4-tire } \\
\text { Truck VMT (billions) }\end{array}$ \\
\hline 2009 & $2,633.2$ & $40.5 \%$ & $1,066.5$ \\
2010 & $2,648.5$ & $43.5 \%$ & $1,152.1$ \\
2011 & $2,650.5$ & $45.0 \%$ & $1,192.7$ \\
2012 & $2,664.5$ & $46.0 \%$ & $1,225.6$ \\
\hline
\end{tabular}

Miles per Vehicle - Vehicle travel divided by registrations.

Fuel Use - Vehicle travel divided by fuel economy.

Fuel Economy - DOE, EIA, Annual Energy Outlook 2014, April 2014 and annual editions back to 2012. 
APPENDIX B

CONVERSIONS 
B-2

TRANSPORTATION ENERGy DATA BOOK: EDITION 33-2014 


\section{CONVERSIONS}

\section{A Note about Heating Values}

The heat content of a fuel is the quantity of energy released by burning a unit amount of that fuel. However, this value is not absolute and can vary according to several factors. For example, empirical formulae for determining the heating value of liquid fuels depend on the fuels' American Petroleum Institute (API) gravity. The API gravity varies depending on the percent by weight of the chemical constituents and impurities in the fuel, both of which are affected by the combination of raw materials used to produce the fuel and by the type of manufacturing process. Temperature and climatic conditions are also factors.

Because of these variations, the heating values in Table B.4 may differ from values in other publications. The figures in this report are representative or average values, not absolute ones. The gross (higher) heating values used here agree with those used by the Energy Information Administration (EIA).

Heating values fall into two categories, usually referred to as "higher" (or gross) and "lower" (or net). If the products of fuel combustion are cooled back to the initial fuel-air or fuel-oxidizer mixture temperature and the water formed during combustion is condensed, the energy released by the process is the higher (gross) heating value. If the products of combustion are cooled to the initial fuel-air temperature, but the water is considered to remain as a vapor, the energy released by the process is the lower (net) heating value. Usually the difference between the gross and net heating values for fuels used in transportation is around 5 to 8 percent; however, it is important to be consistent in their use. 
Table B.1

Hydrogen Heat Content

\begin{tabular}{cc}
\hline \multicolumn{2}{c}{1 kilogram hydrogen $=$} \\
\hline Higher heating value & Lower heating value \\
$134,200 \mathrm{Btu}$ & $113,400 \mathrm{Btu}$ \\
$39.3 \mathrm{kWhr}$ & $33.2 \mathrm{kWhr}$ \\
$141,600 \mathrm{~kJ}$ & $119,600 \mathrm{~kJ}$ \\
$33,800 \mathrm{kCal}$ & $28,560 \mathrm{kCal}$ \\
\hline
\end{tabular}

Table B.2

Hydrogen Conversions

\begin{tabular}{|c|c|c|c|c|c|c|}
\hline & \multicolumn{2}{|c|}{ Weight } & \multicolumn{2}{|c|}{ Gas } & \multicolumn{2}{|c|}{ Liquid } \\
\hline & $\begin{array}{c}\text { Pounds } \\
\text { (lb) }\end{array}$ & $\begin{array}{c}\text { Kilograms } \\
(\mathrm{kg})\end{array}$ & $\begin{array}{c}\text { Standard } \\
\text { cubic feet } \\
(\mathrm{SCF})\end{array}$ & $\begin{array}{c}\text { Normal } \\
\text { cubic meter } \\
\left(\mathrm{Nm}^{3}\right)\end{array}$ & $\begin{array}{c}\text { Gallons } \\
\text { (gal) }\end{array}$ & $\begin{array}{c}\text { Liters } \\
\text { (L) }\end{array}$ \\
\hline $1 \mathrm{lb}$ & 1.0 & 0.4536 & 192.00 & 5.047 & 1.6928 & 6.408 \\
\hline $1 \mathrm{~kg}$ & 2.205 & 1.0 & 423.3 & 11.126 & 3.733 & 14.128 \\
\hline 1 SCF gas & 0.005209 & 0.002363 & 1.0 & 0.02628 & 0.00882 & 0.0339 \\
\hline $1 \mathrm{Nm}^{3}$ gas & 0.19815 & 0.08988 & 38.04 & 1.0 & 0.3355 & 1.2699 \\
\hline 1 gal liquid & 0.5906 & 0.2679 & 113.41 & 2.981 & 1.0 & 3.785 \\
\hline $1 \mathrm{~L}$ liquid & 0.15604 & 0.07078 & 29.99 & 0.77881 & 0.2642 & 1.0 \\
\hline
\end{tabular}

Table B.3

Pressure Conversions

\begin{tabular}{lccc}
\hline & Bar & Atmosphere & $\mathrm{lb}_{\text {in }}^{2}($ or psi) \\
\hline Bar & 1.0 & 0.987 & 14.5 \\
Atmosphere & 1.013 & 1.0 & 14.696 \\
$\mathrm{lb}_{\mathrm{in}}{ }^{2}$ (or psi) & 0.0689 & 0.0680 & 1.0 \\
\hline
\end{tabular}


Table B.4

Heat Content for Various Fuels

\begin{tabular}{|c|c|}
\hline Conventional gasoline & $125,000 \mathrm{Btu} / \mathrm{gal}($ gross $)=115,400 \mathrm{Btu} / \mathrm{gal}$ (net) \\
\hline E10 & $120,900 \mathrm{Btu} / \mathrm{gal}(\mathrm{gross})=112,400 \mathrm{Btu} / \mathrm{gal}(\mathrm{net})$ \\
\hline E15 & $119,000 \mathrm{Btu} / \mathrm{gal}($ gross $)=109,400 \mathrm{Btu} / \mathrm{gal}($ net $)$ \\
\hline Hydrogen & $134,200 \mathrm{Btu} / \mathrm{kg}($ gross $)=113,400 \mathrm{Btu} / \mathrm{kg}$ (net) \\
\hline Low-sulfur diesel & $138,700 \mathrm{Btu} / \mathrm{gal}($ gross $)=128,700 \mathrm{Btu} / \mathrm{gal}($ net $)$ \\
\hline Biodiesel & $126,200 \mathrm{Btu} / \mathrm{gal}($ gross $)=117,100 \mathrm{Btu} / \mathrm{gal}($ net $)$ \\
\hline Methanol & $64,600 \mathrm{Btu} / \mathrm{gal}($ gross $)=56,600 \mathrm{Btu} / \mathrm{gal}($ net $)$ \\
\hline Ethanol & $84,600 \mathrm{Btu} /$ gal $($ gross $)=75,700 \mathrm{Btu} / \mathrm{gal}$ (net) \\
\hline E85 & $90,700 \mathrm{Btu} /$ gal $($ gross $)=81,600 \mathrm{Btu} / \mathrm{gal}($ net $)$ \\
\hline Aviation gasoline & $120,200 \mathrm{Btu} / \mathrm{gal}(\mathrm{gross})=112,000 \mathrm{Btu} / \mathrm{gal}($ net $)$ \\
\hline Liquefied petroleum gas (LPG) & $91,300 \mathrm{Btu} /$ gal $($ gross $)=83,500 \mathrm{Btu} / \mathrm{gal}$ (net) \\
\hline Butane & $103,000 \mathrm{Btu} / \mathrm{gal}(\mathrm{gross})=93,000 \mathrm{Btu} / \mathrm{gal}(\mathrm{net})$ \\
\hline Jet fuel (naphtha) & $127,500 \mathrm{Btu} / \mathrm{gal}($ gross $)=118,700 \mathrm{Btu} / \mathrm{gal}($ net $)$ \\
\hline Jet fuel (kerosene) & $135,000 \mathrm{Btu} / \mathrm{gal}($ gross $)=128,100 \mathrm{Btu} / \mathrm{gal}($ net $)$ \\
\hline Lubricants & $144,400 \mathrm{Btu} / \mathrm{gal}($ gross $)=130,900 \mathrm{Btu} / \mathrm{gal}($ net $)$ \\
\hline Waxes & $131,800 \mathrm{Btu} / \mathrm{gal}($ gross $)=120,200 \mathrm{Btu} / \mathrm{gal}($ net $)$ \\
\hline Asphalt and road oil & $158,000 \mathrm{Btu} / \mathrm{gal}($ gross $)=157,700 \mathrm{Btu} / \mathrm{gal}($ net $)$ \\
\hline Liquefied natural gas (LNG) & $23,700 \mathrm{Btu} / \mathrm{lb}($ gross $)=20,900 \mathrm{Btu} / \mathrm{lb}($ net $)$ \\
\hline Compressed natural gas (CNG) & $22,500 \mathrm{Btu} / \mathrm{lb}($ gross $)=20,200 \mathrm{Btu} / \mathrm{lb}($ net $)$ \\
\hline Crude petroleum & $138,100 \mathrm{Btu} / \mathrm{gal}($ gross $)=131,800 \mathrm{Btu} / \mathrm{gal}($ net $)$ \\
\hline \multicolumn{2}{|l|}{ Fuel Oils } \\
\hline Residual & $149,700 \mathrm{Btu} / \mathrm{gal}($ gross $)=138,400 \mathrm{Btu} / \mathrm{gal}($ net $)$ \\
\hline Distillate & $138,700 \mathrm{Btu} / \mathrm{gal}($ gross $)=131,800 \mathrm{Btu} / \mathrm{gal}($ net $)$ \\
\hline \multicolumn{2}{|l|}{ Coal } \\
\hline Production average & $20.187 \times 10^{6} \mathrm{Btu} / \mathrm{short}$ ton \\
\hline Consumption average & $19.548 \times 10^{6} \mathrm{Btu} / \mathrm{short}$ ton \\
\hline
\end{tabular}

Note: Heat content values are approximate. Data are rounded to the nearest hundred. 


\section{Table B.5}

\section{Fuel Equivalents}

\begin{tabular}{|c|c|}
\hline 1 million bbl crude oil/day & $\begin{array}{l}=0.365 \text { billion bbl crude oil/year } \\
=2.117 \text { quadrillion Btu/year } \\
=108.298 \text { million short tons coal/year } \\
=98.248 \text { million metric tons coal/year } \\
=2.065{\text { trillion } \mathrm{ft}^{3} \text { natural gas/year }} \\
=2,233 \text { petajoules/year }\end{array}$ \\
\hline 1 billion bbl crude oil/year & $\begin{array}{l}=2.740 \text { million bbl crude oil/day } \\
=5.800 \text { quadrillion Btu/year } \\
=296.706 \text { million short tons coal/year } \\
=269.171 \text { million metric tons coal/year } \\
=5.659 \text { trillion } \mathrm{ft}^{3} \text { natural gas/year } \\
=6,119 \text { petajoules/year }\end{array}$ \\
\hline 1 quadrillion Btu/year & $\begin{array}{l}=8.000 \text { million gasoline gallon equivalents } \\
=0.472 \text { million bbl crude oil/day } \\
=172.414 \text { million bbl crude oil/year } \\
=51.156 \text { million short tons coal/year } \\
=46.409 \text { million metric tons coal/year } \\
=975.610 \text { billion } \mathrm{ft}^{3} \text { natural gas/year } \\
=1,055 \text { petajoules/year }\end{array}$ \\
\hline 1 billion short tons coal/year & $\begin{array}{l}=0.907 \text { billion metric tons coal/year } \\
=9.234 \text { million bbl crude oil/day } \\
=3.370 \text { billion bbl crude oil/year } \\
=19.548 \text { quadrillion Btu/year } \\
=19.071 \text { trillion } \mathrm{ft}^{3} \text { natural gas/year } \\
=20,623 \text { petajoules/year }\end{array}$ \\
\hline 1 billion metric tons coal/year & $\begin{array}{l}=1.102 \text { billion short tons coal/year } \\
=8.377 \text { million bbl crude oil/day } \\
=3.058 \text { billion bbl crude oil/year } \\
=17.734 \text { quadrillion btu/year } \\
=17.301 \text { trillion } \mathrm{ft}^{3} \text { natural gas/year } \\
=18,709 \text { petajoules/year }\end{array}$ \\
\hline 1 trillion $\mathrm{ft}^{3}$ natural gas/year & $\begin{array}{l}=0.484 \text { million bbl crude oil/day } \\
=0.177 \text { billion bbl crude oil/year } \\
=1.025 \text { quadrillion Btu/year } \\
=52.435 \text { million short tons coal/year } \\
=47.569 \text { million metric tons coal/year } \\
=1,081 \text { petajoules/year }\end{array}$ \\
\hline 1 petajoule/year & $\begin{array}{l}=447.741 \mathrm{bbl} \text { crude oil/day } \\
=163.425 \text { thousand bbl crude oil/year } \\
=0.948 \text { trillion Btu/year } \\
=48.489 \text { thousand short tons coal/year } \\
=43.989 \text { thousand metric tons coal/year } \\
=0.925 \text { billion } \mathrm{ft}^{3} \text { natural gas/year }\end{array}$ \\
\hline
\end{tabular}


Table B.6

Energy Unit Conversions

\begin{tabular}{|c|c|c|c|}
\hline $1 \mathrm{Btu}$ & $\begin{array}{l}=778.2 \mathrm{ft}-\mathrm{lb} \\
=107.6 \mathrm{~kg}-\mathrm{m} \\
=1055 \mathrm{~J} \\
=39.30 \times 10^{-5} \mathrm{hp}-\mathrm{h} \\
=39.85 \times 10^{-5} \text { metric hp-h } \\
=29.31 \times 10^{-5} \mathrm{kWhr}\end{array}$ & $1 \mathrm{kWhr}$ & $\begin{array}{l}=3412 \mathrm{Btu}^{\mathrm{a}} \\
=2.655 \times 10^{6} \mathrm{ft}-\mathrm{lb} \\
=3.671 \times 10^{5} \mathrm{~kg}-\mathrm{m} \\
=3.600 \times 10^{6} \mathrm{~J} \\
=1.341 \mathrm{hp}-\mathrm{h} \\
=1.360 \text { metric hp-h }\end{array}$ \\
\hline $1 \mathrm{~kg}-\mathrm{m}$ & $\begin{array}{l}=92.95 \times 10^{-4} \mathrm{Btu} \\
=7.233 \mathrm{ft}-\mathrm{lb} \\
=9.806 \mathrm{~J} \\
=36.53 \times 10^{-7} \mathrm{hp}-\mathrm{h} \\
=37.04 \times 10^{-7} \text { metric hp-h } \\
=27.24 \times 10^{-7} \mathrm{kWhr}\end{array}$ & 1 Joule & $\begin{array}{l}=94.78 \times 10^{-5} \mathrm{Btu} \\
=0.7376 \mathrm{ft}-\mathrm{lb} \\
=0.1020 \mathrm{~kg}-\mathrm{m} \\
=37.25 \times 10^{-8} \mathrm{hp}-\mathrm{h} \\
=37.77 \times 10^{-8} \text { metric hp-h } \\
=27.78 \times 10^{-8} \mathrm{kWhr}\end{array}$ \\
\hline $1 \mathrm{hp}-\mathrm{h}$ & $\begin{array}{l}=2544 \mathrm{Btu} \\
=1.98 \times 10^{6} \mathrm{ft}-\mathrm{lb} \\
=2.738 \times 10^{6} \mathrm{kgm} \\
=2.685 \times 10^{6} \mathrm{~J} \\
=1.014 \text { metric } \mathrm{hp}-\mathrm{h} \\
=0.7475 \mathrm{kWhr}\end{array}$ & 1 metric hp-h & $\begin{array}{l}=2510 \mathrm{Btu} \\
=1.953 \times 10^{6} \mathrm{ft}-\mathrm{lb} \\
=27.00 \times 10^{4} \mathrm{~kg}-\mathrm{m} \\
=2.648 \times 10^{6} \mathrm{~J} \\
=0.9863 \mathrm{hp}-\mathrm{h} \\
=0.7355 \mathrm{kWhr}\end{array}$ \\
\hline
\end{tabular}

${ }^{a}$ This figure does not take into account the fact that electricity generation and distribution efficiency is approximately $33 \%$. If generation and distribution efficiency are taken into account, $1 \mathrm{kWhr}=10,339 \mathrm{Btu}$.

Table B.7

International Energy Conversions

\begin{tabular}{rccccc}
\hline To: & Petajoules & $\begin{array}{c}\text { Giga- } \\
\text { calories }\end{array}$ & $\begin{array}{c}\text { Million } \\
\text { tonnes of oil } \\
\text { equivalent }\end{array}$ & $\begin{array}{c}\text { Million } \\
\text { Btu }\end{array}$ & $\begin{array}{c}\text { Gigawatt- } \\
\text { hours }\end{array}$ \\
\hline $\begin{array}{r}\text { From: } \\
\text { Petajoules }\end{array}$ & 1 & $238.8 \times 10^{3}$ & $2.388 \times 10^{-2}$ & $947.8 \times 10^{3}$ & 277.8 \\
$\begin{array}{r}\text { Gigacalories } \\
\text { Million tonnes }\end{array}$ & $4.1868 \times 10^{-6}$ & 1 & $10^{-7}$ & 3.968 & $1.163 \times 10^{-3}$ \\
$\begin{array}{r}\text { of oil equivalent } \\
\text { Million Btu }\end{array}$ & $1.0551 \times 10^{-6}$ & 0.252 & $2.52 \times 10^{-8}$ & 1 & $2.931 \times 10^{-4}$ \\
Gigawatthours & $3.6 \times 10^{-3}$ & 860 & $8.6 \times 10^{-5}$ & 3412 & 1 \\
\hline
\end{tabular}


Table B.8

Distance and Velocity Conversions

\begin{tabular}{|c|c|c|c|}
\hline $1 \mathrm{in.}$ & $\begin{array}{l}=83.33 \times 10^{-3} \mathrm{ft} \\
=27.78 \times 10^{-3} \mathrm{yd} \\
=15.78 \times 10^{-6} \mathrm{mile} \\
=25.40 \times 10^{-3} \mathrm{~m} \\
=0.2540 \times 10^{-6} \mathrm{~km}\end{array}$ & $1 \mathrm{ft}$ & $\begin{array}{l}=12.0 \mathrm{in} . \\
=0.33 \mathrm{yd} \\
=189.4 \times 10^{-3} \mathrm{mile} \\
=0.3048 \mathrm{~m} \\
=0.3048 \times 10^{-3} \mathrm{~km}\end{array}$ \\
\hline 1 mile & $\begin{array}{l}=63360 \mathrm{in} . \\
=5280 \mathrm{ft} \\
=1760 \mathrm{yd} \\
=1609 \mathrm{~m} \\
=1.609 \mathrm{~km}\end{array}$ & $1 \mathrm{~km}$ & $\begin{array}{l}=39370 \mathrm{in} . \\
=3281 \mathrm{ft} \\
=1093.6 \mathrm{yd} \\
=0.6214 \mathrm{mile} \\
=1000 \mathrm{~m}\end{array}$ \\
\hline & $\begin{array}{l}1 \mathrm{ft} / \mathrm{sec}= \\
1 \mathrm{~m} / \mathrm{sec}= \\
1 \mathrm{~km} / \mathrm{h}=0 \\
1 \mathrm{mph}=1 .\end{array}$ & $\begin{array}{l}72 \mathrm{~km} / \mathrm{h} \\
\mathrm{m} / \mathrm{h} \\
\mathrm{mph} \\
\mathrm{n} / \mathrm{h}\end{array}$ & \\
\hline
\end{tabular}

Table B.9

Alternative Measures of Greenhouse Gases

1 pound methane, measured in carbon units $\left(\mathrm{CH}_{4}\right)$

1 pound carbon dioxide, measured in carbon units $\left(\mathrm{CO}_{2}-\mathrm{C}\right)$

1 pound carbon monoxide, measured in carbon units $(\mathrm{CO}-\mathrm{C})$

1 pound nitrous oxide, measured in nitrogen units $\left(\mathrm{N}_{2} \mathrm{O}-\mathrm{N}\right)$
1.333 pounds methane, measured at full

$=$ molecular weight $\left(\mathrm{CH}_{4}\right)$

3.6667 pounds carbon dioxide, measured at full $=$ molecular weight $\left(\mathrm{CO}_{2}\right)$

2.333 pounds carbon monoxide, measured at $=\quad$ full molecular weight $(\mathrm{CO})$

1.571 pounds nitrous oxide, measured at full $=$ molecular weight $\left(\mathrm{N}_{2} \mathrm{O}\right)$ 
Table B.10

Volume and Flow Rate Conversions ${ }^{\mathrm{a}}$

\begin{tabular}{|c|c|c|c|}
\hline \multirow[t]{6}{*}{1 U.S. gal } & $=231 \mathrm{in}^{3}$ & 1 liter & $=61.02 \mathrm{in}^{3}$ \\
\hline & $=0.1337 \mathrm{ft}^{3}$ & & $=3.531 \times 10^{-2} \mathrm{ft}^{3}$ \\
\hline & $=3.785$ liters & & $=0.2624$ U.S. gal \\
\hline & $=0.8321$ imperial gal & & $=0.2200$ imperial gal \\
\hline & $=0.0238 \mathrm{bbl}$ & & $=6.29 \times 10^{-3} \mathrm{bbl}$ \\
\hline & $=0.003785 \mathrm{~m}^{3}$ & & $=0.001 \mathrm{~m}^{3}$ \\
\hline \multicolumn{4}{|c|}{ A U.S. gallon of gasoline weighs 6.2 pounds } \\
\hline \multirow[t]{6}{*}{1 imperial gal } & $=277.4$ in. $^{3}$ & $1 \mathrm{bbl}$ & $=9702$ in. $^{3}$ \\
\hline & $=0.1606 \mathrm{ft}^{3}$ & & $=5.615 \mathrm{ft}^{3}$ \\
\hline & $=4.545$ liters & & $=158.97$ liters \\
\hline & $=1.201$ U.S. gal & & $=42$ U.S. gal \\
\hline & $=0.0286 \mathrm{bbl}$ & & $=34.97$ imperial gal \\
\hline & $=0.004546 \mathrm{~m}^{3}$ & & $=0.15897 \mathrm{~m}^{3}$ \\
\hline \multirow[t]{4}{*}{1 U.S. gal/hr } & $=3.209 \mathrm{ft}^{3} /$ day & & $=1171 \mathrm{ft}^{3} /$ year \\
\hline & $=90.84$ liter $/$ day & & $=33157$ liter/year \\
\hline & $=19.97 \mathrm{imperial}$ gal $/$ day & & $=7289 \mathrm{imperial}$ gal/year \\
\hline & $=0.5712 \mathrm{bbl} / \mathrm{day}$ & & $=207.92 \mathrm{bbl} /$ year \\
\hline \multicolumn{4}{|c|}{ For Imperial gallons, multiply above values by 1.201} \\
\hline \multirow[t]{4}{*}{1 liter/hr } & $=0.8474 \mathrm{ft}^{3} /$ day & & $=309.3 \mathrm{ft}^{3} /$ year \\
\hline & $=6.298$ U.S. gal/day & & $=2299$ U.S. gal $/$ year \\
\hline & $=5.28$ imperial gal $/$ day & & $=1927 \mathrm{imperial} \mathrm{gal} /$ year \\
\hline & $=0.1510 \mathrm{bbl} / \mathrm{day}$ & & $=55.10 \mathrm{bbl} /$ year \\
\hline \multirow[t]{4}{*}{$1 \mathrm{bbl} / \mathrm{hr}$} & $=137.8 \mathrm{ft}^{3} /$ year & & $=49187 \mathrm{ft}^{3}$ year \\
\hline & $=1008$ U.S. gal/day & & $=3.679 \times 10^{5}$ U.S. gal $/$ year \\
\hline & $=839.3 \mathrm{imperial} \mathrm{gal} /$ day & & $=3.063 \times 10^{5} \mathrm{imperial}$ gal $/$ year \\
\hline & $=3815$ liter/day & & $=1.393 \times 10^{6}$ liter $/$ day \\
\hline
\end{tabular}

${ }^{\mathrm{a}}$ The conversions for flow rates are identical to those for volume measures, if the time units are identical. 
Table B.11

Power Conversions

\begin{tabular}{|c|c|c|c|c|c|c|}
\hline \multirow[b]{2}{*}{ FROM } & \multicolumn{6}{|c|}{ TO } \\
\hline & Horsepower & Kilowatts & $\begin{array}{c}\text { Metric } \\
\text { horsepower }\end{array}$ & Ft-lb per sec & $\begin{array}{c}\text { Kilocalories } \\
\text { per sec }\end{array}$ & Btu per sec \\
\hline Horsepower & 1 & 0.7457 & 1.014 & 550 & 0.1781 & 0.7068 \\
\hline Kilowatts & 1.341 & 1 & 1.360 & 737.6 & 0.239 & 0.9478 \\
\hline $\begin{array}{l}\text { Metric } \\
\text { horsepower }\end{array}$ & 0.9863 & 0.7355 & 1 & 542.5 & 0.1757 & 0.6971 \\
\hline Ft-lb per sec & $1.36 \times 10^{-3}$ & $1.356 \times 10^{-3}$ & $1.84 \times 10^{-3}$ & 1 & $0.3238 \times 10^{-3}$ & $1.285 \times 10^{-3}$ \\
\hline $\begin{array}{l}\text { Kilocalories } \\
\text { per sec }\end{array}$ & 5.615 & 4.184 & 5.692 & 3088 & 1 & 3.968 \\
\hline Btu per sec & 1.415 & 1.055 & 1.434 & 778.2 & 0.2520 & 1 \\
\hline
\end{tabular}

Table B.12

Mass Conversions

\begin{tabular}{llllll}
\hline & \multicolumn{5}{c}{ TO } \\
\cline { 2 - 6 } FROM & Pound & Kilogram & Short ton & Long ton & Metric ton \\
\hline Pound & 1 & 0.4536 & $5.0 \times 10^{-4}$ & $4.4643 \times 10^{-4}$ & $4.5362 \times 10^{-4}$ \\
Kilogram & 2.205 & 1 & $1.1023 \times 10^{-3}$ & $9.8425 \times 10^{-4}$ & $1.0 \times 10^{-3}$ \\
Short ton & 2,000 & 907.2 & 1 & 0.8929 & 0.9072 \\
Long ton & 2,240 & 1,106 & 1.12 & 1 & 1.016 \\
Metric ton & 2,205 & 1,000 & 1.102 & 0.9842 & 1 \\
\hline
\end{tabular}


Table B.13

Fuel Efficiency Conversions

\begin{tabular}{|c|c|c|c|c|c|}
\hline MPG & Miles/liter & Kilometers/L & $\begin{array}{c}\text { L/100 } \\
\text { kilometers }\end{array}$ & $\begin{array}{l}\text { Grams of } \\
\mathrm{CO}_{2} \\
\text { per mile }\end{array}$ & $\begin{array}{c}\text { Pounds of } \mathrm{CO}_{2} \\
\text { per mile }\end{array}$ \\
\hline 10 & 2.64 & 4.25 & 23.52 & 877.80 & 1.94 \\
\hline 15 & 3.96 & 6.38 & 15.68 & 585.20 & 1.29 \\
\hline 20 & 5.28 & 8.50 & 11.76 & 438.90 & 0.97 \\
\hline 25 & 6.60 & 10.63 & 9.41 & 351.12 & 0.78 \\
\hline 30 & 7.92 & 12.75 & 7.84 & 292.60 & 0.65 \\
\hline 35 & 9.25 & 14.88 & 6.72 & 250.80 & 0.55 \\
\hline 40 & 10.57 & 17.00 & 5.88 & 219.45 & 0.49 \\
\hline 45 & 11.89 & 19.13 & 5.23 & 195.07 & 0.43 \\
\hline 50 & 13.21 & 21.25 & 4.70 & 175.56 & 0.39 \\
\hline 55 & 14.53 & 23.38 & 4.28 & 159.60 & 0.35 \\
\hline 60 & 15.85 & 25.51 & 3.92 & 146.30 & 0.32 \\
\hline 65 & 17.17 & 27.63 & 3.62 & 135.05 & 0.30 \\
\hline 70 & 18.49 & 29.76 & 3.36 & 125.40 & 0.28 \\
\hline 75 & 19.81 & 31.88 & 3.14 & 117.04 & 0.26 \\
\hline 80 & 21.13 & 34.01 & 2.94 & 109.73 & 0.24 \\
\hline 85 & 22.45 & 36.13 & 2.77 & 103.27 & 0.23 \\
\hline 90 & 23.77 & 38.26 & 2.61 & 97.53 & 0.22 \\
\hline 95 & 25.09 & 40.38 & 2.48 & 92.40 & 0.20 \\
\hline 100 & 26.42 & 42.51 & 2.35 & 87.78 & 0.19 \\
\hline 105 & 27.74 & 44.64 & 2.24 & 83.60 & 0.18 \\
\hline 110 & 29.06 & 46.76 & 2.14 & 79.80 & 0.18 \\
\hline 115 & 30.38 & 48.89 & 2.05 & 76.33 & 0.17 \\
\hline 120 & 31.70 & 51.01 & 1.96 & 73.15 & 0.16 \\
\hline 125 & 33.02 & 53.14 & 1.88 & 70.22 & 0.16 \\
\hline 130 & 34.34 & 55.26 & 1.81 & 67.52 & 0.15 \\
\hline 135 & 35.66 & 57.39 & 1.74 & 65.02 & 0.14 \\
\hline 140 & 36.98 & 59.51 & 1.68 & 62.70 & 0.14 \\
\hline 145 & 38.30 & 61.64 & 1.62 & 60.54 & 0.13 \\
\hline 150 & 39.62 & 63.76 & 1.57 & 58.52 & 0.13 \\
\hline Formula & MPG/3.785 & MPG/[3.785/1.609] & 235.24/MPG & 8,778/MPG & 19.4/MPG \\
\hline
\end{tabular}

${ }^{\mathrm{a}}$ For gasoline-fueled vehicles. 
Table B.14

SI Prefixes and Their Values

\begin{tabular}{llll}
\hline & Value & Prefix & Symbol \\
\hline One million million millionth & $10^{-18}$ & atto & $\mathrm{a}$ \\
One thousand million millionth & $10^{-15}$ & femto & $\mathrm{f}$ \\
One million millionth & $10^{-12}$ & pico & $\mathrm{p}$ \\
One thousand millionth & $10^{-9}$ & nano & $\mathrm{n}$ \\
One millionth & $10^{-6}$ & micro & $\mathrm{m}$ \\
One thousandth & $10^{-3}$ & milli & $\mathrm{c}$ \\
One hundredth & $10^{-2}$ & centi & \\
One tenth & $10^{-1}$ & deci & \\
One & $10^{0}$ & & \\
Ten & $10^{1}$ & deca & \\
One hundred & $10^{2}$ & hecto & $\mathrm{k}$ \\
One thousand & $10^{3}$ & kilo & $\mathrm{M}$ \\
One million & $10^{6}$ & mega & $\mathrm{G}$ \\
One billion $^{\mathrm{a}}$ & $10^{9}$ & giga & $\mathrm{T}$ \\
One trillion $^{\mathrm{a}}$ & $10^{12}$ & tera & $\mathrm{P}$ \\
One quadrillion $^{\mathrm{a}}$ & $10^{15}$ & peta & $\mathrm{E}$ \\
One quintillion $^{\mathrm{a}}$ & $10^{18}$ & exa & \\
\hline
\end{tabular}

${ }^{a}$ Care should be exercised in the use of this nomenclature, especially in foreign correspondence, as it is either unknown or carries a different value in other countries. A "billion," for example, signifies a value of $10^{12}$ in most other countries.

Table B.15

Metric Units and Abbreviations

\begin{tabular}{lll}
\hline \multicolumn{1}{c}{ Quantity } & \multicolumn{1}{c}{ Unit name } & \multicolumn{1}{c}{ Symbol } \\
\hline & & $\mathrm{J}$ \\
Energy & joule & $\mathrm{J} / \mathrm{kg}$ \\
Specific energy & joule/kilogram & $\mathrm{J} /(\mathrm{kg} \bullet \mathrm{km})$ \\
Specific energy consumption & joule/kilogram•kilometer & $\mathrm{J} / \mathrm{km}$ \\
Energy consumption & joule/kilometer & $\mathrm{km} / \mathrm{kJ}$ \\
Energy economy & kilometer/kilojoule & $\mathrm{kW}$ \\
Power & kilowatt & $\mathrm{W} / \mathrm{kg}$ \\
Specific power & watt/kilogram & $\mathrm{W} / \mathrm{m}^{3}$ \\
Power density & watt/meter & $\mathrm{km} / \mathrm{h}$ \\
Speed & kilometer/hour & $\mathrm{m} / \mathrm{s}^{2}$ \\
Acceleration & meter/second ${ }^{2}$ & $\mathrm{~km}$ \\
Range (distance) & kilometer & $\mathrm{kg}$ \\
Weight & kilogram & $\mathrm{N} \bullet \mathrm{m}$ \\
Torque & newton•meter & $\mathrm{m} 3$ \\
Volume & meter & $\mathrm{kg}$ \\
Mass; payload & kilogram & $\mathrm{m}$ \\
Length; width & meter & $\mathrm{kg} / \mathrm{J}$ \\
Brake specific fuel consumption & kilogram/joule & $\mathrm{L} / 100 \mathrm{~km}$ \\
Fuel economy (heat engine) & liters/100 km & \\
\hline
\end{tabular}


Table B.16

Carbon Coefficients, 2002

(Million metric tons carbon per quadrillion Btu)

\begin{tabular}{ll}
\hline \multicolumn{1}{c}{ Fuel Type } & \\
\hline Coal & \\
Coal (residential) & 26.04 \\
Coal (commercial) & 26.04 \\
Coal (industrial coking) & 25.63 \\
Coal (industrial other) & 25.74 \\
Coal (electric utility) & 25.98 \\
Natural gas & \\
Natural gas (pipeline) & 14.47 \\
Natural gas (flared) & 14.92 \\
Petroleum & \\
Asphalt and road oil & 20.62 \\
Aviation gasoline & 18.87 \\
Crude oil & 20.30 \\
Distillate fuel & 19.95 \\
Jet fuel & 19.33 \\
Kerosene & 19.72 \\
LPG & 16.99 \\
Lubricants & 20.24 \\
Motor gasoline & 19.34 \\
Petrochemical feed & 19.37 \\
Petroleum coke & 27.85 \\
Residual fuel & 21.49 \\
Waxes & 19.81 \\
\hline
\end{tabular}

Note: All coefficients based on Higher Heating (Gross Calorific) Value and assume 100 percent combustion. 


\section{Conversion of Constant Dollar Values}

Many types of information in this data book are expressed in dollars. Generally, constant dollars are used-that is, dollars of a fixed value for a specific year, such as 2010 dollars. Converting current dollars to constant dollars, or converting constant dollars for one year to constant dollars for another year, requires conversion factors (Table B.17 and Table B.18). Table B.17 shows conversion factors for the Consumer Price Index inflation factors. Table B.18 shows conversion factors using the Gross National Product inflation factors. 
Table B.17

Consumer Price Inflation (CPI) Index

\begin{tabular}{|c|c|c|c|c|c|c|c|c|c|c|}
\hline From: & 1970 & 1971 & 1972 & 1973 & 1974 & 1975 & 1976 & 1977 & 1978 & 1979 \\
\hline 1970 & 1.000 & 1.044 & 1.077 & 1.144 & 1.271 & 1.387 & 1.466 & 1.562 & 1.680 & 1.871 \\
\hline 1971 & 0.958 & 1.000 & 1.032 & 1.096 & 1.217 & 1.328 & 1.405 & 1.496 & 1.610 & 1.793 \\
\hline 1972 & 0.928 & 0.969 & 1.000 & 1.062 & 1.179 & 1.287 & 1.361 & 1.450 & 1.560 & 1.737 \\
\hline 1973 & 0.874 & 0.912 & 0.941 & 1.000 & 1.110 & 1.212 & 1.282 & 1.365 & 1.468 & 1.635 \\
\hline 1974 & 0.787 & 0.822 & 0.848 & 0.901 & 1.000 & 1.091 & 1.154 & 1.229 & 1.323 & 1.473 \\
\hline 1975 & 0.721 & 0.753 & 0.777 & 0.825 & 0.916 & 1.000 & 1.058 & 1.126 & 1.212 & 1.349 \\
\hline 1976 & 0.682 & 0.712 & 0.735 & 0.780 & 0.866 & 0.946 & 1.000 & 1.065 & 1.146 & 1.276 \\
\hline 1977 & 0.640 & 0.668 & 0.690 & 0.733 & 0.814 & 0.888 & 0.939 & 1.000 & 1.076 & 1.198 \\
\hline 1978 & 0.595 & 0.621 & 0.641 & 0.681 & 0.756 & 0.825 & 0.873 & 0.929 & 1.000 & 1.113 \\
\hline 1979 & 0.534 & 0.558 & 0.576 & 0.612 & 0.679 & 0.741 & 0.784 & 0.835 & 0.898 & 1.000 \\
\hline 1980 & 0.471 & 0.492 & 0.507 & 0.539 & 0.598 & 0.653 & 0.691 & 0.735 & 0.791 & 0.881 \\
\hline 1981 & 0.427 & 0.446 & 0.460 & 0.488 & 0.542 & 0.592 & 0.626 & 0.667 & 0.717 & 0.799 \\
\hline 1982 & 0.402 & 0.420 & 0.433 & 0.460 & 0.511 & 0.558 & 0.590 & 0.628 & 0.676 & 0.752 \\
\hline 1983 & 0.390 & 0.407 & 0.420 & 0.446 & 0.495 & 0.540 & 0.571 & 0.608 & 0.655 & 0.729 \\
\hline 1984 & 0.373 & 0.390 & 0.402 & 0.427 & 0.474 & 0.518 & 0.548 & 0.583 & 0.628 & 0.699 \\
\hline 1985 & 0.361 & 0.376 & 0.388 & 0.413 & 0.458 & 0.500 & 0.529 & 0.563 & 0.606 & 0.675 \\
\hline 1986 & 0.354 & 0.370 & 0.381 & 0.405 & 0.450 & 0.491 & 0.519 & 0.553 & 0.595 & 0.662 \\
\hline 1987 & 0.342 & 0.357 & 0.368 & 0.391 & 0.434 & 0.474 & 0.501 & 0.533 & 0.574 & 0.639 \\
\hline 1988 & 0.328 & 0.342 & 0.353 & 0.375 & 0.417 & 0.455 & 0.481 & 0.512 & 0.551 & 0.614 \\
\hline 1989 & 0.313 & 0.327 & 0.337 & 0.358 & 0.398 & 0.434 & 0.459 & 0.489 & 0.526 & 0.585 \\
\hline 1990 & 0.297 & 0.310 & 0.320 & 0.340 & 0.377 & 0.412 & 0.435 & 0.464 & 0.499 & 0.555 \\
\hline 1991 & 0.285 & 0.297 & 0.307 & 0.326 & 0.362 & 0.395 & 0.418 & 0.445 & 0.479 & 0.533 \\
\hline 1992 & 0.277 & 0.289 & 0.298 & 0.316 & 0.351 & 0.383 & 0.406 & 0.432 & 0.465 & 0.517 \\
\hline 1993 & 0.269 & 0.280 & 0.289 & 0.307 & 0.341 & 0.372 & 0.394 & 0.419 & 0.451 & 0.502 \\
\hline 1994 & 0.262 & 0.273 & 0.282 & 0.300 & 0.333 & 0.363 & 0.384 & 0.409 & 0.440 & 0.490 \\
\hline 1995 & 0.255 & 0.266 & 0.274 & 0.291 & 0.323 & 0.353 & 0.373 & 0.398 & 0.428 & 0.476 \\
\hline 1996 & 0.247 & 0.258 & 0.266 & 0.283 & 0.314 & 0.343 & 0.363 & 0.386 & 0.416 & 0.463 \\
\hline 1997 & 0.242 & 0.252 & 0.260 & 0.277 & 0.307 & 0.335 & 0.355 & 0.378 & 0.406 & 0.452 \\
\hline 1998 & 0.238 & 0.248 & 0.256 & 0.272 & 0.302 & 0.330 & 0.349 & 0.372 & 0.400 & 0.445 \\
\hline 1999 & 0.233 & 0.243 & 0.251 & 0.267 & 0.296 & 0.323 & 0.342 & 0.364 & 0.391 & 0.436 \\
\hline 2000 & 0.225 & 0.235 & 0.243 & 0.258 & 0.286 & 0.312 & 0.330 & 0.352 & 0.379 & 0.422 \\
\hline 2001 & 0.219 & 0.229 & 0.236 & 0.251 & 0.278 & 0.304 & 0.321 & 0.342 & 0.368 & 0.410 \\
\hline 2002 & 0.216 & 0.225 & 0.232 & 0.247 & 0.274 & 0.299 & 0.316 & 0.337 & 0.362 & 0.404 \\
\hline 2003 & 0.211 & 0.220 & 0.227 & 0.241 & 0.268 & 0.292 & 0.309 & 0.329 & 0.354 & 0.395 \\
\hline 2004 & 0.205 & 0.214 & 0.221 & 0.235 & 0.261 & 0.285 & 0.301 & 0.321 & 0.345 & 0.384 \\
\hline 2005 & 0.199 & 0.207 & 0.214 & 0.227 & 0.252 & 0.275 & 0.291 & 0.310 & 0.334 & 0.372 \\
\hline 2006 & 0.192 & 0.201 & 0.207 & 0.220 & 0.245 & 0.267 & 0.282 & 0.301 & 0.323 & 0.360 \\
\hline 2007 & 0.187 & 0.195 & 0.202 & 0.214 & 0.238 & 0.259 & 0.274 & 0.292 & 0.314 & 0.350 \\
\hline 2008 & 0.180 & 0.188 & 0.194 & 0.206 & 0.229 & 0.250 & 0.264 & 0.281 & 0.303 & 0.337 \\
\hline 2009 & 0.181 & 0.189 & 0.195 & 0.207 & 0.230 & 0.251 & 0.265 & 0.282 & 0.304 & 0.338 \\
\hline 2010 & 0.178 & 0.186 & 0.192 & 0.204 & 0.226 & 0.247 & 0.261 & 0.278 & 0.299 & 0.333 \\
\hline 2011 & 0.172 & 0.180 & 0.186 & 0.197 & 0.219 & 0.239 & 0.253 & 0.269 & 0.290 & 0.323 \\
\hline 2012 & 0.169 & 0.176 & 0.182 & 0.193 & 0.215 & 0.234 & 0.248 & 0.264 & 0.284 & 0.316 \\
\hline 2013 & 0.167 & 0.174 & 0.179 & 0.191 & 0.212 & 0.231 & 0.244 & 0.260 & 0.280 & 0.312 \\
\hline
\end{tabular}


Table B.17

Consumer Price Inflation (CPI) Index (Continued)

\begin{tabular}{|c|c|c|c|c|c|c|c|c|c|c|}
\hline From: & 1980 & 1981 & 1982 & 1983 & 1984 & 1985 & 1986 & 1987 & 1988 & 1989 \\
\hline 1970 & 2.124 & 2.343 & 2.487 & 2.567 & 2.678 & 2.773 & 2.825 & 2.928 & 3.049 & 3.196 \\
\hline 1971 & 2.035 & 2.244 & 2.383 & 2.459 & 2.565 & 2.657 & 2.706 & 2.805 & 2.921 & 3.062 \\
\hline 1972 & 1.971 & 2.175 & 2.309 & 2.383 & 2.486 & 2.574 & 2.622 & 2.718 & 2.830 & 2.967 \\
\hline 1973 & 1.856 & 2.047 & 2.173 & 2.243 & 2.340 & 2.423 & 2.468 & 2.559 & 2.664 & 2.793 \\
\hline 1974 & 1.671 & 1.844 & 1.957 & 2.020 & 2.108 & 2.183 & 2.223 & 2.304 & 2.400 & 2.515 \\
\hline 1975 & 1.532 & 1.690 & 1.794 & 1.851 & 1.931 & 2.000 & 2.037 & 2.112 & 2.199 & 2.305 \\
\hline 1976 & 1.448 & 1.598 & 1.696 & 1.750 & 1.826 & 1.891 & 1.926 & 1.996 & 2.079 & 2.179 \\
\hline 1977 & 1.360 & 1.500 & 1.592 & 1.644 & 1.715 & 1.776 & 1.809 & 1.875 & 1.952 & 2.046 \\
\hline 1978 & 1.264 & 1.394 & 1.480 & 1.528 & 1.594 & 1.650 & 1.681 & 1.742 & 1.814 & 1.902 \\
\hline 1979 & 1.135 & 1.252 & 1.329 & 1.372 & 1.431 & 1.482 & 1.510 & 1.565 & 1.629 & 1.708 \\
\hline 1980 & 1.000 & 1.103 & 1.171 & 1.209 & 1.261 & 1.306 & 1.330 & 1.379 & 1.436 & 1.505 \\
\hline 1981 & 0.906 & 1.000 & 1.062 & 1.096 & 1.143 & 1.184 & 1.206 & 1.250 & 1.301 & 1.364 \\
\hline 1982 & 0.854 & 0.942 & 1.000 & 1.032 & 1.077 & 1.115 & 1.136 & 1.177 & 1.226 & 1.285 \\
\hline 1983 & 0.827 & 0.913 & 0.969 & 1.000 & 1.043 & 1.080 & 1.100 & 1.141 & 1.188 & 1.245 \\
\hline 1984 & 0.793 & 0.875 & 0.929 & 0.959 & 1.000 & 1.036 & 1.055 & 1.093 & 1.139 & 1.193 \\
\hline 1985 & 0.766 & 0.845 & 0.897 & 0.926 & 0.966 & 1.000 & 1.019 & 1.056 & 1.099 & 1.152 \\
\hline 1986 & 0.752 & 0.829 & 0.880 & 0.909 & 0.948 & 0.982 & 1.000 & 1.036 & 1.079 & 1.131 \\
\hline 1987 & 0.725 & 0.800 & 0.849 & 0.877 & 0.915 & 0.947 & 0.965 & 1.000 & 1.041 & 1.092 \\
\hline 1988 & 0.697 & 0.768 & 0.816 & 0.842 & 0.878 & 0.910 & 0.926 & 0.960 & 1.000 & 1.048 \\
\hline 1989 & 0.665 & 0.733 & 0.778 & 0.803 & 0.838 & 0.868 & 0.884 & 0.916 & 0.954 & 1.000 \\
\hline 1990 & 0.630 & 0.695 & 0.738 & 0.762 & 0.795 & 0.823 & 0.839 & 0.869 & 0.905 & 0.949 \\
\hline 1991 & 0.605 & 0.667 & 0.709 & 0.731 & 0.763 & 0.790 & 0.805 & 0.834 & 0.869 & 0.910 \\
\hline 1992 & 0.587 & 0.648 & 0.688 & 0.710 & 0.741 & 0.767 & 0.781 & 0.810 & 0.843 & 0.884 \\
\hline 1993 & 0.570 & 0.629 & 0.668 & 0.689 & 0.719 & 0.745 & 0.758 & 0.786 & 0.819 & 0.858 \\
\hline 1994 & 0.556 & 0.613 & 0.651 & 0.672 & 0.701 & 0.726 & 0.740 & 0.767 & 0.798 & 0.837 \\
\hline 1995 & 0.541 & 0.596 & 0.633 & 0.654 & 0.682 & 0.706 & 0.719 & 0.745 & 0.776 & 0.814 \\
\hline 1996 & 0.525 & 0.579 & 0.615 & 0.635 & 0.662 & 0.686 & 0.699 & 0.724 & 0.754 & 0.790 \\
\hline 1997 & 0.513 & 0.566 & 0.601 & 0.621 & 0.647 & 0.670 & 0.683 & 0.708 & 0.737 & 0.773 \\
\hline 1998 & 0.506 & 0.558 & 0.592 & 0.611 & 0.637 & 0.660 & 0.672 & 0.697 & 0.726 & 0.761 \\
\hline 1999 & 0.495 & 0.546 & 0.579 & 0.598 & 0.624 & 0.646 & 0.658 & 0.682 & 0.710 & 0.744 \\
\hline 2000 & 0.479 & 0.528 & 0.560 & 0.578 & 0.603 & 0.625 & 0.636 & 0.660 & 0.687 & 0.720 \\
\hline 2001 & 0.465 & 0.513 & 0.545 & 0.562 & 0.587 & 0.608 & 0.619 & 0.641 & 0.668 & 0.700 \\
\hline 2002 & 0.458 & 0.505 & 0.536 & 0.554 & 0.578 & 0.598 & 0.609 & 0.631 & 0.658 & 0.689 \\
\hline 2003 & 0.448 & 0.494 & 0.524 & 0.541 & 0.565 & 0.585 & 0.596 & 0.617 & 0.643 & 0.674 \\
\hline 2004 & 0.436 & 0.481 & 0.511 & 0.527 & 0.550 & 0.570 & 0.580 & 0.601 & 0.626 & 0.656 \\
\hline 2005 & 0.422 & 0.465 & 0.494 & 0.510 & 0.532 & 0.551 & 0.561 & 0.582 & 0.606 & 0.635 \\
\hline 2006 & 0.409 & 0.451 & 0.479 & 0.494 & 0.515 & 0.534 & 0.544 & 0.563 & 0.587 & 0.615 \\
\hline 2007 & 0.397 & 0.438 & 0.465 & 0.480 & 0.501 & 0.519 & 0.529 & 0.548 & 0.571 & 0.598 \\
\hline 2008 & 0.383 & 0.422 & 0.448 & 0.463 & 0.483 & 0.500 & 0.509 & 0.528 & 0.549 & 0.576 \\
\hline 2009 & 0.384 & 0.424 & 0.450 & 0.464 & 0.484 & 0.502 & 0.511 & 0.530 & 0.551 & 0.578 \\
\hline 2010 & 0.378 & 0.417 & 0.443 & 0.457 & 0.476 & 0.493 & 0.503 & 0.521 & 0.543 & 0.569 \\
\hline 2011 & 0.366 & 0.404 & 0.429 & 0.443 & 0.462 & 0.478 & 0.487 & 0.505 & 0.526 & 0.551 \\
\hline 2012 & 0.359 & 0.396 & 0.420 & 0.434 & 0.453 & 0.469 & 0.477 & 0.495 & 0.515 & 0.540 \\
\hline 2013 & 0.354 & 0.390 & 0.414 & 0.428 & 0.446 & 0.462 & 0.470 & 0.488 & 0.508 & 0.532 \\
\hline
\end{tabular}


Table B.17

Consumer Price Inflation (CPI) Index (Continued)

\begin{tabular}{|c|c|c|c|c|c|c|c|c|c|c|}
\hline From: & 1990 & 1991 & 1992 & 1993 & 1994 & 1995 & 1996 & 1997 & 1998 & 1999 \\
\hline 1970 & 3.369 & 3.510 & 3.616 & 3.724 & 3.820 & 3.928 & 4.044 & 4.137 & 4.201 & 4.294 \\
\hline 1971 & 3.227 & 3.363 & 3.464 & 3.568 & 3.659 & 3.763 & 3.874 & 3.963 & 4.025 & 4.114 \\
\hline 1972 & 3.127 & 3.258 & 3.356 & 3.457 & 3.545 & 3.646 & 3.754 & 3.840 & 3.900 & 3.986 \\
\hline 1973 & 2.944 & 3.068 & 3.160 & 3.255 & 3.338 & 3.432 & 3.534 & 3.615 & 3.671 & 3.752 \\
\hline 1974 & 2.651 & 2.763 & 2.846 & 2.931 & 3.006 & 3.091 & 3.183 & 3.256 & 3.306 & 3.379 \\
\hline 1975 & 2.429 & 2.532 & 2.608 & 2.686 & 2.755 & 2.833 & 2.916 & 2.983 & 3.030 & 3.097 \\
\hline 1976 & 2.297 & 2.394 & 2.466 & 2.540 & 2.605 & 2.678 & 2.757 & 2.821 & 2.865 & 2.928 \\
\hline 1977 & 2.157 & 2.248 & 2.315 & 2.384 & 2.446 & 2.515 & 2.589 & 2.649 & 2.690 & 2.749 \\
\hline 1978 & 2.005 & 2.089 & 2.152 & 2.216 & 2.273 & 2.337 & 2.406 & 2.462 & 2.500 & 2.555 \\
\hline 1979 & 1.800 & 1.876 & 1.933 & 1.990 & 2.041 & 2.099 & 2.161 & 2.211 & 2.245 & 2.295 \\
\hline 1980 & 1.586 & 1.653 & 1.703 & 1.754 & 1.799 & 1.850 & 1.904 & 1.948 & 1.978 & 2.022 \\
\hline 1981 & 1.438 & 1.498 & 1.543 & 1.590 & 1.630 & 1.677 & 1.726 & 1.766 & 1.793 & 1.833 \\
\hline 1982 & 1.354 & 1.411 & 1.454 & 1.497 & 1.536 & 1.579 & 1.626 & 1.663 & 1.689 & 1.726 \\
\hline 1983 & 1.312 & 1.367 & 1.409 & 1.451 & 1.488 & 1.530 & 1.575 & 1.611 & 1.637 & 1.673 \\
\hline 1984 & 1.258 & 1.311 & 1.350 & 1.391 & 1.426 & 1.467 & 1.510 & 1.545 & 1.569 & 1.603 \\
\hline 1985 & 1.215 & 1.266 & 1.304 & 1.343 & 1.377 & 1.416 & 1.458 & 1.492 & 1.515 & 1.548 \\
\hline 1986 & 1.193 & 1.243 & 1.280 & 1.318 & 1.352 & 1.391 & 1.432 & 1.464 & 1.487 & 1.520 \\
\hline 1987 & 1.151 & 1.199 & 1.235 & 1.272 & 1.305 & 1.342 & 1.381 & 1.413 & 1.435 & 1.467 \\
\hline 1988 & 1.105 & 1.151 & 1.186 & 1.221 & 1.253 & 1.288 & 1.326 & 1.357 & 1.378 & 1.408 \\
\hline 1989 & 1.054 & 1.098 & 1.131 & 1.165 & 1.195 & 1.229 & 1.265 & 1.294 & 1.315 & 1.344 \\
\hline 1990 & 1.000 & 1.042 & 1.073 & 1.106 & 1.134 & 1.166 & 1.200 & 1.228 & 1.247 & 1.275 \\
\hline 1991 & 0.960 & 1.000 & 1.030 & 1.061 & 1.088 & 1.119 & 1.152 & 1.178 & 1.197 & 1.223 \\
\hline 1992 & 0.932 & 0.971 & 1.000 & 1.030 & 1.056 & 1.086 & 1.118 & 1.144 & 1.162 & 1.187 \\
\hline 1993 & 0.904 & 0.943 & 0.971 & 1.000 & 1.026 & 1.055 & 1.086 & 1.111 & 1.128 & 1.153 \\
\hline 1994 & 0.882 & 0.919 & 0.947 & 0.975 & 1.000 & 1.028 & 1.059 & 1.083 & 1.100 & 1.124 \\
\hline 1995 & 0.858 & 0.894 & 0.921 & 0.948 & 0.972 & 1.000 & 1.030 & 1.053 & 1.070 & 1.093 \\
\hline 1996 & 0.833 & 0.868 & 0.894 & 0.921 & 0.945 & 0.971 & 1.000 & 1.023 & 1.039 & 1.062 \\
\hline 1997 & 0.814 & 0.849 & 0.874 & 0.900 & 0.923 & 0.950 & 0.978 & 1.000 & 1.016 & 1.038 \\
\hline 1998 & 0.802 & 0.836 & 0.861 & 0.887 & 0.909 & 0.935 & 0.963 & 0.985 & 1.000 & 1.022 \\
\hline 1999 & 0.785 & 0.818 & 0.842 & 0.867 & 0.890 & 0.915 & 0.942 & 0.963 & 0.978 & 1.000 \\
\hline 2000 & 0.759 & 0.791 & 0.815 & 0.839 & 0.861 & 0.885 & 0.911 & 0.932 & 0.947 & 0.967 \\
\hline 2001 & 0.738 & 0.769 & 0.792 & 0.816 & 0.837 & 0.861 & 0.886 & 0.906 & 0.920 & 0.941 \\
\hline 2002 & 0.727 & 0.757 & 0.780 & 0.803 & 0.824 & 0.847 & 0.872 & 0.892 & 0.906 & 0.926 \\
\hline 2003 & 0.710 & 0.740 & 0.763 & 0.785 & 0.805 & 0.828 & 0.853 & 0.872 & 0.886 & 0.905 \\
\hline 2004 & 0.692 & 0.721 & 0.743 & 0.765 & 0.785 & 0.807 & 0.831 & 0.850 & 0.863 & 0.882 \\
\hline 2005 & 0.669 & 0.697 & 0.718 & 0.740 & 0.759 & 0.780 & 0.803 & 0.822 & 0.835 & 0.853 \\
\hline 2006 & 0.648 & 0.676 & 0.696 & 0.717 & 0.735 & 0.756 & 0.778 & 0.796 & 0.809 & 0.826 \\
\hline 2007 & 0.630 & 0.657 & 0.677 & 0.697 & 0.715 & 0.735 & 0.757 & 0.774 & 0.786 & 0.804 \\
\hline 2008 & 0.607 & 0.633 & 0.652 & 0.671 & 0.688 & 0.708 & 0.729 & 0.745 & 0.757 & 0.774 \\
\hline 2009 & 0.602 & 0.635 & 0.654 & 0.674 & 0.691 & 0.710 & 0.731 & 0.748 & 0.760 & 0.777 \\
\hline 2010 & 0.599 & 0.625 & 0.643 & 0.663 & 0.680 & 0.699 & 0.720 & 0.736 & 0.748 & 0.764 \\
\hline 2011 & 0.581 & 0.605 & 0.624 & 0.642 & 0.659 & 0.678 & 0.698 & 0.714 & 0.725 & 0.741 \\
\hline 2012 & 0.569 & 0.593 & 0.611 & 0.629 & 0.645 & 0.664 & 0.683 & 0.699 & 0.710 & 0.726 \\
\hline 2013 & 0.561 & 0.585 & 0.602 & 0.620 & 0.636 & 0.654 & 0.674 & 0.689 & 0.700 & 0.715 \\
\hline
\end{tabular}


Table B.17

Consumer Price Inflation (CPI) Index (Continued)

\begin{tabular}{|c|c|c|c|c|c|c|c|c|c|c|}
\hline From: & 2000 & 2001 & 2002 & 2003 & 2004 & 2005 & 2006 & 2007 & 2008 & 2009 \\
\hline 1970 & 4.438 & 4.564 & 4.637 & 4.742 & 4.869 & 5.034 & 5.196 & 5.344 & 5.549 & 5.529 \\
\hline 1971 & 4.252 & 4.373 & 4.442 & 4.543 & 4.664 & 4.822 & 4.978 & 5.120 & 5.316 & 5.297 \\
\hline 1972 & 4.120 & 4.237 & 4.304 & 4.402 & 4.519 & 4.672 & 4.823 & 4.960 & 5.151 & 5.132 \\
\hline 1973 & 3.878 & 3.989 & 4.052 & 4.144 & 4.255 & 4.399 & 4.541 & 4.670 & 4.849 & 4.832 \\
\hline 1974 & 3.493 & 3.592 & 3.649 & 3.732 & 3.832 & 3.961 & 4.089 & 4.206 & 4.367 & 4.352 \\
\hline 1975 & 3.201 & 3.292 & 3.344 & 3.420 & 3.511 & 3.630 & 3.747 & 3.854 & 4.002 & 3.988 \\
\hline 1976 & 3.026 & 3.112 & 3.162 & 3.234 & 3.320 & 3.432 & 3.543 & 3.644 & 3.784 & 3.770 \\
\hline 1977 & 2.842 & 2.922 & 2.969 & 3.036 & 3.117 & 3.223 & 3.327 & 3.421 & 3.553 & 3.540 \\
\hline 1978 & 2.641 & 2.716 & 2.759 & 2.822 & 2.897 & 2.995 & 3.092 & 3.180 & 3.302 & 3.290 \\
\hline 1979 & 2.372 & 2.439 & 2.478 & 2.534 & 2.602 & 2.690 & 2.777 & 2.856 & 2.966 & 2.955 \\
\hline 1980 & 2.090 & 2.149 & 2.183 & 2.233 & 2.292 & 2.370 & 2.447 & 2.516 & 2.613 & 2.604 \\
\hline 1981 & 1.894 & 1.948 & 1.979 & 2.024 & 2.078 & 2.149 & 2.218 & 2.281 & 2.369 & 2.360 \\
\hline 1982 & 1.784 & 1.835 & 1.864 & 1.907 & 1.958 & 2.024 & 2.089 & 2.149 & 2.231 & 2.223 \\
\hline 1983 & 1.729 & 1.778 & 1.806 & 1.847 & 1.897 & 1.961 & 2.024 & 2.082 & 2.162 & 2.154 \\
\hline 1984 & 1.657 & 1.705 & 1.731 & 1.771 & 1.818 & 1.880 & 1.940 & 1.996 & 2.072 & 2.065 \\
\hline 1985 & 1.600 & 1.646 & 1.672 & 1.710 & 1.756 & 1.815 & 1.874 & 1.927 & 2.001 & 1.994 \\
\hline 1986 & 1.571 & 1.616 & 1.641 & 1.679 & 1.724 & 1.782 & 1.839 & 1.892 & 1.964 & 1.957 \\
\hline 1987 & 1.516 & 1.559 & 1.584 & 1.620 & 1.663 & 1.719 & 1.775 & 1.825 & 1.895 & 1.889 \\
\hline 1988 & 1.456 & 1.497 & 1.521 & 1.555 & 1.597 & 1.651 & 1.704 & 1.753 & 1.820 & 1.813 \\
\hline 1989 & 1.389 & 1.428 & 1.451 & 1.484 & 1.523 & 1.575 & 1.626 & 1.672 & 1.736 & 1.730 \\
\hline 1990 & 1.318 & 1.355 & 1.376 & 1.408 & 1.445 & 1.494 & 1.542 & 1.586 & 1.647 & 1.641 \\
\hline 1991 & 1.264 & 1.300 & 1.321 & 1.351 & 1.387 & 1.434 & 1.480 & 1.522 & 1.581 & 1.575 \\
\hline 1992 & 1.227 & 1.262 & 1.282 & 1.311 & 1.346 & 1.392 & 1.437 & 1.478 & 1.535 & 1.529 \\
\hline 1993 & 1.192 & 1.226 & 1.245 & 1.273 & 1.307 & 1.352 & 1.395 & 1.435 & 1.490 & 1.485 \\
\hline 1994 & 1.162 & 1.195 & 1.214 & 1.242 & 1.275 & 1.318 & 1.360 & 1.399 & 1.453 & 1.448 \\
\hline 1995 & 1.130 & 1.162 & 1.180 & 1.207 & 1.240 & 1.281 & 1.323 & 1.360 & 1.413 & 1.408 \\
\hline 1996 & 1.098 & 1.129 & 1.147 & 1.173 & 1.204 & 1.245 & 1.285 & 1.321 & 1.372 & 1.367 \\
\hline 1997 & 1.073 & 1.103 & 1.121 & 1.146 & 1.177 & 1.217 & 1.256 & 1.292 & 1.341 & 1.337 \\
\hline 1998 & 1.056 & 1.087 & 1.104 & 1.129 & 1.159 & 1.198 & 1.237 & 1.272 & 1.321 & 1.316 \\
\hline 1999 & 1.034 & 1.063 & 1.080 & 1.104 & 1.134 & 1.172 & 1.210 & 1.245 & 1.292 & 1.288 \\
\hline 2000 & 1.000 & 1.028 & 1.045 & 1.069 & 1.097 & 1.134 & 1.171 & 1.204 & 1.250 & 1.246 \\
\hline 2001 & 0.972 & 1.000 & 1.016 & 1.039 & 1.067 & 1.103 & 1.138 & 1.171 & 1.216 & 1.211 \\
\hline 2002 & 0.957 & 0.984 & 1.000 & 1.023 & 1.050 & 1.086 & 1.121 & 1.153 & 1.197 & 1.193 \\
\hline 2003 & 0.936 & 0.963 & 0.978 & 1.000 & 1.027 & 1.061 & 1.096 & 1.127 & 1.170 & 1.166 \\
\hline 2004 & 0.912 & 0.938 & 0.952 & 0.974 & 1.000 & 1.034 & 1.067 & 1.098 & 1.140 & 1.136 \\
\hline 2005 & 0.882 & 0.907 & 0.921 & 0.942 & 0.967 & 1.000 & 1.032 & 1.062 & 1.102 & 1.098 \\
\hline 2006 & 0.854 & 0.878 & 0.892 & 0.913 & 0.937 & 0.969 & 1.000 & 1.028 & 1.068 & 1.064 \\
\hline 2007 & 0.831 & 0.854 & 0.868 & 0.887 & 0.911 & 0.942 & 0.972 & 1.000 & 1.038 & 1.035 \\
\hline 2008 & 0.800 & 0.823 & 0.836 & 0.855 & 0.877 & 0.907 & 0.936 & 0.963 & 1.000 & 0.996 \\
\hline 2009 & 0.803 & 0.825 & 0.839 & 0.858 & 0.881 & 0.910 & 0.940 & 0.966 & 1.004 & 1.000 \\
\hline 2010 & 0.790 & 0.812 & 0.825 & 0.844 & 0.866 & 0.896 & 0.925 & 0.951 & 0.987 & 0.984 \\
\hline 2011 & 0.766 & 0.787 & 0.800 & 0.818 & 0.840 & 0.868 & 0.896 & 0.922 & 0.957 & 0.954 \\
\hline 2012 & 0.750 & 0.771 & 0.784 & 0.801 & 0.823 & 0.851 & 0.878 & 0.903 & 0.938 & 0.934 \\
\hline 2013 & 0.739 & 0.760 & 0.772 & 0.790 & 0.811 & 0.838 & 0.865 & 0.890 & 0.924 & 0.921 \\
\hline
\end{tabular}


Table B.17

Consumer Price Inflation (CPI) Index (Continued)

\begin{tabular}{ccccc}
\hline From: & $\mathbf{2 0 1 0}$ & $\mathbf{2 0 1 1}$ & $\mathbf{2 0 1 2}$ & $\mathbf{2 0 1 3}$ \\
\hline $\mathbf{1 9 7 0}$ & 5.620 & 5.797 & 5.917 & 6.004 \\
$\mathbf{1 9 7 1}$ & 5.384 & 5.554 & 5.669 & 5.752 \\
$\mathbf{1 9 7 2}$ & 5.217 & 5.381 & 5.493 & 5.573 \\
$\mathbf{1 9 7 3}$ & 4.911 & 5.066 & 5.171 & 5.247 \\
$\mathbf{1 9 7 4}$ & 4.423 & 4.563 & 4.657 & 4.725 \\
$\mathbf{1 9 7 5}$ & 4.053 & 4.181 & 4.268 & 4.330 \\
$\mathbf{1 9 7 6}$ & 3.832 & 3.953 & 4.035 & 4.094 \\
$\mathbf{1 9 7 7}$ & 3.598 & 3.712 & 3.789 & 3.844 \\
$\mathbf{1 9 7 8}$ & 3.344 & 3.450 & 3.521 & 3.573 \\
$\mathbf{1 9 7 9}$ & 3.004 & 3.098 & 3.162 & 3.209 \\
$\mathbf{1 9 8 0}$ & 2.646 & 2.730 & 2.786 & 2.827 \\
$\mathbf{1 9 8 1}$ & 2.399 & 2.475 & 2.526 & 2.563 \\
$\mathbf{1 9 8 2}$ & 2.260 & 2.331 & 2.379 & 2.414 \\
$\mathbf{1 9 8 3}$ & 2.189 & 2.258 & 2.305 & 2.339 \\
$\mathbf{1 9 8 4}$ & 2.099 & 2.165 & 2.210 & 2.242 \\
$\mathbf{1 9 8 5}$ & 2.027 & 2.091 & 2.134 & 2.165 \\
$\mathbf{1 9 8 6}$ & 1.990 & 2.052 & 2.095 & 2.126 \\
$\mathbf{1 9 8 7}$ & 1.920 & 1.980 & 2.021 & 2.051 \\
$\mathbf{1 9 8 8}$ & 1.843 & 1.901 & 1.941 & 1.969 \\
$\mathbf{1 9 8 9}$ & 1.759 & 1.814 & 1.852 & 1.879 \\
$\mathbf{1 9 9 0}$ & 1.668 & 1.721 & 1.757 & 1.782 \\
$\mathbf{1 9 9 1}$ & 1.601 & 1.652 & 1.686 & 1.710 \\
$\mathbf{1 9 9 2}$ & 1.554 & 1.603 & 1.636 & 1.660 \\
$\mathbf{1 9 9 3}$ & 1.509 & 1.557 & 1.589 & 1.612 \\
$\mathbf{1 9 9 4}$ & 1.471 & 1.518 & 1.549 & 1.572 \\
$\mathbf{1 9 9 5}$ & 1.431 & 1.476 & 1.507 & 1.529 \\
$\mathbf{1 9 9 6}$ & 1.390 & 1.434 & 1.463 & 1.485 \\
$\mathbf{1 9 9 7}$ & 1.359 & 1.401 & 1.430 & 1.451 \\
$\mathbf{1 9 9 8}$ & 1.338 & 1.380 & 1.409 & 1.429 \\
$\mathbf{1 9 9 9}$ & 1.309 & 1.350 & 1.378 & 1.398 \\
$\mathbf{2 0 0 0}$ & 1.266 & 1.306 & 1.333 & 1.353 \\
$\mathbf{2 0 0 1}$ & 1.231 & 1.270 & 1.296 & 1.315 \\
$\mathbf{2 0 0 2}$ & 1.212 & 1.250 & 1.276 & 1.295 \\
$\mathbf{2 0 0 3}$ & 1.185 & 1.222 & 1.248 & 1.266 \\
$\mathbf{2 0 0 4}$ & 1.154 & 1.191 & 1.215 & 1.233 \\
$\mathbf{2 0 0 5}$ & 1.117 & 1.152 & 1.176 & 1.193 \\
$\mathbf{2 0 0 6}$ & 1.082 & 1.116 & 1.139 & 1.156 \\
$\mathbf{2 0 0 7}$ & 1.052 & 1.085 & 1.107 & 1.124 \\
$\mathbf{2 0 0 8}$ & 1.013 & 1.045 & 1.066 & 1.082 \\
$\mathbf{2 0 0 9}$ & 1.016 & 1.048 & 1.070 & 1.086 \\
$\mathbf{2 0 1 0}$ & 1.000 & 1.032 & 1.053 & 1.068 \\
$\mathbf{2 0 1 1}$ & 0.969 & 1.000 & 1.021 & 1.036 \\
$\mathbf{2 0 1 2}$ & 0.950 & 0.980 & 1.000 & 1.015 \\
$\mathbf{2 0 1 3}$ & 0.936 & 0.966 & 0.986 & 1.000 \\
\hline & & & &
\end{tabular}

Source:

U.S. Bureau of Labor Statistics. 
Table B.18

Gross National Product Implicit Price Deflator

\begin{tabular}{|c|c|c|c|c|c|c|c|c|c|c|}
\hline From: & 1970 & 1971 & 1972 & 1973 & 1974 & 1975 & 1976 & 1977 & 1978 & 1979 \\
\hline 1970 & 1.000 & 1.051 & 1.097 & 1.156 & 1.260 & 1.377 & 1.453 & 1.543 & 1.651 & 1.788 \\
\hline 1971 & 0.952 & 1.000 & 1.043 & 1.100 & 1.199 & 1.310 & 1.382 & 1.468 & 1.571 & 1.701 \\
\hline 1972 & 0.912 & 0.958 & 1.000 & 1.055 & 1.149 & 1.256 & 1.325 & 1.407 & 1.506 & 1.630 \\
\hline 1973 & 0.865 & 0.909 & 0.948 & 1.000 & 1.090 & 1.191 & 1.256 & 1.334 & 1.428 & 1.546 \\
\hline 1974 & 0.794 & 0.834 & 0.870 & 0.918 & 1.000 & 1.093 & 1.153 & 1.224 & 1.311 & 1.419 \\
\hline 1975 & 0.726 & 0.763 & 0.796 & 0.840 & 0.915 & 1.000 & 1.055 & 1.121 & 1.199 & 1.298 \\
\hline 1976 & 0.688 & 0.723 & 0.755 & 0.796 & 0.867 & 0.948 & 1.000 & 1.062 & 1.137 & 1.231 \\
\hline 1977 & 0.648 & 0.681 & 0.711 & 0.749 & 0.817 & 0.892 & 0.942 & 1.000 & 1.070 & 1.159 \\
\hline 1978 & 0.606 & 0.636 & 0.664 & 0.700 & 0.763 & 0.834 & 0.880 & 0.934 & 1.000 & 1.083 \\
\hline 1979 & 0.559 & 0.588 & 0.613 & 0.647 & 0.705 & 0.770 & 0.813 & 0.863 & 0.924 & 1.000 \\
\hline 1980 & 0.513 & 0.539 & 0.563 & 0.593 & 0.647 & 0.707 & 0.745 & 0.792 & 0.847 & 0.917 \\
\hline 1981 & 0.469 & 0.493 & 0.515 & 0.543 & 0.591 & 0.646 & 0.682 & 0.724 & 0.775 & 0.839 \\
\hline 1982 & 0.442 & 0.464 & 0.485 & 0.511 & 0.557 & 0.608 & 0.642 & 0.682 & 0.730 & 0.790 \\
\hline 1983 & 0.425 & 0.447 & 0.466 & 0.492 & 0.536 & 0.585 & 0.617 & 0.656 & 0.702 & 0.760 \\
\hline 1984 & 0.411 & 0.431 & 0.450 & 0.475 & 0.517 & 0.565 & 0.596 & 0.633 & 0.678 & 0.734 \\
\hline 1985 & 0.398 & 0.418 & 0.436 & 0.460 & 0.501 & 0.548 & 0.578 & 0.614 & 0.657 & 0.711 \\
\hline 1986 & 0.390 & 0.410 & 0.428 & 0.451 & 0.491 & 0.537 & 0.566 & 0.602 & 0.644 & 0.697 \\
\hline 1987 & 0.380 & 0.399 & 0.417 & 0.440 & 0.479 & 0.523 & 0.552 & 0.586 & 0.628 & 0.679 \\
\hline 1988 & 0.367 & 0.386 & 0.403 & 0.425 & 0.463 & 0.506 & 0.533 & 0.567 & 0.606 & 0.656 \\
\hline 1989 & 0.353 & 0.371 & 0.388 & 0.409 & 0.445 & 0.487 & 0.513 & 0.545 & 0.584 & 0.632 \\
\hline 1990 & 0.341 & 0.358 & 0.374 & 0.394 & 0.429 & 0.469 & 0.495 & 0.526 & 0.563 & 0.609 \\
\hline 1991 & 0.330 & 0.346 & 0.362 & 0.381 & 0.415 & 0.454 & 0.479 & 0.509 & 0.544 & 0.589 \\
\hline 1992 & 0.322 & 0.339 & 0.353 & 0.373 & 0.406 & 0.444 & 0.468 & 0.497 & 0.532 & 0.576 \\
\hline 1993 & 0.315 & 0.331 & 0.345 & 0.364 & 0.397 & 0.434 & 0.457 & 0.486 & 0.520 & 0.563 \\
\hline 1994 & 0.308 & 0.324 & 0.338 & 0.357 & 0.389 & 0.425 & 0.448 & 0.476 & 0.509 & 0.551 \\
\hline 1995 & 0.302 & 0.317 & 0.331 & 0.349 & 0.381 & 0.416 & 0.439 & 0.466 & 0.499 & 0.540 \\
\hline 1996 & 0.297 & 0.312 & 0.325 & 0.343 & 0.374 & 0.408 & 0.431 & 0.458 & 0.490 & 0.530 \\
\hline 1997 & 0.292 & 0.306 & 0.320 & 0.337 & 0.367 & 0.401 & 0.424 & 0.450 & 0.481 & 0.521 \\
\hline 1998 & 0.288 & 0.303 & 0.316 & 0.334 & 0.363 & 0.397 & 0.419 & 0.445 & 0.476 & 0.516 \\
\hline 1999 & 0.284 & 0.299 & 0.312 & 0.329 & 0.358 & 0.392 & 0.413 & 0.439 & 0.470 & 0.508 \\
\hline 2000 & 0.278 & 0.292 & 0.305 & 0.322 & 0.350 & 0.383 & 0.404 & 0.429 & 0.459 & 0.497 \\
\hline 2001 & 0.272 & 0.286 & 0.298 & 0.314 & 0.343 & 0.374 & 0.395 & 0.419 & 0.449 & 0.486 \\
\hline 2002 & 0.268 & 0.281 & 0.294 & 0.310 & 0.337 & 0.369 & 0.389 & 0.413 & 0.442 & 0.479 \\
\hline 2003 & 0.262 & 0.276 & 0.288 & 0.303 & 0.331 & 0.361 & 0.381 & 0.405 & 0.433 & 0.469 \\
\hline 2004 & 0.255 & 0.268 & 0.280 & 0.295 & 0.322 & 0.352 & 0.371 & 0.394 & 0.422 & 0.457 \\
\hline 2005 & 0.248 & 0.260 & 0.271 & 0.286 & 0.312 & 0.341 & 0.360 & 0.382 & 0.409 & 0.442 \\
\hline 2006 & 0.240 & 0.252 & 0.263 & 0.278 & 0.303 & 0.331 & 0.349 & 0.370 & 0.397 & 0.429 \\
\hline 2007 & 0.234 & 0.246 & 0.256 & 0.270 & 0.295 & 0.322 & 0.340 & 0.361 & 0.386 & 0.418 \\
\hline 2008 & 0.229 & 0.241 & 0.252 & 0.265 & 0.289 & 0.316 & 0.333 & 0.354 & 0.379 & 0.410 \\
\hline 2009 & 0.228 & 0.239 & 0.250 & 0.263 & 0.287 & 0.313 & 0.331 & 0.351 & 0.376 & 0.407 \\
\hline 2010 & 0.225 & 0.236 & 0.246 & 0.260 & 0.283 & 0.309 & 0.326 & 0.347 & 0.371 & 0.402 \\
\hline 2011 & 0.220 & 0.232 & 0.242 & 0.255 & 0.278 & 0.303 & 0.320 & 0.340 & 0.364 & 0.394 \\
\hline 2012 & 0.217 & 0.228 & 0.237 & 0.250 & 0.273 & 0.298 & 0.315 & 0.334 & 0.358 & 0.387 \\
\hline 2013 & 0.213 & 0.224 & 0.234 & 0.247 & 0.269 & 0.294 & 0.310 & 0.329 & 0.352 & 0.381 \\
\hline
\end{tabular}


Table B.18

Gross National Product Implicit Price Deflator (Continued)

\begin{tabular}{|c|c|c|c|c|c|c|c|c|c|c|}
\hline From: & 1980 & 1981 & 1982 & 1983 & 1984 & 1985 & 1986 & 1987 & 1988 & 1989 \\
\hline 1970 & 1.949 & 2.131 & 2.263 & 2.353 & 2.436 & 2.514 & 2.565 & 2.631 & 2.723 & 2.830 \\
\hline 1971 & 1.854 & 2.028 & 2.153 & 2.239 & 2.318 & 2.392 & 2.441 & 2.504 & 2.591 & 2.693 \\
\hline 1972 & 1.777 & 1.943 & 2.064 & 2.145 & 2.222 & 2.293 & 2.339 & 2.399 & 2.484 & 2.580 \\
\hline 1973 & 1.685 & 1.843 & 1.957 & 2.034 & 2.107 & 2.174 & 2.218 & 2.275 & 2.355 & 2.447 \\
\hline 1974 & 1.547 & 1.691 & 1.796 & 1.867 & 1.933 & 1.995 & 2.036 & 2.088 & 2.161 & 2.246 \\
\hline 1975 & 1.415 & 1.548 & 1.644 & 1.709 & 1.769 & 1.826 & 1.863 & 1.911 & 1.978 & 2.055 \\
\hline 1976 & 1.341 & 1.467 & 1.558 & 1.619 & 1.677 & 1.731 & 1.766 & 1.811 & 1.875 & 1.948 \\
\hline 1977 & 1.263 & 1.381 & 1.467 & 1.525 & 1.579 & 1.629 & 1.662 & 1.705 & 1.765 & 1.834 \\
\hline 1978 & 1.180 & 1.290 & 1.370 & 1.425 & 1.475 & 1.522 & 1.553 & 1.593 & 1.649 & 1.714 \\
\hline 1979 & 1.090 & 1.192 & 1.266 & 1.316 & 1.363 & 1.406 & 1.435 & 1.472 & 1.523 & 1.583 \\
\hline 1980 & 1.000 & 1.093 & 1.161 & 1.207 & 1.250 & 1.290 & 1.316 & 1.350 & 1.398 & 1.452 \\
\hline 1981 & 0.915 & 1.000 & 1.062 & 1.104 & 1.143 & 1.180 & 1.204 & 1.235 & 1.278 & 1.328 \\
\hline 1982 & 0.861 & 0.942 & 1.000 & 1.040 & 1.076 & 1.111 & 1.133 & 1.163 & 1.203 & 1.250 \\
\hline 1983 & 0.828 & 0.906 & 0.962 & 1.000 & 1.035 & 1.069 & 1.090 & 1.118 & 1.158 & 1.203 \\
\hline 1984 & 0.800 & 0.875 & 0.929 & 0.966 & 1.000 & 1.032 & 1.053 & 1.080 & 1.118 & 1.162 \\
\hline 1985 & 0.775 & 0.848 & 0.900 & 0.936 & 0.969 & 1.000 & 1.020 & 1.047 & 1.083 & 1.126 \\
\hline 1986 & 0.760 & 0.831 & 0.882 & 0.917 & 0.950 & 0.980 & 1.000 & 1.026 & 1.062 & 1.103 \\
\hline 1987 & 0.741 & 0.810 & 0.860 & 0.894 & 0.926 & 0.956 & 0.975 & 1.000 & 1.035 & 1.075 \\
\hline 1988 & 0.716 & 0.782 & 0.831 & 0.864 & 0.895 & 0.923 & 0.942 & 0.966 & 1.000 & 1.039 \\
\hline 1989 & 0.689 & 0.753 & 0.800 & 0.831 & 0.861 & 0.888 & 0.906 & 0.930 & 0.962 & 1.000 \\
\hline 1990 & 0.664 & 0.726 & 0.771 & 0.802 & 0.830 & 0.857 & 0.874 & 0.897 & 0.928 & 0.964 \\
\hline 1991 & 0.643 & 0.703 & 0.746 & 0.776 & 0.803 & 0.829 & 0.846 & 0.867 & 0.898 & 0.933 \\
\hline 1992 & 0.628 & 0.687 & 0.730 & 0.758 & 0.785 & 0.810 & 0.827 & 0.848 & 0.878 & 0.912 \\
\hline 1993 & 0.614 & 0.671 & 0.713 & 0.741 & 0.767 & 0.792 & 0.808 & 0.828 & 0.858 & 0.891 \\
\hline 1994 & 0.601 & 0.657 & 0.698 & 0.725 & 0.751 & 0.775 & 0.791 & 0.811 & 0.840 & 0.872 \\
\hline 1995 & 0.588 & 0.644 & 0.683 & 0.710 & 0.736 & 0.759 & 0.775 & 0.795 & 0.822 & 0.855 \\
\hline 1996 & 0.578 & 0.632 & 0.671 & 0.698 & 0.722 & 0.746 & 0.761 & 0.780 & 0.808 & 0.839 \\
\hline 1997 & 0.568 & 0.621 & 0.660 & 0.686 & 0.710 & 0.733 & 0.748 & 0.767 & 0.794 & 0.825 \\
\hline 1998 & 0.562 & 0.615 & 0.653 & 0.679 & 0.703 & 0.725 & 0.740 & 0.759 & 0.786 & 0.816 \\
\hline 1999 & 0.554 & 0.606 & 0.644 & 0.669 & 0.693 & 0.715 & 0.729 & 0.748 & 0.774 & 0.805 \\
\hline 2000 & 0.542 & 0.592 & 0.629 & 0.654 & 0.677 & 0.699 & 0.713 & 0.732 & 0.757 & 0.787 \\
\hline 2001 & 0.530 & 0.579 & 0.615 & 0.639 & 0.662 & 0.683 & 0.697 & 0.715 & 0.740 & 0.769 \\
\hline 2002 & 0.522 & 0.570 & 0.606 & 0.630 & 0.652 & 0.673 & 0.687 & 0.704 & 0.729 & 0.757 \\
\hline 2003 & 0.511 & 0.559 & 0.594 & 0.617 & 0.639 & 0.660 & 0.673 & 0.691 & 0.715 & 0.743 \\
\hline 2004 & 0.498 & 0.544 & 0.578 & 0.601 & 0.622 & 0.642 & 0.655 & 0.672 & 0.696 & 0.723 \\
\hline 2005 & 0.482 & 0.527 & 0.560 & 0.582 & 0.603 & 0.622 & 0.635 & 0.651 & 0.674 & 0.700 \\
\hline 2006 & 0.468 & 0.512 & 0.543 & 0.565 & 0.585 & 0.604 & 0.616 & 0.632 & 0.654 & 0.679 \\
\hline 2007 & 0.456 & 0.498 & 0.529 & 0.550 & 0.570 & 0.588 & 0.600 & 0.615 & 0.637 & 0.662 \\
\hline 2008 & 0.447 & 0.489 & 0.519 & 0.540 & 0.559 & 0.577 & 0.588 & 0.604 & 0.625 & 0.649 \\
\hline 2009 & 0.444 & 0.485 & 0.515 & 0.536 & 0.555 & 0.572 & 0.584 & 0.599 & 0.620 & 0.644 \\
\hline 2010 & 0.438 & 0.479 & 0.508 & 0.529 & 0.547 & 0.565 & 0.576 & 0.591 & 0.612 & 0.636 \\
\hline 2011 & 0.429 & 0.469 & 0.499 & 0.518 & 0.537 & 0.554 & 0.565 & 0.580 & 0.600 & 0.623 \\
\hline 2012 & 0.422 & 0.461 & 0.490 & 0.509 & 0.527 & 0.544 & 0.555 & 0.570 & 0.590 & 0.613 \\
\hline 2013 & 0.416 & 0.455 & 0.483 & 0.502 & 0.520 & 0.536 & 0.547 & 0.561 & 0.581 & 0.604 \\
\hline
\end{tabular}


Table B.18

Gross National Product Implicit Price Deflator (Continued)

\begin{tabular}{|c|c|c|c|c|c|c|c|c|c|c|}
\hline From: & 1990 & 1991 & 1992 & 1993 & 1994 & 1995 & 1996 & 1997 & 1998 & 1999 \\
\hline 1970 & 2.935 & 3.033 & 3.102 & 3.176 & 3.243 & 3.311 & 3.372 & 3.429 & 3.467 & 3.517 \\
\hline 1971 & 2.793 & 2.886 & 2.952 & 3.022 & 3.086 & 3.151 & 3.209 & 3.263 & 3.299 & 3.346 \\
\hline 1972 & 2.676 & 2.766 & 2.829 & 2.896 & 2.958 & 3.020 & 3.075 & 3.128 & 3.162 & 3.207 \\
\hline 1973 & 2.538 & 2.623 & 2.683 & 2.746 & 2.805 & 2.863 & 2.916 & 2.966 & 2.998 & 3.041 \\
\hline 1974 & 2.329 & 2.407 & 2.462 & 2.520 & 2.574 & 2.628 & 2.676 & 2.722 & 2.751 & 2.791 \\
\hline 1975 & 2.131 & 2.203 & 2.253 & 2.307 & 2.356 & 2.405 & 2.449 & 2.491 & 2.518 & 2.554 \\
\hline 1976 & 2.020 & 2.088 & 2.135 & 2.186 & 2.233 & 2.279 & 2.321 & 2.361 & 2.387 & 2.421 \\
\hline 1977 & 1.902 & 1.966 & 2.011 & 2.058 & 2.102 & 2.146 & 2.185 & 2.223 & 2.247 & 2.279 \\
\hline 1978 & 1.777 & 1.837 & 1.879 & 1.923 & 1.964 & 2.005 & 2.042 & 2.077 & 2.099 & 2.130 \\
\hline 1979 & 1.642 & 1.697 & 1.735 & 1.776 & 1.814 & 1.852 & 1.886 & 1.918 & 1.939 & 1.967 \\
\hline 1980 & 1.506 & 1.556 & 1.592 & 1.630 & 1.664 & 1.699 & 1.730 & 1.760 & 1.779 & 1.805 \\
\hline 1981 & 1.377 & 1.423 & 1.456 & 1.490 & 1.522 & 1.554 & 1.582 & 1.609 & 1.627 & 1.650 \\
\hline 1982 & 1.297 & 1.340 & 1.371 & 1.403 & 1.433 & 1.463 & 1.490 & 1.516 & 1.532 & 1.554 \\
\hline 1983 & 1.247 & 1.289 & 1.319 & 1.350 & 1.379 & 1.408 & 1.433 & 1.458 & 1.474 & 1.495 \\
\hline 1984 & 1.205 & 1.245 & 1.273 & 1.304 & 1.331 & 1.359 & 1.384 & 1.408 & 1.423 & 1.444 \\
\hline 1985 & 1.167 & 1.206 & 1.234 & 1.263 & 1.290 & 1.317 & 1.341 & 1.364 & 1.379 & 1.399 \\
\hline 1986 & 1.144 & 1.182 & 1.209 & 1.238 & 1.264 & 1.291 & 1.315 & 1.337 & 1.352 & 1.371 \\
\hline 1987 & 1.115 & 1.153 & 1.179 & 1.207 & 1.233 & 1.259 & 1.282 & 1.303 & 1.318 & 1.337 \\
\hline 1988 & 1.078 & 1.114 & 1.139 & 1.166 & 1.191 & 1.216 & 1.238 & 1.259 & 1.273 & 1.291 \\
\hline 1989 & 1.037 & 1.072 & 1.096 & 1.122 & 1.146 & 1.170 & 1.192 & 1.212 & 1.225 & 1.243 \\
\hline 1990 & 1.000 & 1.033 & 1.057 & 1.082 & 1.105 & 1.128 & 1.149 & 1.169 & 1.181 & 1.198 \\
\hline 1991 & 0.968 & 1.000 & 1.023 & 1.047 & 1.069 & 1.092 & 1.112 & 1.131 & 1.143 & 1.159 \\
\hline 1992 & 0.946 & 0.978 & 1.000 & 1.024 & 1.046 & 1.067 & 1.087 & 1.106 & 1.118 & 1.134 \\
\hline 1993 & 0.924 & 0.955 & 0.977 & 1.000 & 1.021 & 1.043 & 1.062 & 1.080 & 1.092 & 1.107 \\
\hline 1994 & 0.905 & 0.935 & 0.956 & 0.979 & 1.000 & 1.021 & 1.040 & 1.057 & 1.069 & 1.084 \\
\hline 1995 & 0.886 & 0.916 & 0.937 & 0.959 & 0.979 & 1.000 & 1.018 & 1.036 & 1.047 & 1.062 \\
\hline 1996 & 0.870 & 0.899 & 0.920 & 0.942 & 0.962 & 0.982 & 1.000 & 1.017 & 1.028 & 1.043 \\
\hline 1997 & 0.856 & 0.884 & 0.905 & 0.926 & 0.946 & 0.966 & 0.983 & 1.000 & 1.011 & 1.025 \\
\hline 1998 & 0.847 & 0.875 & 0.895 & 0.916 & 0.936 & 0.955 & 0.973 & 0.989 & 1.000 & 1.014 \\
\hline 1999 & 0.835 & 0.862 & 0.882 & 0.903 & 0.922 & 0.942 & 0.959 & 0.975 & 0.986 & 1.000 \\
\hline 2000 & 0.816 & 0.843 & 0.863 & 0.883 & 0.902 & 0.921 & 0.938 & 0.954 & 0.964 & 0.978 \\
\hline 2001 & 0.798 & 0.824 & 0.843 & 0.863 & 0.882 & 0.900 & 0.917 & 0.932 & 0.942 & 0.956 \\
\hline 2002 & 0.786 & 0.812 & 0.830 & 0.850 & 0.868 & 0.886 & 0.903 & 0.918 & 0.928 & 0.941 \\
\hline 2003 & 0.770 & 0.796 & 0.814 & 0.834 & 0.851 & 0.869 & 0.885 & 0.900 & 0.910 & 0.923 \\
\hline 2004 & 0.750 & 0.775 & 0.792 & 0.811 & 0.829 & 0.846 & 0.861 & 0.876 & 0.886 & 0.898 \\
\hline 2005 & 0.726 & 0.751 & 0.768 & 0.786 & 0.803 & 0.820 & 0.835 & 0.849 & 0.858 & 0.870 \\
\hline 2006 & 0.705 & 0.728 & 0.745 & 0.763 & 0.779 & 0.795 & 0.810 & 0.824 & 0.832 & 0.844 \\
\hline 2007 & 0.686 & 0.709 & 0.726 & 0.743 & 0.759 & 0.775 & 0.789 & 0.802 & 0.811 & 0.823 \\
\hline 2008 & 0.673 & 0.696 & 0.712 & 0.729 & 0.744 & 0.760 & 0.773 & 0.787 & 0.795 & 0.807 \\
\hline 2009 & 0.668 & 0.690 & 0.706 & 0.723 & 0.738 & 0.754 & 0.768 & 0.781 & 0.789 & 0.800 \\
\hline 2010 & 0.659 & 0.681 & 0.697 & 0.713 & 0.729 & 0.744 & 0.758 & 0.770 & 0.779 & 0.790 \\
\hline 2011 & 0.647 & 0.668 & 0.683 & 0.700 & 0.715 & 0.729 & 0.743 & 0.756 & 0.764 & 0.775 \\
\hline 2012 & 0.635 & 0.657 & 0.672 & 0.688 & 0.702 & 0.717 & 0.730 & 0.743 & 0.751 & 0.761 \\
\hline 2013 & 0.626 & 0.647 & 0.662 & 0.677 & 0.692 & 0.706 & 0.719 & 0.732 & 0.740 & 0.750 \\
\hline
\end{tabular}


Table B.18

Gross National Product Implicit Price Deflator (Continued)

\begin{tabular}{|c|c|c|c|c|c|c|c|c|c|c|}
\hline From: & 2000 & 2001 & 2002 & 2003 & 2004 & 2005 & 2006 & 2007 & 2008 & 2009 \\
\hline 1970 & 3.596 & 3.679 & 3.735 & 3.810 & 3.915 & 4.040 & 4.164 & 4.275 & 4.359 & 4.393 \\
\hline 1971 & 3.422 & 3.501 & 3.555 & 3.626 & 3.725 & 3.845 & 3.963 & 4.068 & 4.148 & 4.180 \\
\hline 1972 & 3.280 & 3.355 & 3.407 & 3.475 & 3.570 & 3.685 & 3.798 & 3.899 & 3.976 & 4.006 \\
\hline 1973 & 3.110 & 3.181 & 3.230 & 3.295 & 3.385 & 3.494 & 3.601 & 3.697 & 3.770 & 3.799 \\
\hline 1974 & 2.854 & 2.920 & 2.965 & 3.024 & 3.107 & 3.206 & 3.305 & 3.393 & 3.460 & 3.487 \\
\hline 1975 & 2.612 & 2.672 & 2.713 & 2.767 & 2.843 & 2.934 & 3.025 & 3.105 & 3.166 & 3.191 \\
\hline 1976 & 2.476 & 2.532 & 2.571 & 2.623 & 2.695 & 2.781 & 2.867 & 2.943 & 3.001 & 3.024 \\
\hline 1977 & 2.331 & 2.384 & 2.421 & 2.470 & 2.537 & 2.619 & 2.699 & 2.771 & 2.825 & 2.847 \\
\hline 1978 & 2.178 & 2.228 & 2.262 & 2.307 & 2.371 & 2.447 & 2.522 & 2.589 & 2.640 & 2.660 \\
\hline 1979 & 2.012 & 2.058 & 2.090 & 2.131 & 2.190 & 2.260 & 2.330 & 2.392 & 2.439 & 2.457 \\
\hline 1980 & 1.846 & 1.888 & 1.917 & 1.955 & 2.009 & 2.073 & 2.137 & 2.194 & 2.237 & 2.254 \\
\hline 1981 & 1.688 & 1.726 & 1.753 & 1.788 & 1.837 & 1.896 & 1.954 & 2.006 & 2.046 & 2.062 \\
\hline 1982 & 1.589 & 1.626 & 1.651 & 1.684 & 1.730 & 1.785 & 1.840 & 1.889 & 1.926 & 1.941 \\
\hline 1983 & 1.529 & 1.564 & 1.588 & 1.620 & 1.664 & 1.717 & 1.770 & 1.817 & 1.853 & 1.867 \\
\hline 1984 & 1.476 & 1.510 & 1.533 & 1.564 & 1.607 & 1.659 & 1.710 & 1.755 & 1.789 & 1.803 \\
\hline 1985 & 1.431 & 1.463 & 1.486 & 1.516 & 1.557 & 1.607 & 1.657 & 1.701 & 1.734 & 1.747 \\
\hline 1986 & 1.402 & 1.434 & 1.456 & 1.485 & 1.526 & 1.575 & 1.624 & 1.667 & 1.700 & 1.713 \\
\hline 1987 & 1.367 & 1.398 & 1.420 & 1.448 & 1.488 & 1.536 & 1.583 & 1.625 & 1.657 & 1.670 \\
\hline 1988 & 1.321 & 1.351 & 1.372 & 1.399 & 1.437 & 1.484 & 1.529 & 1.570 & 1.601 & 1.613 \\
\hline 1989 & 1.271 & 1.300 & 1.320 & 1.347 & 1.383 & 1.428 & 1.472 & 1.511 & 1.541 & 1.553 \\
\hline 1990 & 1.225 & 1.254 & 1.273 & 1.298 & 1.334 & 1.377 & 1.419 & 1.457 & 1.485 & 1.497 \\
\hline 1991 & 1.186 & 1.213 & 1.232 & 1.256 & 1.291 & 1.332 & 1.373 & 1.410 & 1.437 & 1.448 \\
\hline 1992 & 1.159 & 1.186 & 1.204 & 1.228 & 1.262 & 1.302 & 1.343 & 1.378 & 1.405 & 1.416 \\
\hline 1993 & 1.132 & 1.158 & 1.176 & 1.200 & 1.233 & 1.272 & 1.311 & 1.346 & 1.373 & 1.383 \\
\hline 1994 & 1.109 & 1.134 & 1.152 & 1.175 & 1.207 & 1.246 & 1.284 & 1.318 & 1.344 & 1.355 \\
\hline 1995 & 1.086 & 1.111 & 1.128 & 1.151 & 1.182 & 1.220 & 1.258 & 1.291 & 1.317 & 1.327 \\
\hline 1996 & 1.067 & 1.091 & 1.108 & 1.130 & 1.161 & 1.198 & 1.235 & 1.268 & 1.293 & 1.303 \\
\hline 1997 & 1.049 & 1.073 & 1.089 & 1.111 & 1.141 & 1.178 & 1.214 & 1.247 & 1.271 & 1.281 \\
\hline 1998 & 1.037 & 1.061 & 1.077 & 1.099 & 1.129 & 1.165 & 1.201 & 1.233 & 1.257 & 1.267 \\
\hline 1999 & 1.023 & 1.046 & 1.062 & 1.083 & 1.113 & 1.149 & 1.184 & 1.216 & 1.240 & 1.249 \\
\hline 2000 & 1.000 & 1.023 & 1.039 & 1.059 & 1.088 & 1.123 & 1.158 & 1.189 & 1.212 & 1.222 \\
\hline 2001 & 0.978 & 1.000 & 1.015 & 1.036 & 1.064 & 1.098 & 1.132 & 1.162 & 1.185 & 1.194 \\
\hline 2002 & 0.963 & 0.985 & 1.000 & 1.020 & 1.048 & 1.082 & 1.115 & 1.145 & 1.167 & 1.176 \\
\hline 2003 & 0.944 & 0.966 & 0.980 & 1.000 & 1.027 & 1.060 & 1.093 & 1.122 & 1.144 & 1.153 \\
\hline 2004 & 0.919 & 0.940 & 0.954 & 0.973 & 1.000 & 1.032 & 1.064 & 1.092 & 1.114 & 1.122 \\
\hline 2005 & 0.890 & 0.911 & 0.925 & 0.943 & 0.969 & 1.000 & 1.031 & 1.058 & 1.079 & 1.087 \\
\hline 2006 & 0.864 & 0.883 & 0.897 & 0.915 & 0.940 & 0.970 & 1.000 & 1.027 & 1.047 & 1.055 \\
\hline 2007 & 0.841 & 0.861 & 0.874 & 0.891 & 0.916 & 0.945 & 0.974 & 1.000 & 1.020 & 1.028 \\
\hline 2008 & 0.825 & 0.844 & 0.857 & 0.874 & 0.898 & 0.927 & 0.955 & 0.981 & 1.000 & 1.008 \\
\hline 2009 & 0.819 & 0.837 & 0.850 & 0.867 & 0.891 & 0.920 & 0.948 & 0.973 & 0.992 & 1.000 \\
\hline 2010 & 0.808 & 0.826 & 0.839 & 0.856 & 0.879 & 0.908 & 0.936 & 0.960 & 0.979 & 0.987 \\
\hline 2011 & 0.792 & 0.810 & 0.823 & 0.839 & 0.862 & 0.890 & 0.917 & 0.942 & 0.960 & 0.968 \\
\hline 2012 & 0.779 & 0.797 & 0.809 & 0.825 & 0.848 & 0.875 & 0.902 & 0.926 & 0.944 & 0.951 \\
\hline 2013 & 0.767 & 0.785 & 0.797 & 0.813 & 0.835 & 0.862 & 0.888 & 0.912 & 0.930 & 0.937 \\
\hline
\end{tabular}


Table B.18

Gross National Product Implicit Price Deflator (Continued)

\begin{tabular}{ccccc}
\hline From: & $\mathbf{2 0 1 0}$ & $\mathbf{2 0 1 1}$ & $\mathbf{2 0 1 2}$ & $\mathbf{2 0 1 3}$ \\
\hline $\mathbf{1 9 7 0}$ & 4.451 & 4.539 & 4.618 & 4.688 \\
$\mathbf{1 9 7 1}$ & 4.236 & 4.319 & 4.395 & 4.461 \\
$\mathbf{1 9 7 2}$ & 4.059 & 4.140 & 4.212 & 4.275 \\
$\mathbf{1 9 7 3}$ & 3.849 & 3.925 & 3.994 & 4.054 \\
$\mathbf{1 9 7 4}$ & 3.533 & 3.602 & 3.665 & 3.720 \\
$\mathbf{1 9 7 5}$ & 3.233 & 3.297 & 3.354 & 3.405 \\
$\mathbf{1 9 7 6}$ & 3.064 & 3.125 & 3.179 & 3.227 \\
$\mathbf{1 9 7 7}$ & 2.885 & 2.942 & 2.993 & 3.038 \\
$\mathbf{1 9 7 8}$ & 2.696 & 2.749 & 2.797 & 2.839 \\
$\mathbf{1 9 7 9}$ & 2.490 & 2.539 & 2.583 & 2.622 \\
$\mathbf{1 9 8 0}$ & 2.284 & 2.329 & 2.370 & 2.406 \\
$\mathbf{1 9 8 1}$ & 2.089 & 2.130 & 2.167 & 2.200 \\
$\mathbf{1 9 8 2}$ & 1.967 & 2.006 & 2.041 & 2.072 \\
$\mathbf{1 9 8 3}$ & 1.892 & 1.929 & 1.963 & 1.993 \\
$\mathbf{1 9 8 4}$ & 1.827 & 1.863 & 1.896 & 1.924 \\
$\mathbf{1 9 8 5}$ & 1.771 & 1.806 & 1.837 & 1.865 \\
$\mathbf{1 9 8 6}$ & 1.735 & 1.770 & 1.801 & 1.828 \\
$\mathbf{1 9 8 7}$ & 1.692 & 1.725 & 1.755 & 1.782 \\
$\mathbf{1 9 8 8}$ & 1.634 & 1.667 & 1.696 & 1.721 \\
$\mathbf{1 9 8 9}$ & 1.573 & 1.604 & 1.632 & 1.657 \\
$\mathbf{1 9 9 0}$ & 1.517 & 1.547 & 1.574 & 1.597 \\
$\mathbf{1 9 9 1}$ & 1.468 & 1.497 & 1.523 & 1.546 \\
$\mathbf{1 9 9 2}$ & 1.435 & 1.463 & 1.489 & 1.511 \\
$\mathbf{1 9 9 3}$ & 1.402 & 1.429 & 1.454 & 1.476 \\
$\mathbf{1 9 9 4}$ & 1.372 & 1.400 & 1.424 & 1.445 \\
$\mathbf{1 9 9 5}$ & 1.344 & 1.371 & 1.395 & 1.416 \\
$\mathbf{1 9 9 6}$ & 1.320 & 1.346 & 1.370 & 1.390 \\
$\mathbf{1 9 9 7}$ & 1.298 & 1.324 & 1.347 & 1.367 \\
$\mathbf{1 9 9 8}$ & 1.284 & 1.309 & 1.332 & 1.352 \\
$\mathbf{1 9 9 9}$ & 1.266 & 1.291 & 1.313 & 1.333 \\
$\mathbf{2 0 0 0}$ & 1.238 & 1.262 & 1.284 & 1.303 \\
$\mathbf{2 0 0 1}$ & 1.210 & 1.234 & 1.255 & 1.274 \\
$\mathbf{2 0 0 2}$ & 1.192 & 1.215 & 1.236 & 1.255 \\
$\mathbf{2 0 0 3}$ & 1.168 & 1.191 & 1.212 & 1.230 \\
$\mathbf{2 0 0 4}$ & 1.137 & 1.160 & 1.180 & 1.198 \\
$\mathbf{2 0 0 5}$ & 1.102 & 1.123 & 1.143 & 1.160 \\
$\mathbf{2 0 0 6}$ & 1.069 & 1.090 & 1.109 & 1.126 \\
$\mathbf{2 0 0 7}$ & 1.041 & 1.062 & 1.080 & 1.097 \\
$\mathbf{2 0 0 8}$ & 1.021 & 1.041 & 1.059 & 1.075 \\
$\mathbf{2 0 0 9}$ & 1.013 & 1.033 & 1.051 & 1.067 \\
$\mathbf{2 0 1 0}$ & 1.000 & 1.020 & 1.038 & 1.053 \\
$\mathbf{2 0 1 1}$ & 0.981 & 1.000 & 1.017 & 1.033 \\
$\mathbf{2 0 1 2}$ & 0.964 & 0.983 & 1.000 & 1.015 \\
\hline & 0.949 & 0.968 & 0.990 & 1.000 \\
\hline & & & &
\end{tabular}

Source:

U.S. Department of Commerce, Bureau of Economic Analysis, Survey of Current Business, Washington, DC, monthly. 
APPENDIX C

MAPS 
C-2

TRANSPORTATION ENERGY DATA BOOK: EDITION 33-2014 
Table C.1

Census Regions and Divisions

\begin{tabular}{|c|c|c|c|}
\hline \multicolumn{4}{|c|}{ Northeast Region } \\
\hline \multicolumn{2}{|c|}{ Mid-Atlantic division } & \multicolumn{2}{|c|}{ New England division } \\
\hline $\begin{array}{l}\text { New Jersey } \\
\text { New York }\end{array}$ & Pennsylvania & $\begin{array}{l}\text { Connecticut } \\
\text { Maine } \\
\text { Massachusetts }\end{array}$ & $\begin{array}{l}\text { New Hampshire } \\
\text { Rhode Island } \\
\text { Vermont }\end{array}$ \\
\hline \multicolumn{4}{|c|}{ South Region } \\
\hline $\begin{array}{l}\text { West South Central } \\
\text { division }\end{array}$ & $\begin{array}{c}\text { East South Central } \\
\text { division }\end{array}$ & \multicolumn{2}{|c|}{$\begin{array}{c}\text { South Atlantic } \\
\text { division }\end{array}$} \\
\hline $\begin{array}{l}\text { Arkansas } \\
\text { Louisiana } \\
\text { Oklahoma } \\
\text { Texas }\end{array}$ & $\begin{array}{l}\text { Alabama } \\
\text { Kentucky } \\
\text { Mississippi } \\
\text { Tennessee }\end{array}$ & $\begin{array}{l}\text { Delaware } \\
\text { Florida } \\
\text { Georgia } \\
\text { Maryland } \\
\text { North Carolina }\end{array}$ & $\begin{array}{l}\text { South Carolina } \\
\text { Virginia } \\
\text { Washington, DC } \\
\text { West Virginia }\end{array}$ \\
\hline \multicolumn{4}{|c|}{ West Region } \\
\hline \multicolumn{2}{|c|}{ Pacific division } & \multicolumn{2}{|c|}{ Mountain division } \\
\hline $\begin{array}{l}\text { Alaska } \\
\text { California } \\
\text { Hawaii }\end{array}$ & $\begin{array}{l}\text { Oregon } \\
\text { Washington }\end{array}$ & $\begin{array}{l}\text { Arizona } \\
\text { Colorado } \\
\text { Idaho } \\
\text { Montana }\end{array}$ & $\begin{array}{l}\text { Nevada } \\
\text { New Mexico } \\
\text { Utah } \\
\text { Wyoming }\end{array}$ \\
\hline \multicolumn{4}{|c|}{ Midwest Region } \\
\hline \multicolumn{2}{|c|}{ West North Central division } & \multicolumn{2}{|c|}{ East North Central division } \\
\hline $\begin{array}{l}\text { Iowa } \\
\text { Kansas } \\
\text { Minnesota } \\
\text { Missouri }\end{array}$ & $\begin{array}{l}\text { Nebraska } \\
\text { North Dakota } \\
\text { South Dakota }\end{array}$ & $\begin{array}{l}\text { Illinois } \\
\text { Indiana } \\
\text { Michigan }\end{array}$ & $\begin{array}{l}\text { Ohio } \\
\text { Wisconsin }\end{array}$ \\
\hline
\end{tabular}

Source:

U.S. Census Bureau. 
Figure C.1. Census Regions and Divisions

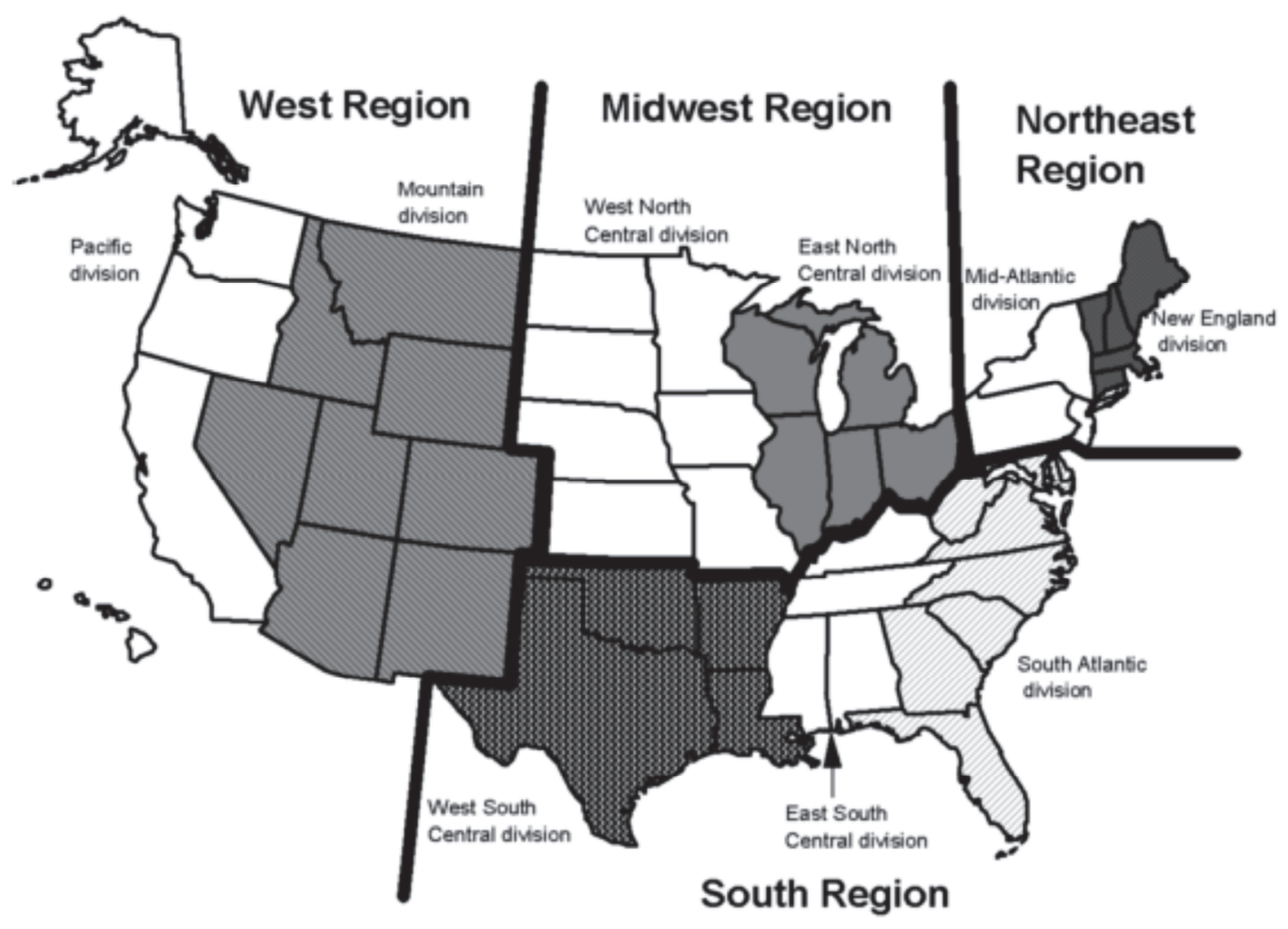

Source:

See Table C.1. 
Table C.2

Petroleum Administration for Defense Districts (PADD)

\begin{tabular}{|l|l|l|}
\hline District & Subdistrict & States \\
\hline $\begin{array}{l}\text { PAD District 1 } \\
\text { East Coast }\end{array}$ & $\begin{array}{l}\text { Subdistrict 1X } \\
\text { New England }\end{array}$ & $\begin{array}{l}\text { Connecticut, Maine, Massachusetts, New } \\
\text { Hampshire, Rhode Island, Vermont }\end{array}$ \\
\hline $\begin{array}{l}\text { Subdistrict 1Y } \\
\text { Central Atlantic }\end{array}$ & $\begin{array}{l}\text { Delaware, District of Columbia, Maryland, New } \\
\text { Jersey, New York, Pennsylvania }\end{array}$ \\
\hline $\begin{array}{l}\text { PAD District 2 } \\
\text { Midwest }\end{array}$ & $\begin{array}{l}\text { Subdistrict 1Z } \\
\text { Lower Atlantic }\end{array}$ & $\begin{array}{l}\text { Florida, Georgia, North Carolina, South Carolina, } \\
\text { Virginia, West Virginia }\end{array}$ \\
\hline $\begin{array}{l}\text { PAD District 3 } \\
\text { Gulf Coast }\end{array}$ & $\begin{array}{l}\text { Illinois, Indiana, Iowa, Kansas, Kentucky, } \\
\text { Michigan, Minnesota, Missouri, Nebraska, North } \\
\text { Dakota, South Dakota, Ohio, Oklahoma, } \\
\text { Tennessee, Wisconsin }\end{array}$ \\
\hline $\begin{array}{l}\text { PAD District 4 } \\
\text { Rocky Mountains }\end{array}$ & $\begin{array}{l}\text { Alabama, Arkansas, Louisiana, Mississippi, New } \\
\text { Mexico, Texas }\end{array}$ \\
\hline $\begin{array}{l}\text { PAD District 5 } \\
\text { West Coast }\end{array}$ & \begin{tabular}{l} 
Colorado Idaho, Montana, Utah, Wyoming \\
\hline
\end{tabular} & $\begin{array}{l}\text { Alaska, Arizona, California, Hawaii, Nevada, } \\
\text { Oregon, Washington }\end{array}$ \\
\hline
\end{tabular}

\section{Source:}

Energy Information Administration web site: http://tonto.eia.doe.gov/oog/info/twip/padddef.html 
Figure C.2. Petroleum Administration for Defense Districts

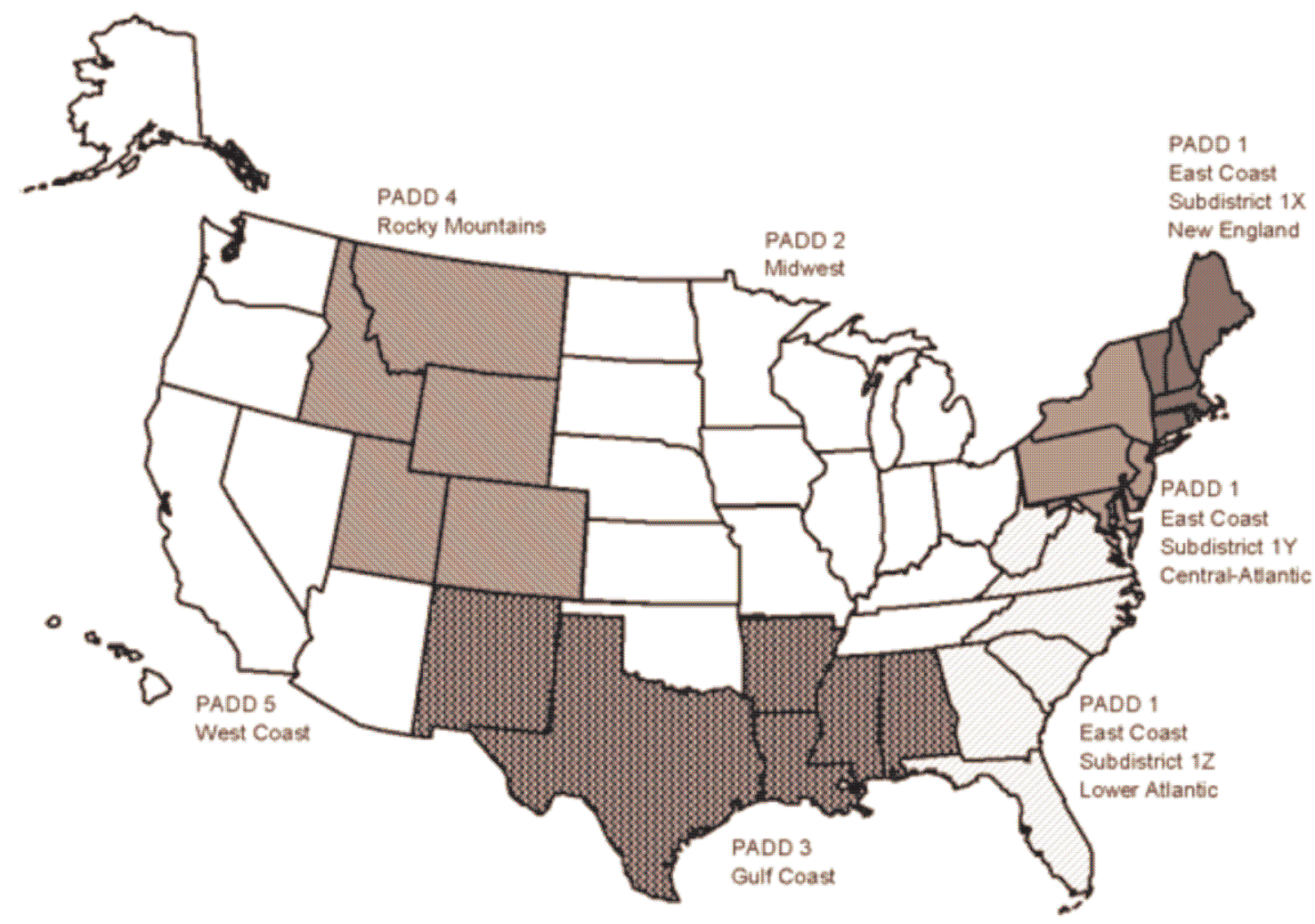

Source:

See Table C.2. 
Figure C.3. Map of Places where Reformulated Gasoline is Sold

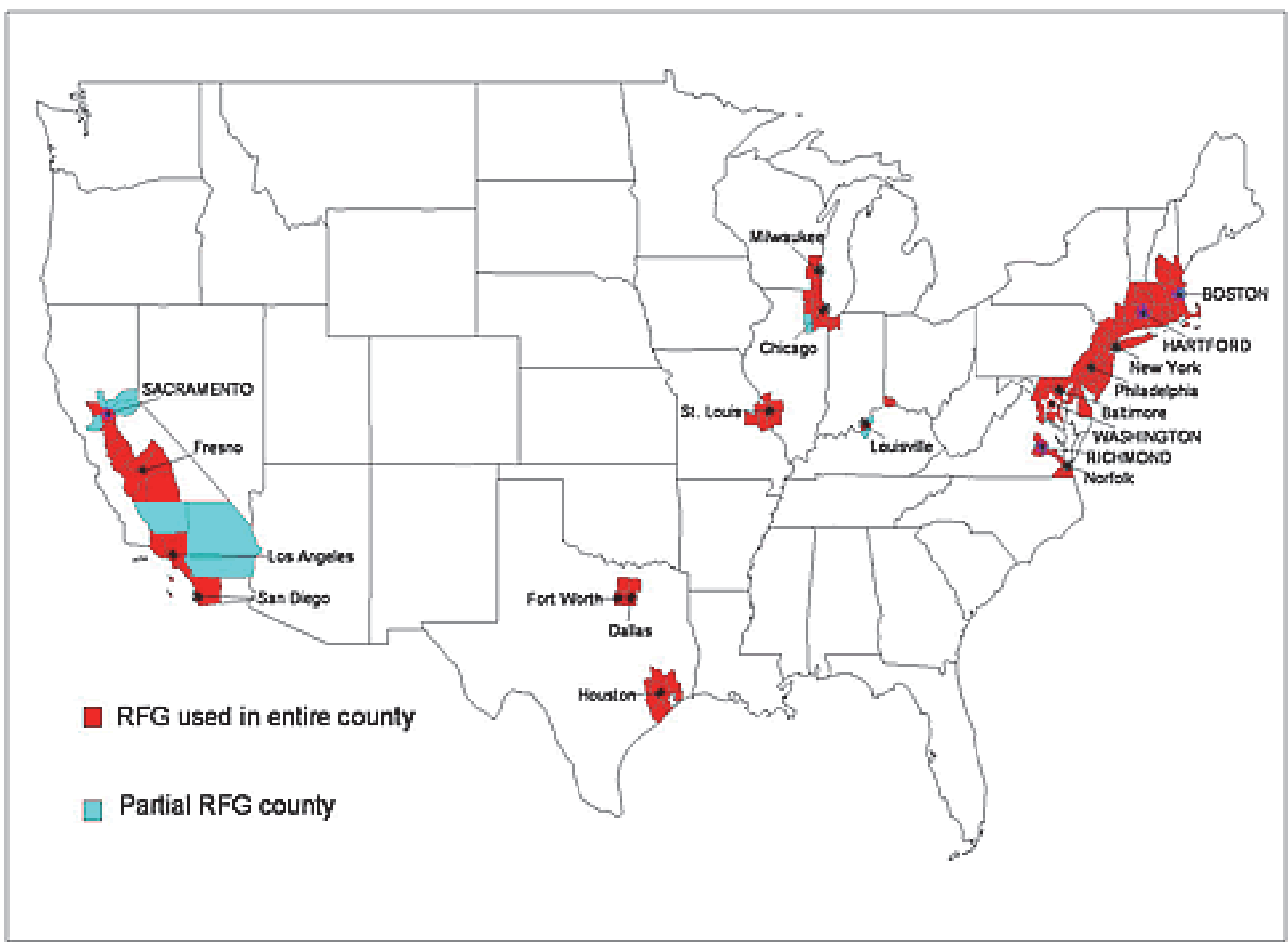

Source:

U.S. Environmental Protection Agency, www.epa.gov/otaq/fuels/gasolinefuels/rfg/areas.htm.

Note: Reformulated gasoline is a motor gasoline specially formulated to achieve significant reductions in vehicle emissions of ozone-forming and toxic air pollutants. The Clean Air Act of 1990 mandates reformulated gasoline use in areas with ozone-air pollution problems. 
C-8

TRANSPORTATION ENERGY DATA BOOK: EDITION 33-2014 


\section{GLOSSARY}

Acceleration power - Measured in kilowatts. Pulse power obtainable from a battery used to accelerate a vehicle. This is based on a constant current pulse for 30 seconds at no less than $2 / 3$ of the maximum open-circuit-voltage, at $80 \%$ depth-of-discharge relative to the battery's rated capacity and at $20^{\circ} \mathrm{C}$ ambient temperature.

Air Carrier - The commercial system of air transportation consisting of certificated air carriers, air taxis (including commuters), supplemental air carriers, commercial operators of large aircraft, and air travel clubs.

Certificated route air carrier: An air carrier holding a Certificate of Public Convenience and Necessity issued by the Department of Transportation to conduct scheduled interstate services. Nonscheduled or charter operations may also be conducted by these carriers. These carriers operate large aircraft (30 seats or more, or a maximum payload capacity of 7,500 pounds or more) in accordance with Federal Aviation Regulation part 121.

Domestic air operator: Commercial air transportation within and between the 50 States and the District of Columbia. Includes operations of certificated route air carriers, Pan American, local service, helicopter, intra-Alaska, intra-Hawaii, all-cargo carriers and other carriers. Also included are transborder operations conducted on the domestic route segments of U.S. air carriers. Domestic operators are classified based on their operating revenue as follows:

Majors - over $\$ 1$ billion

Nationals - \$100-1,000 million

Large Regionals - \$10-99.9 million

Medium Regionals - \$0-9.99 million

International air operator: Commercial air transportation outside the territory of the United States, including operations between the U.S. and foreign countries and between the U.S. and its territories and possessions.

Supplemental air carrier: A class of air carriers which hold certificates authorizing them to perform passenger and cargo charter services supplementing the scheduled service of the certificated route air carriers. Supplemental air carriers are often referred to as nonscheduled air carriers or "nonskeds."

Alcohol - The family name of a group of organic chemical compounds composed of carbon, hydrogen, and oxygen. The molecules in the series vary in chain length and are composed of a hydrocarbon plus a hydroxyl group. Alcohol includes methanol and ethanol.

Alternative fuel - For transportation applications, includes the following: methanol; denatured ethanol, and other alcohols; fuel mixtures containing 85 percent or more by volume of methanol, denatured ethanol, and other alcohols with gasoline or other fuels; natural gas; liquefied petroleum gas (propane); hydrogen; coal-derived liquid fuels; fuels (other than alcohol) derived from biological materials (biofuels such as soy diesel fuel); and electricity (including electricity from solar energy). The term "alternative fuel" does not include alcohol or other blended portions of primarily petroleum-based fuels used as oxygenates or extenders, i.e. MTBE, ETBE, other ethers, and the 10-percent ethanol portion of gasohol.

Amtrak - See Rail. 
Anthropogenic - Human made. Usually used in the context of emissions that are produced as the result of human activities.

Aviation - See General aviation.

Aviation gasoline - All special grades of gasoline for use in aviation reciprocating engines, as given in the American Society for Testing and Materials (ASTM) Specification D 910. Includes all refinery products within the gasoline range that are to be marketed straight or in blends as aviation gasoline without further processing (any refinery operation except mechanical blending). Also included are finished components in the gasoline range which will be used for blending or compounding into aviation gasoline.

Barges - Shallow, nonself-propelled vessels used to carry bulk commodities on the rivers and the Great Lakes.

Battery efficiency - Measured in percentage. Net DC energy delivered on discharge, as a percentage of the total DC energy required to restore the initial state-of-charge. The efficiency value must include energy losses resulting from self-discharge, cell equalization, thermal loss compensation, and all battery-specific auxiliary equipment.

Btu - British thermal unit. The amount of energy required to raise the temperature of 1 pound of water 1 degree Fahrenheit at or near 39.2 degrees Fahrenheit. An average Btu content of fuel is the heat value per quantity of fuel as determined from tests of fuel samples.

Bunker - A storage tank.

Bunkering fuels - Fuels stored in ship bunkers.

Bus -A mode of transit service characterized by roadway vehicles powered by diesel, gasoline, battery, or alternative fuel engines contained within the vehicle.

Intercity bus: A standard size bus equipped with front doors only, high backed seats, luggage compartments separate from the passenger compartment and usually with restroom facilities, for high-speed long distance service.

Motor bus: Rubber-tired, self-propelled, manually-steered bus with fuel supply on board the vehicle. Motor bus types include intercity, school, and transit.

School and other nonrevenue bus: Bus services for which passengers are not directly charged for transportation, either on a per passenger or per vehicle basis.

Transit bus: A bus designed for frequent stop service with front and center doors, normally with a rear-mounted diesel engine, low-back seating, and without luggage storage compartments or restroom facilities.

Trolley coach: Rubber-tired electric transit vehicle, manually-steered, propelled by a motor drawing current, normally through overhead wires, from a central power source not on board the vehicle.

Calendar year - The period of time between January 1 and December 31 of any given year.

Captive imports - Products produced overseas specifically for domestic manufacturers. 
Car size classifications - Size classifications of cars are established by the Environmental Protection Agency (EPA) as follows:

Minicompact - less than 85 cubic feet of passenger and luggage volume.

Subcompact - between 85 to 100 cubic feet of passenger and luggage volume.

Compact - between 100 to 110 cubic feet of passenger and luggage volume.

Midsize - between 110 to 120 cubic feet of passenger and luggage volume.

Large - more than 120 cubic feet of passenger and luggage volume.

Two seater - cars designed primarily to seat only two adults.

Station wagons are included with the size class for the sedan of the same name.

Carbon dioxide $\left(\mathrm{CO}_{2}\right)$ - A colorless, odorless, non-poisonous gas that is a normal part of the ambient air. Carbon dioxide is a product of fossil fuel combustion.

Carbon monoxide (CO) - A colorless, odorless, highly toxic gas that is a by-product of incomplete fossil fuel combustion. Carbon monoxide, one of the major air pollutants, can be harmful in small amounts if breathed over a certain period of time.

Car-mile (railroad) - A single railroad car moved a distance of one mile.

Cargo ton-mile - See Ton-mile.

Certificated route air carriers - See Air carriers.

Class I freight railroad - See Rail.

Coal slurry - Finely crushed coal mixed with sufficient water to form a fluid.

Combination trucks - Consist of a power unit (a truck tractor) and one or more trailing units (a semitrailer or trailer). The most frequently used combination is popularly referred to as a "tractorsemitrailer" or "tractor trailer".

Commercial sector - An energy-consuming sector that consists of service-providing facilities of: businesses; Federal, State, and local governments; and other private and public organizations, such as religious, social or fraternal groups. Includes institutional living quarters.

Commuter rail - A mode of transit service (also called metropolitan rail, regional rail, or suburban rail) characterized by an electric or diesel propelled railway for urban passenger train service consisting of local short distance travel operating between a central city and adjacent suburbs.

Compact car - See car size classifications.

Compression ignition - The form of ignition that initiates combustion in a diesel engine. The rapid compression of air within the cylinders generates the heat required to ignite the fuel as it is injected.

Constant dollars - A time series of monetary figures is expressed in constant dollars when the effect of change over time in the purchasing power of the dollar has been removed. Usually the data are expressed in terms of dollars of a selected year or the average of a set of years.

Consumer Price Index (CPI) - A measure of the average change over time in the prices paid by urban consumers for a market basket of consumer goods and services. 
Continuous discharge capacity - Measured as percent of rated energy capacity. Energy delivered in a constant power discharge required by an electric vehicle for hill climbing and/or high-speed cruise, specified as the percent of its rated energy capacity delivered in a one hour constant-power discharge.

Conventional Refueling Station - An establishment for refueling motor vehicles with traditional transportation fuels, such as gasoline and diesel fuel.

Corporate Average Fuel Economy (CAFE) Standards - CAFE standards were originally established by Congress for new cars, and later for light trucks, in Title V of the Motor Vehicle Information and Cost Savings Act (15 U.S.C.1901, et seq.) with subsequent amendments. Under CAFE, car manufacturers are required by law to produce vehicle fleets with a composite sales-weighted fuel economy which cannot be lower than the CAFE standards in a given year, or for every vehicle which does not meet the standard, a fine of $\$ 5.00$ is paid for every one-tenth of a mpg below the standard.

Criteria pollutant - A pollutant determined to be hazardous to human health and regulated under EPA's National Ambient Air Quality Standards. The 1970 amendments to the Clean Air Act require EPA to describe the health and welfare impacts of a pollutant as the "criteria" for inclusion in the regulatory regime.

Crude oil - A mixture of hydrocarbons that exists in the liquid phase in natural underground reservoirs and remains liquid at atmospheric pressure after passing through surface separating facilities. Crude oil production is measured at the wellhead and includes lease condensate.

Crude oil imports - The volume of crude oil imported into the 50 States and the District of Columbia, including imports from U.S. territories, but excluding imports of crude oil into the Hawaiian Foreign Trade Zone.

Curb weight - The weight of a vehicle including all standard equipment, spare tire and wheel, all fluids and lubricants to capacity, full tank of fuel, and the weight of major optional accessories normally found on the vehicle.

Current dollars - Represents dollars current at the time designated or at the time of the transaction. In most contexts, the same meaning would be conveyed by the use of the term "dollars." See also constant dollars.

Demand Response - A transit mode that includes passenger cars, vans, and small buses operating in response to calls from passengers to the transit operator who dispatches the vehicles. The vehicles do not operate over a fixed route on a fixed schedule. Can also be known as paratransit or dial-a-ride.

Diesel fuel - See Distillate fuel oil.

Disposable personal income - See Income.

Distillate fuel oil - The lighter fuel oils distilled off during the refining process. Included are products known as ASTM grades numbers 1 and 2 heating oils, diesel fuels, and number 4 fuel oil. The major uses of distillate fuel oils include heating, fuel for on-and off-highway diesel engines, and railroad diesel fuel.

Domestic air operator - See Air carrier. 
Domestic water transportation - See Internal water transportation.

E85 - 85\% ethanol and 15\% gasoline.

E95 - 95\% ethanol and 5\% gasoline.

Electric utilities sector - Consists of privately and publicly owned establishments which generate electricity primarily for resale.

Emission standards - Limits or ranges established for pollution levels emitted by vehicles as well as stationary sources. The first standards were established under the 1963 Clean Air Act.

End-use sector - See Sector.

Energy capacity - Measured in kilowatt hours. The energy delivered by the battery, when tested at C/3 discharge rate, up to termination of discharge specified by the battery manufacturer. The required acceleration power must be delivered by the battery at any point up to $80 \%$ of the battery's energy capacity rating.

Energy efficiency - In reference to transportation, the inverse of energy intensiveness: the ratio of outputs from a process to the energy inputs; for example, miles traveled per gallon of fuel (mpg).

Energy intensity - In reference to transportation, the ratio of energy inputs to a process to the useful outputs from that process; for example, gallons of fuel per passenger-mile or Btu per ton-mile.

Ethanol $\left(\mathbf{C}_{2} \mathbf{H}_{5} \mathbf{O H}\right)$ - Otherwise known as ethyl alcohol, alcohol, or grain-spirit. A clear, colorless, flammable oxygenated hydrocarbon with a boiling point of 78.5 degrees Celsius in the anhydrous state. In transportation, ethanol is used as a vehicle fuel by itself (E100 - 100\% ethanol by volume), blended with gasoline (E85 - 85\% ethanol by volume), or as a gasoline octane enhancer and oxygenate ( $10 \%$ by volume).

Excise tax - Paid when purchases are made on a specific good, such as gasoline. Excise taxes are often included in the price of the product. There are also excise taxes on activities, such as highway usage by trucks.

Ferry boat - A transit mode comprising vessels carrying passengers and in some cases vehicles over a body of water, and that are generally steam or diesel-powered.

Fixed operating cost - See Operating cost.

\section{Fleet vehicles -}

Private fleet vehicles: Ideally, a vehicle could be classified as a member of a fleet if it is:

a) operated in mass by a corporation or institution,

b) operated under unified control, or

c) used for non-personal activities.

However, the definition of a fleet is not consistent throughout the fleet industry. Some companies make a distinction between cars that were bought in bulk rather than singularly, or whether they are operated in bulk, as well as the minimum number of vehicles that constitute a fleet (i.e. 4 or $10)$.

Government fleet vehicles: Includes vehicles owned by all Federal, state, county, city, and metro units of government, including toll road operations. 
Foreign freight - Movements between the United States and foreign countries and between Puerto Rico, the Virgin Islands, and foreign countries. Trade between U.S. territories and possessions (e.g. Guam, Wake, American Samoa) and foreign countries is excluded. Traffic to or from the Panama Canal Zone is included.

Gas Guzzler Tax - Originates from the 1978 Energy Tax Act (Public Law 95-618). A new car purchaser is required to pay the tax if the car purchased has a combined city/highway fuel economy rating that is below the standard for that year. For model years 1986 and later, the standard is $22.5 \mathrm{mpg}$.

Gasohol - A mixture of $10 \%$ anhydrous ethanol and $90 \%$ gasoline by volume; $7.5 \%$ anhydrous ethanol and $92.5 \%$ gasoline by volume; or $5.5 \%$ anhydrous ethanol and $94.5 \%$ gasoline by volume. There are other fuels that contain methanol and gasoline, but these fuels are not referred to as gasohol.

Gasoline - See Motor gasoline.

General aviation - That portion of civil aviation which encompasses all facets of aviation except air carriers. It includes any air taxis, commuter air carriers, and air travel clubs which do not hold Certificates of Public Convenience and Necessity.

Global warming potential (GWP) - An index used to compare the relative radiative forcing of different gases without directly calculating the changes in atmospheric concentrations. GWPs are calculated as the ratio of the radiative forcing that would result from the emission of one kilogram of a greenhouse gas to that from the emission of one kilogram of carbon dioxide over a fixed period of time, such as 100 years.

Greenhouse gases - Those gases, such as water vapor, carbon dioxide, nitrous oxide, methane, hydrofluorocarbons (HFCs), perfluorocarbons (PFCs) and sulfur hexafluoride, that are transparent to solar (short-wave) radiation but opaque to long-wave (infrared) radiation, thus preventing long-wave radiant energy from leaving Earth's atmosphere. The net effect is a trapping of absorbed radiation and a tendency to warm the planet's surface.

Gross National Product - A measure of monetary value of the goods and services becoming available to the nation from economic activity. Total value at market prices of all goods and services produced by the nation's economy. Calculated quarterly by the Department of Commerce, the Gross National Product is the broadest available measure of the level of economic activity.

Gross vehicle weight (gvw) - The weight of the empty truck plus the maximum anticipated load weight.

Gross vehicle weight rating (gvwr) - The gross vehicle weight which is assigned to each new truck by the manufacturer. This rating may be different for trucks of the same model because of certain features, such as heavy-duty suspension. Passenger cars do not have gross vehicle weight ratings.

\section{Heavy-heavy truck - See Truck size classifications.}

Heavy rail - A mode of transit service (also called metro, subway, rapid transit, or rapid rail) operating on an electric railway with the capacity for a heavy volume of traffic. Characterized by high speed and rapid acceleration of passenger rail cars.

Household - Consists of all persons who occupy a housing unit, including the related family members and all unrelated persons, if any, who share the housing unit. 
Housing unit - A house, apartment, a group of rooms, or a single room occupied or intended for occupancy as separate living quarters. Separate living quarters are those in which the occupants do not live and eat with any other persons in the structure and which have either (1) direct access from the outside of the building or through a common hallway intended to be used by the occupants of another unit or by the general public, or (2) complete kitchen facilities for the exclusive use of the occupants. The occupants may be a single family, one person living alone, two or more families living together, or any other group of related or unrelated persons who share living arrangements.

Hybrid-electric vehicles - Combines the benefits of gasoline engines and electric motors and can be configured to obtain different objectives, such as improved fuel economy, increased power, or additional auxiliary power for electronic devices and power tools.

Hydrocarbon (HC) - A compound that contains only hydrogen and carbon. The simplest and lightest forms of hydrocarbon are gaseous. With greater molecular weights they are liquid, while the heaviest are solids.

\section{Income -}

Disposable personal income: Personal income less personal tax and non-tax payments.

National income: The aggregate earnings of labor and property which arise in the current production of goods and services by the nation's economy.

Personal income: The current income received by persons from all sources, net of contributions for social insurance.

Industrial sector - Construction, manufacturing, agricultural and mining establishments.

Inertia weight - The curb weight of a vehicle plus 300 pounds.

Intercity bus - See Bus.

Intermodal - Transportation activities involving more than one mode of transportation, including transportation connections and coordination of various modes.

Internal water transportation - Includes all local (intraport) traffic and traffic between ports or landings wherein the entire movement takes place on inland waterways. Also termed internal are movements involving carriage on both inland waterways and the water of the Great Lakes, and inland movements that cross short stretches of open water that link inland systems.

International air operator - See Air carrier.

International freight - See Foreign freight.

Jet fuel - Includes both naphtha-type and kerosene-type fuels meeting standards for use in aircraft turbine engines. Although most jet fuel is used in aircraft, some is used for other purposes such as generating electricity in gas turbines.

Kerosene-type jet fuel: A quality kerosene product with an average gravity of 40.7 degrees API and $10 \%$ to $90 \%$ distillation temperatures of 217 to 261 degrees centigrade. Used primarily as fuel for commercial turbojet and turboprop aircraft engines. It is a relatively low freezing point distillate of the kerosene type. 
Naphtha-type jet fuel: A fuel in the heavy naphtha boiling range with an average gravity of 52.8 degrees API and 10\% to $90 \%$ distillation temperatures of 117 to 233 degrees centigrade used for turbojet and turboprop aircraft engines, primarily by the military. Excludes ramjet and petroleum.

Kerosene - A petroleum distillate in the 300 to 500 degrees Fahrenheit boiling range and generally having a flash point higher than 100 degrees Fahrenheit by the American Society of Testing and Material (ASTM) Method D56, a gravity range from 40 to 46 degrees API, and a burning point in the range of 150 to 175 degrees Fahrenheit. It is a clean-burning product suitable for use as an illuminant when burned in wick lamps. Includes grades of kerosene called range oil having properties similar to Number 1 fuel oil, but with a gravity of about 43 degrees API and an end point of 625 degrees Fahrenheit. Used in space heaters, cooking stoves, and water heaters.

Kerosene-type jet fuel - See Jet fuel.

Large car - See Car size classifications.

Lease Condensate - A liquid recovered from natural gas at the well or at small gas/oil separators in the field. Consists primarily of pentanes and heavier hydrocarbons (also called field condensate).

Light duty vehicles - Cars and light trucks combined.

Light truck - Unless otherwise noted, light trucks are defined in this publication as two-axle, four-tire trucks. The U.S. Bureau of Census classifies all trucks with a gross vehicle weight less than 10,000 pounds as light trucks (See Truck size classifications).

Light-heavy truck - See Truck size classifications.

Light rail - Mode of transit service (also called streetcar, tramway or trolley) operating passenger rail cars singly (or in short, usually two-car or three-car trains) on fixed rails in right-of-way that is often separated from other traffic for part or much of the way.

Liquified petroleum gas (lpg) - Consists of propane and butane and is usually derived from natural gas. In locations where there is no natural gas and the gasoline consumption is low, naphtha is converted to $\operatorname{lpg}$ by catalytic reforming.

Load factor - Total passenger miles divided by total vehicle miles.

Low emission vehicle - Any vehicle certified to the low emission standards which are set by the Federal government and/or the state of California.

M85 - 85\% methanol and 15\% gasoline.

M100 - 100\% methanol.

Medium truck - See Truck size classifications.

Methanol $\left(\mathrm{CH}_{3} \mathrm{OH}\right)$ - A colorless highly toxic liquid with essentially no odor and very little taste. It is the simplest alcohol and boils at 64.7 degrees Celsius. In transportation, methanol is used as a vehicle fuel by itself (M100), or blended with gasoline (M85).

Midsize car - See Car size classifications. 
Minicompact car - See Car size classifications.

Model year - In this publication, model year is referring to the "sales" model year, the period from October 1 to the next September 31.

Motor bus - See Bus.

Motor gasoline - A mixture of volatile hydrocarbons suitable for operation of an internal combustion engine whose major components are hydrocarbons with boiling points ranging from 78 to 217 degrees centigrade and whose source is distillation of petroleum and cracking, polymerization, and other chemical reactions by which the naturally occurring petroleum hydrocarbons are converted into those that have superior fuel properties.

Regular gasoline: Gasoline having an antiknock index, i.e., octane rating, greater than or equal to 85 and less than 88 . Note: Octane requirements may vary by altitude.

Midgrade gasoline: Gasoline having an antiknock index, i.e., octane rating, greater than or equal to 88 and less than or equal to 90 . Note: Octane requirements may vary by altitude.

Premium gasoline: Gasoline having an antiknock index, i.e., octane rating, greater than 90 . Note: Octane requirements may vary by altitude.

Reformulated gasoline: Finished motor gasoline formulated for use in motor vehicles, the composition and properties of which meet the requirements of the reformulated gasoline regulations promulgated by the U.S. Environmental Protection Agency under Section 211(k) of the Clean Air Act. For more details on this clean fuel program see http://www.epa.gov/otaq/fuels/gasolinefuels/rfg/index.htm. Note: This category includes oxygenated fuels program reformulated gasoline (OPRG) but excludes reformulated gasoline blendstock for oxygenate blending (RBOB).

MTBE - Methyl Tertiary Butyl Ether-a colorless, flammable, liquid oxygenated hydrocarbon containing 18.15 percent oxygen.

Naphtha-type jet fuel - See Jet fuel.

National income - See Income.

Nationwide Personal Transportation Survey (NPTS) - A nationwide survey of households that provides information on the characteristics and personal travel patterns of the U.S. population. Surveys were conducted in 1969, 1977, 1983, 1990, and 1995 by the U.S. Bureau of Census for the U.S. Department of Transportation.

Natural gas - A mixture of hydrocarbon compounds and small quantities of various non-hydrocarbons existing in the gaseous phase or in solution with crude oil in natural underground reservoirs at reservoir conditions.

Natural gas, dry: Natural gas which remains after: 1) the liquefiable hydrocarbon portion has been removed from the gas stream; and 2) any volumes of nonhydrocarbon gases have been removed where they occur in sufficient quantity to render the gas unmarketable. Dry natural gas is also known as consumer-grade natural gas. The parameters for measurement are cubic feet at 60 degrees Fahrenheit and 14.73 pounds per square inch absolute. 
Natural gas, wet: The volume of natural gas remaining after removal of lease condensate in lease and/or field separation facilities, if any, and after exclusion of nonhydrocarbon gases where they occur in sufficient quantity to render the gas unmarketable. Natural gas liquids may be recovered from volumes of natural gas, wet after lease separation, at natural gas processing plants.

Natural gas plant liquids: Natural gas liquids recovered from natural gas in processing plants and from natural gas field facilities and fractionators. Products obtained include ethane, propane, normal butane, isobutane, pentanes plus, and other products from natural gas processing plants.

Nitrogen oxides $\left(\mathbf{N O}_{\mathbf{x}}\right)$ - A product of combustion of fossil fuels whose production increases with the temperature of the process. It can become an air pollutant if concentrations are excessive.

Nonattainment area - Any area that does not meet the national primary or secondary ambient air quality standard established by the Environmental Protection Agency for designated pollutants, such as carbon monoxide and ozone.

Oil Stocks - Oil stocks include crude oil (including strategic reserves), unfinished oils, natural gas plant liquids, and refined petroleum products.

\section{Operating cost -}

Fixed operating cost: In reference to passenger car operating cost, refers to those expenditures that are independent of the amount of use of the car, such as insurance costs, fees for license and registration, depreciation and finance charges.

Variable operating cost: In reference to passenger car operating cost, expenditures which are dependent on the amount of use of the car, such as the cost of gas and oil, tires, and other maintenance.

Organization for Economic Cooperation and Development (OECD) - Consists of Australia, Austria, Belgium, Canada, Czech Republic, Denmark, Finland, France, Germany, Greece, Hungary, Iceland, Ireland, Italy, Japan, Luxembourg, Mexico, Netherlands, New Zealand, Norway, Poland, Portugal, South Korea, Spain, Sweden, Switzerland, Turkey, United Kingdom, and United States. Total OECD includes the United States Territories (Guam, Puerto Rico, and the U.S. Virgin Islands). Total OECD excludes data for Czech Republic, Hungary, Mexico, Poland, and South Korea which are not yet available.

OECD Europe: Consists of Austria, Belgium, Czech Republic, Denmark, Finland, France, Germany, Greece, Hungary, Iceland, Ireland, Italy, Luxembourg, Netherlands, Norway, Poland, Portugal, Spain, Sweden, Switzerland, Turkey, and United Kingdom. OECD Europe excludes data for Czech Republic, Hungary, and Poland which are not yet available.

OECD Pacific: Consists of Australia, Japan, and New Zealand.

Organization for Petroleum Exporting Countries (OPEC) - Includes Saudi Arabia, Iran, Venezuela, Libya, Indonesia, United Arab Emirates, Algeria, Nigeria, Ecuador, Gabon, Iraq, Kuwait, and Qatar. Data for Saudi Arabia and Kuwait include their shares from the Partitioned Zone (formerly the Neutral Zone). Angola joined OPEC in December 2006, thus, beginning in 2007, data on OPEC will include Angola.

Arab OPEC - Consists of Algeria, Iraq, Kuwait, Libya, Qatar, Saudi Arabia and the United Arab Emirates.

Other single-unit truck - See Single-unit truck. 
Oxygenate - A substance which, when added to gasoline, increases the amount of oxygen in that gasoline blend. Includes fuel ethanol, methanol, and methyl tertiary butyl ether (MTBE).

Paratransit - Mode of transit service (also called demand response or dial-a-ride) characterized by the use of passenger cars, vans or small buses operating in response to calls from passengers or their agents to the transit operator, who then dispatches a vehicle to pick up the passengers and transport them to their destinations.

Particulates - Carbon particles formed by partial oxidation and reduction of the hydrocarbon fuel. Also included are trace quantities of metal oxides and nitrides, originating from engine wear, component degradation, and inorganic fuel additives. In the transportation sector, particulates are emitted mainly from diesel engines.

Passenger-miles traveled (PMT) - One person traveling the distance of one mile. Total passenger-miles traveled, thus, give the total mileage traveled by all persons.

Passenger rail - See Rail, "Amtrak" and "Transit Railroad".

Persian Gulf countries - Consists of Bahrain, Iran, Iraq, Kuwait, Qatar, Saudi Arabia, and the United Emirates.

Personal Consumption Expenditures (PCE) - As used in the national accounts, the market value of purchases of goods and services by individuals and nonprofit institutions and the value of food, clothing, housing, and financial services received by them as income in kind. It includes the rental value of owner-occupied houses but excludes purchases of dwellings, which are classified as capital goods (investment).

Personal income - See Income.

Petroleum - A generic term applied to oil and oil products in all forms, such as crude oil, lease condensate, unfinished oil, refined petroleum products, natural gas plant liquids, and nonhydrocarbon compounds blended into finished petroleum products.

Petroleum consumption: A calculated demand for petroleum products obtained by summing domestic production, imports of crude petroleum and natural gas liquids, imports of petroleum products, and the primary stocks at the beginning of the period and then subtracting the exports and the primary stocks at the end of the period.

Petroleum exports: Shipments of petroleum products from the 50 States and the District of Columbia to foreign countries, Puerto Rico, the Virgin Islands, and other U.S. possessions and territories.

Petroleum imports: All imports of crude petroleum, natural gas liquids, and petroleum products from foreign countries and receipts from Guam, Puerto Rico, the Virgin Islands, and the Hawaiian Trade Zone. The commodities included are crude oil, unfinished oils, plant condensate, and refined petroleum products.

Petroleum inventories: The amounts of crude oil, unfinished oil, petroleum products, and natural gas liquids held at refineries, at natural gas processing plants, in pipelines, at bulk terminals operated by refining and pipeline companies, and at independent bulk terminals. Crude oil held in storage on leases is also included; these stocks are known as primary stocks. 
Secondary stocks-those held by jobbers dealers, service station operators, and consumers-are excluded. Prior to 1975, stock held at independent bulk terminals were classified as secondary stocks.

Petroleum products supplied: For each petroleum product, the amount supplied is calculated by summing production, crude oil burned directly, imports, and net withdrawals from primary stocks and subtracting exports.

Plug-in hybrid-electric vehicles (PHEVs) - Hybrid-electric vehicles with high capacity batteries that can be charged by plugging them into an electrical outlet or charging station. There are two basic PHEV configurations:

Parallel or Blended PHEV: Both the engine and electric motor are mechanically connected to the wheels, and both propel the vehicle under most driving conditions. Electric-only operation usually occurs only at low speeds.

Series PHEVs, also called Extended Range Electric Vehicles (EREVs): Only the electric motor turns the wheels; the gasoline engine is only used to generate electricity. Series PHEVs can run solely on electricity until the battery needs to be recharged. The gasoline engine will then generate the electricity needed to power the electric motor. For shorter trips, these vehicles might use no gasoline at all.

Processing Gain - The amount by which the total volume of refinery output is greater than the volume of input for given period of time. The processing gain arises when crude oil and other hydrocarbons are processed into products that are, on average, less dense than the input.

Processing Loss - The amount by which the total volume of refinery output is less than the volume of input for given period of time. The processing loss arises when crude oil and other hydrocarbons are processed into products that are, on average, more dense than the input.

Proved Reserves of Crude Oil - The estimated quantities of all liquids defined as crude oil, which geological and engineering data demonstrate with reasonable certainty to be recoverable in future years from known reservoirs under existing economic and operating conditions.

Quad - Quadrillion, $10^{15}$. In this publication, a Quad refers to Quadrillion Btu.

Rail -

Amtrak (American Railroad Tracks): Operated by the National Railroad Passenger Corporation of Washington, DC. This rail system was created by President Nixon in 1970, and was given the responsibility for the operation of intercity, as distinct from suburban, passenger trains between points designated by the Secretary of Transportation.

Class I freight railroad: Defined by the Interstate Commerce Commission each year based on annual operating revenue. A railroad is dropped from the Class I list if it fails to meet the annual earnings threshold for three consecutive years.

Commuter railroad: Those portions of mainline railroad (not electric railway) transportation operations which encompass urban passenger train service for local travel between a central city and adjacent suburbs. Commuter railroad service-using both locomotive-hauled and selfpropelled railroad passenger cars-is characterized by multi-trip tickets, specific station-to-station fares, and usually only one or two stations in the central business district. Also known as suburban railroad. 
Transit railroad: Includes "heavy" and "light" transit rail. Heavy transit rail is characterized by exclusive rights-of-way, multi-car trains, high speed rapid acceleration, sophisticated signaling, and high platform loading. Also known as subway, elevated railway, or metropolitan railway (metro). Light transit rail may be on exclusive or shared rights-of-way, high or low platform loading, multi-car trains or single cars, automated or manually operated. In generic usage, light rail includes streetcars, trolley cars, and tramways.

Refiner sales price - Sales from the refinery made directly to ultimate consumers, including bulk consumers (such as agriculture, industry, and electric utilities) and residential and commercial consumers.

Reformulated gasoline (RFG) - See Motor gasoline.

RFG area - An ozone nonattainment area designated by the Environmental Protection Agency which requires the use of reformulated gasoline.

Residential sector - An energy consuming sector that consists of living quarters for private households. Excludes institutional living quarters.

Residential Transportation Energy Consumption Survey (RTECS) - This survey was designed by the Energy Information Administration of the Department of Energy to provide information on how energy is used by households for personal vehicles. It has been conducted five times since 1979, the most recent being 1991.

Residual fuel oil - The heavier oils that remain after the distillate fuel oils and lighter hydrocarbons are boiled off in refinery operations. Included are products know as ASTM grade numbers 5 and 6 oil, heavy diesel oil, Navy Special Fuel Oil, Bunker C oil, and acid sludge and pitch used as refinery fuels. Residual fuel oil is used for the production of electric power, for heating, and for various industrial purposes.

Rural - Usually refers to areas with population less than 5,000.

Sales period - October 1 of the previous year to September 30 of the given year. Approximately the same as a model year.

Sales-weighted miles per gallon (mpg) - Calculation of a composite vehicle fuel economy based on the distribution of vehicle sales.

Scrappage rate - As applied to motor vehicles, it is usually expressed as the percentage of vehicles of a certain type in a given age class that are retired from use (lacking registration) in a given year.

School and other nonrevenue bus - See Bus.

Sector - A group of major energy-consuming components of U.S. society developed to measure and analyze energy use. The sectors most commonly referred to are: residential, commercial, industrial, transportation, and electric power.

Single-unit truck - Includes two-axle, four-tire trucks and other single-unit trucks.

Two-axle, four-tire truck: A motor vehicle consisting primarily of a single motorized device with two axles and four tires. 
Other single-unit truck: A motor vehicle consisting primarily of a single motorized device with more than two axles or more than four tires.

Spark ignition engine - An internal combustion engine in which the charge is ignited electrically (e.g., with a spark plug).

Special fuels - Consist primarily of diesel fuel with small amount of liquified petroleum gas, as defined by the Federal Highway Administration.

Specific acceleration power - Measured in watts per kilogram. Acceleration power divided by the battery system weight. Weight must include the total battery system.

Specific energy - Measured in watt hours per kilogram. The rated energy capacity of the battery divided by the total battery system weight.

Subcompact car - See Car size classifications.

Supplemental air carrier - See Air carrier.

Survival rate - As applied to motor vehicles, it is usually expressed as the percentage of vehicles of a certain type in a given age class that will be in use at the end of a given year.

Tax incentives - In general, a means of employing the tax code to stimulate investment in or development of a socially desirable economic objective without direct expenditure from the budget of a given unit of government. Such incentives can take the form of tax exemptions or credits.

Test weight - The weight setting at which a vehicle is tested on a dynomometer by the U.S. Environmental Protection Agency (EPA). This weight is determined by the EPA using the inertia weight of the vehicle.

Ton-mile - The movement of one ton of freight the distance of one mile. Ton-miles are computed by multiplying the weight in tons of each shipment transported by the distance hauled.

Transmission types -

A3 - Automatic three speed

A4 - Automatic four speed

A5 - Automatic five speed

L4 - Automatic lockup four speed

M5 - Manual five speed

Transit bus - See Bus.

Transit railroad - See Rail.

Transportation sector - Consists of both private and public passenger and freight transportation, as well as government transportation, including military operations.

Truck Inventory and Use Survey (TIUS) - Survey designed to collect data on the characteristics and operational use of the nation's truck population. It is conducted every five years by the U.S. Bureau of the Census. Surveys were conducted in 1963, 1967, 1972, 1977, 1982, 1987, and 1992. For the 1997 survey, it was renamed the Vehicle Inventory and Use Survey in anticipation 
of including additional vehicle types. However, no additional vehicle types were added to the 1997 survey.

Trolleybus - Mode of transit service (also called transit coach) using vehicles propelled by a motor drawing current from overhead wires via connecting poles called a trolley pole, from a central power source not onboard the vehicle.

Truck size classifications - U.S. Bureau of the Census has categorized trucks by gross vehicle weight (gvw) as follows:

Light - Less than 10,000 pounds gvw (Also see Light Truck.)

Medium - 10,001 to 20,000 pounds gvw

Light-heavy - 20,001 to 26,000 pounds gvw

Heavy-heavy - 26,001 pounds gvw or more.

Two-axle, four-tire truck - See Single-unit truck.

Two seater car - See Car size classifications.

Ultra-low emission vehicle - Any vehicle certified to the ultra-low emission standards which are set by the Federal government and/or the state of California.

Urban - Usually refers to areas with population of 5,000 or greater.

Vanpool: A ridesharing prearrangement using vans or small buses providing round-trip transportation between the participants's prearranged boarding points and a common and regular destination.

Variable operating cost - See Operating cost.

Vehicle Inventory and Use Survey - See Truck Inventory and Use Survey.

Vehicle-miles traveled (vmt) - One vehicle traveling the distance of one mile. Total vehicle miles, thus, is the total mileage traveled by all vehicles.

Volatile organic compounds (VOCs) - Organic compounds that participate in atmospheric photochemical reactions.

\section{Waterborne Commerce -}

Coastwise: Domestic traffic receiving a carriage over the ocean, or the Gulf of Mexico. Traffic between Great Lakes ports and seacoast ports, when having a carriage over the ocean, is also termed Coastwise.

Domestic: Includes coastwise, lakewise, and internal waterborne movements.

Foreign: Waterborne import, export, and in-transit traffic between the United States, Puerto Rico and the Virgin Islands and any foreign country.

Internal: Vessel movements (origin and destination) which take place solely on inland waterways. An inland waterway is one geographically located within the boundaries of the contiguous 48 states or within the boundaries of the State of Alaska. 
Lakewise: Waterborne traffic between the United States ports on the Great Lakes System. The Great Lakes System is treated as a separate waterway system rather than as a part of the inland waterway system. In comparing historical data for the Great Lakes System, one should note that prior to calendar year 1990, marine products, sand and gravel being moved from the Great Lakes to Great Lake destinations were classified as local traffic. From 1990-on, these activities are classified as lakewise traffic.

Well-to-wheel - A life cycle analysis used in transportation to consider the entire energy cycle for a given mode, rather than just tailpipe emissions. The analysis starts at the oil well and ends with the turning wheels of the vehicle.

Zero-emission vehicle - Any vehicle certified to the zero emission standards which are set by the Federal government and/or the state of California. These standards apply to the vehicle emissions only. 


\section{TITLE INDEX}


Acquisitions

Federal Fleet Vehicle Acquisitions by Fuel Type, FY 2002- 2012 …....................................... 7-6

Advanced

Alternative Fuel and Advanced Technology Vehicles and Characteristics ................................. 6-1

Federal and State Advanced Technology Incentives, 2014 ................................................ 10-13

Age

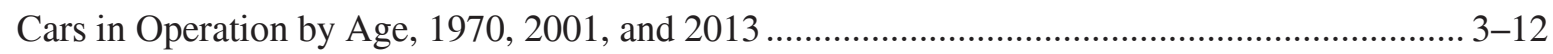

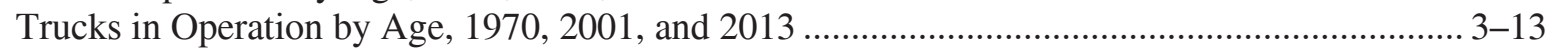

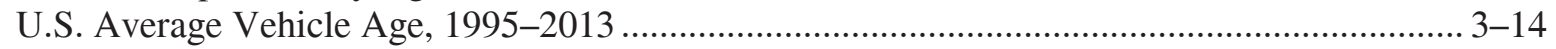

Share of Vehicles by Annual Miles of Travel and Vehicle Age, 2009 NHTS........................... 8-16

Daily and Annual Vehicle Miles of Travel and Average Age for Each Vehicle in a

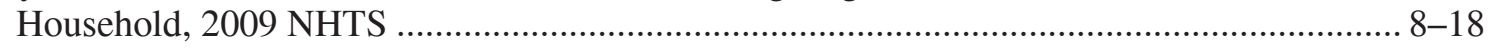

Agency

Federal Government Vehicles by Agency, FY 2012 …............................................................ 7-7

Air

Air Conditioning (SC03) Driving Cycle ….................................................................................. 4-33

Summary Statistics for U.S. Domestic and International Certificated Route Air Carriers

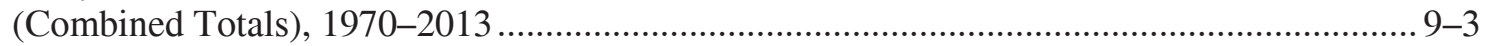

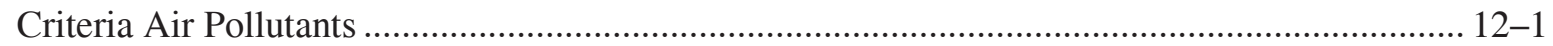

Total National Emissions of the Criteria Air Pollutants by Sector, 2013 .................................. 12-2

Aircraft

Aircraft - Exhaust Emission Standards ............................................................................ 12-24

All

United States Petroleum Production and Consumption - All Sectors, 1973-2040 .................... 1-18

All Highway Vehicles Characteristics .................................................................................. 3-1

Alternative

Alternative Fuel and Oxygenate Consumption, 2005-2011 …................................................ 2-5

Alternative Fuel and Advanced Technology Vehicles and Characteristics ................................ 6-1

Estimates of Alternative Fuel Highway Vehicles in Use, 1995-2011 .......................................... 6-3

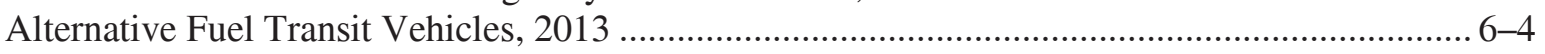

Non-E85 Alternative Fuel Vehicles Available by Manufacturer, Model Year 2014 ..................... 6-7

Number of Alternative Refuel Sites by State and Fuel Type, 2014 …..................................... 6-13

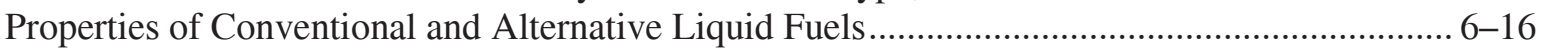

Properties of Conventional and Alternative Gaseous Fuels .................................................. 6-17

Federal and State Alternative Fuel Incentives, 2014 .......................................................... 10-12

Amtrak

Summary Statistics for the National Railroad Passenger Corporation (Amtrak),

$1971-2012$ $9-11$

Annual

Average Annual Vehicle-Miles of Travel for Commercial Fleet Vehicles, 2012 ....................... 7-3

Average Annual Expenditures of Households by Income, 2012 .................................................. 8-4

Annual Household Expenditures for Transportation, 1985-2012 …........................................... 8-5

Average Annual Vehicle-Miles, Vehicle Trips and Trip Length per Household 1969, 1977,

1983, 1990, 1995 NPTS and 2001, 2009 NHTS .... $8-8$ 
Annual (continued)

Average Annual Miles per Household Vehicle by Vehicle Age............................................... 8-13

Self-Reported vs. Odometer Average Annual Miles, 1995 NPTS and 2001 NHTS................... 8-14

Share of Vehicles by Annual Miles of Travel and Vehicle Age, 2009 NHTS ............................ 8-16

Daily and Annual Vehicle Miles of Travel and Average Age for Each Vehicle in a

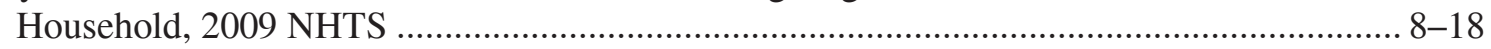

Annual Vehicle Miles of Travel for Each Vehicle in a Household, 2009 NHTS ....................... 8-19

Production-Weighted Annual Carbon Footprint of New Domestic and Import Cars,

Model Years 1975-2013 .................................................................................................. 11-13

Production-Weighted Annual Carbon Footprint of New Domestic and Import Light

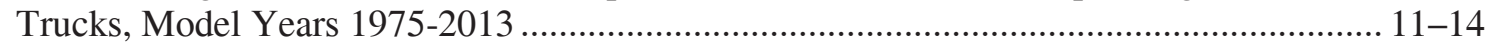

Average Annual Carbon Footprint by Vehicle Classification, 1975 and 2013 .......................... 11-15

Attributes

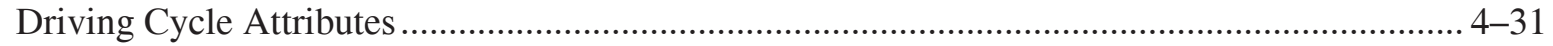

Comparison of U.S., European, and Japanese Driving Cycles Attributes .................................. 4-36

Available

E85 Flex-Fuel Vehicles Available by Manufacturer, Model Year 2014....................................... 6-5

Non-E85 Alternative Fuel Vehicles Available by Manufacturer, Model Year 2014 .................... 6-7

Electric Drive Vehicles Available by Manufacturer, Model Year 2014 ..................................... 6-10

Electric Drive Medium/Heavy Trucks and Buses Available by Manufacturer, 2014 .................. 6-12

Average

U.S. Average Vehicle Age, 1995-2013 ....................................................................................... 3-14

Average Material Consumption for a Domestic Light Vehicle, Model Years 1995, 2000,

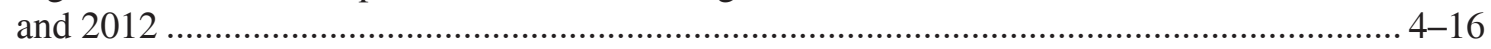

Car Corporate Average Fuel Economy (CAFE) Standards versus Sales-Weighted Fuel Economy Estimates, 1978-2013 ....................................................................................... 4-21

Light Truck Corporate Average Fuel Economy (CAFE) Standards versus Sales-Weighted Fuel Economy Estimates, 1978-2013 ....................................................................... 4-22

Corporate Average Fuel Economy (CAFE) Fines Collected, 1983-2012 ................................. 4-23

Average Miles per Shipment in the United States: Comparison of the 1997, 2002, 2007,

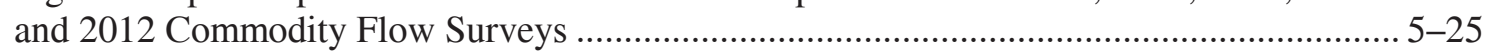

Average Annual Vehicle-Miles of Travel for Commercial Fleet Vehicles, 2012 2...................... 7-3

Average Length of Time Commercial Fleet Vehicles are in Service, 2012 ................................ 7-3

Average Miles per Domestic Federal Vehicle by Vehicle Type, 2012 ..................................... 7-4

Average Annual Expenditures of Households by Income, 2012 ............................................... 8-4

Average Annual Vehicle-Miles, Vehicle Trips and Trip Length per Household 1969, 1977,

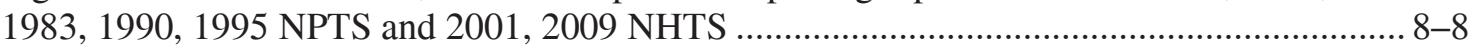

Average Number of Vehicles and Vehicle Travel per Household, 1990 NPTS and 2001

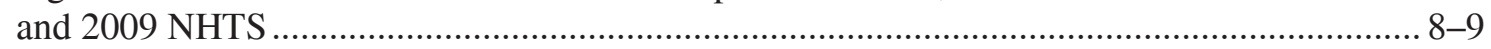

Average Vehicle Occupancy by Vehicle Type, 1995 NPTS and 2009 NHTS .......................... 8-11

Average Vehicle Occupancy by Trip Purpose, 1977 NPTS and 2009 NHTS ........................... 8-12

Average Annual Miles per Household Vehicle by Vehicle Age ................................................. 8-13

Self-Reported vs. Odometer Average Annual Miles, 1995 NPTS and 2001 NHTS................... 8-14

Average Daily Miles Driven (per Driver), 2009 NHTS ............................................................. 8-17

Daily and Annual Vehicle Miles of Travel and Average Age for Each Vehicle in a

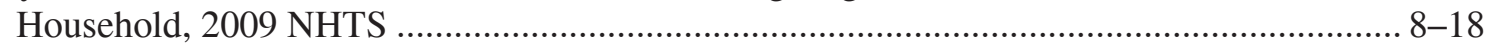

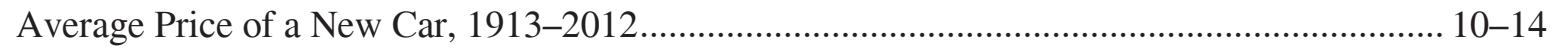

Average Price of a New Car (Domestic and Import), 1970-2012 …...................................... 10-15

Average Annual Carbon Footprint by Vehicle Classification, 1975 and 2013 .......................... 11-15 
Aviation

Summary Statistics for General Aviation, 1970-2012 _........................................................ 9-4

Refiner Sales Prices for Aviation Gasoline and Jet Fuel, 1978-2013..................................... 10-10

Barrel

U.S. Refinery Yield of Petroleum Products from a Barrel of Crude Oil, 1978-2013................. 1-15

Prices for a Barrel of Crude Oil and a Gallon of Gasoline, 1978-2013 ..................................... 10-7

Bicycle

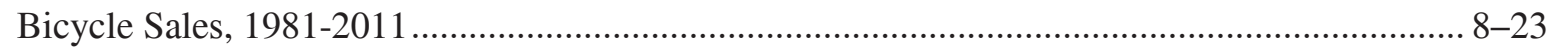

Bike

Walk and Bike Trips by Trip Purpose, 2009 NHTS .............................................................. 8-24

Boat

Recreational Boat Energy Use, 1970-2012 ….......................................................................... 9-7

Bus

Truck and Bus Registrations for Selected Countries, 1960-2012 ............................................ 3-4

Buses

Summary Statistics on Transit Buses and Trolleybuses, 1994-2012 _......................................... 5-26

\section{CAFE}

Car Corporate Average Fuel Economy (CAFE) Standards versus Sales-Weighted Fuel

Economy Estimates, 1978-2013 ....................................................................................... 4-21

Light Truck Corporate Average Fuel Economy (CAFE) Standards versus Sales-Weighted

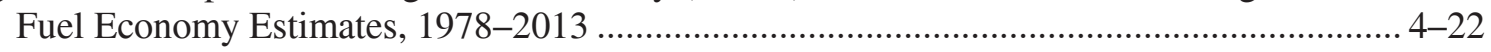

Corporate Average Fuel Economy (CAFE) Fines Collected, 1983-2012 …............................. 4-23

California

California Car, Light Truck and Medium Truck Emission Certification Standards .................. 12-23

Car

Car Registrations for Selected Countries, 1960-2012 ........................................................... 3-3

Car Scrappage and Survival Rates, 1970, 1980 and 1990 Model Years .................................... 3-16

New Retail Car Sales in the United States, 1970-2013 .......................................................... 4-5

Definition of Car Sport Utility Vehicles in Model Year 2013 ................................................... 4-8

Car Corporate Average Fuel Economy (CAFE) Standards versus Sales-Weighted Fuel

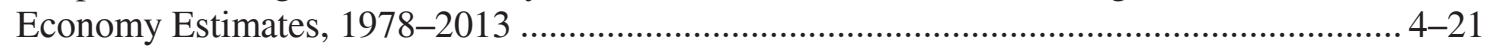

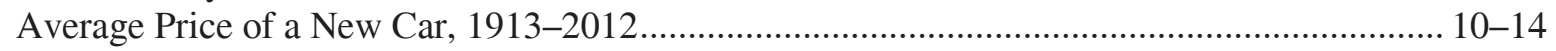

Average Price of a New Car (Domestic and Import), 1970-2012 .......................................... 10-15

Car Operating Cost per Mile, 1985-2013 ........................................................................ 10-16

Fixed Car Operating Costs per Year, 1975-2013 ................................................................. 10-17

California Car, Light Truck and Medium Truck Emission Certification Standards .................. 12-23

Carbon

U.S. Carbon Emissions from Fossil Fuel Consumption by End-Use Sector, 1990-2012............ 11-6

U.S. Carbon Emissions from Fossil Fuel Combustion in the Transportation End-Use

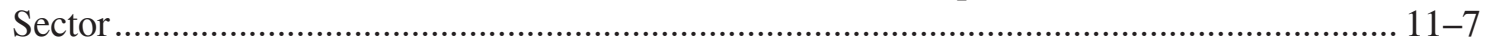

Production-Weighted Annual Carbon Footprint of New Domestic and Import Cars,

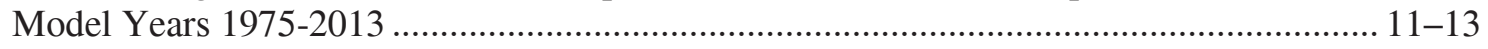


Carbon (continued)

Production-Weighted Annual Carbon Footprint of New Domestic and Import Light

Trucks, Model Years 1975-2013 …........................................................................................11-14

Average Annual Carbon Footprint by Vehicle Classification, 1975 and 2013 ......................... 11-15

Carbon Dioxide

Fuel Economy and Carbon Dioxide Emissions Standards, MY 2012-2025.............................. 4-19

Fuel Economy and Carbon Dioxide Targets for Model Year 2025 ......................................... 4-20

Numerical Estimates of Global Warming Potentials Compared with Carbon Dioxide ................. 11-3

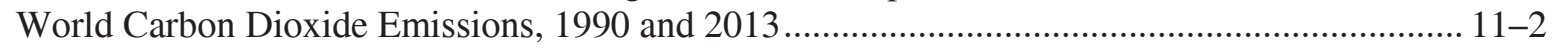

Direct Carbon Dioxide Emissions from a Gallon of Fuel ...................................................... 11-16

Carbon Monoxide

Total National Emissions of Carbon Monoxide, 1970-2013 ...................................................... 12-3

Emissions of Carbon Monoxide from Highway Vehicles, 1970-2005 ......................................... 12-4

Carriers

Summary Statistics for U.S. Domestic and International Certificated Route Air Carriers

(Combined Totals), 1970-2013 ............................................................................................... 9-3

Cars

World Production of Cars and Trucks, 2000-2012 …................................................................ 3-2

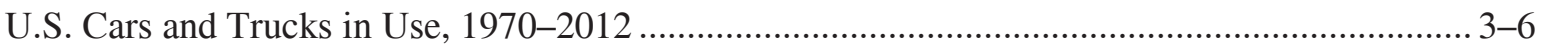

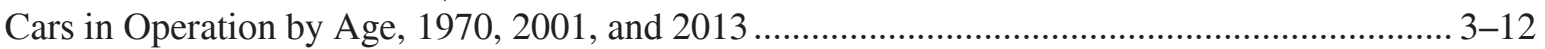

Summary Statistics for Cars, 1970-2012 .................................................................................... 4-2

Production Sales, Production Shares, and Sales-Weighted Fuel Economies of New

Domestic and Import Cars, Selected Model Years 1975-2013 .............................................. 4-7

Production and Production-Weighted Fuel Economies of New Domestic and Import Cars, Light Trucks, and Light Vehicles, Model Years 1975-2013 .............................................. 4-10

Production-Weighted Engine Size of New Domestic and Import Cars, Model Years

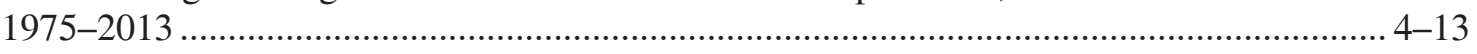

Production-Weighted Curb Weight of New Domestic and Import Cars, Model Years

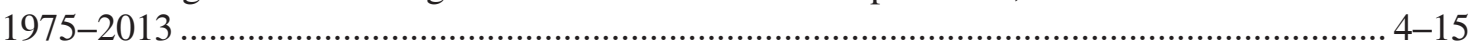

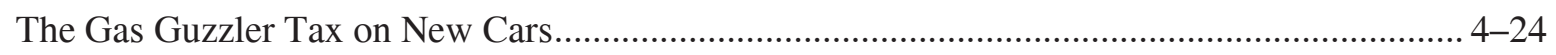

List of Model Year 2013 Cars with Gas Guzzler Taxes ............................................................ 4-25

Production-Weighted Annual Carbon Footprint of New Domestic and Import Cars,

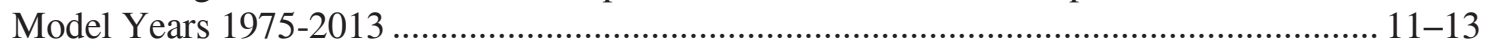

Census

Household Vehicle Ownership, 1960-2012 Census ............................................................... 8-6

Certificated

Summary Statistics for U.S. Domestic and International Certificated Route Air Carriers

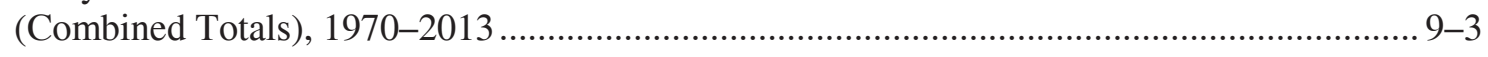

Certification

California Car, Light Truck and Medium Truck Emission Certification Standards $12-23$

Characteristics

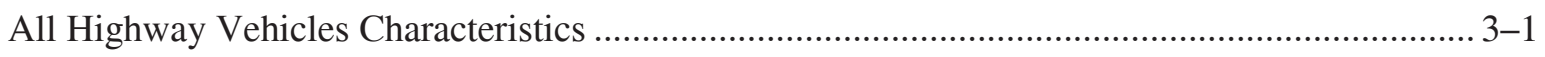

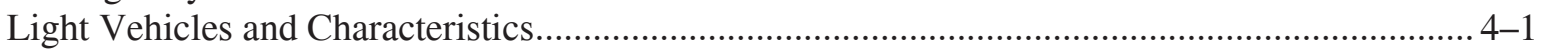

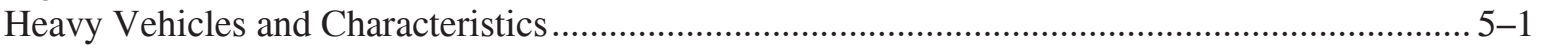

Alternative Fuel and Advanced Technology Vehicles and Characteristics .................................. 6-1 
Characteristics (continued)

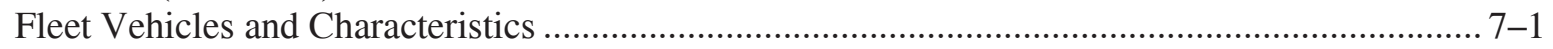

Household Vehicles and Characteristics ............................................................................ 8-1

Characteristics of U.S. Daily per Vehicle Driving vs. Dwelling Unit Type and Density ............ 8-21

Housing Unit Characteristics, 2010 ........................................................................................ 8-21

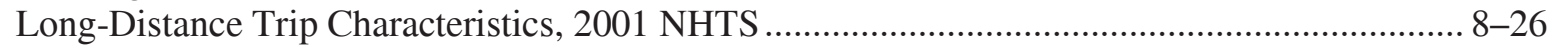

Cities

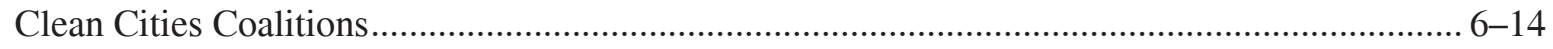

City

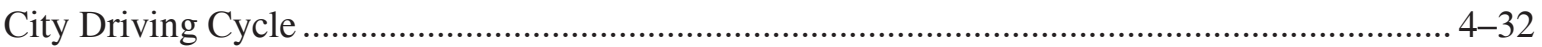

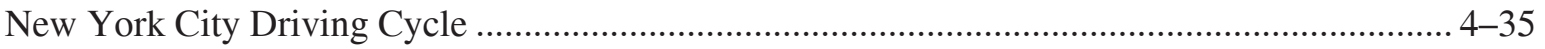

Class

Truck Harmonic Mean Fuel Economy by Size Class, 1992, 1997, and 2002............................. 5-6

Truck Statistics by Gross Vehicle Weight Class, 2002 ................................................................ 5-6

Class 1

Sales Estimates of Class 1, Class 2a, and Class 2b Light Trucks, 1989-1999 ............................. 4-4

Summary Statistics on Class 1, Class 2a, and Class 2b Light Trucks......................................... 4-4

Class 2a

Sales Estimates of Class 1, Class 2a, and Class 2b Light Trucks, 1989-1999 .............................. 4-4

Summary Statistics on Class 1, Class 2a, and Class 2b Light Trucks.......................................... 4-4

Class $2 b$

Sales Estimates of Class 1, Class 2a, and Class 2b Light Trucks, 1989-1999 .............................. 4-4

Summary Statistics on Class 1, Class 2a, and Class 2b Light Trucks.......................................... 4-4

Class 3-8

Summary Statistics for Class 3-8 Single-Unit Trucks, 1970-2012 .......................................... 5-2

Class 7-8

Summary Statistics for Class 7-8 Combination Trucks, 1970-2012 …..................................... 5-3

Class 8

Effect of Terrain on Class 8 Truck Fuel Economy ................................................................. 5-14

Fuel Economy for Class 8 Trucks as Function of Speed and Tractor-Trailer Tire

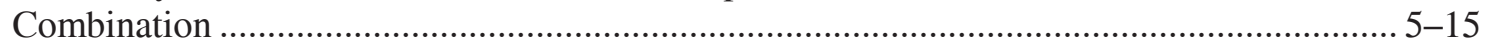

Class 8 Truck Fuel Economy as a Function of Speed and Tractor-Trailer Tire Combination and Percentage of Total Distance Traveled as a Function of Speed..................................... 5-16

Class 8 Truck Percent of Total Fuel Consumed as a Function of Speed and Tractor-Trailer

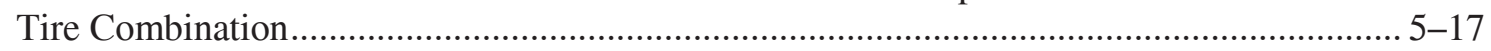

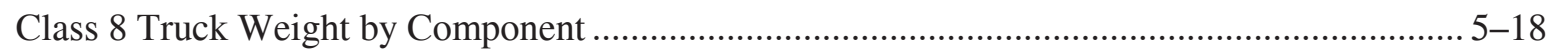

Distribution of Class 8 Trucks by On-Road Vehicle Weight, 2008 ......................................... 5-20

Class I

Class I Railroad Freight Systems in the United States Ranked by Revenue Ton-Miles, 2012 ...... 9-8

Summary Statistics for Class I Freight Railroads, 1970-2012 ............................................... 9-9

Classification

Average Annual Carbon Footprint by Vehicle Classification, 1975 and 2013 _......................... 11-15 
Clean

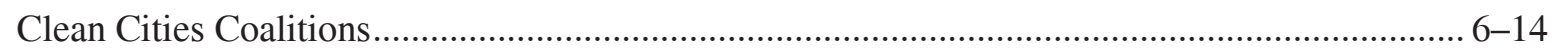

Coalitions

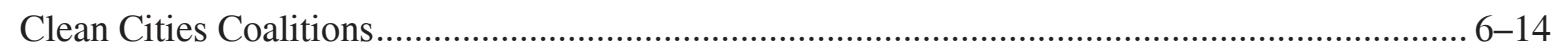

Cold

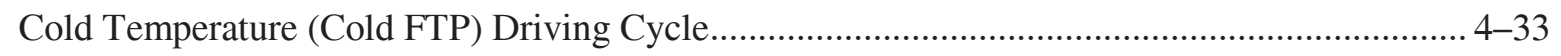

Collected

Corporate Average Fuel Economy (CAFE) Fines Collected, 1983-2012 ................................. 4-23

Combination

Summary Statistics for Class 7-8 Combination Trucks, 1970-2012 …...................................... 5-3

Fuel Economy for Class 8 Trucks as Function of Speed and Tractor-Trailer Tire

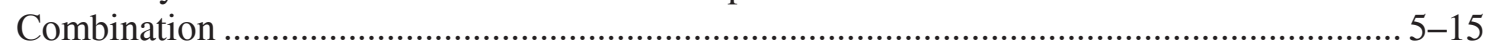

Class 8 Truck Fuel Economy as a Function of Speed and Tractor-Trailer Tire Combination and Percentage of Total Distance Traveled as a Function of Speed......................................5-16

Class 8 Truck Percent of Total Fuel Consumed as a Function of Speed and Tractor-Trailer

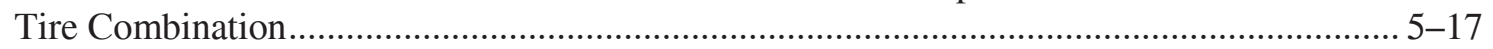

Combined

Summary Statistics for U.S. Domestic and International Certificated Route Air Carriers (Combined Totals), 1970-2013...

Combustion

U.S. Carbon Emissions from Fossil Fuel Combustion in the Transportation End-Use Sector $11-7$

Commerce

Tonnage Statistics for Domestic and International Waterborne Commerce, 1970-2012 ............. 9-5

Summary Statistics for Domestic Waterborne Commerce, 1970-2012 ....................................... 9-6

Commercial

Average Annual Vehicle-Miles of Travel for Commercial Fleet Vehicles, 2012 2....................... 7-3

Average Length of Time Commercial Fleet Vehicles are in Service, 2012 ................................. 7-3

Commodity

Value of Goods in the United States: Comparison of the 1997, 2002, 2007, and 2012

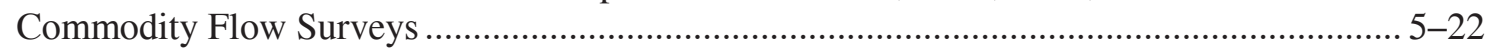

Tons of Freight in the United States: Comparison of the 1997, 2002, 2007, and 2012

Commodity Flow Surveys .......................................................................................... 5-23

Growth of Ton Miles in the United States: Comparison of the 1997, 2002, 2007, and 2012

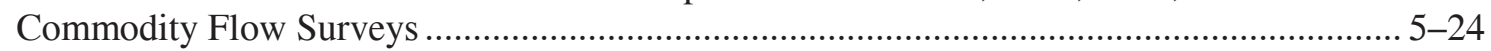

Average Miles per Shipment in the United States: Comparison of the 1997, 2002, 2007,

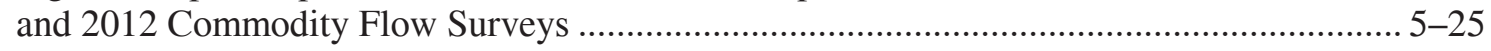

Commute

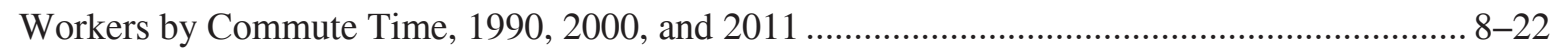

Commuter

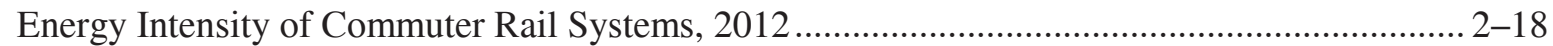

Summary Statistics for Commuter Rail Operations, 1984-2012 ….......................................... 9-12 
Compared

Vehicles per Thousand People: U.S. (Over Time) Compared to Other Countries (in 2002

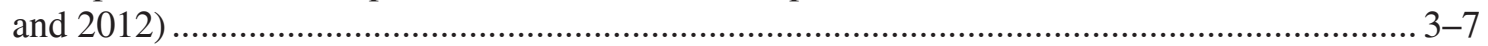

Numerical Estimates of Global Warming Potentials Compared with Carbon Dioxide ................ 11-3

Comparison

Comparison of U.S., European, and Japanese Driving Cycles Attributes .................................. 4-36

Value of Goods in the United States: Comparison of the 1997, 2002, 2007, and 2012

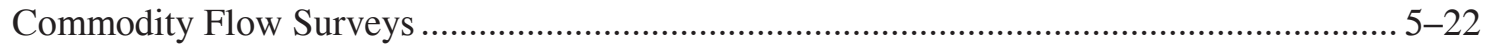

Tons of Freight in the United States: Comparison of the 1997, 2002, 2007, and 2012

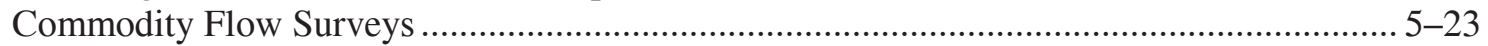

Growth of Ton Miles in the United States: Comparison of the 1997, 2002, 2007, and 2012

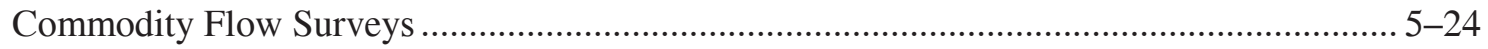

Average Miles per Shipment in the United States: Comparison of the 1997, 2002, 2007,

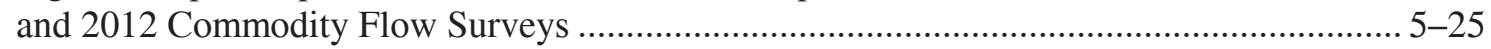

Component

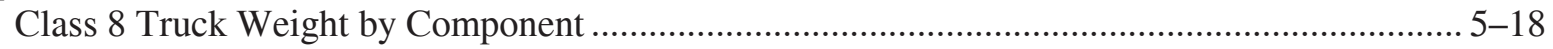

Compounds

Total National Emissions of Volatile Organic Compounds, 1970-2013 ................................... 12-7

Emissions of Volatile Organic Compounds from Highway Vehicles, 1970-2005 ...................... 12-8

Compression-Ignition

Heavy-Duty Highway Compression-Ignition Engines and Urban Buses - Exhaust

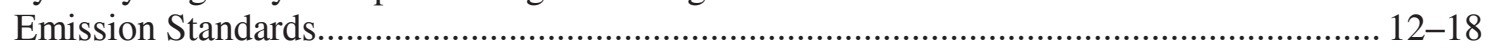

Heavy-Duty Highway Compression-Ignition and Spark-Ignition Engines - Evaporative

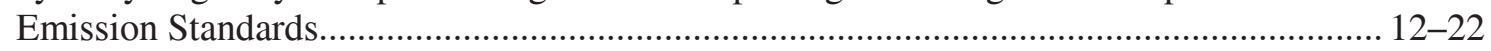

Nonroad Compression-Ignition Engines - Exhaust Emission Standards................................. 12-25

Marine Compression-Ignition (CI) Engines - Exhaust Emission Standards ............................ 12-30

Conditioning

Air Conditioning (SC03) Driving Cycle ............................................................................... 4-33

Consumed

Class 8 Truck Percent of Total Fuel Consumed as a Function of Speed and Tractor-Trailer

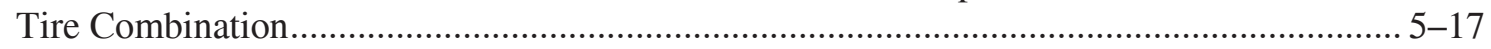

Fuel Consumed by Federal Government Fleets, FY 2000-2012 ….......................................... 7-6

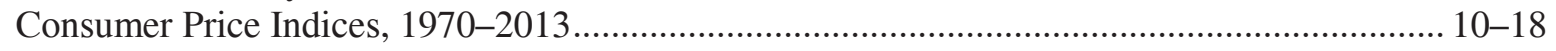

Consumption

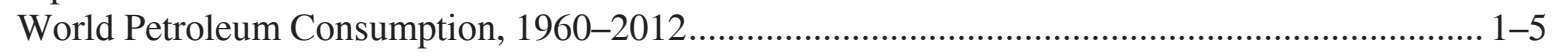

World Oil Reserves, Production and Consumption, 2012 ................................................... 1-6

World Natural Gas Reserves, Production and Consumption, 2012 ........................................... 1-7

Petroleum Production and Transportation Petroleum Consumption in Context, 1950-2013 ....... 1-17

United States Petroleum Production and Consumption - All Sectors, 1973-2040 .................... 1-18

United States Petroleum Production, and Transportation Consumption, 1970-2040.................. 1-19

Consumption of Petroleum by End-Use Sector, 1973-2013 ................................................... 1-20

Highway Transportation Petroleum Consumption by Mode, 1970-2012................................. 1-21

Nonhighway Transportation Petroleum Consumption by Mode, 1970-2012 …......................... 1-22

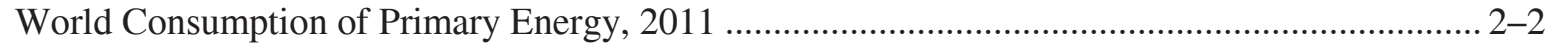

U. S. Consumption of Total Energy by End-Use Sector, 1973-2013 …...................................... 2-3

Distribution of Energy Consumption by Source, 1973 and 2013 ................................................ 2-4 
Consumption (continued)

Alternative Fuel and Oxygenate Consumption, 2005-2011 ................................................ 2-5

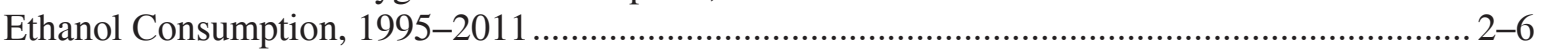

Domestic Consumption of Transportation Energy by Mode and Fuel Type, 2012 ...................... 2-7

Highway Transportation Energy Consumption by Mode, 1970-2012 …................................... 2-9

Nonhighway Transportation Energy Consumption by Mode, 1970-2012 …............................. 2-10

Off-Highway Transportation-Related Fuel Consumption from the Nonroad Model, 2012 .......... 2-11

Fuel Consumption from Lawn and Garden Equipment, 2012 .............................................. 2-12

Average Material Consumption for a Domestic Light Vehicle, Model Years 1995, 2000,

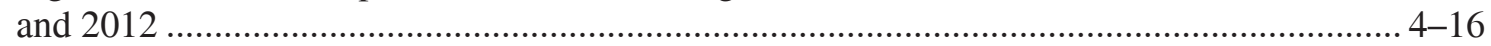

Personal Consumption Expenditures, 1970-2013 ............................................................. 10-18

U.S. Carbon Emissions from Fossil Fuel Consumption by End-Use Sector, 1990-2012............ 11-6

Context

Petroleum Production and Transportation Petroleum Consumption in Context, 1950-2013 ....... 1-17

Conventional

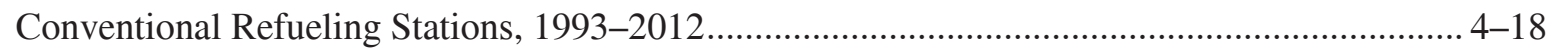

Properties of Conventional and Alternative Liquid Fuels ........................................................ 6-16

Properties of Conventional and Alternative Gaseous Fuels ...................................................... 6-17

Corporate

Car Corporate Average Fuel Economy (CAFE) Standards versus Sales-Weighted Fuel

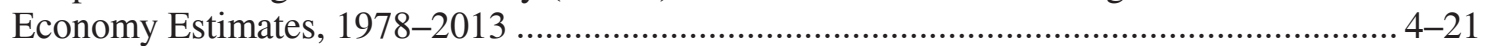

Corporate Average Fuel Economy (CAFE) Fines Collected, 1983-2012 ................................. 4-23

Corporation

Summary Statistics for the National Railroad Passenger Corporation (Amtrak), 1971-2012 $9-11$

Cost

Car Operating Cost per Mile, 1985-2013 ......................................................................... 10-16

Costs

Costs of Oil Dependence to the U.S. Economy, 1970-2012 …................................................ 1-12

Fixed Car Operating Costs per Year, 1975-2013 ............................................................ 10-17

Countries

Car Registrations for Selected Countries, 1960-2012 …........................................................... 3-3

Truck and Bus Registrations for Selected Countries, 1960-2012 ............................................. 3-4

Vehicles per Thousand People: U.S. (Over Time) Compared to Other Countries (in 2002

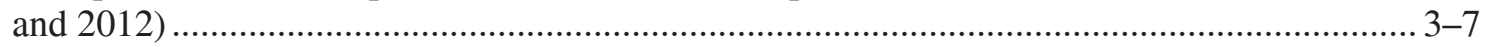

Vehicles per Thousand People in Other Countries, 2002 and 2012 …....................................... 3-9

Top Ten Natural Gas Producing Countries, 2012 …............................................................. 6-15

Gasoline Prices for Selected Countries, 1990-2013 ............................................................ 10-3

Diesel Fuel Prices for Selected Countries, 1998-2013 ........................................................ 10-4

Gasoline Prices for Selected Countries, 1990 and 2013 ......................................................... 10-5

Diesel Prices for Selected Countries, 1990 and 2013 ............................................................. 10-6

Country

Imported Crude Oil by Country of Origin, 1973-2013 ............................................................ 1-9 


\section{Criteria}

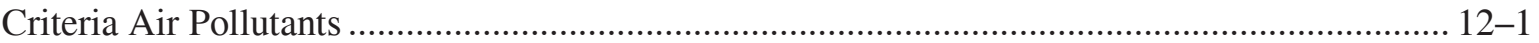

Total National Emissions of the Criteria Air Pollutants by Sector, 2013 .................................. 12-2

Crude

Proved Reserves of Crude Oil and Natural Gas, 1980-2012 …................................................ 1-2

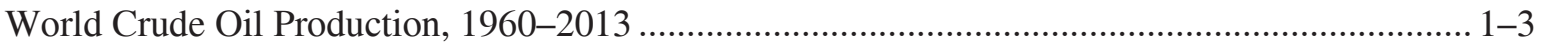

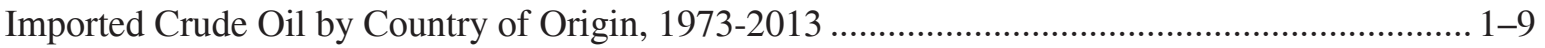

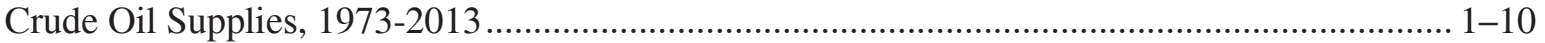

U.S. Refinery Input of Crude Oil and Petroleum Products, 1987-2012 …................................ 1-14

U.S. Refinery Yield of Petroleum Products from a Barrel of Crude Oil, 1978-2013................. 1-15

Prices for a Barrel of Crude Oil and a Gallon of Gasoline, 1978-2013 ..................................... 10-7

Curb

Production-Weighted Curb Weight of New Domestic and Import Cars, Model Years

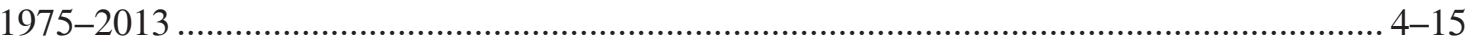

Cycle

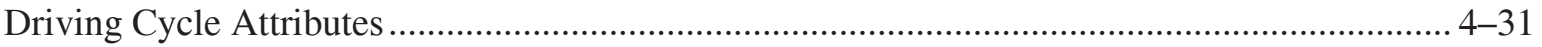

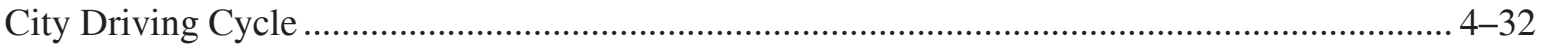

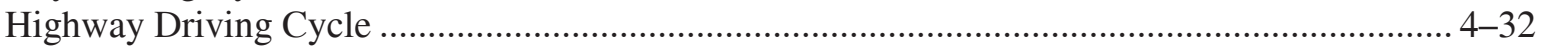

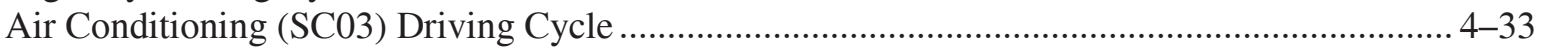

Cold Temperature (Cold FTP) Driving Cycle........................................................................ 4-33

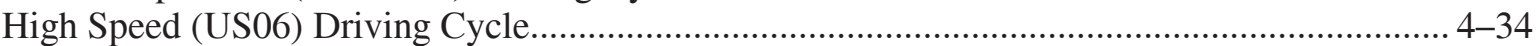

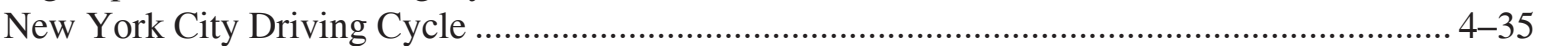

Representative Number Five Driving Cycle ........................................................................... 4-35

Cycles

Comparison of U.S., European, and Japanese Driving Cycles Attributes .................................. 4-36

Example of Differing Results Using the U.S., European, and Japanese Driving Cycles............. 4-36

Daily

Average Daily Miles Driven (per Driver), 2009 NHTS............................................................ 8-17

Daily and Annual Vehicle Miles of Travel and Average Age for Each Vehicle in a

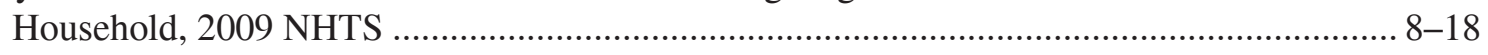

Daily Vehicle Miles of Travel (per Vehicle) by Number of Vehicles in the Household,

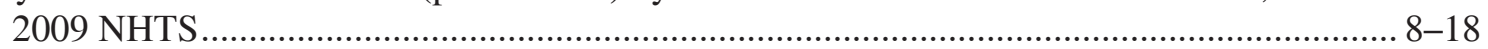

Daily Vehicle Miles of Travel for Each Vehicle in a Household, 2009 NHTS .......................... 8-19

Characteristics of U.S. Daily per Vehicle Driving vs. Dwelling Unit Type and Density ............ 8-21

Dealerships

New Light Vehicle Dealerships and Sales, 1970-2012 …..................................................... 4-17

Definition

Definition of Car Sport Utility Vehicles in Model Year 2013 ................................................ 4-8

Demand

Summary Statistics on Demand Response Vehicles, 1994-2012 ........................................... 4-37

Demographic

Demographic Statistics from the 1969, 1977, 1983, 1990, 1995 NPTS and 2001, 2009 NHTS. 
Density

Characteristics of U.S. Daily per Vehicle Driving vs. Dwelling Unit Type and Density.....

Dependence

Costs of Oil Dependence to the U.S. Economy, 1970-2012 $1-12$

Diesel

Highway Usage of Gasoline and Diesel, 1973-2012 _........................................................... 2-13

Diesel Fuel Prices for Selected Countries, 1998-2013 ............................................................. 10-4

Diesel Prices for Selected Countries, 1990 and 2013 ........................................................... 10-6

Refiner Sales Prices for Propane and No. 2 Diesel, 1978-2013 ............................................. 10-9

Highway, Nonroad, Locomotive, and Marine (NRLM) Diesel Fuel Sulfur Standards .............. 12-39

Differing

Example of Differing Results Using the U.S., European, and Japanese Driving Cycles............. 4-36

Direct

Direct Carbon Dioxide Emissions from a Gallon of Fuel $11-16$

Distance

Class 8 Truck Fuel Economy as a Function of Speed and Tractor-Trailer Tire Combination and Percentage of Total Distance Traveled as a Function of Speed..................................... 5-16

Share of Vehicle Trips by Trip Distance, 2009 NHTS ............................................................... 8 8-15

Share of Vehicle Trips to Work by Trip Distance, 2009 NHTS ................................................ 8-15

Distribution

Distribution of Energy Consumption by Source, 1973 and 2013 ...............................................2-4

Distribution of Trucks over 26,000 lbs. by Vehicle-Miles Traveled .......................................... 5-11

Distribution of Class 8 Trucks by On-Road Vehicle Weight, 2008 ........................................... 5-20

Domestic

Domestic Consumption of Transportation Energy by Mode and Fuel Type, 2012 ...................... 2-7

Production Sales, Production Shares, and Sales-Weighted Fuel Economies of New Domestic and Import Cars, Selected Model Years 1975-2013 .............................................. 4-7

Production, Production Shares, and Production-Weighted Fuel Economies of New

Domestic and Import Light Trucks, Model Years 1975-2013 ............................................ 4-9

Production and Production-Weighted Fuel Economies of New Domestic and Import

Cars, Light Trucks, and Light Vehicles, Model Years 1975-2013 …..................................... 4-10

Production-Weighted Engine Size of New Domestic and Import Cars, Model Years

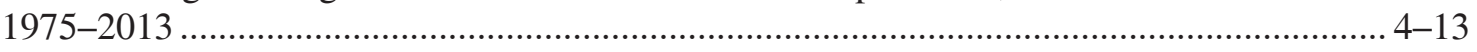

Production-Weighted Engine Size of New Domestic and Import Light Trucks, Model

Years 1975-2013 ......................................................................................................... 4-14

Production-Weighted Curb Weight of New Domestic and Import Cars, Model Years

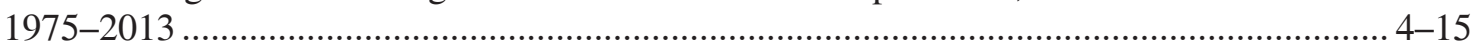

Average Material Consumption for a Domestic Light Vehicle, Model Years 1995, 2000,

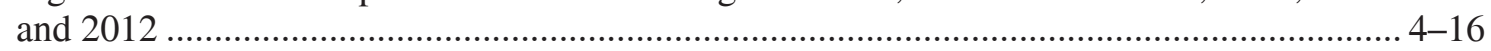

Average Miles per Domestic Federal Vehicle by Vehicle Type, 2012 ..................................... 7-4

Summary Statistics for U.S. Domestic and International Certificated Route Air

Carriers (Combined Totals), 1970-2013 .......................................................................... 9-3

Tonnage Statistics for Domestic and International Waterborne Commerce, 1970-2012 ............. 9-5

Summary Statistics for Domestic Waterborne Commerce, 1970-2012 ...................................... 9-6

Average Price of a New Car (Domestic and Import), 1970-2012 …...................................... 10-15 
Domestic (continued)

Production-Weighted Annual Carbon Footprint of New Domestic and Import Cars,

Model Years 1975-2013

Production-Weighted Annual Carbon Footprint of New Domestic and Import Light

Trucks, Model Years 1975-2013 $11-14$

Drive

Electric Drive Vehicles Available by Manufacturer, Model Year 2014 …................................ 6-10

Electric Drive Medium/Heavy Trucks and Buses Available by Manufacturer, 2014 .................. 6-12

Driven

Average Daily Miles Driven (per Driver), 2009 NHTS .............................................................. 8-17

Driver

Average Daily Miles Driven (per Driver), 2009 NHTS ............................................................ 8-17

Driving

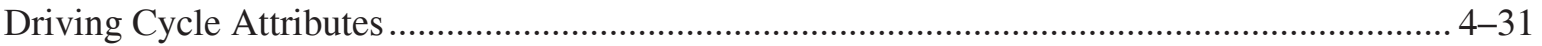

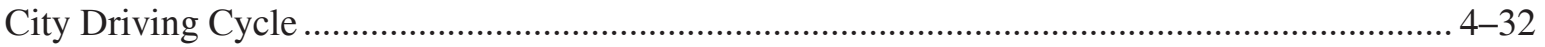

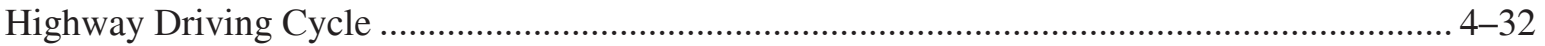

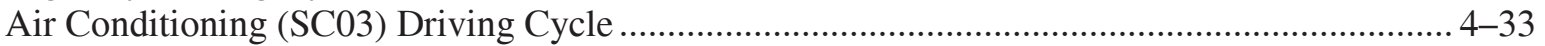

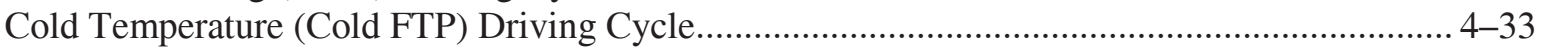

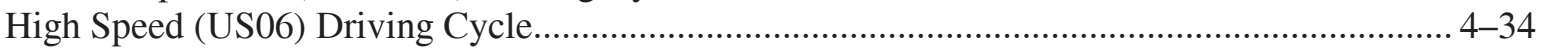

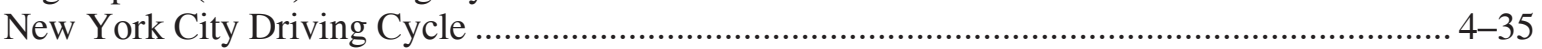

Representative Number Five Driving Cycle ............................................................................ 4-35

Comparison of U.S., European, and Japanese Driving Cycles Attributes .................................. 4-36

Example of Differing Results Using the U.S., European, and Japanese Driving Cycles............. 4-36

Characteristics of U.S. Daily per Vehicle Driving vs. Dwelling Unit Type and Density ............ 8-21

Dwelling

Characteristics of U.S. Daily per Vehicle Driving vs. Dwelling Unit Type and Density ............ 8-21

E85

E85 Flex-Fuel Vehicles Available by Manufacturer, Model Year 2014...................................... 6-5

Non-E85 Alternative Fuel Vehicles Available by Manufacturer, Model Year 2014 .................... 6-7

Each

Annual Vehicle Miles of Travel for Each Vehicle in a Household, 2009 NHTS ....................... 8-19

Daily Vehicle Miles of Travel for Each Vehicle in a Household, 2009 NHTS ........................... 8-19

Economic

Oil Price and Economic Growth, 1970-2013 .......................................................................... 1-11

Economies

Production Sales, Production Shares, and Sales-Weighted Fuel Economies of New

Domestic and Import Cars, Selected Model Years 1975-2013 .............................................. 4-7

Production, Production Shares, and Production-Weighted Fuel Economies of New

Domestic and Import Light Trucks, Model Years 1975-2013 ............................................. 4-9

Production and Production-Weighted Fuel Economies of New Domestic and Import

Cars, Light Trucks, and Light Vehicles, Model Years 1975-2013 $4-10$ 
Economy

Costs of Oil Dependence to the U.S. Economy, 1970-2012 …................................................ 1-12

Fuel Economy and Carbon Dioxide Emissions Standards, MY 2012-2025............................ 4-19

Fuel Economy and Carbon Dioxide Targets for Model Year 2025 ........................................... 4-20

Car Corporate Average Fuel Economy (CAFE) Standards versus Sales-Weighted Fuel

Economy Estimates, 1978-2013 ....................................................................................... 4-21

Light Truck Corporate Average Fuel Economy (CAFE) Standards versus Sales-Weighted

Fuel Economy Estimates, 1978-2013 .................................................................................22

Corporate Average Fuel Economy (CAFE) Fines Collected, 1983-2012 ….............................. 4-23

Fuel Economy by Speed, PSAT Model Results.................................................................. 4-28

Fuel Economy by Speed, 1973, 1984, 1997, and 2012 Studies .............................................. 4-29

Truck Harmonic Mean Fuel Economy by Size Class, 1992, 1997, and 2002 ............................ 5-6

Effect of Terrain on Class 8 Truck Fuel Economy ..................................................................... 5-14

Fuel Economy for Class 8 Trucks as Function of Speed and Tractor-Trailer Tire

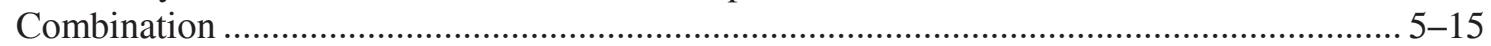

Class 8 Truck Fuel Economy as a Function of Speed and Tractor-Trailer Tire Combination and Percentage of Total Distance Traveled as a Function of Speed...................................... 5-16

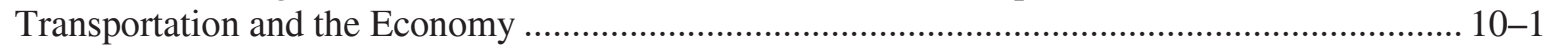

Effect

Effect of Terrain on Class 8 Truck Fuel Economy …................................................................ 5-14

Electric

Electric Drive Vehicles Available by Manufacturer, Model Year 2014 .................................... 6-10

Electric Drive Medium/Heavy Trucks and Buses Available by Manufacturer, 2014 .................. 6-12

Electronic

Share of Heavy Trucks with Selected Electronic Features, 2002 …........................................ 5-12

Emission

Tier 3 Evaporative Emission Standards .......................................................................... 12-15

Tier 3 Particulate Matter Emission Standards for Light Gasoline Vehicles, MY 2017

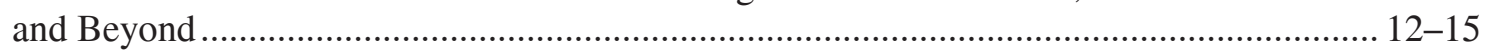

Light-Duty Vehicle, Light-Duty Truck, and Medium-Duty Passenger Vehicle - Tier 2

Exhaust Emission Standards ........................................................................................... 12-16

Light-Duty Vehicle, Light-Duty Truck, and Medium-Duty Passenger Vehicle - Tier 2

Evaporative Emission Standards ................................................................................... 12-17

Heavy-Duty Highway Compression-Ignition Engines and Urban Buses - Exhaust

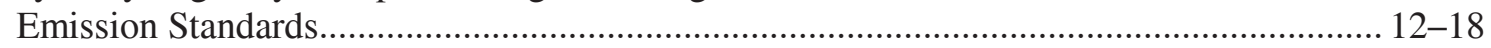

Heavy-Duty Highway Spark-Ignition Engines - Exhaust Emission Standards ........................ 12-20

Heavy-Duty Highway Compression-Ignition and Spark-Ignition Engines - Evaporative

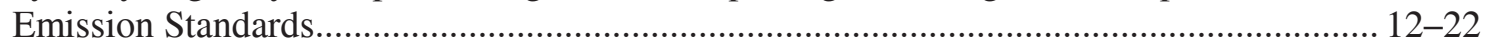

California Car, Light Truck and Medium Truck Emission Certification Standards .................. 12-23

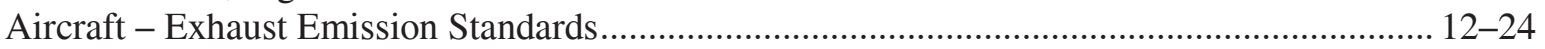

Nonroad Compression-Ignition Engines - Exhaust Emission Standards................................. 12-25

Nonroad Large Spark-Ignition Engines - Exhaust and Evaporative Emission Standards .......... 12-27

Locomotives - Exhaust Emission Standards …................................................................. 12-28

Marine Compression-Ignition (CI) Engines - Exhaust Emission Standards ............................ 12-30

Marine Spark-Ignition Engines and Vessels - Exhaust Emission Standards ............................ 12-34

Nonroad Recreational Engines and Vehicles - Exhaust Emission Standards............................ 12-36 
Emissions

Fuel Economy and Carbon Dioxide Emissions Standards, MY 2012-2025............................ 4-19

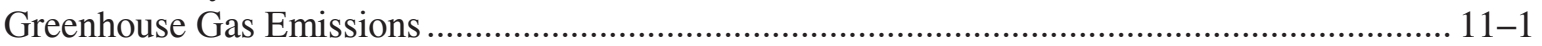

World Carbon Dioxide Emissions, 1990 and 2013 .................................................................. 11-2

U.S. Emissions of Greenhouse Gases, based on Global Warming Potential, 1990-2012 ........... 11-4

Total U.S. Greenhouse Gas Emissions by End-Use Sector, 2012 ........................................... 11-5

U.S. Carbon Emissions from Fossil Fuel Consumption by End-Use Sector, 1990-2012 ............. 11-6

U.S. Carbon Emissions from Fossil Fuel Combustion in the Transportation

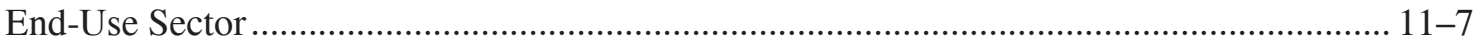

Transportation Greenhouse Gas Emissions by Mode, 1990 and 2012 ..................................... 11-8

Well-to-Wheel Emissions for Various Fuels and Vehicle Technologies .................................. 11-11

Direct Carbon Dioxide Emissions from a Gallon of Fuel ...................................................... 11-16

Total National Emissions of the Criteria Air Pollutants by Sector, 2013 ................................... 12-2

Total National Emissions of Carbon Monoxide, 1970-2013 ................................................... 12-3

Emissions of Carbon Monoxide from Highway Vehicles, 1970-2005 ..................................... 12-4

Total National Emissions of Nitrogen Oxides, 1970-2013 ................................................. 12-5

Emissions of Nitrogen Oxides from Highway Vehicles, 1970-2005 ........................................ 12-6

Total National Emissions of Volatile Organic Compounds, 1970-2013 ................................... 12-7

Emissions of Volatile Organic Compounds from Highway Vehicles, 1970-2005 ...................... 12-8

Total National Emissions of Particulate Matter (PM-10), 1970-2013 ...................................... 12-9

Total National Emissions of Particulate Matter (PM-2.5), 1990-2013 .................................... 12-11

Emissions of Particulate Matter (PM-2.5) from Highway Vehicles, 1990-2005 ...................... 12-12

Employment

Transportation-Related Employment, 2000 and 2013 .......................................................... 10-19

U.S. Employment for Motor Vehicles and Motor Vehicle Parts Manufacturing,

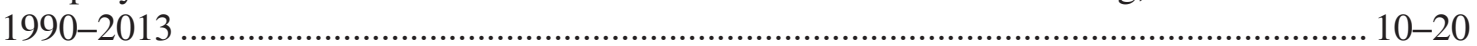

Empty

Gross Vehicle Weight vs. Empty Vehicle Weight ................................................................. 5-19

End-Use

Consumption of Petroleum by End-Use Sector, 1973-2013 .................................................... 1-20

U. S. Consumption of Total Energy by End-Use Sector, 1973-2013 .......................................... 2-3

Total U.S. Greenhouse Gas Emissions by End-Use Sector, 2012 .......................................... 11-5

U.S. Carbon Emissions from Fossil Fuel Consumption by End-Use Sector, 1990-2012............ 11-6

U.S. Carbon Emissions from Fossil Fuel Combustion in the Transportation

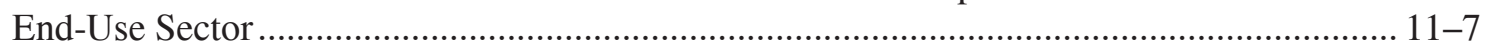

Energy

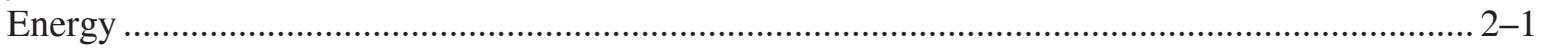

World Consumption of Primary Energy, 2011 ...................................................................... 2-2

U. S. Consumption of Total Energy by End-Use Sector, 1973-2013 .......................................... 2-3

Distribution of Energy Consumption by Source, 1973 and 2013 ............................................. 2-4

Domestic Consumption of Transportation Energy by Mode and Fuel Type, 2012 ...................... 2-7

Transportation Energy Use by Mode, 2011-2012 …............................................................... 2-8

Highway Transportation Energy Consumption by Mode, 1970-2012 ….................................... 2-9

Nonhighway Transportation Energy Consumption by Mode, 1970-2012 ….............................. 2-10

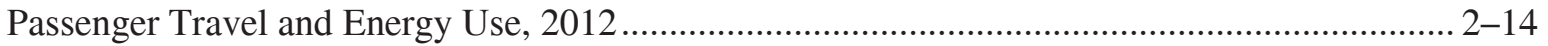

Energy Intensities of Highway Passenger Modes, 1970-2012 .............................................. 2-15

Energy Intensities of Nonhighway Passenger Modes, 1970-2012 …........................................ 2-16

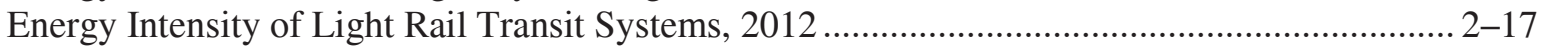

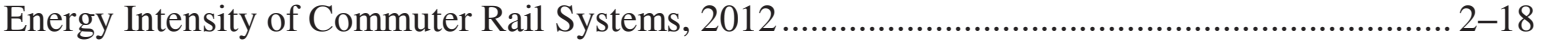


Energy (continued)

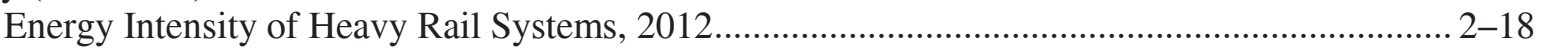

Energy Intensities of Freight Modes, 1970-2012 …............................................................. 2-19

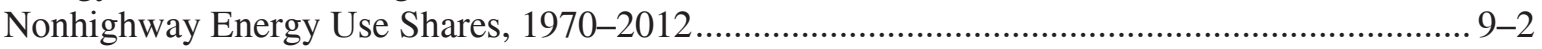

Recreational Boat Energy Use, 1970-2012 …................................................................ 9-7

Engine

Production-Weighted Engine Size of New Domestic and Import Cars, Model Years

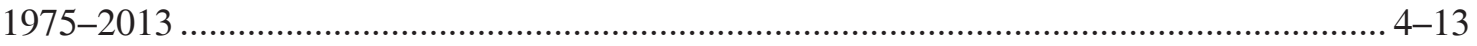

Production-Weighted Engine Size of New Domestic and Import Light Trucks, Model

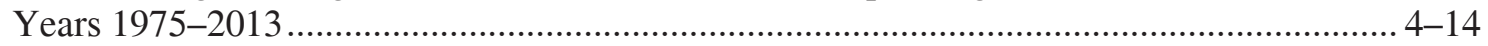

Engines

Heavy-Duty Highway Compression-Ignition Engines and Urban Buses - Exhaust

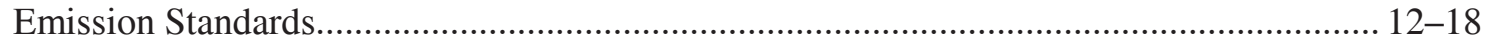

Heavy-Duty Highway Spark-Ignition Engines - Exhaust Emission Standards ......................... 12-20

Heavy-Duty Highway Compression-Ignition and Spark-Ignition Engines - Evaporative

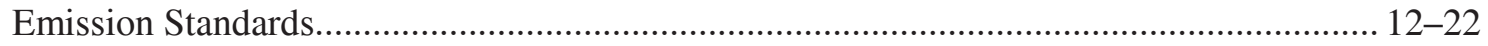

Nonroad Compression-Ignition Engines - Exhaust Emission Standards.................................. 12-25

Nonroad Large Spark-Ignition Engines - Exhaust and Evaporative Emission Standards .......... 12-27

Marine Compression-Ignition (CI) Engines - Exhaust Emission Standards ........................... 12-30

Marine Spark-Ignition Engines and Vessels - Exhaust Emission Standards ............................ 12-34

Nonroad Recreational Engines and Vehicles - Exhaust Emission Standards............................ 12-36

Equipment

Fuel Consumption from Lawn and Garden Equipment, 2012 ............................................... 2-12

Estimates

Sales Estimates of Class 1, Class 2a, and Class 2b Light Trucks, 1989-1999 .............................. 4-4

Car Corporate Average Fuel Economy (CAFE) Standards versus Sales-Weighted Fuel

Economy Estimates, 1978-2013 .............................................................................. 4-21

Light Truck Corporate Average Fuel Economy (CAFE) Standards versus Sales-Weighted

Fuel Economy Estimates, 1978-2013 ........................................................................... 4-22

Estimates of Alternative Fuel Highway Vehicles in Use, 1995-2011 ........................................ 6-3

Numerical Estimates of Global Warming Potentials Compared with Carbon Dioxide ................ 11-3

Ethanol

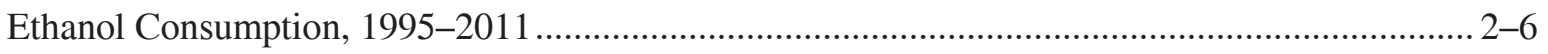

European

Comparison of U.S., European, and Japanese Driving Cycles Attributes ................................. 4-36

Example of Differing Results Using the U.S., European, and Japanese Driving Cycles............. 4-36

Evaporative

Tier 3 Evaporative Emission Standards ............................................................................. 12-15

Light-Duty Vehicle, Light-Duty Truck, and Medium-Duty Passenger Vehicle - Tier 2

Evaporative Emission Standards

Heavy-Duty Highway Compression-Ignition and Spark-Ignition Engines - Evaporative

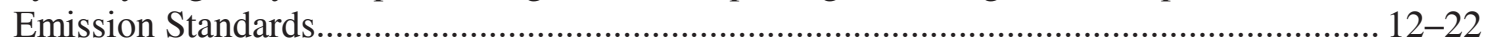

Nonroad Large Spark-Ignition Engines - Exhaust and Evaporative Emission Standards .......... 12-27

Example

Example of Differing Results Using the U.S., European, and Japanese Driving Cycles............. 4-36 
Excise

Federal Excise Taxes on Motor Fuels, 2011 .......................................................................... 10-11

Exhaust

Light-Duty Vehicle, Light-Duty Truck, and Medium-Duty Passenger Vehicle - Tier 2

Exhaust Emission Standards ............................................................................................. 12-16

Heavy-Duty Highway Compression-Ignition Engines and Urban Buses - Exhaust

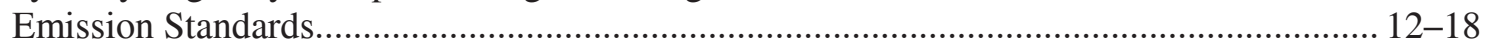

Heavy-Duty Highway Spark-Ignition Engines - Exhaust Emission Standards ........................ 12-20

Aircraft - Exhaust Emission Standards......................................................................... 12-24

Nonroad Compression-Ignition Engines - Exhaust Emission Standards................................. 12-25

Nonroad Large Spark-Ignition Engines - Exhaust and Evaporative Emission Standards .......... 12-27

Locomotives - Exhaust Emission Standards ....................................................................... 12-28

Marine Compression-Ignition (CI) Engines - Exhaust Emission Standards ............................ 12-30

Marine Spark-Ignition Engines and Vessels - Exhaust Emission Standards............................ 12-34

Nonroad Recreational Engines and Vehicles - Exhaust Emission Standards............................ 12-36

Expenditures

Average Annual Expenditures of Households by Income, 2012 ................................................. 8-4

Annual Household Expenditures for Transportation, 1985-2012 …........................................ 8-5

Personal Consumption Expenditures, 1970-2013 .............................................................. 10-18

Exports

United States Petroleum Production, Imports and Exports, 1950-2013 ................................... 1-16

Facility

Percentage of Trucks by Fleet Size and Primary Fueling Facility, 2002 …................................ 5-9

Share of Trucks by Major Use and Primary Fueling Facility, 2002 ........................................ 5-10

Features

Share of Heavy Trucks with Selected Electronic Features, 2002 ........................................... 5-12

Federal

Average Miles per Domestic Federal Vehicle by Vehicle Type, 2012 ..................................... 7-4

Federal Government Vehicles, 2001-2012 ..................................................................... 7-5

Federal Fleet Vehicle Acquisitions by Fuel Type, FY 2002-2012 _..................................... 7-6

Fuel Consumed by Federal Government Fleets, FY 2000-2012 …...................................... 7-6

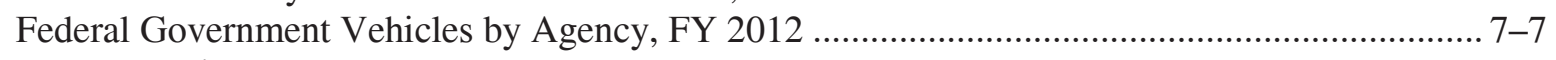

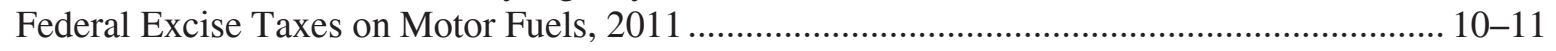

Federal and State Alternative Fuel Incentives, 2014 ......................................................... 10-12

Federal and State Advanced Technology Incentives, 2014 .................................................. 10-13

Feedstocks

GREET Model Feedstocks and Fuels …...................................................................... 11-10

Fines

Corporate Average Fuel Economy (CAFE) Fines Collected, 1983-2012 …............................ 4-23

Five

Representative Number Five Driving Cycle .................................................................... 4-35

Fixed

Fixed Car Operating Costs per Year, 1975-2013 ….............................................................. 10-17 
Fleet

Percentage of Trucks by Fleet Size and Primary Fueling Facility, 2002 ................................... 5-9

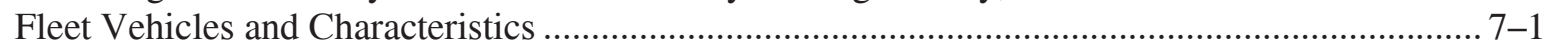

Fleet Vehicles in Service as of January 1, 2013 .................................................................... 7-2

Average Annual Vehicle-Miles of Travel for Commercial Fleet Vehicles, 2012 ......................... 7-3

Average Length of Time Commercial Fleet Vehicles are in Service, 2012 .................................. 7-3

New Light Fleet Vehicle Registrations by Vehicle Type, Model Year 2012 ................................ 7-3

Federal Fleet Vehicle Acquisitions by Fuel Type, FY 2002- 2012 .......................................... 7-6

Fleets

Fuel Consumed by Federal Government Fleets, FY 2000-2012 ….......................................... 7-6

Flex-Fuel

E85 Flex-Fuel Vehicles Available by Manufacturer, Model Year 2014...................................... 6-5

Flow

Value of Goods in the United States: Comparison of the 1997, 2002, 2007, and 2012

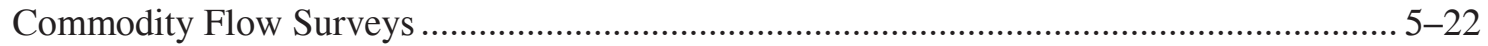

Tons of Freight in the United States: Comparison of the 1997, 2002, 2007, and 2012

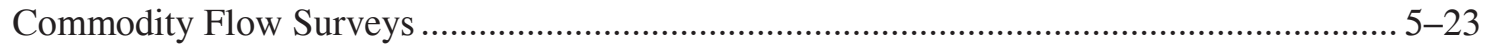

Growth of Ton Miles in the United States: Comparison of the 1997, 2002, 2007, and 2012

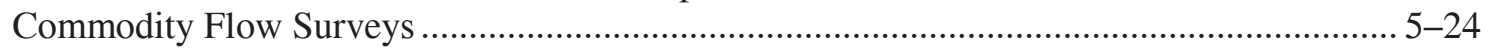

Average Miles per Shipment in the United States: Comparison of the 1997, 2002, 2007,

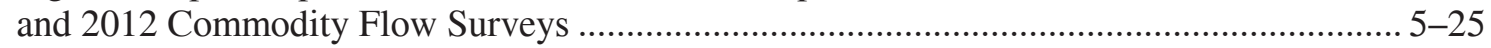

Footprint

Production-Weighted Annual Carbon Footprint of New Domestic and Import Cars,

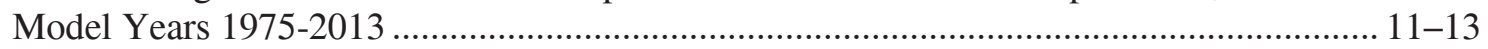

Production-Weighted Annual Carbon Footprint of New Domestic and Import Light

Trucks, Model Years 1975-2013 ..................................................................................... 11-14

Average Annual Carbon Footprint by Vehicle Classification, 1975 and 2013 ......................... 11-15

Fossil

U.S. Carbon Emissions from Fossil Fuel Consumption by End-Use Sector, 1990-2012............ 11-6

U.S. Carbon Emissions from Fossil Fuel Combustion in the Transportation

End-Use Sector

Four-Tire

Summary Statistics for Two-Axle, Four-Tire Trucks, 1970-2012 …........................................ 4-3

Freight

Energy Intensities of Freight Modes, 1970-2012 ................................................................. 2-19

Tons of Freight in the United States: Comparison of the 1997, 2002, 2007, and 2012

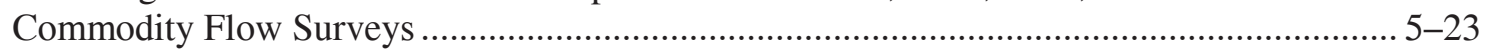

Class I Railroad Freight Systems in the United States Ranked by Revenue Ton-Miles, 2012 ...... 9-8

Summary Statistics for Class I Freight Railroads, 1970-2012 …................................................ 9-9

FTP

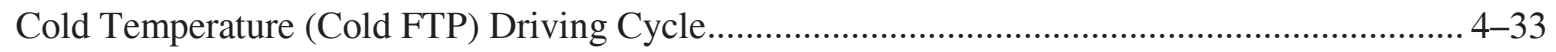

Fuel

Alternative Fuel and Oxygenate Consumption, 2005-2011 ................................................... 2-5

Domestic Consumption of Transportation Energy by Mode and Fuel Type, 2012 ...................... 2-7 
Fuel (continued)

Off-Highway Transportation-Related Fuel Consumption from the Nonroad Model, 2012......... 2-11

Fuel Consumption from Lawn and Garden Equipment, 2012 ................................................. 2-12

Production Sales, Production Shares, and Sales-Weighted Fuel Economies of New

Domestic and Import Cars, Selected Model Years 1975-2013 .............................................. 4-7

Production, Production Shares, and Production-Weighted Fuel Economies of New

Domestic and Import Light Trucks, Model Years 1975-2013 ............................................... 4-9

Production and Production-Weighted Fuel Economies of New Domestic and Import Cars,

Light Trucks, and Light Vehicles, Model Years 1975-2013 ….......................................... 4-10

Fuel Economy and Carbon Dioxide Emissions Standards, MY 2012-2025.............................. 4-19

Fuel Economy and Carbon Dioxide Targets for Model Year 2025 .......................................... 4-20

Car Corporate Average Fuel Economy (CAFE) Standards versus Sales-Weighted Fuel

Economy Estimates, 1978-2013 ...................................................................................... 4-21

Light Truck Corporate Average Fuel Economy (CAFE) Standards versus Sales-Weighted

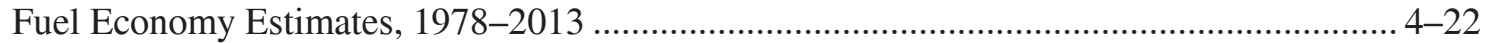

Corporate Average Fuel Economy (CAFE) Fines Collected, 1983-2012 ................................. 4-23

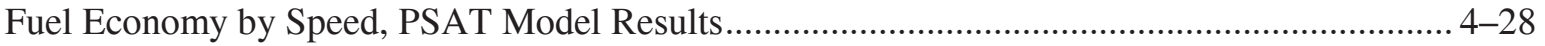

Fuel Economy by Speed, 1973, 1984, 1997, and 2012 Studies ............................................. 4-29

Truck Harmonic Mean Fuel Economy by Size Class, 1992, 1997, and 2002 .............................. 5-6

Effect of Terrain on Class 8 Truck Fuel Economy ................................................................. 5-14

Fuel Economy for Class 8 Trucks as Function of Speed and Tractor-Trailer Tire

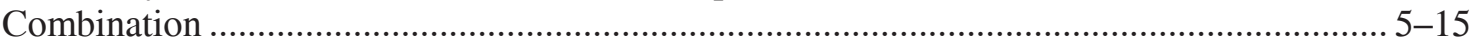

Class 8 Truck Fuel Economy as a Function of Speed and Tractor-Trailer Tire Combination and Percentage of Total Distance Traveled as a Function of Speed..................................... 5-16

Class 8 Truck Percent of Total Fuel Consumed as a Function of Speed and Tractor-Trailer

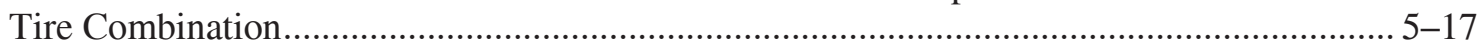

Alternative Fuel and Advanced Technology Vehicles and Characteristics ................................. 6-1

Estimates of Alternative Fuel Highway Vehicles in Use, 1995-2011 ....................................... 6-3

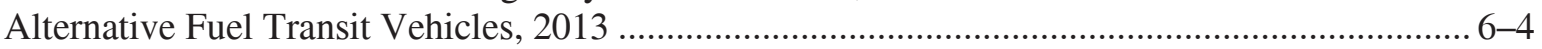

Non-E85 Alternative Fuel Vehicles Available by Manufacturer, Model Year 2014 .................... 6-7

Number of Alternative Refuel Sites by State and Fuel Type, 2014 ......................................... 6-13

Federal Fleet Vehicle Acquisitions by Fuel Type, FY 2002- 2012 _..................................... 7-6

Fuel Consumed by Federal Government Fleets, FY 2000-2012 ….......................................... 7-6

Diesel Fuel Prices for Selected Countries, 1998-2013 ........................................................... 10-4

Retail Prices for Motor Fuel, 1978-2013 .................................................................................. 10-8

Refiner Sales Prices for Aviation Gasoline and Jet Fuel, 1978-2013 ...................................... 10-10

Federal and State Alternative Fuel Incentives, 2014 .......................................................... 10-12

U.S. Carbon Emissions from Fossil Fuel Consumption by End-Use Sector, 1990-2012............ 11-6

U.S. Carbon Emissions from Fossil Fuel Combustion in the Transportation End-Use

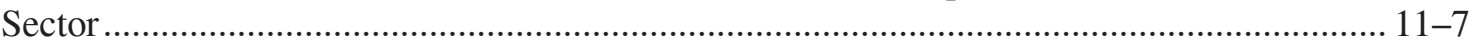

Direct Carbon Dioxide Emissions from a Gallon of Fuel ..................................................... 11-16

Highway, Nonroad, Locomotive, and Marine (NRLM) Diesel Fuel Sulfur Standards ............. 12-39

Fueling

Percentage of Trucks by Fleet Size and Primary Fueling Facility, 2002 .................................... 5-9

Share of Trucks by Major Use and Primary Fueling Facility, 2002 .......................................... 5-10

Fuels

Properties of Conventional and Alternative Liquid Fuels .......................................................... 6-16

Properties of Conventional and Alternative Gaseous Fuels .................................................. 6-17

Federal Excise Taxes on Motor Fuels, 2011 ..........................................................................10

GREET Model Feedstocks and Fuels ............................................................................. 11-10

Well-to-Wheel Emissions for Various Fuels and Vehicle Technologies ................................... 11-11 
Function

Fuel Economy for Class 8 Trucks as Function of Speed and Tractor-Trailer Tire

Combination

Class 8 Truck Fuel Economy as a Function of Speed and Tractor-Trailer Tire Combination and Percentage of Total Distance Traveled as a Function of Speed.

Class 8 Truck Percent of Total Fuel Consumed as a Function of Speed and Tractor-Trailer Tire Combination.

Gallon

Prices for a Barrel of Crude Oil and a Gallon of Gasoline, 1978-2013. $10-7$

Direct Carbon Dioxide Emissions from a Gallon of Fuel $11-16$

Garden

Fuel Consumption from Lawn and Garden Equipment, 2012 $2-12$

Gas

Proved Reserves of Crude Oil and Natural Gas, 1980-2012 ….............................................. 1-2

World Natural Gas Reserves, Production and Consumption, 2012 …...................................... 1-7

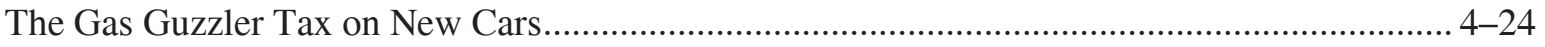

List of Model Year 2013 Cars with Gas Guzzler Taxes .......................................................... 4-25

Tax Receipts from the Sale of Gas Guzzlers, 1980-2012 ....................................................... 4-27

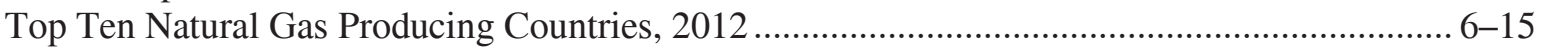

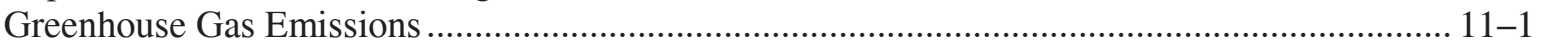

Total U.S. Greenhouse Gas Emissions by End-Use Sector, 2012 ........................................... 11-5

Transportation Greenhouse Gas Emissions by Mode, 1990 and 2012 ...................................... 11-8

Gaseous

Properties of Conventional and Alternative Gaseous Fuels ................................................... 6-17

Gases

U.S. Emissions of Greenhouse Gases, based on Global Warming Potential, 1990-2012 ........... 11-4

Tier 3 Non-Methane Organic Gases and Nitrogen Oxide Standards ...................................... 12-14

Gasoline

Highway Usage of Gasoline and Diesel, 1973-2012 ........................................................... 2-13

Gasoline Prices for Selected Countries, 1990-2013 ........................................................... 10-3

Gasoline Prices for Selected Countries, 1990 and 2013 ...................................................... 10-5

Prices for a Barrel of Crude Oil and a Gallon of Gasoline, 1978-2013................................... 10-7

Refiner Sales Prices for Aviation Gasoline and Jet Fuel, 1978-2013 ...................................... 10-10

Tier 3 Particulate Matter Emission Standards for Light Gasoline Vehicles, MY 2017

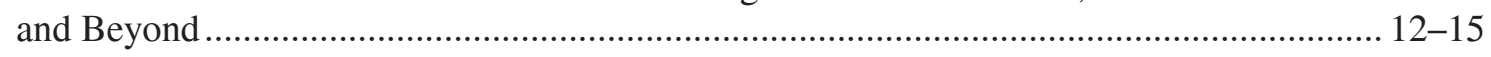

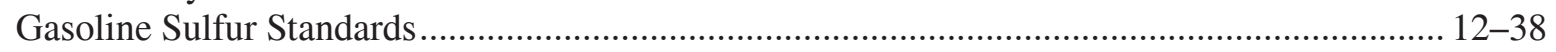

General

Summary Statistics for General Aviation, 1970-2012 _......................................................... 9-4

Global

Numerical Estimates of Global Warming Potentials Compared with Carbon Dioxide ................ 11-3

U.S. Emissions of Greenhouse Gases, based on Global Warming Potential, 1990-2012 ........... 11-4

Goods

Value of Goods in the United States: Comparison of the 1997, 2002, 2007, and 2012

Commodity Flow Surveys . 
Government

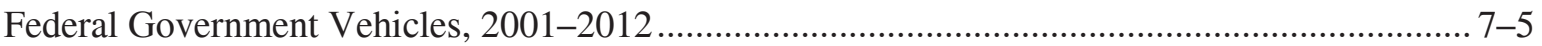

Fuel Consumed by Federal Government Fleets, FY 2000-2012 …........................................ 7-6

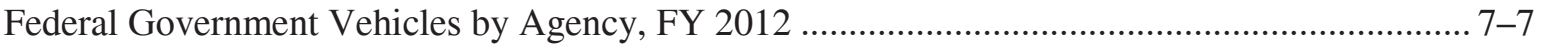

Greenhouse

Greenhouse Gas Emissions ................................................................................................ 11-1

U.S. Emissions of Greenhouse Gases, based on Global Warming Potential, 1990-2012 _.......... 11-4

Total U.S. Greenhouse Gas Emissions by End-Use Sector, 2012 …..................................... 11-5

Transportation Greenhouse Gas Emissions by Mode, 1990 and 2012 ...................................... 11-8

\section{GREET}

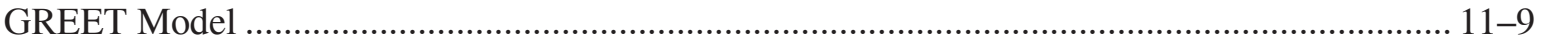

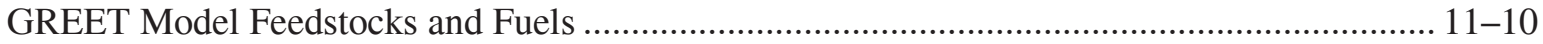

Gross

Refinery Gross Output by World Region, 2003 and 2013 ..................................................... 1-13

New Retail Sales of Trucks 10,000 Pounds GVW and Less in the United States, 1970-2013 ..... 4-6

New Retail Truck Sales by Gross Vehicle Weight, 1970-2013 ................................................. 5-4

Truck Statistics by Gross Vehicle Weight Class, 2002 .............................................................. 5-6

Gross Vehicle Weight vs. Empty Vehicle Weight .................................................................... 5-19

Growth

Oil Price and Economic Growth, 1970-2013 $1-11$

Growth of Ton Miles in the United States: Comparison of the 1997, 2002, 2007, and 2012

Commodity Flow Surveys

Guzzler

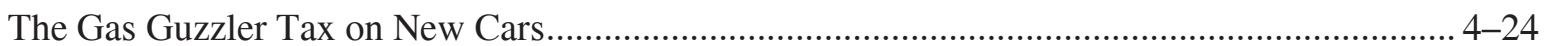

List of Model Year 2013 Cars with Gas Guzzler Taxes ............................................................ 4-25

Guzzlers

Tax Receipts from the Sale of Gas Guzzlers, 1980-2012 ......................................................... 4-27

Harmonic

Truck Harmonic Mean Fuel Economy by Size Class, 1992, 1997, and 2002 ............................ 5-6

Heavy

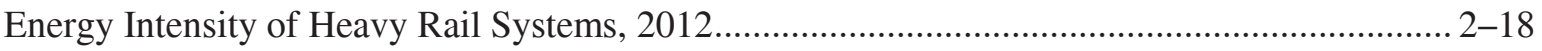

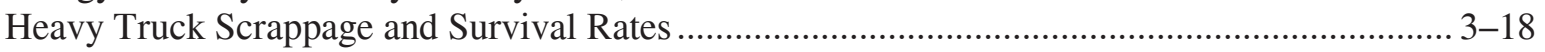

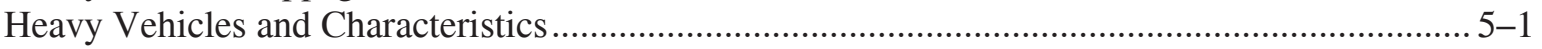

Share of Heavy Trucks with Selected Electronic Features, 2002 ............................................. 5-12

Electric Drive Medium/Heavy Trucks and Buses Available by Manufacturer, 2014 .................. 6-12

Heavy-Duty

Heavy-Duty Highway Compression-Ignition Engines and Urban Buses - Exhaust

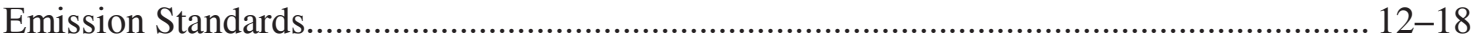

Heavy-Duty Highway Spark-Ignition Engines - Exhaust Emission Standards ......................... 12-20

Heavy-Duty Highway Compression-Ignition and Spark-Ignition Engines - Evaporative

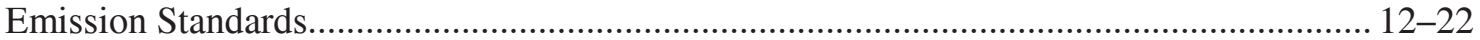

High

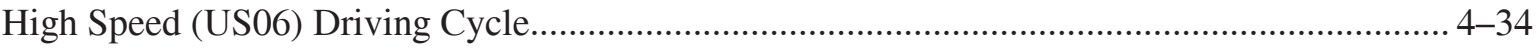


Highway

Highway Transportation Petroleum Consumption by Mode, 1970-2012................................. 1-21

Highway Transportation Energy Consumption by Mode, 1970-2012 ...................................... 2-9

Highway Usage of Gasoline and Diesel, 1973-2012 ............................................................ 2-13

Energy Intensities of Highway Passenger Modes, 1970-2012 .............................................. 2-15

All Highway Vehicles Characteristics .....................................................................................

Shares of Highway Vehicle-Miles Traveled by Vehicle Type, 1970-2012 _.............................. 3-11

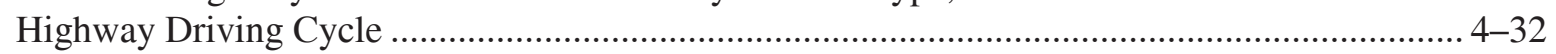

Estimates of Alternative Fuel Highway Vehicles in Use, 1995-2011 ......................................... 6-3

Emissions of Carbon Monoxide from Highway Vehicles, 1970-2005...................................... 12-4

Emissions of Nitrogen Oxides from Highway Vehicles, 1970-2005 ...................................... 12-6

Emissions of Volatile Organic Compounds from Highway Vehicles, 1970-2005 ...................... 12-8

Emissions of Particulate Matter (PM-2.5) from Highway Vehicles, 1990-2005 ...................... 12-12

Heavy-Duty Highway Compression-Ignition Engines and Urban Buses - Exhaust

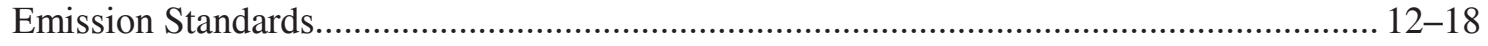

Heavy-Duty Highway Spark-Ignition Engines - Exhaust Emission Standards ......................... 12-20

Heavy-Duty Highway Compression-Ignition and Spark-Ignition Engines - Evaporative

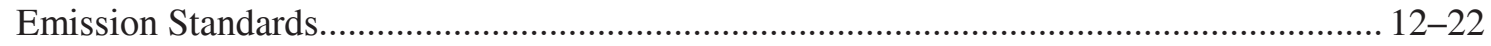

Highway, Nonroad, Locomotive, and Marine (NRLM) Diesel Fuel Sulfur Standards ............. 12-39

Household

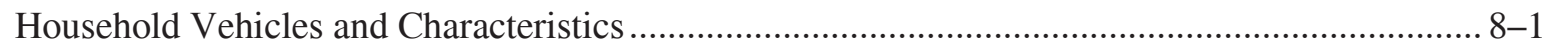

Annual Household Expenditures for Transportation, 1985-2012 ............................................ 8-5

Household Vehicle Ownership, 1960-2012 Census ................................................................... 8-6

Average Annual Vehicle-Miles, Vehicle Trips and Trip Length per Household 1969, 1977,

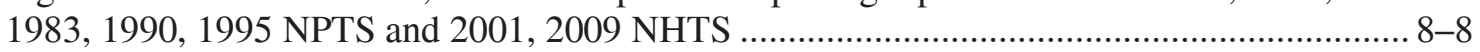

Average Number of Vehicles and Vehicle Travel per Household, 1990 NPTS and 2001

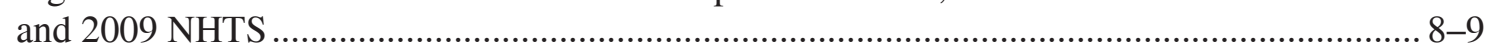

Average Annual Miles per Household Vehicle by Vehicle Age.................................................. 8 8-13

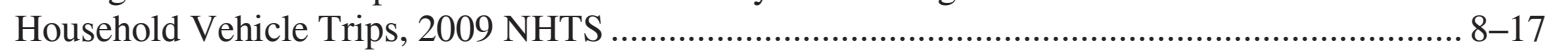

Daily and Annual Vehicle Miles of Travel and Average Age for Each Vehicle in a

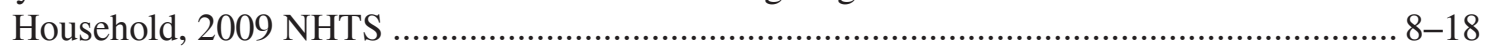

Daily Vehicle Miles of Travel (per Vehicle) by Number of Vehicles in the Household,

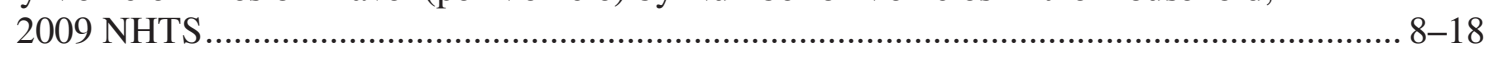

Annual Vehicle Miles of Travel for Each Vehicle in a Household, 2009 NHTS ....................... 8-19

Daily Vehicle Miles of Travel for Each Vehicle in a Household, 2009 NHTS .......................... 8-19

Households

Average Annual Expenditures of Households by Income, 2012 ............................................ 8-4

Housing

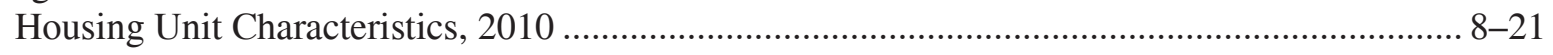

Hybrid

Hybrid and Plug-In Vehicle Sales, 1999-2013 ....................................................................... 6-9

Import

Production Sales, Production Shares, and Sales-Weighted Fuel Economies of New

Domestic and Import Cars, Selected Model Years 1975-2013 .............................................. 4-7

Production, Production Shares, and Production-Weighted Fuel Economies of New

Domestic and Import Light Trucks, Model Years 1975-2013 .............................................. 4-9

Production and Production-Weighted Fuel Economies of New Domestic and Import Cars,

Light Trucks, and Light Vehicles, Model Years 1975-2013 ................................................ 4-10 
Import (continued)

Production-Weighted Engine Size of New Domestic and Import Cars, Model Years 1975-2013

Production-Weighted Engine Size of New Domestic and Import Light Trucks, Model Years 1975-2013

Production-Weighted Curb Weight of New Domestic and Import Cars, Model Years 1975-2013

Average Price of a New Car (Domestic and Import), 1970-2012 ... $10-15$

Production-Weighted Annual Carbon Footprint of New Domestic and Import Cars, Model Years 1975-2013

Production-Weighted Annual Carbon Footprint of New Domestic and Import Light

Trucks, Model Years 1975-2013

Imported

Imported Crude Oil by Country of Origin, 1973-2013 $1-9$

Imports

U.S. Petroleum Imports, 1960-2013.... $1-8$

United States Petroleum Production, Imports and Exports, 1950-2013. $1-16$

Incentives

Federal and State Alternative Fuel Incentives, 2014 $10-12$

Federal and State Advanced Technology Incentives, 2014 $10-13$

Income

Average Annual Expenditures of Households by Income, 2012 $8-4$

Index

Transportation Services Index, January 1990-January 2014 $10-2$

Indices

Consumer Price Indices, 1970-2013. $10-18$

Input

U.S. Refinery Input of Crude Oil and Petroleum Products, 1987-2012 .................................... 1-14

Intensities

Energy Intensities of Highway Passenger Modes, 1970-2012 .............................................. 2-15

Energy Intensities of Nonhighway Passenger Modes, 1970-2012 ........................................... 2-16

Energy Intensities of Freight Modes, 1970-2012 ….............................................................. 2-19

Intensity

Energy Intensity of Light Rail Transit Systems, 2012 ............................................................ 2-17

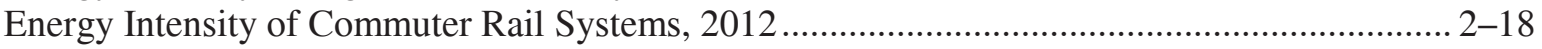

Energy Intensity of Heavy Rail Systems, 2012 ...................................................................... 2-18

Intermodal

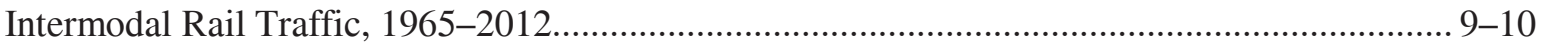

International

Summary Statistics for U.S. Domestic and International Certificated Route Air Carriers

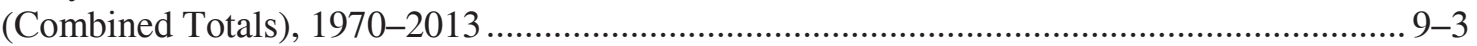

Tonnage Statistics for Domestic and International Waterborne Commerce, 1970-2012 ............. 9-5 
Japanese

Comparison of U.S., European, and Japanese Driving Cycles Attributes ................................. 4-36

Example of Differing Results Using the U.S., European, and Japanese Driving Cycles............. 4-36

Jet

Refiner Sales Prices for Aviation Gasoline and Jet Fuel, 1978-2013...................................... 10-10

Large

Nonroad Large Spark-Ignition Engines - Exhaust and Evaporative Emission Standards

Lawn

Fuel Consumption from Lawn and Garden Equipment, 2012 $2-12$

Length

Average Length of Time Commercial Fleet Vehicles are in Service, 2012. $7-3$

Average Annual Vehicle-Miles, Vehicle Trips and Trip Length per Household 1969, 1977, 1983, 1990, 1995 NPTS and 2001, 2009 NHTS .... $8-8$

Light

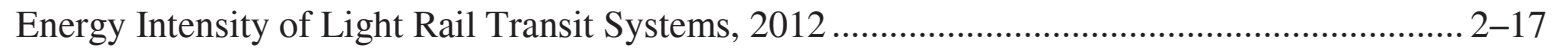

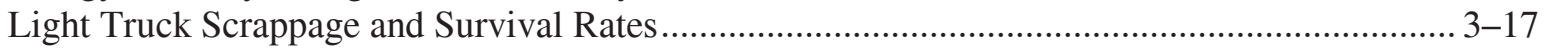

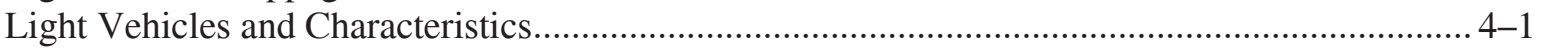

Sales Estimates of Class 1, Class 2a, and Class 2b Light Trucks, 1989-1999 ............................... 4-4

Summary Statistics on Class 1, Class 2a, and Class 2b Light Trucks......................................... 4-4

Production, Production Shares, and Production-Weighted Fuel Economies of New Domestic and Import Light Trucks, Model Years 1975-2013 .............................................. 4-9

Production and Production-Weighted Fuel Economies of New Domestic and Import Cars, Light Trucks, and Light Vehicles, Model Years 1975-2013 .............................................. 4-10

Light Vehicle Production Shares, Model Years 1975-2013 ........................................................ 4-12

Production-Weighted Engine Size of New Domestic and Import Light Trucks, Model

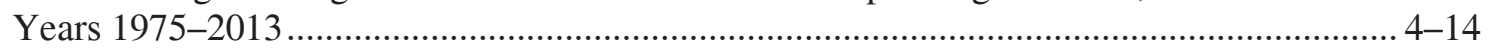

Average Material Consumption for a Domestic Light Vehicle, Model Years 1995, 2000,

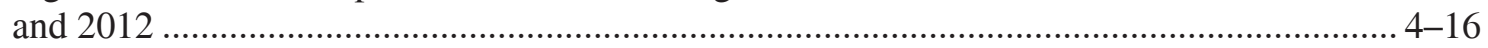

New Light Vehicle Dealerships and Sales, 1970-2012 ............................................................ 4-17

Light Truck Corporate Average Fuel Economy (CAFE) Standards versus Sales-Weighted

Fuel Economy Estimates, 1978-2013 ……......................................................................... 4-22

New Light Fleet Vehicle Registrations by Vehicle Type, Model Year 2012 ................................ 7-3

Production-Weighted Annual Carbon Footprint of New Domestic and Import Light

Trucks, Model Years 1975-2013 ...................................................................................... 11-14

Tier 3 Particulate Matter Emission Standards for Light Gasoline Vehicles, MY 2017

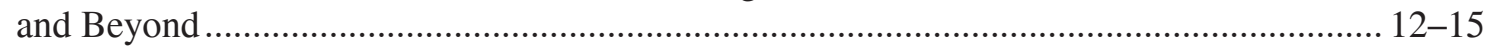

California Car, Light Truck and Medium Truck Emission Certification Standards .................. 12-23

Light-Duty

Light-Duty Vehicle, Light-Duty Truck, and Medium-Duty Passenger Vehicle - Tier 2

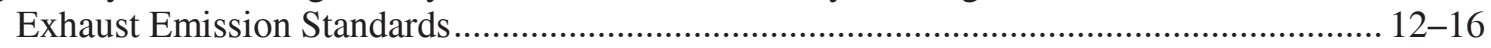

Light-Duty Vehicle, Light-Duty Truck, and Medium-Duty Passenger Vehicle - Tier 2

Evaporative Emission Standards ..................................................................................... 12-17

Liquid

Properties of Conventional and Alternative Liquid Fuels $6-16$ 
List

List of Model Year 2013 Cars with Gas Guzzler Taxes

Locomotive

Locomotives - Exhaust Emission Standards

Highway, Nonroad, Locomotive, and Marine (NRLM) Diesel Fuel Sulfur Standards

Long-Distance

Long-Distance Trip Characteristics, 2001 NHTS

Major

Percentage of Trucks by Size Ranked by Major Use, 2002 .................................................... 5-8

Share of Trucks by Major Use and Primary Fueling Facility, 2002 .......................................... 5-10

Manufacturer

E85 Flex-Fuel Vehicles Available by Manufacturer, Model Year 2014..................................... 6-5

Non-E85 Alternative Fuel Vehicles Available by Manufacturer, Model Year 2014 ..................... 6-7

Electric Drive Vehicles Available by Manufacturer, Model Year 2014 .................................... 6-10

Electric Drive Medium/Heavy Trucks and Buses Available by Manufacturer, 2014.................. 6-12

Manufacturing

U.S. Employment for Motor Vehicles and Motor Vehicle Parts Manufacturing, 1990-2013

Marine

Marine Compression-Ignition (CI) Engines - Exhaust Emission Standards ........................... 12-30

Marine Spark-Ignition Engines and Vessels - Exhaust Emission Standards ............................. 12-34

Highway, Nonroad, Locomotive, and Marine (NRLM) Diesel Fuel Sulfur Standards ............. 12-39

Material

Average Material Consumption for a Domestic Light Vehicle, Model Years 1995, 2000,

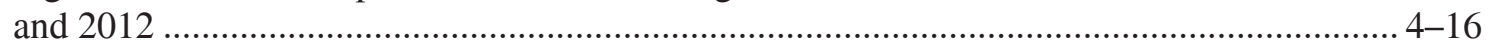

Matter

Total National Emissions of Particulate Matter (PM-10), 1970-2013 ..................................... 12-9

Total National Emissions of Particulate Matter (PM-2.5), 1990-2013 .................................... 12-11

Emissions of Particulate Matter (PM-2.5) from Highway Vehicles, 1990-2005 ...................... 12-12

Tier 3 Particulate Matter Emission Standards for Light Gasoline Vehicles, MY 2017 and Beyond

Mean

Truck Harmonic Mean Fuel Economy by Size Class, 1992, 1997, and 2002 ............................. 5-6

Means

Means of Transportation to Work, 1980, 1990, 2000 and 2012 ............................................. 8-20

Medium

Electric Drive Medium/Heavy Trucks and Buses Available by Manufacturer, 2014................. 6-12

California Car, Light Truck and Medium Truck Emission Certification Standards $12-23$

Medium-Duty

Light-Duty Vehicle, Light-Duty Truck, and Medium-Duty Passenger Vehicle - Tier 2

Exhaust Emission Standards $12-16$ 
Medium-Duty (continued)

Light-Duty Vehicle, Light-Duty Truck, and Medium-Duty Passenger Vehicle - Tier 2

Evaporative Emission Standards $12-17$

Mile

Car Operating Cost per Mile, 1985-2013 $10-16$

Miles

Growth of Ton Miles in the United States: Comparison of the 1997, 2002, 2007, and 2012

Commodity Flow Surveys $5-24$

Average Miles per Shipment in the United States: Comparison of the 1997, 2002, 2007,

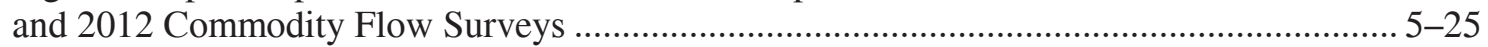

Average Miles per Domestic Federal Vehicle by Vehicle Type, 2012 …................................... 7-4

Average Annual Miles per Household Vehicle by Vehicle Age.................................................. 8-13

Self-Reported vs. Odometer Average Annual Miles, 1995 NPTS and 2001 NHTS................... 8-14

Share of Vehicles by Annual Miles of Travel and Vehicle Age, 2009 NHTS............................ 8-16

Average Daily Miles Driven (per Driver), 2009 NHTS.............................................................. 8-17

Daily and Annual Vehicle Miles of Travel and Average Age for Each Vehicle in a

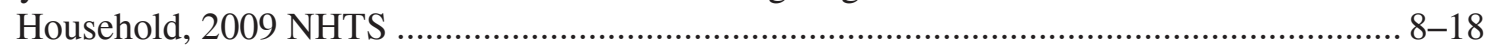

Daily Vehicle Miles of Travel (per Vehicle) by Number of Vehicles in the Household,

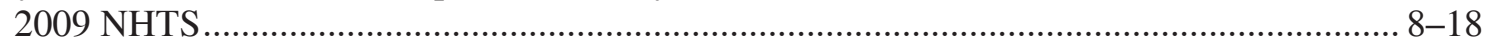

Annual Vehicle Miles of Travel for Each Vehicle in a Household, 2009 NHTS ....................... 8-19

Daily Vehicle Miles of Travel for Each Vehicle in a Household, 2009 NHTS ........................... 8-19

Mode

Highway Transportation Petroleum Consumption by Mode, 1970-2012.................................. 1-21

Nonhighway Transportation Petroleum Consumption by Mode, 1970-2012 ............................. 1-22

Transportation Petroleum Use by Mode, 2011-2012 .............................................................. 1-23

Domestic Consumption of Transportation Energy by Mode and Fuel Type, 2012 ...................... 2-7

Transportation Energy Use by Mode, 2011-2012 ................................................................ 2-8

Highway Transportation Energy Consumption by Mode, 1970-2012 ….................................. 2-9

Nonhighway Transportation Energy Consumption by Mode, 1970-2012 ............................... 2-10

Transportation Greenhouse Gas Emissions by Mode, 1990 and 2012 ...................................... 11-8

Model

Off-Highway Transportation-Related Fuel Consumption from the Nonroad Model, 2012 ......... 2-11

Car Scrappage and Survival Rates, 1970, 1980 and 1990 Model Years ...................................... 3-16

Production Sales, Production Shares, and Sales-Weighted Fuel Economies of New

Domestic and Import Cars, Selected Model Years 1975-2013 …........................................... 4-7

Definition of Car Sport Utility Vehicles in Model Year 2013 .................................................... 4-8

Production, Production Shares, and Production-Weighted Fuel Economies of New

Domestic and Import Light Trucks, Model Years 1975-2013 ............................................. 4-9

Production and Production-Weighted Fuel Economies of New Domestic and Import Cars,

Light Trucks, and Light Vehicles, Model Years 1975-2013 ............................................. 4-10

Light Vehicle Production Shares, Model Years 1975-2013 .................................................... 4-12

Production-Weighted Engine Size of New Domestic and Import Cars, Model Years

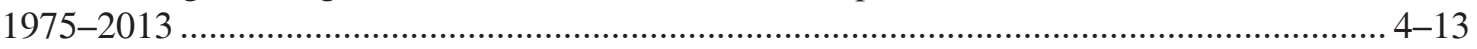

Production-Weighted Engine Size of New Domestic and Import Light Trucks, Model

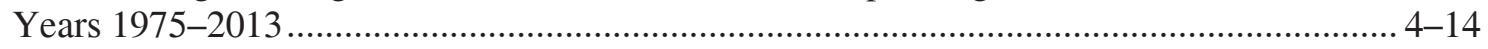

Production-Weighted Curb Weight of New Domestic and Import Cars, Model Years

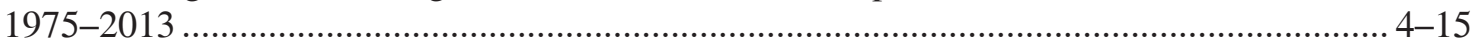

Average Material Consumption for a Domestic Light Vehicle, Model Years 1995, 2000,

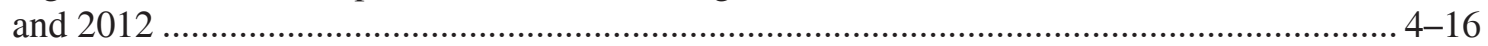


Model (continued)

Fuel Economy and Carbon Dioxide Emissions Standards, MY 2012-2025............................. 4-19

Fuel Economy and Carbon Dioxide Targets for Model Year 2025 ......................................... 4-20

List of Model Year 2013 Cars with Gas Guzzler Taxes ............................................................. 4-25

Fuel Economy by Speed, PSAT Model Results............................................................. 4-28

E85 Flex-Fuel Vehicles Available by Manufacturer, Model Year 2014...................................... 6-5

Non-E85 Alternative Fuel Vehicles Available by Manufacturer, Model Year 2014 ..................... 6-7

Electric Drive Vehicles Available by Manufacturer, Model Year 2014 …................................ 6-10

New Light Fleet Vehicle Registrations by Vehicle Type, Model Year 2012 ................................ 7-3

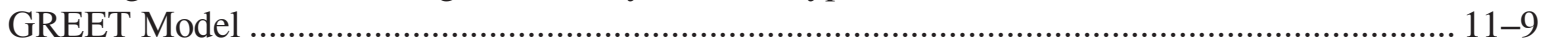

GREET Model Feedstocks and Fuels .............................................................................. 11-10

Production-Weighted Annual Carbon Footprint of New Domestic and Import Cars,

Model Years 1975-2013 ................................................................................................... 11-13

Production-Weighted Annual Carbon Footprint of New Domestic and Import Light

Trucks, Model Years 1975-2013 …................................................................................. 11-14

Tier 3 Particulate Matter Emission Standards for Light Gasoline Vehicles, MY 2017

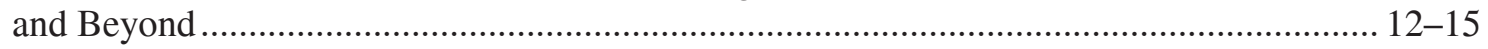

Modes

Energy Intensities of Highway Passenger Modes, 1970-2012 ............................................... 2-15

Energy Intensities of Nonhighway Passenger Modes, 1970-2012 …...................................... 2-16

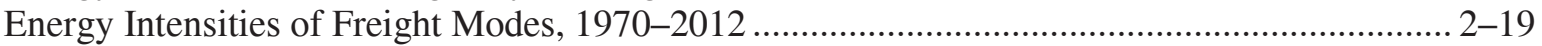

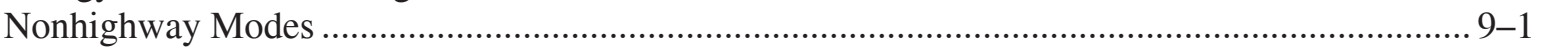

Motor

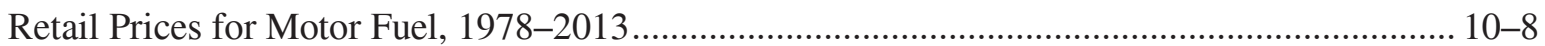

Federal Excise Taxes on Motor Fuels, 2011 .......................................................................... 10-11

U.S. Employment for Motor Vehicles and Motor Vehicle Parts Manufacturing,

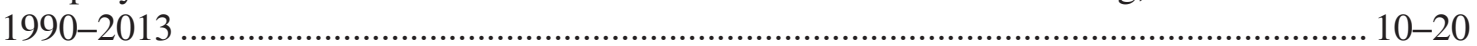

National

Summary Statistics for the National Railroad Passenger Corporation (Amtrak),

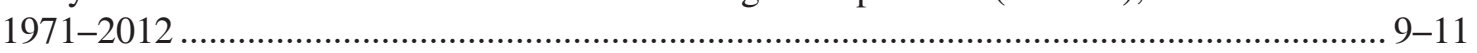

Total National Emissions of the Criteria Air Pollutants by Sector, 2013 .................................. 12-2

Total National Emissions of Carbon Monoxide, 1970-2013 ...................................................... 12-3

Total National Emissions of Nitrogen Oxides, 1970-2013 ...................................................... 12-5

Total National Emissions of Volatile Organic Compounds, 1970-2013 .................................... 12-7

Total National Emissions of Particulate Matter (PM-10), 1970-2013 ….................................. 12-9

Total National Emissions of Particulate Matter (PM-2.5), 1990-2013 ..................................... 12-11

Natural

Proved Reserves of Crude Oil and Natural Gas, 1980-2012 ................................................... 1-2

World Natural Gas Reserves, Production and Consumption, 2012 ............................................ 1-7

Top Ten Natural Gas Producing Countries, 2012 ............................................................... 6-15

New

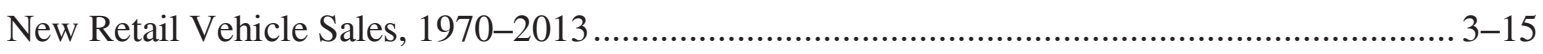

New Retail Car Sales in the United States, 1970-2013 …......................................................... 4-5

New Retail Sales of Trucks 10,000 Pounds GVW and Less in the United States, 1970-2013 ..... 4-6

Production Sales, Production Shares, and Sales-Weighted Fuel Economies of New

Domestic and Import Cars, Selected Model Years 1975-2013 ............................................. 4-7

Production, Production Shares, and Production-Weighted Fuel Economies of New

Domestic and Import Light Trucks, Model Years 1975-2013 ................................................ 4-9 
New (continued)

Production and Production-Weighted Fuel Economies of New Domestic and Import Cars,

Light Trucks, and Light Vehicles, Model Years 1975-2013 ................................................ 4-10

Production-Weighted Engine Size of New Domestic and Import Cars, Model Years

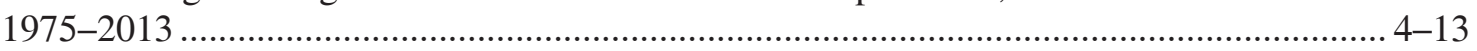

Production-Weighted Engine Size of New Domestic and Import Light Trucks, Model

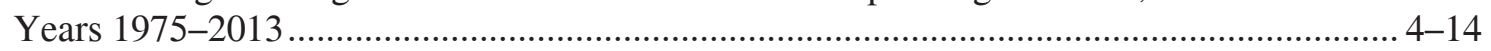

Production-Weighted Curb Weight of New Domestic and Import Cars, Model Years

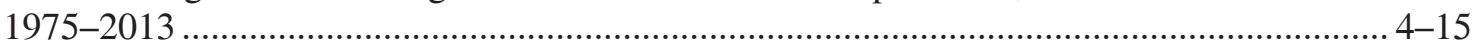

New Light Vehicle Dealerships and Sales, 1970-2012 ........................................................ 4-17

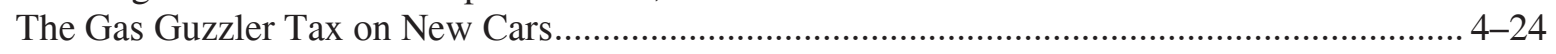

New Retail Truck Sales by Gross Vehicle Weight, 1970-2013 ............................................... 5-4

New Light Fleet Vehicle Registrations by Vehicle Type, Model Year 2012 .............................. 7-3

Average Price of a New Car, 1913-2012 ................................................................................. 10-14

Average Price of a New Car (Domestic and Import), 1970-2012 …...................................... 10-15

Production-Weighted Annual Carbon Footprint of New Domestic and Import Cars,

Model Years 1975-2013 .............................................................................................. 11-13

Production-Weighted Annual Carbon Footprint of New Domestic and Import Light

Trucks, Model Years 1975-2013 …........................................................................................ 11-14

New York

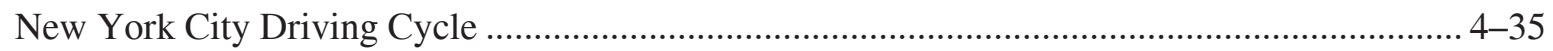

NHTS

Demographic Statistics from the 1969, 1977, 1983, 1990, 1995 NPTS and 2001,

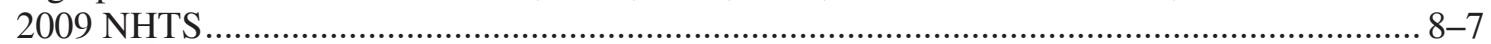

Average Annual Vehicle-Miles, Vehicle Trips and Trip Length per Household 1969, 1977,

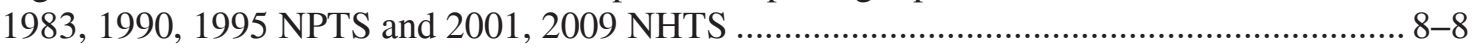

Average Number of Vehicles and Vehicle Travel per Household, 1990 NPTS and 2001

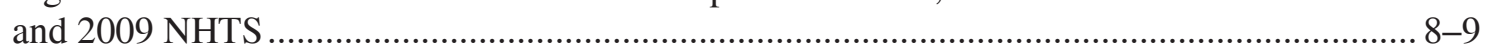

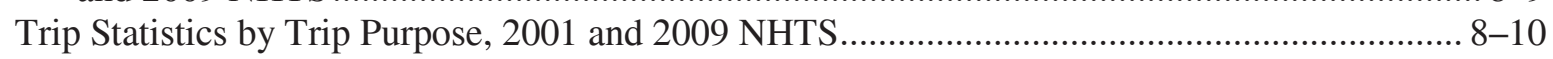

Average Vehicle Occupancy by Vehicle Type, 1995 NPTS and 2009 NHTS ......................... 8-11

Average Vehicle Occupancy by Trip Purpose, 1977 NPTS and 2009 NHTS ........................... 8-12

Self-Reported vs. Odometer Average Annual Miles, 1995 NPTS and 2001 NHTS.................... 8-14

Share of Vehicle Trips by Trip Distance, 2009 NHTS ............................................................ 8-15

Share of Vehicle Trips to Work by Trip Distance, 2009 NHTS ............................................. 8-15

Share of Vehicles by Annual Miles of Travel and Vehicle Age, 2009 NHTS............................ 8-16

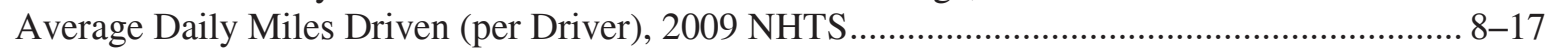

Household Vehicle Trips, 2009 NHTS ......................................................................... 8-17

Daily and Annual Vehicle Miles of Travel and Average Age for Each Vehicle in a

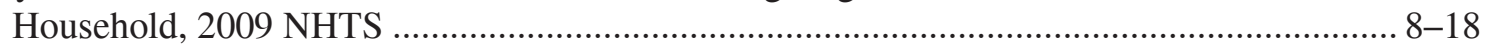

Daily Vehicle Miles of Travel (per Vehicle) by Number of Vehicles in the Household,

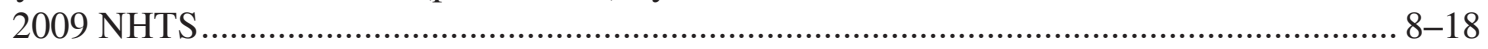

Annual Vehicle Miles of Travel for Each Vehicle in a Household, 2009 NHTS ....................... 8-19

Daily Vehicle Miles of Travel for Each Vehicle in a Household, 2009 NHTS ......................... 8-19

Walk and Bike Trips by Trip Purpose, 2009 NHTS …................................................................ 8-24

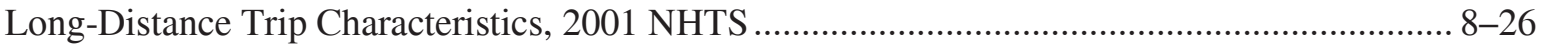

Nitrogen

Tier 3 Non-Methane Organic Gases and Nitrogen Oxide Standards ...................................... 12-14 
Nitrogen Oxides

Total National Emissions of Nitrogen Oxides, 1970-2013 ................................................... 12-5

Emissions of Nitrogen Oxides from Highway Vehicles, 1970-2005 ....................................... 12-6

Non-E85

Non-E85 Alternative Fuel Vehicles Available by Manufacturer, Model Year 2014 .................... 6-7

Nonhighway

Nonhighway Transportation Petroleum Consumption by Mode, 1970-2012 …......................... 1-22

Nonhighway Transportation Energy Consumption by Mode, 1970-2012 …........................... 2-10

Energy Intensities of Nonhighway Passenger Modes, 1970-2012 …........................................ 2-16

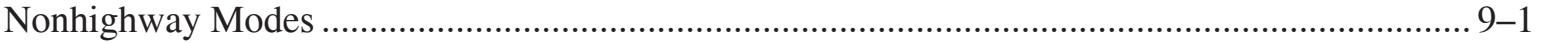

Nonhighway Energy Use Shares, 1970-2012 ........................................................................... 9-2

Non-Methane

Tier 3 Non-Methane Organic Gases and Nitrogen Oxide Standards ...................................... 12-14

Nonroad

Off-Highway Transportation-Related Fuel Consumption from the Nonroad Model, 2012 ......... 2-11

Nonroad Compression-Ignition Engines - Exhaust Emission Standards................................ 12-25

Nonroad Large Spark-Ignition Engines - Exhaust and Evaporative Emission Standards .......... 12-27

Nonroad Recreational Engines and Vehicles - Exhaust Emission Standards........................... 12-36

Highway, Nonroad, Locomotive, and Marine (NRLM) Diesel Fuel Sulfur Standards .............. 12-39

NPTS

Demographic Statistics from the 1969, 1977, 1983, 1990, 1995 NPTS and 2001,

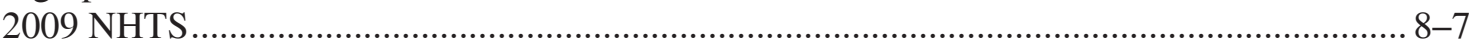

Average Annual Vehicle-Miles, Vehicle Trips and Trip Length per Household 1969, 1977,

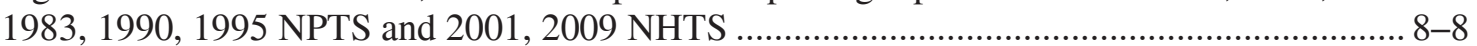

Average Number of Vehicles and Vehicle Travel per Household, 1990 NPTS and 2001

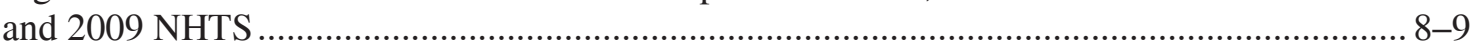

Average Vehicle Occupancy by Vehicle Type, 1995 NPTS and 2009 NHTS .......................... 8-11

Average Vehicle Occupancy by Trip Purpose, 1977 NPTS and 2009 NHTS ............................ 8-12

Self-Reported vs. Odometer Average Annual Miles, 1995 NPTS and 2001 NHTS.................... 8-14

NRLM

Highway, Nonroad, Locomotive, and Marine (NRLM) Diesel Fuel Sulfur Standards ............. 12-39

Number

Representative Number Five Driving Cycle ............................................................................ 4-35

Number of Alternative Refuel Sites by State and Fuel Type, 2014 ........................................ 6-13

Average Number of Vehicles and Vehicle Travel per Household, 1990 NPTS and 2001

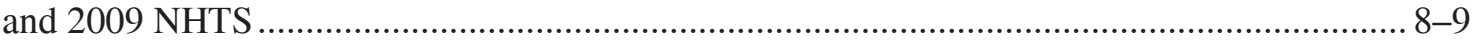

Daily Vehicle Miles of Travel (per Vehicle) by Number of Vehicles in the Household, 2009 NHTS

Refiner Sales Prices for Propane and No. 2 Diesel, 1978-2013 $10-9$

Numerical

Numerical Estimates of Global Warming Potentials Compared with Carbon Dioxide $11-3$

Occupancy

Average Vehicle Occupancy by Vehicle Type, 1995 NPTS and 2009 NHTS $8-11$

Average Vehicle Occupancy by Trip Purpose, 1977 NPTS and 2009 NHTS $8-12$ 
Odometer

Self-Reported vs. Odometer Average Annual Miles, 1995 NPTS and 2001 NHTS. $8-14$

Off-Highway

Off-Highway Transportation-Related Fuel Consumption from the Nonroad Model, 2012 ......... 2-11

Oil

Proved Reserves of Crude Oil and Natural Gas, 1980-2012 ................................................... 1-2

World Crude Oil Production, 1960-2013 ..................................................................................... 1-3

World Oil Reserves, Production and Consumption, 2012 ....................................................... 1-6

Imported Crude Oil by Country of Origin, 1973-2013 .......................................................... 1-9

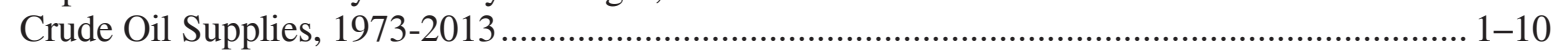

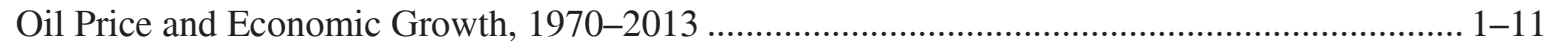

Costs of Oil Dependence to the U.S. Economy, 1970-2012 ................................................. 1-12

U.S. Refinery Input of Crude Oil and Petroleum Products, 1987-2012 .................................... 1-14

U.S. Refinery Yield of Petroleum Products from a Barrel of Crude Oil, 1978-2013................. 1-15

Prices for a Barrel of Crude Oil and a Gallon of Gasoline, 1978-2013 ...................................... 10-7

On-Road

Distribution of Class 8 Trucks by On-Road Vehicle Weight, 2008 ......................................... 5-20

Operating

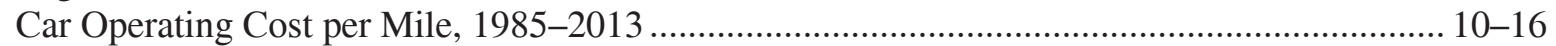

Fixed Car Operating Costs per Year, 1975-2013 .................................................................. 10-17

Operation

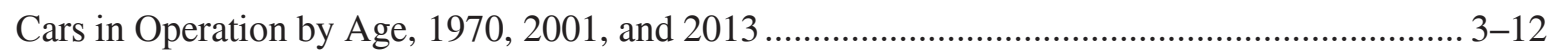

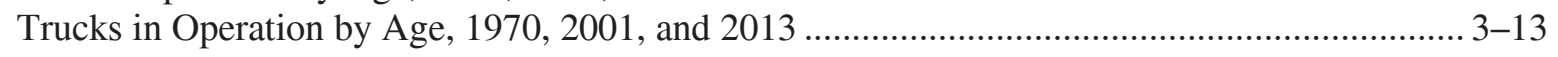

Operations

Summary Statistics for Commuter Rail Operations, 1984-2012 …....................................... 9-12

Summary Statistics for Rail Transit Operations, 1970-2012 ................................................... 9-13

Organic

Total National Emissions of Volatile Organic Compounds, 1970-2013 ................................... 12-7

Emissions of Volatile Organic Compounds from Highway Vehicles, 1970-2005 ..................... 12-8

Tier 3 Non-Methane Organic Gases and Nitrogen Oxide Standards ....................................... 12-14

Origin

Imported Crude Oil by Country of Origin, 1973-2013 .......................................................... 1-9

Other

Vehicles per Thousand People: U.S. (Over Time) Compared to Other Countries (in 2002

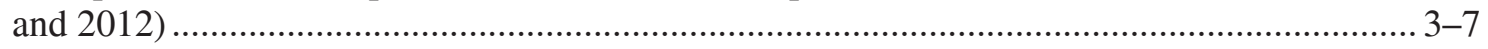

Vehicles per Thousand People in Other Countries, 2002 and 2012 ......................................... 3-9

Output

Refinery Gross Output by World Region, 2003 and 2013 ..................................................... 1-13

Ownership

Household Vehicle Ownership, 1960-2012 Census ................................................................... 8-6 
Oxide

Tier 3 Non-Methane Organic Gases and Nitrogen Oxide Standards $12-14$

Oxygenate

Alternative Fuel and Oxygenate Consumption, 2005-2011 $2-5$

Particulate

Total National Emissions of Particulate Matter (PM-10), 1970-2013 $12-9$

Total National Emissions of Particulate Matter (PM-2.5), 1990-2013 $12-11$

Emissions of Particulate Matter (PM-2.5) from Highway Vehicles, 1990-2005 .... $12-12$

Tier 3 Particulate Matter Emission Standards for Light Gasoline Vehicles, MY 2017 and Beyond $12-15$

Parts

U.S. Employment for Motor Vehicles and Motor Vehicle Parts Manufacturing, 1990-2013 $10-20$

Passenger

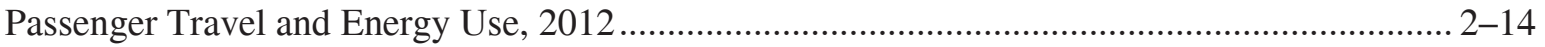

Energy Intensities of Highway Passenger Modes, 1970-2012 …........................................... 2-15

Energy Intensities of Nonhighway Passenger Modes, 1970-2012 ........................................... 2-16

Summary Statistics for the National Railroad Passenger Corporation (Amtrak),

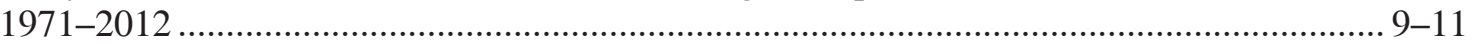

Light-Duty Vehicle, Light-Duty Truck, and Medium-Duty Passenger Vehicle - Tier 2 Exhaust Emission Standards ..................................................................................................... 12-16

Light-Duty Vehicle, Light-Duty Truck, and Medium-Duty Passenger Vehicle - Tier 2 Evaporative Emission Standards ..................................................................................... 12-17

People

Vehicles per Thousand People: U.S. (Over Time) Compared to Other Countries (in 2002

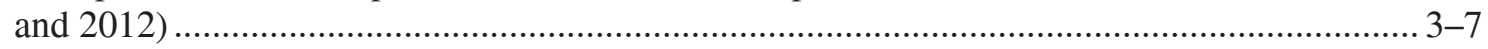

Vehicles per Thousand People in Other Countries, 2002 and 2012 2....................................... 3-9

Vehicles per Thousand People in the United States, 1990-2012 ............................................... 3-10

Per Capita

Vehicles and Vehicle-Miles per Capita, 1950-2012 …............................................................. 8-3

Percent

Class 8 Truck Percent of Total Fuel Consumed as a Function of Speed and Tractor-Trailer

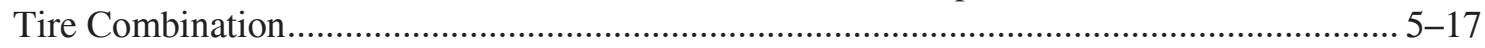

Percentage

Percentage of Trucks by Size Ranked by Major Use, 2002 .................................................... 5-8

Percentage of Trucks by Fleet Size and Primary Fueling Facility, 2002 ................................... 5-9

Class 8 Truck Fuel Economy as a Function of Speed and Tractor-Trailer Tire Combination and Percentage of Total Distance Traveled as a Function of Speed ...................................... 5-16

Personal

Personal Consumption Expenditures, 1970-2013 ................................................................. 10-18

Petroleum

World Petroleum Production, 1973-2013 ….......................................................................... 1-4

World Petroleum Consumption, 1960-2012 ......................................................................... 1-5 
Petroleum (continued)

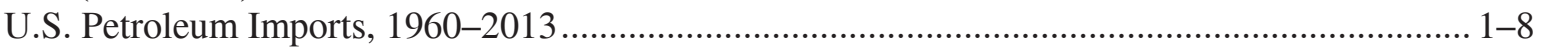

U.S. Refinery Input of Crude Oil and Petroleum Products, 1987-2012 ................................... 1-14

U.S. Refinery Yield of Petroleum Products from a Barrel of Crude Oil, 1978-2013.................. 1-15

United States Petroleum Production, Imports and Exports, 1950-2013 ..................................... 1-16

Petroleum Production and Transportation Petroleum Consumption in Context, 1950-2013 ....... 1-17

United States Petroleum Production and Consumption - All Sectors, 1973-2040 .................... 1-18

United States Petroleum Production, and Transportation Consumption, 1970-2040.................. 1-19

Consumption of Petroleum by End-Use Sector, 1973-2013 ................................................... 1-20

Highway Transportation Petroleum Consumption by Mode, 1970-2012................................ 1-21

Nonhighway Transportation Petroleum Consumption by Mode, 1970-2012 ............................ 1-22

Transportation Petroleum Use by Mode, 2011-2012 ................................................................ 1-23

Plug-In

Hybrid and Plug-In Vehicle Sales, 1999-2013 _........................................................................... 6-9

PM-10

Total National Emissions of Particulate Matter (PM-10), 1970-2013 …................................... 12-9

PM-2.5

Total National Emissions of Particulate Matter (PM-2.5), 1990-2013 .................................... 12-11

Emissions of Particulate Matter (PM-2.5) from Highway Vehicles, 1990-2005 ...................... 12-12

Pollutants

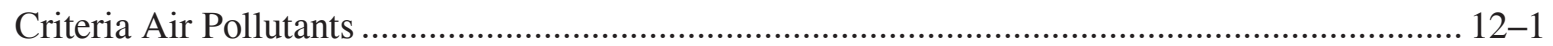

Total National Emissions of the Criteria Air Pollutants by Sector, 2013 ................................. 12-2

Population

Population and Vehicle Profile, 1950-2012 ............................................................................... 8-2

Potential

Numerical Estimates of Global Warming Potentials Compared with Carbon Dioxide ................ 11-3

U.S. Emissions of Greenhouse Gases, based on Global Warming Potential, 1990-2012 ........... 11-4

Pounds

Distribution of Trucks over 26,000 lbs. by Vehicle-Miles Traveled .......................................... 5-11

Price

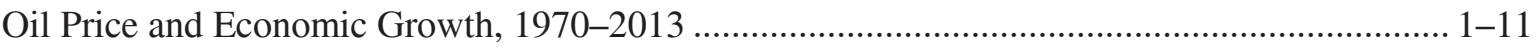

Average Price of a New Car, 1913-2012 ............................................................................ 10-14

Average Price of a New Car (Domestic and Import), 1970-2012 ......................................... 10-15

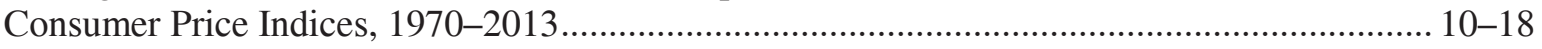

Prices

Gasoline Prices for Selected Countries, 1990-2013 .............................................................. 10-3

Diesel Fuel Prices for Selected Countries, 1998-2013 ........................................................ 10-4

Gasoline Prices for Selected Countries, 1990 and 2013 ......................................................... 10-5

Diesel Prices for Selected Countries, 1990 and 2013 ............................................................ 10-6

Prices for a Barrel of Crude Oil and a Gallon of Gasoline, 1978-2013 ...................................... 10-7

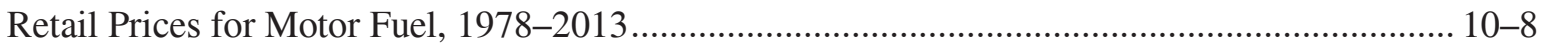

Refiner Sales Prices for Propane and No. 2 Diesel, 1978-2013 ............................................. 10-9

Refiner Sales Prices for Aviation Gasoline and Jet Fuel, 1978-2013 ....................................... 10-10 
Primary

World Consumption of Primary Energy, 2011 ........................................................................... 2-2

Percentage of Trucks by Fleet Size and Primary Fueling Facility, 2002 ....................................5-9

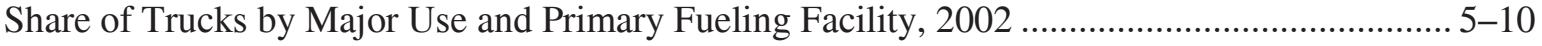

Producing

Top Ten Natural Gas Producing Countries, 2012 .................................................................... 6-15

Production

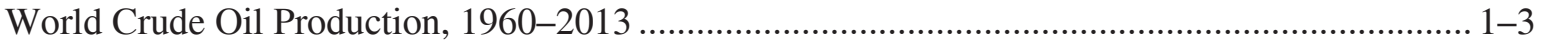

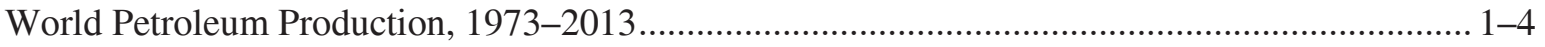

World Oil Reserves, Production and Consumption, 2012 ...................................................... 1-6

World Natural Gas Reserves, Production and Consumption, 2012 ........................................... 1-7

United States Petroleum Production, Imports and Exports, 1950-2013 …............................... 1-16

Petroleum Production and Transportation Petroleum Consumption in Context, 1950-2013 ....... 1-17

United States Petroleum Production and Consumption - All Sectors, 1973-2040 .................... 1-18

United States Petroleum Production, and Transportation Consumption, 1970-2040.................. 1-19

World Production of Cars and Trucks, 2000-2012 …............................................................... 3-2

Production Sales, Production Shares, and Production-Weighted Fuel Economies of New

Domestic and Import Cars, Selected Model Years 1975-2013 ............................................ 4-7

Light Vehicle Production Shares, Model Years 1975-2013 ..................................................... 4-12

Production-Weighted

Production, Production Shares, and Production-Weighted Fuel Economies of New

Domestic and Import Light Trucks, Model Years 1975-2013 ................................................. 4-9

Production-Weighted Engine Size of New Domestic and Import Cars, Model Years

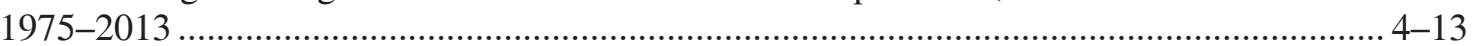

Production-Weighted Engine Size of New Domestic and Import Light Trucks, Model

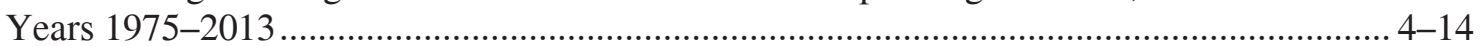

Production-Weighted Curb Weight of New Domestic and Import Cars, Model Years

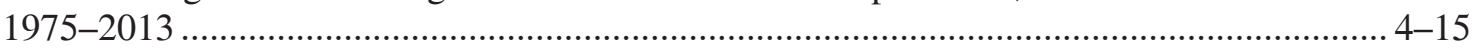

Production-Weighted Annual Carbon Footprint of New Domestic and Import Cars, Model Years 1975-2013 ................................................................................................... 11-13

Production-Weighted Annual Carbon Footprint of New Domestic and Import Light

Trucks, Model Years 1975-2013 ................................................................................................ 11-14

Products

U.S. Refinery Input of Crude Oil and Petroleum Products, 1987-2012 ................................... 1-14

U.S. Refinery Yield of Petroleum Products from a Barrel of Crude Oil, 1978-2013.................. 1-15

Profile

Population and Vehicle Profile, 1950-2012 ….......................................................................... 8-2

Propane

Refiner Sales Prices for Propane and No. 2 Diesel, 1978-2013 ............................................... 10-9

Properties

Properties of Conventional and Alternative Liquid Fuels ....................................................... 6-16

Properties of Conventional and Alternative Gaseous Fuels ..................................................... 6-17

Proved

Proved Reserves of Crude Oil and Natural Gas, 1980-2012 .................................................... 1-2 
PSAT

Fuel Economy by Speed, PSAT Model Results............................................................... 4-28

Purpose

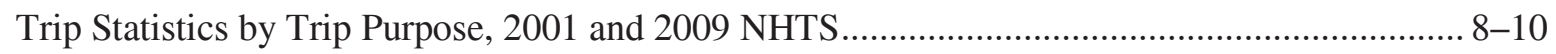

Average Vehicle Occupancy by Trip Purpose, 1977 NPTS and 2009 NHTS ............................. 8-12

Walk and Bike Trips by Trip Purpose, 2009 NHTS ...................................................................... 8-24

Rail

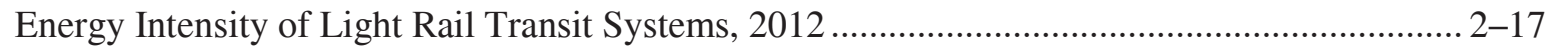

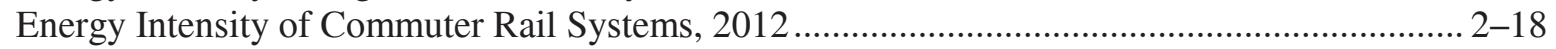

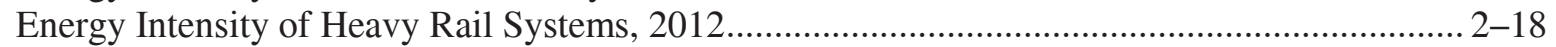

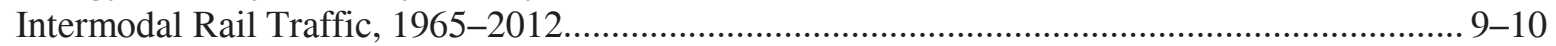

Summary Statistics for Commuter Rail Operations, 1984-2012 …......................................... 9-12

Summary Statistics for Rail Transit Operations, 1970-2012 .................................................... 9-13

Railroad

Class I Railroad Freight Systems in the United States Ranked by Revenue Ton-Miles, 2012 ...... 9-8

Summary Statistics for the National Railroad Passenger Corporation (Amtrak),

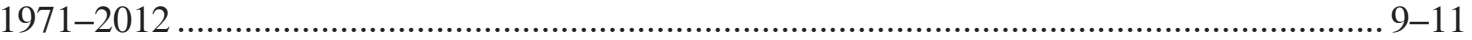

Railroads

Summary Statistics for Class I Freight Railroads, 1970-2012 _.............................................. 9-9

Ranked

Percentage of Trucks by Size Ranked by Major Use, 2002 ..................................................... 5-8

Class I Railroad Freight Systems in the United States Ranked by Revenue Ton-Miles, 2012 ...... 9-8

Rates

Car Scrappage and Survival Rates, 1970, 1980 and 1990 Model Years................................... 3-16

Light Truck Scrappage and Survival Rates........................................................................... 3-17

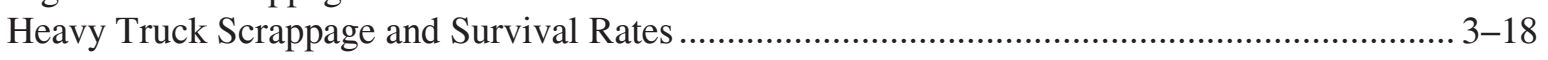

Receipts

Tax Receipts from the Sale of Gas Guzzlers, 1980-2012 ...................................................... 4-27

Recreational

Recreational Boat Energy Use, 1970-2012 …........................................................................... 9-7

Nonroad Recreational Engines and Vehicles - Exhaust Emission Standards........................... 12-36

Refiner

Refiner Sales Prices for Propane and No. 2 Diesel, 1978-2013 ............................................. 10-9

Refiner Sales Prices for Aviation Gasoline and Jet Fuel, 1978-2013 ....................................... 10-10

Refinery

Refinery Gross Output by World Region, 2003 and 2013 ...................................................... 1-13

U.S. Refinery Input of Crude Oil and Petroleum Products, 1987-2012 .................................... 1-14

U.S. Refinery Yield of Petroleum Products from a Barrel of Crude Oil, 1978-2013.................. 1-15

Refuel

Number of Alternative Refuel Sites by State and Fuel Type, 2014 ....................................... 6-13 
Refueling

Conventional Refueling Stations, 1993-2012 ....................................................................... 4-18

Region

Refinery Gross Output by World Region, 2003 and 2013 ..................................................... 1-13

Registrations

Car Registrations for Selected Countries, 1960-2012 …......................................................... 3-3

Truck and Bus Registrations for Selected Countries, 1960-2012 .............................................. 3-4

New Light Fleet Vehicle Registrations by Vehicle Type, Model Year 2012 ............................... 7-3

Representative

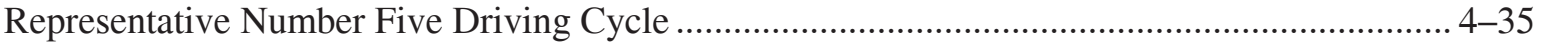

Reserves

Proved Reserves of Crude Oil and Natural Gas, 1980-2012 …............................................. 1-2

World Oil Reserves, Production and Consumption, 2012 ....................................................... 1-6

World Natural Gas Reserves, Production and Consumption, 2012 ............................................ 1-7

Response

Summary Statistics on Demand Response Vehicles, 1994-2012 ............................................ 4-37

Results

Fuel Economy by Speed, PSAT Model Results................................................................. 4-28

Example of Differing Results Using the U.S., European, and Japanese Driving Cycles............. 4-36

Retail

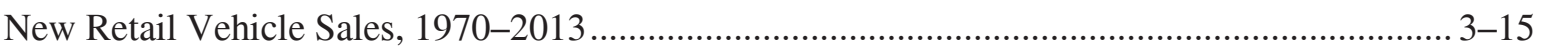

New Retail Car Sales in the United States, 1970-2013 .............................................................. 4-5

New Retail Sales of Trucks 10,000 Pounds GVW and Less in the United States, 1970-2013 ...... 4-6

New Retail Truck Sales by Gross Vehicle Weight, 1970-2013 ….......................................... 5-4

Retail Prices for Motor Fuel, 1978-2013 _............................................................................ 10-8

Revenue

Class I Railroad Freight Systems in the United States Ranked by Revenue Ton-Miles, 2012 ...... 9-8

Route

Summary Statistics for U.S. Domestic and International Certificated Route Air Carriers

(Combined Totals), 1970-2013 ............................................................................................... 9-3

Sale

Tax Receipts from the Sale of Gas Guzzlers, 1980-2012 ........................................................ 4-27

Sales

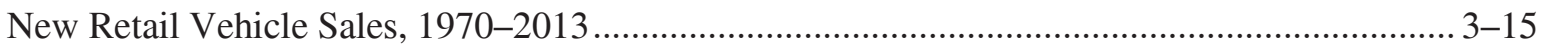

Sales Estimates of Class 1, Class 2a, and Class 2b Light Trucks, 1989-1999 …........................... 4-4

New Retail Car Sales in the United States, 1970-2013 …......................................................... 4-5

New Retail Sales of Trucks 10,000 Pounds GVW and Less in the United States, 1970-2013 ..... 4-6

Production Sales, Production Shares, and Sales-Weighted Fuel Economies of New

Domestic and Import Cars, Selected Model Years 1975-2013 ............................................ 4-7

New Light Vehicle Dealerships and Sales, 1970-2012 …......................................................17

New Retail Truck Sales by Gross Vehicle Weight, 1970-2013 ................................................ 5-4

Hybrid and Plug-In Vehicle Sales, 1999-2013 .......................................................................... 6-9 
Sales (continued)

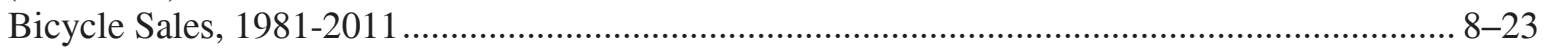

Refiner Sales Prices for Propane and No. 2 Diesel, 1978-2013 .............................................. 10-9

Refiner Sales Prices for Aviation Gasoline and Jet Fuel, 1978-2013 ....................................... 10-10

Sales-Weighted

Production Sales, Production Shares, and Sales-Weighted Fuel Economies of New

Domestic and Import Cars, Selected Model Years 1975-2013 ............................................. 4-7

Car Corporate Average Fuel Economy (CAFE) Standards versus Sales-Weighted Fuel

Economy Estimates, 1978-2013

Light Truck Corporate Average Fuel Economy (CAFE) Standards versus Sales-Weighted

Fuel Economy Estimates, 1978-2013 ................................................................................ 4-22

SC03

Air Conditioning (SC03) Driving Cycle 4-33

Scrappage

Car Scrappage and Survival Rates, 1970, 1980 and 1990 Model Years .................................... 3-16

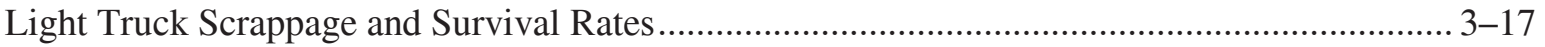

Heavy Truck Scrappage and Survival Rates ........................................................................... 3-18

Sector

Consumption of Petroleum by End-Use Sector, 1973-2013 .................................................... 1-20

U.S. Consumption of Total Energy by End-Use Sector, 1973-2013 ......................................... 2-3

Total U.S. Greenhouse Gas Emissions by End-Use Sector, 2012 ............................................ 11-5

U.S. Carbon Emissions from Fossil Fuel Consumption by End-Use Sector, 1990-2012............ 11-6

U.S. Carbon Emissions from Fossil Fuel Combustion in the Transportation End-Use

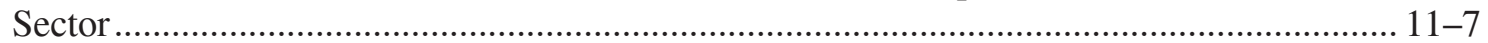

Total National Emissions of the Criteria Air Pollutants by Sector, 2013 .................................. 12-2

Sectors

United States Petroleum Production and Consumption - All Sectors, 1973-2040 .................... 1-18

Selected

Car Registrations for Selected Countries, 1960-2012 …........................................................ 3-3

Truck and Bus Registrations for Selected Countries, 1960-2012 ................................................ 3-4

Production Sales, Production Shares, and Sales-Weighted Fuel Economies of New

Domestic and Import Cars, Selected Model Years 1975-2013 ............................................. 4-7

Share of Heavy Trucks with Selected Electronic Features, 2002 ............................................ 5-12

Gasoline Prices for Selected Countries, 1990-2013 .............................................................. 10-3

Diesel Fuel Prices for Selected Countries, 1998-2013 ........................................................ 10-4

Gasoline Prices for Selected Countries, 1990 and 2013 ...................................................... 10-5

Diesel Prices for Selected Countries, 1990 and 2013 .......................................................... 10-6

Self-Reported

Self-Reported vs. Odometer Average Annual Miles, 1995 NPTS and 2001 NHTS.................... 8-14

Service

Fleet Vehicles in Service as of January 1, 2013 ......................................................................

Average Length of Time Commercial Fleet Vehicles are in Service, 2012 .................................. 7-3

Services

Transportation Services Index, January 1990-January 2014 $10-2$ 
Share

Share of Trucks by Major Use and Primary Fueling Facility, 2002 ......................................... 5-10

Share of Heavy Trucks with Selected Electronic Features, 2002 ............................................ 5-12

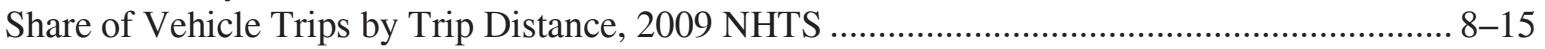

Share of Vehicle Trips to Work by Trip Distance, 2009 NHTS ................................................ 8-15

Share of Vehicles by Annual Miles of Travel and Vehicle Age, 2009 NHTS............................ 8-16

Shares

Shares of Highway Vehicle-Miles Traveled by Vehicle Type, 1970-2012 ................................ 3-11

Production Sales, Production Shares, and Sales-Weighted Fuel Economies of New

Domestic and Import Cars, Selected Model Years 1975-2013 _............................................. 4-7

Production, Production Shares, and Production-Weighted Fuel Economies of New

Domestic and Import Light Trucks, Model Years 1975-2013 …........................................ 4-9

Light Vehicle Production Shares, Model Years 1975-2013 …................................................ 4-12

Nonhighway Energy Use Shares, 1970-2012 ............................................................................. 9-2

Shipment

Average Miles per Shipment in the United States: Comparison of the 1997, 2002, 2007, and 2012 Commodity Flow Surveys

Single-Unit

Summary Statistics for Class 3-8 Single-Unit Trucks, 1970-2012 _............................................. 5-2

Sites

Number of Alternative Refuel Sites by State and Fuel Type, 2014 $6-13$

Size

Production-Weighted Engine Size of New Domestic and Import Cars, Model Years 1975-2013 $4-13$

Production-Weighted Engine Size of New Domestic and Import Light Trucks, Model

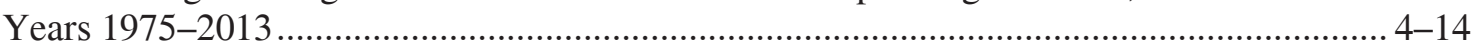

Truck Harmonic Mean Fuel Economy by Size Class, 1992, 1997, and 2002 ........................... 5-6

Truck Statistics by Size, 2002 ............................................................................................ $5-7$

Percentage of Trucks by Size Ranked by Major Use, 2002 ................................................... 5-8

Percentage of Trucks by Fleet Size and Primary Fueling Facility, 2002 ................................... 5-9

Source

Distribution of Energy Consumption by Source, 1973 and 2013 ............................................ 2-4

Spark-Ignition

Heavy-Duty Highway Spark-Ignition Engines - Exhaust Emission Standards........................ 12-20

Heavy-Duty Highway Compression-Ignition and Spark-Ignition Engines - Evaporative

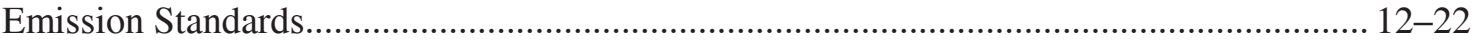

Nonroad Large Spark-Ignition Engines - Exhaust and Evaporative Emission Standards .......... 12-27

Marine Spark-Ignition Engines and Vessels - Exhaust Emission Standards............................ 12-34

Speed

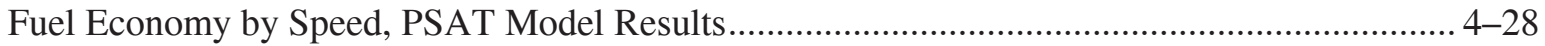

Fuel Economy by Speed, 1973, 1984, 1997, and 2012 Studies ................................................29

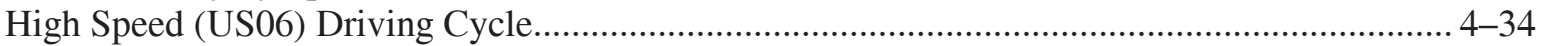

Fuel Economy for Class 8 Trucks as Function of Speed and Tractor-Trailer Tire

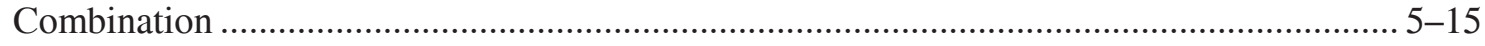


Speed (continued)

Class 8 Truck Fuel Economy as a Function of Speed and Tractor-Trailer Tire Combination and Percentage of Total Distance Traveled as a Function of Speed.................................... 5-16

Class 8 Truck Percent of Total Fuel Consumed as a Function of Speed and Tractor-Trailer Tire Combination.

Sport

Definition of Car Sport Utility Vehicles in Model Year 2013 ................................................. 4-8

Standards

Fuel Economy and Carbon Dioxide Emissions Standards, MY 2012-2025.............................. 4-19

Car Corporate Average Fuel Economy (CAFE) Standards versus Sales-Weighted Fuel

Economy Estimates, 1978-2013 ............................................................................................ 4-21

Light Truck Corporate Average Fuel Economy (CAFE) Standards versus Sales-Weighted

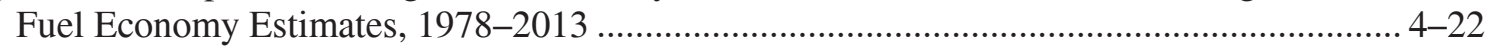

Tier 3 Non-Methane Organic Gases and Nitrogen Oxide Standards .................................... 12-14

Tier 3 Evaporative Emission Standards ................................................................................ 12-15

Tier 3 Particulate Matter Emission Standards for Light Gasoline Vehicles, MY 2017

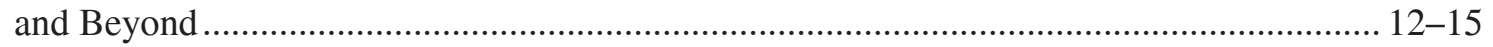

Light-Duty Vehicle, Light-Duty Truck, and Medium-Duty Passenger Vehicle - Tier 2

Exhaust Emission Standards ............................................................................................... 12-16

Light-Duty Vehicle, Light-Duty Truck, and Medium-Duty Passenger Vehicle - Tier 2

Evaporative Emission Standards .................................................................................... 12-17

Heavy-Duty Highway Compression-Ignition Engines and Urban Buses - Exhaust

Emission Standards.................................................................................................... 12-18

Heavy-Duty Highway Spark-Ignition Engines - Exhaust Emission Standards ........................ 12-20

Heavy-Duty Highway Compression-Ignition and Spark-Ignition Engines - Evaporative

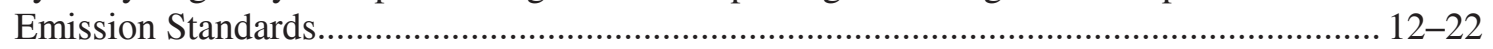

California Car, Light Truck and Medium Truck Emission Certification Standards .................. 12-23

Aircraft - Exhaust Emission Standards ........................................................................ 12-24

Nonroad Compression-Ignition Engines - Exhaust Emission Standards................................ 12-25

Nonroad Large Spark-Ignition Engines - Exhaust and Evaporative Emission Standards .......... 12-27

Locomotives - Exhaust Emission Standards ................................................................... 12-28

Marine Compression-Ignition (CI) Engines - Exhaust Emission Standards ............................ 12-30

Marine Spark-Ignition Engines and Vessels - Exhaust Emission Standards ........................... 12-34

Nonroad Recreational Engines and Vehicles - Exhaust Emission Standards.......................... 12-36

Gasoline Sulfur Standards ........................................................................................... 12-38

Highway, Nonroad, Locomotive, and Marine (NRLM) Diesel Fuel Sulfur Standards .............. 12-39

State

Number of Alternative Refuel Sites by State and Fuel Type, 2014 ....................................... 6-13

Federal and State Alternative Fuel Incentives, 2014 ...................................................... 10-12

Federal and State Advanced Technology Incentives, 2014 .................................................. 10-13

States

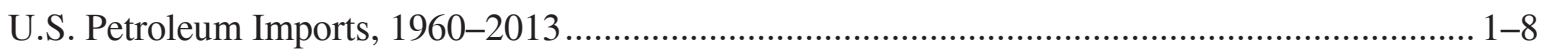

Costs of Oil Dependence to the U.S. Economy, 1970-2012 ….............................................. 1-12

U.S. Refinery Input of Crude Oil and Petroleum Products, 1987-2012 ..................................... 1-14

U.S. Refinery Yield of Petroleum Products from a Barrel of Crude Oil, 1978-2013................. 1-15

United States Petroleum Production, Imports and Exports, 1950-2013 ................................... 1-16

United States Petroleum Production and Consumption - All Sectors, 1973-2040 ..................... 1-18

United States Petroleum Production, and Transportation Consumption, 1970-2040.................. 1-19

U. S. Consumption of Total Energy by End-Use Sector, 1973-2013 ......................................... 2-3 
States (continued)

U.S. Cars and Trucks in Use, 1970-2012 ............................................................................ 3-6

Vehicles per Thousand People: U.S. (Over Time) Compared to Other Countries (in 2002 and 2012)

Vehicles per Thousand People in the United States, 1990-2012 ............................................... 3-10

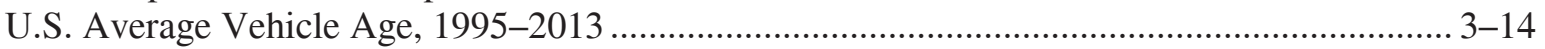

New Retail Car Sales in the United States, 1970-2013 ….......................................................... 4-5

New Retail Sales of Trucks 10,000 Pounds GVW and Less in the United States, 1970-2013 ..... 4-6

Comparison of U.S., European, and Japanese Driving Cycles Attributes ................................. 4-36

Example of Differing Results Using the U.S., European, and Japanese Driving Cycles............. 4-36

Value of Goods in the United States: Comparison of the 1997, 2002, 2007, and 2012

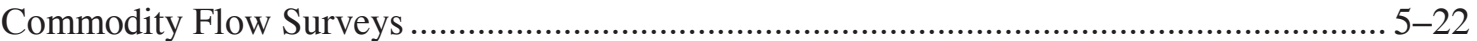

Tons of Freight in the United States: Comparison of the 1997, 2002, 2007, and 2012

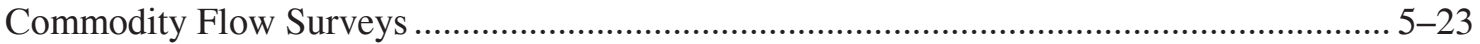

Growth of Ton Miles in the United States: Comparison of the 1997, 2002, 2007, and 2012

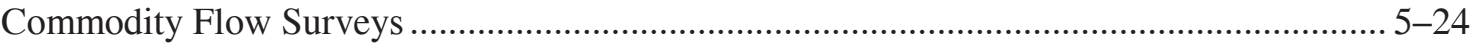

Average Miles per Shipment in the United States: Comparison of the 1997, 2002, 2007,

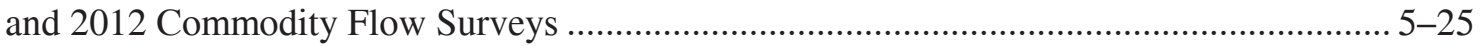

Characteristics of U.S. Daily per Vehicle Driving vs. Dwelling Unit Type and Density ............ 8-21

Summary Statistics for U.S. Domestic and International Certificated Route Air Carriers (Combined Totals), 1970-2013 .......................................................................................

Class I Railroad Freight Systems in the United States Ranked by Revenue Ton-Miles, 2012 ...... 9-8

U.S. Employment for Motor Vehicles and Motor Vehicle Parts Manufacturing,

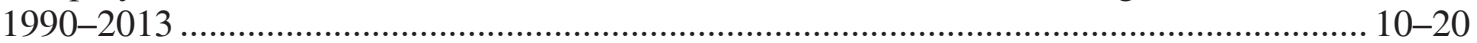

U.S. Emissions of Greenhouse Gases, based on Global Warming Potential, 1990-2012 ........... 11-4

Total U.S. Greenhouse Gas Emissions by End-Use Sector, 2012 ......................................... 11-5

U.S. Carbon Emissions from Fossil Fuel Consumption by End-Use Sector, 1990-2012............ 11-6

U.S. Carbon Emissions from Fossil Fuel Combustion in the Transportation End-Use

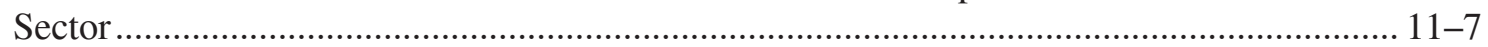

Stations

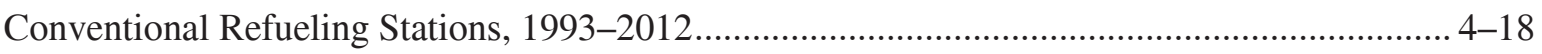

Statistics

Summary Statistics for Cars, 1970-2012 ............................................................................... 4-2

Summary Statistics for Two-Axle, Four-Tire Trucks, 1970-2012 ….......................................... 4-3

Summary Statistics on Class 1, Class 2a, and Class 2b Light Trucks.......................................... 4-4

Summary Statistics on Demand Response Vehicles, 1994-2012 ............................................ 4-37

Summary Statistics for Class 3-8 Single-Unit Trucks, 1970-2012 ............................................. 5-2

Summary Statistics for Class 7-8 Combination Trucks, 1970-2012 ....................................... 5-3

Truck Statistics by Gross Vehicle Weight Class, 2002 .............................................................. 5-6

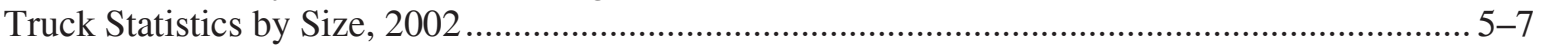

Summary Statistics on Transit Buses and Trolleybuses, 1994-2012 …..................................... 5-26

Demographic Statistics from the 1969, 1977, 1983, 1990, 1995 NPTS and 2001,

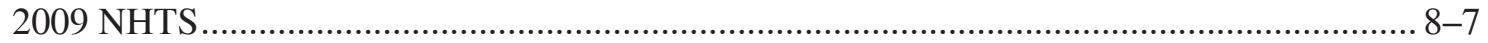

Trip Statistics by Trip Purpose, 2001 and 2009 NHTS ................................................................ 8-10

Summary Statistics for U.S. Domestic and International Certificated Route Air Carriers

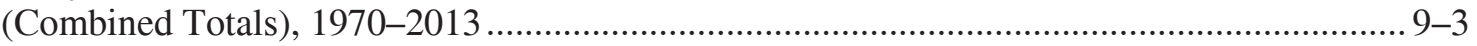

Summary Statistics for General Aviation, 1970-2012 …....................................................... 9-4

Tonnage Statistics for Domestic and International Waterborne Commerce, 1970-2012 ............. 9-5

Summary Statistics for Domestic Waterborne Commerce, 1970-2012 ...................................... 9-6

Summary Statistics for Class I Freight Railroads, 1970-2012 …............................................. 9-9 
Statistics (continued)

Summary Statistics for the National Railroad Passenger Corporation (Amtrak),

$1971-2012$............................................................................................................... 9-11

Summary Statistics for Commuter Rail Operations, 1984-2012 .......................................... 9-12

Summary Statistics for Rail Transit Operations, 1970-2012 ................................................. 9-13

Studies

Fuel Economy by Speed, 1973, 1984, 1997, and 2012 Studies ............................................. 4-29

Sulfur

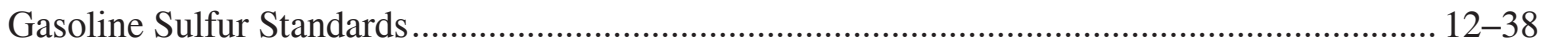

Highway, Nonroad, Locomotive, and Marine (NRLM) Diesel Fuel Sulfur Standards .............. 12-39

Summary

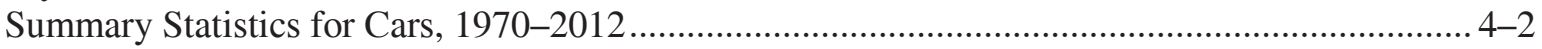

Summary Statistics for Two-Axle, Four-Tire Trucks, 1970-2012 …...................................... 4-3

Summary Statistics on Class 1, Class 2a, and Class 2b Light Trucks........................................... 4-4

Summary Statistics on Demand Response Vehicles, 1994-2012 …...................................... 4-37

Summary Statistics for Class 3-8 Single-Unit Trucks, 1970-2012 ............................................ 5-2

Summary Statistics for Class 7-8 Combination Trucks, 1970-2012 ….................................... 5-3

Summary Statistics on Transit Buses and Trolleybuses, 1994-2012 _......................................... 5-26

Summary Statistics for U.S. Domestic and International Certificated Route Air Carriers

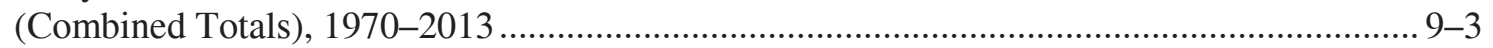

Summary Statistics for General Aviation, 1970-2012 .............................................................. 9-4

Summary Statistics for Domestic Waterborne Commerce, 1970-2012 _....................................... 9-6

Summary Statistics for Class I Freight Railroads, 1970-2012 ................................................. 9-9

Summary Statistics for the National Railroad Passenger Corporation (Amtrak),

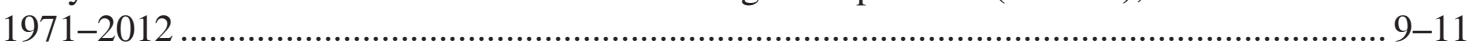

Summary Statistics for Commuter Rail Operations, 1984-2012 …......................................... 9-12

Summary Statistics for Rail Transit Operations, 1970-2012 ................................................. 9-13

Supplies

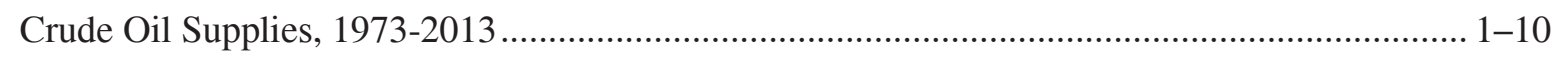

Surveys

Value of Goods in the United States: Comparison of the 1997, 2002, 2007, and 2012

Commodity Flow Surveys .......................................................................................... 5-22

Tons of Freight in the United States: Comparison of the 1997, 2002, 2007, and 2012

Commodity Flow Surveys ............................................................................................. $5-23$

Growth of Ton Miles in the United States: Comparison of the 1997, 2002, 2007, and 2012

Commodity Flow Surveys ..................................................................................................... 5-24

Average Miles per Shipment in the United States: Comparison of the 1997, 2002, 2007,

and 2012 Commodity Flow Surveys ……….................................................................... 5-25

Survival

Car Scrappage and Survival Rates, 1970, 1980 and 1990 Model Years ................................... 3-16

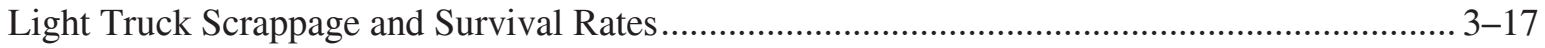

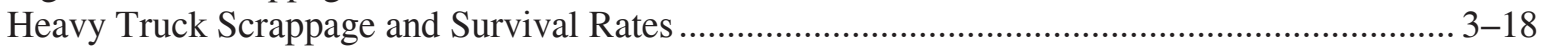

Systems

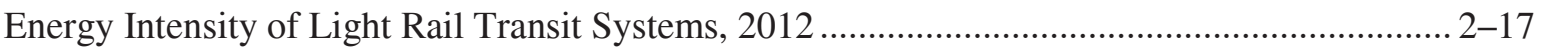

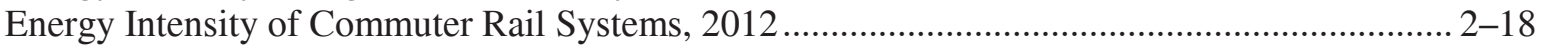

Energy Intensity of Heavy Rail Systems, 2012 ...................................................................... 2-18 
Systems (continued)

Class I Railroad Freight Systems in the United States Ranked by Revenue Ton-Miles, 2012 ...... 9-8

Targets

Fuel Economy and Carbon Dioxide Targets for Model Year 2025

Tax

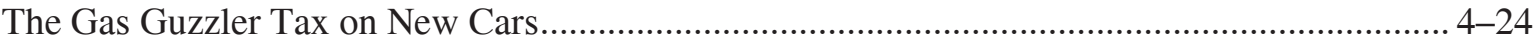

Tax Receipts from the Sale of Gas Guzzlers, 1980-2012 ........................................................ 4-27

Taxes

List of Model Year 2013 Cars with Gas Guzzler Taxes ........................................................ 4-25

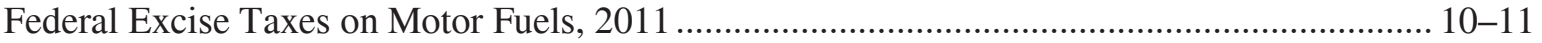

Technologies

Well-to-Wheel Emissions for Various Fuels and Vehicle Technologies .................................. 11-11

Technology

Alternative Fuel and Advanced Technology Vehicles and Characteristics ................................. 6-1

Federal and State Advanced Technology Incentives, 2014 .................................................. 10-13

Temperature

Cold Temperature (Cold FTP) Driving Cycle............................................................................ 4-33

Ten

Top Ten Natural Gas Producing Countries, 2012 ........................................................................ 6-15

Terrain

Effect of Terrain on Class 8 Truck Fuel Economy .................................................................. 5-14

Thousand

Vehicles per Thousand People: U.S. (Over Time) Compared to Other Countries (in 2002

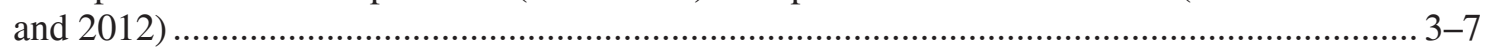

Vehicles per Thousand People in Other Countries, 2002 and 2012 _.......................................... 3-9

Vehicles per Thousand People in the United States, 1990-2012 ............................................... 3-10

Tier 2

Light-Duty Vehicle, Light-Duty Truck, and Medium-Duty Passenger Vehicle - Tier 2

Exhaust Emission Standards ................................................................................................ 12-16

Light-Duty Vehicle, Light-Duty Truck, and Medium-Duty Passenger Vehicle - Tier 2

Evaporative Emission Standards ..................................................................................... 12-17

Tier 3

Tier 3 Non-Methane Organic Gases and Nitrogen Oxide Standards ....................................... 12-14

Tier 3 Evaporative Emission Standards ............................................................................... 12-15

Tier 3 Particulate Matter Emission Standards for Light Gasoline Vehicles, MY 2017

and Beyond

Time

Vehicles per Thousand People: U.S. (Over Time) Compared to Other Countries (in 2002

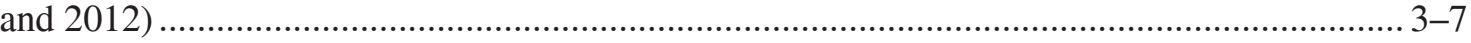

Average Length of Time Commercial Fleet Vehicles are in Service, 2012 .............................. 7-3

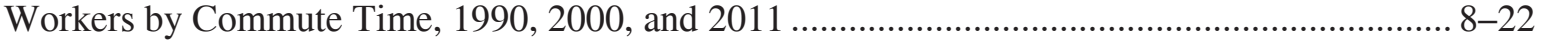


Tire

Fuel Economy for Class 8 Trucks as Function of Speed and Tractor-Trailer Tire Combination

Class 8 Truck Fuel Economy as a Function of Speed and Tractor-Trailer Tire Combination and Percentage of Total Distance Traveled as a Function of Speed.

Class 8 Truck Percent of Total Fuel Consumed as a Function of Speed and Tractor-Trailer Tire Combination.

Ton

Growth of Ton Miles in the United States: Comparison of the 1997, 2002, 2007, and 2012

Commodity Flow Surveys

Ton-Miles

Class I Railroad Freight Systems in the United States Ranked by Revenue Ton-Miles, 2012 ...... 9-8

Tonnage

Tonnage Statistics for Domestic and International Waterborne Commerce, 1970-2012 $9-5$

Tons

Tons of Freight in the United States: Comparison of the 1997, 2002, 2007, and 2012

Commodity Flow Surveys. $5-23$

Top

Top Ten Natural Gas Producing Countries, 2012 $6-15$

Total

U. S. Consumption of Total Energy by End-Use Sector, 1973-2013. $2-3$

Class 8 Truck Fuel Economy as a Function of Speed and Tractor-Trailer Tire Combination and Percentage of Total Distance Traveled as a Function of Speed. $5-16$

Class 8 Truck Percent of Total Fuel Consumed as a Function of Speed and Tractor-Trailer

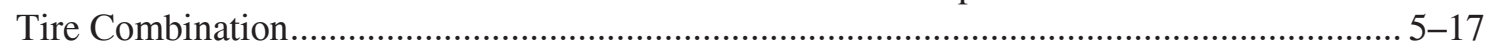

Total U.S. Greenhouse Gas Emissions by End-Use Sector, 2012 .......................................... 11-5

Total National Emissions of the Criteria Air Pollutants by Sector, 2013 ................................... 12-2

Total National Emissions of Carbon Monoxide, 1970-2013 .................................................... 12-3

Total National Emissions of Nitrogen Oxides, 1970-2013 ....................................................... 12-5

Total National Emissions of Volatile Organic Compounds, 1970-2013 …................................. 12-7

Total National Emissions of Particulate Matter (PM-10), 1970-2013 ...................................... 12-9

Total National Emissions of Particulate Matter (PM-2.5), 1990-2013 ..................................... 12-11

Totals

Summary Statistics for U.S. Domestic and International Certificated Route Air Carriers

(Combined Totals), 1970-2013

Tractor-Trailer

Fuel Economy for Class 8 Trucks as Function of Speed and Tractor-Trailer Tire Combination $5-15$

Class 8 Truck Fuel Economy as a Function of Speed and Tractor-Trailer Tire Combination and Percentage of Total Distance Traveled as a Function of Speed. $5-16$

Class 8 Truck Percent of Total Fuel Consumed as a Function of Speed and Tractor-Trailer Tire Combination.

Traffic

Intermodal Rail Traffic, 1965-2012 $9-10$ 
Transit

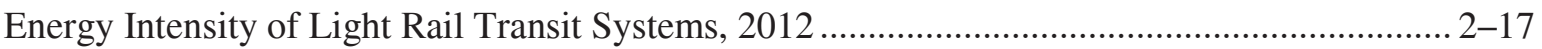

Summary Statistics on Transit Buses and Trolleybuses, 1994-2012 _....................................... 5-26

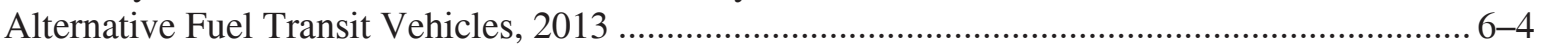

Summary Statistics for Rail Transit Operations, 1970-2012 ................................................... 9-13

Transportation

Petroleum Production and Transportation Petroleum Consumption in Context, 1950-2013 ....... 1-17

United States Petroleum Production, and Transportation Consumption, 1970-2040.................. 1-19

Highway Transportation Petroleum Consumption by Mode, 1970-2012................................. 1-21

Nonhighway Transportation Petroleum Consumption by Mode, 1970-2012 …........................ 1-22

Transportation Petroleum Use by Mode, 2011-2012 ......................................................... 1-23

Domestic Consumption of Transportation Energy by Mode and Fuel Type, 2012 ....................... 2-7

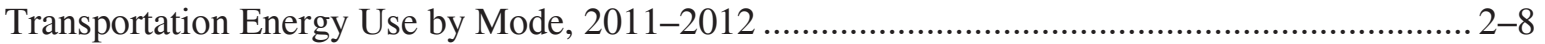

Highway Transportation Energy Consumption by Mode, 1970-2012 ….................................... 2-9

Nonhighway Transportation Energy Consumption by Mode, 1970-2012 …............................. 2-10

Annual Household Expenditures for Transportation, 1985-2012 …....................................... 8-5

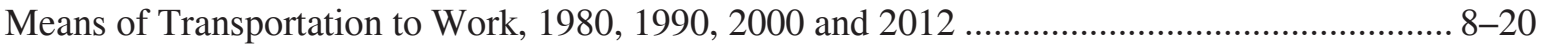

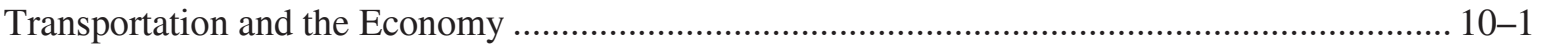

Transportation Services Index, January 1990-January 2014 …............................................. 10-2

U.S. Carbon Emissions from Fossil Fuel Combustion in the Transportation End-Use

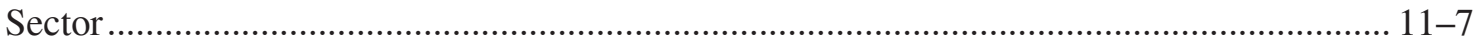

Transportation Greenhouse Gas Emissions by Mode, 1990 and 2012 ....................................... 11-8

Transportation-Related

Off-Highway Transportation-Related Fuel Consumption from the Nonroad Model, 2012......... 2-11

Transportation-Related Employment, 2000 and 2013 ........................................................... 10-19

Travel

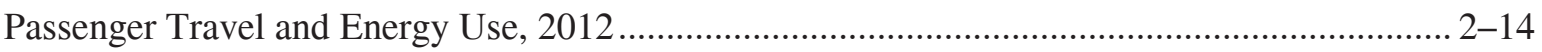

Average Annual Vehicle-Miles of Travel for Commercial Fleet Vehicles, 2012 ........................ 7-3

Average Number of Vehicles and Vehicle Travel per Household, 1990 NPTS and 2001

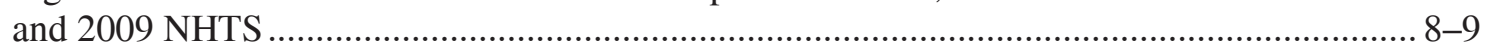

Share of Vehicles by Annual Miles of Travel and Vehicle Age, 2009 NHTS............................ 8-16

Daily and Annual Vehicle Miles of Travel and Average Age for Each Vehicle in a

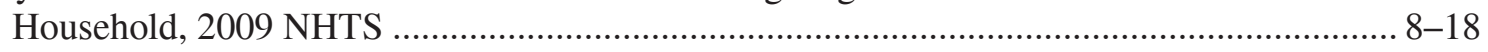

Daily Vehicle Miles of Travel (per Vehicle) by Number of Vehicles in the Household,

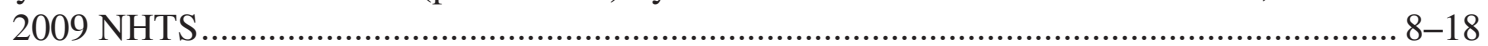

Annual Vehicle Miles of Travel for Each Vehicle in a Household, 2009 NHTS ....................... 8-19

Daily Vehicle Miles of Travel for Each Vehicle in a Household, 2009 NHTS .......................... 8-19

Traveled

Shares of Highway Vehicle-Miles Traveled by Vehicle Type, 1970-2012 …........................... 3-11

Distribution of Trucks over 26,000 lbs. by Vehicle-Miles Traveled ........................................... 5-11

Class 8 Truck Fuel Economy as a Function of Speed and Tractor-Trailer Tire Combination and Percentage of Total Distance Traveled as a Function of Speed......................................5-16

Trip

Average Annual Vehicle-Miles, Vehicle Trips and Trip Length per Household 1969, 1977,

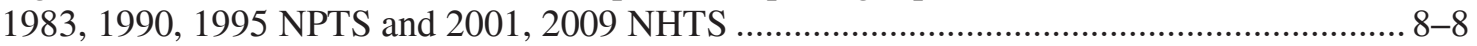

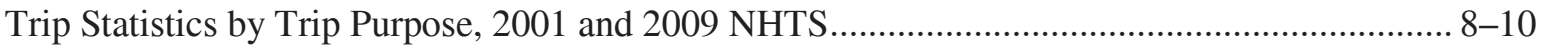

Average Vehicle Occupancy by Trip Purpose, 1977 NPTS and 2009 NHTS ............................ 8-12

Share of Vehicle Trips by Trip Distance, 2009 NHTS ............................................................. 8-15 
Trip (continued)

Share of Vehicle Trips to Work by Trip Distance, 2009 NHTS .............................................. 8-15

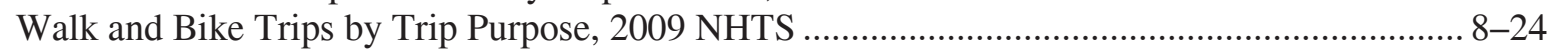

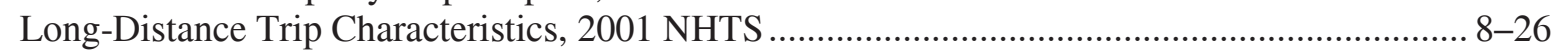

Trips

Average Annual Vehicle-Miles, Vehicle Trips and Trip Length per Household 1969, 1977,

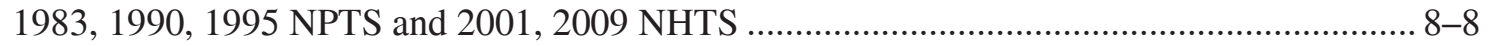

Share of Vehicle Trips by Trip Distance, 2009 NHTS ............................................................. 8-15

Share of Vehicle Trips to Work by Trip Distance, 2009 NHTS ................................................. 8-15

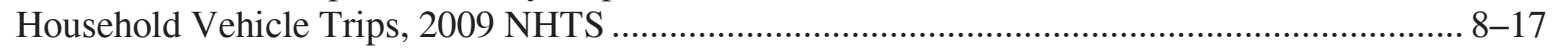

Walk and Bike Trips by Trip Purpose, 2009 NHTS ................................................................. 8-24

Trolleybuses

Summary Statistics on Transit Buses and Trolleybuses, 1994-2012 ........................................ 5-26

Truck

Truck and Bus Registrations for Selected Countries, 1960-2012 _............................................. 3-4

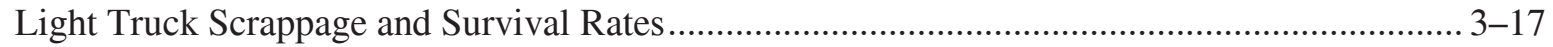

Heavy Truck Scrappage and Survival Rates .......................................................................... 3-18

Light Truck Corporate Average Fuel Economy (CAFE) Standards versus Sales-Weighted

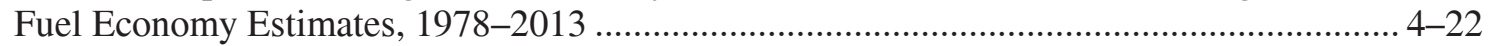

New Retail Truck Sales by Gross Vehicle Weight, 1970-2013 ................................................... 5-4

Truck Harmonic Mean Fuel Economy by Size Class, 1992, 1997, and 2002 .............................. 5-6

Truck Statistics by Gross Vehicle Weight Class, 2002 …........................................................ 5-6

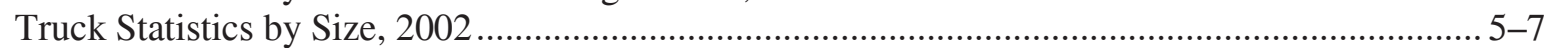

Effect of Terrain on Class 8 Truck Fuel Economy ................................................................. 5-14

Class 8 Truck Fuel Economy as a Function of Speed and Tractor-Trailer Tire Combination and Percentage of Total Distance Traveled as a Function of Speed..................................... 5-16

Class 8 Truck Percent of Total Fuel Consumed as a Function of Speed and Tractor-Trailer

Tire Combination....................................................................................................... $5-17$

Class 8 Truck Weight by Component .............................................................................. 5-18

Light-Duty Vehicle, Light-Duty Truck, and Medium-Duty Passenger Vehicle - Tier 2

Exhaust Emission Standards ............................................................................................ 12-16

Light-Duty Vehicle, Light-Duty Truck, and Medium-Duty Passenger Vehicle - Tier 2

Evaporative Emission Standards .................................................................................. 12-17

California Car, Light Truck and Medium Truck Emission Certification Standards .................. 12-23

Trucks

World Production of Cars and Trucks, 2000-2012 ............................................................. 3-2

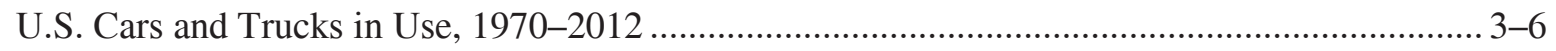

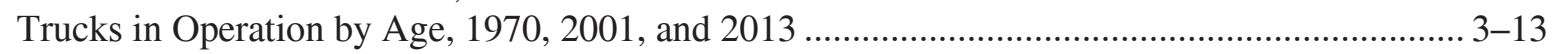

Summary Statistics for Two-Axle, Four-Tire Trucks, 1970-2012 ......................................... 4-3

Sales Estimates of Class 1, Class 2a, and Class 2b Light Trucks, 1989-1999 .............................. 4-4

Summary Statistics on Class 1, Class 2a, and Class 2b Light Trucks......................................... 4-4

New Retail Sales of Trucks 10,000 Pounds GVW and Less in the United States, 1970-2013 ...... 4-6

Production, Production Shares, and Production-Weighted Fuel Economies of New

Domestic and Import Light Trucks, Model Years 1975-2013 …........................................... 4-9

Production and Production-Weighted Fuel Economies of New Domestic and Import Cars,

Light Trucks, and Light Vehicles, Model Years 1975-2013 ............................................... 4-10

Production-Weighted Engine Size of New Domestic and Import Light Trucks, Model

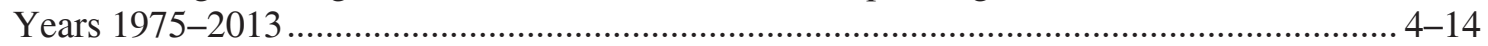

Summary Statistics for Class 3-8 Single-Unit Trucks, 1970-2012 ............................................ 5-2 
Trucks (continued)

Summary Statistics for Class 7-8 Combination Trucks, 1970-2012 ….................................... 5-3

Percentage of Trucks by Size Ranked by Major Use, 2002 .................................................... 5-8

Percentage of Trucks by Fleet Size and Primary Fueling Facility, 2002 ................................... 5-9

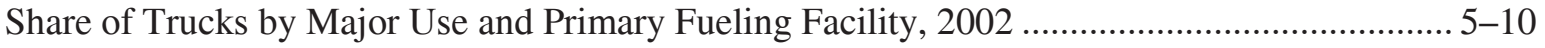

Distribution of Trucks over 26,000 lbs. by Vehicle-Miles Traveled .......................................... 5-11

Share of Heavy Trucks with Selected Electronic Features, 2002 ............................................. 5-12

Fuel Economy for Class 8 Trucks as Function of Speed and Tractor-Trailer Tire

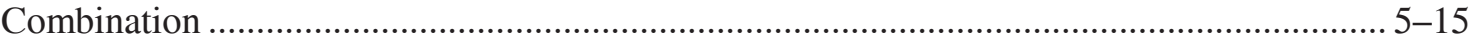

Distribution of Class 8 Trucks by On-Road Vehicle Weight, 2008 …..................................... 5-20

Electric Drive Medium/Heavy Trucks and Buses Available by Manufacturer, 2014................. 6-12

Production-Weighted Annual Carbon Footprint of New Domestic and Import Light

Trucks, Model Years 1975-2013 …................................................................................... 11-14

Two-Axle

Summary Statistics for Two-Axle, Four-Tire Trucks, 1970-2012 …......................................... 4-3

Type

Domestic Consumption of Transportation Energy by Mode and Fuel Type, 2012 ...................... 2-7

Shares of Highway Vehicle-Miles Traveled by Vehicle Type, 1970-2012 …............................ 3-11

Number of Alternative Refuel Sites by State and Fuel Type, 2014 ........................................ 6-13

New Light Fleet Vehicle Registrations by Vehicle Type, Model Year 2012 .............................. 7-3

Average Miles per Domestic Federal Vehicle by Vehicle Type, 2012 ........................................ 7-4

Federal Fleet Vehicle Acquisitions by Fuel Type, FY 2002- 2012 _......................................... 7-6

Average Vehicle Occupancy by Vehicle Type, 1995 NPTS and 2009 NHTS ........................... 8-11

Characteristics of U.S. Daily per Vehicle Driving vs. Dwelling Unit Type and Density ............. 8-21

Unit

Characteristics of U.S. Daily per Vehicle Driving vs. Dwelling Unit Type and Density ............ 8-21

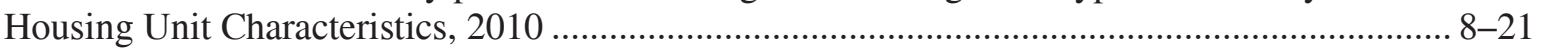

United

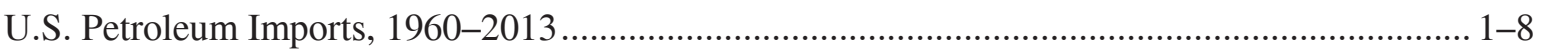

Costs of Oil Dependence to the U.S. Economy, 1970-2012 .................................................. 1-12

U.S. Refinery Input of Crude Oil and Petroleum Products, 1987-2012 ................................... 1-14

U.S. Refinery Yield of Petroleum Products from a Barrel of Crude Oil, 1978-2013.................. 1-15

United States Petroleum Production, Imports and Exports, 1950-2013 ................................... 1-16

United States Petroleum Production and Consumption - All Sectors, 1973-2040 .................... 1-18

United States Petroleum Production, and Transportation Consumption, 1970-2040.................. 1-19

U.S. Consumption of Total Energy by End-Use Sector, 1973-2013 ........................................ 2-3

U.S. Cars and Trucks in Use, 1970-2012 ......................................................................... 3-6

Vehicles per Thousand People: U.S. (Over Time) Compared to Other Countries (in 2002

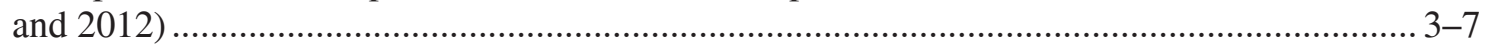

Vehicles per Thousand People in the United States, 1990-2012 ............................................ 3-10

U.S. Average Vehicle Age, 1995-2013 ….............................................................................. 3-14

New Retail Car Sales in the United States, 1970-2013 ….......................................................... 4-5

New Retail Sales of Trucks 10,000 Pounds GVW and Less in the United States, 1970-2013 ..... 4-6

Comparison of U.S., European, and Japanese Driving Cycles Attributes ................................. 4-36

Example of Differing Results Using the U.S., European, and Japanese Driving Cycles............. 4-36

Value of Goods in the United States: Comparison of the 1997, 2002, 2007, and 2012

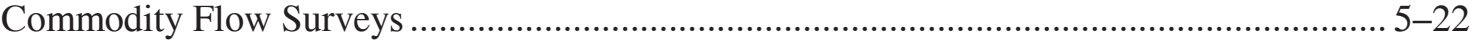

Tons of Freight in the United States: Comparison of the 1997, 2002, 2007, and 2012

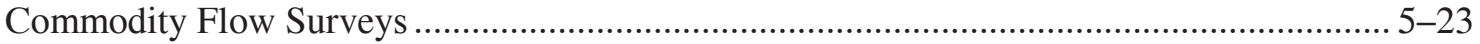


United (continued)

Growth of Ton Miles in the United States: Comparison of the 1997, 2002, 2007, and 2012

Commodity Flow Surveys ..

Average Miles per Shipment in the United States: Comparison of the 1997, 2002, 2007,

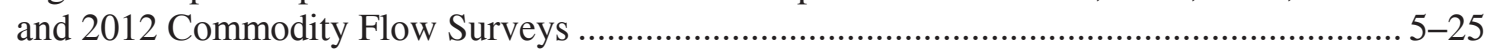

Characteristics of U.S. Daily per Vehicle Driving vs. Dwelling Unit Type and Density............ 8-21

Summary Statistics for U.S. Domestic and International Certificated Route Air Carriers (Combined Totals), 1970-2013 ............................................................................................. 9-3

Class I Railroad Freight Systems in the United States Ranked by Revenue Ton-Miles, 2012 ...... 9-8

U.S. Employment for Motor Vehicles and Motor Vehicle Parts Manufacturing,

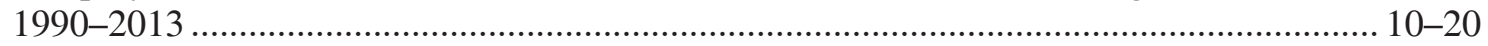

U.S. Emissions of Greenhouse Gases, based on Global Warming Potential, 1990-2012 ........... 11-4

Total U.S. Greenhouse Gas Emissions by End-Use Sector, 2012 ........................................... 11-5

U.S. Carbon Emissions from Fossil Fuel Consumption by End-Use Sector, 1990-2012............ 11-6

U.S. Carbon Emissions from Fossil Fuel Combustion in the Transportation End-Use Sector..... $11-7$

Urban

Heavy-Duty Highway Compression-Ignition Engines and Urban Buses - Exhaust Emission Standards. $12-18$

US06

High Speed (US06) Driving Cycle. $4-34$

Usage

Highway Usage of Gasoline and Diesel, 1973-2012. $2-13$

Use

Transportation Petroleum Use by Mode, 2011-2012 …........................................................... 1-23

Transportation Energy Use by Mode, 2011-2012 ............................................................. 2-8

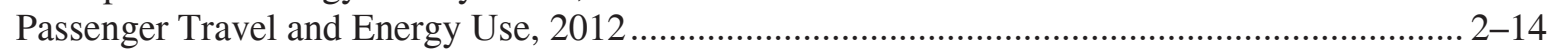

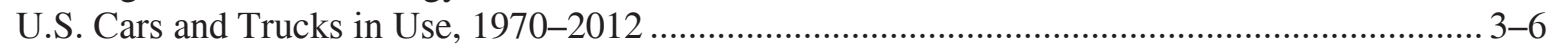

Percentage of Trucks by Size Ranked by Major Use, 2002 .................................................... 5-8

Share of Trucks by Major Use and Primary Fueling Facility, 2002 ........................................ 5-10

Estimates of Alternative Fuel Highway Vehicles in Use, 1995-2011 ....................................... 6-3

Nonhighway Energy Use Shares, 1970-2012 _................................................................ 9-2

Recreational Boat Energy Use, 1970-2012 …........................................................................ 9-7

Utility

Definition of Car Sport Utility Vehicles in Model Year 2013 ................................................. 4-8

Value

Value of Goods in the United States: Comparison of the 1997, 2002, 2007, and 2012

Commodity Flow Surveys

Various

Well-to-Wheel Emissions for Various Fuels and Vehicle Technologies $11-11$

Vehicle

Shares of Highway Vehicle-Miles Traveled by Vehicle Type, 1970-2012 _.............................. 3-11

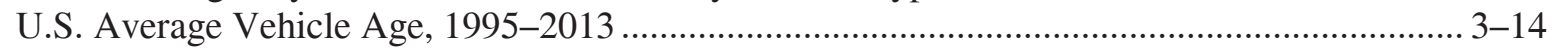

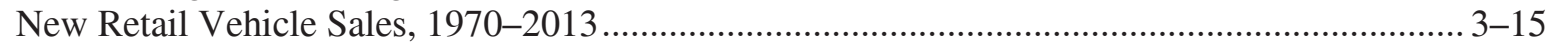

New Retail Sales of Trucks 10,000 Pounds GVW and Less in the United States, 1970-2013 ..... 4-6 
Vehicle (continued)

Light Vehicle Production Shares, Model Years 1975-2013 ..................................................... 4-12

Average Material Consumption for a Domestic Light Vehicle, Model Years 1995, 2000,

and 2012

New Light Vehicle Dealerships and Sales, 1970-2012 …...................................................... 4-17

New Retail Truck Sales by Gross Vehicle Weight, 1970-2013 …............................................... 5-4

Truck Statistics by Gross Vehicle Weight Class, 2002 …......................................................... 5-6

Gross Vehicle Weight vs. Empty Vehicle Weight ................................................................. 5-19

Distribution of Class 8 Trucks by On-Road Vehicle Weight, 2008......................................... 5-20

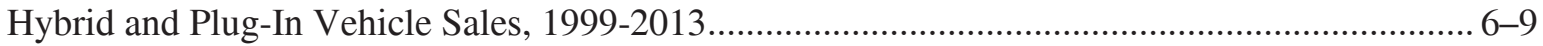

New Light Fleet Vehicle Registrations by Vehicle Type, Model Year 2012 ............................. 7-3

Average Miles per Domestic Federal Vehicle by Vehicle Type, 2012 ...................................... 7-4

Federal Fleet Vehicle Acquisitions by Fuel Type, FY 2002- 2012 …..................................... 7-6

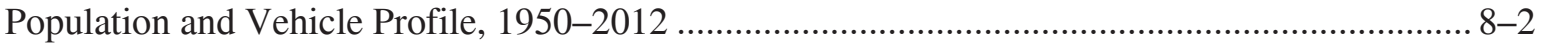

Household Vehicle Ownership, 1960-2012 Census .............................................................. 8-6

Average Annual Vehicle-Miles, Vehicle Trips and Trip Length per Household 1969, 1977,

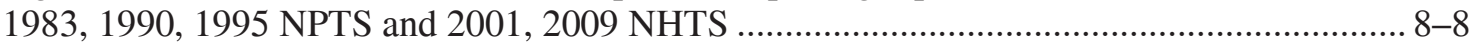

Average Number of Vehicles and Vehicle Travel per Household, 1990 NPTS and 2001

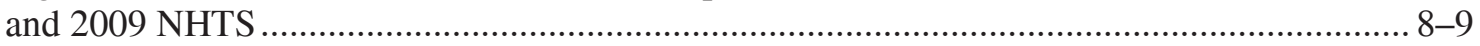

Average Vehicle Occupancy by Vehicle Type, 1995 NPTS and 2009 NHTS .......................... 8-11

Average Vehicle Occupancy by Trip Purpose, 1977 NPTS and 2009 NHTS ........................... 8-12

Average Annual Miles per Household Vehicle by Vehicle Age............................................... 8-13

Share of Vehicle Trips by Trip Distance, 2009 NHTS ............................................................... 8 8-15

Share of Vehicle Trips to Work by Trip Distance, 2009 NHTS ................................................ 8-15

Share of Vehicles by Annual Miles of Travel and Vehicle Age, 2009 NHTS............................. 8-16

Household Vehicle Trips, 2009 NHTS ............................................................................... 8-17

Daily and Annual Vehicle Miles of Travel and Average Age for Each Vehicle in a

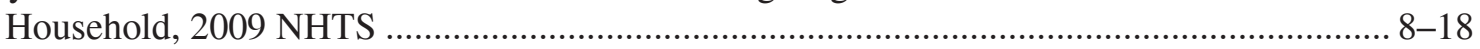

Daily Vehicle Miles of Travel (per Vehicle) by Number of Vehicles in the Household,

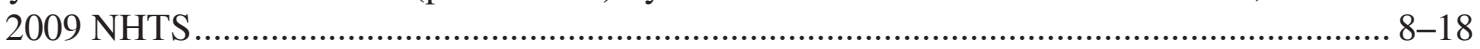

Annual Vehicle Miles of Travel for Each Vehicle in a Household, 2009 NHTS ....................... 8-19

Daily Vehicle Miles of Travel for Each Vehicle in a Household, 2009 NHTS .......................... 8-19

Characteristics of U.S. Daily per Vehicle Driving vs. Dwelling Unit Type and Density ............ 8-21

U.S. Employment for Motor Vehicles and Motor Vehicle Parts Manufacturing,

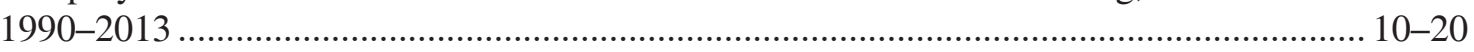

Well-to-Wheel Emissions for Various Fuels and Vehicle Technologies ................................... 11-11

Average Annual Carbon Footprint by Vehicle Classification, 1975 and 2013 .......................... 11-15

Light-Duty Vehicle, Light-Duty Truck, and Medium-Duty Passenger Vehicle - Tier 2

Exhaust Emission Standards ................................................................................................ 12-16

Light-Duty Vehicle, Light-Duty Truck, and Medium-Duty Passenger Vehicle - Tier 2

Evaporative Emission Standards ......................................................................................... 12-17

Vehicle-Miles

Shares of Highway Vehicle-Miles Traveled by Vehicle Type, 1970-2012 ............................... 3-11

Distribution of Trucks over 26,000 lbs. by Vehicle-Miles Traveled ..........................................5-11

Average Annual Vehicle-Miles of Travel for Commercial Fleet Vehicles, 2012 ......................... 7-3

Vehicles and Vehicle-Miles per Capita, 1950-2012 .................................................................. 8-3

Average Annual Vehicle-Miles, Vehicle Trips and Trip Length per Household 1969, 1977,

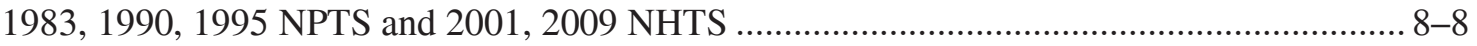


Vehicles

All Highway Vehicles Characteristics ................................................................................. 3-1

Vehicles per Thousand People: U.S. (Over Time) Compared to Other Countries (in 2002

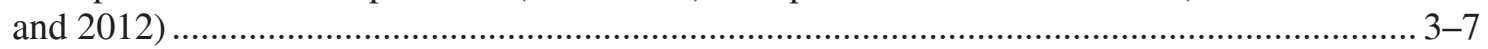

Vehicles per Thousand People in Other Countries, 2002 and 2012 ......................................... 3-9

Vehicles per Thousand People in the United States, 1990-2012 ............................................. 3-10

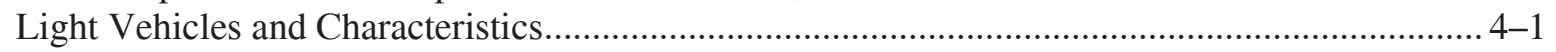

Definition of Car Sport Utility Vehicles in Model Year 2013 ................................................... 4-8

Production and Production-Weighted Fuel Economies of New Domestic and Import Cars,

Light Trucks, and Light Vehicles, Model Years 1975-2013 .............................................. 4-10

Summary Statistics on Demand Response Vehicles, 1994-2012 ............................................ 4-37

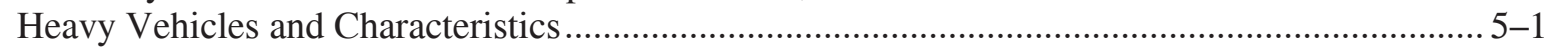

Alternative Fuel and Advanced Technology Vehicles and Characteristics ................................ 6-1

Estimates of Alternative Fuel Highway Vehicles in Use, 1995-2011 ....................................... 6-3

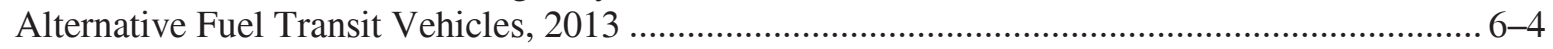

E85 Flex-Fuel Vehicles Available by Manufacturer, Model Year 2014..................................... 6-5

Non-E85 Alternative Fuel Vehicles Available by Manufacturer, Model Year 2014.................... 6-7

Electric Drive Vehicles Available by Manufacturer, Model Year 2014 …................................. 6-10

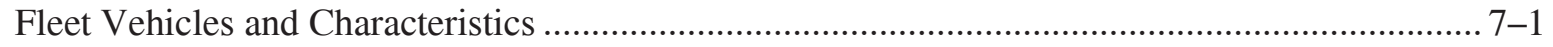

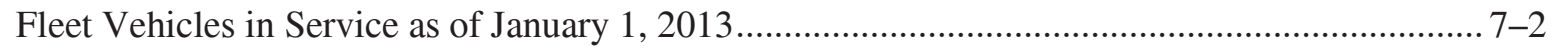

Average Annual Vehicle-Miles of Travel for Commercial Fleet Vehicles, 2012 ........................ 7-3

Average Length of Time Commercial Fleet Vehicles are in Service, 2012 ................................ 7-3

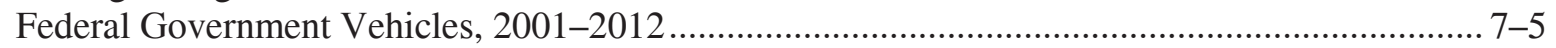

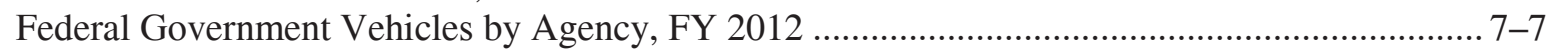

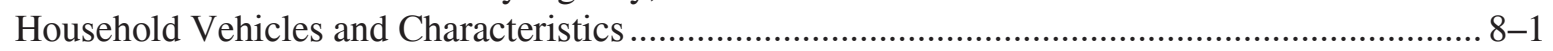

Vehicles and Vehicle-Miles per Capita, 1950-2012 ............................................................. 8-3

Average Number of Vehicles and Vehicle Travel per Household, 1990 NPTS and 2001

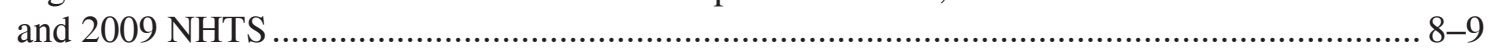

Share of Vehicles by Annual Miles of Travel and Vehicle Age, 2009 NHTS............................ 8-16

Daily Vehicle Miles of Travel (per Vehicle) by Number of Vehicles in the Household,

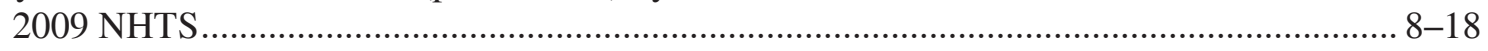

U.S. Employment for Motor Vehicles and Motor Vehicle Parts Manufacturing,

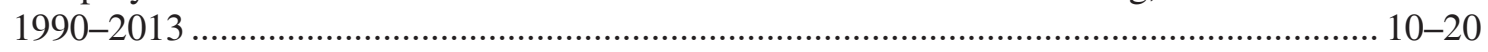

Emissions of Carbon Monoxide from Highway Vehicles, 1970-2005....................................... 12-4

Emissions of Nitrogen Oxides from Highway Vehicles, 1970-2005 ....................................... 12-6

Emissions of Volatile Organic Compounds from Highway Vehicles, 1970-2005 ..................... 12-8

Emissions of Particulate Matter (PM-2.5) from Highway Vehicles, 1990-2005 ...................... 12-12

Tier 3 Particulate Matter Emission Standards for Light Gasoline Vehicles, MY 2017

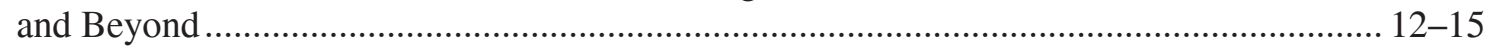

Nonroad Recreational Engines and Vehicles - Exhaust Emission Standards.......................... 12-36

Vessels

Marine Spark-Ignition Engines and Vessels - Exhaust Emission Standards ............................ 12-34

Volatile

Total National Emissions of Volatile Organic Compounds, 1970-2013 ................................... 12-7

Emissions of Volatile Organic Compounds from Highway Vehicles, 1970-2005 ...................... 12-8

Walk

Walk and Bike Trips by Trip Purpose, 2009 NHTS $8-24$ 
Warming

Numerical Estimates of Global Warming Potentials Compared with Carbon Dioxide ................ 11-3

U.S. Emissions of Greenhouse Gases, based on Global Warming Potential, 1990-2012 ........... 11-4

Waterborne

Tonnage Statistics for Domestic and International Waterborne Commerce, 1970-2012 .............. 9-5

Summary Statistics for Domestic Waterborne Commerce, 1970-2012 ....................................... 9-6

Weight

New Retail Sales of Trucks 10,000 Pounds GVW and Less in the United States, 1970-2013 ..... 4-6

Production-Weighted Curb Weight of New Domestic and Import Cars, Model Years

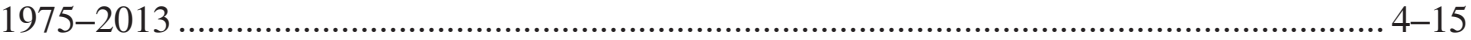

New Retail Truck Sales by Gross Vehicle Weight, 1970-2013 …............................................ 5-4

Truck Statistics by Gross Vehicle Weight Class, 2002 ............................................................. 5-6

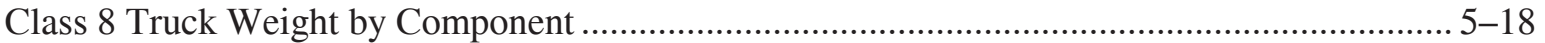

Gross Vehicle Weight vs. Empty Vehicle Weight ................................................................. 5-19

Distribution of Class 8 Trucks by On-Road Vehicle Weight, 2008 ........................................... 5-20

Well-to-Wheel

Well-to-Wheel Emissions for Various Fuels and Vehicle Technologies .................................. 11-11

Work

Share of Vehicle Trips to Work by Trip Distance, 2009 NHTS ................................................ 8-15

Means of Transportation to Work, 1980, 1990, 2000 and 2012 ................................................ 8-20

Workers

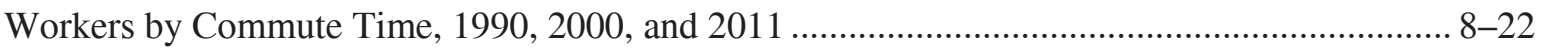

World

World Crude Oil Production, 1960-2013 .............................................................................. 1-3

World Petroleum Production, 1973-2013 ................................................................................ 1-4

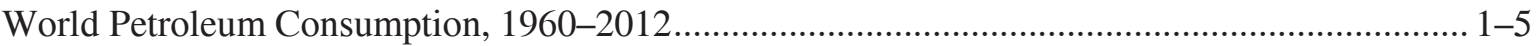

World Oil Reserves, Production and Consumption, 2012 ....................................................... 1-6

World Natural Gas Reserves, Production and Consumption, 2012 ........................................... 1-7

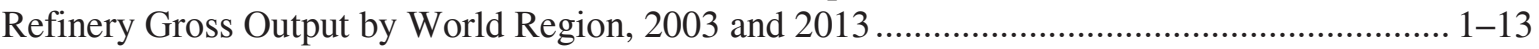

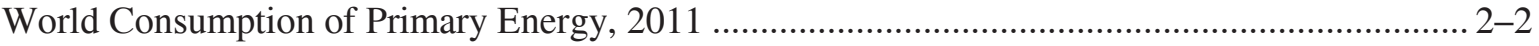

World Production of Cars and Trucks, 2000-2012 …................................................................. 3-2

World Carbon Dioxide Emissions, 1990 and 2013 …............................................................ 11-2

Year

Definition of Car Sport Utility Vehicles in Model Year 2013 ................................................. 4-8

Fuel Economy and Carbon Dioxide Emissions Standards, MY 2012-2025............................... 4-19

Fuel Economy and Carbon Dioxide Targets for Model Year 2025 .......................................... 4-20

List of Model Year 2013 Cars with Gas Guzzler Taxes ............................................................. 4-25

E85 Flex-Fuel Vehicles Available by Manufacturer, Model Year 2014 ..................................... 6-5

Non-E85 Alternative Fuel Vehicles Available by Manufacturer, Model Year 2014 ..................... 6-7

Electric Drive Vehicles Available by Manufacturer, Model Year 2014 …................................. 6-10

New Light Fleet Vehicle Registrations by Vehicle Type, Model Year 2012 .............................. 7-3

Fixed Car Operating Costs per Year, 1975-2013 …............................................................... 10-17

Tier 3 Particulate Matter Emission Standards for Light Gasoline Vehicles, MY 2017 and Beyond

$12-15$ 
Years

Car Scrappage and Survival Rates, 1970, 1980 and 1990 Model Years.................................... 3-16

Production Sales, Production Shares, and Sales-Weighted Fuel Economies of New

Domestic and Import Cars, Selected Model Years 1975-2013 ............................................... 4-7

Production, Production Shares, and Production-Weighted Fuel Economies of New

Domestic and Import Light Trucks, Model Years 1975-2013 ............................................ 4-9

Production and Production-Weighted Fuel Economies of New Domestic and Import Cars,

Light Trucks, and Light Vehicles, Model Years 1975-2013 …........................................ 4-10

Light Vehicle Production Shares, Model Years 1975-2013 ….................................................. 4-12

Production-Weighted Engine Size of New Domestic and Import Cars, Model Years

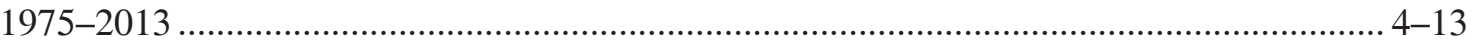

Production-Weighted Engine Size of New Domestic and Import Light Trucks, Model

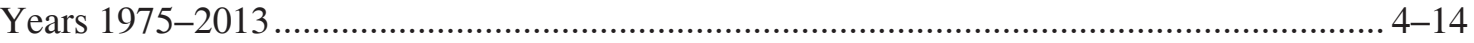

Production-Weighted Curb Weight of New Domestic and Import Cars, Model Years 1975-2013

Average Material Consumption for a Domestic Light Vehicle, Model Years 1995, 2000,

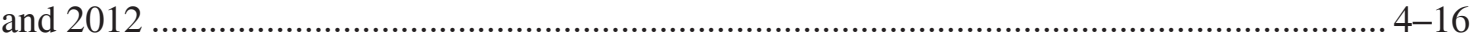

Production-Weighted Annual Carbon Footprint of New Domestic and Import Cars, Model Years 1975-2013 .................................................................................................. 11-13

Production-Weighted Annual Carbon Footprint of New Domestic and Import Light

Trucks, Model Years 1975-2013 ....................................................................................... 11-14

Yield

U.S. Refinery Yield of Petroleum Products from a Barrel of Crude Oil, 1978-2013.................. 1-15 


\section{USEFUL WEB SITES}

U.S. DEPARTMENT OF ENERGY

Energy Efficiency and Renewable Energy

Vehicle Technologies Office

Fuel Cells Technologies Office

Bioenergy Technologies Office

Alternative Fuels Data Center

Clean Cities

Transportation Fact of the Week

Fuel Economy

Hybrid and Vehicle Systems

Buildings Energy Data Book

Energy Information Administration

OAK RIDGE NATIONAL LABORATORY

Center for Transportation Analysis

Transportation Energy Data Book

Vehicle Technologies Market Report

ARGONNE NATIONAL LABORATORY

GREET Model

FEDERAL GOVERNMENT - FEDSTATS

USA.GOV

U.S. ARMY CORPS OF ENGINEERS

U.S. BUREAU OF LABOR STATISTICS

U.S. CENSUS BUREAU

Vehicle Inventory and Use Survey

U.S. DEPARTMENT OF COMMERCE

Bureau of Economic Analysis

U.S. DEPARTMENT OF TRANSPORTATION

Bureau of Transportation Statistics

Commodity Flow Survey Program

National Transportation Library

TranStats

Federal Aviation Administration

Federal Highway Administration

Office of Highway Policy Information

Nationwide Household Travel Survey

Federal Motor Carrier Safety Administration

Federal Railroad Administration

Federal Transit Administration

Maritime Administration

National Highway Traffic Safety Administration

Research and Innovative Technology Administration

John A. Volpe National Transportation Systems Center

U.S. Coast Guard

U.S. ENVIRONMENTAL PROTECTION AGENCY

Office of Air and Radiation

Office of Transportation and Air Quality www.energy.gov

www.eere.doe.gov

www.eere.energy.gov/vehiclesandfuels

www.eere.energy.gov/hydrogenandfuelcells

www.eere.energy.gov/biomass

www.afdc.energy.gov/afdc

www.eere.energy.gov/cleancities

www.eere.energy.gov/vehiclesandfuels/facts

www.fueleconomy.gov

www.eere.energy.gov/vehiclesandfuels/technologies/systems

www.btscoredatabook.net

www.eia.gov

www.ornl.gov

cta.ornl.gov

cta.ornl.gov/data

cta.ornl.gov/vtmarketreport

www.anl.gov

greet.es.anl.gov

www.fedstats.gov

www.usa.gov

www.iwr.usace.army.mil/ndc

www.bls.gov

www.census.gov

www.census.gov/svsd/www/tiusview.html

www.commerce.gov

www.bea.gov

www.dot.gov

www.bts.gov

www.bts.gov/publications/commodity_flow_survey

ntl.bts.gov

www.transtats.bts.gov

www.faa.gov

www.fhwa.dot.gov

www.fhwa.dot.gov/policyinformation

nhts.ornl.gov

www.fmcsa.dot.gov

www.fra.dot.gov

www.fta.dot.gov

www.marad.dot.gov

www.nhtsa.dot.gov

www.rita.dot.gov

www.volpe.dot.gov

www.uscg.mil

www.epa.gov

www.epa.gov/oar

www.epa.gov/otaq 